\title{
Differente Lernkulturen - regional, national, transnational
}

Olaf Dörner, Carola Iller

Henning Pätzold, Steffi Robak (Hrsg.)

Schriftenreihe der Sektion Erwachsenenbildung der Deutschen Gesellschaft für Erziehungswissenschaft

DGfE Deutsche Gesellschaft 
Schriftenreihe der Sektion

Erwachsenenbildung

in der Deutschen Gesellschaft für

Erziehungswissenschaft (DGfE) 


\section{Olaf Dörner}

Carola Iller

Henning Pätzold

Steffi Robak (Hrsg.)

\section{Differente Lernkulturen -}

regional, national,

transnational

Verlag Barbara Budrich

Opladen • Berlin • Toronto 2016 
Bibliografische Information der Deutschen Nationalbibliothek

Die Deutsche Nationalbibliothek verzeichnet diese Publikation in der Deutschen Nationalbibliografie; detaillierte bibliografische Daten sind im Internet über

http://dnb.d-nb.de abrufbar.

Gedruckt auf säurefreiem und alterungsbeständigem Papier

Alle Rechte vorbehalten

(C) 2016 Verlag Barbara Budrich, Opladen, Berlin \& Toronto

www.budrich-verlag.de

$$
\text { ISBN 978-3-8474-0788-1 (Paperback) }
$$

eISBN 978-3-8474-0911-3

Das Werk einschließlich aller seiner Teile ist urheberrechtlich geschützt. Jede Verwertung außerhalb der engen Grenzen des Urheberrechtsgesetzes ist ohne Zustimmung des Verlages unzulässig und strafbar. Das gilt insbesondere für Vervielfältigungen, Übersetzungen, Mikroverfilmungen und die Einspeicherung und Verarbeitung in elektronischen Systemen.

Umschlaggestaltung: Bettina Lehfeldt, Kleinmachnow - www.lehfeldtgraphic.de Lektorat: Karina Siuda, Berlin

Satz: Judith Henning, Hamburg - www.buchfinken.com 


\section{Inhalt}

Steffi Robak/Olaf Dörner/Carola Iller/Henning Pätzold

Differente Lernkulturen - regional, national, transnational. Eine

Einleitung

Ingeborg Schüßler

Lernkulturen in Transformationsgesellschaften. Paradoxien,

Herausforderungen und Gestaltungsoptionen

\section{Panel I: Lernkulturen und Sozialraum}

Anne Schlüter

Lernkulturwandel über die Herstellung von Transparenz für

Bildungsberatung? Strategien im Rahmen des kommunalen

Bildungsmanagements zur Gestaltung der Bildungsregionen...

Olaf Dörner/Christoph Damm

Lernkulturen im Spannungsfeld von Institutionenkulturen. Das

Beispiel „Alphabetisierung im Stadtteil“ einer städtischen

Volkshochschule

Julia Franz

Regionale Lehr- und Lernkulturen ländlicher Erwachsenenbildung.

Ergebnisse einer explorativ-rekonstruktiven Forschungsarbeit

Klaus Buddeberg/Wibke Riekmann

Wirkt das Bild von Lernkulturen in der Grundbildung als

Teilnahmehemmnis?

Maria Stimm

Lernkulturaspekte im Science Slam. Aufriss der Konzeptualisierung

einer mehrperspektivischen Untersuchung

Daniela Rothe

Lebensgeschichte(n) im Museum. Empirische Lernkulturforschung im

Feld der kulturellen Erwachsenenbildung

Sara Becker/Daniel Otto

Lernkulturen im (Klima-)Wandel. Digital Storytelling zur

Kompetenzvermittlung in interkulturellen Lehr-Lernsettings 101 


\section{Panel II: Träger, Organisationen und Lernkulturen}

Helmar Hanak/Nico Sturm

Annäherung differenter Lernkulturen als Voraussetzung für

Durchlässigkeit

Ewelina Mania/Monika Tröster

Programm- und Angebotsentwicklung in der Finanziellen

Grundbildung als Gestaltungselement einer ,neuen` Lernkultur

Aiga von Hippel

Programmplanungskulturen in der betrieblichen Weiterbildung. Eine

Programmanalyse zu Funktionen betrieblicher

Weiterbildungsprogramme

Steffi Robak/Lisa Marie Lorenz/Eike Asche

Zur Modellierung transnationaler Lernkulturen anhand der

Institutionalisierung von Professionalitäts- und

Organisationsstrukturen

Sabine Schöb/Matthias Rohs/Carmen Biel/Tim Scholze

Professionalisierung von Lehrhandeln in einer digital geprägten

Lernkultur. Bedarf und Ansatzpunkte der Entwicklung einer Online-

Lernumgebung.

\section{Panel III: Lernkulturen in Hochschulen}

Max Reinhardt

Lernkulturwandel. Eine Diskursanalyse der Stellungnahmen von ausgewählten zentralen hochschulpolitischen Akteuren zum

Deutschen Qualifikationsrahmen (DQR)

Rüdiger Rhein

Die Universität als Lernort.

Lisa Freieck/Tatjana Kasatschenko

„Kinder der anderen Kultur“. Zur Bedeutung von Kulturalisierungs-

und Rassismuskritik für die universitäre Lehramtsausbildung

Claudia Lobe/Markus Walber

Innovationen in den Lehr-Lernkulturen an Hochschulen 


\section{Panel IV: Politik, multi-nationale Projekte}

Martin Dust/Henning Marquardt

Kultur als Praxis in europäischen Vergleichsstudien. Kritische

Reflexion eines multilateralen Projekts

Julia Gillen/Marius Herzog

Transnationale Lernkulturen beruflicher Aus- und Weiterbildung in

Deutschland und China am Beispiel handlungsorientierten Lernens

Christian Bernhard

Regionalität von Erwachsenenbildung. Eine qualitative Untersuchung aus Organisationssicht in den Euroregionen Großregion SaarLorLux und Neiße-Nysa-Nisa.

Caroline Euringer

Differente Sichtweisen auf Grundbildung Erwachsener in der

öffentlichen Bildungsverwaltung

Marion Fleige

Zum Zusammenhang von Lernkulturen, Programmen und

Organisationen in der Erwachsenenbildung/Weiterbildung.

Begriffsbestimmungen und Befunde

Autorinnen und Autoren .305 



\section{Differente Lernkulturen - regional, national, transnational. Eine Einleitung}

Der Begriff der „Lernkulturen“ scheint, was die Dichte der Veröffentlichungen betrifft, seinen Zenit überschritten zu haben. Die Ursache dafür könnte vordergründig darin bestehen, dass nicht mehr für ,neue " Lernkulturen argumentiert werden muss, weil sie wahlweise akzeptiert, abgewehrt oder ignoriert werden, in jedem Fall aber nicht mehr diskutiert werden müssten. Gleichwohl sind Akzeptanz, Abwehr und Ignorieren gleichermaßen Formen des Umgangs mit lernkulturellen Veränderungen und Herausforderungen, die auch gegenwärtig von nicht zu unterschätzender Bedeutung für die Erwachsenenbildung sind.

Mit der Dokumentation der Jahrestagung „Differente Lernkulturen: regional, national, transnational“ der Sektion Erwachsenenbildung der Deutschen Gesellschaft für Erziehungswissenschaft, die 2015 von der Abteilung Erwachsenenbildung der Leibniz Universität Hannover ausgerichtet wurde, greifen wir den Diskurs deshalb wieder auf und gehen dabei von Lernkulturen als einem doppelten Konstrukt aus: Einerseits erlaubt er die Analyse und empirische Erschließung gestalteter Programme, Arrangements und Lernmöglichkeiten sowie faktischer, gelebter Lernhandlungen, Bildungs- und Kompetenzentwicklungsprozesse und andererseits bietet der Begriff „Lernkultur" Möglichkeiten der professionellen Gestaltung von Bildungsrealitäten. Dabei zeichnen sich Lernkulturen unserem Verständnis nach einerseits durch erwachsenenpädagogisch gestaltete Binnenstrukturen bzw. Auslegungen von Lernen und Bildung aus. Wir blicken damit z.B. auf träger- und institutionenspezifische Lernkulturen (z.B. Volkshochschulen, Betriebe). Andererseits sind sie in soziokulturelle Milieus eingebettet, ohne als solche bezeichnet sein zu müssen. Vor allem Globalisierungs- und Transnationalisierungsprozesse sind gegenwärtig verbunden mit komplexen systemischen, bildungspolitischen und strukturellen Fragestellungen der Gestaltung und Konzipierung von Bildung und Qualifizierung, sei es für Geflüchtete, Expatriates oder andere Gruppen mit und ohne Migrationsbiographie. Hier gewinnen, neben den Bildungs- und Qualifizierungsaspekten, kulturelle Aspekte der Gestaltung von Lernkulturen eine neue Bedeutung.

Betrachtet man Lernkulturen als Bindeglied zwischen gesellschaftlichen Entwicklungen, Lernbedarfen und -bedürfnissen, den organisationalen und professionellen Gestaltungsanforderungen in den (Weiterbildungs)institutionen und den individuellen Orientierungen Lernender, so zeigt sich eine Vielfalt an aktuellen Forschungs- und Gestaltungsherausforderungen. 
Inwieweit hat die disziplinspezifische Beschäftigung mit Lernkulturen Impulse für die Erwachsenenbildung geliefert? Eine Debatte zu Lernkulturen in der Erwachsenen- und Weiterbildung startete in den 1990er Jahren (vgl. Arnold/Schüßler 1998; Pätzold/Lang 1999). Rückblickend betrachtet kann sie als wichtiger Vorstoß gewertet werden, in dem bestimmte, bis dahin getrennt verlaufende Diskursstränge miteinander verbunden und so neue Gestaltungsoptionen, Forschungsfragestellungen und Systematisierungen erschlossen wurden. So ist es nicht mehr ungewöhnlich, beispielsweise bildungspolitische Debatten durch lerntheoretische Empirie zu befruchten oder erwachsenenpädagogische Organisationsforschung unter der Perspektive von Teilnehmendenmilieus zu betreiben. Insbesondere die Lehr-/Lernforschung, die Didaktik, die Erforschung von erwachsenenpädagogischen Institutionen und Organisationen sowie deren professionelle Gestaltungsanforderungen haben neue Impulse, aber auch neue Anwendungsfelder erhalten. Auch die Teilnehmenden und deren Anteil an der Ausformung von Lernkulturen rücken wieder ins Blickfeld. Dabei verweisen die Entwicklungen um den Begriff auf im programmatischen und empirischen Sinne grundlegende Funktionsveränderungen von Bildung und Kompetenzentwicklung, die Auswirkungen auf Institutionen, pädagogisches Handeln sowie Forschungsperspektiven haben.

Der Begriff der Lernkulturen kann somit als Diskurs-, Analyse- und Gestaltungskategorie genutzt werden. Rückblickend lassen sich in der Entwicklung im deutschsprachigen Raum fünf große Entwicklungsabschnitte unterscheiden (siehe dazu umfassend Fleige/Robak 2016 und Schüßler in diesem Band):

1. Faktische Veränderungen der Lehr-Lernformen in Organisationen der Erwachsenen- und Weiterbildung in den 1970er und 80er Jahren: Hier sind Veränderungen in den Zugängen und Lernformen zu nennen, die mit der gesellschaftlichen Formierung der „,neuen sozialen Bewegungen“ (Frieden, Ökologie, Gleichberechtigung u.a.m.) einhergingen (vgl. Heuer u.a. 2001), aber auch mit Veränderungen im Kontext der „Humanisierung der Arbeitswelt" (vgl. Vetter 1973: 6). Bei den Lernformen im Betrieb wurden etwa neue Formen des arbeitsplatzbezogenen Lernens entwickelt (vgl. Dehnbostel 2008), in den „,neuen sozialen Bewegungen“ u.a. partizipative Lernformen.

2. Bildungspolitische Gestaltungs(an-)forderungen in den 1990er Jahren: Bildungspolitische Intentionen der Gestaltung und Durchsetzung von Lernkulturen flankieren umfassende gesellschaftliche Veränderungen. Lernkulturen sollten Teil dieser Veränderungen werden. Anpassungen, gerade vor dem Hintergrund der Wiedervereinigung Deutschlands, sollen über sozialisatorisches Lernen und Kompetenzentwicklung Beiträge $\mathrm{zu}$ einer gesellschaftlichen Transformation leisten (Erpenbeck/Weinberg 1993). 
3. Diskurs und Entwicklungsforschung zu ,neuen Lernkulturen “: Insbesondere seit Mitte der 1990er Jahre finden sich Auslegungen „,neuer Lernkulturen“ im Diskurs und in konzeptionellen Ansätzen des selbstorganisierten und selbstgesteuerten Lernens (Dietrich 2000; Forneck 2003) sowie in Ansätzen des digitalen bzw. medialen Lernens (z.B. Kerres/ Gorhan 1998). Es folgten mikrodidaktische Neuorientierungen, die bereits systematisiert wurden (Schüßler/Thurnes 2005). Modernisierungstheoretische und lerntheoretische Begründungen, hier sind insbesondere konstruktivistische Auslegungen zu nennen (Arnold/Schüßler 1998), strukturieren die verschiedenen Lesarten von Lernkultur. Schüßler und Thurnes (2005) rücken stärker Rahmungen für Gestaltungsprozesse in den Blick und berücksichtigen auch die Unterschiede für den öffentlichen Bereich der Erwachsenen- und Weiterbildung sowie den betrieblichen Bereich.

4. Analysen zu Lernkulturen auf verschiedenen didaktischen Handlungsebenen: Aktuelle Beiträge fokussieren Lernkulturen auf verschiedenen didaktischen Handlungsebenen und in spezifischen Institutionalformen (Fleige 2011; Schicke 2012). Es ergeben sich differenzierte Einsichten in die professionell gestalteten und gelebten Realitäten des Lehrens und Lernens (Gieseke/Robak/Wu 2009; Robak 2012) sowie Perspektiven der Verschränkung von Lern- und Organisationskulturen (Dewe/Schwarz 2012).

5. Neuere Stränge der Erforschung von Lernkulturen: Dazu gehören theorieentwickelnde Auslegungen, vor allem im Bereich von Lehr-Lernarrangements und Lernformen (vgl. Klingovsky 2009) und auch informellem Lernen (Fahrenwald 2011). Weiterhin wird zunehmend die Raumkategorie für Betrachtungen der Realisierung von Angeboten, Programmen und Lernformen im weiteren Sinne herangezogen und nach erweiterten Zugängen der Bildungspartizipation gefragt (vgl. Bernhard u.a. 2015) und es werden Regionen gezielt in den Blick genommen (vgl. den Beitrag von Bernhard in diesem Band).

Bei alledem ist der Lernkulturbegriff mit einem allgemeineren Verständnis von Kultur verbunden, das ebenfalls im Wandel ist. Nicht selten wurde in früheren Arbeiten wie selbstverständlich der Kontext eines national geprägten Bildungssystems vorausgesetzt, innerhalb dessen Lernkulturen weiter entwickelt werden können, etwa solche des selbstgesteuerten oder des medialen Lernens. Mit den gegenwärtigen gesellschaftlichen Veränderungen (demografischer Wandel, Europäisierung und Transnationalisierung usw.) werden Gestaltung und institutionelle Rahmung von Erwachsenenlernen komplexer und es wird offensichtlich, dass eine nationale Systemperspektive zu kurz greift. Kulturelle Ausformungen von Lernkulturen können Regionalspezifika aufweisen, können sich in transnationalen Zusammenhängen im 
Spannungsfeld von Diversifizierung und Standardisierung entwickeln und durch verschiedenste Auslegungen kultureller Differenz geprägt sein.

Diesbezüglich fordern das Paradigma der Interkulturalität und jüngere Auslegungen von Transkulturalität dazu heraus, über deren Bedeutung für Lernkulturen nachzudenken sowie Lern- und Bildungsmöglichkeiten zu erforschen. Der Begriff der Transkulturalität wurde von Welsch (1988, 2005) geprägt und grenzt sich bewusst vom Begriff der Interkulturalität ab. Er geht davon aus, dass abgegrenzte Einzelkulturen nicht mehr existieren, sondern Kulturen längst Produkte von Vermischungen und Verschmelzungen sind, die Elemente, Merkmale und Praktiken verschiedener vermeintlich trennbarer Ausgangskulturen in sich binden und neu platzieren. Für die Gestaltung von Lernkulturen bedeutet das, dass die Präsenz von Fremdheit und Differenzerfahrung innerhalb eines transkulturell geteilten Raums der Regelfall ist. Bildungsangebote, die dem Transkulturalitätsansatz folgen, thematisieren oder bearbeiten z.B. Anforderungen der Identitätsentwicklung im Kontext verschiedener kultureller Zugehörigkeiten und unterstützen in vielfältigen Angebotsformen die Erarbeitung und Reflexion von (kulturdifferenten) Deutungen, Wahrnehmungsformen und Möglichkeiten individueller Bedeutungsproduktion. Sie thematisieren Wirkungsweisen kultureller Differenz dabei nicht direkt, sie stellen die Individuen ins Zentrum, in der Annahme, dass alle über Potenziale transkultureller Prägungen verfügen, die kulturelle Neuformungen bewirken können. Was dies für Lernkulturgestaltungen und die in ihr möglichen Lern- und Bildungsprozesse bedeutet, ist weiterführend zu erforschen.

Auf der Jahrestagung 2015 wurden diese und weitere Diskursstränge und Forschungsoptionen aufgenommen und mit Blick auf aktuelle Herausforderungen und gesellschaftliche Problemlagen empirische, theoretische und methodologische Implikationen für die Erwachsenenbildungsforschung diskutiert. Die doppelte Perspektive der Gestaltung und Analyse von Lernkulturen eröffnet auch verschiedene Aspekte und Perspektiven der Erforschung von Lernkulturen, die in diesem Band versammelt sind. In ihnen bestätigt sich, dass das Konzept Lernkultur weiterhin das Potenzial hat, erwachsenenpädagogisch relevante Teildiskurse zusammenzuführen, für die Gestaltung von Bildungsangeboten $\mathrm{zu}$ erschließen und darüber hinausgehende Forschung anzuregen.

\section{Literatur}

Arnold, R./Schüßler, I. (1998): Wandel der Lernkulturen. Ideen und Bausteine für ein lebendiges Lernen. Darmstadt.

Bernhard, C./Kraus, K./Schreiber-Barsch, S./Stang, R. (Hrsg.): Erwachsenenbildung und Raum: theoretische Perspektiven - professionelles Handeln - Rahmungen des Lernens. Bielefeld 2015. 
Dehnbostel, Peter (2008): Berufliche Weiterbildung. Grundlagen aus arbeitnehmerorientierter Sicht. Berlin.

Dewe, B./Schwarz, M. (2012): Lernen in Organisationen: individuell-intentionale Lernprozesse und ,Eintritt" in organisationale Wissens- und Lernkultur. In: von Felden, H./Hof, C./Schmidt-Lauff, S. (Hrsg.): Erwachsenenbildung und Lernen. Baltmannsweiler, S. 243-254.

Dietrich, S. (2000): Selbstgesteuertes Lernen im institutionellen Kontext - Entwicklung einer neuen Lernkultur. In: Dokumentation: 2. Konstanzer Lehr- und Lerntage. Konstanz: Förderkreis Wirtschaft im Landkreis Konstanz, S. 63-69.

Erpenbeck, J./Weinberg, J. (1993): Menschenbild und Menschenbildung. Münster, New York.

Fahrenwald, C. (2011): Erzählen im Kontext neuer Lernkulturen. Eine bildungstheoretische Analyse im Spannungsfeld von Bildung, Lernen und Subjekt. Wiesbaden.

Fleige, M. (2011): Lernkulturen in der öffentlichen Erwachsenenbildung. Theorieentwickelnde und empirische Betrachtungen am Beispiel evangelischer Träger. Münster u.a.

Fleige, M./Robak, S. (2016): Lehr-Lernkulturen in der Erwachsenenbildung/Weiterbildung. Erscheint in: Tippelt, R./Hippel, A. von (Hrsg.): Handbuch Erwachsenenbildung/Weiterbildung. Frankfurt a.M.

Forneck, H. J. (2003): Selbstlernarchitekturen, Lernprozesssteuerung und individualisiertes Lernen. In: Gary, C. (Hrsg.): Erwachsenenbildung im Wandel. Theoretische Aspekte und Praxiserfahrungen zu Individualisierung und Selbststeuerung. Wien: ÖIBF, S. 161-167.

Gieseke, W./Robak, S./ Wu, M.L. (2009): Transkulturelle Perspektiven auf Kulturen des Lernens. Transcript: Bielefeld.

Heuer, U./Botzat, T./Meisel, K. (Hrsg.) (2001): Neue Lehr- und Lernkulturen in der Weiterbildung. Bielefeld.

Kerres, M./Gorhan, E. (1998): Multimediale und telemediale Lernangebote. In: Baethge, M. u.a. (Hrsg.): Kompetenzentwicklung '98. Forschungsstand und Forschungsperspektiven. Münster u.a., S. 143-164.

Klingovsky, U. (2009): Schöne Neue Lernkultur. Transformationen der Macht in der Weiterbildung. Bielefeld.

Pätzold, G./Lang, M.: Lernkulturen im Wandel. Didaktische Konzepte für eine wissensbasierte Organisation. Bielefeld 1999.

Robak, S. (2012): Kulturelle Formationen des Lernens. Zum Lernen deutscher Expatriates in kulturdifferenten Arbeitskontexten in China - die versäumte Weiterbildung. Habilitationsschrift. Münster/New York/München/Berlin: Waxmann.

Schicke, H. (2012): Organisation als Kontext der Professionalität: Beruflichkeit pädagogischer Arbeit in der Transformationsgesellschaft. Bielefeld: Bertelsmann.

Schüßler, I./Thurnes, C. M. (2005): Lernkulturen in der Weiterbildung. Bielefeld.

Welsch, W. (1988): Wege aus der Moderne. Schlüsseltexte der Postmoderne-Diskussion. Weinheim.

Welsch, W. (2005): Auf dem Weg zu transkulturellen Gesellschaften. In: Allolio-Näcke, L./Kalscheurer, B./Manzeschke, A. (Hrsg.): Differenzen anders denken. Bausteine zu einer Kulturtheorie der Transdifferenz. Frankfurt a.M., S. 314-341.

Vetter, H. O. (1973): Humanisierung der Arbeitswelt als gewerkschaftliche Aufgabe. Gewerkschaftliche Monatshefte 1, S. 1-13. 

Ingeborg Schüßler

\section{Lernkulturen in Transformationsgesellschaften. Paradoxien, Herausforderungen und Gestaltungsoptionen ${ }^{1}$}

\section{Einleitung}

Der folgende Beitrag geht von der These aus, dass wir in einer Transformationsgesellschaft leben, deren Veränderungsdynamik durch ein mehr oder weniger intendiertes Zusammenspiel ökonomischer, politischer, wirtschaftlicher und kultureller Faktoren gesteuert wird. Gesellschaftliche Transformationsprozesse sind nicht einfach durch sozialen Wandel gekennzeichnet, sondern im Vordergrund stehen gezielte Anstöße, z.B. seitens Politik und Wirtschaft, und eine absehbare, durch bestimmte Medien beziehungsweise Organisationen und Institutionen getragene Entwicklung. Hinzu kommt eine Plan- und Steuerbarkeit im Hinblick auf bestimmte Ziele der erwünschten Transformationen (vgl. Universallexikon 2013, Reißig 2009). Für den Bildungsbereich wird dies durch spezifische Förder- und Forschungsprogramme deutlich, die aus Land- oder Bundesmitteln oder dem ESF finanziert werden und damit bestimmte Wandlungsimpulse auch im Bereich der Lernkultur setzen.

So steht das Konzept Lernkultur in einem direkten Zusammenhang mit dem Diskurs über die Vorstellungen einer modernen, globalisierten Welt, die von den Menschen insbesondere Selbstorganisationsfähigkeiten sowohl im beruflichen wie privaten Bereich erfordert (vgl. Veith 2003; Ricken 2011: 15). Zudem bewegt sich die Diskussion um neue Lernkulturen im selben diskursiven Kontext, auf den sich auch das Bologna-Abkommen und weitere bildungspolitische Reformprogramme beziehen. Auf dieser Basis werden für die Gestaltung von Lernprozessen konkrete Veränderungen abgeleitet und mit Elementen einer kompetenzorientierten Didaktik verknüpft. Es ist also interessant, diesen Transformationsprozessen etwas kritisch nachzugehen und $\mathrm{zu}$ analysieren, was das für Erwachsenenbildung/Weiterbildung (EB/WB) und insbesondere deren Lernkulturen bedeutet und welche Paradoxien, Herausforderungen und Gestaltungsoptionen sich hier zeigen.

In einem ersten Schritt wird versucht, den Diskurs zu Lernkulturen in der EB/WB historisch nachzuzeichnen, um vor allem die Stoßrichtungen, mit

Der Beitrag ist die gekürzte Fassung der Abschlusskeynote der Sektionstagung 2015. 
denen das Thema diskutiert wurde, aber auch die daraus entstandenen Paradoxien und Herausforderungen aufzuzeigen.

In einem zweiten Schritt wird der Frage nachgegangen, wie sich Lernkulturen weiterentwickeln müssten in einer Gesellschaft, in der vielfältige Transformationsprozesse nicht die Ausnahme, sondern die Regel sind, und welche Fragen sich daraus für die Forschung ableiten.

\section{Lernkulturen und ihre Diskurse in der Erwachsenenbildung}

Schaut man genauer hin, könnte man meinen, dass der Diskurs zu diesem Thema seinen Höhepunkt überschritten hat, zumindest deutet die Publikationsrate darauf hin. Dieses Ergebnis bringt eine Auswertung der Veröffentlichungen zu diesem Thema in der Literaturdatenbank fis Bildung (Stand 9/2015).

Dazu wurde über die Freitextsuche nach den Themen Lernkultur/Lernkulturen mit einem besonderen Blick auf EB/WB recherchiert. Das Ergebnis zeigt, dass die meisten Veröffentlichungen zwischen Ende 1990 und 2010, vor allem in den Jahren 2005-2009, erschienen sind. In den letzten fünf Jahren hat sich die Publikationswelle zu diesem Thema merklich beruhigt und zeigt, dass der Diskurs seinen Höhepunkt überschritten hat. Anhand der Veröffentlichungen lässt sich auch die Historie des Diskurses gut nachzeichnen, der vor allem auch in der schulischen und beruflichen Bildung (z.B. Pätzold/Lang 1999) geführt wurde. Neben dem Buch „Wandel der Lernkulturen“ (Arnold/Schüßler 1998) war es vor allem der Literatur- und Forschungsreport, der das Thema „Neue Lernkulturen“ (Report 44/1999) für die EB/ WB aufgriff. Im gleichen Jahr gab Schlutz den Band zu Lernkulturen heraus, in dem es um den Preis für Innovation in der Erwachsenenbildung ging, den das DIE seit 1997 alle zwei Jahre auslobt und dessen Impuls auf innovative Lernkulturen zielt.

Schon damals wurde die Gleichzeitigkeit der Ungleichzeitigkeit deutlich, d.h. innovative wie auch eher traditionelle Lernkulturen existieren zeitgleich nebeneinander, worauf auch Weinberg (1999) deutlich hinwies. Er erinnerte zudem daran, Lernkulturen nicht nur auf der mikrodidaktischen Ebene zu untersuchen, sondern auch in ihren makro- und mesodidaktischen Zusammenhängen zu beleuchten und damit die gesellschaftlich-historische Kontextualisierung von Weiterbildungsorganisationen stärker in den Blick zu nehmen. Ein Aspekt, dem Fleige (2011) in ihrer Studie zu Lernkulturen in der öffentlichen Erwachsenenbildung am Beispiel evangelischer Träger nachgegangen ist.

Das DIE griff zu dieser Zeit ebenfalls das Thema auf. Neben Workshops und Forschungsprojekten zum Thema entstand in diesem Zuge auch eine 
Reihe von Veröffentlichungen (Heuer/Botzat/Meisel 2001; Dietrich 2001, 2004; Dietrich/Herr 2005; Schüßler/Thurnes 2005). Es ging hier vor allem um einen Perspektivenwechsel vom Lehren zum Lernen, eine - man könnte fast sagen - zweite reflexive Wende. Angestoßen wurde diese Diskussion u.a. durch die lerntheoretische Forschung, insb. die Rezeption der systemisch-konstruktivistischen (z.B. Arnold 2007, Siebert 2009) sowie subjektwissenschaftlichen Lerntheorie (z.B. Ludwig 2005; Faulstich/Ludwig 2004), die damit verknüpfte Bedeutungszunahme des selbstgesteuerten Lernens und den daraus begründeten Wandel der Lehrerrolle hin zum Lernberater. Dazwischen gab es eine Reihe weiterer Veröffentlichungen zum Thema, die auch bereits spezifische Fokusse zu regionalen Lernkulturen, der Bedeutung von Lernberatung, E-Learning etc. setzten, meist verknüpft mit dem Wörtchen „,neu“, da es im Rahmen bildungspolitischer Programme (z.B. Forum Bildung 2001) vorrangig um die Innovation von Bildungseinrichtungen ging.

Wurde diese Entwicklung bereits stark von Wissenschaft und Praxis der EB/WB forciert, gibt es noch eine zweite Entwicklungslinie zu diesem Thema, die ihren Ursprung in den 1990er Jahren durch die Transformationsprozesse im Osten nach dem Mauerfall nahm. Hier setzte die Bildungspolitik zentrale Akzente durch das von 2001 bis 2007 mit Mitteln des BMBF und ESF geförderte Programm „Lernkultur Kompetenzentwicklung”, das durch entsprechende Programme wie z.B. „Kompetenzentwicklung für den wirtschaftlichen Wandel - Strukturveränderungen betrieblicher Weiterbildung“ vorbereitet wurde. Es handelte sich dabei nicht nur um Forschung, sondern vor allem um die konkrete Umsetzung in die Praxis bzw. deren Umbau nach folgender Zielsetzung: „Es soll dem Aufbau effizienter beruflicher Lernstrukturen als Motor für gesellschaftliche Innovation und Wettbewerbsfähigkeit dienen. Zugleich soll es die Stärkung von individueller beruflicher Kompetenz, von Beschäftigungsfähigkeit, Flexibilität und Unternehmergeist fördern" (Programmflyer).

In den geförderten Projekten ging es ebenso um das selbstorganisierte Lernen, ob im Unternehmen, in der Weiterbildung oder im sozialen Umfeld. Die Fokusse waren indes vor allem auf die Modellierung, Messung und Bilanzierung von Kompetenzen, einen Wandel von der Input- zur Outputorientierung und den Ausbau neuer Lerndienstleistungen gerichtet. In diesem Zusammenhang wurde der Begriff der Weiterbildung abgelehnt, da er mit Qualifizierung gleichgesetzt wurde. Man betonte gegenüber dem Qualifikationsbegriff die Wertedimension und den Subjektbezug bei den Kompetenzen. Mit der bekannten Reihe „Kompetenzentwicklung“ im Waxmann-Verlag, die von 1996 bis 2006 fast jährlich erschien, wurde somit auch ein begrifflicher Umbau forciert. Die anfangs geäußerte Kritik (vgl. Arnold 1997) verblasste aber mit den Jahren.

Bedeutsam war in diesem Diskurs sicherlich die Betonung des informellen gegenüber dem formalen Lernen (z.B. Wittwer/ Kirchhof 2003). Begrün- 
det wurde dies damit, dass Erwachsene ohnehin das meiste außerhalb von formalisierten Bildungsprozessen lernen, weshalb dieses stärker gefördert und anerkannt werden müsse. Auch die EB/WB war sich des Lernens en passant schon davor bewusst (vgl. Reischmann 1995). Der Unterschied mag darin liegen, dass diese den privaten und Freizeitbereich als unverfügbar betrachtete und nur darauf Bezug nahm, wenn sich daraus Suchbewegungen im Lernprozess - im Sinne eines erfahrungsorientierten Lernens - ergaben. Der Kompetenzdiskurs hingegen versuchte mit dem Feld des informellen Lernens und der Formel vom Lebenslangen Lernen auch noch diese Enklave der Selbstbestimmung für das Transformationsvorhaben einzufangen und das informelle gegenüber dem formalen Lernen aufzuwerten. Veröffentlichungen wie z.B. „Weiterbildung: Ein Mythos zerbricht“ (Staudt/Kriegsmann 2000) stellten zudem den Nutzen formalisierter Weiterbildung zusätzlich in Frage.

Wie angedeutet, zeigt sich allerdings in dem vor allem in der beruflichen Weiterbildung geführten Diskurs eine andere Stoßrichtung als in den Arbeiten der EB/WB. So lässt sich in dem Programmvorhaben klar die Handschrift der europäischen Bildungspolitik und des 2000 verabschiedeten LissabonProgramms erkennen, in dem die EU sich das Ziel setzte, die Gemeinschaft bis 2010 zum wettbewerbsfähigsten und dynamischsten wissensgestützten Wirtschaftsraum der Welt zu machen. Dieses politische Programm scheint zudem nur eine Richtung zu kennen: Wachstum und Innovation. Das zeigt auch das Nachfolgeprogramm Europa 2020, hier gilt als Ziel ein ,intelligentes, nachhaltiges und integratives Wachstum" mit einer besseren Koordinierung der nationalen und europäischen Wirtschaft. Wirtschaftliches Wachstum und ein schnellerer, auf freien Märkten basierender Innovationszyklus werden mit Prosperität und wiederum mit sozialer Stabilität assoziiert.

Erinnert sei hier auch an die Kritik, die mit den Bestrebungen zum selbstgesteuerten oder auch selbstorganisierten Lernen aufkam und die zeigt, dass es hier um zwei unterschiedliche Diskurse zum darauf bezogenen Wandel der Lernkulturen im Zuge lebenslangen Lernens ging. Die Zielsetzungen eines selbstgesteuerten und lebenslangen Lernens stießen zunächst auf weitgehende Zustimmung in der EB/WB, weil sie sich mit dem Bildungsverständnis eines autonomen, selbstverantwortlichen Subjekts vereinbaren lassen. Nachdem allerdings das Konzept des Lebenslangen Lernens zunehmend stärker in den Dienst der vor allem seitens der Bildungspolitik und der Betriebe geforderten Employability gestellt wurde, meldete sich zunehmend ein Unbehagen auf Seiten der EB/WB. Im Kontext neoliberaler Regierungen werde der Lerner zum Entrepreneur seines eigenen Lebens konfiguriert und sein Lernen diene vor allem der Selbstökonomisierung, so die Kritik (vgl. Forneck 2005; Pongratz 2005).

Mit dieser Verbetrieblichung der alltäglichen Lebensführung löst sich zudem die Trennung von Arbeit und Freizeit sukzessive auf. Dies führt zum einen zu den zunehmenden Belastungsphänomenen wie Depression oder gar 
Burnout, zum anderen zu Selbstoptimierungsprozessen, die durch die umfassende Computerisierung noch forciert werden. Zwar schafft diese Work-LifeIntegration auch Chancen, selbständig und eigenverantwortlich die eigene Employability zu beeinflussen, allerdings setzt dies einen mündigen und emanzipierten Menschen voraus sowie eine Lernkultur ,digitaler Mündigkeit“ (Kurz/Rieger 2011).

Ohne auf diese Entwicklungen weiter eingehen zu können, wird dennoch deutlich, wie bildungspolitische Entscheidungen und Gesetzgebungen auch indirekt eine Lernkultur mit beeinflussen können. Denn mit den Empfehlungen oder Erklärungen der Bildungspolitik sind mögliche Gestaltungsintentionen des Weiterbildungssektors verknüpft, die kurz- oder auch mittelfristig über die Gesetzgebung ganz konkrete praktische Relevanz erhalten und den Rahmen für die Entwicklung von Lernkulturen abstecken, teilweise mit positiven Ergebnissen, teilweise auch mit negativen Vorzeichen.

Dabei bildet die bestehende Lernkultur in den Einrichtungen einen Filter, von dessen Durchlässigkeit die Sensibilität im Umgang mit internen und externen Anforderungen abhängt und der letztlich bestimmt, welche Handlungsalternativen als richtig oder falsch gelten. Insbesondere in Transformationsprozessen, die von Unsicherheit, Komplexität und Unübersichtlichkeit geprägt sind, greifen Menschen auf ihre handlungsleitenden Wertvorstellungen zurück. Dazu zählen auch die in einer Lernkultur entwickelten Wahrnehmungs- und Verhaltensmuster. Für Bildungseinrichtungen ist das so lange kein Problem, solange das normative Gerüst dieser Lernkultur mit den Umfeldbedingungen harmonisiert. Allerdings zeigt sich, dass die Transformationsprozesse, mit denen wir es heute zu tun haben, mit den Problemlösungen und den Lernroutinen von gestern nicht mehr adäquat zu bewältigen sind. Die Frage stellt sich somit, wie sich Lernkulturen angesichts der Herausforderungen unserer Transformationsgesellschaften wandeln müssten.

\section{Lernkulturen in Transformationsgesellschaften - Mögliche Gestaltungsoptionen}

Erinnert sei in diesem Zusammenhang an die von Ortfried Schäffter differenzierten Transformationsmodelle, um deutlich zu machen, vor welchen Herausforderungen Bildungseinrichtungen und ihre Lernkulturentwicklung stehen.

Schäffter (2001: $17 \mathrm{ff}$.) differenziert zwischen zielbestimmten und zieloffenen Transformationen, wobei die Ausgangslagen entweder bekannt oder unbekannt sein können. Daraus leitet er vier unterschiedliche Transformationsmodelle mit jeweils unterschiedlichen Transformationslogiken ab: 
Ingeborg Schüßler

\begin{tabular}{llll}
\hline Ausgangslage & Zielwert & Didaktisches Modell & Transformationsmodell \\
\hline bekannt & bekannt & Qualifizierungs-Modell & Lineare Transformation \\
\hline unbekannt & bekannt & Aufklärungs-Modell & $\begin{array}{l}\text { Zielbestimmte } \\
\text { Transformation }\end{array}$ \\
\hline \multirow{2}{*}{ bekannt } & unbekannt & Suchbewegungs- & Zieloffene \\
& & Modell & Transformation \\
& & & \\
\hline unbekannt & unbekannt & Selbstvergewisserungs- & $\begin{array}{l}\text { Reflexive } \\
\text { Transformation }\end{array}$ \\
& & Modell & R
\end{tabular}

Abb. 1: Vier Modelle struktureller Transformation (in Anlehnung an Schäffter 2001:17ff.)

Ohne hier näher auf die einzelnen Transformationsmodelle eingehen zu können, zeigt sich, dass wir es heute zunehmend mit den im vierten Modell beschriebenen Herausforderungen zu tun haben. Während in den ersten drei Modellen davon ausgegangen wird, dass durch Lernen das Moment des Unbestimmten wieder ,,in den Griff“ zu bekommen ist und man eine Orientierungssicherheit zurückgewinnt, ist diese prinzipielle Bestimmbarkeit in unserer heutigen Transformationsgesellschaft selbst fragwürdig geworden (vgl. Reißig 2009). Genau daraus leitet sich das vierte Modell ab, in dem Ausgang wie auch Ergebnis von Veränderungsprozessen unbestimmbar geworden sind, wie dies auch als Kennzeichen reflexiver Moderne beschrieben wird (vgl. Beck/Giddens/Lash 1996). Solche Phasen fordern letztlich eine permanente Selbstvergewisserung durch eine reflexive Haltung. Schäffter (2001: 28) spricht hier von einer ,unabschließbaren Iteration permanenter Veränderungen“, die letztlich nur durch Distanzierung von diesem Veränderungsdruck und einen Widerstand gegenüber blinden Beschleunigungsmustern zu bewältigen sei. Für die „Institutionalisierung von Dauerreflexion“ - wie er es nennt - schlägt er solche Angebote wie Supervision, kollegiale Praxisberatung, Qualitätszirkel oder Selbsthilfegruppen vor. Was heißt das dann aber für die Erwachsenenbildung? Bleibt ihr dann nur noch die Aufgabe der Moderation solcher Reflexionsangebote? Welche Aufgaben haben dann Bildungseinrichtungen? Welche Lernkulturen würden eine solche reflexive Haltung fördern? Lässt sich eine solche Dauerreflexion überhaupt kultivieren, ist sie alltagspraktisch? Welche Forschungsfragen leiten sich daraus ab?

Die angesprochene Distanzierung hat viel mit den Formen eines „Deutungslernens" (vgl. Schüßler 2000) bzw. „transformativen Lernens“" (vgl. Mezirow 1997; Illeris 2014) gemein. Es geht dabei um die Bewusstmachung und Transformation der hinter den Handlungen liegenden Deutungs- und Emotionsmuster. Diese sind immer in Bildungsveranstaltungen virulent und 
wirken als Motiv wie auch Filter von Lernanstrengungen. Tietgens (1992: 10) sprach daher auch von einem Lehren und Lernen im Modus der Auslegung, bei dem man sich um die „Kommunikation von Deutungssystemen“ bemühen müsse. Und die erwachsenenpädagogische Aufgabe bestünde dann darin, „Vermittlungsversuche einzuleiten bzw. zu unterstützen, die zu Offenheit und zu Differenzierung der Deutungsmuster führen“ (ebd.: 19).

Empirische Untersuchungen zum nachhaltigen Lernen (vgl. Schüßler 2007) zeigen aber, dass durch Wissensvermittlung, Aufklärung oder einen rationalen Diskurs, wie ihn Jack Mezirow im Konzept des transformativen Lernens vorschlägt, sich die tief verwurzelten Überzeugungen, Vorurteile oder auch Ängste kaum auflösen lassen. Auch ist eine „Dauerreflexion“ wie sie Schäffter fordert - kaum umsetzbar und auch gar nicht gewünscht. Denn angesichts der steigenden Veränderungsdynamik, des unaufhaltsamen Einströmens von Informationen, der Multioptionalität und einer Dauerverfügbarkeit durch die neuen Medien sehnen sich viele Menschen heute zunehmend nach Orten der Ruhe und Muße. Es geht auch hier um eine Distanz zum Alltag, aber auch um ein Nicht-Denken, Nicht-Wissen, Nicht-Gefordertsein. Das erklärt, warum neben der EB/WB sich ein wachsender Bereich spiritueller Angebote entwickelt.

In unserer Kultur haben sich vor allem die Religionen dieser Frage angenommen, aber den Menschen geht es immer weniger um religiöse Riten, sondern vielmehr um eine alltagspraktische Spiritualität. Dabei suchen sie seriöse Angebote jenseits von Esoterik und New Age. Wenn Schäffter mit Blick auf die Transformationsherausforderungen daher die Aufgabe der Erwachsenenbildung darin sieht, „Möglichkeiten der Selbstvergewisserung und der Wiedergewinnung von Erlebnisfähigkeit für die Gegenwart" (Schäffter 2001: 28) bereitzustellen, um dadurch auch zu einer ,gesellschaftlichen Entschleunigung beizutragen“, dann liegt genau darin die Herausforderung für unsere Lernkulturen der Zukunft. Und wir müssen uns fragen, ob die aktuellen Lernkulturen eines propagierten Lebenslangen Lernens mit ihrer Outputorientierung und einem Accelerated Learning dafür den richtigen Weg weisen oder nicht vielmehr einen weiteren Beitrag zu unserer heutigen „erschöpften Gesellschaft" (Grünewald 2013) leisten.

Wir wissen mittlerweile durch empirische Studien (vgl. z.B. Ott 2010; Kabat Zinn 2013), dass die Kultivierung von Achtsamkeit im Alltag eine positive Wirkung auf die psychische und physische Gesundheit hat. Gefördert wird dadurch eine nicht wertende und akzeptierende Haltung gegenüber jeder Art von Stress im Alltag. Das steigende Stressempfinden in unserer Gesellschaft spiegelt sich in zunehmenden Burnout- und depressiven Erkrankungen und interessanterweise sind Arbeitslose besonders davon betroffen (vgl. Gesundheitsreport der Techniker Krankenkasse 2015). Es hat somit nicht nur etwas mit der Arbeitsverdichtung zu tun, sondern auch mit unserer Arbeits- und Leistungskultur und den damit verknüpften Bewertungsmustern. 
Die Achtsamkeitspraxis kultiviert nicht nur ein größeres Maß an Gelassenheit gegenüber den steigenden Anforderungen und der Unbestimmbarkeit des Alltags, sondern fördert auch ein Wachstum an Empathie und Zufriedenheit, wie wissenschaftliche Studien an Menschen mit Meditationspraxis zeigen (vgl. Singer/Bolz 2013). Es geht dabei nicht darum, nun alle Menschen zu Meditierenden auszubilden, sondern darum, ein größeres Gewahrsein, sprich nicht urteilendes Bewusstsein gegenüber dem gegenwärtigen Augenblick, in dem man lebt, zu entwickeln. Dadurch wird die Anhaftung an routinierte Handlungsmuster gelockert und die von Tietgens geforderte Offenheit und Differenzierung der Emotions- und Deutungsmuster gefördert. Das schafft im Alltag größere Denk- und Handlungsfreiheit und gleichzeitig eine vorurteilsfreiere, weil differenziertere und damit zugleich kritischere Wahrnehmung des gegenwärtigen Augenblicks. Scharmer (2011) nennt das „Listening by downloading“. Ich beobachte mich selbst dabei, wenn ich meine routinierten Denkmuster „downloade“: Diesen Prozess nennt er „Presencing“, eine Wortschöpfung aus den Wörtern ,presence“ (Gegenwart bzw. Anwesenheit) und „sensing“ (Hinspüren). Erst durch diese Achtsamkeitspraxis gewinne ich eine differenziertere Deutungskompetenz. Es ist eine Haltung des Staunens, des Wahrnehmens ohne Bewertung und sofortige Einordnung. Erwachsenenbildung könnte ein Ort sein, an dem diese Achtsamkeitspraxis kultiviert und damit den Menschen ein professioneller Rahmen für ihre Suchbewegungen nach Sinn bereitgestellt wird. Solche „Lernkulturen der Achtsamkeit" wären zugleich eine wichtige Voraussetzung zur Entwicklung von Selbstbestimmungs-, Mitbestimmungs- und Solidaritätsfähigkeit (vgl. Klafki 1993: 52): ein Aspekt, der in der gegenwärtigen kompetenzlastigen Diskussion fast schon in Vergessenheit geraten ist. Zugleich ist diese Achtsamkeit als ein „Wissen-in-Praxis“ im Rahmen hochtechnologischer und komplexer Arbeitsprozesse, die ein Handeln in eher unbekannten und unplanbaren Zusammenhängen erfordern, ebenso bedeutsam, wie das die Studie von Langemeyer (2015) zeigt. Um eine Lernkultur der Achtsamkeit zu etablieren, braucht es allerdings auch Erwachsenenbildner/innen, die selbst ein Maß an Achtsamkeit mitbringen, um offen und aufmerksam für das zu sein, was sich im Bildungsprozess situativ zeigt. Gerade diese situativ-hermeneutische Handlungskompetenz gilt als Kennzeichen der Professionalität (vgl. Tietgens 1988).

Wenn wir also davon ausgehen, dass wir in einer Transformationsgesellschaft leben, in der Unbestimmtheit bzw. Kontingenz die Regel ist, dann gilt es, Menschen zu einem souveränen und mündigen Umgang damit zu befähigen. Nur so können sie dem Wunsch nach Bestimmtheit sowie Eindeutigkeit und damit der Unterwerfung unter Sicherheit versprechende Kontrollstrategien widerstehen. Mündigkeit entsteht allerdings nur dann, wenn ich verstehe, anerkenne und mich darauf einlassen kann, die Dinge auch anders zu sehen, und den Raum für andere Möglichkeiten öffne. Wie sehr allerdings im 
Zeitalter unseres Turbokapitalismus die Entwicklung solcher reflexiven Fähigkeiten gefährdet ist, beschreibt Sennett (1998) in seinem Buch „Der flexible Mensch“, das im Original den passenden Titel „The Corrosion of Character" trägt. Ohne an dieser Stelle diese Aspekte weiter ausführen zu können, sollen die hier aufgezeigten Überlegungen noch einmal in drei Thesen verdichtet und mit Fragestellung für die Forschung zusammengeführt werden:

\section{Systemik der Lernkulturentwicklung verstehen}

Lernkulturentwicklung vollzieht sich immer in einer Kontinuität bzw. in Kohärenz zur Organisations- und Führungskultur. Dafür braucht es ein „dichtes Kommunikationsgeflecht“, um sich über die handlungsleitenden Werte und Überzeugungen im Lehren und Lernen auszutauschen. Das bedeutet auch, alle Mitglieder und Akteure einer Organisation in diesen Entwicklungsprozess mit einzubeziehen, also nicht nur das pädagogische, sondern auch das Verwaltungspersonal (vgl. Franz 2013) und ggf. weitere Stakeholder.

Für die Erwachsenenbildungsforschung wäre es daher weiterführend, die Systemik von Lehr-, Lern-, Organisations-, Führungskultur sowie regionalen Kulturen mit ihren jeweiligen (bildungs-)politischen Diskursen, z.B. in Form „dichter Beschreibungen“ (Geertz 2003), zu erfassen. Damit ließen sich auch die Ausbalancierungsprozesse von professioneller und organisationaler Logik rekonstruieren und in den erweiterten Kontext sozialer und regionaler Entwicklungen stellen.

\section{Lernkulturen der Achtsamkeit entwickeln}

Die Transformationsherausforderungen erfordern einen souveränen Umgang mit Kontingenz, Reflexionsfähigkeit, eine Zurückhaltung gegenüber simplifizierenden Erklärungsansätzen, Bereitschaft zur Flexibilität und Infragestellung von Routinen. Bildungseinrichtungen müssten sich daher stärker ihrer eigentlichen Kernaufgabe besinnen und selbst „Transformationskompetenz“ (Tietgens 1988; Ludwig 2011) aufbauen, um Menschen in ihren vielfältigen Entwicklungsprozessen zu unterstützen, die auch andere Entfaltungswege eröffnen als nur die Orientierung an einem wirtschaftlichen Wachstum. Lernkulturen wären dann eher an einer Idee von Achtsamkeit (vgl. Hyland 2010) und Sustainable Development (vgl. Schüßler 2007) auszurichten.

Um besser zu verstehen, welche Mechanismen für die Lernkulturentwicklung bedeutsam sind, welche Wirklichkeitsmodelle sich durchsetzen und in sozialen Praktiken manifestieren, wären weitere Lernkulturanalysen in Weiterbildungseinrichtungen wichtig, vor allem auch in solchen, die sich den oben beschriebenen Ideen öffnen. 


\section{Lernkulturen des „Sich-Einmischens“ etablieren}

Neben der Kultivierung von Achtsamkeit für ein vorurteilsfreies Gewahrsein geht es auch um die Entwicklung einer Praxis des "Sich-Einmischens“. Nur dadurch können moderne Sicherheitspraktiken, wie wir sie derzeit in unserer hoch kompetitiven und beschleunigten Gesellschaft erleben, überhaupt als subtile Regierungs- und Selbstführungstechniken wahrgenommen und verändert werden. Voraussetzung dafür sind Lernkulturen, die von einem professionellen Selbstverständnis der Bildungsverantwortlichen getragen sind, die sich in Kenntnis bildungstheoretischer Diskurse auch mit den durch Bildungspolitik und Wirtschaft gesetzten Wachstums- und Innovationsanforderungen kritisch auseinandersetzen.

Dazu gehört es auch, die „diskursive Mangelwirtschaft" (vgl. Hartung 2015) in unseren Bildungspraxen zu überwinden, um die Gestaltung unserer Lernkulturen wieder selbst in die Hand zu nehmen. Deshalb geht es zum anderen auch um die Frage, wie sich Erwachsenenbildung innerhalb dieser Veränderungsprozesse positioniert und aktiv an der lernkulturellen Entwicklung der Gesellschaft - nicht nur innerhalb ihres institutionellen Kontexts mitwirkt.

Für unsere Community heißt das auch, sich mehr in die gesellschaftlichen und bildungspolitischen Themen einzumischen, damit wir nicht nur diskursanalytisch den Status quo entlarven, sondern engagiert und ganz praktisch die Bildungslandschaft in all ihren Facetten mitgestalten. Denn wenn das Wissen selbst zu einer zweifelhaften Ware geworden ist, wenn wir weniger nach dem Wert des Wissens als nach seiner möglichen Politisiertheit fragen müssen, dann können wir uns nicht mehr auf die Wissenschaftsproduktion und -vermittlung allein zurückziehen. Dann geht es darum, wissenschaftliche Theoriebildung und öffentliches Engagement mehr miteinander zu verzahnen. Was wir brauchen, ist eine aktualisierte kritische Theorie, die mutig genug ist, Modelle zu entwickeln, in und mit Praxis zu experimentieren, und die sich gleichzeitig damit verknüpfter normativer Aufladungen bewusst ist. Es geht darum, die Räume für eine Lernkulturentwicklung zu öffnen, die den Transformationsherausforderungen von heute gewachsen ist.

\section{Literatur}

ABWF (Hrsg.) (1998 ff.): Kompetenzentwicklung. Münster.

Arnold, R. (1997): Von der Weiterbildung zur Kompetenzentwicklung. Neue Denkmodelle und Gestaltungsansätze in einem sich verändernden Handlungsfeld. In: Albrecht, G. u.a.: Kompetenzentwicklung '97. Berufliche Weiterbildung in der Transformation - Fakten und Visionen. Hrsg. v. QUEM. Münster, S. 253-300.

Arnold, R. (2007): Ich lerne, also bin ich. Eine systemisch-konstruktivistische Didaktik. Heidelberg.

Arnold, R./Schüßler, I. (1998): Wandel der Lernkulturen. Darmstadt. 
Beck, U./Giddens, A./Lash, S. (1996): Reflexive Modernisierung. Eine Kontroverse. Frankfurt a.M.

Dietrich, S. (Hrsg.) (2001): Selbstgesteuertes Lernen in der Weiterbildungspraxis. Bielefeld.

Dietrich, S. (2004): Neue Lernkultur erfordert neue Lehrkultur. Zur Veränderung professionellen Handelns. In: Hessische Blätter für Volksbildung, 1, S. 57-66.

Dietrich, S./Herr, M. (Hrsg.) (2005): Support für neue Lehr- und Lernkulturen. Bielefeld.

Faulstich, P./Ludwig, J. (Hrsg.) (2004): Expansives Lernen. Baltmannsweiler.

Fleige, M. (2011): Lernkulturen in der öffentlichen Erwachsenenbildung. Theorieentwickelnde und empirische Betrachtungen am Beispiel evangelischer Träger. Münster u.a.

Forneck, H. J. (2005): Das „unregierte“ Subjekt - Lernen in der Weiterbildung. In: REPORT. Literatur- und Forschungsreport Weiterbildung, 1, S. 122-127.

Forum Bildung (Hrsg.) (2001): Neue Lern- und Lehrkultur - Vorläufige Empfehlungen und Expertenbericht. Bonn.

Franz, J. (2013): Verwaltungskräfte in pädagogischen Organisationen. Erste empirische Ergebnisse zur Perspektive von Verwaltungskräften auf die pädagogische Praxis von Organisationen Allgemeiner Erwachsenenbildung. bildungsforschung, 13, 1, S. 111-134. http://www.bildungsforschung.org/index.php/bildungsforschung/ article/view/164/190 [Zugriff: 09.2015].

Geertz, C. (2003): Dichte Beschreibung. Beiträge zum Verstehen kultureller Systeme. Frankfurt a.M.

Gesundheitsreport der Techniker Krankenkasse (2015): https:/www.tk.de/centaurus/ servlet/contentblob/718612/Datei/143830/Gesundheitsreport-2015.pdf [Zugriff: 09.2015].

Gieseke, W./Robak, S./Wu, M.-L. (Hrsg.) (2009): Transkulturelle Perspektiven auf Kulturen des Lernens. Bielefeld.

Grünewald, S. (2013): Die erschöpfte Gesellschaft von Stephan. Frankfurt a.M.

Hartung, M. J. (2015): Was tut ihr, Hochschulen? In: ZEIT Online, 08.10.2015. http://www.zeit.de/2015/39/hochschule-intellektuelle-professoren-schweigen [Zugriff: 24.07.2016].

Heuer, U./Botzat, T./Meisel, K. (Hrsg.) (2001): Neue Lehr- und Lernkulturen in der Weiterbildung. Bielefeld.

Hyland, T. (2010): Mindfulness, adult learning and therapeutic education: Integrating the cognitive and affective domains of learning. In: International Journal of Lifelong Education 29, 5, S. 517-532. https://www.researchgate.net/publication/ 47463938_Mindfulness_adult_learning_and_therapeutic_education_Integrating_t he_cognitive_and_affective_domains_of_learning [Zugriff: 09.2015].

Illeris, K. (2014): Transformative Learning and Identity. Routledge, Abingdon und New York.

Kabat Zinn, J. (2013): Gesund durch Meditation. Voll. überarb. Aufl. München.

Klafki, W. (1993): Neue Studien zur Bildungstheorie und Didaktik. Beiträge zur kritisch-konstruktiven Didaktik. Weinheim.

Langemeyer, I. (2015): Das Wissen der Achtsamkeit. Kooperative Kompetenz in komplexen Arbeitsprozessen. Münster.

Ludwig, J. (2005): Modelle subjektorientierter Didaktik. In: Report - Zeitschrift für Weiterbildungsforschung 28, 1, S. 75-80. 
Ludwig, J. (2011): Transformationskompetenz für Professionalität in der Erwachsenenbildung. In: Wiltrud Gieseke/ders. (Hrsg.): Hans Tietgens. Ein Leben für die Erwachsenenbildung. Berlin.

Mezirow, J. (1997): Transformative Erwachsenenbildung. Baltmannsweiler (Org.: Transformative Dimension of Adult Learning. San Francisco, Oxford 1991).

Ott, U. (2010): Meditation für Skeptiker: Ein Neurowissenschaftler erklärt den Weg zum Selbst. Roßdorf.

Pätzold, G./Lang, M. (1999): Lernkulturen im Wandel. Didaktische Konzepte für eine wissensbasierte Organisation. Bielefeld.

Pongratz, L. A. (2005): Subjektivität und Gouvernementalität. In: Hafeneger, B. (Hrsg.): Subjektdiagnosen. Subjekt, Modernisierung und Bildung. Schwalbach, S. 25-38.

Reischmann, J. (1995): Lernen en passant. Die vergessene Dimension. Die Kehrseite der Professionalisierung in der Erwachsenenbildung. In: Grundlagen der Weiterbildung (GdWZ) 6, 4, S. 200-204.

Reißig, R. (2009): Gesellschafts-Transformation im 21. Jahrhundert. Ein neues Konzept sozialen Wandels. Wiesbaden.

REPORT - Literatur- und Forschungsreport 44, 1999.

Ricken, J. (2011). Universitäre Lernkultur. Fallstudien aus Deutschland und Schweden. Wiesbaden.

Schäffter, O. (2001): Weiterbildung in der Transformationsgesellschaft. Baltmannsweiler.

Scharmer, O. (2011): Theorie U - Von der Zukunft her führen. Presencing als soziale Technik. 2. erw. Aufl. Heidelberg.

Schlutz, E. (Hrsg.) (1999): Lernkulturen. Frankfurt a.M.

Schüßler, I. (2000): Deutungslernen. Baltmannsweiler.

Schüßler, I. (2007): Nachhaltigkeit in der Weiterbildung. Baltmannsweiler.

Schüßler, I./Thurnes, C.M. (2005): Lernkulturen in der Weiterbildung. Bielefeld.

Siebert, H. (2009): Didaktisches Handeln in der Erwachsenenbildung. Didaktik aus konstruktivistischer Sicht. 6., überarb. Aufl., Augsburg.

Sennett, R. (1998): Der flexible Mensch. Die Kultur des neuen Kapitalismus. Berlin.

Singer, R./Bolz, M. (2013): Mitgefühl. In Alltag und Forschung. E-Book (Download unter: http://www.compassion-training.org/).

Staudt, E./Kriegesmann, B. (2000): Weiterbildung: Ein Mythos zerbricht. In: Grundlagen der Weiterbildung (GdWZ): „Kompetenzentwicklung und Qualifikation“, 4, S. 174-176.

Tietgens, H. (1988): Professionalität für die Erwachsenenbildung. In: Gieseke, W. u.a. (Hrsg.): Professionalität und Professionalisierung. Bad Heilbrunn/Obb. 1988, S. 28-75.

Tietgens, H. (1992): Reflexionen zur Erwachsenendidaktik. Bad Heilbrunn/Obb.

Universallexikon (2013): Transformationsgesellschaften. http://universal_lexikon.de academic.com/311057/Transformationsgesellschaften [Zugriff: 09.2015].

Veith, H. (2003): Kompetenzen und Lernkulturen. Münster.

Weinberg, J. (1999): Lernkultur - Begriff, Geschichte, Perspektiven. In: Kompetenzentwicklung 1999. Münster, S. 81-143.

Wittwer, W./ Kirchhof, S. (Hrsg.) (2003). Informelles Lernen und Weiterbildung: Neue Wege zur Kompetenzentwicklung. München. 
Panel I:

Lernkulturen und Sozialraum 



\section{Lernkulturwandel über die Herstellung von Transparenz für Bildungsberatung? Strategien im Rahmen des kommunalen Bildungsmanagements zur Gestaltung der Bildungsregionen}

\section{Lernkultur und Bildungsregion - über kommunales Bildungsmanagement zum Lernkulturwandel?}

Wir sprechen im Feld der Erwachsenenbildung häufig von Lernkultur, aber nicht von regionaler Weiterbildungskultur. Der Begriff der Lernkultur bezieht sich meistens lediglich auf die Lernkultur in pädagogischen Einrichtungen, die durch den sozialen Umgang zwischen Lehrenden und Lernenden als Akteurinnen und Akteure entsteht. Um die Institutionenvielfalt des (Weiter-) Bildungswesens übergreifend $\mathrm{zu}$ beschreiben, sprechen wir von der Bildungs- bzw. Weiterbildungslandschaft (Schlüter 2010) und bezogen auf konkrete räumliche Verortungen aktuell von der Bildungsregion. Mit dem Begriff der Bildungsregion lässt sich die Vorstellung von verschiedenen Lebenswelten - auch sozialen Milieus - assoziieren, die in der Lernbereitschaft ständig Neu- und Weiterzulernen sehr unterschiedliche Niveaus aufweisen. Um eine Weiterbildungskultur des lebenslangen Lernens zu schaffen, braucht es „Impulse für eine neue Lernkultur durch Forschung und Entwicklung“, wie beispielsweise unter diesem Titel von Rainer Brödel auf der Basis eines Modellprojektes zur „Kompetenzentwicklung im Auf- und Ausbau regionaler Infrastruktur" (LisU) (Brödel 2001) thematisiert. Daran anknüpfend lässt sich Lernkultur im Folgenden auf Region als soziale Realität beziehen, denn es ist davon auszugehen, dass regionale Bedingungen einerseits Rahmen für das Niveau und die Beteiligung der Bevölkerung an Lernen, Bildung und Weiterbildung und andererseits auch Rahmen für Akteurinnen und Akteure schaffen, die über Kooperationen die Infrastruktur für Bildung und Weiterbildung gestalten.

Meine Überlegungen und Ausführungen zum Lernkulturwandel über kommunales Bildungsmanagement verstehe ich als Beitrag zum Diskurs „Raum als gemeinsamer Aktions- und Planungsraum“ (vgl. Mania u.a. 2015). Mit diesem Rezeptionsstrang im wissenschaftlichen Diskurs ist ein erweiterter Bildungsbegriff verbunden, der Bildung als Querschnittsaufgabe für die regionale Entwicklung versteht und diese an die ,Kommune“ bindet. $\mathrm{Zu}$ dieser verortenden Einbindung lässt sich die Bundesministerin Johanna Wanka mit der Aussage zitieren: „Wir lernen lebenslang und an unterschiedlichen 
Orten. Der zentrale Ort, an dem wir alle Menschen erreichen, sind die Kommunen“" (BMBF 2014: 2).

Da die Aneignung von Bildung in jeder individuellen Lebensphase in der Gesellschaft als wichtige Voraussetzung für Wirtschaftswachstum und Wohlstand eingeschätzt wird, müssen kommunale Akteure sich nicht allein einig im Handeln für mehr Bildung sein, sondern auch die Einstellung teilen, dass Lern- und Bildungskulturen in einer Region als gestaltete und gestaltende Struktur zu begreifen sind.

Staatlich finanzierte Bildungspolitik greift seit Jahren auf der Basis von Projekten ein, um letztlich (Weiter-)Bildungschancen für Bürgerinnen und Bürger zu erhöhen. Am Beispiel der Kommune Duisburg soll in diesem Beitrag gefragt werden, auf welche Weise und mit welchen Strategien Akteure vorhandene regionale Strukturen beeinflussen, um einen Lernkulturwandel zugunsten des lebenslangen Lernens zu erreichen. Das Erkenntnisinteresse besteht darin, mit einem erweiterten Blick auf Bildung zu fragen, wie und mit welcher Gestaltungskompetenz für Lern- und Bildungsprozesse auf kommunaler Ebene die örtlichen Bedingungen geschaffen werden, damit individuelle Bildungsbiographien häufiger als bisher gelingen. Die Hypothese bezieht sich auf die Überlegung, dass vergemeinschaftende Lernprozesse wesentlich sind, um in einer kommunalen Lernkulturentwicklung Wirkungen zu erzeugen. Diese wiederum sind nur vorstellbar mit einer Entscheidung für bildungspolitische Ziele, die von vielen kommunalen Akteuren und Akteurinnen getragen und kommuniziert werden.

\section{Zur Situation für kommunales Bildungsmanagement in der Bildungsregion}

Die Akteure aus den Projekten „Lernende Regionen“ und „Lernen vor Ort“ sowie den Anschlussprojekten haben in den letzten Jahren in den Regionen die Vernetzung von (Weiter-)Bildungsakteuren vorangetrieben, um die $\mathrm{Zu}-$ gänge zu Bildung und Weiterbildung kommunizierbar zu machen. Ein langfristiges Ziel ist, über leicht erreichbare Bildungsberatung die Bildungswege in der Bevölkerung generell zu verbessern. Die Herstellung von Transparenz zu den Bildungsberatungsangeboten am Ort ist ein erster, aber wesentlicher Schritt aus der Sicht der Akteure. Denn eine Erhöhung der Bildungsnachfrage - angeregt durch Beratung - bedeutet eine Begleitung biographischer Übergänge im Lebenslauf. Dieses Vorhaben ist als Antwort auf den sozialen Wandel zu verstehen, der charakterisiert wird durch die Beschreibung der Transformation der Lebenswelten vor dem Hintergrund des sozioökonomischen Wandels und der veränderten Organisations- und Managementkonzepte vieler Einrichtungen (Schlüter 2014). Entsprechend lässt sich diese Transparenzwerdung von Angeboten als Rekurs auf einen politisch geführten 
Lernkulturdiskurs interpretieren, der u.a. auch ein kompetenzorientiertes Professionalisierungskonzept für die Organisation von Beratungsmöglichkeiten durch Bildungsakteure am Ort unterstellt.

Bildungspolitische Diskurse zum lebenslangen Lernen zielen auf die Internalisierung durch die Individuen. Politisch wurde daraus die Vorstellung, dass Lernen in den verschiedenen Lebensphasen aufeinander abgestimmt sein sollte, um erfolgreiche Bildungsbiographien für alle Bürgerinnen und Bürger zu ermöglichen (vgl. Schlüter 2015). Konkret bedeutet dies gegenwärtig, Lernende werden als Konstrukteure der eigenen professionellen Kompetenz entworfen, die durch Institutionen begleitet werden können. D. h. in der Konsequenz das in dieser Weise entworfene individuelle Subjekt muss regionale bzw. lokale Strukturen vorfinden, um seine eigene Gestaltungskompetenz kreativ ausleben zu können. Existierende regionale Strategien sind daher die Optimierung der Bildungsberatung für eine Ermöglichung der Bildungsübergänge sowie die Weiterbildung des Beratungspersonals. Erst wenn Bildung als Querschnittsaufgabe bezogen auf die individuellen Übergänge in Lebensläufen gesteuert werden kann, können Bildungsakteure in ihren je eigenen Aktionsfeldern erfolgreich agieren.

Eine Fragestellung im Kontext des Aufsatzes ist daher auch: Welche Entscheidungen mussten gefällt werden und welcher Diskurs wurde geführt, um Nachhaltigkeit für diese Art der bildungspolitischen Unterstützung in den Kommunen zu etablieren?

\section{Vernetzung in der kommunalen (Weiter-)Bildungslandschaft}

Die Kommune ist ein politischer und geografischer Raum, ein lokaler administrativer Raum für Bildungsplanung. Die Kommune ist ein Ort für Gestaltung durch Akteure, die sich auf diesen Raum beziehen bzw. konzentrieren. Der Raum ,Kommune" ist die Bezugseinheit für Bildungsziele und -aktivitäten. Der geografisch und administrativ begrenzte Raum wurde von politisch denkenden Akteuren aus Wissenschaft und Politik bereits in der Vergangenheit als ein Lern- und Bildungsort entworfen, für den sie in verschiedener Weise Vorschläge formulierten. So schrieb Peter Faulstich 2001 im Rahmen des Forums Bildung:

„Durch Kooperationsverbünde - als auf Kontinuität gestellte Netzwerke - sollen regionale Infrastrukturen gefördert und gesichert werden, deren Hauptziel es ist, Anbieter und Nachfrager sowie andere Interessierte zusammenzuführen, um so Verbesserungen der Lernangebote zu erreichen und gleichzeitig die Lernbeteiligung zu steigern“ (Faulstich 2001: 136). 
Es ist in seinen Ausführungen darüber hinaus nachvollziehbar, dass, wenn die jeweilige Region über Weiterbildungspolitik entwickelt werden soll, dies auch voraussetzt, regionale Kulturen ,als Gesamtheiten unterschiedlicher, räumlich differenzierter Lebensweisen“ (Faulstich 2001: 137), also als entwicklungsrelevant für Lernkulturen zu setzen. Innovationsstrategien, transportiert und reguliert über Netzwerkbildung, dienen unter diesem Blickwinkel der Kompetenzentwicklung in der Region (ebd.).

Etliche Projekte haben den Zusammenhang von kommunalem Engagement und Erwachsenenbildung für eine professionelle Vernetzung der Bildungsakteure modellhaft untersucht (beispielsweise Faulseit-Stübner u.a. 2009). Diese und viele andere Initiativen haben im Ergebnis dazu geführt, dass die Chancen zur Realisierung bildungspolitischer Ziele in der Region bzw. Kommune im Aufbau von Supportstrukturen gesehen werden. Supportstrukturen werden seit 2001 über das ,größte regionale Bildungs- und Entwicklungsprogramm“ (Nuissl/Nuissl 2015: 9) geschaffen, nämlich über die Projekte der „lernenden Regionen“ und „Lernen vor Ort“, die allerdings nicht flächendeckend, sondern an ausgewählten Orten durchgeführt wurden. Sie verstanden sich als Lernmodelle, die langfristig über die kommunalen Abgrenzungen, also eher über geografische und politische Grenzziehungen hinaus kommuniziert wurden, um überall sichtbar zu werden. Sie sind mittlerweile politisch meist in strategische Gesamtziele der kommunalen Akteure eingebunden worden, um das kommunale Bildungsmanagement $\mathrm{zu}$ befördern. Aufgrund bisheriger Erfahrungen lässt sich darauf verweisen, dass die Netzwerkbildung in der Kommune als Einflussgröße für die Gestaltung einer Bildungsregion als wesentlich betrachtet wird (Feld 2011). Die folgenden Ausführungen werden sich beispielhaft nur auf die Entwicklung einer kommunalen Bildungslandschaft seit diesem Zeitpunkt konzentrieren können.

\section{Zur Gestaltung des kommunalen Bildungsmanagements - Beispiel Duisburg}

Duisburg ist eine Stadt, die unter Haushaltssicherung steht. Das bedeutet, dass die Kommune lediglich die gesetzlich verpflichteten Leistungen zahlt. Obgleich sie aufgrund der Bevölkerungsstruktur viele soziale Aufgaben hat (DuisburgBildung 2011: 34), kann sie vieles nicht bezahlen. Kompensation verspricht die Akquise von Projekten.

Duisburg hat sich 2007 um ein Projekt aus dem Programm „Lernen vor Ort" beworben und auch einen Zuschlag dafür erhalten. Ein Akteur auf kommunaler Ebene ist der Leiter der Volkshochschule in Duisburg, Dr. Gerhard Jahn. Er gab mir im Juni 2015 dazu rückblickend folgende Einschätzung: 
„Aufbauend auf den Erfahrungen in anderen Kommunen mit dem Projekt ,Lernende Regionen' bestand die Absicht den Netzwerkgedanken auch in Duisburg auf die Kommunale Bildungsarbeit zu übertragen und die Versäulung im Bildungsbereich zu überwinden. Im Rahmen des Projektes ,Lernen vor Ort' sowie der Beteiligung der Kommune aus dem vom Land NRW ausgerufenen ,Regionalen Bildungsnetzwerken' wurde ein kommunales Bildungsmanagement eingerichtet sowie Verwaltungsstrukturen im Blick auf den Netzwerkgedanken verändert. Es entstand in der Kernverwaltung die Bildungsholding, die sämtliche am Bildungsprozess beteiligte Fachbereiche (Schulverwaltung, Schulamt, Jugendamt, Stadtbibliothek und Volkshochschule) vereinigte. Auch das Modellprojekt ,Lernen vor Ort" wurde in die Bildungsholding integriert"(Z 32-42).

Die Stadt Duisburg hat auf der Verwaltungsebene 2007 verschiedene Gesamtstrategien, in die Bildungsziele eingebunden sind, verabschiedet, z.B. die Sicherung des ökonomischen Wachstums und die Erhöhung der urbanen Lebensqualität (DuisburgBildung 2011: 17). Eine Strategie lautet: ,aktive Wahrnehmung der Verantwortung als Bildungsregion“" (ebd.). Zur Realisierung der Gesamtstrategien wurden entsprechende Handlungsforen eingesetzt, um die Ziele operationalisieren zu können. Über die gegründete Bildungsholdung wurde kommuniziert: „Duisburg wird seine Verantwortung als Bildungsregion aktiv wahrnehmen“ (a.a.O.: 18). Sie versicherte:

„Die Bürger finden in Duisburg eine sehr gute und vielfältige Bildungslandschaft.

Duisburg ist eine der führenden Bildungsstädte der Region. Deshalb unternimmt die Stadt alle Anstrengungen, um die Bildungsakteure zu vernetzen" (ebd.).

Das benannte strategische Ziel konnte in Leitziele für die Stadt Duisburg ausdifferenziert werden. In den weiteren Ausführungen wurde formuliert:

„1. Wir gestalten erfolgreiche Bildungslaufbahnen und fördern die Entfaltung der Persönlichkeit.

2. Wir gestalten Bildung so, dass alle Bürger/innen entsprechend ihren Voraussetzungen und Potentialen individuell und optimal gefördert werden.

3. Wir gestalten Bildung so, dass sich die Potentiale aller Herkünfte individuell und optimal entfalten können." (ebd.).

Im Zuge der weiteren Operationalisierung wurden für den gesamten Bildungsbereich in der Kommune zwölf Ziele erarbeitet. Dabei wurde ersichtlich, dass die empirische Datenlage zum gesamten Bildungsbereich höchst problematisch ist. Konstatiert wurde in den weiteren Diskussionen, dass sich viele ausformulierte Ziele lediglich auf den schulischen Bereich beziehen. 
Ein neu aufzubauendes Bildungsmonitoring sollte von vornherein neben den schulischen auch die außerschulischen Bildungsdaten mit erheben, um einerseits eine Beurteilung der Stärken und Schwächen im Bildungssystem vornehmen zu können, andererseits eine Perspektive für umfassende Entwicklungen aufzeigen zu können. In der genannten Zielformulierung bezog sich lediglich ein Ziel auf Weiterbildung. Dieses lautete: ,Jede/r Duisburger Bürger/in nimmt wenigstens einmal im Lebenslauf die Chance der Weiterbildung wahr" (a.a.O.: 19).

Diese Schwerpunktsetzung ist auch in anderen Kommunen zu finden, wie Michael Schemmann für andere Regionen feststellte, in denen vergleichbare Projekte des Programms „Lernen vor Ort" durchgeführt wurden (Schemmann 2015).

Zum Zeitpunkt der Verabschiedung der Bildungsziele und der Einrichtung der Projekte „Lernen vor Ort“" war auch der Hinweis wichtig, dass die Ausrichtung des zukünftig zu erstellenden Bildungsberichts für Duisburg sich an der Bundesbildungsberichterstattung „Bildung in Deutschland“ von 2010 orientieren (Duisburg Bildung 2011: 20) und außerdem der Handlungsleitlinie der Stadt folgen sollte. Die Handlungsleitlinie trägt den Namen: „Bildung - ein Leben lang" (a.a.0.: 22). Mit dieser Leitlinie war zweierlei verbunden: erstens die „Betrachtung von Bildungsthemen entlang der Bildungsstationen im Lebenslauf eines Menschen" und zweitens die Entscheidung, die Bearbeitung der Handlungsfelder entlang des Lebenslaufs vorzunehmen. Dies wurde als Entscheidung für ein politisches Vorgehen legitimiert, eingeschränkt mit dem Hinweis, dass damit keine ,Zusammenhänge zwischen Problemen und ihren möglichen Ursachen oder auch Lösungen“ (ebd.) dargestellt werden. Es diene lediglich dazu, eine „Ausgangsbasis für die Berichterstattung und Bestandsaufnahme von Stand und Entwicklung von Bildungsbeteiligung und strukturellen Bedingungen innerhalb von Kommunen“" (ebd.) zu schaffen.

Die Bildungsakteure in der kommunalen Bildungslandschaft waren von nun an aufgerufen, die angegebenen Ziele zu erreichen. Die Ausrichtung wurde über die Behandlung in den geschaffenen politischen Handlungsforen weiterhin bearbeitet.

\subsection{Handlungsfelder in der Kommune}

Das übergreifende Ziel, den Bürgerinnen und Bürgern Duisburgs das Durchlaufen einer erfolgreichen Bildungsbiographie von der frühkindlichen Bildung bis hin zur Erwachsenenbildung zu ermöglichen, erfolgt also Schritt für Schritt in den verschiedenen Handlungsfeldern, die je nach Kommune neben den generellen Handlungsfeldern einige Modifikationen aufweisen. Duisburg entschied sich für folgende: Bildungsmanagement, Bildungsmonitoring, 
Bildungsberatung, Bildungsübergänge, Eltern- und Familienbildung sowie Demographie.

Die inhaltlichen Schwerpunkte des Programms in Duisburg waren und sind:

- der Aufbau und Ausbau eines kommunalen Bildungsmonitoringsystems als Grundlage einer kontinuierlichen Bildungsberichterstattung

- die Einrichtung eines Zentrums für Bildungs- und Lernberatung, das Ratsuchende an den verschiedenen Bildungsübergängen unterstützt

- Bereitstellung eines qualitativen Bildungsberatungsangebotes vor Ort unter Berücksichtigung des demographischen Wandels

- inhaltliche Einbindung und Vernetzung der Familienzentren und weiteren Bildungseinrichtungen, um durch zielorientierte Elternarbeit den Einstieg in eine erfolgreiche Bildungsbiographie zu erleichtern.

Was wurde kommuniziert? Das Team des Projektes „Lernen vor Ort“ - so lässt sich bislang resümieren - hat einen wichtigen Beitrag für den Bildungsstandort Duisburg geleistet. Damit beispielsweise das Handlungsfeld Bildungsberatung in der Kommune bearbeitet werden konnte, also Bildungsberatung in seinen Funktionen transparent wird, wurden nach Aussage des Koordinators des Projektes „Lernen vor Ort“, Hans Peter Müller, der für die Gesamtprojektkoordination verantwortlich war, folgende Argumente für die bildungspolitische Diskussion in der Kommune am häufigsten verwendet:

- fehlende Kenntnis über existierende Angebote

- unterdurchschnittliche Wahrnehmung /Inanspruchnahme von Weiterbildungsangeboten und -förderung

- erste Anzeichen eines Fachkräftemangels

- unterdurchschnittliche Bildungsabschlüsse in der Kommune (zum Landesdurchschnitt)

- zurückgehende Fördermittel in der Arbeitsmarktpolitik (ARGE/Agentur für Arbeit) soll so neue Nachfrage bei Bildungsanbietern generiert werden

- fehlendes Wissen einzelner Akteure über die eigene Bildungsmarktsegmentgrenzen hinaus

- selbstgesetztes Ziel der Kommune soll realisiert werden, Duisburg zu einer Stadt der Bildung weiterzuentwickeln (Z46-56).

Solche Argumente in der örtlichen Debatte waren absolut notwendig, denn zu Beginn der Aktivitäten - so Müller im Juni 2015 im Gespräch - fehlte die Akzeptanz der Projektziele bei wichtigen Entscheidern vor Ort. Erst mit der Neuaufstellung des zuständigen Dezernats und der Neubesetzung des Amtes des Oberbürgermeisters sowie der Neuansiedlung des Bildungsbüros im Dezernat (nicht wie vorher bei einem Amt) änderte sich dies. Damit wurden die Ziele des Projektes umsetzbar. Neue Kommunikationsstrukturen wurden etabliert. Die Kommunikation findet nun in der Akteurslandschaft über Bil- 
dungskonferenzen und Mitteilungsvorlagen für Ausschüsse und Rat auf der politischen Ebene statt. Die einmal im Jahr stattfindende Bildungskonferenz wird durch den Oberbürgermeister geleitet. Die Ergebnisse der Bildungskonferenzen werden als weitere strategische Ziele für die kommunale Bildungsarbeit betrachtet. Diese Neuordnung ist der Arbeit des Modellprojektes „Lernen vor Ort" zu verdanken.

Sie lässt sich in folgender Struktur veranschaulichen:

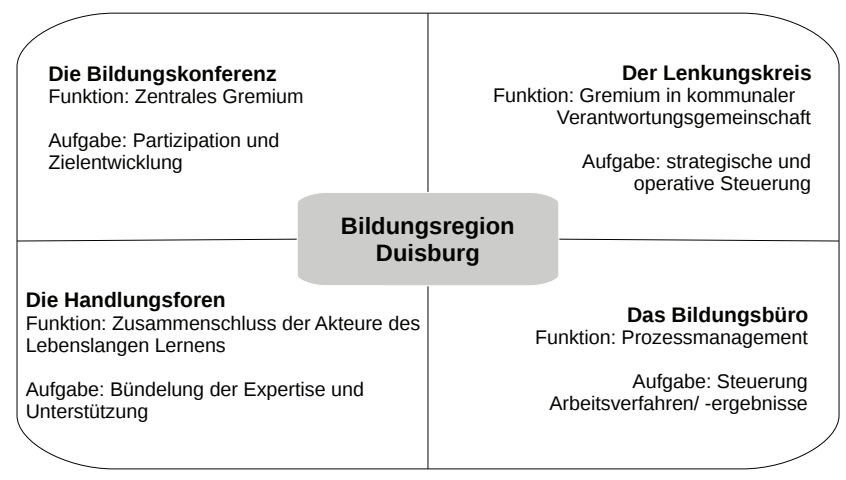

Abb. 1: Bildungsregion Duisburg (Schaubild: Schlüter/Hundorf 2015: 2)

\subsection{Transparenz für Bildungsberatung in allen Lebensphasen}

Um das Ziel, Transparenz für Bildungsberatung herzustellen, zu befördern, diskutierten die Akteure aus dem Duisburger Netzwerk, Strategien zu dessen Erreichung. Eine Befragung wurde zu Beginn des Jahres 2015 an das Fachgebiet „Erwachsenenbildung/Bildungsberatung“ an der Universität DuisburgEssen in Auftrag gegeben. Angeschrieben wurden 602 Institutionen, von denen im ersten Anlauf $19 \%$ antworteten. Eine zweite zeitlich spätere Aufforderung brachte weitere Antworten, die für die Erstellung einer Bildungsberatungslandkarte einbezogen werden.

Die Ergebnisse der ersten Runde der Befragung wurden auf der zweiten Bildungskonferenz „Starke Bildung stärkt die Stadt" im Mai 2015 in Duisburg präsentiert. Eingeladen waren alle Akteure der Bildungsregion Duisburg ${ }^{1}$.

1 Neben dem Handlungsforum „Bildungsberatung im Lebenslauf“ wurde in drei weiteren Foren diskutiert. Die Foren waren: „Duisburg als inklusive Bildungsstadt“, „Verkehrs- und Bildungssprache Deutsch stärken“, ,Scheitern von Bildungslaufbahnen vermeiden“. 
Auf der Sitzung des Forums „Bildungsberatung im Lebenslauf“ konnten aufgrund der Erhebung durch den Fragebogen Beratungsstellen mit ihren Beratungsschwerpunkten vorgestellt werden. Diese verteilen sich über das ganze Stadtgebiet, häufen sich jedoch erwartungsgemäß in der Stadtmitte. Werden die Angebote inhaltlich gebündelt, lassen sich im Wesentlichen vier Bereiche unterscheiden: vorschulische, schulische, nachschulische und nonformale Bildungsberatungsangebote. Der Fragebogen hielt zu den genannten Bereichen eine unterschiedliche Anzahl von Antwortmöglichkeiten bereit. Die meisten Wahlmöglichkeiten zeigten sich im nachschulischen Bereich. Nennungen bezogen sich vor allem auf die berufliche Orientierung, auf die Weiterbildungsberatung und Laufbahnberatung sowie auf die Beratung zum Nachholen von Schulabschlüssen. Beratungsangebote im schulischen Bereich lassen sich untergliedern in: Schullaufbahn, berufliche Orientierung, Vermeidung von Schulabbruch. Zum Thema Integration und Inklusion werden die meisten Angebote bereitgehalten. Für den vorschulischen Bereich sind die Angebote relativ gering. Auffällig ist jedoch die hohe Zahl der Nennungen für die Elternberatung.

$\mathrm{Zu}$ den größten Zielgruppen der Beratung gehören eindeutig die Eltern (72\%) und Schüler/innen (63\%). Es folgen Personen mit Migrationshintergrund $(47 \%)$ und Arbeitnehmer/innen (32\%). Ersichtlich wurde auch, dass viele der Institutionen bereits regional vernetzt sind und sich in einem Beratungsnetzwerk engagieren. Zum Ausdruck kam ein großes Interesse der noch nicht vernetzten Akteure, sich zukünftig dem Netzwerk anzuschließen. Die Erhebung der Daten zu den Beratungsstellen wird zur Erstellung einer interaktiven Online-Beratungslandkarte führen, auf der nach Stichworten auffindbar die verschiedenartigen Beratungsangebote zur Information aufgerufen werden können (ausführlicher in Schüter/Hundorf 2015 dargestellt).

\section{Ausblick zum Lernkulturwandel durch kommunales Bildungsmanagement}

Die konkrete Ausrichtung in der Kommune im Selbstverständnis als Bildungsregion setzt auf eine kontinuierliche Entwicklung über politisch verabschiedete Bildungsziele. Um für die Umsetzung der politisch verabschiedeten Ziele handlungsfähig zu werden, bedurfte es erst einer Neuordnung der Akteurslandschaft. Die Annahme ist, dass durch die Gestaltung der kommunalen Bildungslandschaft Voraussetzungen geschaffen werden können, um Bildungsbiographien zum Gelingen zu bringen und zwar insbesondere solche, die an den biographischen Übergängen durch Brüche bedroht sein könnten. Inwieweit Bildungsberatung von Bürgern und Bürgerinnen angenommen wird, wenn diese als Angebot sichtbarer als bisher gemacht wird und inwieweit damit längerfristig Verbesserungen für die jeweilige Arbeitsmarktregion 
verbunden sind, sind offene Fragen. Sie zu beantworten, ist nicht allein eine Forschungs-, sondern auch eine Entwicklungsfrage. Auf der Basis der Befragung lässt sich konstatieren, dass die Strategie der Herstellung von Transparenz für die Beratungsvielfalt in Duisburg als wertvoll eingeschätzt wird. Bezogen auf die Bildungsregion wird diese Einschätzung als Motivation verstanden, regional für deren Optimierung zu sorgen. Über die Strategie, Transparenz über die potentiell nutzbaren Beratungsstellen in der Stadt herzustellen, lieferten einige der zentralen Akteure Einschätzungen zu den damit eingeleiteten Perspektiven.

So erläuterte Dr. Gerhard Jahn - Leiter der Volkshochschule - im Juni 2015 im Interview:

„Wir stehen am Anfang. Bildungsmonitoring und Bildungsberichterstattung werden es uns ermöglichen, die Evaluation von Bildungsprozessen systematisch vorzunehmen. Bildungsberatung muss sehr niederschwellig betrieben werden und an allen Übergängen ansetzen. Die Digitalisierung in der Erwachsenenbildung ist eine der großen Herausforderungen. In diesem Kontext kann der Aufbau und die Pflege einer Bildungsberatungslandkarte digital wie analog zur Verbesserung des organisierten autonomen Lernens beitragen“"(Z 85-91).

Die Einschätzung von Hans Peter Müller, Koordinator, im Juni 2015 lautet:

„Meine Einschätzung ist, dass bei richtiger und regelmäßiger Präsentation der Online-Beratungskarte und regelmäßiger Öffentlichkeitsarbeit mehr Bürger/innen selbständig Beratung in Bildungsfragen in Anspruch nehmen werden. Aufgrund des überdurchschnittlichen Anteils von Bürger/innen mit bildungsfernen Ausgangssituationen, unterdurchschnittlichen Einkommen und überdurchschnittlichen vielen Bürger/innen mit Migrationshintergrund (drei von einander unabhängige Ausgangssituationen!) ist dennoch eine Form der niederschwelligen Beratungsweiterleitung (z.B. in Form von Beratungslotsen) unabdingbar notwendig“"(Z 59$65)$.

Mit der Implementierung der interaktiven Landkarte mit Beratungsangeboten wird die Arbeit nicht beendet sein. Das regionale Bildungsnetzwerk diskutierte 2015 die Einführung von Beratungslotsen. Dieses Modell wird als Chance für diejenigen gesehen, die eine Unterstützung bei der Suche nach dem individuell passenden Beratungsangebot brauchen. Diese Idee wird in Zukunft noch weiter verfolgt werden. Auf der Basis der Fragebogenergebnisse lässt sich sagen, dass es dafür in der Kommune Duisburg Interesse gibt. Um allerdings von einer Kultur des Lebenslangen Lernens bzw. von einer Weiterbildungskultur sprechen zu können, braucht es wohl noch mehr Impulse für weitere Entwicklungen vor Ort. 


\section{Literatur}

BMBF (2014) (Hg.): Transferinitiative Kommunales Bildungsmanagement: Bildung gemeinsam gestalten. Berlin.

Brödel, Rainer (2001): Impulse für eine neue Lernkultur durch Forschung und Entwicklung. In: QUEM-report, H.67, Berlin: AG Betriebliche Weiterbildungsforschung e. V., S. 73-80.

DuisburgBildung/Stadt Duisburg/Dezernat für Familie, Bildung, Kultur (2011) (Hg.):

- Bildung in Duisburg, 1. Teilbericht: Elementar-/Primarbereich. Duisburg.

Faulseit-Stüber, Andrea/Gernentz, Johanna/Kron, Ulrike/Weiss, Karin (2009) (Hg.): Weiterbildung und kommunales Management. Anregungen für die Praxis. Bielefeld: Bertelsmann.

Faulstich, Peter (2001): Regionale Netzwerke lebenslangen Lernens. In: Lernen - ein Leben lang. Vorläufige Empfehlungen und Expertenbericht. Materialien des Forum Bildung. Bonn, S. 136-145.

Feld, Timm C. (2011): Netzwerke und Organisationsentwicklung in der Weiterbildung. Bielefeld: Bertelsmann.

Mania, Ewelina/Bernhard, Christian/Fleige, Marion (2015): Raum in der Erwachsenen-/Weiterbildung. Rezeptionsstränge im wissenschaftlichen Diskurs. In: Bernhard, C./Kraus, K./ Schreiber-Barsch, S. (Hg.): Erwachsenenbildung und Raum, Bielefeld: Bertelsmann, S. 29-39.

Nuissl, Ekkehard/Nuissl, Henning (2015) (Hg.): Bildung im Raum. Baltmannsweiler: Schneider Hohengehren.

Schemmann, Michael (2015): Lokale (Weiter-)Bildungsberichterstattung als Fokussierung von Weiterbildung und Raum. Konzepte, Befunde, Akzeptanz. In: Nuissl, E./Nuissl, H. (Hg.): Bildung im Raum. Baltmannsweiler, S. 11-134.

Schlüter, Anne (2010): Die Weiterbildungslandschaft - Tätigkeitsfelder und Orte der Erwachsenenbildung und Bildungsberatung. In: Dies.: Bildungsberatung. Opladen und Farmington Hills, MI: Barbara Budrich, S. 19-29.

Schlüter, Anne (2014): Von der Transparenz der Weiterbildung zur lebensbegleitenden Übergangsberatung - zum Funktionswandel von Weiterbildungsberatung. In: Felden, H. v./Schäffter, O./Schicke, H. (Hg.): Denken in Übergängen. Weiterbildung in transitorischen Lebenslagen. Wiesbaden: Springer VS, S. 253-266.

Schlüter, Anne (2015): Übergänge von Erwachsenen. 10 Fragen zum Thema. Hg. von der Transferagentur Kommunales Bildungsmanagement Agentur NRW. Münster.

Schlüter, Anne/Hundorf, Sarah (2015): Evaluationsbericht. Ergebnisse der Erhebung der Struktur und Verteilung der Bildungsberatungsangebote in der Bildungsregion Duisburg. Unveröffentlichter Bericht, Essen. 



\section{Lernkulturen im Spannungsfeld von Institutionenkulturen. Das Beispiel „Alphabetisierung im Stadtteil" einer städtischen Volkshochschule}

Lernkulturen als pädagogisch gestaltete Strukturen sind zunächst eingebettet in soziokulturelle Milieus. Im Zusammenwirken der Milieus konstituiert sich eine Praxis eigener Logik, die in Bezug auf pädagogische Intentionen als gelungen oder nicht gewertet wird. In der Regel wird zumindest im Evaluierungsprozess Optimierungspotential identifiziert. Inwieweit gestaltete Lernkulturen dann auch zu gelingenden Nutzungskulturen werden, ist letztlich das Kriterium für den Erfolg der konzeptionellen Arbeit. Aber auch die konzeptionelle Arbeit selbst konstituiert eine eigene spannungsreiche Praxis. In besonderer Weise bergen Kooperationen zwischen erwachsenenpädagogischen Institutionen und nicht-erwachsenenpädagogischen Institutionen Herausforderungen für die beteiligten Akteure. In der Gestaltung von Lernkulturen treffen verschiedene Institutionen-Milieus aufeinander (vergleichbar mit Organisations-Milieus, Nohl 2006: 186 ff.), die eine eigene Kooperationslogik hervorbringen. Um diese soll es im Folgenden gehen, auch hinsichtlich der Frage, inwieweit unterschiedliche Institutionen im Bemühen um eine Lernkultur eine eigene Kooperationslogik konstituieren und ferner darum, inwieweit diese zum Gelingen des gemeinsamen Projektes führt. Wir beziehen uns auf empirische Ergebnisse der wissenschaftlichen Begleitung des stadtteilbezogenen Angebotes für funktionale Analphabet_innen einer Volkshochschule. Das stadtteilbezogene Angebot ist verbunden mit der Idee einer neuen Lernkultur im städtischen Raum, deren Kern die Zusammenarbeit zwischen der Volkshochschule als klassische Institution der Erwachsenenbildung und soziokulturellen Einrichtungen in den Stadtteilen ist.

Zunächst gehen wir auf das Projekt als eine Form stadtteilbezogener Lernkultur (1) ein, beleuchten anschließend institutionenbezogene Orientierungen der Kooperationspartner (2), die zur Konstitution einer spezifischen Kooperationspraxis beitragen, und beenden unsere Ausführungen mit einer Überlegung zur modulierten Kooperation (3).

\section{Stadtteilbezogene Alphabetisierung als Lernkultur}

Lernkulturen werden (nicht nur) in der Erwachsenenbildung gemeinhin als gestaltbare Lehr-Lernsettings verstanden und unter dem Stichwort „Wandel der Lernkulturen" in der Dualität von traditionellen und neuen Lernkulturen 
debattiert: Traditionell sind dann jene, deren Grundannahmen, Gewissheiten und Aktionsmuster kritisch betrachtet und ggf. widerlegt werden, neu sind jene, die an deren Stelle treten (vgl. etwa Wittpoth; Meyer 2013). Es geht dabei um die Bezweifelung der Koppelung von Lehren und Lernen mit etwaigen Konsequenzen für Diskussionen um Fragen der Wissensaneignung, Entgrenzungen, Entschulungen oder Selbstorganisation bzw. Selbstmanagement von Lernen (vgl. etwa Schüßler/Thurnes 2005: 9). In jüngerer Zeit haben Raumkonzepte in der Erwachsenenbildung an Bedeutung gewonnen, wie sie etwa unter dem Thema „Regionale Bildungslandschaften“ (Fischbach 2011) diskutiert werden und ihren bildungspolitischen Ausdruck in Programmen wie „Lernen vor Ort“ oder „Lernende Regionen“ finden (vgl. Bundesministerium für Bildung und Forschung 2015, 2008). Mit der ursprünglich aus der Sozialen Arbeit stammenden Grundidee sozialraumorientierter Arbeit wird von der Einzelfallorientierung abgerückt und die Lebenswelt der Betroffenen in den Handlungsfokus gestellt. Unterschiedliche Akteure - Bildungseinrichtungen, Gemeinden, Kommunen, Stadteile u.Ä. - sollen miteinander kooperieren, um die Lebenslagen der jeweiligen Betroffenen verbessern zu können. Vernetzungen über Fachgrenzen hinweg und ehrenamtliches Engagement sind zentrale Merkmale einer sozialraumorientierten Arbeit. Als wesentlich für die erfolgreiche Gestaltung einer Bildungslandschaft gilt Kooperation: Es sei notwendig, so Fischbach (2011), dass die multiprofessionellen Akteure eine „gleiche Sprache“ sprechen, ,um gemeinsame Ziele mit einer gewissen Verbindlichkeit $\mathrm{zu}$ formulieren und konkrete Handlungsschritte zu planen“ (Fischbach 2011: 201). Hier möchten wir ansetzen und unser Interesse darauf richten, wie Kooperationen von Erwachsenenbildungsmit anderen Einrichtungen gestaltet sind und inwieweit sie in welcher Art und Weise eine spezifische Kooperationspraxis konstituieren, die wiederum die Gestaltung von Lernkulturen rahmt. Unsere These ist, dass Erwachsenenbildungseinrichtungen im Rahmen der Debatte um Lernkulturen auf Bildung im Raum im Sinne ihrer institutionellen Handlungslogik, weniger im Sinne ihrer Programmatik rekurrieren. Oder anders: Der Zugriff auf Debatten um Lernkulturen folgt weniger einer pädagogischen als vielmehr einer institutionellen Logik.

Im Folgenden möchten wir den Blick auf das Projekt „Alphabetisierung im Stadtteil" einer städtischen Volkshochschule richten und zentrale Orientierungen der Kooperationspartner darstellen. Das Projekt „Alphabetisierung im Stadtteil" ist Teil der gemeinsamen nationalen Strategie für Alphabetisierung und Grundbildung Erwachsener vom Bundesministerium für Bildung und Forschung (BMBF) und der Kultusministerkonferenz (KMK) und wird mit Mitteln aus dem Europäischen Sozialfonds (ESF) gefördert. Konzeptionell wird von einer geringen Beteiligung an den Alphabetisierungskursen der 
Volkshochschule ausgegangen ${ }^{1}$, für die zwei Hauptgründe im Konzeptpapier angeführt werden: Angst vor Stigmatisierung und negative Lernerfahrungen (Schule). Diese Barrieren würden erst dann überwunden werden, wenn „eingeübte Kompensationsstrategien aufgrund von beruflichen oder privaten Krisen nicht mehr greifen und Bloßstellung droht." Damit wird an zentrale empirische Befunde der Forschung zum Analphabetismus angeschlossen (vgl. etwa Egloff 2007), wonach Analphabetismus nicht per se ein Alltagsproblem ist, das Betroffene im Rahmen von Erwachsenenbildung bearbeiten. Für notwendig erachtet werden Angebote, die über herkömmliche Alphabetisierungskurse hinausgehen, diese ,aufbrechen“ und mit denen die genannten Barrieren - Stigmatisierung und negative Lernerfahrungen - abgebaut werden können. Dazu wird „das“ Raumkonzept aufgegriffen: Alphabetisierungsarbeit soll dem Konzept zufolge räumlich und thematisch stärker an die Lebenswelt der Betroffenen anschließen, Anonymität gewährleisten und negative Assoziationen mit Schule vermeiden.

Kern des Projektes ist die Einrichtung sog. „Schreibstuben“ als niedrigschwellige Angebote. Hier sollen Betroffene sowohl Unterstützung beim Ausfüllen von Behördenbriefen erhalten als auch Lese- und Schreibfähigkeiten aufbauen und festigen. Insgesamt wurden vier Schreibstuben in verschiedenen Sozialeinrichtungen und unterschiedlichen Stadtteilen eingerichtet. Die Aktivitäten werden von einer pädagogischen Fachkraft koordiniert, die bereits viele Jahre als freiberufliche Dozentin Alphabetisierungskurse an der Volkshochschule durchführt. Geleitet wird das Projekt vom Leiter der Volkshochschule, der zudem den Bereich Grundbildung und Alphabetisierung verantwortet.

Im Rahmen der Evaluationsforschung konzentrieren wir uns primär auf Orientierungen der beteiligten Kooperationsakteure (vgl. zu dokumentarischen Evaluationsforschung Bohnsack/Nentwig-Gesemann 2010). Im Folgenden möchten wir fallbezogenen zeigen, inwieweit unterschiedliche Bedeutungen, die der Kooperationsgegenstand für die Interviewten hat, dieses Spannungsfeld (mit) konstituieren. Wir beziehen uns dabei auf Experteninterviews (vgl. Dörner 2012) und Gruppendiskussionen (vgl. Schäffer 2012a), die wir mit den Leitungen der beteiligten Einrichtungen geführt bzw. durchgeführt haben. Insgesamt haben wir während des laufenden Projektes neun Interviews und zwei Gruppendiskussionen mit verantwortlichen Akteuren sowie vier teilnehmende Beobachtungen in den Schreibstuben durchgeführt. Die Auswertung erfolgt mit Hilfe der dokumentarischen Methode (vgl. Schäffer 2012b), deren Kern die Rekonstruktion von Orientierungen ist. Durch Kontrastierungen verschiedener Interviews je Einrichtungen werden 
methodologisch abgesicherte institutionsbezogene Orientierungen über die der einzelnen Expert_innen hinaus für eine komparative Analyse der Kooperationsorientierungen zugänglich gemacht.

\section{Ein Kooperationsprojekt, unterschiedliche Kooperationsorientierungen}

Insgesamt haben wir es mit fünf Einrichtungen ${ }^{2}$ und institutionsbezogenen Orientierungen zu tun. Die Ergebnisse zeigen, dass der Dreh- und Angelpunkt des Projektes die Kooperation selbst ist, nicht das Projekt ,Schreibstube" bzw. die Grundbildungsarbeit. Auf der kommunikativen Ebene bewerten die Interviewten das Projekt als positiv und die Idee des Stadtteilbezugs als interessant. Die geringe Nutzung des Angebots verunsichert, wird allerdings von den Interviewten überwiegend in wohlmeinende Optimierungsvorschläge gewendet: Verbesserungswürdig sei die aktive Rolle der VHS als Projektträger, insbesondere müsse kontinuierlich über Stand und Entwicklung des Projektes informiert sowie Kontakt zu den Kooperationspartnern gehalten werden. Ausbaufähig seien die Öffentlichkeitsarbeit, die Ansprache der Zielgruppe sowie die Bewerbung der Schreibstube. Jenseits dieser grundsätzlich positiv konnotierten Bekundungen und Optimierungsideen zeigen sich Orientierungen, die die Kooperation in grundsätzlicher Weise formen bzw. zur Konstituierung einer Kooperationskultur beitragen und die die Bemühungen zur Verwirklichung der gewollten Lernkultur gewissermaßen unterlaufen bzw. eingrenzen.

\subsection{Analphabetismus: ein bedingt relevantes Thema bei den Kooperationspartnern}

Es fällt zunächst auf, dass Analphabetismus (und auch Alphabetisierung) bei den Kooperationspartnern der VHS erst im Rahmen des Kooperationsprojektes ein Thema wurde. Die Bilder, die sich die Kooperationspartner von Analphabet_innen und Analphabetismus machen, sind aus der öffentlichen Debatte gespeist und von habituellen Orientierungen geformt. Hinzu kommen vereinzelt Erfahrungen mit Personen, die in bestimmten Situationen vermuten lassen, nicht lesen und schreiben zu können. In einem Fall gibt eine Interviewpartnerin an, Analphabeten näher zu kennen. Speziell zum Thema Analphabetismus geschulte Personen gibt es in den Kooperationseinrichtungen nicht.

2 Die VHS als projektverantwortliche Institution kooperiert mit vier Einrichtungen aus dem Bereich der sozialen Arbeit. 
Wir haben es nicht mit professionellen Expertenverständnissen zu tun, sondern vielmehr mit Orientierungen von Personen, die als Expert_innen zu einem Thema angesprochen werden, für welches sie keine Expert_innen sind. Einziges Kriterium für die Auswahl der Einrichtungen ist deren institutionelles Selbstverständnis als soziale Dienstleister, in deren Klientel auch jene vermutet werden, die selbst zur Gruppe der Analphabet_innen gehören oder aber Analphabet_innen kennen. Da Analphabetismus und Alphabetisierung erst über das Projekt relevant wurde, möchten wir von projektbedingter Relevanz sprechen. Die bedingte Relevanz der Kooperationspartner rahmt die Zusammenarbeit mit der Volkshochschule in der Weise, als dass das Projekt in das eigene Angebot mit aufgenommen und bekannt gemacht wird und für die zur Verfügung gestellten Räume Mieteinnahmen zu verzeichnen sind - nicht mehr und nicht weniger. Als zentrales Charakteristikum zeigt sie sich zugleich in den institutionenbezogenen Orientierungen der interviewten Kooperationspartner in unterschiedlichen Ausprägungen und Formen.

\subsection{Sicherung und Behauptung der eigenen Angebote und Position: „für unsere Herangehensweise [...] zu werben"}

Für die Volkshochschule selbst dokumentiert sich eine Orientierung, wonach es um die Sicherung und Behauptung der eigenen Angebote und Position im Feld der Erwachsenengrundbildung geht. Konzeptionell wird die Schreibstube als Schulalternative betont - „,der funktionale Analphabet geht nicht wirklich gern zurück in die Schule ". Jedoch verliert dieses Argument in der Praxis an Gewicht. So äußert die Projektkoordinatorin die Hoffnung, über die stadtteilbezogene Arbeit Teilnehmer_innen für die herkömmlichen Grundbildungskurse der VHS zu gewinnen.

„Das ist ja auch ein Hintergedanke vielleicht, dass es doch einige gibt, die wenn sie den ersten Schritt gemacht haben und dann in unsere Schreibstube vielleicht Vertrauen gefasst haben [...] warum soll daraus nicht eine Gruppe hier wieder in der Schule wachsen?" (Koordinatorin VHS).

Anders stellt sich die Funktion des Alphabetisierungsprojektes für den VHSLeiter dar, für den es nicht nur darum geht, Öffentlichkeit herzustellen, Partner zu finden und davon zu überzeugen, wie sie vom Projekt profitieren können. Sein Interesse liegt in der Positionierung der eigenen Einrichtung im bundeslandspezifischen Feld der Erwachsenenbildung.

„meine Aufgabe ist es [dieses Projekt, Anm. d. Verf.] innerhalb der Volkshochschulen oder in der Erwachsenenbildungslandschaft in [Bundesland] zu vertreten und da eben auch für unsere Herangehensweise [...] zu werben“" (Leiter VHS). 
Es fällt auf, dass der Leiter die Erreichung der Zielgruppe kaum erwähnt. Vielmehr werden Didaktik, Organisation, Steuerung, Werbung und Legitimation angesprochen. Es dokumentiert sich eine erfahrungsgesättigte Haltung, wonach die Erreichung bzw. Gewinnung der Zielgruppe nicht nur ein ungewisses Spiel ist - „Und das ist nach wie vor ungewiss, wie stark das Interesse daran sein kann [...] wir brauchen sicherlich einen sehr sehr langen Atem." -, sondern vor allem ein hoffnungsloses. Dies zeigt sich recht eindrucksvoll an Stellen im Interview, bei denen durch Lachen dem Gesagten die Ernsthaftigkeit genommen wird.

„was wir halt viel stärker machen müssen ist zu organisieren (lacht), dass die Angebote auch die Adressaten erreichen und dass die dann auch diese Angebote wahrnehmen“ (Leiter VHS).

Wenn sich nicht erklärt werden kann, warum etwas nicht funktioniert, dann wird auf gängige und akzeptierte Scheinlösungen verwiesen: Man muss eben besser und mehr organisieren.

Wir haben es mit einer Orientierung zu tun, bei der Alphabetisierungsarbeit zumindest nicht von der Überzeugung getragen wird, erfolgreich zu mehr Beteiligung an Grundbildungskursen zu führen. Einige Äußerungen des Leiters verweisen darauf, dass das Projekt auch als Möglichkeit gesehen wird, sich im Feld der Alphabetisierungsarbeit gegenüber jenen Einrichtungen zu behaupten, die im Rahmen von EU-Förderprogrammen dieses Feld neu betreten.

„Es gibt ein [Gremium] auf Landesebene wo wir zwar nicht direkt drin sind, aber wo eben auch zu diesem Thema ein Austausch erfolgt und da jetzt viele verschiedene Projekte laufen“(Leiter VHS).

\subsection{Hauptsache kooperieren: „das Projekt ist schon zu Ende, aber der Kontakt ist erst mal da"}

Bei diesem Typus geht es vor allem um die Möglichkeit, mit der Volkshochschule überhaupt zu kooperieren, nicht aber um Alphabetisierungsarbeit. Für das beteiligte Altenservicezentrum ist die Volkshochschule wegen der Zielgruppe „Ältere Menschen“ von Interesse. „Die machen ja so verschiedene Kurse für Senioren “, so die Leiterin. Das Klientel-Motiv mag generell für solche Einrichtungen zutreffen, relevanter ist jedoch das Interesse, überhaupt mit der Volkshochschule zu kooperieren. Und zwar auch vor dem Hintergrund der Einschätzung, dass die Zusammenarbeit mit anderen Einrichtungen der Gemeinwesenarbeit, dem eigentlichen Bereich der Einrichtung, im Stadtviertel insgesamt nachgelassen habe und schwieriger geworden sei. 
„Wir versuchen immer wieder [...] mit verschiedenen Einrichtungen Kontakt aufzubauen [...] Es ist sehr schwer. Und irgendwann gibt man dann auch mal auf, wenn man merkt, es kommt nichts zurück, weil man auch keine Kraft mehr hat, ja. [...] viele Einrichtungen sind auch einfach von ihren eigenen Aufgaben dermaßen zugeschossen, dass die den Kopf nicht wirklich frei haben für andere Sachen“ (Leiterin Altenservicezentrum).

Die Projektkooperation mit der Volkshochschule als Bildungseinrichtung ist dann nicht nur hinsichtlich der Erweiterung eigener Angebote relevant, sondern überhaupt als Möglichkeit bzw. erster Schritt zur Zusammenarbeit mit einer bereichsfremden Einrichtung, die wiederum die Position im eigenen Professionsfeld stärkt.

\subsection{Institutionelle Handlungsexpansion: „Wir versuchen ja auch diesen Ansatz"}

Hingegen geht es beim Typus Handlungsexpansion primär um Kooperation als Möglichkeit zur Erweiterung des eigenen Handlungsfeldes um den Bereich Bildung. So möchte das beteiligte Familienzentrum seinen eigentlichen Aufgabenbereich Familienhilfe um den der Familienbildung erweitern. Das Problem Analphabetismus ist bekannt, wird aber als Randbereich der eigenen Arbeit gesehen, als Phänomen, mit dem man irgendwie umgehen muss, etwa durch Hilfen beim Ausfüllen von Formularen. Das Projekt der Volkshochschule ist insofern von Bedeutung, als dass die Einrichtung seit etwa zwei Jahren ein breites Spektrum von Veranstaltungen im Bereich Familienbildung anbietet - von der „Babymassage“ bis zum „Wohnen im Alter“. Ferner engagiert sie sich mit öffentlichen, familienpolitischen Diskussionsrunden. Deutlich werden zwei Orientierungen: Die Expansionsorientierung ist kommunikativ von einer Idee- und Programm-Solidarität (s.u.) gerahmt. Die „Schreibstube“ wird als zusätzliches Bildungsangebot und zudem als passend zur eigenen Maxime verstanden, jene Menschen ,niedrigschwellig“ anzusprechen, die gemeinhin als bildungsfern gelten.

„,fanden die Idee sehr gut, an Orte zu gehen, wo es für für Betroffene einfacher ist, vielleicht den Zugang zu finden, als die Volkshochschule [...] Wir versuchen ja auch diesen Ansatz Familienbildung so zu gestalten [...], dass es Menschen nicht so schwer fällt, an Familienbildungsveranstaltungen teilzunehmen [...] Das sind auch Menschen, die würden nie einen Kurs für Pädagogik in der Volkshochschule besuchen“" (Leiter Familieneinrichtung). 


\subsection{Programm-Solidarität vs. Projektskepsis: „Finde das Projekt sehr gut, aber ..."}

Beim Typ Programm-Solidarität vs. Projektskepsis ist die Kooperation bei aller Skepsis gegenüber dem Projektanliegen Ausdruck einer solidarischen Haltung gegenüber Bildungseinrichtungen, die sich um gesellschaftlich tabuisierte Gruppen bemühen und dafür neue Wege gehen. Begrüßt wird, dass die Volkshochschule sich mit ihrem Bildungsangebot „Schreibstube“ in die soziale Stadtteilarbeit bewegt. Damit würdige sie Sozialarbeit und jene Einrichtungen, die in diesem Bereich tätig sind. Wenn die Volkshochschule sagt, dass Alphabetisierungskurse aufgrund ihres Schulcharakters nur in geringem Maße von Analphabet_innen genutzt werden bzw. diese abschrecken, dann wird anerkannt, dass sich die VHS davon nicht abhalten lässt, (a) weiterhin Alphabetisierungsarbeit zu leisten, also niemanden aufzugeben, und (b) neue Wege zu gehen.

„Dieser Ansatz jetzt mal aus dieser Volkshochschule aus dem Kurs raus $\mathrm{zu}$ gehen und zu sagen, wir gucken mal, dass wir das probieren, die Leute dazu animieren so was zu machen [...] das ist schon mal ganz gut“ (Gruppediskussion Familieneinrichtung).

Letztlich sehe man sich am Projekt zwar beteiligt, für dessen Gelingen aber nicht verantwortlich. Im Gegenteil: Vom Gelingen ist man auch nicht überzeugt. Ähnlich wie bei den Anonymen Alkoholiker_innen, die nur einen geringen Teil der trockenen Alkoholiker_innen ausmachten, würde man auch bei den Analphabet_innen immer nur wenige erreichen.

\subsection{Kompetenz-Lob: „es gibt ja auch viele andere Einrichtungen, die man hätte fragen können"}

Schließlich haben wir es beim Typ Kompetenz-Lob mit einer Orientierung darauf zu tun, wie die eigene Einrichtung im kommunalen Feld der sozialen Dienstleister und Bildungseinrichtungen wahrgenommen wird. Die Leiterin einer Wohlfahrtseinrichtung sieht sich durch die Kooperationsanfrage der Volkshochschule als kompetenter und wirklicher Partner ernstgenommen. Es dokumentiert sich eine auf Außenwahrnehmung orientierte Handlungsfacette, deren negativer Gegenhorizont schlimmstenfalls das Gefühl ist, von anderen Einrichtungen ignoriert zu werden. Im Falle der Ansprache sieht sie sich und ihre Einrichtung als Auserwählte unter anderen.

„Ich habe mich gefreut, also, dass Herr $\mathrm{X}$ uns als Partner raussucht und denkt, dass wir kompetente Partner sind für die Arbeit. [...] Naja, es zeigt ja auch Vertrauen für die Zusammenarbeit und wir arbeiten ja auch beim Bereich Familienpaten auch zusammen und dann hat man das Ge- 
fühl, das bewährt sich. Man hat auf der Arbeitsebene einen guten Kontakt und es gibt ja auch viele andere Einrichtungen, die man hätte fragen können, vielleicht [...] Ich habe mich gefreut? (lacht) Ist ein gutes Gefühl." (Leiterin Wohlfahrtseinrichtung).

Auf eine Besonderheit im Vergleich zu den anderen Kooperationseinrichtungen möchten wir an dieser Stelle hinweisen: Wir haben es zudem mit einer Orientierung zu tun, deren Erfahrungsbasis ein Minimum an bewährter Praxis und Vertrauen in den Kooperationspartner VHS ist. Vertrauen und Bewährung in diesem Fall kamen in einem Projekt zustande, in dem beide Einrichtungen als teilnehmende Partner mitwirkten. Im Grundbildungsprojekt wirkt die VHS als leitender, die Wohlfahrtseinrichtung als teilnehmender Kooperationspartner. Die Erwartungen der teilnehmenden an die leitende Einrichtung sind deutlich auf Verantwortung und Steuerung gerichtet, nicht auf Mitwirken im Rahmen der eigenen institutionellen Möglichkeiten. Insofern unterscheidet sich die Wohlfahrtseinrichtung von den anderen Einrichtungen in einem Punkt. Aufgrund der bewährten und vertrauten Zusammenarbeit in früherer Zeit werden die institutionellen Möglichkeiten nicht nur darauf begrenzt, Räume zur Verfügung zu stellen (für die man auch Miete nimmt) und als Multiplikator zu agieren, sondern auch auf inhaltlicher Ebene gesehen. Es geht gewissermaßen darum, auch interprofessionell zusammen zu arbeiten, um dann nicht nur als kompetenter Partner angesprochen zu werden, sondern auch als wirklicher Partner.

„Aber da würde ich schon sagen, dass die Volkshochschule auch uns mehr in Anspruch nehmen könnte. Ich denke, die Zusammenarbeit ist so gut, dass sie sagen könnte: ,Das und das haben wir vor. Könnt ihr euch in irgendeiner Form mit beteiligen?‘ [...] Ist jetzt auch, wenn wir jetzt sagen, das ist ja jetzt nicht unsere Hauptbaustelle, aber wenn dann ein Partner, ein wirklicher Partner einen um Hilfe bittet, sagt man ja auch nicht nein. Wenn es jetzt nicht zwanzig Stunden sind oder so, aber ja, das was man machen kann, macht man schon." (Leiterin Wohlfahrtseinrichtung).

\section{Fazit: Modulierte Kooperation}

Insgesamt ist das Ergebnis des Grundbildungsprojektes ein Kursangebot „Schreibstube“, das zwar in den Einrichtungen stattfindet, jedoch keine handlungspraktische Einbindung in die vorhandenen Strukturen erfährt. Der Stadtteilbezug wird stadtteilplanerisch, nicht aber auch sozialräumlich im pädagogischen praktiziert. Die Schreibstuben sind in ihrer praktischen Ausformung ein VHS-Kurs en miniature in einem bestimmten Stadtteil - mit dem Unterschied, dass die VHS mit ihrem traditionellen Angebot bekannt ist und die Schreibstuben bisher nicht. 
Wir haben es mit einer Kooperationspraxis zu tun, bei der die beteiligten Einrichtungen ihrer eigenen Institutionenlogik so sehr verhaftet sind, dass das eigentliche Projektanliegen - der Aufbau einer kooperativen, stadtteilbezogenen Grundbildungsstruktur - nicht in das handlungsrelevante Repertoire der Einrichtungen integriert werden kann. Bei aller Programm- und Ideensolidarität, deren Kern die Ansprache von Randgruppen mit Hilfe alternativer Angebote ist, sehen die Akteure ihre begrenzten institutionellen Möglichkeiten und verweisen ausdrücklich auf ihre Verantwortungsgrenzen. Gemeinsam ist dabei allen Kooperationseinrichtungen die Betonung eines eigenen Hauptarbeitsfeldes, das nicht Alphabetisierungsarbeit heißt: Alphabetisierung und das stadtteilbezogene Grundbildungsprojekt sind von projektbedingter Relevanz. Die VHS hingegen kooperiert mit den Einrichtungen, um dadurch die Gewinnung der Zielgruppe zu gestalten. Die Einbindung ist mit dem Kooperationsschluss scheinbar ausreichend gegeben. Darüber hinaus wird aber auch deutlich, dass mit dem Projekt Möglichkeiten ausgelotet werden, die Position der eigenen Einrichtung im kommunalen Feld auszubauen und zu stärken. Das VHS-Projekt wird als Möglichkeit gesehen, im Spiel um kommunale und stadtteilbezogene Legitimation zu sein und zu bleiben. Die VHS als städtische Einrichtung übernimmt eine Brückenfunktion zur Erschließung neuer Angebotsformate im Interesse der eigenen Adressaten- und Teilnehmergruppen sowie zur Erschließung neuer Spielfelder, auf denen verschiedene kommunale Akteure zusammen- und aufeinandertreffen, um die eigenen Handlungsspielräume unter je spezifischen, sich verändernden Bedingungen und Gegebenheit zu gestalten und zu verbessern. Im Goffman'schen Sinne haben wir es mit Modulationen der Kooperation zu tun (vgl. Goffman 1980: 54): Die beteiligten Partner spielen zwar äußerlich ein und dasselbe bzw. ein sinnvolles Spiel, transferieren es jedoch in etwas, das dem Spiel nur nachgebildet ist, indem sie es als etwas ganz anderes sehen bzw. rahmen. Wir haben es mit einer Debatte zu tun, deren diskursive Kraft derart ausgeprägt ist, das Institutionen auf die Debatte handlungs- bzw. projektleitend Bezug nehmen, deren Kraft jedoch dort endet, wo sie in die jeweiligen institutionellen Eigenlogiken gerät. In Bezug auf Kooperation als Kernelement stadteilbezogener Grundbildungsarbeit und die Anforderung einer gemeinsamen Sprache der beteiligten Kooperationspartner ist Skepsis angebracht. Zu prüfen wäre, inwieweit bspw. eine rationale Kommunikation, wie sie etwa von Tippelt (2010) vorgeschlagen wird, eine Möglichkeit wäre, wie Kooperationen zwischen Erwachseneneinrichtungen und Nicht-Erwachseneneinrichtungen gestaltet werden können. 


\section{Literatur}

Bohnsack, Ralf/Nentwig-Gesemann, Iris (Hrsg.) (2010): Dokumentarische Evaluationsforschung. Theoretische Grundlagen und Beispiele aus der Praxis. Opladen/ Farmington Hills: Barbara Budrich.

Bundesministerium für Bildung und Forschung (2008): Lernende Regionen Förderung von Netzwerken. Bonn/Berlin.

Bundesministerium für Bildung und Forschung (2015): http://www.lernen-vorort.info/121.php [Zugriff 20.9.2015].

Dörner, Olaf/Liebe, Ralf (2014): ,Die‘ Volkshochschule als Bildungsort mit hohem Anspruch. Zu hoch? In: Dippelhofer-Stiem, B./Dippehofer, S. (Hrsg.): Enzyklopädie Erziehungswissenschaft Online. Erziehungs- und Bildungssoziologie. Bad Heilbrunn: Beltz Juventa, S. 1-24.

Dörner, Olaf (2012): Experteninterviews. In: Schäffer, B./Dörner, O. (Hrsg.): Handbuch Qualitative Erwachsenen- und Weiterbildungsforschung. Opladen, Berlin \& Toronto: Barbara Budrich, S. 321-333.

Egloff, Birte (1997): Biographische Muster „funktionaler Analphabeten“. Eine biographieanalytische Studie zu Entstehungsbedingungen und Bewältigungsstrategien von ,funktionalem Analphabetismus“. Bonn: Deutsches Institut für Erwachsenenbildung (DIE).

Fischbach, Robert (2011): Bildungslandschaften. Reflexionskategorien und ihre professionspraktischen Konsequenzen. In: Der pädagogische Blick 19, 4, S. 196-205.

Goffman, Erving (1980): Rahmen-Analyse. Ein Versuch über die Organisation von Alltagserfahrungen. Frankfurt/M.: Suhrkamp.

Gogolin, Ingrid/Kuper, Harm/Krüger, Heinz-Hermann/Baumert, Jürgen (2013): Stichwort: Zeitschrift für Erziehungswissenschaft. Wiesbaden: Springer VS.

Huntemann, Hella (2014): Volkshochschul-Statistik 2013 - Zahlen in Kürze. www.diebonn.de/doks/2014-volkshochschule-statistik-02.pdf [Zugriff: 14.07.2016].

Meyer, Meinert A.(2013): Alter oder neue Lernkultur? In: Zeitschrift für Erziehungswissenschaft, S. 223-249.

Nohl, Arnd-Michael (2006): Konzepte interkultureller Pädagogik. Eine systematische Einführung. Bad Heilbrunn: Klinkhardt.

Schäffer, Burkhard (2012): Dokumentarische Methode. Einordnung, Prinzipien und Arbeitsschritte einer praxeologischen Methodologie. In: Schäffer, B./Dörner, O. (Hrsg.): Handbuch Qualitative Erwachsenen- und Weiterbildungsforschung. Opladen/Berlin/Toronto: Barbara Budrich, S. 196-211.

Schäffer, Burkhard (2012): Gruppendiskussionsverfahren und Focus Groups. In: Schäffer, B./Dörner, O. (Hrsg.): Handbuch Qualitative Erwachsenen- und Weiterbildungsforschung, Opladen/Berlin/Toronto: Barbara Budrich, S. 347-362.

Schüßler, Ingeborg/Thurnes, Christian M. (2005): Lernkulturen in der Weiterbildung. Bielefeld: W. Bertelsmann.

Tippelt, Rudolf (2010): Institutionenforschung in der Erwachsenenbildung/Weiterbildung. In: Tippelt, R./Hippel, A. v. (Hrsg.): Handbuch Erwachsenenbildung/Weiterbildung. Wiesbaden: VS Verlag für Sozialwissenschaften, S. 453-471.

Wittpoth, Jürgen (2003): „Lernkulturen“ einst und jetzt. In: Brödel, R./Siebert, H. (Hrsg.): Ansichten zur Lerngesellschaft. Festschrift für Josef Olbrich. Baltmannsweiler: Schneider-Verl. Hohengehren, S. 155-164. 



\section{Regionale Lehr- und Lernkulturen ländlicher Erwachsenenbildung. Ergebnisse einer explorativ- rekonstruktiven Forschungsarbeit}

\section{Einführung}

In den Diskursen um Lehr- und Lernkulturen wurde anfänglich der Blick vorwiegend normativ auf die Entwicklung neuer Lernkulturen gerichtet (vgl. Arnold/Schüßler 1998; Schüßler in diesem Band). Damit verbunden war das Anliegen, eine Entwicklung von einer lehr- zu einer lernerzentrierten Lernkultur anzuregen bzw. einen Wechsel von einer Erzeugungs- zu einer Ermöglichungsdidaktik (vgl. Arnold/Schüßler 2015).

Forschungen zu Lehr- und Lernkulturen in pädagogischen Organisationen weisen darauf hin, dass diese als performativ erzeugte soziale Praxen des Lehrens und Lernens verstanden werden können, die symbolische Ordnungen schaffen (vgl. Helsper 2008). Lehrkulturen entstehen dabei in einer Praxisgemeinschaft von Lehrenden und prägen ihre Orientierungen (Hargreaves 1994; Pratt/Nesbit 2000), während Lernkulturen in der Interaktion in Lehrund Lernsituationen emergieren (vgl. James et al. 2007; Nolda 1996; Kade et al. 2014). Die jeweilige pädagogische Organisation wird dabei als ein ,institutional frame““ (vgl. Nesbit 1998) oder als prägender Kontext (Franz 2016a; zu Schulkulturen siehe Helsper 2008) betrachtet, auf den Träger- und Verbandsstrukturen bzw. -kulturen einwirken (vgl. Fleige 2011), die wiederum Lehr- und Lernkulturen indirekt beeinflussen können.

Inwiefern der „Raum“ als Kontextbedingung von pädagogischen Organisationen für die Herausbildung spezifischer regionaler Lehr- und Lernkulturen eine Rolle spielt, ist bislang empirisch nicht hinreichend geklärt. In der empirischen Forschung werden im Hinblick auf den Raum vorwiegend regionale Differenzierungen im Weiterbildungsangebot und im Weiterbildungsverhalten herausgearbeitet (vgl. Martin u.a. 2015; Weishaupt/Böhm-Kasper 2011). Dabei wird mitunter deutlich, dass der Bereich Allgemeiner Erwachsenenbildung in städtischen Gebieten stärker ausgebaut ist als in urbanen Regionen, während ländliche Regionen stärker mit demographischen und strukturellen Herausforderungen konfrontiert erscheinen (vgl. Klemm 2015; Universität Trier/LEB/asw e.V. 2007). Dem ländlichen Raum wird zudem in entsprechenden Diskussionen der Erwachsenenbildung - zumindest konzeptionell - eine relative Besonderheit zugeschrieben. Diese äußere sich in einer intensiven Form des sozialen Zusammenlebens, spezifischen traditionellen Wertvorstellungen und einer Nähe zur Landschaft (vgl. Faber 1989; Ritchey 
2008). Gerade im Kontext einer zunehmenden Bedeutung räumlicher Kategorien in der Erziehungswissenschaft und Erwachsenenbildung (vgl. Nugel 2014, 2016; Bernhard u.a. 2015) und der Diskussion um regionale Bildungslandschaften (vgl. Fischbach 2011) stellt sich die Frage, ob sich eine relative Besonderheit des ländlichen Raums auch in Organisationen ländlicher Erwachsenenbildung zeigt und auf ihre Lehr- und Lernkulturen einwirkt. Im Anschluss an dieses Forschungsdesiderat wird in diesem Beitrag die Frage verfolgt, woran sich erwachsenenpädagogische Bildungsanbieter in ländlichen Regionen orientieren, wenn sie Bildungsveranstaltungen anbieten und welche Lehrkulturen ${ }^{1}$ sich dabei andeuten. Dazu wird zunächst das methodische Vorgehen der Studie beschrieben, bevor die empirischen Ergebnisse anhand von zwei Fallbeispielen ländlicher Erwachsenenbildung vorgestellt werden. Abschließend erfolgt eine Diskussion zur Ausdifferenzierung regionaler Lehrkulturen in der Erwachsenenbildung.

\section{Methodisches Vorgehen}

Die Fragestellung nach kollektiven Lehrorientierungen in Organisationen ländlicher Erwachsenenbildung wurde im Rahmen einer Reanalyse von Daten bearbeitet, die aus dem Kontext einer umfangreicheren qualitativ-rekonstruktiven Studie zum „Lehren in Organisationen der Allgemeinen Erwachsenenbildung" stammen (vgl. Franz 2016a).

In der Datenerhebung der zugrundeliegenden Studie wurde darauf abgezielt, Einblicke in die konjunktiven Erfahrungsräume (vgl. Mannheim 1980) von Organisationen Allgemeiner Erwachsenenbildung zu erhalten. Dazu wurden in jeder Organisation leitfadengestützte Interviews mit Leitungen sowie je eine Gruppendiskussion (vgl. Loos/Schäffer 2001) mit hauptamtlich pädagogischen Mitarbeitenden, freiberuflichen Kursleitenden und Verwaltungskräften geführt.

Das Sample wurde nach dem Prinzip des ,theoretical sampling“ (vgl. Glaser/Strauss 1967) aufgebaut und beinhaltet neun Organisationen der Allgemeinen Erwachsenenbildung, in unterschiedlicher Trägerschaft, Angebotsstruktur und räumlicher Situierung.

Die Auswertung des Datenmaterials erfolgte mit Hilfe der dokumentarischen Methode (vgl. Bohnsack et al. 2007), mit der es möglich wird, implizite von expliziten Wissensbeständen zu unterscheiden. In mehreren komparativen Interpretationsschritten wurde darauf abgezielt, Aspekte des impliziten organisationalen Erfahrungsraums zum Lehren zu erforschen. Dazu wurden in jedem Fall die Orientierungen der hauptamtlich pädagogischen

1 In diesem Beitrag wird der Begriff der Lehrkultur verwendet, da Organisationen und damit die Anbieterseite in den Blick genommen werden. 
Mitarbeitenden und der freiberuflichen Kursleitenden durch formulierende und reflektierende Interpretationen rekonstruiert und mit den Perspektiven der Leitungen und Verwaltungskräfte in Beziehung gesetzt. Im Rahmen der Studie konnten durch eine mehrdimensionale Typenbildung (vgl. Nohl 2013) vier organisationale Lehrorientierungen herausgearbeitet werden (vgl. Franz 2016a). Diese entstehen in der Relation von drei Orientierungsdimensionen. Dies sind die normative Grundlage der Bildungsarbeit, das Verständnis von Lehr- und Lernprozessen im engeren Sinne und die Wahrnehmung des umgebenden Umfelds der Einrichtungen für das Lehren.

Anknüpfend an diese Ergebnisse wird in einer aktuellen Anschlussstudie ${ }^{2}$ das bestehende empirische Material reanalysiert. Hier wird unter anderem die Frage bearbeitet, ob und inwiefern die Wahrnehmung des umgebenden Raums Einfluss auf das Lehren nimmt. In diesem Beitrag wird daher der Blick explizit auf die Organisationen ländlicher Erwachsenenbildung im Sample gerichtet und danach gefragt, inwieweit sich eine ,relative Besonderheit" des ländlichen Raums auch in den Lehrorientierungen ländlicher Organisationen niederschlägt.

\section{Ergebnisse: Fallbeispiele ländlicher Erwachsenenbildung}

In der Reanalyse wurde untersucht, woran sich Organisationen ländlicher Erwachsenenbildung orientieren und welche Lehrkulturen dabei sichtbar werden. Gemeinsam ist ländlichen Organisationen der Erwachsenenbildung im Sample, dass hier die Wahrnehmung des umgebenden Umfeldes eine große Rolle spielt. Der Blick wird nach außen gerichtet und das eigene Lehrangebot daraufhin angepasst und entfaltet. In dieser Gemeinsamkeit lassen sich im Material zwei unterschiedliche Perspektiven differenzieren. Diese werden im Folgenden anhand von Fallbeispielen beschrieben.

\section{Marktorientierte Perspektive ländlicher Erwachsenenbildung}

Eine marktorientierte Perspektive ländlicher Erwachsenenbildung lässt sich am Fallbeispiel der Organisation E herausarbeiten. Organisation E ist eine Einrichtung in öffentlicher Trägerschaft und zeichnet sich dadurch aus, dass sie in einer ländlichen, strukturstarken Region in der Peripherie einer Groß-

2 Für die Förderung dieses Anschlussprojekts ,Formen der Ausdifferenzierung in Organisationen Allgemeiner Erwachsenenbildung. Zwei qualitativ-rekonstruktive Reanalysen zu kollektiven Orientierungen im Hinblick auf Mitarbeiter- und Raumformationen " (FR 2716/2-2) bedanke ich mich herzlich bei der Deutschen Forschungsgemeinschaft (DFG). 
stadt situiert ist. Sie verfügt über eine zentrale Geschäftsstelle und mehrere Außenstellen, um das inhaltlich breit gefächerte Angebot in der Region bereitzustellen. Die Lehrorientierung dieser Organisation setzt sich wie folgt zusammen.

- Wahrnehmung des umgebenden Umfelds: Das umgebende Umfeld wird in dieser Einrichtung als Weiterbildungsmarkt wahrgenommen. Dies zeigt sich daran, dass alle Akteure immer wieder ihre eigenen innovativen Angebote betonen. Dabei blicken sie aus einer vergleichenden Perspektive auf das Umfeld und betrachten die Angebote anderer Einrichtungen in der Region um - wie es die Leitung formuliert - „Benchmarks“ zu setzen. Auch in der Diskussion der Hauptamtlichen wird die eigene Situation im Vergleich zur Bildungssituation in der Stadt beschrieben. Dadurch, dass sie „,vor Ort“ wären, hätten sie einen Standortvorteil, da sich gute Angebote schnell herumsprechen würden. Das „Vor Ort“-Sein wird marktstrategisch interpretiert und der Vergleich mit anderen Einrichtungen zur eigenen Positionierung auf einem Weiterbildungsmarkt genutzt. Durch diese Interpretation werden mögliche Spezifika hinsichtlich der Teilnehmendenstruktur oder geographische und soziostrukturelle Aspekte im regionalen Umfeld kaum wahrgenommen.

- Normative Fundierung: Dieser marktorientierte Blick zeigt sich auch im Hinblick auf die normative Fundierung der Lehrorientierung. Während im Leitbild die Perspektive offen für alle zu sein expliziert wird, zeigt sich in den Gesprächen mit den Akteuren, dass diese sich nicht an einer partizipatorischen, sondern eher an einer formalen normativen Grundlage orientieren. So betonen Leitungen und Verwaltungskräfte, dass es Ziel der Einrichtung sei, innovativ zu sein, Trends zu erkennen und zu setzen. Dies zeigt sich beispielsweise in der Diskussion mit den hauptamtlich pädagogischen Mitarbeitenden.

Dw (.) also da sieht man einfach wie die Themen also wir sind irgendwie mehr so Vorreiter. hab ich so den Eindruck; ich muss immer gucken also was is jetzt hier neu, wo geht ne Entwicklung hin, dass ich halt was biete was die sonst noch nich kriegen [...]

Aw aber ich glaub des können wer alle hier bestätigen weil wir alle schon sehr lange hier sind und des spiegelt den Wandel der Gesellschaft wieder;

Dw

$L_{\text {mhm }}[\ldots]$

Em wir sind als Einrichtung viel mehr in der Lage schnell zu reagieren; grade die staatlichen behördlichen (.) Gremien des dauert ja ätzend lange bis da mal was in Bewegung kommt, da sind wir viel $\underline{\text { schneller }}$

Organisation E, HPM Z. 901-926, Passage: Vorreiter

In dieser Passage zeigt sich, dass die Gruppe die Einrichtung als Vorreiter versteht, die Angebote an neue Entwicklungen, die hier im Sinne von Trends 
verstanden werden, schnell anpassen kann. Deutlich wird, dass es nicht darum geht, normativ für die Region bedeutsame Themen zu setzen, sondern darum, auf der Höhe der Zeit zu agieren und den Markt zu bedienen. Daher wird hier der Wandel der Gesellschaft marktstrategisch interpretiert.

- Lehr- und Lernprozesse: Entsprechend werden in dieser Einrichtung Lehr- und Lernprozesse als selbstverständlicher, aber im Vergleich zur Planung von innovativen Angeboten nachgeordneter Bereich gefasst. Danach gefragt, wie ein gelungenes Seminar aussehen sollte, betont die Leitung, dass die Teilnehmenden vor allem Spaß haben sollten. Es geht darum, zufriedene Kunden zu gewinnen und zu halten. In der Diskussion mit den hauptamtlich pädagogischen Mitarbeitenden wird hervorgehoben, dass die Frage, wie gelehrt werden solle, erst nach der Frage beantwortet werden könne, um was es eigentlich gehen solle und ob es in der Region bei den potenziellen Teilnehmenden Anklang finden würde. Erst danach würden sie sich überlegen, wie ein Lehrangebot gestaltet werden könne. Dabei wird in der Diskussion eine Hierarchisierung zwischen makrodidaktischer Programmplanung und mikrodidaktischer Lehrpraxis deutlich: Lehren wird als Selbstverständlichkeit im Modus der Aus- und Durchführung betrachtet, während die Planung von Angeboten in den Vordergrund gerückt wird.

Die am Beispiel des Falles der Organisation E herausgearbeitete marktorientierte Perspektive zeichnet sich zusammenfassend dadurch aus, dass das umgebende Umfeld als Markt betrachtet wird und es normativ und didaktisch darum geht, zeitgemäße und innovative Angebote bereitzustellen, mit denen die Wünsche der Kunden befriedigt werden. Mit dieser Perspektive werden regionale Strukturbedingungen oder die Hintergründe der Teilnehmenden für diese Organisation kaum beobachtbar.

\section{Versorgungsorientierte Perspektive ländlicher Erwachsenenbildung}

Eine zweite Perspektive ländlicher Erwachsenenbildung lässt sich als versorgungsorientiert beschreiben und kann anhand der Organisationen F und G herausgearbeitet werden. Beide Organisationen stehen in öffentlicher Trägerschaft. Sie sind für die Bildungsarbeit in einer großen geographischen Fläche zuständig und jeweils in einer ländlichen, strukturschwachen Region situiert. Die inhaltlichen Angebote sind breit gefächert. In Organisation G werden zudem längerfristige berufliche Qualifizierungen angeboten.

- Wahrnehmung des Umfelds: Anders als in Organisation E wird von den Organisationen F und $\mathrm{G}$ das umgebende Umfeld als sozialer Raum verstanden. Dies zeigt sich in der Art und Weise, wie die Region beobachtet wird. Das regionale Umfeld wird insbesondere von Organisation G vor dem Hin- 
tergrund sozialer und demographischer Entwicklungen betrachtet. So sei die Region durch eine hohe Arbeitslosenquote sowie durch Abwanderungsbewegungen jüngerer Generationen gekennzeichnet. In dieser Situation nehmen die einzelnen Akteure die als schwierig eingeschätzten sozioökonomischen Hintergründe der Teilnehmenden differenziert wahr. Für die eigene Bildungsarbeit bedeutet dies ganz selbstverständlich mit anderen Institutionen der Region - wie Vereinen, Kommunen, Betrieben, Arbeitsagenturen - zusammenzuarbeiten, um so für die Teilnehmenden ein Versorgungsnetz bereitzustellen.

Bm naja und wenn mans wenn mans insgesamt sieht, Bildungsveranstaltungen
entstehen eigentlich nur eh auf entsprechenden Bedarfen, (.)das sind zum
einen hier jetzt was die Aw gesagt zum einen (.) die Bedarfe in den Vereinen
und Gruppen, beziehungsweise auch die Interessenlagen, die dort ne Rolle
spielen, wie und wo so ne Veranstaltung durchgeführt wird, zum anderen
spielen Interessen von Institutionen ne Rolle, sprich also jobcenter oder
Agentur für Arbeit; die dann ma sagen hier könntet ihr nich schnell ma ne
Bildungsmaßnahme in dem und dem Bereich aufbauen; wo mer sagen, (.)die
sollen das und das lernen, sollen praktisch meinetwegen (.) äh mit nem äh
verstärkten öh Selbstbewusstsein rausgehen, mit (.) kommunikativen
Fähigkeiten, die meistens (.) ein Vermittlungshemmnis darstellen heutzutage,
bei den Leuten die jetzt noch im Hartz 4 sind, des muss ma wirklich so sagen;
dort ham wers ja nich me:hr (.) wie vor 567 Jahren mit Leuten zu tun, die (.)
äh sag mer ma ein Hemmnis hatten, sondern heute ham wir ja multiple (.)
Vermittlungshemmnisse, (.) äh wo man sagen muss da kommt zum Alkohol
vielleicht dann noch ä:h das Analphabetentum dazu, äh des wär jetz der
Extremstfall; ne, also; aber ham wer auch schon gehabt.
Aw Lham wer auch schon
Bm Lham
auch schon gehabt (.)
Organisation G, HPM Z. $312-314$, Passage: Bedarf

Die Mitarbeitenden heben hier hervor, sich an regionalen Bedarfen zu orientieren. Während die allgemeine Bildungsarbeit in Kooperation mit Vereinen stattfindet, geht es bei der beruflichen Bildung um die konkrete Anfrage von Vermittlungsagenturen. Implizit wird dabei deutlich, dass diese Bedarfsorientierungen als Möglichkeit der Stärkung sozialer Kohäsion gesehen werden. Dies wird mitunter auch damit zum Ausdruck gebracht, wenn davon gesprochen wird, auch schwer vermittelbare Personen ein Stück ,weiter zu bringen".

Andere regionale Akteure werden als Partner betrachtet, mit denen soziale Strukturen der Region aufrechterhalten werden können. In Organisation F 
wird diese partnerschaftliche Perspektive auch als Chance für organisationale Lernprozesse gedeutet.

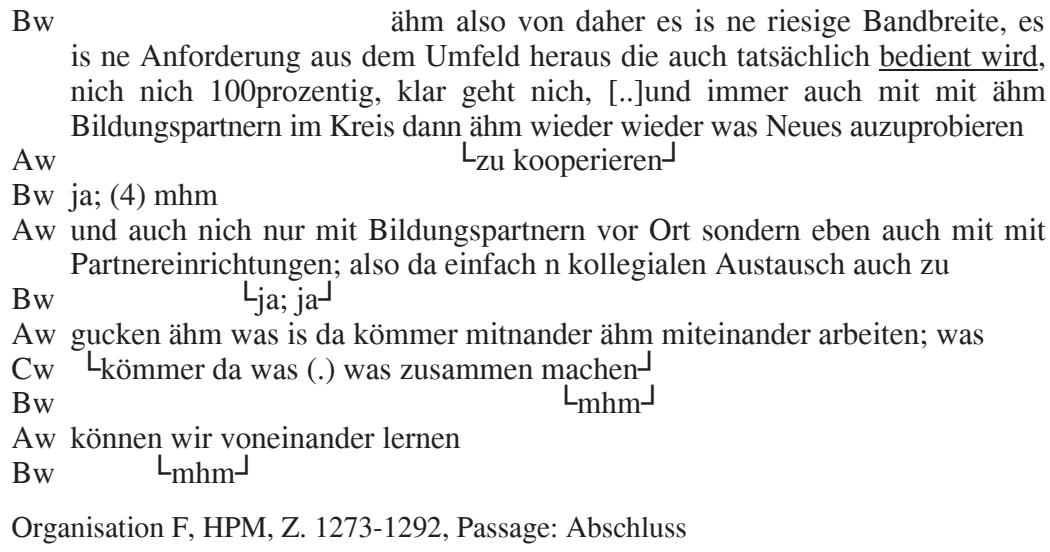

- Normative Fundierung: Mit Blick auf die normative Fundierung der Lehrorientierung zeigt sich, dass in Organisation F der eigene Bildungsauftrag thematisiert wird, mit dem Bildungsangebot in die Fläche zu wirken und die Region zu versorgen. Damit verbunden ist - wie insbesondere bei Organisation G sichtbar wird - die Orientierung, durch Bildungsarbeit die Sozialstruktur der Region zu stärken und die Teilnehmenden zu unterstützen, wie im Interview mit der Leitung deutlich wird. Diese spricht davon, dass insbesondere der soziale Zusammenhalt in der Region gestärkt werden müsse, da es kaum noch informelle Treffpunkte (wie den Einkaufsladen, das Lokal oder den Bäcker) gäbe. Daher nehme die Bildungsarbeit die Funktion ein, eine Sozialstruktur bereitzustellen. In den berufsqualifizierenden Angeboten wird sich normativ zudem an der Vermittlung zentraler Beschäftigungskompetenzen wie Team- und Arbeitsfähigkeit orientiert. Zusammenfassend zeigt sich im Gegensatz zur marktorientierten Perspektive ländlicher Erwachsenenbildung, dass Bildungsarbeit ausgehend von der Unterstützung von Teilnehmenden her gedacht wird.

- Lehr- und Lernprozesse: Entsprechend orientieren sich diese Organisationen in der Gestaltung von Lehr- und Lernprozessen vorwiegend daran, Teilnehmenden Hilfestellungen und Anleitungen im Hinblick auf die jeweiligen Lerngegenstände zu geben. In Organisation G zeigt sich dies in zwei Hinsichten. Auf der einen Seite sind die didaktischen Vorstellungen vom Prinzip der Anleitung geprägt, das sich durch ein Wechselspiel von Erklärung, Vormachen und Übung auszeichnet. Auf der anderen Seite wirkt auf die erwachsenenpädagogische Arbeit eine sozialpädagogische Hilfsperspek- 
tive prägend, die über die Kurse hinaus Bedeutung einnimmt. So beschreiben Verwaltungskräfte und hauptamtlich pädagogische Mitarbeitende, dass arbeitssuchende Teilnehmende auch beim Schreiben von Bewerbungen unterstützt werden. In Organisation $\mathrm{F}$ zeigt sich dies in einer kontinuierlichen Weiterentwicklung der bislang frontalorientierten Lehrpraxis. So werden im Kontext von Alphabetisierungskursen Formate der Lernberatung erprobt, um Teilnehmende individueller in ihren Lernprozessen zu begleiten. Mit einer versorgungsorientierten Perspektive werden die Teilnehmenden als Ausgangspunkte der didaktischen Gestaltung betrachtet, die auch durch sozialpädagogisch orientierte Hilfestellungen unterstützt werden.

Die durch die Fallbeispiele $\mathrm{G}$ und $\mathrm{F}$ aufgezeigte versorgungsorientierte Perspektive ländlicher Erwachsenenbildung basiert darauf, dass das umgebende Umfeld als sozialer Raum in seiner strukturellen Besonderheit wahrgenommen wird und andere Institutionen der Region als Partner wahrgenommen werden. Normativ fundiert wird diese Perspektive durch das Ziel, Teilnehmende der Region mit Bildung zu versorgen und in ihrer individuellen Entwicklung zu unterstützen. Entsprechend sind die Vorstellungen der Gestaltung von Lehr- und Lernprozessen durch die Prinzipien der Anleitung und Hilfestellung gekennzeichnet und sozialpädagogisch angereichert.

\section{Diskussion: Zur Ausdifferenzierung regionaler Lehrkulturen}

Die Befunde zur Ausdifferenzierung einer markt- und einer versorgungsorientierten Perspektive ländlicher Erwachsenenbildung werden im Folgenden lehrkulturtheoretisch ausgedeutet und diskutiert.

Unter Berücksichtigung einer systemtheoretischen Perspektive kann (Lehr-)Kultur als eine Beobachtungsleistung zweiter Ordnung verstanden werden, mit deren Hilfe Organisationen Komplexität im Hinblick auf Lehrentscheidungen reduzieren können, um gemeinsam geteilten Sinn zu generieren (Scheunpflug et al. 2012). Auf diese Weise wird es für Organisationen möglich, Entscheidungen nicht immer neu treffen zu müssen, sondern sie können vielmehr auf einen „Themenvorrat“ (vgl. Luhmann 1995: 579) zurückgreifen, der das alltägliche Arbeiten im Hinblick auf das Lehren entlastet. Lehrkulturen gründen dabei in erwachsenenpädagogischen Organisationen auf der Verbindung sachlicher, sozialer, zeitlicher und räumlicher Sinndimensionen (Luhmann 1984), mit denen jeweils unterschiedliche Facetten des Lehrens betrachtet werden.

Die empirisch herausgearbeiteten Perspektiven ländlicher Erwachsenenbildung lassen sich zunächst als Ausdruck einer spezifischen Lehrkultur interpretieren, die die relative Besonderheit ländlicher Erwachsenenbildung integriert. Diese ländliche Lehrkultur basiert im Hinblick auf eine sachliche 
Sinndimension auf einer Entscheidung für Bedarfsorientierung. Hinsichtlich der zeitlichen Sinndimension zeichnet sich die Lehrkultur durch eine Orientierung an aktuellen Entwicklungen aus. Im Kontext einer sozialen Sinndimension wird die Orientierung an den Adressaten der Einrichtung hervorgehoben. Und schließlich zeigt sich im Hinblick auf die räumliche Sinndimension ein territoriales Raumverständnis, bei dem von einem ontologischen gegebenen Handlungsraum ausgegangen wird (Treml 2000). Mit den jeweils geographisch abgegrenzten Regionen stecken die Organisationen die Reichweite ihres Handlungsraums ab.

Interessant erscheint nun, dass sich die zwei vorgestellten Perspektiven als Ausdifferenzierungen dieser ländlichen Lehrkultur interpretieren lassen (siehe Tab. 1).

Tab. 1: Ausdifferenzierung ländlicher Lehrkulturen (Quelle: Eigene Erstellung)

\begin{tabular}{|l|l|l|l|}
\hline $\begin{array}{l}\text { Sinn- } \\
\text { dimensionen }\end{array}$ & $\begin{array}{l}\text { ländliche } \\
\text { Lehrkulturen }\end{array}$ & $\begin{array}{l}\text { Versorgungsorientierte } \\
\text { Perspektive }\end{array}$ & $\begin{array}{l}\text { Marktorientierte } \\
\text { Perspektive }\end{array}$ \\
\hline $\begin{array}{l}\text { Sachliche } \\
\text { Sinndimension: }\end{array}$ & $\begin{array}{l}\text { Bedarfs- } \\
\text { orientierung }\end{array}$ & $\begin{array}{l}\text { Unterstützung, Stärkung, } \\
\text { Hilfe }\end{array}$ & $\begin{array}{l}\text { Innovation, } \\
\text { Kundenwünsche }\end{array}$ \\
\hline $\begin{array}{l}\text { Zeitliche } \\
\text { Sinndimension: }\end{array}$ & Aktualität & $\begin{array}{l}\text { Wandel der Bedingungen } \\
\text { in der Region }\end{array}$ & Zeitgeist, Trends \\
\hline $\begin{array}{l}\text { Soziale } \\
\text { Sinndimension }\end{array}$ & $\begin{array}{l}\text { Adressaten- } \\
\text { orientierung }\end{array}$ & $\begin{array}{l}\text { Teilnehmende als } \\
\text { hilfsbedürftige Individuen }\end{array}$ & $\begin{array}{l}\text { Teilnehmende als } \\
\text { zufriedenzustellende } \\
\text { Kunden }\end{array}$ \\
\hline $\begin{array}{l}\text { Räumliche } \\
\text { Sinndimension }\end{array}$ & $\begin{array}{l}\text { Territorialer } \\
\text { Raum }\end{array}$ & Kooperativer Sozialraum & $\begin{array}{l}\text { Konkurrenzgeprägter } \\
\text { Marktraum }\end{array}$ \\
\hline
\end{tabular}

Die sachliche Bedarfsorientierung erscheint im Kontext einer versorgungsorientierten Perspektive an der Idee der Unterstützung, Stärkung, Hilfe und Stabilisation der Region ausgerichtet, während bei der marktorientierten Perspektive Bedarfsorientierungen im Hinblick auf Innovation und Kundenwünsche gedeutet wird. Bei der Zeitdimension stehen bei der versorgungsorientierten Perspektive im Hinblick auf die Orientierung an Aktualität zeitliche Wandlungsprozesse der Region im Mittelpunkt, während bei der marktorientierten Perspektive eine Orientierung am Zeitgeist und an Trends fokussiert wird. Die adressatenorientierte Ausrichtung der sozialen Sinndimension geht bei der versorgungsorientierten Perspektive mit einem Teilnehmendenbild einher, bei dem diese als hilfsbedürftige Individuen betrachtet werden. Im Kontext einer marktorientierten Perspektive werden Teilnehmende als Kunden betrachtet. Die räumliche Sinndimension differenziert sich in der versorgungsorientierten Perspektive im Hinblick auf einen kooperativen Sozial- 
raum aus und im Hinblick auf eine marktorientierte Perspektive auf einen konkurrenzgeprägten Marktraum.

Abschließend lässt sich die These formulieren, dass diese Ausdifferenzierung abhängig von der wahrgenommenen Strukturstärke oder Strukturschwäche einer Region erscheint. So findet sich eine versorgungsorientierte Perspektive ländlicher Lehrkulturen im empirischen Material in Organisationen, die in strukturschwachen, peripheren Regionen situiert sind. Eine marktorientierte Perspektive ländlicher Lehrkulturen findet sich im Material hingegen bei einer Organisation, die in einer strukturstarken zentralen Region situiert ist. Darin deutet sich an, dass die sozioökonomische Verfasstheit von Regionen einen entscheidenden Faktor zur Herausbildung regionaler Lehrkulturen darstellt. Dies impliziert wiederum, dass hier nicht die Differenz von Stadt und Land handlungsleitend wird, schließlich finden sich markt- und versorgungsorientierte Perspektiven auch bei städtischen Organisationen (vgl. Franz 2016b). Vor diesem Hintergrund bietet es sich für die weitere Analyse von regionalen Lehr- und Lernkulturen an, insbesondere die Strukturstärke oder Strukturschwäche einer regionalen Bildungslandschaft (siehe z.B. Martin u.a. 2015) als Ausgangspunkt für weitere komparative Analysen zu nutzen.

\section{Literatur}

Arnold, R./Schüßler, I. (1998): Wandel der Lernkulturen. Ideen und Bausteine für ein lebendiges Lernen. Darmstadt: wissenschaftliche Buchgesellschaft.

Arnold, R./Schüßler, I. (2015): Ermöglichungsdidaktik: Erwachsenenpädagogische Grundlagen und Erfahrungen (2. Aufl.). Baltmannsweiler: Schneider Verlag Hohengehren.

Bernhard, C./Kraus, K./Schreiber-Barsch, S./Stang, R. (Hrsg.) (2015): Erwachsenenbildung und Raum. Theoretische Perspektiven - professionelles Handeln Rahmungen des Lernens. Bielefeld: wbv.

Bohnsack, R./Nentwig-Gesemann, I./Nohl, A.-M. (Hrsg.) (2007): Die dokumentarische Methode und ihre Forschungspraxis. Grundlagen qualitativer Sozialforschung. (2. Auf.). Wiesbaden: VS-Verlag.

Faber, W. (1989): Gegenwartsfragen und Zukunftsperspektiven ländlicher Erwachsenenbildung. Erwachsenenbildung 2, 88-83. Wiederabdruck in Faber, W. (1990). Für eine zeitgerechte Erwachsenenbildung. Gesammelte Aufsätze. Bamberg, S. 115-124.

Fischbach, R. (2011): Bildungslandschaften. Reflexionskategorien und ihre professionspraktischen Konsequenzen. Der pädagogische Blick 19, 4, S. 196-205.

Fleige, M. (2011): Lernkulturen in der öffentlichen Erwachsenenbildung. Theorieentwickelnde und empirische Betrachtungen am Beispiel evangelischer Träger. Münster: Waxmann.

Franz, J. (2016a): Kulturen des Lehrens. Eine Studie zu kollektiven Lehrorientierungen in Organisationen Allgemeiner Erwachsenenbildung. Bielefeld: W. Bertelsmann Verlag. 
Franz, J. (2016b): Zur Bedeutung von „Raum“ in Organisationen Allgemeiner Erwachsenenbildung - Ergebnisse einer qualitativ-rekonstruktiven Studie. Zeitschrift für Erziehungswissenschaft 19, 1, S. 31-53.

Glaser, B. G./Strauss, A. L. (1967): The Discovery Of Grounded Theory: Strategies For Qualitative Research. Hawthorne, NY: Aldine de Gruyter.

Hargreaves, A. (1994): Changing Teachers, Changing Times. Teachers Work and Culture in the Postmodern Age. London: Cassell.

Helsper, W. (2008): Schulkulturen als symbolische Sinnordnungen und ihre Bedeutung für pädagogische Professionalität. In: Helsper, W./Busse, S./Hummrich, M./ Kramer, R.-T. (Hrsg.): Pädagogische Professionalität in Organisationen. Neue Verhältnisbestimmungen am Beispiel der Schule. Wiesbaden: Springer, S. 115-145.

James, D./Biesta, G. (Eds.) (2007): Improving learning cultures in further education. London: Routledge Falmer.

Kade, J./Nolda, S./Dinkelaker, J./Herrle, M. (2014): Videographische Kursforschung: Empirie des Lehrens und Lernens Erwachsener. Stuttgart: Kohlhammer.

Klemm, U. (2015): Eigenständige Regionalentwicklung als Leitidee für die Erwachsenenbildung im ländlichen Raum: Erfahrungen seit den 1980er Jahren und Perspektiven für das 21. Jahrhundert. In: Nuissl, E./Nuissl, H. (Hrsg.): Bildung im Raum. Baltmannsweiler: Schneider Verlag Hohengehren, S. 75-100.

Loos, P./Schäffer, B. (2001): Das Gruppendiskussionsverfahren. Theoretische Grundlagen und empirische Anwendung. Opladen: Budrich Verlag.

Luhmann, N. (1984): Soziale Systeme. Grundriß einer allgemeinen Theorie. Frankfurt a.M.: Suhrkamp.

Luhmann, N. (1995): Kultur als historischer Begriff. In: Luhmann, N.: Gesellschaftsstruktur und Semantik. Studien zur Wissenssoziologie der modernen Gesellschaft. Band 4. Frankfurt a.M.: Suhrkamp, S. 31-54.

Nohl, A.-M. (2013): Relationale Typenbildung und Mehrebenenvergleich: Neue Wege der dokumentarischen Methode. Wiesbaden: VS-Verlag.

Nolda, S. (1996): Interaktion und Wissen. Eine qualitative Studie zum Lehr-/Lernverhalten in Veranstaltungen der Allgemeinen Erwachsenenbildung. Frankfurt a.M.: Deutscher Volkshochschulverband.

Nesbit, T. (1998): Teaching in Adult Education: Opening the Black Box. Adult Education Quarterly 48, 3, S. 157-170.

Nugel, M. (2014): Erziehungswissenschaftliche Diskurse über Räume der Pädagogik. Wiesbaden: VS-Verlag.

Nugel, M. (2016): Stichwort: Bildungsräume - Bildung und Raum. Zeitschrift für Erziehungswissenschaft 19, 1.

Mannheim, K. (1980): Strukturen des Denkens. Frankfurt a.M.: Suhrkamp.

Martin,A./Schömann,K./Schrader, J./Kuper, H. (Hrsg.) (2015): Deutscher Weiterbildungsatlas. Bielefeld: wbv.

Pratt, D. D./Nesbit, T. (2000): Discourses and cultures of teaching. In: Hayes, E./ Wilson, A. (Eds.): Handbook of Adult and Continuing Education. San Francisco: Jossey Bass, S. 117-131.

Ritchey, J. (2008): Rural Adult Education: Current Status. In: New Directions for Adult and Continuing Education 117, S. 5-12.

Schüßler, Ingeborg (2016): Lernkulturen in Transformationsgesellschaften - Paradoxien, Herausforderungen und Gestaltungsoptionen. In diesem Band, S. 11-22. 


\section{$64 \quad$ Julia Franz}

Scheunpflug, A.,/Franz, J./Stadler-Altmann, U. (2012): Zur „Kultur“ in pädagogischen Zusammenhängen. In: Fink, T./Hill, B./Reinwand, V./Wenzlik, A. (Hrsg.): Die Kunst, über Kulturelle Bildung zu forschen. Theorie- und Forschungsansätze. München, S. 99-110.

Treml, A. K. (2000): Allgemeine Pädagogik. Stuttgart: Kohlhammer.

Universität Trier/LEB (Landesvereinigung für ländliche Erwachsenenbildung in Rheinland-Pfalz/asw e.V., AG sozialwissenschaftliche Forschung und Weiterbildung) (2007): Demographischer und sozialer Wandel im ländlichen Raum. Herausforderungen und Perspektiven für die ländliche Erwachsenenbildung. Trier.

Weishaupt, H./Böhm-Kasper (2011): Weiterbildung in regionaler Differenzierung. In: Tippelt, R./ Hippel, A. von (Hrsg.): Handbuch Erwachsenenbildung/Weiterbildung. Wiesbaden, S. 789-799. 


\section{Wirkt das Bild von Lernkulturen in der Grundbildung als Teilnahmehemmnis? ${ }^{1}$}

Unter der Überschrift „Töpfern war gestern. Volkshochschulen haftet immer noch ein altbackenes Image an" (Wißmann 2013) thematisierte die Wochenzeitung Die Zeit im Herbst 2013 den Widerspruch zwischen dem öffentlich häufig rezipierten Image von allgemeiner Weiterbildung und deren didaktischer Realität.

In diesem Beitrag geht es um dieses Image, um die Bilder und Vorstellungen, die Menschen von den Lernkulturen im Bereich der Grundbildung haben. Sind diese Bilder dazu angetan - so die zentrale Frage des Beitrags Menschen mit geringer Literalität für die Kursteilnahme zu gewinnen? Oder lassen sich im Gegenteil die sehr geringen Teilnahmequoten im Bereich der Grundbildung Erwachsener auch dadurch erklären, dass die dort herrschenden - oder die unterstellten - Lernkulturen potenzielle Kursteilnehmende von der Teilnahme abhalten? Der Beitrag basiert auf Ergebnissen der ,Studie zum mitwissenden Umfeld funktionaler Analphabetinnen und Analphabeten" ${ }^{2}$ (Kurztitel: „Umfeldstudie“). Die Studie untersucht die unterstützenden Netzwerke von Erwachsenen mit geringen bis sehr geringen Schriftsprachkenntnissen.

\section{Forschungsstand: Von der Adressatenforschung zur Umfeldforschung}

Die bisherige Forschung zu Erwachsenen mit geringer Literalität konzentrierte sich entweder qualitativ auf die betreffenden Erwachsenen selbst oder zog ihre Erkenntnisse aus repräsentativen Bevölkerungsstudien, deren Bedeutung in den letzten Jahren stark zugenommen hat. Die Ergebnisse dieser quantitativen Studien zeigen, dass die Zahl der Erwachsenen mit geringen Lese- und Schreibfertigkeiten sehr viel größer ist, als gemeinhin angenom-

1 Das diesem Artikel zugrundeliegende Vorhaben wurde mit Mitteln des Bundesministeriums für Bildung und Forschung (BMBF) unter dem Förderkennzeichen W138000 gefördert. Die Verantwortung für den Inhalt dieser Veröffentlichung liegt bei den Autor/inn/en.

2 Der Begriff „funktionaler Analphabetismus“ wird kontrovers diskutiert. Er hat eine lange Begriffstradition im deutschen Grundbildungsdiskurs, ist aber insbesondere aufgrund einer stigmatisierenden Konnotation umstritten. Zur Diskussion des Begriffs vgl. u.a. Grotlüschen/Riekmann/Buddeberg (2012: $15 \mathrm{ff}$.), Rosenbladt (2012) und Nickel (2011: $53 \mathrm{ff}$.). In diesem Beitrag wird der Begriff - obwohl Teil des Titels der vorgestellten Studie - weitgehend vermieden. 
men wurde. Nationale Studien in Frankreich (Jeantheau 2015), England (Department for Business 2011) und Deutschland (Grotlüschen/Riekmann 2012) zeigten dies für die jeweiligen Länder. Das „Programme for the International Assessment of Adult Competencies“ (PIAAC) bestätigt dies über den Bereich der Literalität hinaus für weitere Grundkompetenzen im internationalen Vergleich (Zabal et al. 2013).

Wenn PIAAC für Deutschland berichtet, dass über 17 Prozent der Erwachsenen auf niedrigen Kompetenzniveaus lesen, wenn aber gleichzeitig nur sehr wenige Erwachsene an Lese- und Schreibkursen der Volkshochschulen teilnehmen, so ergeben sich daraus mindestens zwei Schlussfolgerungen. Die erste betrifft die Frage der Erreichbarkeit, also eine der Grundproblematiken der Erwachsenenbildung (Hippel/Tippelt 2011). Die Zahlen der VHS-Statistik von 2015 ergeben etwa 30.000 Kursbelegungen (Huntemann/Reichart 2015: 30), das sind weniger als ein Prozent der Betroffenen (siehe auch Rosenbladt 2011: 98; Frieling/Rustemeyer 2011: 238).

Die zweite Schlussfolgerung betrifft die Forschungslage. Forschung zu Erwachsenen mit geringen Lese- und Schreibfertigkeiten richtete sich in der Vergangenheit in Form von qualitativen Studien (vgl. exemplarisch Projektträger im Deutschen Zentrum für Luft- und Raumfahrt e.V. 2011a, 2011b; Egloff 1997; Döbert/Hubertus 2000; Linde 2008; Schladebach 2007), seltener auf Basis quantitativer Untersuchungen (Rosenbladt/Bilger 2011) vor allem auf Teilnehmende von Alphabetisierungs- und Grundbildungskursen. Sie fokussierte auf die betroffene Person selbst, auf deren Handlungsstrategien (Nienkemper 2015: 80-88; Döbert/Hubertus 2000: 70; Egloff 1997: 161) und Einbettung in Unterstützungsstrukturen (Wagner/Schneider 2008: 56; Fingeret 1983; Hodge/Barton/Pearce 2010; St. Clair/Tett/Maclachlan 2010). Über Erwachsene, die sich zum Kursbesuch entschieden haben (,Lernende Analphabeten“: Rosenbladt 2011), liegt somit umfangreiches Wissen vor, nicht jedoch über die Gruppe der Adressat/inn/en insgesamt. Diese Informationslücke ist nicht durch die Übertragung der Resultate aus der Teilnehmendenforschung auf die Gesamtgruppe der Adressat/inn/en möglich, da dies zu unzulässigen Generalisierungen führt (Grotlüschen/Riekmann/Buddeberg 2015). Die oben erwähnten nationalen und internationalen Large-ScaleAssessment-Studien können gegenüber den qualitativen Befragungen Aussagen über die gesamte Adressat/inn/engruppe ermöglichen, also auch über die nicht in Kursen Lernenden, allerdings sind die Resultate dieser quantitativen - messenden - Forschungsrichtung abstrakt und ermöglichen kaum Rückschlüsse auf die zugrundeliegenden subjektiven Gegebenheiten und die konkrete literale Praxis der Betroffenen.

Dies verweist auf ein grundsätzliches Spannungsverhältnis zwischen Large-Scale-Assessments und deren zugrundeliegenden theoretischen Annahmen zur Literalität und ihrer Messbarkeit einerseits und der jeweils spezifischen praktischen Realität von Subjekten und der sozialen Einbettung lite- 
raler Praktiken andererseits, die theoretisch besonders pointiert seitens der New Literacy Studies beschrieben werden (aktuell diskutiert in Hamilton/ Maddox/Addey 2015; vgl. auch Barton/Hamilton 2000; Pabst/Zeuner 2011).

Die Umfeldstudie nimmt neben diesen beiden ,klassischen' Forschungssträngen zur Literalität eine neue Perspektive ein. Die Perspektive richtet sich nicht auf die betroffene Person selbst, sondern auf jene Personen, die das persönliche oder berufliche Netzwerk bilden. Von diesen Personen, so die Grundannahme, müssten eine oder mehrere in die Lese- und Schreibschwierigkeiten eingeweiht sein. Sie wissen davon, sie sind „Mitwissende“. Anliegen der Studie war es, herauszuarbeiten, wie das Verhältnis zwischen diesen beiden Personen beschaffen ist und wie die Mitwissenden ihre Rolle interpretieren. Ein Teilaspekt war die Frage, ob durch die Mitwissenden Lernprozesse initiiert werden, etwa in Form informeller Lernsettings oder durch die Vermittlung in non-formale Bildung. Die Studie folgt der Annahme, dass Mitwissende, die soziales Kapital (Bourdieu 1983: 190) im Sinne von bridging social capital (Putnam 2001: 16ff.) zur Verfügung stellen, in der Lage sein sollten, neue Felder zu erschließen und somit auch Lernprozesse anzuregen oder zu unterstützen. Dabei können sie prinzipiell eine Scharnierfunktion übernehmen zwischen Betroffenen und Anbietern von Grundbildung.

\section{Forschungsdesign}

Die Studie basiert auf einer quantitativen und einer qualitativen Teilstudie, die in allen Projektphasen im Sinne eines Triangulations-Ansatzes aufeinander bezogen wurden. Dies entspricht der Empfehlung von Mayring, der nahelegt, „Analysestrategien differenziert dort einzusetzen, wo sie ihre Stärken entfalten können, und damit auch nach einer Kombination qualitativer und quantitativer Strategien zu suchen“ (Mayring 2001). Die Studie beleuchtet somit ihren Gegenstand aus unterschiedlichen Richtungen mit dem Ziel eines umfassenderen und valideren Bildes. Da beide Studienteile gleichberechtigt, also nicht hierarchisch angeordnet sind, hält der Ansatz auch den strengen Anforderungen stand, die Flick für den Begriff der Triangulation vorschlägt (Flick 2011: 12).

Die standardisierte Telefonbefragung unter Erwachsenen in Hamburg umfasst 1.511 Interviews. Die qualitative Teilstudie umfasst 30 leitfadengestützte Interviews. Aus diesen 30 Interviews ließen sich 41 Fälle von Mitwissen herausarbeiten, aus denen eine Typenbildung von Mitwisserschaft resultierte (Riekmann 2015: 166-167). Sowohl bei der Entwicklung des Erhebungsinstruments als auch bei der Auswertung hat sich eine enge Verzahnung beider Teilstudien als sehr produktiv erwiesen. 


\section{Potenzial des Umfelds zur Initiierung von Lernprozessen}

Gut 40 Prozent der Erwachsenen in Hamburg kennen andere Erwachsene, die auffällige Probleme beim Lesen und Schreiben haben. Die Schwierigkeiten reichen von funktionalem Analphabetismus in der Diktion der leo.-Studie (Grotlüschen/Riekmann/Buddeberg 2012: 19f.) bis zu einer auffällig fehlerhaften Schreibung bei gebräuchlichem Wortschatz. Die hohe Inzidenz verdeutlicht, dass fehlerhaftes Lesen und Schreiben im gesellschaftlichen Miteinander durchaus auffällt und thematisiert wird. Darüber hinaus wissen praktisch alle Mitwissenden, dass es Lese- und Schreibkurse für Erwachsene gibt. Allerdings ist es eher die Ausnahme als die Regel, dass die Mitwissenden ihre Kenntnis von Lernmöglichkeiten nutzen, um eine konkrete Empfehlung zu geben, einen solchen Kurs auch aufzusuchen. Gut 20 Prozent der Mitwissenden geben ihr Wissen über Kursmöglichkeiten weiter, aber knapp 80 Prozent tun dies nicht (Buddeberg 2015: 221f.). Das fraglos vorhandene Potenzial, Betroffene auf Kurse hinzuweisen, wird also nicht annähernd ausgeschöpft. Dies bedeutet freilich nicht, dass das Umfeld keinerlei Lernprozesse initiiert. Für den Aufbau informeller Lernsettings lassen sich vor allem aus dem qualitativen Datenmaterial verschiedene Hinweise herausarbeiten. Die Aussage gilt aber fraglos für die Vermittlung in organisierte nonformale Weiterbildung.

Mitwissende, die zwar über das Angebot von Kursen prinzipiell informiert sind, jedoch keine Kursempfehlung abgeben, begründen dies häufig damit, dass sie sich nicht zuständig fühlen, da sie der betroffenen Person nicht nahe genug stehen, dass sie der Meinung sind, das Problem sei für die betroffene Person nicht gravierend genug, oder dass sie (i.e. die Mitwissenden) nicht genug über die Kursmöglichkeiten wissen (ebd.: 222). Die Befragten sind also zwar grundsätzlich über die Existenz von Kursen im Bilde, sie sind aber vermutlich nicht detailliert genug informiert über didaktische Ansätze, Kursformate, Kursgrößen, Lernformen und dergleichen, um eine qualifizierte Empfehlung abgeben zu können. Mitwissende haben mehrheitlich kein oder ein sehr unscharfes Konzept der in Grundbildungskursen vorherrschenden Lernkultur.

\section{Das Bild des Umfelds von der Lernkultur in der Grundbildung für Erwachsene}

Der Begriff der Lernkultur zeichnet sich durch seine begriffliche Offenheit und Unschärfe aus (Arnold/Pätzold 2008: 184), die es gelegentlich erschwert, ihn auf eine bestimmte Fragestellung zu konkretisieren. Lernkulturen sind in Anlehnung an Siebert als durch die Institutionen und gesellschaftliche Realität beeinflussbar anzusehen, sie werden aber auch durch die Lernenden mitgestaltet (Schüßler/Thurnes 2005: 14). Lernkulturen sind demnach etwas, das 
erst im Handeln der Akteure als Teil einer pädagogischen Praktik entsteht (Reh et al. 2015: 19). Aus dem Datenmaterial der Umfeldstudie sind selbstverständlich keine direkten Beschreibungen von konkret beobachteten Lernkulturen zu entnehmen, vielmehr lässt sich in Anlehnung an Schüßler und Thurnes mehr von individuellen und kollektiven Bildern über das Lehren und Lernen sprechen, die sich in den Daten widerspiegeln (Schüßler/Thurnes 2005: 14). Bezogen auf die Lernkulturen im Mehrebenensystem der Erwachsenenbildung, wie Fleige es ordnend vorgeschlagen hat (Fleige 2011), geht es dabei vorrangig um Bilder von Lehren und Lernen auf der Mikroebene. Diese Bilder der Lernkulturen in der Erwachsenenbildung - so die diesem Beitrag zugrundeliegende These - können dazu führen, dass Mitwissende die Lernmöglichkeiten in Lese- und Schreibkursen als für die betreffende Person ungeeignet wahrnehmen und deshalb darauf verzichten, eine Kursteilnahme nahezulegen.

Im Kontext von Teilnahme bzw. Nicht-Teilnahme an Weiterbildung bringen Bremer und Kleemann-Göhring (Bremer/Kleemann-Göhring 2011) auf Basis von Milieuforschung die Begriffe der Selbst- und Fremdexklusion in Bezug auf die Erreichbarkeit von so genannten bildungsfernen Gruppen mit Weiterbildung ins Spiel. Durch die Ergebnisse der Umfeldstudie lässt sich dieser Sichtweise nun möglicherweise eine dritte Form von Exklusion hinzufügen. Nicht nur das System Weiterbildung schließt Betroffene aus und Betroffene schließen sich selber aus, weil sie Weiterbildung für sich nicht als passend empfinden. Auch das Umfeld schließt potenzielle Teilnehmende aus, bzw. es bringt sein Potenzial an bridging social capital nicht zur Geltung, indem es seine vorhandene Beratungskompetenz nicht nutzt.

Im qualitativen Material lassen sich für diese Form der Exklusion Begründungsmuster finden. So sehen Mitwissende bei ihrem Gegenüber entweder keinen Bedarf an Weiterbildung (,Er kommt ja auch so ganz gut zurecht im Leben ", Interview 3, Abs. 23) oder sie erkennen in Weiterbildung keinen Nutzen (,,Und ob das überhaupt mit ihr dann Sinn gehabt hätte, weiß ich auch nicht, das muss ich eher bezweifeln", Interview 6, Abs. 70). Der Schutz der Betroffenen vor peinlichen Situationen ist ebenso eine Begründung, wie der Wunsch, sich nicht als besser informiert und somit überlegen hervorzutun. Zwei weitere Begründungsmuster, die Möglichkeit der Kursteilnahme nicht anzusprechen, korrespondieren mit klassischen situationsbezogenen Teilnahmehindernissen: mit der Tatsache, dass die betroffene Person entweder keine Zeit dafür hat (,die sind ja auch Schichtarbeiter, das würden sie überhaupt nicht hinkriegen", Interview 11, Abs. 99) oder aber dass Weiterbildung räumlich schlecht erreichbar ist (,Ich wüsste gar nicht, dass solche Kurse irgendwo angeboten werden, die auch vernünftig erreichbar wären ", Interview 15, Abs. 20).

Weitere Interviewpassagen stehen hingegen in recht engem Zusammenhang zur Frage der wahrgenommenen oder vermuteten Lernkultur bzw. feh- 
lenden Vorstellungen über die Lernkultur im Bereich der Grundbildung für Erwachsene. Wie im quantitativen Studienteil deutlich wurde, gibt es zwar ein breites grundsätzliches Wissen über die Existenz von Lese- und Schreibkursen für Erwachsene, gleichzeitig erweist sich dieses Wissen als unvollständig und lückenhaft. Fehlende Vorstellung über die Bildungspraxis in diesem Segment - das zeigen auch die qualitativen Interviews - hindert manche Mitwissende daran, eine Kursteilnahme nahezulegen.

„Nee, ich hatte damit nichts zu tun, und warum soll ich mich über irgendwas schlau machen, auf die Idee wäre ich gar nicht gekommen“ (Interview 6, Abs. 35).

Fehlende Informationen über Lernmöglichkeiten in Verbindung mit einer generellen Verunsicherung, wie mit dem Wissen über die Lese- und Schreibschwierigkeiten einzelner Personen umgegangen werden kann, ist ein Charakteristikum des Typus der verunsicherten Mitwisserschaft (Riekmann 2015: 167-173). Eine Leiterin gewerkschaftlicher Bildungsseminare äußerte beispielsweise in einem Interview, dass sie ,in dem Moment nicht gewusst (habe), wie kann ich ihm richtig helfen“ (Interview 11, Abs. 53). Mitwissende bringen aber auch zum Ausdruck, dass sie sehr wohl eine Vorstellung vom Lernen im Kontext Erwachsenenbildung haben, die Lernkultur aber als unpassend für die betroffene Person erachten. Dabei ist das Image von Weiterbildung, dies zeigte das Berichtssystem Weiterbildung, grundsätzlich besser bei denen, die bereits Erfahrung mit Weiterbildung haben, als bei jenen, die keine Erfahrungen haben (Kuwan/Thebis 2006: 258). In diesem Fall unterbleibt die Kursempfehlung, da die Perzeption von Weiterbildung einem bestimmten - möglicherweise klischeehaften - Image folgt. Eine interviewte Person äußert deutlich, dass sie die Empfehlung, einen VHS-Kurs zu besuchen, für gänzlich ungeeignet halten würde:

„Ich glaube, das hat damit was zu tun, dass eine gewisse Klientel halt angesprochen wird davon; dass es ein gewisses Image hat, also die Volkshochschule."

Frage: „Was für ein Image?““

„Hausfrauenkurse. Es ist kein professionelles Umfeld würde ich sagen“ (Interview 23, Abs. 64-66).

Diese Haltung ließe sich in Anlehnung an die von Siebert vorgenommene Typisierung von Nicht-Teilnahme (Siebert 2004: 12f.) und dem dort beschriebenen Typus der „offensiven Nicht-Teilnahme“ (Volkshochschulkurse, aber auch berufliche Weiterbildung werden entschieden abgelehnt, Volkshochschulbesucher werden negativ stigmatisiert) als ,offensive Nicht-Empfehlung" umschreiben. Die hier zitierte Person arbeitet zusammen mit dem Betroffenen in einem kleinen Unternehmen der Kreativbranche. Die Aussage, dass das Image von Volkshochschulen nur eine bestimmte Gruppe von Er- 
wachsenen anspricht, nicht jedoch jüngere Angehörige der Kreativszene, bezieht der Interviewpartner nicht allein auf die betroffene Person allein, sondern er generalisiert sie auf das gesamte kreative Milieu. Es liegt also ein Bild vom Lehren und Lernen in der Weiterbildung vor (Schüßler/Thurnes 2005: 14), das ungeachtet der Frage, ob es zutreffend ist oder nicht, von einer Kursempfehlung aus grundsätzlichen Erwägungen absehen lässt.

Interessanterweise berichtet der zuletzt zitierte Interviewpartner davon, dass er mit dem Betroffenen ein informelles Lernarrangement geschaffen hat, eine Struktur, die von der zuvor zitierten Leiterin gewerkschaftlicher Bildungsseminare in ähnlicher Weise beschrieben wird. Beide empfehlen nicht die Teilnahme an Weiterbildung, haben aber durch konsequentes gemeinsames Bearbeiten von Schreibarbeiten mit den betroffenen Personen eine signifikante Verbesserung der Schreibfähigkeit erreicht, also durch „Selbstgesteuerte Lernprozesse in ,ungewöhnlichen" Lernarrangements" (Schüßler/ Thurnes 2005: 18). Exklusion bezieht sich also hier nicht auf das Lernen, sondern lediglich auf die organisierte Weiterbildung.

Eine weitere Perzeption von Weiterbildung setzt diese mit Schule gleich.

„Alles, was an Schule erinnert, und was an Prüfung und allein das Wort Kurs: das weckt irgendwie schon Hemmnisse“ (Interview 11, Abs. 101).

Ein weiterer Interviewpartner, der über einen Mitarbeiter in einem Ein-EuroJob berichtet, verwendet selbst ausdrücklich das Wort Schule und nicht das Wort Volkshochschule, um Anbieter von Lese- und Schreibkursen zu beschreiben. Die Ablehnung durch den Betroffenen aufgrund dessen schlechter Schulerfahrung kann er folglich nicht mit einem Verweis auf die erwachsenenspezifische Lernkultur im Bereich der Grundbildung Erwachsener kontern.

„Ich hab ihn auch direkt darauf angesprochen, weil wir hatten ein sehr gutes Verhältnis miteinander, und da hat er dann auch gesagt: ,Ja, so ist es. Und ich brauche da unbedingt Hilfe'. Und dann habe ihn versucht, irgendwie dort zu bekommen, Schule zu besuchen, es gibt da diverse Schulen, die sich um die Menschen kümmern. Das hat er kategorisch abgelehnt und hat gesagt, er geht nicht mehr zur Schule, er hat da schlechte Erfahrungen gemacht, aufgrund dieser Behinderung mit anderen Mitschülern.“" (Interview 10, Abs. 8).

Lernen in der Erwachsenenbildung wird gewissermaßen als Verlängerung der Schuldidaktik interpretiert. Mitwissende Personen, die bei den Betroffenen von negativen Schulerfahrungen ausgehen, verzichtet aus diesem Grund darauf, den Besuch eines Kurses nahezulegen. 


\section{Fazit}

In der Praxis existieren viele unterschiedliche Lernkulturen nebeneinander. „In manchen Bildungsinstitutionen oder auch einzelnen Kursen beherrscht Frontalunterricht das Geschehen - in anderen hingegen eine von Selbstorganisation geprägte Lernpraxis“ (Schüßler/Thurnes 2005: 19).

Das Bild von allgemeiner Weiterbildung als antiquiert und verschult mag also auf bestimmte Kurse zutreffen. Vielleicht ist es ja sogar so - dies wäre empirisch zu überprüfen -, dass in vielen Bereichen der allgemeinen Erwachsenenbildung eher, alte " Lernkulturen herrschen, im Bereich der Grundbildung aber eher ,neue' Lernkulturen. So würde eine Gleichzeitigkeit des Ungleichzeitigen die verzerrte Sichtweise (Schüßler/Thurnes 2005: 18) stärken.

Gezeigt werden konnte, dass das Umfeld eine Beratungs- und Brückenfunktion im Sinne einer Vermittlung in die non-formale Erwachsenenbildung für Menschen mit geringen Lese- und Schreibkenntnissen annehmen kann und in diesen Fällen Unterstützung benötigt, um nicht die Vorstellungen von Grundbildung als erneuten „Schulbesuch“ zu reproduzieren. Die vorherrschenden Bilder von Lernkulturen in der Erwachsenenbildung können also eine Begründung für die Nicht-Teilnahme und damit ein Hemmnis sein. Anbieter von Alphabetisierungs- und Grundbildungskursen haben sich in jedem Fall die Frage zu stellen, wie Bilder von Lernkulturen verändert werden können und wie Informationen über Kurse nicht nur an die Betroffenen gelangen, sondern im Sinne einer doppelten Adressierung auch an das Umfeld, das dann diese Betroffenen beraten kann. Im Rahmen der Nationalen Dekade für Alphabetisierung und Grundbildung ist eine umfassende Medienkampagne im Entstehen. Hier wäre es also geboten, auch auf die Art und Weise des Lehrens und Lernens im Erwachsenenalter zu verweisen, um bestehende - berechtigte oder unberechtigte - Vorstellungen der dortigen Lernkulturen zu relativieren.

Weitere Forschungsergebnisse der Umfeldstudie weisen aber auch darauf hin, dass die Nicht-Teilnahme an einem Kurs nicht bedeutet, dass mit dem und durch das Umfeld nicht trotzdem gelernt wird. So kann das Umfeld auch dazu beitragen, dass informelle Lerngelegenheiten geschaffen werden und Betroffene auf diese Weise an der Verbesserung ihrer Kompetenzen arbeiten (vgl. Riekmann/Buddeberg 2016).

Damit stellt sich im Sinne der Zielgruppenanalyse auch die Frage, in welchen Bereichen das Umfeld möglicherweise besser unterstützt als organisierte Weiterbildung. Für die Forschung bestätigt die Umfeldstudie die Leistung der Umfeldforschung für die Alphabetisierung und Grundbildung. Es besteht Potenzial für weitere Bereiche, wenn es zum Beispiel um die Sichtbarmachung von informellen Lernprozessen bei möglicherweise anderen schwer erreichbaren Zielgruppen geht oder auch wenn es das Ziel ist, Über- 
gänge zwischen non-formalen und informellen Lernformaten zu formal organisierten Lernformaten zu erforschen.

\section{Literatur}

Arnold, Rolf/Pätzold, Henning (2008): Bausteine zur Erwachsenenbildung. Grundlagen der Berufs- und Erwachsenenbildung, Bd. 53. Baltmannsweiler: Schneider Hohengehren.

Barton, David/Hamilton, Mary (2000): Literacy Practices. In: Barton, D./Hamilton, M./Ivanič, R. (Hrsg.): Situated literacies. Reading and writing in context. Literacies. London, New York: Routledge, S. 7-15.

Bourdieu, Pierre (1983): Ökonomisches Kapital, kulturelles Kapital, soziales Kapital. In: Kreckel, R. (Hrsg.): Soziale Ungleichheiten. Soziale Welt. Sonderband, Band 2. Göttingen: Schwartz, S. 183-198.

Bremer, Helmut/Kleemann-Göhring, Mark (2011): Weiterbildung und „Bildungsferne“. Forschungsbefunde, theoretische Einsichten und Möglichkeiten für die Praxis. Abschlussbericht der wissenschaftlichen Begleitung des Projektes „Bildungsferne - Ferne Bildung“.

Buddeberg, Klaus (2015): Beratung durch das persönliche Umfeld funktionaler Analphabeten und Analphabetinnen. In: Report - Zeitschrift für Weiterbildungsforschung 38, 2, S. 213-226.

Department for Business, Innovation and Skills (2011): 2011 Skills for Life Survey: Headline findings. London.

Döbert, Marion/Hubertus, Peter (Hrsg.) (2000): Ihr Kreuz ist die Schrift. Analphabetismus und Alphabetisierung in Deutschland. Münster [u.a.]: Bundesverband Alphabetisierung und Grundbildung.

Egloff, Birte (1997): Biographische Muster „funktionaler Analphabeten“. Eine biographieanalytische Studie zu Entstehungsbedingungen und Bewältigungsstrategien von „funktionalem Analphabetismus“. DIE-Analysen für Erwachsenenbildung. Frankfurt/M.: DIE.

Fingeret, Arlene (1983): Social Networks. A New Perspective on Independence and Illiterate Adults. In: Adult Education Quarterly 33, 3, S. 133-146.

Fleige, Marion (2011): Lernkulturen in der öffentlichen Erwachsenenbildung. Theorieentwickelnde und empirische Betrachtungen am Beispiel evangelischer Träger. Humboldt-Univ., Diss.- Berlin, 2009. Internationale Hochschulschriften, Band 554. Münster: Waxmann.

Flick, Uwe (2011): Triangulation. Eine Einführung. Band 12. Wiesbaden: VS, Verlag für Sozialwissenschaften.

Frieling, Gundula/Rustemeyer, Angela (2011): Die Volkshochschulen, der Forschungstransfer und das Recht auf Alphabetisierung. In: Egloff, B./Grotlüschen, A. (Hrsg.): Forschen im Feld der Alphabetisierung und Grundbildung. Ein Werkstattbuch. Münster [u.a.]: Waxmann Verlag, S. 237-241.

Grotlüschen, Anke/Riekmann, Wibke (Hrsg.) (2012): Funktionaler Analphabetismus in Deutschland. Ergebnisse der ersten leo. - Level-One Studie. Alphabetisierung und Grundbildung, Band 10. Münster [u.a.]: Waxmann Verlag. 
Grotlüschen, Anke/Riekmann, Wibke/Buddeberg, Klaus (2012): Hauptergebnisse der leo. - Level-One Studie. In: Grotlüschen, A./Riekmann, W. (Hrsg.): Funktionaler Analphabetismus in Deutschland. Ergebnisse der ersten leo. - Level-One Studie. Alphabetisierung und Grundbildung, Band 10. Münster [u.a.]: Waxmann Verlag, S. 13-53.

Grotlüschen, Anke/Riekmann, Wibke/Buddeberg, Klaus (2015): Stereotypes versus Research Results regarding Functionally Illiterate Adults. Conclusions from the first German Level-One Survey and the Learner Panel Study. In: Grotlüschen, A./Zimper, D. (Hrsg.): Literalitäts- und Grundlagenforschung. Alphabetisierung und Grundbildung, Band 11. Münster: Waxmann Verlag, S. 105-122.

Hamilton, Mary/Maddox, Bryan/Addey, Camilla (Hrsg.) (2015): Literacy as numbers. Researching the politics and practices of international literary assessment. Cambridge, United Kingdom: Cambridge University Press.

Hippel, Aiga von/Tippelt, Rudolf (2011): Adressaten-, Teilnehmer- und Zielgruppenforschung. In: Tippelt, R./Hippel, A. v. (Hrsg.): Handbuch Erwachsenenbildung/Weiterbildung. Wiesbaden, S. 801-811.

Hodge, Rachel/Barton, David/Pearce, Linda (2010): Progression. Moving on in life and learning. London.

Huntemann, Hella/Reichart, Elisabeth (2015): Volkshochschul-Statistik: 53. Folge, Arbeitsjahr 2014.

Jeantheau, Jean-Pierre (2015): IVQ 2011. What lessons can be drawn from the evolution of the state of adult literacy in France? In: Grotlüschen, A./Zimper, D. (Hrsg.): Literalitäts- und Grundlagenforschung. Alphabetisierung und Grundbildung, Band 11. Münster: Waxmann Verlag, S. 177-196.

Kuwan, Helmut/Thebis, Frauke (Hrsg.) (2006): Berichtssystem Weiterbildung IX. Ergebnisse der Repräsentativbefragung zur Weiterbildungssituation in Deutschland. Bonn: BMBF.

Linde, Andrea (2008): Literalität und Lernen. Eine Studie über das Lesen- und Schreibenlernen im Erwachsenenalter. Internationale Hochschulschriften, Bd. 503. Münster, München [u.a.]: Waxmann Verlag.

Mayring, Philipp (2001): Kombination und Integration qualitativer und quantitativer Analyse. In: Forum Qualitative Sozialforschung/Forum Qualitative Social 2, 1.

Nickel, Sven (2011): Literalität - Familie - family literacy: die Transmission schriftkultureller Praxis und generationenübergreifende Bildungsprogramme als Schlüsselstrategie. In: Psychologie \& Gesellschaftskritik 35, 3, S. 53-77.

Nienkemper, Barbara (2015): Lernstandsdiagnostik bei funktionalem Analphabetismus. Akzeptanz und Handlungsstrategien. 1. Aufl. Theorie und Praxis der Erwachsenenbildung. Bielefeld: Bertelsmann, W.

Pabst, Antje/Zeuner, Christine (2011): Literalität als soziale Praxis. Bedeutungen von Schriftsprachlichkeit. In: Report - Zeitschrift für Weiterbildungsforschung 34, 3, S. 36-47.

Projektträger im Deutschen Zentrum für Luft- und Raumfahrt e.V. (Hrsg.) (2011a): Lernprozesse in Alphabetisierung und Grundbildung Erwachsener. Diagnostik, Vermittlung, Professionalisierung. Band 2. Bielefeld: WBV, Bertelsmann.

Projektträger im Deutschen Zentrum für Luft- und Raumfahrt e.V. (Hrsg.) (2011b): Zielgruppen in Alphabetisierung und Grundbildung Erwachsener. Bestimmung, Verortung, Ansprache. Bielefeld: WBV, Bertelsmann. 
Putnam, Robert D. (Hrsg.) (2001): Gesellschaft und Gemeinsinn. Sozialkapital im internationalen Vergleich. Gütersloh: Bertelsmann Stiftung.

Reh, Sabine et al. (2015): Lernkulturen. Rekonstruktion pädagogischer Praktiken an Ganztagsschulen. Reihe Schule und Gesellschaft, Bd. 47. Wiesbaden: Springer VS.

Riekmann, Wibke (2015): Das mitwissende Umfeld funktionaler Analphabetinnen und Analphabeten - Ergebnisse der qualitativen Teilstudie. In: Grotlüschen, A./Zimper, D. (Hrsg.): Literalitäts- und Grundlagenforschung. Alphabetisierung und Grundbildung, Band 11. Münster: Waxmann Verlag, S. 157-176.

Riekmann, Wibke/Buddeberg, Klaus (2016): Hilfe und Lernen im mitwissenden Umfeld. In: Riekmann, W./Buddeberg, K./Grotlüschen, A. (Hrsg.): Das mitwissende Umfeld von Erwachsenen mit geringen Lese- und Schreibkompetenzen. Alphabetisierung und Grundbildung, Band 12. Münster: Waxmann Verlag, S. 107-130.

Rosenbladt, Bernhard von (2011): Lernende Analphabetinnen und Analphabeten. Wen erreicht das Kursangebot der Volkshochschulen? In: Egloff, B./Grotlüschen, A. (Hrsg.): Forschen im Feld der Alphabetisierung und Grundbildung. Ein Werkstattbuch. Alphabetisierung und Grundbildung, Band 7. Münster [u.a.]: Waxmann Verlag, S. 89-99.

Rosenbladt, Bernhard von (2012): Der sogenannte funktionale Analphabetismus eine sprachkritische Bestandsaufnahme. In: Alfa-Forum 79, S. 10-13.

Rosenbladt, Bernhard von/Bilger, Frauke (2011): Erwachsene in Alphabetisierungskursen der Volkshochschulen. Ergebnisse einer repräsentativen Befragung (AlphaPanel). Bonn.

Schladebach, Almuth (2007): Ein rotes Tuch: Formulare und Fragebögen! Auswertung der Teilnehmerbefragung im 2. Semester 2004 im Grundbildungszentrum der Hamburger Volkshochschule. In: Grotlüschen, A./Linde, A. (Hrsg.): Literalität, Grundbildung oder Lesekompetenz? Beiträge zu einer Theorie-Praxis-Diskussion $=$ Literacy, basic education or reading competencies? Münster [u.a.]: Waxmann Verlag, S. 140-146.

Schüßler, Ingeborg/Thurnes, Christian M. (2005): Lernkulturen in der Weiterbildung. Studientexte für Erwachsenenbildung. Bielefeld: Bertelsmann.

Siebert, Horst (2004): Weiterbildungsbeteiligung und Lernmotivation. In: Report Zeitschrift für Weiterbildungsforschung 27, 3, S. 9-14.

St. Clair, Ralph/Tett, Lyn/Maclachlan, Kathy (2010): Scottish Survey of adult literacies 2009: Report of findings. Edinburgh.

Wagner, Harald/Schneider, Johanna (2008): Charakteristika spezifischer Gruppen von Menschen mit unzureichender Schriftsprachkompetenz. In: Schneider, J./Gintzel, U./Wagner, H. (Hrsg.): Sozialintegrative Alphabetisierungsarbeit. Bildungs- und sozialpolitische sowie fachliche Herausforderungen. Münster, München [u.a.]: Waxmann Verlag, S. 47-62.

Wißmann, Constantin (2013): Töpfern war gestern. Volkshochschulen haftet immer noch ein altbackenes Image an. In: Die Zeit.

Zabal, Anouk et al. (2013): Grundlegende Kompetenzen der erwachsenen Bevölkerung in Deutschland im internationalen Vergleich. In: Rammstedt, B. (Hrsg.): Grundlegende Kompetenzen Erwachsener im internationalen Vergleich. Ergebnisse von PIAAC 2012. Münster [u.a.]: Waxmann Verlag, S. 31-59. 



\section{Lernkulturaspekte im Science Slam. Aufriss der Konzeptualisierung einer mehrperspektivischen Untersuchung}

Wissenschaftler_innen sehen sich den stetig zunehmenden Anforderungen einer inhaltlich umfassenden Wissenschaftskommunikation gegenüber, die zwischen Wissenschaften, zwischen gesellschaftspolitischen Systemen, aber vor allem im Austausch mit der Öffentlichkeit einsetzt. Fokussiert geht es um die Wissensvermittlung zunehmend komplexer werdender wissenschaftlicher Zusammenhänge. Kriterien für eine damit einhergehende Wissenstransformation innerhalb der Wissenschaftskommunikation lassen sich unter den Aspekten Verständlichkeit, Ästhetik, Attraktivität und Emotionalität (Janich 2006) bündeln. Aus erwachsenenpädagogischer Perspektive erfolgt dies grundlegend unter dem Anspruch, dass das wissenschaftliche Wissen zielgruppenspezifisch sowie didaktisch aufbereitet wird und eine am Dialog ausgerichtete Wissenschaftsvermittlung (auch Faulstich/Trumann 2016) zielführend ist.

Mit den Entwicklungen von (dialogischen) Vermittlungsformaten im Rahmen der Wissenschaftskommunikation entstehen spezifische Lernkulturen. Diese sind geprägt durch eine Vielzahl sie charakterisierender Perspektiven und Aspekte. In der empirischen Erschließung ist daher der Fokus auf die Herausarbeitung des Da-Zwischens (Gieseke 2007b) zu setzen, um die jeweils spezifisch gestaltete Lernkultur in ihrer Ganzheitlichkeit zu erfassen. In den folgenden Ausführungen wird ein Aspekt - Raumbetrachtung - mit dem ihn charakterisierenden theoretischen sowie aus dem empirischen Material erschlossenen Perspektiven herausgegriffen. Anhand dieses einen Aspektes wird die Besonderheit der empirischen Erschließung von Lernkulturen deutlich, die einer detaillierten analytischen Betrachtung bedarf.

\section{Wissenschaftskommunikation: Wissenschaften als Bühnenstück im Science Slam}

Der Begriff „Wissenschaftskommunikation“ kann unter verschiedenen Perspektiven betrachtet werden (Pansegrau et al. 2011): Zum einen wird er in der Politik und im Event- bzw. Marketingbereich genutzt, um die Kommunikation der Wissenschaftler_innen ,mit einer unspezifizierten, aber möglichst breiten Öffentlichkeit“" (Pansegrau et al. 2011: 2) zu beschreiben. Zum anderen gibt es die mediale Berichterstattung über wissenschaftliche Themen. Es geht nicht selten um die Legitimation von Wissenschaftsbereichen, For- 
schungsprojekten oder thematischer Ausrichtungen im Sinne politischer und/ oder ökonomischer Vorgaben. „Vielmehr muss Wissenschaft in der Demokratie [jedoch] ein Interesse daran haben, dass Prioritäten- und Ressourcenentscheidungen auf der Grundlage vernünftiger Argumente gefällt werden“ (Faulstich/Trumann 2016: 02-6). Dazu bedarf es der Einbindung einer Öffentlichkeit, die als Teilhaberin an der Wissensproduktion anerkannt wird. „Wissenschaftskommunikation [ist daher] keine Einbahnstraße der passiven Wissensaufnahme“ (Antos 2005: XII), sondern durch einen dialogischen Charakter $^{1}$ zwischen Wissenschaften und Öffentlichkeit geprägt (Weingart/ Voß 2008). Wie diese Öffentlichkeit charakterisiert wird, ist dabei zunächst offen. Die Zielgruppe muss jeweils spezifisch für das entsprechende Format der Wissenschaftskommunikation bestimmt werden. Wissenschaftler_innen verändern ihr wissenschaftliches Wissen somit zielgruppenspezifisch und nutzen didaktisch aufbereitete Vermittlungspraktiken. Hier sind Transformationsprozesse des Wissens (erster Zugang zu Transformationsprozessen im Science Slam $)^{2}$ gefordert.

Im Rahmen gesellschaftspolitischer sowie wissenschaftlicher Entwicklungen ist zusammenfassend festzuhalten, ,dass ein großer Bedarf an alternativen wissenschaftlichen Formaten besteht, weil das Interesse in der Öffentlichkeit groß ist, das Unwissen aber ebenso" (MS10201: 428-430)³. Daher haben sich Veranstaltungsformate der Wissenschaftskommunikation mit jeweils unterschiedlichen Zielsetzungen und (Vermittlungs-)Praktiken etabliert: Wissenschaftsmagazine im Fernsehen, bundesweite oder EU-übergreifende Wissenschaftsjahre oder Preise für erfolgreiche Wissenschaftskommunikation. Allen Formaten ist gemeinsam, dass sie einen Zugang für unterschiedliche Zielgruppen zu wissenschaftlichen Inhalten schaffen und teilweise Wissenschaften aus ihrem (universitären) Forschungsumfeld herauslösen, so dass Wissenschaftskommunikation Flexibilisierungs- und Verschiebungstendenzen einfordert (Stimm 2016).

1 Die begriffliche Trennung zwischen Vermittlung und Dialog wird im zugrunde liegenden Verständnis der Wissenschaftskommunikation aufgehoben. An sie wird der Anspruch gestellt, einen dialogischen Charakter einzunehmen, der auch vermittelnde Elemente enthält.

2 Ebenso können Transformationsprozesse zwischen dem wissenschaftlichen Wissen und dem Alltagswissen erkannt werden (zweiter Zugang zu Transformationsprozessen im Science Slam). Diese Transformationsprozesse werden als Figuren der Überführung zwischen den Wissensstrukturen charakterisiert, so dass die möglichen unterschiedlichen Übergänge zwischen Alltagswissen und wissenschaftlichem Wissen deutlich werden.

3 Das genutzte Interviewmaterial stammt aus dem Forschungsprojekt „Wissenschaft in zehn Minuten. Eine ethnografische Erkundung pädagogischer Lernanlässe zwischen Wissen und Event". Es handelt sich bei dem Forschungsprojekt um die Dissertation der Autorin, in deren Rahmen Interviews mit Organisierenden des Science Slams, mit Wissenschaftler_innen, die während des Science Slams als Slammende teilnahmen, und mit Personen aus dem Publikum geführt wurden. 
Anschließend an Veranstaltungsformate der Wissenschaftskommunikation ist der Science Slam das im Forschungsprojekt gewählte Beispiel, welches die Flexibilisierungs- und Verschiebungstendenzen deutlich kennzeichnet. Als Gegenbewegung zum akademischen Elfenbeinturm kann er als dessen exponierteste Form angesehen werden. Zudem geht es in dem Veranstaltungsformat um eine Setzung von Lern- und Bildungsimpulsen, auch für „Zuhörerschaften [...], die ansonsten kaum erreicht werden können“ (MS10201: 174-175).

Während der Science-Slam-Veranstaltung präsentieren Wissenschaftler_innen Forschungsergebnisse und Forschungsthemen. Sie vermitteln, ,was sie gemacht haben, warum sie es gemacht haben und was rausgekommen ist" (MS10201: 438). Dabei gelten „,drei Regeln: zehn Minuten, eigene Forschungsergebnisse und Spaß haben auf der Bühne. Was nicht heißt, dass die da den Hampelmann machen müssen oder den Comedian, das dürfen auch ernsthafte oder spannende oder traurige, was auch immer sie für Vorträge haben, welche Emotionen sie berühren, das ist eine andere Sache" (MS10201: 237-240). In der Verbindung von wissenschaftlichen Inhalten und mündlicher Vortragskunst, die durch die Vorführung eines Experiments, die Nutzung von Visualisierungen oder das Vortragen des Forschungsthemas als Gedicht unterstützt werden kann, entsteht mit jedem einzelnen Slam eine spezifische Performance:

„Beim Vortrag, der auch gerne als Performance bezeichnet wird, gilt es vor allem, das Publikum mittels Mündlichkeit von den eigenen Texten zu überzeugen - dabei gibt es viele Methoden und Techniken, vor allem auch das situative Moment und die Interaktion, die zwischen Publikum und Vortragendem herrscht, auszunutzen“ (Westermayr 2004: 9).

Die zehnminütigen Slams der Wissenschaftler_innen während einer ScienceSlam-Veranstaltung werden grundlegend als Performance eingeordnet. Einfluss auf die Slam-Performance nehmen die im Gesamten die Lernkultur konstituierenden „,komplexe[n] Strukturen, Orte, Räume, Bedingungen, Atmosphären und Arrangements“ (Gieseke 2007a: 223), die in den theoretischen Ausführungen zur Performance (Fischer-Lichte 2004) durch leibliche Ko-Präsenz, Körperlichkeit, Räumlichkeit, Lautlichkeit sowie Zeitlichkeit aufgegriffen werden. Während einer Performance werden Handlungen vollzogen, die deutlich machen, dass ,sprachliche Äußerungen nicht nur dem Zweck dienen, einen Sachverhalt zu beschreiben oder eine Tatsache zu behaupten" (Fischer-Lichte 2004: 31). Performative Handlungen können vielmehr als selbstreferentiell und wirklichkeitskonstituierend beschrieben werden. Darüber entsteht eine Interaktionsästhetik (Preckwitz 2002), die die Personen im Publikum zu handelnden Akteur_innen in der Performance werden lässt. Während des Science Slams bestimmt das Publikum in der abschließenden Applausabstimmung oder Punktevergabe am Veranstaltungs- 
abend die_den Science-Slam-Sieger_in. Bei der Einordnung des einzelnen Slams als Performance steht jedoch vielmehr die aktive Einbindung der einzelnen Personen in den jeweiligen Slam über Rückmeldungen und Interaktionen im Vordergrund.

Die Trennung zwischen Subjekt und Objekt wird damit aufgehoben, es handelt sich vielmehr um ein oszillierendes Verhältnis, ,in dem sich Subjektund Objektposition kaum mehr klar bestimmen noch auch deutlich voneinander unterscheiden ließen“ (Fischer-Lichte 2004: 20). Durch die damit einhergehende veränderte Rollenzuschreibung der Zuschauenden, aber auch Wissenschaftler_innen - ergo die Auflösung der Rollenzuschreibungen zwischen Produzent_innen und Rezipient_innen - entsteht mit jeder Slam-Performance ein spezifisches Bühnenstück, in das alle Beteiligten involviert sind (FischerLichte 2004). Diese Akteur_innenkonstellation innerhalb der Performance lässt einen Zwischenraum sichtbar werden, der „Intensität und Reibung“ (Seitz 1996: 344) und damit Produktivität ermöglicht, indem durch die Konfrontationen mit Wissen, Erfahrungen, Deutungen dazu angeregt wird, den Standpunkt zu ändern, loszulassen, irritiert zu sein (Seitz 1996) (zweiter Zugang zu Transformationsprozessen im Science Slam).

Ausgehend von dieser theoretischen Grundlegung wird aus einer erwachsenenpädagogischen Forschungsperspektive das Veranstaltungsformat Science Slam im Ganzen innerhalb des Forschungsprojektes als Lernkultur eingeordnet, die im Veranstaltungsformat durch eine Entgrenzung zwischen Inszenierung und Bildung charakterisiert ist. Dabei wird erst durch das Einbeziehen aller das Veranstaltungsformat Science Slam kennzeichnenden Perspektiven und Aspekte diese spezifische Lernkultur kenntlich. Die einzelne Slam-Performance ist dabei jeweils ein Aspekt der Lernkultur Science Slam, die hier angesetzten Betrachtungen gehen über ,die rein mikrodidaktische (Lehr/Lern)-Ebene hinaus“" (Schmidt-Lauf et al. 2013: 23), es geht um einen komplexeren und damit umfassenderen Zugang zur Lernkultur Science Slam. ${ }^{4}$

4 Im Forschungsprojekt liegt der Fokus ausgehend vom ersten Zugang zu Transformationsprozessen im Science Slam (zielgruppenspezifisch methodisch-didaktische Aufbereitung des Wissens durch die Wissenschaftler_innen), welcher eher einer Lehrkultur Science Slam entspricht, verstärkt auf dem zweiten Zugang zu Transformationsprozessen (Figuren der Überführung im Übergang zwischen wissenschaftlichem Wissen und Alltagswissen), so dass hier von einer Lernkultur Science Slam ausgegangen wird. Die Perspektive der Personen aus dem Publikum, die zu Akteur_innen und damit Teilnehmenden werden, wird zum zentralen Ausgangspunkt und stützt dabei gleichzeitig den dialogischen Anspruch in der Wissenschaftskommunikation, der über die ,reine“ Vermittlung hinausgeht. 


\section{Gestaltete Lernkulturen: Sichtbarmachung durch Perspektivenverschränkung}

Fernab von schulnahen Angeboten differenzieren sich Lernkulturen aus, so dass eine homogene Abbildung von Lernkulturen nicht mehr möglich ist. Substantiell ist daher nicht die Bestimmung, was Lernkulturen sind oder in welchen Definitionen sie sich widerspiegeln, sondern vorrangig sollen Lernkulturen zunächst wahrgenommen und vernetzt werden. Veranstaltungsformate der Wissenschaftskommunikation können als gestaltete Lernkulturen interpretiert werden, wenn sie didaktische und zielgruppenspezifische Aspekte für den Gesamtkontext konzeptionell beachten und in die Umsetzung einfließen lassen. „Lernkulturen umfassen [dabei grundlegend] komplexe Strukturen, Orte, Räume, Bedingungen, Atmosphären und Arrangements, die Wissen für die verschiedenen Lehr-/Lernsituationen umsetzen" (Gieseke 2007a: 223). Als gestaltbares Konstrukt unterliegen diese Aspekte der Lernkultur offenen Prozessen der Entfaltung und sind mit einer hohen Dynamik versehen. Daher sind Lernkulturen nur über eine Vielzahl von Aspekten zu beschreiben, die erst im Zusammenspiel ein Muster ergeben, welches als Lernkultur spezifiziert werden kann (Gieseke 2009). Daraus ergibt sich, dass Lernkulturen empirisch nicht unmittelbar zugänglich sind.

Auf Basis der Annahmen zur ,dichten Beschreibung“ (Geertz 2001) wird das Veranstaltungsformat Science Slam daher in einer Perspektivenverschränkung (Gieseke 2007b) analysiert. Erst durch die Erschließung von beeinflussenden Aspekten auf die Lernkultur, durch das Einbeziehen aller beteiligten Akteure und Akteurinnen sowie der Verschränkung ihrer Perspektiven wird das inhärente Konstrukt der Lernkultur fassbar und ihre vielschichtigen Ebenen können ausdifferenziert werden (Gieseke 2007b). Daraus ergibt sich gleichzeitig der Fokus des Forschungsprojektes, denn durch die Verschränkung der verschiedenen Perspektiven und Ebenen wird ein DaZwischen - der das Geschehen herstellende Moment - herausgearbeitet (Gieseke 2007b). Die Wahrnehmung des Da-Zwischens erlaubt, den Science Slam als gestaltete Lernkultur umfassend zu beschreiben.

Dieser Zielstellung des Forschungsprojektes gehen folgende Fragestellungen, die sich an den Zugängen $\mathrm{zu}$ den Transformationsprozessen (Schäffter 2001) innerhalb des Science Slams orientieren, voraus: Welche Inszenierungsstrategien und Vermittlungspraktiken von Wissen sowie Wissensbeständen werden in den Slam-Performances genutzt (erster Zugang)? Wie lassen sich Figuren der Überführung zwischen Alltagswissen und wissenschaftlichem Wissen beschreiben (zweiter Zugang)?

Die Bearbeitung der Fragestellungen erfolgte im Forschungsprojekt über den Einbezug aller am Veranstaltungsformat beteiligten Akteur_innen Organisator_innen des Science Slams, Slammende und Personen aus dem Publikum - in die Datenerhebung. Dazu wurden zum einen Expert_innen- 
interviews (Bogner et al. 2009; Gläser/Laudel 2009; Meuser/Nagel 2010) mit den Organisator_innen des Science Slams geführt. ${ }^{5}$ Zum anderen wurden fokussierte Interviews (Merton et al. 1956) mit Personen aus dem Publikum und den Slammenden durchgeführt, die sich inhaltlich explizit an der jeweiligen Science-Slam-Veranstaltung ausrichteten. Vervollständigt werden die Perspektiven durch Videomitschnitte der jeweiligen Slams und Beobachtungsprotokolle, erstellt in Anknüpfung an Annahmen zur fokussierten Ethnografie (Knoblauch 2001; Oeser 2008). Dem empirischen Material wird sich in der anschließenden Analyse aus drei divergenten Richtungen genähert. Es stehen in einem Auswertungsblock jeweils die Interviews mit Personen aus dem Publikum und die Interviews mit den Slammenden im Fokus. Dabei bewegen sich beide Annäherungen an das Material zunächst auf einer formulierenden Ebene als Perspektiven auf Science Slam und die Slam-Performances. Um eine anschließend reflektierende Interpretation zu ermöglichen, wird die wechselseitige Unbestimmtheit der sich gegenseitig ansteuernden Wissensstrukturen angenommen. In der Zusammenführung der Interviewsegmente als dritten Analyseschritt werden die Figuren der Überführung zwischen den Wissensstrukturen in ihren Schattierungen erkennbar. Für die Verschränkung der beiden Interviewsegmente wird neben den Interviewanalysen auf Beobachtungsprotokolle und Slam-Videomitschnitte zurückgegriffen, um die Perspektivenverschränkung zur Erschließung des Da-Zwischens der Lernkultur zu gewährleisten.

\section{Erschließung eines ausgewählten Lernkulturaspektes im Science Slam: Raumbetrachtungen}

In der Auswertung des Datenmaterials konnte neben anderen, die Lernkultur Science Slam prägenden und damit das Da-Zwischen beeinflussenden Aspekten auch der Aspekt Raum und Atmosphäre herausgefiltert werden. Dieser wird für die vorliegenden Ausführungen beispielhaft als ein die Lernkultur mitprägender Aspekt herausgegriffen. Zudem sind Raum und Atmosphäre im Kontext der Wissenschaftskommunikation zentrale Gestaltungsmomente, da im Science Slam nicht nur ein Zugang für eine spezifische Zielgruppe zu

5 Zum Zeitpunkt der Datenerhebung ergab eine systematische Recherche zum Veranstaltungsformat Science Slam vorwiegend Material aus medialer Berichterstattung, theoretische und empirische Auseinandersetzungen fehlten. Die Organisator_innen wurden daher als Expert_innen - immer jedoch als relationaler Status gedacht - eingestuft, da sie Innensichten auf den Forschungsgegenstand ermöglichen konnten. Mit dem „Begriff ,Experte“ [galt es,] all die Personen zu erfassen, deren Wissen über die zu untersuchenden sozialen Situationen und Prozesse im Interview erschlossen werden“ (Gläser/Laudel 2009: 9). 
wissenschaftlichen Inhalten geschaffen wird, sondern Wissenschaften explizit aus ihrem (universitären) Forschungsumfeld herausgelöst werden.

Der im Forschungszusammenhang untersuchte Science Slam findet monatlich in einem Veranstaltungsort in einer Großstadt statt. Über diese VerOrtung des Science Slams wird eine Verschiebung von räumlichen Zuschreibungen provoziert. Das entspricht dem Ziel der Science-Slam-Organisierenden, die „Wissenschaft da rausholen, wo man sie erwartet, und dahin bringen, wo sie kein Mensch erwartet" (MS10201: 118-119). Der spezifische Veranstaltungsort ist gesellschaftlich mit bestimmten Attributen belegt, die einer Funktionalitätszuschreibung folgen. Der Raum wird in einer theoretischen Fassung aus dieser Perspektive zur Hülle für die darin befindlichen Körper und Objekte (Schroer 2008: 44). Die Verschiebung der wissenschaftlichen Auseinandersetzung in diese unerwartete, fernab der Wissenschaften konnotierte Umgebung lässt ein Spannungsverhältnis zwischen den erwarteten und genutzten Räumlichkeiten entstehen. Wissenschaften werden häufig nicht mit dem gewählten Veranstaltungsort in Verbindung gebracht, ,nein, ich war sehr verwundert, dass sowas im SO36 stattfindet, wo es so viele, sage ich mal, kleine Bühnen oder Kulturvereine, Clubs oder sonst was gibt" (MS171207: 10).

Jedoch wird auch deutlich, dass der Raum nicht einfach nur existiert. Räume bringen Handeln hervor, aber dieses Handeln reproduziert wiederum auch räumliche Strukturen, so dass der Raum in einem Kontinuum der Dualität von Handeln und Struktur spiralförmig entsteht (Löw 2007). Das Verhalten im Raum wird durch die Zuschreibungen, die der Veranstaltungsort hervorruft, gelenkt. Der mit dem Science Slam stattfindende räumliche Verschiebungsprozess führt zu einem Durchbrechen von dem Raum zugeschriebenen „Verhaltensregeln“ (Schroer 2006: 177). Die Anwesenden treffen durch den Science Slam auf Anforderungen und Programmatiken des Veranstaltungsortes, sie beschäftigen sich mit seinen Hintergründen und damit einhergehenden politischen sowie kulturellen Themen. Gleichzeitig öffnet sich der Veranstaltungsort dabei selbst Inhalten und Zielgruppen, die in der bisherigen Funktionalitätsbeschreibung nicht mitgedacht wurden.

Im Zusammenspiel der Akteur_innen und ihren Zuschreibungen zum Ort entsteht eine Atmosphäre, die Emotionen lenkt und emotionale Reibungen hervorruft (Gieseke 2007a). Daher beginnen die Interviewten, sich den Veranstaltungsort losgelöst von seiner bisherigen Konnotation zu erschließen. Diese Ebene ist geprägt durch die Betrachtung der sich zirkulär und im Wechselverhältnis entwickelnden Atmosphäre, welche sich ,als ein nie zu fixierender Zustand des Dazwischen, weder dem Objekt noch dem Subjekt ganz zugehörig, aber von beiden gemeinsam produziert“" (Lehnert 2011: 16) ausbildet. Aus den Interviews wird deutlich, dass die Beschreibung der Atmosphäre zum einen über atmosphärische Gefühlsäußerungen, zum anderen aber auch über die Wirkung des Raumes greifbar wird. Dabei ruft die verän- 
derte Raumkonstruktion auch emotionale Reibungen hervor. Es scheint schwierig, das Veranstaltungsformat in die vorhandenen Zuschreibungen zum erwarteten wissenschaftlichen Veranstaltungsort einzuordnen. Der ,gespürte" Raum bildet sich somit in einer spezifischen Zuschreibungslage aus. Grundlegend für die ,gespürte“ Atmosphäre und ihre Wirkungen ist daher Relationalität (Gieseke 2007a). Die Interviewten setzen sich darüber auch mit ihren eigenen Deutungen und Erfahrungen auseinander.

Die Interviewaussagen aus dem Forschungsprojekt lassen sich daher zwei Ebenen zuordnen: Entweder wird (a) die Verschiebung des Veranstaltungsortes - Wissenschaften herauslösen aus wissenschaftlichen Kontexten und die damit einhergehende Veränderung der sozialen Praktiken thematisiert oder (b) die Interviewten beschreiben, was die veränderte Raumkonstruktion auf einer emotionalen Ebene bei ihnen auslöst, Atmosphäre wird darüber greifbar. Damit wird die Annahme gestützt, dass die Zuschreibungen zum Raum bestimmte soziale Praktiken hervorrufen sowie Erfahrungen und Deutungsmuster offenlegen.

Deutlich wird durch diese punktuellen Beschreibungen der Raumbetrachtungen im Science Slam ein dynamisches Raumkonzept, das den Raum durch Bewegung, Wahrnehmung und soziales sowie symbolisches Handeln entstehen lässt. Der im Science Slam genutzte performative Raum ist daher ein sich ständig verändernder Raum. Die beispielhaften und nicht erschöpfenden Ausführungen zu Raumbetrachtungen zeigen die Komplexität auf, denen die einzelnen Aspekte einer Lernkultur in ihrer analytischen ErschlieBung unterliegen. Es handelt sich bei Raum und Atmosphäre um nur einen Aspekt, der die spezifische Lernkultur kennzeichnet. Die Erschließung und Zusammenführung aller entsprechenden Aspekte charakterisiert dann die jeweilige Lernkultur.

\section{Zusammenführung}

Wie beschrieben sind Lernkulturen nicht unmittelbar empirisch zugänglich. Als gestaltbares Konstrukt unterliegen sie offenen Prozessen der Entfaltung und sind mit einer hohen Dynamik versehen. Daher sind sie nur über eine Vielzahl von Aspekten und Perspektiven zu beschreiben, die erst im Zusammenspiel eine ganzheitliche Betrachtung der Lernkultur ermöglichen. Über Perspektivenverschränkung in der Erhebung und Analyse können die einzelnen Aspekte ausdifferenziert werden. Raum und Atmosphäre bilden einen Aspekt, der auf die spezifische Lernkultur Science Slam Einfluss nimmt, denn Lernende sind niemals unabhängig von ihrer Lernumgebung, welche sich der_dem Einzelnen über ein gefühlsmäßiges Eingelassen-Sein in den Lernraum vermittelt. Die über die Ausführungen angedeutete detaillierte 
empirische Erschließung der Lernkultur macht deren analytische Besonderheit deutlich.

\section{Literatur}

Antos, Gerd (2005): Vorwort. In: Antos, Gerd/Wichert, Sigurd (Hrsg.): Wissenschaftstransfer durch Sprache als gesellschaftliches Problem. Frankfurt a.M.: Peter Lang Verlag, S. IX-XII.

Bogner, Alexander/Littig, Beate/Menz, Wolfgang (Hrsg.) (2009): Experteninterviews. Theorien, Methoden, Anwendungsfelder. Wiesbaden: VS Verlag für Sozialwissenschaften.

Faulstich, Peter/Trumann, Jana (2016): Wissenschaftsvermittlung, Popularisierung und kollektive Wissensproduktion. In: Magazin erwachsenenbildung.at 27, S. 02-1-02-11.

Fischer-Lichte, Erika (2004): Ästhetik des Performativen. Frankfurt a.M.: Suhrkamp Verlag.

Geertz, Clifford (2001): Dichte Beschreibung. Beiträge zum Verstehen kultureller Systeme. Frankfurt a.M.: Suhrkamp Verlag.

Gieseke, Wiltrud (2007a): Lebenslanges Lernen und Emotionen. Wirkungen von Emotionen auf Bildungsprozesse aus beziehungstheoretischer Perspektive. Bielefeld: W. Bertelsmann Verlag.

Gieseke, Wiltrud (2007b): Das Forschungsarrangement in der Perspektivverschränkung. In: Erwachsenenpädagogischer Report 11, S. 10-22.

Gieseke, Wiltrud (2009): Organisationstheoretische Überlegungen zur Lernkultur Der übersehene institutionelle/organisatorische Faktor im Lernkulturdiskurs. In: Gieseke, Wiltrud/Robak, Steffi/Wu, Ming-Lieh (Hrsg.): Transkulturelle Perspektiven auf Kulturen des Lernens. Bielefeld: Transcript Verlag, S. 49-86.

Gieseke, Wiltrud (2010): Atmosphäre in Bildungskontexten - Beziehungstheoretische Überlegungen. In: Egger, Rudolf/Hackl, Bernd (Hrsg.): Sinnliche Bildung? Pädagogische Prozesse zwischen vorprädikativer Situierung und reflexivem Anspruch. Wiesbaden: VS Verlag für Sozialwissenschaften, S. 57-70.

Gläser, Jochen/Laudel, Grit (2009): Experteninterviews und Qualitative Inhaltsanalyse. Wiesbaden: VS Verlag für Sozialwissenschaften.

Janich, Nina (2006): Qualitätsmerkmale von Texten im Wissenstransfer. Theoretische und empirische Perspektiven. In: Wichter, Sigurd/Busch, Albert (Hrsg.): Wissenstransfer - Erfolgskontrolle und Rückmeldungen aus der Praxis. Frankfurt a.M.: Peter Lang, S. 201-212.

Knoblauch, Hubert (2005): Focused Ethnography. In: Forum Qualitative Sozialforschung/Forum: Qualitative Social Research 6, 3. http://nbn-resolving.de/urn:nbn: de:0114-fqs0503440. [Zugriff: 06.08.2014].

Löw, Martina (2007): Zwischen Handeln und Struktur. Grundlagen einer Soziologie des Raums. In: Kessl, Fabian/Otto, Hans-Uwe (Hrsg.): Territorialisierung des Sozialen. Regieren über soziale Nahräume. Opladen \& Farmington Hills: Verlag Barbara Budrich, S. 81-100.

Merton, Robert King/Fiske, Marjorie/Kendall, Patricia L. (1956): The focused interview: a manual of problems and procedures. Glencoe: Free Press. 
Meuser, Michael/Nagel, Ulrike (2010): Experteninterviews - wissensoziologische Voraussetzungen und methodische Durchführung. In: Friebertshäuser, Barbara/ Langer, Antje/Prengel, Annedore (Hrsg.): Handbuch Qualitative Forschungsmethoden in der Erziehungswissenschaft. Weinheim und München: Juventa Verlag, S. 457-471.

Oester, Kathrin (2008): ,Fokussierte Ethnographie‘: Überlegungen zu den Kernansprüchen der Teilnehmenden Beobachtung. In: Hünersdorf, Bettina/Maeder, Christoph/Müller, Burkhard (Hrsg.): Ethnographie und Erziehungswissenschaft. Methodologische Reflexionen und empirische Annäherungen. Weinheim und München: Juventa Verlag, S. 233-243.

Pansegrau, Petra/Taubert, Niels/Weingart, Peter (2011): Wissenschaftskommunikation in Deutschland. Ergebnisse einer Onlinebefragung. Eine Untersuchung im Auftrag des Deutschen Fachjournalisten-Verbandes (DFJV). https://www.dfjv. de/documents/10180/178294/DFJV_Studie_Wissenschaftskommunikation_in_D eutscDeuts.pdf. [Zugriff: 29.11.2015].

Preckwitz, Boris (2002): Slam Poetry - Nachhut der Moderne. Eine literarische Bewegung als Anti-Avantgarde. Hamburg: Books on Demand.

Schäffter, Ortfried (2001): Weiterbildung in der Transformationsgesellschaft: Zur Grundlegung einer Theorie der Institutionalisierung. Baltmannsweiler: Schneider Verlag Hohengehren.

Schmidt-Lauff, Sabine/Lemke, Tobias/Kochan, Marie (2013): Erwachsenengerechte Lernkultur an der Hochschule. In: HDS.Journal 1, S. 22-30, https://www.hds.unileipzig.de/fileadmin/media/HDS.Journal_I_2013_Tagungsedition.pdf. [Zugriff: 07.06.2016].

Schroer, Markus (2006): Räume, Orte, Grenzen. Auf dem Weg zu einer Soziologie des Raumes. Frankfurt a.M.: Suhrkamp Verlag.

Schroer, Markus (2008): Raum: Das Ordnen der Dinge. In: Moebius, Stephan/Reckwitz, Andreas (Hrsg.): Poststrukturalistische Sozialwissenschaften. Frankfurt a.M.: Suhrkamp Verlag, S.141-157.

Seitz, Hanna (1996): Räume im Dazwischen. Bewegung, Spiel und Inszenierung im Kontext ästhetischer Theorie und Praxis. Grundlegung einer Bewegungsästhetik. Essen: Klartext.

Stimm, Maria (2016): Ortsraum. Gefühlsraum? Sozialraum! Ein Brückenschlag zwischen Raumbetrachtungsebenen und Bildungsimpulsen am Beispiel des Veranstaltungsformats Science Slam. In: Ludwig, Joachim/Ebner von Eschenbach, Malte/Kondratjuk, Maria (Hrsg.): Sozialräumliche Forschungsperspektiven verschiedener Disziplinen. Opladen u.a.: Barbara Budrich Verlag, S. 75-89.

Weingart, Peter/Voß, Miriam (2008): Wissenschaftskommunikation. In: Wissenschaftsmanagement Online. http://www.wissenschaftsmanagement-online.de /system/files/downloads-wimoarticle/Themenfeld\% 209_Weingart\%20Voss_Final.pdf. [Zugriff: 02.04.2016].

Westermayr, Stephanie (2004): Poetry Slam in Deutschland. Theorie und Praxis einer multimedialen Kunstform. Marburg: Tectum-Verlag. 


\section{Lebensgeschichte(n) im Museum. Empirische Lernkulturforschung im Feld der kulturellen Erwachsenenbildung}

Die Debatte über Lernkultur wird seit den 1990er Jahren mit zunehmender Intensität geführt. Sie ist verbunden mit Diskussionen über Unternehmenskultur und lernende Organisationen, aber auch mit der Forderung nach einer Modernisierung von Bildungseinrichtungen (Schule, Erwachsenenbildung, betriebliche Aus- und Weiterbildung) und der Politik des Lebenslangen Lernens. Der folgende Text beschäftigt sich mit einem spezifischen Teilbereich von Bildung - der kulturellen Erwachsenenbildung. Hier wird ein weiter Kulturbegriff verwendet, der Alltagskultur und Soziokultur (vgl. Schlutz 2015) ebenso einschließt wie die Auseinandersetzung mit sogenannter Hochkultur. Kulturelle Erwachsenenbildung findet in sehr unterschiedlichen Formen statt, die sich von unterrichtlichen Lernarrangements oft sehr unterscheiden. Sie sind eingebettet in (alltags-)kulturelle Aktivitäten und Tätigkeiten, die um ihrer selbst willen stattfinden und weniger von den sonst üblichen Nützlichkeits- oder Verwertungserwägungen bestimmt sind. Deshalb werden sie von den Teilnehmenden oft auch nicht in erster Linie als Lernen und Bildung beschrieben, was jedoch nicht heißt, dass sie nicht genau das sind: Prozesse der Aneignung von Welt sowie Transformationen im Verhältnis zwischen Selbst, Welt und Anderen. Die inhaltlichen Gegenstände sind vielfältig und entstehen in der Zusammenarbeit von Teilnehmenden, WissenschaftlerInnen, KünstlerInnen, PädagogInnen und anderen Berufsgruppen. Die Pluralität von Organisationsformen, Inhalten und Arrangements macht die kulturelle Erwachsenenbildung - gerade auch für eine empirische Lernkulturforschung - zu einem interessanten Forschungsfeld.

Ich beginne meinen Beitrag mit Beobachtungen dazu, wie der Begriff der Lernkultur in der Erwachsenenbildung gebraucht wird und unterscheide dabei zwischen Positionen, die primär die (Um-)Gestaltung von Lernkultur fokussieren und solchen, die an der Analyse und Rekonstruktion von Lernkultur interessiert sind (1). Anschließend konkretisiere ich letztere am Beispiel eines ethnographischen Forschungsprojekts über ein Lernarrangement in einem stadtgeschichtlichen Museum ${ }^{1}$ (2). Auf der Grundlage der

1 Die hier vorgestellte Forschung ist Teil eines Projektes über Prozesse und Praktiken der Biographisierung in der Erwachsenen- und Weiterbildung. Es ist als multilokale Ethnographie angelegt, d.h. es werden auch noch andere Bildungseinrichtungen und Lernarrangements untersucht. Da sich sozialwissenschaftliche Ethnographie als Kulturforschung ver- 
exemplarischen Analyse spitze ich am Ende zu, welche Erkenntnismöglichkeiten mit einer praxeologischen Perspektive für die Lernkulturforschung in der Erwachsenenbildung verbunden sind (3).

\section{Lernkultur - Gebrauchsweisen des Begriffs in der Erwachsenenbildung}

Die Popularität des Begriffs „Lernkultur“ ist auch eine Folge von in den 1990er Jahren politisch installierten Forschungs- und Entwicklungsprogrammen $^{2}$, deren Ziel es war, in Kooperation mit der Forschung die Erwachsenenund Weiterbildung entsprechend des bildungspolitischen Programms des Lebenslangen Lernens umzugestalten. Der Abschluss dieser Programme ermöglicht eine Neuorientierung in der Lernkulturforschung und die Bearbeitung der nach wie vor bestehenden theoretischen und empirischen Leerstellen (vgl. Dammer 2013, Laschewski 2013).

\section{1 "Neue Lernkultur" in der Erwachsenenbildung}

Mit dem Begriff der Lernkultur werden zumeist in sehr umfassender Weise institutionelle und organisationale Ordnungen, didaktische Gestaltung und praktische Durchführung von Lehren und Lernen bezeichnet. Begründet wird die Notwendigkeit der gezielten Veränderung von Lernkulturen mit gesellschaftlichen Modernisierungsprozessen und damit verbundenen Anforderungen in der Arbeitswelt, denen mit einer Veränderung des Lernens begegnet werden kann und muss (Arnold/Schüßler 1998, Schüßler/Thurnes 2005). Dabei werden reformpädagogische Ideen unter den gesellschaftlichen Bedingungen des Neoliberalismus wiederbelebt (vgl. Dammer 2013), aber auch lerntheoretische Perspektiven wie der Konstruktivismus programmatisch gewendet und als Gestaltungsprinzip neuer Lernkultur profiliert (Arnold/ Schüßler 1998; Arnold/Lermen 2009). Selbststeuerung und Selbstorganisation werden so zu neuen Normen für die didaktische Gestaltung und machen Lernen zu einer Sache individueller Selbstverantwortung. Die Kennzeichnung von Lernkultur als ,neu“ bedeutet zumeist nur eine abgrenzende Unterscheidung gegenüber ,alt", oder auch ,traditionell“. Sie markiert nicht nur eine zeitliche Differenz, sondern beinhaltet auch eine Bewertung, weil die „,neue“ im Vergleich zur „alten“ oder auch „überholten“ Lernkultur als die

steht, die sich mit Ausschnitten der eigenen Kultur beschäftigt, lässt sich dieses Projekt auch als Projekt über verschiedene Lernkulturen in Bildungseinrichtungen verstehen.

2 Ein prominentes Beispiel ist das aus Mitteln des Bundesministeriums für Bildung und Forschung und dem Europäischen Sozialfonds geförderte Programm „Lernkultur-Kompetenzentwicklung“" (Laufzeit 2001-2007). 
einzig zeitgemäße erscheint. Die im Vergleich zum Umfang der Debatte geringfügigen Veränderungen in der Praxis des Lehrens und Lernens werden mit den „biographischen Erfahrungen“ der Lehrenden (Arnold/Lermen 2009: 45) oder der Abwehr von Veränderungsprozessen durch die Lernenden erklärt (Schüßler/Thurnes 2005: 122). Man kann aber auch vermuten, dass die gestalterisch-programmatische Ausrichtung des Lernkulturdiskurses überdeckt, dass wir theoretisch und empirisch zu wenig darüber wissen, wie Lernkulturen „funktionieren“ und welche Rolle die Lernenden dabei spielen. Offenbar entziehen sich Komplexität und Eigenlogik von Lernkulturen der politischen, administrativen und didaktischen Intervention.

Der Begriff „,neue Lernkultur“ teilt darüber hinaus einige der Probleme, die auch mit dem Begriff „Lebenslanges Lernen“ verbunden sind. Dass neue Lernkultur nicht zwangsläufig selbstbestimmtes Lernen bedeutet, sondern auch eine besonders optimale Verschränkung von Fremd- und Selbststeuerung sein kann, wurde insbesondere in Studien gezeigt, die mit dem Konzept der Gouvernementalität arbeiten (vgl. Klingovsky 2009; Rothe 2011). Die soziale Komplexität interaktiver und kommunikativer Ordnungen, in denen Lernen gerade auch in den Gruppensettings der Erwachsenenbildung stattfindet, wird mit der starken Fokussierung auf Selbststeuerung und Selbstorganisation häufig zu wenig differenziert betrachtet.

Auch der meist selbstverständlich benutzte Kulturbegriff verdient eine genauere Betrachtung. Er wird oft für die Bezeichnung von Deutungs- und Wahrnehmungsmustern, Wissensordnungen, kollektiven Sinnsystemen und Institutionen verwendet, die dann auf das Lernen und seine Gestaltung bezogen werden (z.B. Schüßler/Thurnes 2005). Spezifizierungen wie regional, national und international verweisen auf geographische und politische bzw. nationalstaatliche Ordnungen. Dabei wird unterstellt, dass damit lernrelevante Differenzen verbunden sind, die unterscheidbare Lernkulturen hervorbringen. Die theoretische Verknüpfung zwischen geographischem Raum, nationalem Kontext und Lernen bleibt zumeist unbestimmt. Die Rede von der Transkulturalität bleibt dieser Logik letztlich verhaftet, auch wenn hier ein zusätzlicher Bereich identifiziert wird, der aus Gemeinsamkeiten und Überschneidungen zwischen den Kulturen entsteht (Gieseke/Robak 2009). Der Rekurs auf kulturelle Differenz bleibt jedoch bestehen.

Anders als im Begriff des „Lebenslangen Lernens“ liegt in dem Begriff der Lernkultur jedoch ein erhebliches theoretisches und empirisches Potenzial, vor allem wenn es gelingt, an die theoretischen und methodischen Entwicklungen anzuknüpfen, die seit dem „cultural turn“ in den Sozialwissenschaften entstanden sind. Nicht zuletzt in der kritischen Auseinandersetzung mit dem Kulturbegriff haben sich Forschungsansätze entwickelt, die produktive Anknüpfungsmöglichkeiten für die Erwachsenenbildungsforschung bieten. Sie ermöglichen, „Kultur“ nicht als übergeordnete normative Deutungsmuster oder Regeln zu untersuchen, sondern als konkrete, situierte und 
verstetigte Praxis in spezifischen Lernkontexten, die lebensweltlich oder institutionell gerahmt sein kann. Erste Hinweise für eine praxeologische Wendung des Lernkulturbegriffs finden sich auch in der Erwachsenenbildungsforschung (z.B. Gieseke/Robak 2009). Laschewski (2013) entwickelt auf der Grundlage der praxeologisch ausgerichteten Akteur-Netzwerk-Theorie von Bruno Latour ebenfalls einen interessanten Vorschlag für die empirische Untersuchung von lokalen Lernkulturen. Dabei steht im Mittelpunkt, wie E-Learning und die darin eingeschriebenen Handlungsskripte in der Weiterbildung wirksam werden.

\subsection{Eine praxeologische Perspektive in der Lernkulturforschung}

Die praxeologische Perspektive entwickelt einen Kulturbegriff, der sich von einem normativen oder differenzierungstheoretischen Verständnis von Kultur deutlich unterscheidet (vgl. Reckwitz 2003; Moebius 2009; Hörning/Reuter 2004; Schäfer/Daniel/Hillebrandt 2015). Kultur wird hier als soziale Praxis verstanden, durch die „Deutungsstrukturen, Sinnstrukturen, kollektive Wissensschemata und symbolische Machtverhältnisse erst ihre Wirkungen entfalten und überhaupt bestehen können." Soziale Praktiken sind „sozial geregelte, eingeübte, typisierte sowie routinierte Formen körperlicher Darstellungen" (Reckwitz 2006: 36). Sie beruhen auf implizitem praktischen Wissen sowie übersubjektiven Handlungsgefügen bzw. -mustern, die routiniertes soziales Handeln ermöglichen. Diese gilt es mit den Mitteln interpretativer Forschung zu rekonstruieren. Die Ethnographie hat sich dafür als besonders geeigneter Forschungszugang erwiesen, ermöglicht sie doch, soziale Praktiken in ihrem Vollzug zu beobachten und anschließend zum Gegenstand der Analyse zu machen (vgl. Breidenstein u.a. 2013). Dabei geht es nicht allein um das Handeln der Akteure, sondern auch um die materiellen Räume und Strukturen, in denen Handlungsvollzüge stattfinden, und die Artefakte, die Teil dieser Handlungsvollzüge sind. Artefakte werden nicht nur genutzt, verändert und hervorgebracht, sondern rahmen Handlungen und erweisen sich oft genug gegenüber den Handlungsintentionen der Akteure als widerständig (vgl. Hörning/Reuter 2004; Wieser 2004).

Soziale Praktiken und inkorporierte Wissensbestände bestimmen auch das Geschehen in Bildungssituationen. Das gilt für das Handeln der Teilnehmenden und der Professionellen sowie für die Abstimmung zwischen beiden. Entwickelt und verinnerlicht in langen Jahren der routinierten Teilnahme an institutionalisierten Lehr-Lernarrangements in der Schule, Hochschule sowie Aus- und Weiterbildung, bilden diese Praktiken die zentrale Grundlage für das weitgehend reibungslose Funktionieren von organisierten Lernsituationen in der Erwachsenenbildung. Didaktische Strategien und Konzepte ruhen auf 
diesen grundlegenden Routinen des Handelns auf und tragen sowohl zur Verfestigung von Lernpraktiken und Handlungsroutinen als auch zu ihrer Transformation bei. Das Gelingen didaktischer Arrangements beruht nicht zuletzt darauf, dass sie an die habitualisierten Handlungsroutinen der Beteiligten in Lernsituationen anschließen und diese nicht grundsätzlich in Frage stellen, sondern allenfalls neue Varianten von Handlungsroutinen hervorbringen. ${ }^{3}$

Materialitäten und Artefakte spielen eine zentrale Rolle im interaktiven Geschehen. Sie ermöglichen, dass Personen organisierte Bildungssituationen als solche erkennen und in ihnen handlungsfähig sind: die Struktur des Raumes, die Anordnung von Tischen und Stühlen, die Tafel (Flipchart, Whiteboard oder Leinwand), Bücher, Unterlagen, Schreibutensilien, aber auch die Platzierung von Geräten wie Laptop und Beamer.

Besonders die ethnographische Schulforschung hat in den letzten Jahren Erkenntnisse über und Einsichten in die sozialen und pädagogischen Praktiken produziert, die das organisierte Lernen in Bildungsinstitutionen bestimmen und es den Beteiligten - LehrerInnen wie SchülerInnen - erlauben, als kompetente Akteure zu agieren (z.B. Breidenstein 2006; Zaborowski/Meier/ Breidenstein 2011). In der Erwachsenenbildungsforschung sind ethnographische Zugänge noch eher die Ausnahmen (z.B. Ott 2011), auch wenn es durchaus eine Tradition der Interaktionsanalyse gibt (Nolda 1996; Herrle 2012). ${ }^{4}$ Ergänzend dazu interessiert sich eine praxeologische Perspektive auf Lernkultur für die Handlungsroutinen und impliziten Wissensbestände, die geordnete Interaktionen im Lehr-Lerngeschehen überhaupt erst möglich machen. Wie eine solche praxeologische Lernkulturforschung aussehen könnte, erläutere ich im Folgenden exemplarisch an einer ethnographischen Forschung in einem stadtgeschichtlichen Museum.

3 Geht man davon aus, dass „Lernkultur“ auf dieser grundsätzlichen Ebene organisierten Lerngeschehens angesiedelt ist, wird auch deutlich, dass eine gezielte Veränderung der Lernkultur wie auch andere Kulturveränderungen nicht so ohne Weiteres möglich sind. In empirischen Vergleichen würde man vermutlich finden, dass zentrale Praktiken des Lernens in organisierten Settings außerordentlich dauerhaft sind und sich Veränderungen vor allem auf der Ebene didaktischer Konjunkturen abspielen.

4 Daraus hat sich inzwischen auch eine Richtung videographischer Kursforschung entwickelt (vgl. Kade u.a. 2014), die jedoch nicht unbedingt an praxeologische Theorien und Positionen anschließt und sich in der Folge auch nicht als ethnographische Lernkulturforschung versteht. Im Vordergrund steht vielmehr die Analyse von Wissensvermittlung und Wissenserwerb im Rahmen interaktiver Lern- und Bildungsprozesse in Kursgruppen, in denen auch Raumstrukturen, Artefakte und performative Prozesse der Beteiligten von Bedeutung sind. 


\section{Exemplarisch: Ethnographische Feldforschung im stadtgeschichtlichen Museum}

Museen verstehen sich zumeist nicht in erster Linie als Bildungseinrichtungen, sondern als Orte des Sammelns, Archivierens, Forschens und der Präsentation von Objekten. Dass sie etwas zeigen, weist aber schon auf die grundlegende pädagogische Dimension von Museen hin, die dann in der museumspädagogischen Vermittlungsarbeit auch deutlich hervortritt. Das stadtgeschichtliche Museum, in dem ich forsche, verfügt nicht nur über eine Dauerausstellung, sondern behandelt in wechselnden Ausstellungen zeitgeschichtliche Themen, die in der Dauerausstellung nicht oder wenig vorkommen. Es geht dabei nicht nur um große historische Ereignisse, sondern auch um die historische Veränderung der Alltagskultur. Dafür spielt die Auseinandersetzung mit besonderen Orten (das Stadtviertel, der Garten, die Parks, das Meer) und den alltäglichen Tätigkeiten in der Stadt (Mobilität, Konsum) sowie mit ihren BewohnerInnen - den berühmten aber auch den sogenannten kleinen Leuten - eine zentrale Rolle. Das Museum spricht auf diese Weise nicht nur Touristen und das historisch interessierte Publikum an, sondern Menschen, deren Lebensgeschichte und Alltagserfahrungen mit der Stadt und ihrer Geschichte auf ganz unterschiedliche Weise verbunden sind.

\subsection{Das Forschungsfeld: Lebensgeschichtliches Erzählen im Museum}

Das didaktische Arrangement „Gesprächskreis“, in dem ich meine Beobachtungen durchführe, ist ein regelmäßiges Angebot im Frühjahrs- und Herbstprogramm der museumspädagogischen Vermittlungsarbeit. Es richtet sich vor allem an ältere Menschen, die sich für Lebensgeschichten und Zeitgeschichte interessieren, und beruht auf Methoden des moderierten biographischen Erzählens (Hof 1995; Behrens-Cobet/Reichling 1997). In der Vorbereitung aber auch in der Begleitung von Ausstellungen bietet es den Teilnehmenden die Möglichkeit, zu einem festgelegten und mit der Stadt verbundenen Thema über ihre eigenen Erlebnisse und Erfahrungen zu erzählen und die Erzählungen anderer zu hören. Die Veranstaltung wird von externen SozialwissenschaftlerInnen (Geschichte, Soziologie) in Absprache mit dem Museum vorbereitet und moderiert. Entlang eines ausgewählten Themas werden an mehreren Terminen jeweils unterschiedliche inhaltliche Facetten beleuchtet. Häufig entwickeln sich neue thematische Facetten aus den Erzählungen heraus und werden von der Moderation aufgegriffen und weitergeführt. 


\subsection{Das Vermittlungsarrangement: Der Raum, die Akteure, die Dinge}

Das Arrangement lässt sich anhand eines Auszugs aus einem Beobachtungsprotokoll vorstellen und erläutern. Dabei geht es mir in einer ethnographischen Einstellung darum, das räumlich-materielle Arrangement so zu beschreiben, dass deutlich wird, wie es das soziale und interaktive Geschehen rahmt und mitbestimmt.

Kontext: Die 20 bis 35 Teilnehmenden treffen sich wie immer vor Veranstaltungsbeginn im Foyer des Museums und werden dort von den ModeratorInnen erwartet. Auf ein Zeichen des Moderators oder der Moderatorin setzt sich die Gruppe langsam in Bewegung. Sie durchquert das Atrium, geht durch eine Tür, die sonst geschlossen ist, und ein paar Stufen hinauf. Alle betreten nacheinander das „Extrazimmer“ und suchen sich einen Sitzplatz. In einem Protokoll heißt es: Der recht lang gestreckte Raum ist vorbereitet. (...) Eine lange Tafel ist gestellt mit vielen Stühlen, wirklich jeden Platz ausnutzend. Auf dem Tisch stehen kleine Gläser, Wasserflaschen und Salzstangen. An drei Stellen des Tisches liegen Schreibsachen und aufgestapelte, beschriftete Namensaufsteller. An der Seite des Raumes, die der Tür gegenüberliegt, ist noch eine „zweite Reihe“ mit Stühlen und auch zwei kleinen Tischen gestellt. Die Plätze um den großen langen Tisch sind schnell besetzt. Am Ende ist noch ein Platz am großen Tisch frei, die Moderatorin macht die Personen in der zweiten Reihe nachdrücklich darauf aufmerksam. Ich sitze in der zweiten Reihe neben einem Tisch - damit ich schreiben kann (...) (Protokoll/2013/10/10/1,32-1,42).

In dieser Beschreibung werden drei Aspekte des Geschehens besonders gut sichtbar: der Raum, die Akteure und die Dinge. Zunächst ist festgehalten, was sich zu den einzelnen Beobachtungsterminen im Feld wiederholt und auf etablierte Routinen des Settings verweist. Die Teilnehmenden und die ModeratorInnen treffen sich im Foyer des Museums und gehen gemeinsam zum eigentlichen Ort des Geschehens, der als „Extrazimmer“ auch in der Bezeichnung als ein besonderer Ort hervorgehoben ist. Er liegt - durch eine meist geschlossene Tür vom öffentlich zugänglichen Teil des Museums abgetrennt - hinter der Ausstellungsfläche und dem Café, wo Büros und Arbeitsplätze der MitarbeiterInnen die Hinterbühne des Ausstellungsgeschehens bilden. Es handelt sich um einen multifunktionalen Arbeitsraum, der für unterschiedliche Veranstaltungen genutzt wird und in dem unterschiedliche Gegenstände und Materialien in Wandregalen abgelegt sind. Obwohl der Gesprächskreis ein öffentliches, für alle Interessierten zugängliches Angebot ist, erzeugt die Lage des Raumes gleichzeitig eine Form der Abgeschlossenheit. Sind die Türen einmal durchschritten und geschlossen, bleibt die Gruppe unter sich. 
Das Arrangement der Tische und Stühle weist einige für die Erwachsenenbildung typische, aber auch einige ungewöhnliche Merkmale auf. Im Unterschied zu einer frontalen Unterrichtssituation, in der Teilnehmende und Professionelle sichtbar räumlich voneinander getrennt sind, sitzen die Teilnehmenden und ModeratorInnen in einer Art Kreis um einen großen, zusammengestellten Tisch. Dieser dient nicht nur zum Abstellen von Gläsern und Flaschen, sondern auch zum Ablegen und ggf. Ausbreiten von Unterlagen und zur Platzierung der Namensaufsteller. Für gewöhnlich liegen mehrere Aufnahmegeräte auf dem Tisch, die in diesem Protokollauszug nicht erwähnt werden, aber zeigen, dass Aufzeichnung und Archivierung selbstverständlicher Bestandteil des Settings sind. ${ }^{5}$ Die bereits beschrifteten Namensaufsteller machen deutlich, dass man zumindest ungefähr weiß, wer anwesend sein wird. Aber man kennt sich untereinander offenbar nicht so gut, dass Namensschilder verzichtbar sind. Es gibt einen festen Kern an regelmäßig Teilnehmenden, andere kommen unregelmäßig oder punktuell zu Themen, die sie besonders interessieren. Neue Teilnehmende können immer dazu kommen, es gibt weder Zugangsreglementierungen noch Anwesenheitserwartungen für die Dauer einer thematischen Reihe. Obwohl sich einige Teilnehmende schon lange kennen, ist aufgrund der Größe der Gruppe und der unterschiedlichen Grade der Bekanntheit untereinander der Umgang eher formell. Man spricht sich wechselseitig mit Sie an. Sitzordnung und die Nutzung des Tisches für Namensaufsteller, Wassergläser, Unterlagen und Aufnahmegeräte verleihen dem Arrangement den Charakter einer gemeinsamen Arbeitssituation oder einer Konferenz.

Die Beschreibung im Protokoll macht deutlich, dass der Raum eng und die Anzahl der Sitzplätze am Tisch knapp ist, so dass in einer „Zweiten Reihe" noch einige Reserveplätze vorgesehen sind. Personen, die hier sitzen, nehmen eine gewisse räumliche und soziale Distanz zum Geschehen ein. Dazu gehören die Beobachterin, die KuratorInnen des Museums, die als Gäste teilnehmen, KooperationspartnerInnen aus der Universität oder Personen, die sich für biographisches Erzählen interessieren. Diese räumliche Differenzierung ist mit einer impliziten kommunikativen Regel verknüpft, die erst nach längerer Beobachtung erkennbar wird. Personen in der ,zweiten Reihe" bringen sich nur dann erzählend in das Geschehen ein, wenn sie von den ModeratorInnen, die zwischen den Teilnehmenden am Tisch sitzen oder stehen, explizit dazu aufgefordert werden. Manchmal werden sie gebeten,

5 Es besteht eine langjährige Kooperation zwischen dem Museum und einer Universität, an der ein Archiv für lebensgeschichtliche Aufzeichnungen angesiedelt ist, zu dem auch einige der Teilnehmenden schon beigetragen haben. Die Teilnehmenden sind vertraut damit, dass WissenschaftlerInnen anwesend sind, die sich beispielsweise für sozialhistorische Fragen interessieren und Aufzeichnungen für Forschungszwecke machen. 
kurz vor Ende des Gesprächskreises Beobachtungen zu formulieren, die ihnen beim Zuhören aufgefallen sind. Das führt bereits zum nächsten Punkt.

\subsection{Praktiken der Kommunikation: Moderieren, Erzählen und Zuhören}

Erzählen und Zuhören stehen als aufeinander verweisende und verknüpfte Praxisformen im Zentrum des hier beschriebenen Arrangements. Außerdem konnte ich noch drei weitere Praktiken beobachten, die für die Herstellung und Aufrechterhaltung des kommunikativen Arrangements Gesprächskreis von Bedeutung sind: Moderieren, Fragen stellen und Kommentieren. Auch wenn die Einzelheiten der kommunikativen Prozesse hier nicht anhand von Auszügen aus den transkribierten Audioaufzeichnungen dargestellt werden können, möchte ich diese Praktiken knapp skizzieren und einige Fragen andeuten, die sich aus einer praxeologischen Perspektive stellen.

Die Moderation - im Team oder allein - eröffnet den Gesprächskreis und erinnert nach der Begrüßung der Anwesenden an das Thema sowie daran, zu welchen Teilaspekten bei den zurückliegenden Terminen bereits etwas erzählt worden ist. Dann führt sie knapp in eine neue Facette des Themas ein und setzt damit einen Erzählimpuls, der meistens gleich aufgenommen wird. Die Praxis des Erzählens in einem solchen Rahmen beruht auf der im Alltag entwickelten Erzählkompetenz der Teilnehmenden. Anders als in Interviewsituationen handelt es sich bei den Stegreiferzählungen hier jedoch nicht um ausführliche Narrationen, sondern um autobiographische Miniaturen, die einzelne Erlebnisse und Erfahrungen fokussieren und manchmal auch mehrere (kleine) Ereignisse verketten. Darüber hinaus gibt es Erzählfragmente, die keinen vollständigen Erzählbogen bilden, sondern sich auf Teile von Erzählungen, z.B. die Beschreibung bestimmter Orte zu einer bestimmten Zeit, beschränken.

Im Unterschied zu Alltagssituationen reiht die Moderation auf der Grundlage von verbalen und nonverbalen Zeichen der Teilnehmenden (z.B. Handzeichen, zum Sprechen ansetzen) die Redebeiträge. Sie erteilt das Rederecht, interveniert, erinnert wenn notwendig an die kommunikativen Regeln ${ }^{6}$ und führt nach etwa zwei Stunden den kommunikativen Prozess zu einem vorläufigen Abschluss. Inhaltliche Interventionen der Moderation sind selten,

6 Die kommunikativen Regeln für den Gesprächskreis beruhen auf den Grundlagen biographischer Methoden und werden zu Beginn jeder neuen thematischen Reihe in knapper Form vorgestellt. Sie besagen, dass die biographischen Erlebnisse und Erfahrungen der Teilnehmenden, d.h. das Erzählen und Zuhören, im Mittelpunkt des Gesprächskreises stehen und es nicht darum geht, Meinungen oder unterschiedliche Positionen zu diskutieren. Außerdem müssen die Redebeiträge im Umfang begrenzt sein, damit alle, die sich erzählend beteiligen möchten, zu Wort kommen können. 
führen aber zu der Frage, aus welchen Anlässen Interventionen entstehen und welche Folgen sie für den inhaltlichen Verlauf und das Interaktionsgeschehen haben.

Auch wenn das Erzählen den Verlauf eines Gesprächskreises bestimmt, kommen auch andere Arten von Wortbeiträgen durch Teilnehmende vor. Bisher lassen sich zwei Formen unterscheiden: Fragen stellen und Kommentieren. Drei Frageformen sind zu beobachten: Die häufigste Form sind Rückfragen, die sich auf den Zeit und den Ort eines erzählten Geschehens beziehen, wenn diese in Erzählungen unklar bleiben. Diese Frageform verweist insbesondere auf das zeitgeschichtliche Interesse der Teilnehmenden, das eine Verortung lebensgeschichtlicher Erfahrungen in historischen Kontexten notwendig macht. Gelegentlich stellen Teilnehmende für sie offene Fragen in den Raum, die an alle adressiert werden. An erzählende Personen gestellte Verständnisfragen sind hingegen sehr selten, was nicht nur mit der Erzählkompetenz der Teilnehmenden zu tun hat, sondern auch damit, dass die Zeit für einzelne Beiträge durch das Erzählinteresse der anderen Teilnehmenden begrenzt wird, die immer schon darauf warten, dass sie an der Reihe sind. Auch erzählgenerierende Nachfragen sind deshalb selten und werden nahezu ausschließlich von der Moderation gestellt.

Die differenzierte Analyse des Materials zeigt, dass jede Erzählung an eine oder mehrere vorangegangene Erzählungen anschließt und als Variation, Ergänzung oder Gegengeschichte immer auch eine Kommentierung vorheriger Redebeiträge darstellt. Zwei weitere, sehr gegensätzliche, aber wiederkehrende Formen des Kommentierens sind Witze und die Bekundung von Anteilnahme beispielsweise in Reaktion auf Erzählungen über dramatische Erlebnisse und Erfahrungen, die nicht häufig sind, aber auch vorkommen. ${ }^{7}$

Erst im Laufe meiner Beobachtungen ist mir aufgefallen, dass das Zuhören noch bedeutsamer ist als das Erzählen, weil die Teilnehmenden die überwiegende Zeit mit Zuhören verbringen und manche Teilnehmende sogar ausschließlich zum Zuhören kommen und sich nie erzählend beteiligen. Gleichzeitig entzieht sich das Zuhören in erheblichem Maße einer teilnehmenden Beobachtung. Es ließe sich als körperliche Haltung beschreiben und ist der Selbstbeobachtung zugänglich, aber was die TeilnehmerInnen hören, bleibt mir als Ethnographin verborgen. Allerdings lässt sich in der Analyse der Erzählbeiträge durchaus rekonstruieren, wie Themen aufgenommen, weitergeführt, fallengelassen und unter Umständen zu einem späteren Zeit-

7 Hier zeigt sich eine für diesen Kontext spezifische Form des Umgangs mit dramatischen Erlebnissen und Erfahrungen. Sie können erzählt werden und finden Gehör, werden jedoch nicht kommunikativ bearbeitet. Oder anders gesagt, die Bearbeitung solcher Erzählungen ist eher eine beiläufige, sie besteht darin, ihnen einen Platz in diesem kommunikativen Zusammenhang einzuräumen. Sie werden zu einem Teil des in der Gruppe geteilten Wissens, was auch eine Form von Anerkennung darstellt. 
punkt wieder aufgegriffen werden. Die vielfältigen Verbindungen zwischen den individuellen Erzählungen lassen sich auch als Hinweis auf sehr genaues Zuhören lesen und zeigen, wie im Zuhören permanent Verknüpfungen zwischen den eigenen Erfahrungen und den Erfahrungen der anderen hergestellt werden.

\section{Möglichkeiten einer praxeologischen Lernkulturforschung}

Auf der Grundlage der exemplarischen Darstellung und Analyse eines Lernarrangements der kulturellen Erwachsenenbildung möchte ich an dieser Stelle noch einmal die Möglichkeiten einer praxeologischen Lernkulturforschung konkretisieren. Drei Punkte sind mir besonders wichtig:

Eine praxeologische Perspektive, die mit den Mitteln der teilnehmenden Beobachtung arbeitet, ermöglicht es zu verstehen, wie das räumlich-materielle Arrangement einer Bildungssituation die Interaktionsprozesse vorstrukturiert oder - mit Goffman gesprochen - die wesentlichen Parameter der Interaktionsordnung festlegt, die sich darin zwischen den Beteiligten entfalten kann. Am präsentierten Beispiel zeigt sich insbesondere, wie die Sitzordnung und die auf dem Tisch platzierten Dinge die Möglichkeiten des Sprechens und Zuhörens mitbestimmen und damit unter anderem zu einer Formalisierung der wechselseitigen Ansprache beitragen.

Eine praxeologische Perspektive bietet einen Zugang dazu, Handlungsroutinen und habitualisierte Praktiken der an einer organisierten Lernsituation beteiligten Akteure zu rekonstruieren. Diese sind einer konkreten Lernsituation in der Erwachsenenbildung immer schon vorgängig und nicht ohne weiteres veränderbar. Wie am Beispiel deutlich wurde, handelt es sich beim Erzählen, Zuhören, Fragen, Kommentieren und Moderieren um sehr allgemeine Praxisformen, die in nahezu allen Lernsituationen vorkommen. Gleichwohl sind noch andere Praxisformen denkbar, die in der vorgestellten Situation keine Rolle spielen, wie beispielsweise das Schreiben oder das Zeigen. Die Rekonstruktion der in Lernsituationen typischerweise vorkommenden Praxisformen, ihrer Variationen und ihres interaktiven Vollzugs sind auch ein Ansatzpunkt dafür, zu verstehen, warum sich Lernkulturen nur sehr langsam verändern, obwohl es sich keineswegs um starre, unveränderbare Interaktionsroutinen handelt. Inwiefern sich aus einer solchen Analyseperspektive auch Anregungen für die Gestaltung von Lernarrangements entwickeln lassen, kann vorerst nicht gesagt werden.

Mit der für die praxeologische Perspektive typischen Präferenz für die teilnehmende Beobachtung sind jedoch auch bestimmte Erkenntnisgrenzen verbunden. Dies wurde ansatzweise am Beispiel des Zuhörens deutlich. Was Teilnehmende hören, wie sie das Gehörte mit ihren eigenen Erfahrungen 
verbinden und daraus möglicherweise neue Selbst- und Welteinsichten konstruieren, entzieht sich der direkten Beobachtung. Dies kann man als Beschränkung verstehen und nach methodischen Möglichkeiten suchen, individuelle Verstehensprozesse beispielsweise über Interviews doch noch zugänglich zu machen. Die Ethnographie als methodenpluraler Forschungszugang erlaubt das nicht nur, sondern empfiehlt ausdrücklich, über den Methodeneinsatz gegenstandsangemessen zu entscheiden. Man kann in der Beschränkung auf die Beobachtung aber auch das besondere Potenzial eines solchen Ansatzes sehen, der die Analyse auf die räumlich-materielle Situierung und die kommunikativen und interaktiven Prozesse, in denen Lernen stattfindet, fokussiert, und darauf verzichten, sich erneut auf die Perspektive des lernenden Individuums festzulegen.

Insofern versteht sich dieser Beitrag auch als ein Plädoyer dafür, den sich jüngst in der Lernkulturforschung abzeichnenden Richtungswechsel von einer programmatischen Gestaltungsperspektive hin zu einer empirisch-rekonstruktiven Perspektive fortzusetzen, um besser zu verstehen, was Teilnehmende und Professionelle in organisierten Lernsituationen praktisch tun und wie sie dieses Tun aufeinander abstimmen.

\section{Literatur}

Arnold, Rolf/Schüßler, Ingeborg (1998): Wandel der Lernkulturen. Ideen und Bausteine für ein lebendiges Lernen. Darmstadt: Wissenschaftliche Buchgesellschaft.

Arnold, Rolf/Lermen, Markus (2009): Konstruktivistische Lernkulturen. In: Gieseke, Wiltrud/Robak, Steffi/Wu, Ming-Lieh (Hrsg.): Transkulturelle Perspektiven auf Kulturen des Lernens. Bielefeld: Transcript, S. 25-48.

Breidenstein, Georg/Hirschauer, Stefan/Kalthoff, Herbert (2013): Ethnografie. Die Praxis der Feldforschung. Konstanz: UVK, München: Lucius.

Behrens-Cobet, Heidi/Reichling, Norbert (1997): Biographische Kommunikation. Lebensgeschichten im Repertoire der Erwachsenenbildung. Neuwied: Luchterhand.

Breidenstein, Georg (2006): Teilnahme am Unterricht. Ethnographische Studien zum Schülerjob. Wiesbaden: VS.

Dammer, Karl-Heinz (2013): Mythos Neue Lernkultur. In: Pädagogische Korrespondenz 48, S. 27-57.

Herrle, Matthias (2012): Interaktionsprozesse unter Erwachsenen. Zur Mikroethnographie pädagogischen Handelns. In: Report - Zeitschrift für Weiterbildungsforschung 2012, 3, S. 15-29.

Hof, Christiane (1995): Erzählen in der Erwachsenenbildung. Geschichte - Verfahren - Probleme. Neuwied, Kriftel: Luchterhand.

Laschewski, Julia (2013): Vernetzte Lernkultur - Eine Perspektivenerweiterung durch die Akteur-Netzwerk-Theorie nach Bruno Latour. In: Dollhausen, Karin/Feld, Timm C./Seitter, Wolfgang (Hrsg.): Erwachsenenpädagogische Kooperationsund Netzwerkforschung. Wiesbaden: Springer, S. 171-184. 
Gieseke, Wiltrud/Robak, Steffi (2009). Einleitung - Kultur als offenes Konzept aus erwachsenenpädagogischer Perspektive. In: Gieseke, Wiltrud/Robak, Steffi/Wu, Ming-Lieh (Hrsg.): Transkulturelle Perspektiven auf Kulturen des Lernens. Bielefeld: Transcript, S. 7-24.

Hörning, Karl H./Reuter, Julia (2004): Doing Culture. Kultur als Praxis. In: Dies. (Hrsg.): Doing Culture. Neue Positionen zum Verhältnis von Kultur und sozialer Praxis. Bielefeld: Transcript, S. 9-15.

Hörning, Karl H./Reuter, Julia (Hrsg.)(2004): Doing Culture. Neue Positionen zum Verhältnis von Kultur und sozialer Praxis. Bielefeld: Transcript.

Kade, Jochen/Nolda, Sigrid/Dinkelaker, Jörg/Herrle, Matthias (2014): Videographische Kursforschung. Empirie des Lehrens und Lernens Erwachsener. Stuttgart: Kohlhammer.

Klingovsky, Ulla (2009): Schöne Neue Lernkultur. Transformationen der Macht in der Weiterbildung. Eine gouvernementalitätstheoretische Analyse. Bielefeld: Transcript.

Moebius, Stephan (2009): Kultur. Bielefeld: Transcript.

Nolda, Sigrid (1995): Interaktion und Wissen. Eine qualitative Studie zu Lehr-Lernverhalten in Veranstaltungen der allgemeinen Erwachsenenbildung. Frankfurt a.M.: DIE.

Ott, Marion (2011): Aktivierung von (In-)Kompetenz. Praktiken im Profiling - eine machtanalytische Ethnographie. Konstanz: UVK.

Reckwitz, Andreas (2003): Grundelemente einer Theorie sozialer Praktiken. Eine sozialtheoretische Perspektive. In: Zeitschrift für Soziologie 32, 4, S. 282-301.

Reckwitz, Andreas (2006): Das hybride Subjekt. Eine Theorie der Subjektkulturen von der bürgerlichen Moderne bis zur Postmoderne. Weilerswirst: Velbrück.

Reuter, Julia (2004): Postcoloniales Doing Culture. Oder: Kultur als translokale Praxis. In: Hörning, Karl H./Reuter, Julia (Hrsg.): Doing Culture. Neue Positionen zum Verhältnis von Kultur und sozialer Praxis. Bielefeld: Transcript, S. 239-255.

Rothe, Daniela (2011): Lebenslanges Lernen. Eine diskursive Formation in der Erwachsenenbildung. Frankfurt, New York: Campus.

Schäfer, Frank/Daniel, Anna/Hillebrandt, Frank (Hrsg.) (2015): Methoden einer Soziologie der Praxis. Bielefeld: Transcript.

Schlutz, Erhard (2015): Kultur. In: Dinkelaker, Jörg/ Hippel, Aiga von (Hrsg.): Erwachsenenbildung in Grundbegriffen. Stuttgart: Kohlhammer, S. 223-230.

Schüßler, Ingeborg/Thurnes, Christian M. (2005). Lernkulturen in der Weiterbildung. Bielefeld: Bertelsmann.

Wiesner, Matthias (2004): Inmitten der Dinge. Zum Verhältnis von sozialen Praktiken und Artefakten. In: Hörning, Karl H./Reuter, Julia (Hrsg.): Doing Culture. Neue Positionen zum Verhältnis von Kultur und sozialer Praxis. Bielefeld: Transcript, S. 92-107.

Zaborowski, Katrin Ulrike/Meier, Michael/Breidenstein, Georg (2011): Leistungsbewertung und Unterricht. Ethnographische Studien zur Bewertungspraxis in Gymnasien und Sekundarschule. Wiesbaden: VS. 



\section{Lernkulturen im (Klima-)Wandel. Digital Storytelling zur Kompetenzvermittlung in interkulturellen Lehr-Lernsettings}

\section{Einleitung}

Ende des Jahres 2010 begann mit dem Arabischen Frühling ein Prozess des demokratischen Aufstandes in vielen Staaten der Maghreb-Region. Ausgangspunkt dieses Prozesses war Tunesien, das seitdem als (einziger) demokratischer Erfolgsfall eine Sonderrolle im Arabischen Frühling einnimmt.

Bildung nimmt in Gesellschaften, die sich in einem Übergangsprozess hin zur Etablierung einer demokratischen Kultur befinden, eine zentrale Rolle ein und bildet eine notwendige Voraussetzung für demokratische Partizipation. Umweltbildung und das damit verbundene Konzept der Nachhaltigen Entwicklung sind ein Kernelement für den nachhaltigen Umgang mit Ressourcen.

Eine zentrale Aufgabe einer Bildung für Nachhaltige Entwicklung im Rahmen der internationalen Hochschulkooperation sind innovative Lehrkonzepte für den studentischen Austausch und das interkulturelle Lernen. Die unterschiedlichen Lernkulturen müssen dabei als eine besondere Herausforderung berücksichtigt werden. Wir argumentieren in diesem Artikel, dass das Digital Storytelling (DS) eine innovative Lernmethode darstellt, um in interkulturellen Kontexten zur Kompetenzvermittlung für den Umgang mit Themen der Nachhaltigen Entwicklung beizutragen. In Anlehnung an den durch den Bologna-Prozess vollzogenen Wandel zur Lernergebnisorientierung verstehen wir Kompetenzen als „,nachgewiesene Fähigkeiten, Kenntnisse, Fertigkeiten sowie persönliche, soziale und/oder methodische Fähigkeiten in Arbeits- oder Lernsituationen" (Schermutzki 2008: 5).

Die Eignung des DS belegen wir anhand der Auswertung eines Lehr- und Forschungsprojektes im Rahmen einer internationalen universitären Kooperation im Bereich der (Weiter-)Bildung. Für das Projekt wurde als theoriegeleitete Arbeitshypothese davon ausgegangen, dass die Methode des DS in einer herausfordernden Lernkultur, verstanden als interdisziplinäres und interkulturelles Lernarrangement, zum Kompetenzerwerb im Bereich Nachhaltige Entwicklung beiträgt. Das untersuchte Fallbeispiel bildet ein zweijähriges, vom DAAD mit Mitteln des Auswärtigen Amtes gefördertes Kooperationsprojekt Living with Climate Change - a Digital Story zwischen dem Masterstudiengang Umweltwissenschaften der FernUniversität in Hagen und 
dem Masterstudiengang Ökotourismus der Virtuellen Universität Tunis. Beide sind Weiterbildungsstudiengänge und interdisziplinär ausgerichtet.

\section{Der Klimawandel als Kernproblem Nachhaltiger Entwicklung}

Der Klimawandel kann als das bedeutendste internationale Umweltproblem bezeichnet werden und bildet dementsprechend ein Kernthema der Nachhaltigen Entwicklung (de Haan 2002). Eine Erderwärmung über das von der internationalen politischen Gemeinschaft übereinstimmend mit der Klimawissenschaft definierte 2-Grad-Ziel hinaus kann in seinen Auswirkungen kaum überschätzt werden (Otto 2015).

Aus dieser Problemkonstitution und -dimension des Klimawandels ergibt sich ferner ein bildungspolitischer Auftrag, den Klimawandel als Thema in der universitären Bildung zu verankern (Wilson 2012). Dabei muss berücksichtigt werden, dass der Klimawandel nur aus einer holistischen Perspektive heraus verstanden werden kann. Anders als rein lokale Umweltprobleme (z.B. Gewässerschutz) ist der Klimawandel durch seine regionale oder sektorale Entgrenzung gekennzeichnet. An der wissenschaftlichen Erforschung der Gründe und Folgen des Klimawandels sind sowohl Natur- und Ingenieurwissenschaften als auch Rechts-, Sozial- und Wirtschaftswissenschaften beteiligt. Internationale Kooperationsprojekte im Bereich der Weiterbildung stehen deshalb vor der Herausforderung, die Multidimensionalität und Interdisziplinarität des Klimawandels herauszuarbeiten und gezielt auf die unterschiedliche Betroffenheit und das divergierende Problembewusstsein unterschiedlicher Länder einzugehen (Breitmeier/Otto 2012). Unterschiedliche kollektive und individuelle Deutungsmuster betroffener Personen und Gesellschaften sichtbar zu machen, kann zu einem gegenseitigen Lernprozess beitragen (Abbott/Wilson 2015). Besonders letzterer Aspekt muss die Zielvorstellung internationaler Kooperationen bilden, da dort unterschiedliche Deutungen verstärkt zu erwarten sind.

\section{Interkulturelle Umweltbildung und Lernkulturen}

Aus dem oben beschriebenen Anspruch einer interdisziplinären und interkulturellen Kompetenzvermittlung für das Klimawandelproblem an eine heterogene studentische Zielgruppe ergibt sich die Herausforderung, eine entsprechende Lernkultur mit geeigneten didaktischen Methoden für die Kompetenzvermittlung zu etablieren.

Versteht man den sehr uneinheitlich definierten und in vielen Kontexten angewandten Begriff der Lernkultur basal als eine kulturell und gesellschaftspolitisch konstituierte Handlungsorientierung in Lern- und Lehrarran- 
gements, wird die didaktische Herausforderung im internationalen Kontext besonders deutlich. Im nordafrikanischen Raum sowie im Nahen und Mittleren Osten scheint das Defizit einer produktiven und partizipativen Lernkultur evident. So zeigt die Analyse zahlreicher Länder dieser Regionen, dass die schulische und universitäre Lernkultur noch immer (zu) stark auf das reproduktive, autoritative Wissen ausgerichtet ist, während die Befähigung zu kreativen und eigeständigen Denkweisen größtenteils fehlt (Mattes 2004: 222). Aber auch im deutschsprachigen Raum herrscht - abgesehen von einer Debatte über die genaue Begriffsbestimmung - eine Auseinandersetzung darüber, wo und wie neue Lernkulturen entstehen (sollen) (Felbinger 2010). Damit steht die universitäre Weiterbildung vor der Herausforderung, neue Lernkulturen für die an sie gestellten Anforderungen der Kompetenzvermittlung zu etablieren. Der durch die Debatte über neue Lernkulturen initiierte Paradigmenwechsel hat als konsensuale Basis etabliert, dass der Lernende im Mittelpunkt des Lernprozesses steht und dessen Eigenständigkeit sowie die gezielte Kompetenzentwicklung gefördert werden sollen (ebd.: 67).

In der Literatur sind bereits zahlreiche neue Lernkulturen für den Bereich der Weiterbildung identifiziert worden (Schüßler/Thurnes 2012). Dennoch können nicht beliebige Lernkulturen für spezifische Lerninhalte adaptiert werden. Eine unserer Meinung nach bisher im Kontext der Weiterbildung vergleichsweise wenig thematisierte, aber innovative didaktische Methode bildet das DS.

\section{DS für die Kompetenzvermittlung im Bereich Klimawandel}

Wenn der Begriff des Storytellings in den Debatten über die zukünftige Ausgestaltung von neuen Lernkulturen im hochschulischen Bereich Erwähnung findet (König 2009), so scheint dies auf den ersten Blick paradox. Die Grundidee des Storytellings als die narrative Weitergabe expliziten, aber vor allem impliziten Wissens, zählt zu einer der ältesten Methoden der Übermittlung von Wissen. Lebendiges, metaphorisches Erzählen und ein aktives Einbinden der Zuhörenden in die Geschichte führt zu einer verbesserten Erinnerbarkeit bei den Rezipienten. Mit Beginn der 1990er Jahre wurde der Einsatz des Storytellings als didaktische Methode in der Unterrichtsforschung vermehrt diskutiert (Egan 1989). In Schulen und der universitären Lehre wird Storytelling primär eigesetzt, um komplexe Sachverhalte besser zu vermitteln (Floßmann 2014; Schekatz-Schopmeier 2010).

Bezeichnet das Storytelling zusammengefasst eine tradierte und mittlerweile etablierte Form der Wissensvermittlung, so hat sich das Konzept des DS erst in den letzten Jahren etabliert (Robin/McNeil 2012). Der Zusatz Digital erweitert das Storytelling um die Möglichkeit, digitale Techniken wie 
Mobiltelefone oder Videobearbeitungssoftware sowie soziale Netzwerke oder Videoportale für die Produktion und Verbreitung von Geschichten nutzbar zu machen (Otto 2014). Untersuchungen über den didaktischen Mehrwert des DS zeigen einen überwiegend positiven Befund (Robin 2006). Mehrere Studien über die Anwendung von DS in der Lehre verweisen auf eine Kompetenzsteigerung der Teilnehmenden im Umgang mit digitalen Medien, eine erhöhte Lernmotivation sowie gesteigertes Engagement (Dogan/Robin 2008). Gründe hierfür finden sich primär in dem strukturellen Aufbau eines DSProzesses. Der Prozess der Konzeption und praktischen Umsetzung einer Story rückt die selbstgesteuerte studentische Interaktion in den Vordergrund des Lernprozesses. Didaktisch verändert sich damit die Lernsituation von einem lehrerorientierten zu einem studierendenorientierten Lernen. Da der DS-Prozess meist in kleineren Gruppen stattfindet, arbeiten diese überwiegend autonom, die Lehrenden greifen nur bei gravierenden Problemen ein. Gruppenarbeit steigert die Motivation und fördert durch soziale Kontrolle die Verbindlichkeit der Mitarbeit. Die konzeptionelle Freiheit für die inhaltliche Gestaltung führt dazu, dass die Studierenden eigene Ideen und Meinungen einbringen und umsetzen können. Besonders bei heterogenen Gruppen können unterschiedliche Sichtweisen auf ein Thema aufgenommen werden. Die filmische Umsetzung fördert in erster Linie den Umgang mit digitalen Medien. Wichtig ist zu beachten, dass beim DS der Lernprozess im Vordergrund steht und nicht das filmische Ergebnis der digitalen Story selbst. Mit den heute größtenteils kostenlos verfügbaren Techniken lassen sich jedoch bereits anspruchsvolle Projekte umsetzen.

Zusammengefasst bietet DS eine vielversprechende Lernmethode zur Kompetenzentwicklung in vielen Bereichen. Im Rückgriff auf Robin (2006) erhöht der Einsatz des DS u.a. folgende Kompetenzen:

- Forschungskompetenz: Dokumentieren einer Geschichte, Finden und Analysieren von relevanten Informationen.

- Problemlösungskompetenz: Fähigkeit, Entscheidungsfindungsprozesse zu steuern und verschiedene Hürden im Verlauf dieses Prozesses zu überwinden.

- Interpersonale Kompetenz: Fähigkeit, in einer Gruppe zu arbeiten und unterschiedliche Rollenverteilungen festzulegen.

- Technologiekompetenz: Fähigkeit, eine Vielzahl unterschiedlicher digitaler Werkzeuge wie Videokameras, Mikrofone, Videosoftware zu nutzen.

\section{Fallstudie: Living with Climate Change - a Digital Story}

Die praktische Anwendung des DS als Lernmethode erfolgte in dem zweijährigen deutsch-tunesischen Kooperationsprojekt Living with Climate Change- 
a Digital Story. Das Kooperationsmodul wurde in den Jahren 2013 und 2014 als 5-ECTS-Modul in einem dreimonatigen blended learning-Ansatz gemeinsam in beiden Masterstudiengängen angeboten. 2013 nahmen insgesamt 26 Studierende, 201420 Studierende teil. Da der tunesische Masterstudiengang allen Studierenden der Region offen steht, stammten die Teilnehmenden u.a. aus Algerien, Marokko und dem Libanon.

Lernziel war die Kompetenzvermittlung für den Umgang mit dem Klimawandel als einem Kernproblem Nachhaltiger Entwicklung. Angestrebte Lernergebnisse waren:

- Fähigkeit, den Klimawandel als interdisziplinäres Problem zu erfassen und zu kommunizieren;

- Fähigkeit, die unterschiedlichen Wahrnehmungen des Klimawandels überindividuell und gesellschaftsübergreifend zu verstehen;

- Unterschiedliche Wissensformen (Experten, Laien usw.) einordnen und bewerten;

- Zusammenarbeit in interdisziplinären und multikulturellen Gruppen.

DS wurde als didaktische Methode gewählt, um zu einem Kompetenzgewinn in diesen Bereichen und einer allgemein erhöhten Lernmotivation beizutragen. Didaktisch wurde das Modul in drei Phasen untereilt: Eine Vorbereitungsphase, eine Gruppenarbeitsphase und eine Reflexionsphase.

Die Vorbereitungsphase beinhaltete das Erarbeiten von ausgewählten interdisziplinären Texten über das Problem des Klimawandels. Die Texte waren virtuell in einer Lernplattform verfügbar, die ein tunesischer und ein deutscher Tutor gemeinsam betreuten. Die Studierenden konnten sich in der Lernplattform untereinander auszutauschen. Der erste von zwei Workshops in Hamburg bildete den Auftakt der Gruppenarbeitsphase. Im Mittelpunkt stand das Erlernen der grundlegenden Techniken des DS. Weiterhin wurden Expertenvorträge über die Auswirkungen des Klimawandels in Deutschland gehalten. Eine Exkursion zu einem Klimaanpassungsprojekt ermöglichte den Studierenden erste Versuche der Umsetzung eines kleineren Filmprojektes. Zum Ende des Workshops bildeten die Studierenden Gruppen von 4-5 Personen, mit der Aufgabe, eine Digital Story zu einem ausgewählten Themenbereich des Klimawandels zu erstellen. Das gewählte Thema sollte aus unterschiedlichen disziplinären und nationalen Perspektiven dargestellt werden. Die Digital Story sollte unterschiedliche Wissensformen (Experte, Laie etc.) einbeziehen.

Die in der virtuellen Gruppenphase durchgeführte Zusammenarbeit zur Erstellung einer Digital Story fand in Anlehnung an Morras acht Schritte für den DS-Prozess statt (2013). Um den Lernprozess überprüfen zu können, wurde nach 4 Wochen das Storyboard (Schritt 4) bei den Tutoren eingereicht, die eine konstruktive Rückmeldung über Verbesserungsmöglichkeit gaben. 


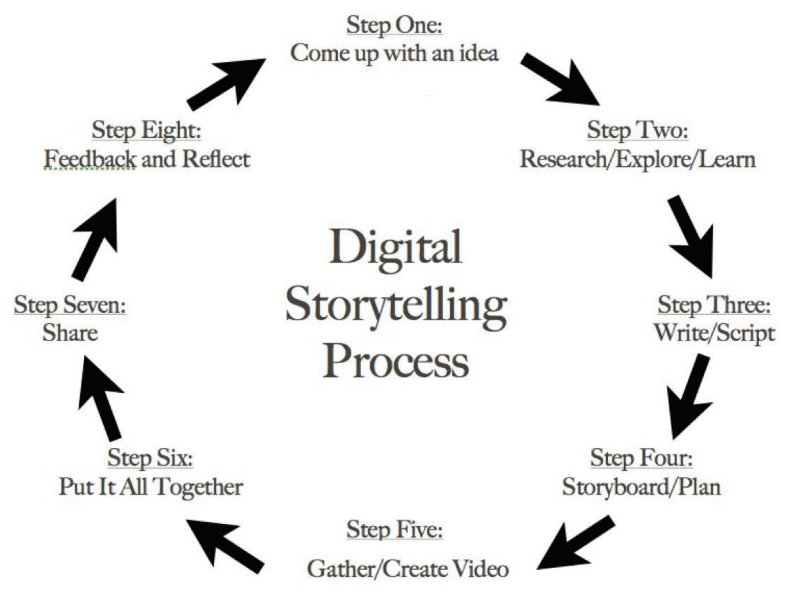

Abb.1: 8 Steps to Great Digital Storytelling (Morra 2013)

Nach der virtuellen Gruppenarbeitsphase fand ein fünftägiger Workshop in Tunis statt. Ziel war die Finalisierung der Digital Story und die gemeinsame Präsentation und Diskussion der Projekte. Darüber hinaus gab es Expertenvorträge über die Folgen des Klimawandels in Tunesien sowie eine Exkursion zu Klimaanpassungsprojekten in der Region.

Der Gruppenarbeitsphase folgte eine Reflexionsphase. Die Gruppenreflexion begann bereits am Ende des Workshops in Tunis mit einer offenen Feedbackrunde. Anschließend sollten die Studierenden ihre Erfahrungen im Modul in einem maximal fünfseitigen Individual Reflection Report (IRR) reflektieren. Das von Petranek (2000) und seinem Konzept des written debriefing inspirierte Vorgehen postuliert, dass eine erneute Auseinandersetzung mit Ereignissen und gemachten Erfahrungen zu einem vertieften Erinnern und dadurch zu einem besseren Verarbeiten von Erlebnissen führt. Um eigenen Gedanken Raum zu lassen, wird für den Bericht keine klare inhaltliche Struktur vorgegeben, sondern nur eine Liste mit möglichen Oberthemen zur Diskussion genannt. Die Studierenden müssen somit nicht auf alle Aspekte eingehen, sondern können eigene inhaltliche Schwerpunkte setzen. Dieses Vorgehen ermöglicht es den Lehrenden, vertiefte Einblicke in die Lernerfahrungen und subjektiven Gedankengänge der Studierenden zu erlangen. Gleichzeitig dienen die Berichte auch als eine zentrale Quelle für die Evaluation der Lernergebnisse. 


\section{Analyse}

Durch eine ex-post-Analyse wird der Mehrwert des DS als Lernmethode für die Kompetenzentwicklung in der Erwachsenenpädagogik kritisch überprüft. Bisher finden sich im deutschsprachigen Raum hierzu keine aussagekräftigen Studien, so dass auf Basis der englischsprachigen Literatur konfirmatorisch vorgegangen wurde. Für die Überprüfung der deduzierten Thesen wurde methodisch auf die teilnehmende Beobachtung der Tutoren während des Moduls (Kawulich 2005) sowie auf die qualitative Inhaltsanalyse der 38 eingereichten IRRs unter Verwendung induktiver Kategorienbildung (Mayring 2010) zurückgegriffen. Die Methode erlaubt sowohl eine Auszählung als auch eine Interpretation der Kodierungen.

Folgende zwei Thesen wurden auf Basis der Literatur entwickelt:

These 1: Die entwickelte Problemlösungs- und Forschungskompetenz im Rahmen des DS-Prozesses führt dazu, die Vernetztheit und Multidimensionalität des Klimaproblems zu erkennen und den Klimawandel als interdisziplinäres Mehrebenenproblem zu begreifen.

These 2: Die interpersonale und interkulturelle Zusammenarbeit bei der Erstellung der Digital Story führt zur Reflexion eigener kultureller Leitbilder und zur Erhöhung interkultureller Sensibilität.

\subsection{These 1: Inhaltliche Kompetenz}

Zwei Drittel der Studierenden gaben an, dass ihr zuvor eher national gefärbter Blick um eine transnationale Mehrebenenperspektive ergänzt wurde (vgl. Abb. 2). Durch ihre Teilnahme an dem Projekt sind sie in der Lage, sich in die klimapolitischen Sichtweisen, Prioritäten und spezifischen Herangehensweisen von Menschen aus dem jeweiligen anderen Kulturkreis hineinzuversetzen und haben ein multidisziplinäres Verständnis des Klimawandels entwickelt. Diese Befunde werden z.B. in folgenden Aussagen deutlich:

,$[\ldots]$ it now seems obvious to me that $[\ldots]$ a purely ,meteorological ${ }^{\star}$ or ,natural sciences ${ }^{6}$ approach to climate change is becoming increasingly inadequate to understand its social and personal consequences" (IRRa, 2013).

"Since we had the opportunity to interact with the local people in North Africa, [...] I have a genuine idea about how they understand climate change and deal with it" (IRRc, 2014). 
口

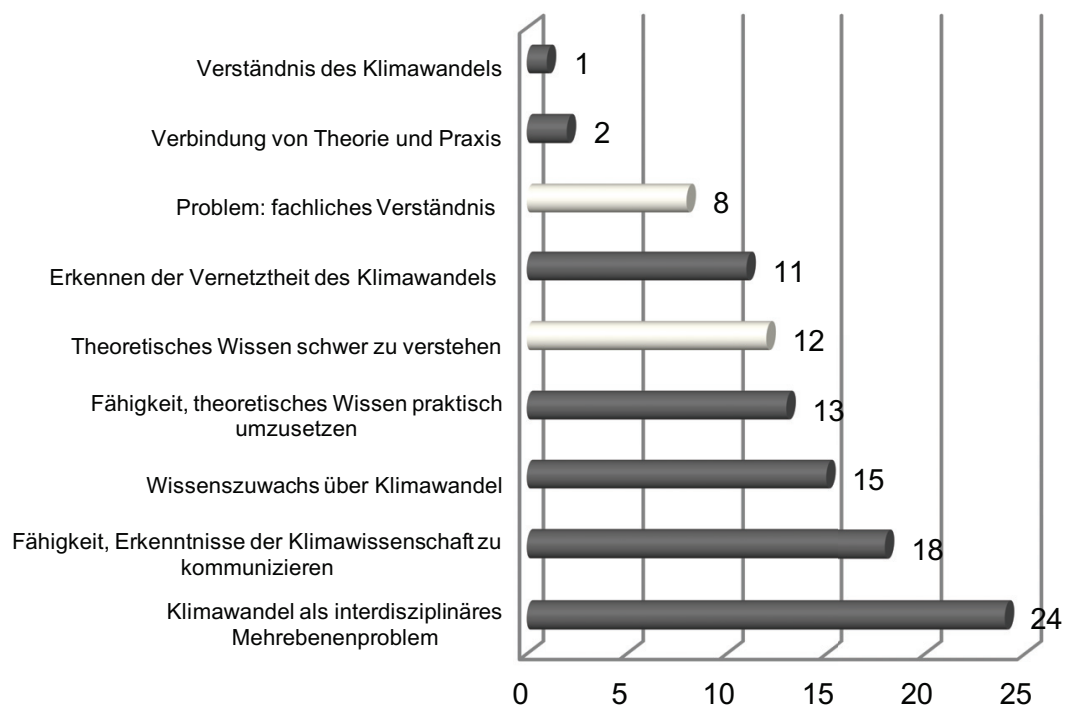

Abb. 2: Problemlösungs- und Forschungskompetenz (eigene Darstellung)

Die von 8 Studierenden hervorgehobene Schwierigkeit, die zum Projektstart bereitgestellten theoretischen Informationen zu verstehen (vgl. Abb. 3), lässt sich insbesondere auf die fachliche Heterogenität der Teilnehmenden zurückführen. Umso bedeutsamer war es, dass in der Gruppenarbeit ein Austausch unterschiedlicher Fachdisziplinen ermöglicht wurde, wodurch eine gemeinsame Verständnisbasis geschaffen wurde.

Rund die Hälfte der Studierenden gab an, dass die Erarbeitung der eigenen Digital Story ihre Fähigkeit gefördert hat, klimawissenschaftliche Fakten zu kommunizieren und damit theoretisches Wissen praktisch umzusetzen (vgl. Abb. 2).

Der Lernerfolg im Hinblick auf die Vernetztheit und Multidimensionalität des Klimawandels sowie hinsichtlich der Kommunikationsfähigkeit der Studierenden wurde auch seitens des Dozententeams bestätigt. So ließ sich im Projektverlauf eine deutliche Veränderung der Diskussionsprozesse feststellen. Argumentierten die Teilnehmenden bei ihrem ersten Treffen häufig noch stark aus der eigenen nationalen und disziplinären Perspektive heraus, wurden die Diskussionen in den Kleingruppen zunehmend differenzierter und interdisziplinärer. 


\subsection{These 2: Interkulturelle Kompetenz}

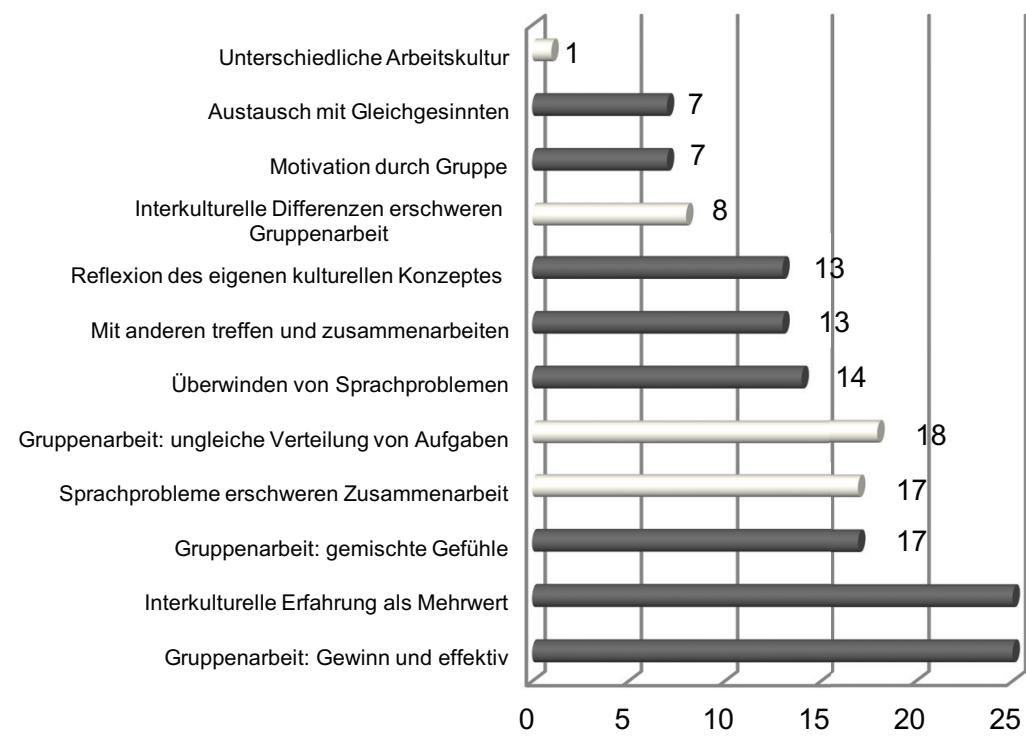

Abb. 3: Interkulturelle und interpersonale Interaktion (n38) (eigene Darstellung)

Neben der Kompetenzvermittlung im Bereich Klimawandel leistete das Projekt einen Beitrag zur Steigerung der interkulturellen Sensibilität sowie zur kritischen Reflexion eigener kultureller Leitbilder. Zwei Drittel der Studierenden hoben in ihren IRRs den Mehrwert der interkulturellen Begegnung hervor und bewerteten die didaktische Methode der Arbeit in internationalen Teams als effektiv und gewinnbringend (vgl. Abb. 3).

„Although the concept of mixing Tunisian and German students when forming the groups added an extra challenge to the workshop, it was one of the most valuable experiences" (IRRh, 2013).

Allen Gruppen ist es - trotz der anfänglich teilweise stark hervorgetretenen kulturellen Unterschiede, Sprachbarrieren sowie unterschiedlicher Arbeitseinstellungen (vgl. Abb. 3) - gelungen, als Team zusammenzuarbeiten, sich auf ein Thema für ihre Digital Story zu einigen, ein gemeinsames Konzeptpapier einzureichen und einen gemeinsamen Film zu produzieren. Viele Studierende hoben den motivierenden Effekt der Gruppenarbeit hervor; die Kleingruppen boten einen Rahmen, Gleichgesinnte zu treffen und zu erleben, 
dass das gemeinsame Interesse am Thema ein verbindendes Element jenseits kultureller Barrieren ermöglichte:

,$[\ldots]$ on the one hand (the group was) very heterogeneous, on the other hand united in a common understanding of the importance to act on environmental issues" (IRRi, 2014).

Viele Studierende reflektierten in ihren Berichten die anfangs (unerwartet) schwierige Zusammenarbeit in den kulturell heterogenen Teams und die Suche nach einem gemeinsamen Weg des Verständnisses:

„At the beginning the culture mix was an obstacle for efficient communication and good group work. [...] It needed some time to find the right way for communication and a mode of operation" (IRRe, 2014).

„Also cultural differences occurred: We could see an affectation to emotional aspects [...] expressed by the ,south" and a more objective [...] point of view shown by the ,north'. So we had to integrate the differences and create a manifold but nevertheless homogeneous film project" (IRRf, 2013).

Den IRRs lässt sich insgesamt entnehmen, dass die Teilnehmenden an der Herausforderung der internationalen Teamarbeit gewachsen sind und wertvolle interkulturelle Kompetenzen entwickelt haben. Diese Sichtweise wurde von den Koordinatorinnen und Koordinatoren einhellig bestätigt.

„From a cultural point of view, I learned a lot about intercultural communication and behavior. Working in intercultural teams opens new points of view“ (IRRg, 2014).

Besonders positiv ist, dass viele Studierende in ihren IRRs die Reflexion des eigenen kulturellen Konzepts als Lernerfolg unterstrichen (vgl. Abb. 3). Insbesondere deutsche Teilnehmende entwickelten ein besseres Verständnis für die Folgen des Klimawandels in Entwicklungsländern und die privilegierte Position des globalen Nordens. Exemplarisch wird dies im folgenden Zitat deutlich:

„My view on the impacts of climate change has changed. [...] In Germany, we are really privileged while working against the impacts of climate change. Other less developed countries have to face the challenges without support from the governments and do not have the financial and technical possibilities“" (IRRb, 2014). 


\section{Fazit}

Ziel dieses Artikels war die Untersuchung des DS als didaktische Methode zur Vermittlung von Bildung für Nachhaltige Entwicklung in interkulturellen Lehr-Lernsettings. Die Ergebnisse der Analyse leisten einen Beitrag zu den in der Erwachsenen- und Weiterbildung geführten Diskursen über die zunehmende Entgrenzung des Lernens (Arnold 2012) und den Einfluss unterschiedlicher Lernkulturen (Gieseke 2009). Wie die Untersuchung zeigt, gelingt es durch die didaktische Methode des DS, eine produktive Lernkultur vor einem interkulturellen und interdisziplinären Hintergrund zu etablieren. Die Analyse bestätigte unsere Thesen hinsichtlich des Potenzials von DS, sowohl inhaltliche als auch interkulturelle Kompetenzen zu vermitteln. Erstens erlangten die Studierenden durch ihre Teilnahme ein besseres Verständnis für den Klimawandel als multidimensionales und interdisziplinäres Mehrebenenproblem. Zweitens förderte die Gruppenarbeit die interkulturelle Sensibilität der Teilnehmenden, ihre Fähigkeit zur Selbstreflektion und zur interkulturellen Kompromissfindung. Die bisher überwiegend englischsprachigen Forschungsbefunde zum DS konnten wir in unserer Untersuchung bestätigen und auf den Bereich der Erwachsenenpädagogik übertragen. Auch in Blended-Learning-Formaten lässt sich DS als didaktische Methode erfolgreich integrieren und umsetzen. Interessant wäre es, in zukünftigen Forschungsvorhaben zu prüfen, ob sich die Methode des DS auch auf andere Themenbereiche der Weiterbildung übertragen ließe. Weiterhin muss der Mehrwert des DS relational zu anderen didaktischen Methoden kritisch geprüft werden.

\section{Literatur}

Abbott, Dina/Wilson, Gordon (2015): The Lived Experience of Climate Change. Knoweldge, Science and Public Action. Cham, CH.

Arnold, Rolf (2012): Entgrenzungen des Lernens : internationale Perspektiven für die Erwachsenenbildung. Bielefeld.

Breitmeier, Helmut/Otto, Daniel (2012): Understanding Political Processes in Climate Change Negotiations by means of an Interdisciplinary Curriculum in Higher Education. In: International Journal on Innovation and Sustainable Development 6, 1, S. 20-30.

de Haan, Gerhard (2002): Die Kernthemen der Bildung für eine nachhaltige Entwicklung. In: Zeitschrift für internationale Bildungsforschung und Entwicklungspädagogik 25, S. 13-20.

Dogan, Bulent/Robin, Bernard (2008): Implementation of Digital Storytelling in the Classroom by Teachers Trained in a Digital Storytelling Workshop. In: McFerrin, Karen/Weber, Roberta/Carlsen, Roger/Willis, Dee Anna (Hrsg.): Proceedings of Society for Information Technology \& Teacher Education International Conference 2008. Las Vegas, Nevada, USA, S. 902-907. 
Egan, Kieran (1989): Teaching as story telling. An alternative approach to teaching and the curriculum. Chicago.

Felbinger, Andrea (2010): Kohärenzorientierte Lernkultur. Wiesbaden.

Floßmann, Bernd (2014): Narrativierung. Im Unterricht und in der Lehre. Berlin.

Gieseke, Wiltrud (2009): Transkulturelle Perspektiven auf Kulturen des Lernens. Bielefeld.

König, Andreas (2009): Von Generationen, Gelehrten und Gestaltern der Zukunft der Hochschulen. Warum die „Digital Native“-Debatte fehlgeht und wie das Modell lebender Systeme das Zukunftsdenken und -handeln von Hochschulen verändern kann. In: Apostolopoulos, Nicolas/Hoffmann, Harriet/Mansmann, Veronika/ Schwill, Andreas (Hrsg.): E-Learning 2009. Lernen im digitalen Zeitalter. Münster [u.a.].

Mattes, Hanspeter (2004): Nahost Jahrbuch 2002: Politik, Wirtschaft und Gesellschaft in Nordafrika und dem Nahen und Mittleren Osten. Deutsches Orient Institut (Hrsg.). Wiesbaden.

Morra, Samantha (2013): 8 Steps To Great Digital Storytelling. http://samanthamorra. com/2013/06/05/edudemic-article-on-digital-storytelling/ [Zugriff: 18.07.2016].

Otto, Daniel (2014): Studentischer Austausch in der Fernlehre? A digital story! In: Zawacki-Richter, Olaf/Kergel, David/Kleinefeld, Norbert/Muckel, Petra/Stöter, Joachim/Brinkmann, Katrin (Hrsg.): Teaching Trends 2014. Münster, S. 137-152.

Otto, Daniel (2015): Potenziale und Grenzen von „epistemic communities“. Eine Analyse des Weltklimarates und der Klimarahmenkonvention. Münster [u.a.].

Petranek, Charles F. (2000): Written Debriefing: The Next Vital Step in Learning with Simulations. In: Simulation \& Gaming 31, 1, S. 108-118.

Robin, Bernard (2006): The Educational Uses of Digital Storytelling. In: Crawford, Caroline M/Carlsen, Roger/McFerrin, Karen/Price, Jerry/Weber, Roberta/Willis, Dee Anna (Hrsg.): Proceedings of Society for Information Technology \& Teacher Education International Conference 2006. Orlando, Florida, USA, S. 709-716.

Robin, Bernard/McNeil, Sara G. (2012): What educators should know about teaching digital storytelling. In: Digital Education Review 22, S. 37-51.

Schekatz-Schopmeier, Sonja (2010): Storytelling - eine narrative Methode zur Vermittlung naturwissenschaftlicher Inhalte im Sachunterricht der Grundschule. Göttingen.

Schermutzki, Margret (2008): Learning outcomes - Lernergebnisse: Begriffe, Zusammenhänge, Umsetzung und Erfolgsermittlung. In: Schmidt, Uwe/Kohler, Jürgen/ Pohlenz, Philipp (Hrsg.): Handbuch Qualität in Studium und Lehre. Berlin.

Schüßler, Ingeborg/Thurnes, Christian M. (2012): Lernkulturen in der Weiterbildung.

Wilson, Gordon (2012): The lived experience of climate change: expanding the knowledge base through collaborative Master's curriculum in the European Union. In: International Journal on Innovation and Sustainable Development 6, 1, S. 43-52. 
Panel II:

Träger, Organisationen und Lernkulturen 



\section{Annäherung differenter Lernkulturen als Voraussetzung für Durchlässigkeit}

\section{Einleitung}

Das Lebenslange Lernen zielt als eines der Kernthemen des Bologna-Prozesses unter anderem auf die horizontale und vertikale Durchlässigkeit in einem vernetzten Bildungssystem ab, das flexible Bildungswege für unterschiedliche Zielgruppen ermöglichen soll. Unmittelbar damit verbunden ist die Frage, wie sich hochschulische und außerhochschulische Lernkulturen mit dem Ziel der Durchlässigkeit aneinander annähern können. Durch die in der Vergangenheit starre Abschottung zwischen hochschulischer und außerhochschulischer Bildung besteht ein hoher Bedarf nach Instrumenten zur Schaffung von Durchlässigkeit. Projekte wie die ,ANKOM Initiative ${ }^{61}$ (20042015) oder der Bundeswettbewerb ,Aufstieg durch Bildung: offene Hochschulen $^{\circ 2}$ (2011-2020) haben bereits einen wichtigen Beitrag zur Öffnung der Hochschulen und zur Durchlässigkeit zwischen den Bildungssystemen geleistet. So wurden vielfältige Verfahren entwickelt, durch die erfolgreich abgeschlossene Ausbildungen aus dem Feld der beruflichen Bildung auf Studienprogramme angerechnet werden können ${ }^{3}$. Auch für den Bereich der non-formal und informell erworbenen Kompetenzen liegen Konzepte vor, die Kompetenzen mit den Zielen der Zulassung zu Studienprogrammen oder der Verkürzung des zu studierenden Workloads für Studienangebote anrechenbar zu machen. ${ }^{4}$ Die Gestaltung von Durchlässigkeit kann demnach als ein Instrument betrachtet werden, der starken gegenseitigen Abgrenzung von hochschulischer und außerhochschulischer Bildung entgegenzuwirken.

Bei den bisher entwickelten Konzepten und Verfahren zur Durchlässigkeit handelt es sich jedoch meist um einzelne Studienangebote und Initiativen. Eine Einbettung dieser einzelnen Angebote in die Hochschullandschaft ist bislang in der Breite nicht gelungen. Hierfür erscheint eine Annäherung der unterschiedlichen Lernkulturen von hochschulischer und außerhochschulischer Bildung notwendig. Dieser Annäherungsprozess stellt sich auf unter-

1 Ausführliche Informationen unter: http://ankom.his.de.

2 Ausführliche Informationen unter: www.wettbewerb-offene-hochschulen-bmbf.de.

3 Vergleiche hierzu auch das ,AnKE-Anrechnungsverfahren. Anrechnung der Kompetenzen von Erzieherinnen und Erziehern auf den Bachelor ,Soziale Arbeit' der Frankfurt University of Applied Sciences. https://www.frankfurt-university.de/?id=5940.

4 Vergleiche hierzu den Weiterbildungsstudiengang ,Innovationsmanagement ' der Technischen Hochschule Mittelhessen. www.weiterbildung.studiumplus.de. 
schiedlichen Ebenen als hoch voraussetzungsreich dar. Nuissl und Dollhausen betonen als zentrale Herausforderung der kooperativen Programmentwicklung ,die kommunikative Konfrontation und Aushandlung von unterschiedlichen Sichtweisen und Auffassungen darüber, welche Ziele mit Angeboten und Programmen verfolgt werden sollen und welche externen und organisationsinternen Bedingungen dabei zu berücksichtigen sind“" (Nuissl/Dollhausen 2011: 121). Gieseke stellt ,,[...] in der Praxis nicht Anpassung, sondern eine Angleichung der Positionen durch Aushandlung, meint Abstimmung, meint, so gesehen, auch Optimierung der Bedarf- und Bedürfniserschließung für die Programmentwicklung“ (Gieseke 2008: 105) in den Vordergrund. Demnach sind Verfahren der Programmentwicklung „,...] dialogische Bedarfsanalysen, Angebotsvergleiche, didaktische Lebensweltanalysen, Beratungskonzepte [und] Zielgruppenplanungen" (ebd. 2003: 196). Zink weist darauf hin, dass sich die kooperative Angebotsentwicklung in der wissenschaftlichen Weiterbildung durch eine Triade auszeichnet, ,die sich aus Hochschulen ${ }^{5}$, institutionellen und individuellen Abnehmenden zusammensetzt und sich durch diese Konstellation auch eindeutig von der grundständigen Lehre unterscheidet" (Zink 2013: 144). Die unterschiedlichen Aspekte machen deutlich, wie hoch komplex und voraussetzungsreich sich eine Annäherung differenter Lernkulturen darstellt.

Anhand der Beschreibung eines kooperativen Programmentwicklungsprozesses in der wissenschaftlichen Weiterbildung, der nachträglich reflektiert und daraufhin auf die lernkulturelle Annäherung von beruflicher und hochschulischer Bildung übertragen wird, soll der Frage nachgegangen werden, welche zentrale Bedeutung die Annäherung differenter Lernkulturen für die Durchlässigkeit zwischen den Bildungsbereichen hat.

$\mathrm{Zu}$ diesem Zweck erfolgt die Darstellung der lernkulturellen Annäherung mit einem besonderen Fokus auf den Aspekt der Durchlässigkeit am Beispiel eines zwischen einer Hochschule und einer Volkshochschule kooperativ entwickelten Zertifikatskurses. Dieses Studienprogramm wurde im Rahmen eines im Bund-Länder-Wettbewerb ,Aufstieg durch Bildung: offene Hochschulen' geförderten Verbundprojektes entwickelt. Ein zentraler Faktor für die Entscheidung zur Programmentwicklung bestand darin, die Durchlässigkeit zwischen dem hochschulischen und dem außerhochschulischen Bildungsbereich voranzutreiben. Dabei ist als herausragendes Merkmal hervorzuheben, dass es nicht nur darum ging, dass eine Hochschule ein Studienprogramm entwickelt, auf welches dann außerhochschulisch erworbene Kompetenzen pauschal angerechnet werden können. Vielmehr stand eine Wissenschaften (of applied science), Fachhochschulen und sonstige fachlichen Hochschulen gemeint. 
gemeinsame und gleichberechtigte Programmentwicklung zwei unterschiedlicher Bildungsanbieter im Mittelpunkt. Daher bietet sich die nähere Betrachtung der kooperativen Programmentwicklung unter dem Aspekt der Annäherung der Lernkulturen und verbunden mit dem Ziel der Erhöhung der Durchlässigkeit an diesem Beispiel besonders an. Diese Betrachtungen beziehen sich zunächst auf den interinstitutionellen kooperativen Entwicklungsprozess, bevor eine Rückspiegelung dieses Vorgangs auf die Annäherung von Lernkulturen vorgenommen wird. Abschließend soll die Bedeutung der lernkulturellen Harmonisierung für die operative Implementierung von Verfahren mit dem Ziel der Durchlässigkeit zwischen hochschulischer und außerhochschulischer Bildung herausgearbeitet werden.

\section{Annäherung differenter Lernkulturen. Ein Entwicklungsprozess}

Ein zentrales Ziel des Zertifikatskurses „Kernkompetenzen für den Beruf: Kommunikation und Betriebswirtschaft", der in einer Kooperation zwischen der Technischen Hochschule Mittelhessen (THM) und der regionalen Volkshochschule des Landkreises Gießen-Friedberg (VHS) entwickelt wurde, bestand darin, explizit nicht-traditionelle Zielgruppen anzusprechen. Darüber hinaus wurde angestrebt, durch eine kooperative Programmentwicklung diese Zielgruppen auf die Hochschulen als Anbieter wissenschaftlicher Weiterbildung aufmerksam zu machen. Im Folgenden wird dieser Entwicklungsprozess rückblickend auf die Annäherung differenter Lernkulturen auf institutioneller Ebene reflektiert. Diese Reflexion ist möglich, da die Programmentwicklung im Kontext eines Prozesses stand, in dem die Entwickelnden gleichzeitig Forschende waren. Daher konnte neben Expertengesprächen mit den für die Entwicklung verantwortlichen Akteurinnen und Akteuren auch auf ein Forschungstagebuch zurückgegriffen werden, welches den Entwicklungsprozess dokumentiert. Seitter et al. machen auf diesen Hybridcharakter aufmerksam, indem sie auf die ,durchgehende Normalität doppelter Beobachtungskonstellationen“ (Seitter et al. 2015: 41f.) verweisen. Diese Konstellation entsteht dadurch, dass die Akteure und Akteurinnen ,einerseits als wissenschaftliche Themenspezialisten das Feld erkenntnisorientiert betrachten und andererseits als hochschulinterne Angebotsentwickler_innen das Feld praxisorientiert gestalten“ (ebd.). Die ständige Vergewisserung und Reflexion der methodologischen und methodischen Konsequenzen dieser Entwicklung erscheint daher als bedeutsames Gütekriterium heutiger Forschung (vgl. Seitter et al. 2015: 41). In der Vergewisserung der besonderen methodologischen Herausforderung wurden neben den Forschungstagebüchern und den Expertengesprächen auch die Protokolle der bildungsanbie- 
terübergreifenden Projekttreffen herangezogen, um die Annäherung der differenten Lernkulturen aus zwei Prozessebenen auszuwerten.

\subsection{Die Annäherung differenter Lernkulturen auf zwei Prozessebenen}

Im Folgenden findet die Darstellung der Programmentwicklung auf zwei unterschiedlichen Prozessebenen statt. Auf inhaltlich-curricularer Ebene wird verdeutlicht, welche strategischen Entscheidungen getroffen und welche Operationalisierungen vorgenommen wurden, um eine Harmonisierung der unterschiedlichen Vorgehensweisen bei der Programmentwicklung mit dem Ziel zu erreichen, ein kooperatives Bildungsangebot anbieten zu können. Auf organisational-prozeduraler Ebene wird die Annäherung der Institutionen durch Interaktion und Kommunikation der beteiligten Akteure und Akteurinnen dargestellt.

\subsubsection{Die inhaltlich-curriculare Prozessebene}

In einem institutionsübergreifenden Team der beiden Bildungseinrichtungen THM und VHS wurde zunächst diskutiert, welches thematische Spektrum durch ein gemeinsames Angebot mit dem Fokus auf die definierte Zielgruppe abgedeckt werden könnte. Dabei wählten beide Institutionen jeweils nach ihrem individuellen Profil entsprechende Themen aus. Es wurde darauf geachtet, dass die Kooperationspartner und -partnerinnen mit ihren individuellen institutionellen Kompetenzen im Curriculum des Zertifikatskurses vertreten sind. Während Module mit einem starken fachlichen Bezug im Hinblick auf Betriebswirtschaftslehre (BWL) und Managementsysteme durch die THM verantwortet und angeboten werden, bringt die VHS ihre Kompetenzen in der Vermittlung von Schlüsselkompetenzen (Kompetenzbilanzierung; Kommunikation im beruflichen Alltag) beziehungsweise auf dem grundlagengenerierenden Sektor (Medienkompetenz, Grundlagen der BWL) zur Vermittlung von Wissen und Fähigkeiten ein. Die Modulübersicht (Abb. 1) verdeutlicht den Aufbau des Zertifikatskurses sowie die Aufteilung der Inhalte auf die beiden Bildungsanbieter. 


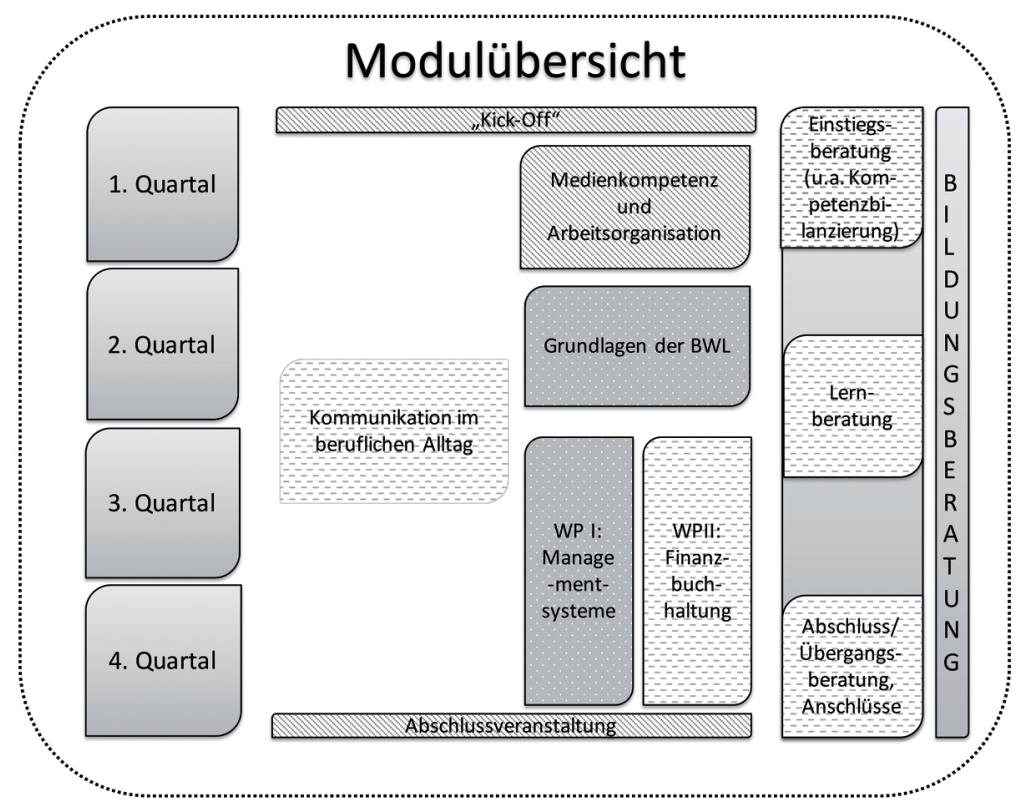

Legende:

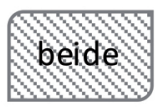

\section{THM}

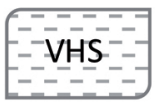

WP $=$ Wahlpflichtmodul

Abb. 1: Modulübersicht des Zertifikatskurses: „Kernkompetenzen für den Beruf: Kommunikation und Betriebswirtschaft“" (eigene Darstellung)

Um die an der VHS erbrachten Leistungen pauschal anzurechnen und in den Zertifikatskurs zu integrieren, war es in einem ersten Schritt notwendig, dass beide Institutionen Modulblätter anfertigen. Zur Erleichterung der Überprüfung der Gleichwertigkeit der an der VHS erbrachten Leistungen durch Fachvertretende seitens der THM nutzte die VHS bei der Anfertigung der Modulblätter die Vorlagen der Hochschule. Auf diese Weise wurde sichergestellt, dass gemäß der Bologna-Vorgaben überprüfbare Lernergebnisse formuliert werden und das Niveau nach dem Deutschen Qualifikationsrahmen (DQR) bestimmbar ist. Während die VHS lediglich Modulblätter für die von ihr angebotenen Module anfertigte, erstellte die THM Modulblätter für alle Module des Zertifikatskurses. Somit wurde sichergestellt, dass durch einen Äquivalenzvergleich die an der VHS erbrachten Leistungen in Form von Anrechnungsmodulen pauschal und in vollem Umfang auf das Weiterbil- 
dungsangebot anerkannt und angerechnet werden können (vgl. Hanak/Sturm 2015: 20). Im Rahmen dieses Äquivalenzvergleiches wurden die von der VHS erstellten Modulblätter durch den programmverantwortlichen Professor an der THM auf Gleichwertigkeit zu den an der Hochschule entwickelten Inhalten überprüft. Die Feststellung eben dieser Gleichwertigkeit der vermittelten Kompetenzen in Form von Lernergebnissen bildete die Grundlage für die Anrechnung ${ }^{6}$ auf den Zertifikatskurs. Somit werden die an der VHS erbrachten Leistungen pauschal und mit Übernahme der Noten auf den Zertifikatskurs der THM angerechnet, ohne dass die Teilnehmenden diese Module an der THM aktiv studieren müssen.

Ein wichtiger Schritt hierbei war, dass sich die VHS bei der Dokumentation der Inhalte und Lernergebnisse an den Modulblättern der THM orientiert hat. Durch diese Ausrichtung wurde die Grundlage für die Anerkennung ${ }^{7}$ von Kompetenzen und damit für die Durchlässigkeit zwischen hochschulischer und außerhochschulischer Bildung geschaffen.

\subsubsection{Die organisational-prozedurale Prozessebene}

Um bewerten zu können, ob sich aus einer kooperativen Angebotsentwicklung unterschiedlicher Bildungsanbieter eine Annäherung der jeweiligen Lernkulturen ergeben kann, die sich wiederum auf eine Begünstigung von Durchlässigkeit rückkoppeln lässt, wird der Entwicklungsprozess im Folgenden auf der organisational-prozeduralen Ebene betrachtet.

Die Betrachtung der Forschungstagebücher, der Gespräche sowie der Protokolle der bildungsanbieterübergreifenden Projekttreffen hat gezeigt, dass sich der gesamte Entwicklungsprozess rückblickend in einem sich wiederholenden kooperativen Verfahrensablauf der Programmentwicklung (Abb. 2) darstellt. Nach den jeweiligen Projekttreffen des institutionsübergreifenden Entwicklungsteams der beiden Bildungseinrichtungen fand im Rahmen der Nachbereitung eine Reflexion sowohl der eigenen als auch der jeweils anderen organisationalen Selbstverständnisse statt. Diese umfassten Aspekte wie beispielsweise die zeitliche Verortung der Lehrangebote. Während an der VHS eine Vielzahl von Kursen an den Nachmittagen und Abenden angeboten wird, um adäquat auf die Bedürfnisse der Zielgruppe zu reagieren, liegen die Kernzeiten der Hochschullehre zwischen vormittags und nachmittags. Auch die didaktische Schulung der Lehrenden wurde von den Institutionen unterschiedlich gehandhabt. Während die VHS traditionell viele

6 Zur Definition von ,Anrechnung' siehe Hanak, H./Sturm, N. (2015): Anerkennung und Anrechnung außerhochschulisch erworbener Kompetenzen. Eine Handreichung für die wissenschaftliche Weiterbildung. Wiesbaden: Springer VS.

7 Zur Definition von ,Anerkennung 'siehe Hanak, H./Sturm, N. (2015): Anerkennung und Anrechnung außerhochschulisch erworbener Kompetenzen. Eine Handreichung für die wissenschaftliche Weiterbildung. Wiesbaden: Springer VS. 
Ressourcen darauf aufwendet, ihre Lehrenden didaktisch zu professionalisieren, ist dies an den Hochschulen noch ein vergleichsweise junges, wenn auch sich schnell entwickelndes Feld. Auch die individuelle Bildungsberatung von (potentiellen) Teilnehmenden ist ein Themenfeld, auf dem die VHS bereits stark professionalisiert ist, was sich auch in der Konzeption des Zertifikatskurses wiederspiegelt (vgl. Abb. 1). Schließlich bestand ein zentraler Punkt bei der Reflexion der gegenseitigen Selbstverständnisse in der Dokumentation des Niveaus und der Inhalte beziehungsweise der Lernergebnisse der jeweiligen Bildungsangebote. Während die Hochschule in ihren Studienprogrammen mit standardisierten Modulblättern arbeitet, in denen Workload (Präsenzzeiten und Selbstlernphase) sowie Niveau und Lernergebnisse (auf fachlicher, überfachlicher und sozialer Ebene) dokumentiert werden, arbeitet die VHS mit unterschiedlichen Darstellungskonzepten. Dies ist auf die Vielfalt des Angebotsspektrums der VHS zurückzuführen.

Mit dem Ziel der Harmonisierung der beiden Lernkulturen als begleitender Prozess bei der kooperativen Angebotsentwicklung war eine Rückspiegelung der eben dargestellten unterschiedlichen Rahmenbedingungen in die eigene Organisation notwendig. In diesem Verfahrensschritt waren institutionelle Entscheidungen von den verantwortlichen Akteuren und Akteurinnen der beiden Organisationen zu treffen. So wurde aufgrund der individuellen institutionellen Kompetenzprofile entschieden, welche Inhalte von wem verantwortet werden. Während die THM eine starke Kompetenz in der Vermittlung betriebswirtschaftlicher Grundlagen hat, verfügt die VHS über langjährige vertiefte Expertenkenntnisse in der individuellen Lernberatung. Die VHS kam in ihrem internen organisationalen Rückkopplungsprozess zu dem Ergebnis, dass sie die Modulblätter der THM zur Konzeption der durch sie verantworteten Blöcke übernehmen kann. An der THM bestand der Rückkopplungsprozess darin, trotz der strukturellen Unterschiedlichkeiten einen kooperativen Programmentwicklungsprozess mit einem außerhochschulischen Bildungsträger in dieser Form einzugehen. Hier standen vor allem die Überlegungen zu einer möglichen pauschalen Anrechnung der durch die VHS angebotenen Inhalte im Vordergrund. Auf Basis dieser strategischen und politischen Entscheidungen innerhalb der jeweiligen Organisationen bereiteten die Mitarbeitenden das nächste institutionsübergreifende Projekttreffen vor. Dabei war es immer wieder notwendig, die institutionellen Besonderheiten der jeweils anderen Institution zu reflektieren und mit den eigenen organisationalen Rahmenbedingungen abzugleichen. Der hier exemplarisch dargestellte Verfahrenskreislauf wurde von der Planung bis zum Ende der kooperativen Entwicklung immer wieder durchlaufen. Es ist davon auszugehen, dass auch während einer operativen Durchführung und Evaluation des Weiterbildungsangebotes diese Schleife bestehen bleibt. 


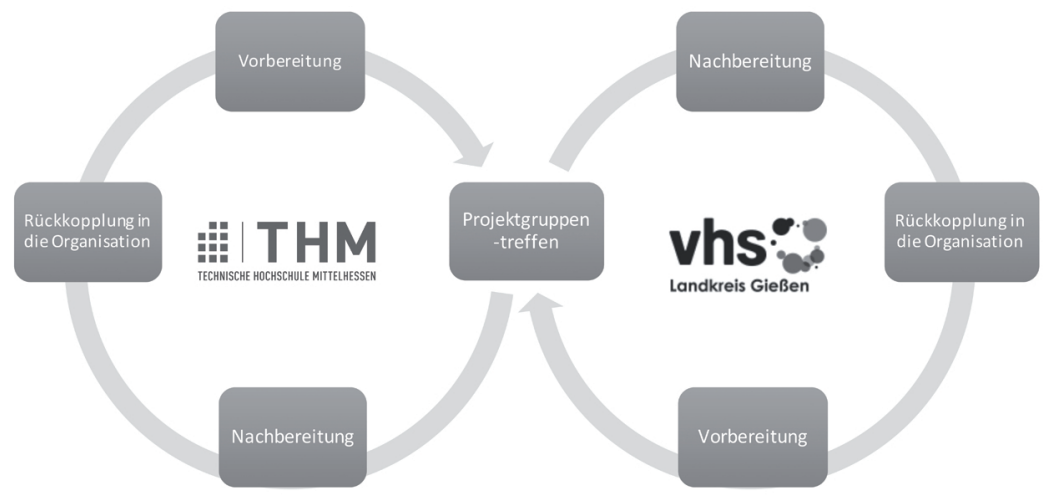

Abb. 2: Kooperativer Verfahrenskreislauf der Programmentwicklung (eigene Darstellung)

Retrospektiv betrachtet kann festgestellt werden, dass mit jedem erneuten Durchlauf des Verfahrenskreislaufes das gegenseitige organisationale Verständnis gestiegen ist und damit auch zunächst zu einer Annäherung der Verständnisse von Lernkulturen geführt hat. Im Ergebnis führte dies dazu, dass es gelungen ist, notwendige Entscheidungen wie Modulverantwortlichkeiten und Zulassungsvoraussetzungen zu treffen, um das Zertifikat markttauglich zu konzeptionieren.

\subsection{Die Rückspiegelung der Prozessschritte auf die Annährung der Lernkulturen}

Nachfolgend werden die beiden zunächst deskriptiv auf inhaltlich-curricularer (2.1.1) und organisational-prozeduraler (2.1.2) Ebene dargestellten Verfahren der Programmentwicklung theoretisch auf die Annäherung institutioneller Lernkulturen übertragen. Der Begriff der ,Lernkultur' wird in diesem Zusammenhang auf die institutionelle Ebene der Erwachsenenbildung fokussiert $^{8}$.

„[...] Lernkulturen haben einen gesellschaftlich-kulturellen Kontext. Bei partikular-gemeinnützigen Institutionen der Erwachsenenbildung äußern sie sich charakteristisch in balancierten Lernkulturformen von Träger-

8 Auf eine vertiefte Auseinandersetzung mit dem Lernkulturbegriff muss aufgrund der Komplexität des Themas an dieser Stelle verzichtet werden. 
kulturen, Zivilkulturen, Partizipationskulturen und Verbandskulturen.“ (Fleige 2011: 52)

Um den Lernkulturbegriff auf institutioneller Ebene um organisational kulturelle Aspekte zu erweitern, werden neben Werten der jeweiligen Institutionen auch organisationsspezifische Basisannahmen betrachtet, die für Organisationen reflexiv gegebenenfalls gar nicht verfügbar sind. Zusätzlich werden lernkulturelle Artefakte als operative Instrumente zur Annäherung von Lernkulturen mit dem Ziel der Steigerung von Durchlässigkeit untersucht. Vor dem Hintergrund der Organisationskulturtheorie (vgl. Merkens 2006: 208f.) wird im Folgenden der Frage nachgegangen, ob das Zusammenspiel von Artefakten mit anerkannten Werten und organisationsspezifischen Basisannahmen (vgl. Schein 2003) zur Annäherung von Lernkulturen beiträgt.

Zunächst wird die Programmentwicklung auf organisational-prozeduraler Ebene in Bezug auf die anerkannten Werte und organisationsspezifischen Basisannahmen in Form der Institutionalisierung von Kommunikationsräumen betrachtet (2.2.1). Des Weiteren wird die inhaltlich-curriculare Ebene mit Blick auf die Erzeugung lernkultureller Artefakte beleuchtet (2.2.2), um abschließend die Bedeutung der lernkulturellen Harmonisierung für die Stärkung der Durchlässigkeit zwischen hochschulischer und außerhochschulischer Bildung aufzuzeigen (3.).

\subsubsection{Institutionalisierung eines Kommunikationsraums}

Die intensive Befassung mit dem jeweils anderen Bildungsanbieter im Rahmen der institutionsübergreifenden Projektgruppen hat einen zentralen Beitrag dazu geleistet, die jeweiligen organisationsspezifischen Basisannahmen zu verstehen. Die fortlaufende offene Kommunikation und das gemeinsame Ziel, ein kooperatives Weiterbildungsangebot zu entwickeln, hat Vertrauen zwischen den handelnden Akteuren und Akteurinnen geschaffen, welches sich im Rahmen des Entwicklungsprozesses auch auf ein Vertrauen in die Stärken und Kompetenzen der jeweils anderen Institution ausgeweitet hat. Mit dem fortwährenden Durchlaufen des kooperativen Verfahrenskreislaufs der Programmentwicklung (vgl. Abb. 2) ist durch die ständige Kommunikation ein Raum zwischen den an dem Prozess beteiligten Akteuren und Akteurinnen entstanden, der es zugelassen hat, dass die unterschiedlichen Institutionen bereit waren, sich der jeweils anderen zu öffnen. Auf Seiten der VHS ist hier als besonderes Beispiel die Bereitschaft hervorzuheben, alle eigenen Angebote im Rahmen des Zertifikatskurses an den, für die eigene Institution vollkommen fremden, Vorgaben der THM in Form von Modulblättern zu konzipieren. Damit verbunden war die Auseinandersetzung von Workloadberechnungen, kompetenzorientierter Prüfungskonzeption und Lernergebnisbeschreibungen. Seitens der THM hat die enge vertrauensvolle Zusammenarbeit zu der Bereitschaft geführt, sich unvoreingenommen mit den an der VHS 
vermittelten Kompetenzen vor dem Hintergrund der Anrechenbarkeit auf Hochschulprogramme auseinanderzusetzen.

Zusammenfassend kann resümiert werden, dass die kooperative Angebotsentwicklung und der intensive Austausch über mehrere Monate hinweg $\mathrm{zu}$ einer starken Wertschätzung der jeweils anderen Institution und derer individuellen Kompetenzen geführt hat.

\subsubsection{Modulblätter als lernkulturelle Artefakte}

Sichtbarer Ausdruck der Wertschätzung sind die Modulblätter als lernkulturelle Artefakte. Diese Modulblätter fungieren als Türöffner zur breiteren Annäherung der differenten Lernkulturen. Durch die Bereitschaft und das Entgegenkommen der VHS, die Modulblätter für die Programmentwicklung einzusetzen, konnte die THM diese nach Inhalt und Niveau auf ihre Anrechenbarkeit auf Hochschulprogramme hin überprüfen. Bei dieser Überprüfung wurde schnell deutlich, dass es in den Kompetenzbereichen der VHS Anrechnungspotentiale auf (Grundlagen)Module von Studienprogrammen gibt, was zu einer weiteren Kooperation der Institutionen und damit zu einer Steigerung von Durchlässigkeit führte. Diese Verbreiterung gelang nur auf der Grundlage der lernkulturellen Verklammerung, welche sich aus der Schaffung gegenseitigen Vertrauens sowie aus der Produktion lernkultureller Artefakte generierte.

Die soziale Interaktion und die aus der gemeinsamen Programmentwicklung resultierende gegenseitige Wertschätzung können daher als Voraussetzung für die Produktion von Artefakten betrachtet werden. Das Generieren eben dieser hatte wiederum zur Folge, dass die auf interpersonaler Ebene entstandene Wertschätzung auf die institutionelle Ebene adaptiert werden konnte. Durch die Objektivierung und Synchronisation der Lernergebnisse in Gestalt der Modulblätter konnte sich die Annäherung der an dem Prozess beteiligten Akteure und Akteurinnen hin zu einer Annäherung der institutionellen Lernkulturen entwickeln.

\section{Annäherung differenter Lernkulturen. Eine Voraussetzung für Durchlässigkeit}

In der Gesamtbetrachtung des Annäherungsprozesses von unterschiedlichen Lernkulturen wurde deutlich, dass die eingangs erwähnten Aushandlungsprozesse (zum Beispiel in Bezug auf die Modulblätter) eine wesentliche Rolle bei der kooperativen Programmentwicklung spielen. Darüber hinaus konnte durch die ,Harmonisierung der Lernkulturen' gezeigt werden, dass nicht eine Anpassung, sondern vielmehr eine Angleichung im Rahmen der Programmentwicklung eine wichtige Bedingung ist. Vor dem Hintergrund der Durchlässigkeit konnte ein Dreischritt identifiziert werden (vgl. Abb. 3). 
Während in einem ersten Schritt die kooperative Programmentwicklung zu einer gegenseitigen Wertschätzung und Anerkennung der an dem Prozess beteiligten Akteure und Akteurinnen führte, musste diese Anerkennung in einem zweiten Schritt organisational vollzogen werden. Dies konnte in dem hier dargestellten Beispiel durch die gemeinsame Festlegung auf einheitliche Modulblätter gelingen. In einem dritten Schritt kann es dann darum gehen, die organisational-wertschätzende Anerkennung ,technisch` zu vollziehen, indem die an der VHS vermittelten und in den Modulblättern dokumentierten Kompetenzen formal auf Hochschulprogramme ,anerkannt` werden.

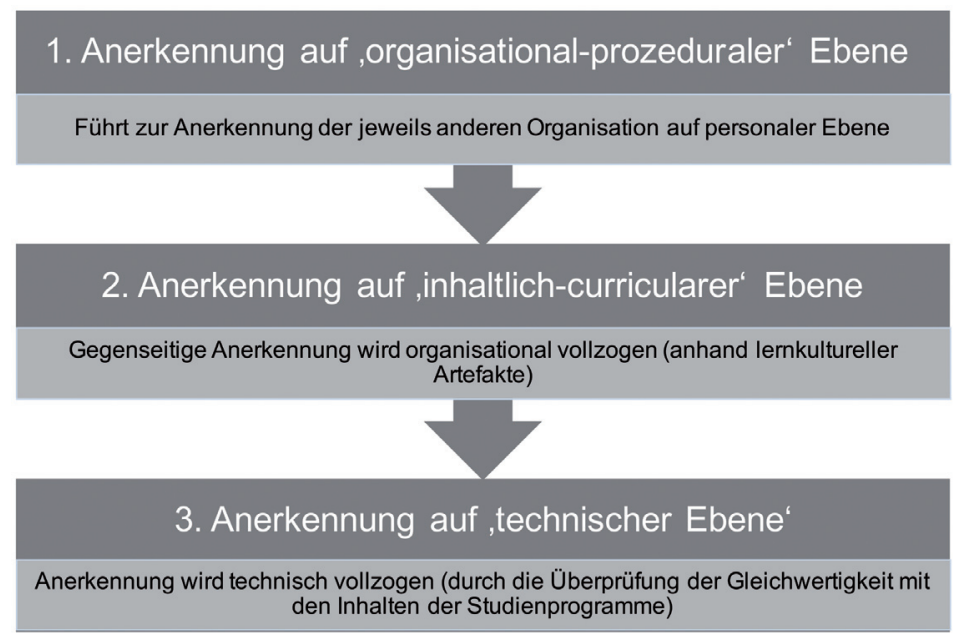

Abb. 3: Dimensionen der Anerkennung (eigene Darstellung)

In dem hier dargestellten Beispiel wurde dies operativ umgesetzt, indem alle an der VHS entwickelten und angebotenen Module (vgl. Abb. 1) in Form von Anrechnungsmodulen pauschal auf den Zertifikatskurs angerechnet werden. Die hierdurch vollzogene Annäherung differenter Lernkulturen fand darüber hinaus darin Ausdruck, dass im weiteren Verlauf auch andere von der VHS angebotene Kurse pauschal auf ein Bachelorprogramm der THM angerechnet werden können.

Vor dem Hintergrund der Betrachtung von Durchlässigkeit bedeutet dies, dass die Entwicklung von Konzepten und Verfahren der Anerkennung und Anrechnung außerhochschulisch erworbener Kompetenzen mit dem Ziel, die Durchlässigkeit zwischen den Bildungssystemen zu erhöhen, nur eine geringe Wirkung entfalten kann, wenn die organisationale Annäherung differenter Lernkulturen nicht aktiv gestaltet wird. Diese ist, wie aus dem hier 
dargestellten Beispiel deutlich wird, höchst voraussetzungsvoll. Wenn sich die in den letzten zehn Jahren erfolgreich entwickelten und erprobten Verfahren zur Anerkennung und Anrechnung außerhochschulisch erworbener Kompetenzen in der Breite der Hochschullandschaft nachhaltig umsetzen lassen sollen, stehen die Hochschulen vor der Aufgabe, sich der außerhochschulischen Bildung zu öffnen, um zu einer Annäherung der unterschiedlichen Lernkulturen zu gelangen. Ein Gelingen dieser Annäherung ist aber ebenso von der Bereitschaft der außerhochschulischen Bildungsträger abhängig, sich für einen solchen Prozess zu öffnen. Besonders Arbeitgebende und Unternehmen stehen hier in der Pflicht, auf die Hochschulen zuzugehen, soll die Durchlässigkeit zwischen den Bildungssystemen erhöht werden.

\section{Literatur}

Fleige, Marion (2011): Lernkulturen in der öffentlichen Erwachsenenbildung. Theorieentwickelnde und empirische Betrachtungen am Beispiel evangelischer Träger. Münster: Waxmann.

Gieseke, W. (2003): Programmplanungshandeln als Angleichungshandeln. Die realisierte Vernetzung in der Abstimmung von Angebot und Nachfrage. In: Gieseke, W. (Hrsg.): Institutionelle Innensichten. Bielefeld, S. 189-211.

Gieseke, W. (Hrsg.) (2008): Bedarfsorientierte Angebotsplanung in der Erwachsenenbildung. Bielefeld.

Hanak, Helmar/Sturm, Nico (2015): Anerkennung und Anrechnung außerhochschulisch erworbener Kompetenzen. Eine Handreichung für die wissenschaftliche Weiterbildung. Wiesbaden: Springer VS.

Merkens, Hans (2006): Pädagogische Institutionen. Pädagogisches Handeln im Spannungsfeld von Individualisierung und Organisation. Wiesbaden: Springer VS.

Nuissl, E./Dollhausen, K. (2011): Kulturen der Programmplanung. In: Zeitschrift für Pädagogik 57, 1, S. 114-129.

Schäffter, Ortfried (2007): Erwachsenenpädagogische Institutionsanalyse. Begründungen für eine lernfördernde Forschungspraxis. In: Heuer, Ulrike/Siebers, Ruth (Hrsg.): Weiterbildung am Beginn des 21. Jahrhunderts. Festschrift für Wiltrud Gieseke. Waxmann, S. 354-370.

Schein, Edgar H. (2010): Organisationskultur. Bergisch-Gladbach: EHP Organisation.

Seitter, W./Schemmann, M./Friese, F. (Hrsg.) (2015): Zielgruppen in der wissenschaftlichen Weiterbildung. Wiesbaden: Springer VS.

Zink, Franziska (2013): Wissenschaftliche Weiterbildung in der Aushandlung. Die Akteure und ihre Themen in interinstitutionellen Aushandlungsprozessen im Kontext kooperativer Angebotsentwicklung. In: Dollhausen, K./Feld, T. C./Seitter, W. (Hrsg.): Erwachsenenpädagogische Kooperations- und Netzwerkforschung. Wiesbaden. Springer VS. 


\section{Programm- und Angebotsentwicklung in der Finanziellen Grundbildung als Gestaltungselement einer, neuen' Lernkultur}

\section{Problemaufriss}

Lernkultur ist ein „schillernder“ Begriff (Schüßler/Thurnes 2005: 13). Die Verwendung erfolgt zumeist im Zusammenhang mit der Beschreibung eines Wandels, indem das ,Neue ' betont wird. Vor diesem Hintergrund hat sich die Bezeichnung ,Neue Lehr-Lernkulturen“ als Schlagwort durchgesetzt (vgl. Fleige 2010: 120). Da sich der Begriff ,Lernkultur" auf verschiedene Bereiche bezieht, wird von einem „Mehrebenenverständnis von Lernkulturen“ (Fleige 2013: 86) gesprochen, sodass es nicht nur um Programmprofile von Institutionen oder Veranstaltungs- und Angebotsformen, sondern auch um Lerninhalte und Lerngegenstände geht (vgl. auch Gieseke 2001; Egetenmeyer 2008; Dietrich/Herr 2003).

Fleige (2011: 5) definiert Lernkulturen als „Kulturen des Lehrens und Lernens, Planens, Disponierens und Partizipierens in Institutionen der Erwachsenenbildung/Weiterbildung im Modus institutionalformenspezifischer Praktiken, Deutungsmuster, Werte/Normen, Interaktionen/Rituale und Beziehungsstrukturen. Lernkulturen werden professionell pädagogisch gestaltet und individuell rekonstruiert, ausgehandelt und gesteuert. Lernkulturen haben einen gesellschaftlich-kulturellen Kontext“. Programmgestaltung kann demnach als eine Praktik der Lernkulturgestaltung verstanden werden (vgl. Fleige 2010).

Darauf Bezug nehmend fokussiert der vorliegende Beitrag darauf, dass Programmplanende und Lehrende in der Alphabetisierung und Grundbildung eine Schlüsselrolle für die Lernkultur in diesem Programmbereich innehaben bzw. die Programm- und Angebotsentwicklung in der Finanziellen Grundbildung ein wesentliches Gestaltungselement für eine ,neue“ Lernkultur darstellt. Als eine (makro-)didaktische Grundlage für die Programm- und Angebotsplanung in diesem Bereich wurde im Projekt $\mathrm{CurVe}^{1}$ ein Modell entwi-

1 Das Projekt „Schuldnerberatung als Ausgangspunkt für Grundbildung - Curriculare Vernetzung und Übergänge“ (CurVe) ist dem Förderschwerpunkt „Arbeitsplatzorientierte Alphabetisierung und Grundbildung Erwachsener" zugeordnet und wird mit Mitteln des Bundesministeriums für Bildung und Forschung (BMBF) unter dem Förderkennzeichen 01AB12009 gefördert. Die Verantwortung für den Inhalt dieser Veröffentlichung liegt bei den Autorinnen. 
ckelt, das die Kompetenzanforderungen im Umgang mit Geld auf Grundbildungsniveau operationalisiert.

Um die Frage zu beantworten, welche Implikationen das Kompetenzmodell für Lernkulturen in der (Finanziellen) Grundbildung hat, wird vor der überblickshaften Vorstellung des Modells auf den bisherigen Forschungsund Diskussionsstand sowie die theoretischen und empirischen Grundlagen des Modells eingegangen.

\section{Ausgangspunkte}

Wird die bisherige Lernkultur in der Grundbildung bzw. der wissenschaftliche Diskussionsstand zu Finanzieller Grundbildung betrachtet, so zeigt sich, dass das Gros der erfassten Veranstaltungen als Alphabetisierung zu bezeichnen ist (vgl. Ambos/Horn 2015), d.h. das Erlernen des Lesens und Schreibens bzw. die ,Beherrschung der Kulturtechniken' fokussiert (siehe dazu auch Abraham/Linde 2011). Grundbildung wird demnach teilweise immer noch auf eine kompensatorische Funktion reduziert; seine komplementäre und erweiternde Funktion sowie die Anwendungsbezüge von Schriftsprache und weitere Alltagskompetenzen stehen nach wie vor im Hintergrund (vgl. Kastner 2011).

In den letzten Jahren wird Finanzielle Grundbildung als Teil des Programmbereichs Alphabetisierung und Grundbildung im (inter-)nationalen Diskurs vermehrt diskutiert: Auf der einen Seite wird Finanzielle Grundbildung im Rahmen der Nationalen Strategie der Alphabetisierung und Grundbildung (vgl. BMBF 2012) und im wissenschaftlichen Diskurs (vgl. u.a. Egloff 2014; Hummelsheim 2010) als Bestandteil eines ganzheitlichen Grundbildungsverständnisses begriffen. Mit Blick auf eine Reihe von sozialen, politischen und ökonomischen Entwicklungen wie dem hohen Verschuldungsgrad von Privathaushalten oder der zunehmenden Erfordernis privater Vorsorge wird die Relevanz und Bedeutungszunahme Finanzieller Grundbildung betont (vgl. Weber et al. 2013; Aprea 2012).

Auf der anderen Seite gibt es bisher kaum Angebote und keine didaktischen Konzepte, die explizit auf Erwachsene als Adressatinnen und Adressaten von Lernangeboten im Bereich Umgang mit Geld auf Grundbildungsniveau ausgerichtet sind (vgl. Bigge 2015; Ambos/Greubel 2012), sondern im Wesentlichen nur ein Konzept für selbstgesteuertes Lernen (vgl. DVV o.J.). Die Analysen der Kompetenzanforderungen im Bereich Umgang mit Geld liegen nicht auf der Ebene der Grundbildung vor, sondern sind in der Regel in der Berufs- und Wirtschaftspädagogik als Grundlage für die Kompetenzentwicklung und -messung von Schülerinnen und Schülern oder jungen Erwachsenen entstanden (vgl. Schürkmann/Schuhen 2013; Seeber et al. 2012). Grundbildungskompetenzen wie Lesen, Schreiben und Rechnen werden 
beispielsweise in dem Modell der ökonomischen (Grund-)Bildung von Remmele et al. (2013), das Finanzielle Grundbildung als Teilbereich vorsieht, vorausgesetzt.

Der Blick in den Forschungs- und Diskussionsstand offenbart eine Vielzahl von Begriffen, die im internationalen Kontext teilweise synonym verwendet werden, wie ,Financial Literacy', ,Financial Capability', ,Finanzkompetenz', ,Finanzielle Alphabetisierung 'oder ,Finanzieller Analphabetismus' (vgl. Aprea 2012; Mania/Tröster 2013). Im Folgenden werden unter Finanzieller Grundbildung die ,existenziell basalen und unmittelbar lebenspraktischen Anforderungen alltäglichen Handelns und der Lebensführung in geldlichen Angelegenheiten“ definiert (Mania/Tröster 2014: 140).

\section{Theoretische Bezüge}

Bei der theoretischen Fundierung des Kompetenzmodells Finanzielle Grundbildung wurde hauptsächlich auf drei theoretische Diskurse zurückgegriffen (vgl. Mania 2015):

- den Didaktikdiskurs in der Erwachsenenbildung,

- den Kompetenzdiskurs in den Bildungswissenschaften

- und den internationalen Literalitätsdiskurs.

Die Gestaltung von Programmen und Angeboten wird als Bestandteil (makro-)didaktischen Handelns von professionell Planenden in der Erwachsenenbildung betrachtet (vgl. u.a. Reich-Claassen/von Hippel 2011; HöfferMehlmer 2011; Gieseke 2008; Arnold 2010). Während im Zuge des Aufkommens konstruktivistischer Ansätze selbstgesteuertes Lernen und Ermöglichungsdidaktik im Vordergrund standen, wird in den letzten Jahren eine Beschäftigung mit der Begründung, Bestimmung und Strukturierung von Inhalten als zentrale Aspekte einer Didaktik für Erwachsenenbildung gefordert (vgl. Frank 2013; Lehner 2013; Schlutz 2006). Die Didaktik der Erwachsenenbildung bedarf daher eines ,inhaltsorientierten Segments" (Lehner 2013: 03-5) oder einer „Kompetenzorientierung“ (Frank/Iller 2013: 32). Im Sinne des Planungsmodells für die Angebotsentwicklung von Schlutz (2006), der wiederum auf die Curriculumtheorie von Robinsohn rekurriert, können die Kompetenzanforderungen in bestimmten Situationen und Lebenslagen zum Ausgangspunkt für die Konzeption von Programmen und Angeboten in der Erwachsenenbildung genommen werden. Lernziele und -inhalte werden dann nicht aus einer abstrakten Bildungsidee abgeleitet, sondern anhand der Analyse von (Handlungs-)Anforderungen in ,Lebens- und Verwendungssituationen" (Schlutz 2006: 86) (empirisch) ermittelt. Durch seine Orientierung an den gewünschten Lernergebnissen sowie den Situations- und Verwen- 
dungsbezug ist das Modell der Angebotsentwicklung von Schlutz (2006) an den Kompetenzdiskurs in den Bildungswissenschaften anschlussfähig.

Ausgehend von der einschlägigen Kompetenzdefinition von Weinert lässt sich analytisch trennen zwischen kognitiven Kompetenzbestandteilen, also dem Wissen und Können, und non-kognitiven Aspekten wie Einstellungen, Werten und Motivationen (Weinert 2001: 27 f.). Der Kern des „Wissens und Könnens" (Baumert/Kunter 2005: 481) in einem bestimmten inhaltlichen Bereich kann mittels Kompetenzmodellen abgebildet werden, wobei die jeweiligen Lernfelder, fachbezogenen Kompetenzbereiche oder Lern- und Handlungsbereiche als Kompetenzdomänen bezeichnet werden können (vgl. Klieme/Leutner 2006).

Der Kompetenzdiskurs ist damit an den Literalitätsansatz von Barton und Hamilton (2003) anschlussfähig, die in ihrem Konzept der ,situated literacies ${ }^{6}$ von unterschiedlichen Literalitäten in unterschiedlichen Domänen, d.h. sozial strukturierten Kontexten oder Lebensbereichen, ausgehen. Literalität wird dabei als soziale Praxis begriffen, sodass die Bedeutungshorizonte und Anwendungskontexte von Schriftsprache betrachtet werden (vgl. u.a. Zeuner/ Papst 2011; Street 2003; The New London Group 1996). So lassen sich verschiedene Grundbildungsbereiche wie Ernährung (Food Literacy), Gesundheit (Health Literacy) und Finanzen (Financial Literacy) unterscheiden.

\section{Methodisches Vorgehen}

Um ein möglichst umfassendes Bild der alltäglichen Kompetenzanforderungen im Umgang mit Geld zu erhalten, wurde im Projekt CurVe ein perspektivverschränkender Ansatz verfolgt, sodass drei Expertengruppen einbezogen wurden, die aufgrund ihrer beruflichen Rolle und persönlichen Erfahrungen jeweils eine besondere Perspektive auf den Gegenstand einnehmen: Weiterbildungspersonal im Bereich Grundbildung, Schuldnerberatende sowie Grundbildungsadressatinnen und -adressaten (vgl. Mania 2015). Mangelnde Finanzielle Grundbildung wird dabei, neben anderen Auslösern wie Arbeitslosigkeit und Krankheit, als eine mögliche Ursache für Überschuldung gesehen, daher wurde die Perspektive der Ratsuchenden aus der Schuldnerberatung als potenziellen Grundbildungsadressatinnen und -adressaten mittels Forschender Lernwerkstätten (vgl. Grell 2004) mit insgesamt 18 Personen einbezogen. Die Auswahl der Ratsuchenden mit vermutetem Grundbildungsbedarf erfolgte in Abstimmung mit den zuständigen Schuldnerberatenden. Charakteristisch für den multimethodischen Ansatz einer Forschenden Lernwerkstatt ist die Veränderung ,von einer Situation der Beforschung zu einem Partizipationsgedanken“ (vgl. Grell 2004: 61). Im Rahmen von insgesamt 24 Experteninterviews (vgl. Gläser/Laudel 2004) wurde die Perspektive von Schuldnerberatenden sowie des Weiterbildungspersonals berücksichtigt. Die 
Auswertung der erhobenen Daten in Anlehnung an die qualitative Inhaltsanalyse (vgl. Gläser/Laudel 2004) zielte auf Rekonstruktion der Komplexität der Kompetenzanforderungen.

\section{Empirische Ergebnisse}

Im Bereich der basalen Anforderungen beim Umgang mit Geld lässt sich zwischen kognitiven und non-kognitiven Aspekten differenzieren. Um die kognitiven Kompetenzanforderungen $\mathrm{zu}$ strukturieren und $\mathrm{zu}$ beschreiben, wurde auf Basis der empirischen Daten ein Kompetenzmodell Finanzielle Grundbildung entwickelt. Es beinhaltet einerseits sechs Domänen, inklusive 23 Subdomänen, Finanzieller Grundbildung und andererseits die vier Dimensionen Wissen, Lesen, Schreiben und Rechnen, die in Form einer Kreuztabelle die Heuristik für die Bestimmung exemplarischer (Handlungs-)Anforderungen in Alltagssituationen bilden (vgl. Abb. 1).

Bei der Formulierung der Kennt- bzw. Kann-Beschreibungen wurde zum einen auf Beispiele aus dem empirischen Material zurückgriffen. Zum anderen wurden weitere (Handlungs-)Anforderungen im Sinne einer ,konsensuellen" (Grotlüschen et al. 2014: 51) Entwicklung von Kompetenzmodellen und einer kommunikativen Validierung in Zusammenarbeit mit Expertinnen und Experten aus der Schuldnerberatung und Erwachsenenbildung formuliert.

Im Folgenden werden am Beispiel der Domäne ,Haushalten“ der Zuschnitt und der Inhalt der Domänen veranschaulicht.

Die Kategorie ,Haushalten“ bezieht sich auf die Relation von Einnahmen und Ausgaben. Die Domäne beschreibt die Kompetenz, das Geld bzw. die Einnahmen so „einzuteilen“ (I 14, A. 87; I 24, A. 208; GD 1, A. 127; GD 2, A. 900), dass keine (erneute) Überschuldung entsteht bzw. dass alle Ausgaben bezahlt werden können. Die jeweilige Person bzw. der Haushalt muss mit dem vorhandenen Geld „kalkulieren“ (I 3, A. 122), „wirtschaften“ (I 12, A. 61) und ,zurechtkommen“ (I 5, A. 93). Eine besondere Rolle spielt hierbei die Fähigkeit ,planen zu können, also (...) abwägen können“ (I 10, A. 191). Die Domäne ,Haushalten“ beinhaltet die Subdomänen ,Überblick', ,Wochenund Monatsplanung', ,Jahresplanung', ,Risiko- und Krisenmanagement sowie ,Sparen', die unterschiedliche Anforderungen mit sich bringen. 


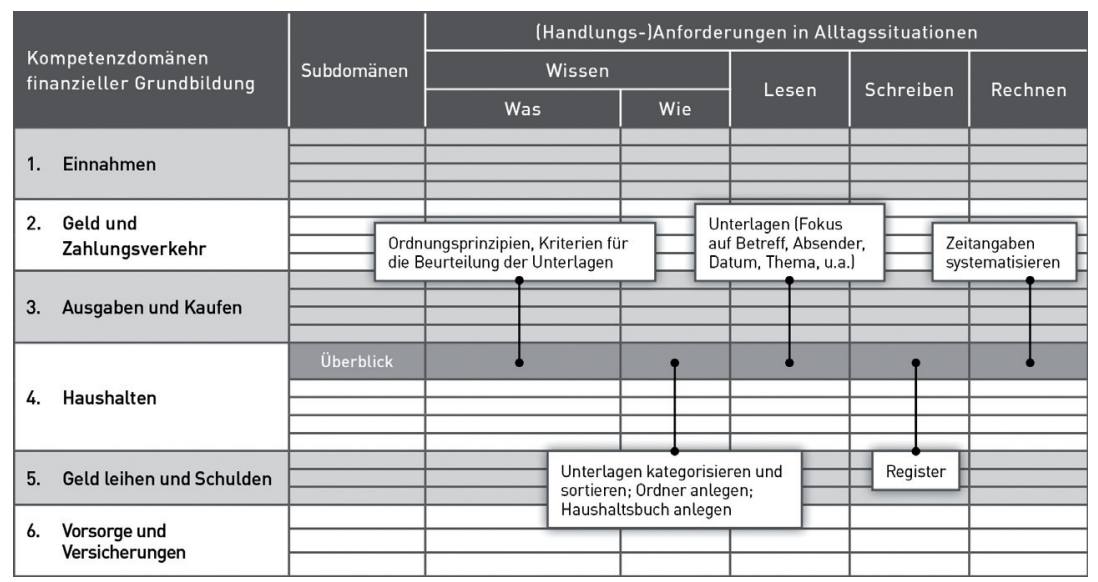

Abb. 1: Das Kompetenzmodell Finanzielle Grundbildung (vgl. Mania 2015: 261)

Als beispielhafte Handlungsanforderungen auf Grundbildungsniveau lassen sich in der Subdomäne , Überblick' folgende Kennt- bzw. Kann-Beschreibungen festhalten (vgl. Mania 2015: 261):

- Deklaratives Wissen: kennt Ordnungsprinzipien; kennt Kriterien für die Beurteilung der Unterlagen;

- Prozedurales Wissen: kann Unterlagen kategorisieren und sortieren; kann Ordner anlegen; kann ein Haushaltsbuch anlegen und führen;

- Lesen: kann aus Unterlagen Informationen entnehmen (z.B. Betreff, Absender, Datum, Thema u.a.);

- Schreiben: kann ein Register schreiben;

- Rechnen: kann Zeitangaben systematisieren.

Das Modell enthält Aufgaben mit unterschiedlichem Schwierigkeitsgrad innerhalb des niedrigsten Kompetenzlevels, des sogenannten Level-One. Kompetentes Handeln in einem bestimmten inhaltlichen Feld Finanzieller Grundbildung ist als komplexes Zusammenspiel der Anforderungen in allen vier Dimensionen definiert.

\section{Fazit und Ausblick}

Das Kompetenzmodell liefert für die Praxis der Alphabetisierung und Grundbildung eine Orientierung zur Formulierung von kompetenzorientierten Lernzielen und -inhalten für Angebote zu Finanzieller Grundbildung. Um die praktischen Umsetzungsmöglichkeiten des Modells zu explorieren, wurden in Zusammenarbeit mit Weiterbildungseinrichtungen, Schuldnerberatungsstel- 
len und einem Betrieb modellhafte Lernangebote konzipiert, durchgeführt und evaluiert. Es hat sich gezeigt, dass das Modell bei der Angebotsentwicklung flexibel eingesetzt werden kann, sodass - jeweils in Abhängigkeit vom Einrichtungsprofil, von der inhaltlichen Ausrichtung, den Adressatinnen und Adressaten sowie den Kooperationspartnerinnen und -partnern - unterschiedliche Angebote entstehen können (vgl. Mania/Tröster 2015).

Im Hinblick auf ,neue‘ Lernkulturen in der (Finanziellen) Grundbildung lassen sich folgende Schlussfolgerungen ableiten: Durch die Wahl partizipativer Forschungsmethoden sowie die aktive Einbeziehung von Expertengruppen aus unterschiedlichen bzw. sich ergänzenden Bereichen kann eine partizipative Programmgestaltung als Element der Lernkulturentwicklung befördert werden. Die Fokussierung auf handlungsbezogene Lernziele und -inhalte stellt - im Unterschied zu einem reduzierten Verständnis von Lesen und Schreiben als Kulturtechnik - die Grundlage für Lernansätze wie das ,problemzentrierte " und ,situative" Lernen dar, die den für die Grundbildung geforderten Prinzipien der Teilnehmer- und Lebensweltorientierung gerecht werden (vgl. Brödel 2012).

Die flexible Handhabung des Modells bietet eine Unterstützung des pädagogischen Handelns bei der Auswahl von Lerngegenständen und Entwicklung neuer Lernformate, ohne jedoch starre Vorgaben oder Richtlinien zu liefern. Damit soll die für die Erwachsenenbildung charakteristische Angebotsvielfalt keinesfalls eingeschränkt werden, sondern es geht perspektivisch vielmehr darum, die Finanzielle Grundbildung als Teil des Programmbereichs Alphabetisierung und Grundbildung wissenschaftlich zu fundieren und zu professionalisieren (vgl. Mania 2015). Zudem sind vielfältige Anschlussmöglichkeiten an bisherige Veranstaltungsformate (Alphabetisierungskurse oder Rechenkurse, Lerncafés, projektförmige Angebote etc.) und Bezüge zu anderen (Grund-)Bildungsbereichen denkbar - bezogen auf Food Literacy beispielsweise die Themen Einkauf, insbesondere Produktauswahl und Preisvergleiche von Lebensmitteln.

Als ein Orientierungsrahmen für die Entwicklung einer ,neuen“ Lernkultur in der (Finanziellen) Grundbildung wird im Rahmen eines Anschlussprojekts $^{2}$ ein flexibles, modulares und trägerübergreifendes Curriculum für den Bereich Finanzielle Grundbildung entwickelt und hinsichtlich Akzeptanz und Auswirkung auf den Lernprozess wissenschaftlich erprobt. Um die Grundbildungslehrenden bei der Adaptation des Kompetenzmodells sowie der Planung und Durchführung konkreter Lernangebote zu unterstützen, wird zudem eine Fortbildung als Beitrag zur Professionalitätsentwicklung konzipiert und evaluiert. 
Die Ergebnisse der Evaluation des Curriculums und der Fortbildung werden zeigen, wie sich die Lernkultur in der Grundbildung durch diese Entwicklungen verändern wird.

\section{Literatur}

Abraham, Ellen/Linde, Andrea (2010): Alphabetisierung/Grundbildung als Aufgabengebiet der Erwachsenenbildung. In: Tippelt, R./Hippel, A. v. (Hrsg.): Handbuch Erwachsenenbildung/Weiterbildung. Wiesbaden: VS Verlag für Sozialwissenschaften, S. 889-903.

Ambos, Ingrid/Greubel, Stefanie (2012): Ökonomische Grundbildung. Themenfeld „Akteurs- und Angebotsanalyse“. Abschlussbericht. http://www.die-bonn.de/ doks/2012-oekonomische-grundbildung-akteurs-und-angebotsanalyse-01.pdf [Zugriff: 26.01.2016].

Ambos, Ingrid/Horn, Heike (2015): Angebotsstrukturen in der Alphabetisierung und Grundbildung für Erwachsene 2014. Ergebnisse der alphamonitor-Anbieterbefragung des DIE. http://www.die-bonn.de/id/31623 [Zugriff: 26.01.2016].

Aprea, Carmela (2012): Messung der Befähigung zum Umgang mit Geld und Finanzthemen. Ausgewählte Instrumente und alternative diagnostische Zugänge. In: Berufs- und Wirtschaftspädagogik - online 22, S. 1-21. http://www.bwpat.de/ ausgabe22/aprea_bwpat22.pdf [Zugriff: 26.01.2016].

Arnold, Rolf (2010): Didaktik - Methodik. In: Arnold, R./Nolda, S./Nuissl, E. (Hrsg.): Wörterbuch Erwachsenenbildung. Bad Heilbrunn: Klinkhardt, S. 64-66.

Barton, David/Hamilton, Mary (2003): Situated literacies. Reading and writing in context. London: Routledge.

Baumert, Jürgen/Kunter, Mareike (2006): Stichwort: Professionelle Kompetenz von Lehrkräften. In: Zeitschrift für Erziehungswissenschaft 9, 4, S. 469-520.

Bigge, Caroline (2015): Finanzielle Grundbildung in Deutschland - Eine Bestandsaufnahme und Systematisierung bisheriger Angebotskonzepte. Programmanalyse im Projekt CurVe. Forschungsbericht (unveröff.).

BMBF - Bundesministerium für Bildung und Forschung (2012): Vereinbarung über eine gemeinsame nationale Strategie für Alphabetisierung und Grundbildung Erwachsener in Deutschland 2012-2016. https://www.bmbf.de/files/NEU strategiepapier_nationale_alphabetisierung.pdf [Zugriff: 26.01.2016].

Brödel, Rainer (2012): Didaktik der Grundbildung. Plädoyer für einen lebensweltorientierten Ansatz. In: Erwachsenenbildung 58, 2, S. 63-66.

Deutscher Volkshochschul-Verband e.V. (DVV) (o.J.): Curriculum Leben \& Geld. Bereich Alphabetisierung. http://grundbildung.de/fileadmin/content/01Projekte/ ich-will-lernen.de/Curricula_Handbuecher/Curriculum_Leben_Geld.pdf [Zugriff: 27.01.2016].

Dietrich, Stephan/Herr, Monika (2003): Zum Begriff der „Neuen Lehr- und Lernkultur“. http://www.die-bonn.de/esprid/dokumente/doc-2003/dietrich03_01.pdf [Zugriff: 26.01.2016].

Egetenmeyer, Regina (2008): Informal Learning in betrieblichen Lernkulturen. Eine interkulturelle Vergleichsstudie. Baltmannsweiler: Schneider Hohengehren. 
Egloff, Birte (2014): Grundbildung - Zur Einführung in den Themenschwerpunkt. In: Hessische Blätter für Volksbildung 2, S. 103-106.

Fleige, Marion (2010): Erwachsenenbildung im demographischen und sozialen Wandel. Befunde und Schlussfolgerungen aus Lernkulturanalysen in der Evangelischen Erwachsenenbildung im Land Brandenburg. In: Hof, C./Ludwig, J./Schäffer, B. (Hrsg.): Erwachsenenbildung im demographischen und sozialen Wandel vom 24. bis 26. September 2009 an der Hochschule für Philosophie München, der Ludwig-Maximilians-Universität München und der Universität der Bundeswehr München. Baltmannsweiler: Schneider Hohengehren, S. 119-133

Fleige, Marion (2011): Lernkulturen in der öffentlichen Erwachsenenbildung. Theorieentwickelnde und empirische Betrachtungen am Beispiel evangelischer Träger. Münster: Waxmann.

Fleige, Marion (2013): Bildungskulturen - Kultur als Thema von Bildung - transkulturelle Bildung: Forschungsperspektiven zum Verhältnis von Erwachsenenbildung und Kultur. In: Käpplinger, B./Robak, S./Schmidt-Lauff, S. (Hrsg.): Engagement für die Erwachsenenbildung. Ethische Bezugnahmen und demokratische Verantwortung. Wiesbaden: Springer VS, S. 85-98.

Frank, Stephen (2013): Kompetente Bildung oder eingebildete Kompetenz? Kompetenzen als inhaltsdidaktische Leitgröße. In: Magazin erwachsenenbildung.at 20, S. 04-1-04-7. http://www.erwachsenenbildung.at/magazin/13-20/meb13-20.pdf [Zugriff: 26.01.2016].

Frank, Stephen/Iller, Carola (2013): Kompetenzorientierung, mehr als ein didaktisches Prinzip. In: Report - Zeitschrift für Weiterbildungsforschung 36, 4, S. 32 44. http://www.die-bonn.de/doks/report/2013-erwachsenenbildung-03.pdf [Zugriff: 26.01.2016].

Gieseke, Wiltrud (2001): Einführungsvortrag: Professionalität und Lernkulturen. In: Heuer, U./Botzat, T./Meisel, K.(Hrsg.): Neue Lehr- und Lernkulturen in der Weiterbildung. Bielefeld: W. Bertelsmann, S. 77-88.

Gieseke, Wiltrud (2008): Bedarfsorientierte Angebotsplanung in der Erwachsenenbildung. Bielefeld: W. Bertelsmann.

Gläser, Jochen/Laudel, Grit (2004): Experteninterviews und qualitative Inhaltsanalyse als Instrumente rekonstruktiver Untersuchungen. Wiesbaden: VS Verlag für Sozialwissenschaften.

Grell, Petra (2004): Teilnehmerforschung zum Thema Lernwiderstand - ein Konzept partizipativer Forschung und seine Ergebnisse. In: Report - Zeitschrift für Weiterbildungsforschung 27,1, S. 60-67. http://www.die-bonn.de/doks/grell0401.pdf [Zugriff: 27.01.2016].

Grotlüschen, Anke/Bonna, Franziska/Euringer, Caroline/Heinemann, Alisha (2014): Konsequenzen der Konstruktion von Literalität hinsichtlich der Vergleichbarkeit der Alpha-Levels mit den Niveaustufen des Europäischen Referenzrahmens Sprachen. In: Pätzold, H./von Felden, H./Schmidt-Lauff, S. (Hrsg.): Programme, Themen und Inhalte in der Erwachsenenbildung Baltmannsweiler: Schneider Hohengehren, S. 51-65.

Höffer-Mehlmer, Markus (2011): Programmplanung und -organisation. In: Tippelt R./Hippel, A. von (Hrsg.): Handbuch Erwachsenenbildung/Weiterbildung. Wiesbaden: VS Verlag für Sozialwissenschaften, S. 989-1002. 
Hummelsheim, Stefan (2010): Ökonomische Grundbildung tut not: Empirische Studien weisen auf erhebliche Defizite in der ökonomischen und finanziellen Grundbildung hin. DIE Fakten. http://www.die-bonn.de/doks/hummelsheim 1001.pdf [Zugriff: 26.01.2016].

Kastner, Monika (2011): Vitale Teilhabe. Bildungsbenachteiligte Erwachsene und das Potenzial von Basisbildung. Wien: Löcker.

Klieme, Eckhard/Leutner, Detlev (2006): Kompetenzmodelle zur Erfassung individueller Lernergebnisse und zur Bilanzierung von Bildungsprozessen. Überarbeitete Fassung des Antrags an die DFG auf Einrichtung eines Schwerpunktprogramms. http://kompetenzmodelle.dipf.de/pdf/rahmenantrag [Zugriff: 26.01.2016].

Lehner, Martin (2013): Inhalte als zentrale Aspekte einer Didaktik der Erwachsenenbildung. In: Magazin erwachsenenbildung.at 20, S. 03-1-03-10. http://www. erwachsenenbildung.at/magazin/13-20/meb13-20.pdf [Zugriff: 26.01.2016].

Mania, Ewelina (2015): Kompetenzorientierung in der Finanziellen Grundbildung als Grundlage für die Programmentwicklung. In: Report - Zeitschrift für Weiterbildungsforschung 38, 2, S. 251-265. http://link.springer.com/content/pdf/10.1007\% 2Fs40955-015-0030-0.pdf [Zugriff: 26.01.2016].

Mania, Ewelina/Tröster, Monika (2013): Finanzielle Grundbildung. Wege einer partizipativen Didaktik im DIE-Projekt CurVe. In: Magazin erwachsenen bildung.at. 20, S. 12-1-12-8. http://erwachsenenbildung.at/magazin/13-20/meb13 -20.pdf\#page=104 [Zugriff: 26.01.2016].

Mania, Ewelina/Tröster, Monika (2014): Finanzielle Grundbildung - Ein Kompetenzmodell entsteht. In: Hessische Blätter für Volksbildung 64, 2, S. 136-145.

Mania, Ewelina/Tröster, Monika (2015): Finanzielle Grundbildung. Programme und Angebote planen. Bielefeld: W. Bertelsmann.

Reich-Claassen, Jutta/Hippel, Aiga von (2011): Angebotsplanung und -gestaltung. In: Tippelt, R./Hippel, A. von (Hrsg.): Handbuch Erwachsenenbildung/Weiterbildung. Wiesbaden: VS Verlag für Sozialwissenschaften, S. 1003-1015.

Remmele, Bernd/Seeber, Günther/Speer, Sandra/Stoller, Friederike (2013): Ökonomische Grundbildung für Erwachsene. Ansprüche - Kompetenzen - Grenzen. Schwalbach/Ts.: Wochenschau.

Schlutz, Erhard (2006): Bildungsdienstleistungen und Angebotsentwicklung. Münster: Waxmann.

Schürkmann, Susanne/Schuhen, Michael (2013): Kompetenzmessung im Bereich financial literacy. Ergebnisse zum Umgang mit Online-Rechnern aus der FILSStudie. In: Zeitschrift für ökonomische Bildung, 1, S. 73-89. http://zfoeb. de/2013_1/schuerkmann_schuhen.pdf [Zugriff: 26.01.2016].

Schüßler, Ingeborg/Thurnes, Christian M. (2005): Lernkulturen in der Weiterbildung. Bielefeld: Bertelsmann Verlag. http://www.die-bonn.de/doks/2005-lernkultur01.pdf [Zugriff: 26.01.2016].

Seeber, Günther/Retzmann, Thomas/Remmele, Bernd/Jongebloed, Hans-Carl (2012): Bildungsstandards der ökonomischen Allgemeinbildung. Kompetenzmodell Aufgaben - Handlungsempfehlungen. Schwalbach/Ts: Wochenschau.

Street, Brian V. (2003): What's ,new“ in New Literacy Studies? In: Current Issues in Comparative Education 2, 5, S. 77-91.

The New London Group (1996): A Pedagogy of Multiliteracies: Designing Social Futures. In: Harvard Educational Review 66, 1, S. 60-92. 
Weber, Birgit/van Eik, Iris/Maier, Petra (Hrsg.) (2013): Ökonomische Grundbildung für Erwachsene. Ansprüche und Grenzen, Zielgruppen, Akteure und Angebote Ergebnisse einer Forschungswerkstatt. Bielefeld: W. Bertelsmann.

Weinert, Franz E. (2001): Vergleichende Leistungsmessung in Schulen. In: Weinert, F. E. (Hrsg.): Leistungsmessung in Schulen. Weinheim et al.: Beltz, S. 17-31.

Zeuner, Christine/Pabst, Antje (2011): „Lesen und Schreiben eröffnen eine neue Welt!“ Literalität als soziale Praxis - Eine ethnographische Studie. Bielefeld: W. Bertelsmann. 

Aiga von Hippel

\section{Programmplanungskulturen in der betrieblichen Weiterbildung. Eine Programmanalyse zu Funktionen betrieblicher Weiterbildungsprogramme}

\section{Einleitung}

Betriebliche Weiterbildung (bWB) kann als „,beigeordnete“ Bildung bezeichnet werden, da der Hauptzweck der Organisation nicht Weiterbildung ist (vgl. Gieseke 2008) (weitere Begriffe mit anderer Akzentuierung: nachgeordnete Bildung (Wittpoth 1997), implizite EB (Kade/Nittel/Seitter 2007), sekundäre Erwachsenenbildunginstitution (Schmitz 1980)). BWB hat aus personalwirtschaftlicher Sicht die Funktion, den Faktor Arbeit - mit dem Ziel der Produktivität des Unternehmens - bereitzustellen und zu sichern, zu problemadäquaten Kosten (vgl. Rodehuth 1999) - d.h. es muss nicht primär um Lernen gehen. Betriebliche Weiterbildung ist damit kontingent, erfüllt unterschiedliche Funktionen und muss innerbetrieblich gerechtfertigt werden. Gerade in der beigeordneten Bildung ist daher die Analyse der jeweiligen Funktionen besonders interessant.

Funktionen, die bWB erfüllen soll bzw. Funktionen, die betrieblichen Weiterbildungsprogrammen durch unterschiedliche Akteure zugeschrieben werden, sind somit auch eine spezifische Auslegung von Weiterbildung. Ein Programm kann definiert werden als ,der zeitgeschichtlich materialisierte Ausdruck gesellschaftlicher Auslegung von Erwachsenenbildung durch einen bestimmten Träger. [...] Es wird ausgelegt und gefiltert durch professionell Handelnde.“ (vgl. Gieseke/Opelt 2003: 46).

Eingebettet in eine Studie zum Programmplanungshandeln in der betrieblichen Weiterbildung differenziert der Beitrag empirisch unterschiedliche Programmarten bWB, stellt die Ergebnisse einer Programmanalyse betrieblicher Weiterbildungsprogramme zu den in ihnen abgebildeten, in gewisser Weise ,ausgeschriebenen“ Funktionen vor und gibt darüber hinaus Ausblicke auf Programmplanungskulturen in der betrieblichen Weiterbildung.

\section{Theoretische Zugänge und Forschungsstand}

Betrachtet man den Forschungsgegenstand der betrieblichen Programmplanung, ergeben sich mehrere Theorie- und Forschungszugänge. Es gibt meh- 
rere Studien, die den Fokus auf die Teilnahme an bWB und die Einflussfaktoren darauf legen. Hier liegen umfangreiche Ergebnisse beispielsweise zum Einfluss von Unternehmensbranche, Größe, soziodemografischen Merkmalen etc. vor (vgl. BMBF 2014; Dobischat/Düsseldorff 2010; Schiersmann 2007). Deutlich weniger Studien beschäftigen sich - mit unterschiedlichen disziplinären Zugängen - mit der Programmplanung bWB. Zu nennen sind hier eher soziologische Studien (vgl. Harney 1998; Schmitz 1980), betriebswirtschaftliche mit Fokus auf Weiterbildungsmanagement (vgl. z.B. Bäumer 1999; Rodehuth 1999) und erwachsenenpädagogische Studien (vgl. Käpplinger 2009; Heuer 2010).

Um die Angebotsstruktur bWB genau untersuchen zu können, reicht die Weiterbildungsstatistik nicht aus, da nicht ersichtlich ist, welche konkreten Angebote erfasst wurden. Hierzu und zur Analyse von Programmen als Ergebnisse von Programmplanungshandeln sind Programmanalysen notwendig, da nur sie Detailanalysen ermöglichen.

Programmanalysen in der bWB gibt es wenige. Dies liegt zum einen daran, dass die Zugänglichkeit zu betrieblichen Weiterbildungsprogrammen eingeschränkt ist und darüber hinaus viele Angebote kurzfristig nach Bedarf geplant werden und nicht alle Angebote beispielsweise in einem Programmheft gebündelt und gedruckt werden, sondern oft nur im Intranet in einer Datenbank vorhanden sind ${ }^{1}$. Schrader (2003a) untersuchte phänomenologisch Anbieter beruflicher und betrieblicher WB in ihren Botschaften an ihre jeweiligen Umwelten. Büchter (2006) analysierte Ankündigungstexte der bWB in der Weimarer Republik. Zwei wirtschaftswissenschaftliche Studien untersuchten inhaltsanalytisch in den 1990er Jahren betriebliche Weiterbildungsprogramme. Ennen/Günther (1996) verzeichnen bei Großunternehmen einen Trend zu weniger Fachkursen und mehr verhaltens- und persönlichkeitsorientierten Angeboten. Pullig (1991) zeigt zwar bereits auf, dass in vielen Unternehmen neben den "Standardprogrammen“ bedarfsorientierte Angebote geplant werden, allerdings wird in dem Beitrag keine systematische Differenzierung der unterschiedlichen Angebotsbereiche vorgenommen. Er weist auf die Spannungsfelder zwischen Individualität - Sozialität und langfristige Personalentwicklung - kurzfristige Bedarfsorientierung in den Weiterbildungskonzepten hin.

Aus betriebswirtschaftlicher Sicht wird die Konzeption von Weiterbildung als Weiterbildungsmanagement verstanden. Als erwachsenenpädagogische Perspektive auf Programmplanungshandeln sind insbesondere die empirisch-theoretischen Analysen von W. Gieseke weiterführend. Sie beschreibt

1 Programmanalysen können daher nur die im Voraus geplanten Weiterbildungsangebote untersuchen, arbeitsplatzintegrierte Lernformen der betrieblichen Weiterbildung, wie z.B. auch Einarbeitungen, sind nicht berücksichtigt. 
Abstimmungsprozesse in der Programmplanung als „Angleichungshandeln“, da in der Erwachsenenbildung nicht „Lehrpläne, sondern Anforderungen beantwortet werden sollen" (Gieseke 2008: 133). Aus internationaler Perspektive eignen sich beispielsweise die Modelle von Caffarella und Ratcliff Daffron (2013) (Interactive Model of Program Planning) und Cervero/Wilson (1994) (negotiating power and interests) zu Analyse und Beschreibung von Programmplanungsprozessen. Abstimmungsprozesse in der betrieblichen Weiterbildung betreffen auch die Zuschreibung von Funktionen an betriebliche Weiterbildung.

Planungsstrategien wiederum sind als ein Teil von Lernkultur zu sehen: „Lernkulturen sind Kulturen des Lehrens und Lernens, Planens, Disponierens und Partizipierens in Institutionen der Erwachsenenbildung im Modus institutionalformenspezifischer Praktiken, Deutungsmuster, Werte/Normen, Interaktionen/Rituale und Beziehungsstrukturen. Lernkulturen werden professionell pädagogisch gestaltet und individuell rekonstruiert, ausgehandelt und gesteuert. Lernkulturen haben einen gesellschaftlich-kulturellen Kontext.“ (Fleige 2011: 15). Planungskulturen umfassen Bedingungen, jeweils relevante Möglichkeitsspielräume der Angebotsplanung und ihre interpretative Ausleuchtung (vgl. Dollhausen 2008). Planungskultur meint einen Ausschnitt „,der Organisationskultur [...], der hilft, den forschenden Blick speziell auf die sozialen Praktiken zu lenken, mit denen die Planung von Angeboten in Weiterbildungsorganisationen als sinnhaftes Geschehen kenntlich und begreifbar gemacht wird.“ (Dollhausen 2008: 34).

Durch den in diesem Beitrag erweiterten Begriff der „Programmplanungskulturen" soll sowohl die interpretative Ausleuchtung bei der Planung (in der vorliegenden Studie mit Fokus zugeschriebene Funktionen) als auch das Ergebnis „Programm(e)“ betont werden und methodisch durch die Verknüpfung von Interviews mit Planenden und Programmanalysen umgesetzt werden. Als Kategorien zur Beschreibung von Programmplanungskulturen können genannt werden: beteiligte planende Akteure (auch: Rolle der WBAbteilung, berufliche Hintergründe der planenden Akteure - erwachsenenpädagogische Zugänge?), zugewiesene Funktionen, Übereinstimmung, Programmarten, Verwendungszusammenhang, Zielperspektive Programme, Begründungslogiken usw.

Insgesamt lässt sich sagen, dass es zu wenig grundlegende theoretische Arbeiten zur Programmplanung betrieblicher Weiterbildung gibt (vgl. Käpplinger 2016), ebenso wenige empirische Detailanalysen der betrieblichen Programmplanung (vgl. Schrader 2003b) beispielsweise mit Analysen zur Programmplanung verknüpfte Programmanalysen².

2 So findet sich beispielsweise auch in der Bibliographie zu Programmanalysen (https://www.die- 
$\mathrm{Zu}$ den Funktionen bWB gibt es mehrere theoretische und empirische Beiträge (vgl. Harney 1998; Schmitz 1980; Diedrich 1988; Weber 1985; Faulstich 1998; Rodehuth 1999; Heuer 2010; Dewe/Feistel 2013; Seyda/ Werner 2014), die - oft systemtheoretisch fundiert - von objektiven Funktionen ausgehen. Eine Differenzierung nach Programmarten und unterschiedlichen Akteuren der Planung, die der bWB interpretativ Funktionen zuschreiben, sowie eine Verknüpfung mit Programmanalysen fehlten. Funktionen können jedoch auch als akteursabhängige Zuschreibungen in der Programmplanung betrieblicher Weiterbildung definiert werden (vgl. von Hippel/Röbel 2016) ${ }^{3}$. Deduktiv aus der Literatur (in der allerdings nur von objektiven Funktionen gesprochen wird und weder Programmarten noch Akteure differenziert werden) und induktiv aus den Interviews haben wir folgende zugeschriebene Funktionen an bWB differenziert (vgl. von Hippel/Röbel 2016).

\section{Funktionen mit hohem Bezug zu den Lernergebnissen}

- Qualifizierungs- und Versorgungsfunktion (Versorgt das Unternehmen mit den notwendigen Qualifikationen/qualifizierten Mitarbeitern, stellt die Arbeitsfähigkeit der Mitarbeiter sicher)

- Bewältigungs- und Flexibilitätsfunktion (Ermöglicht die Bewältigung konkreter technisch-organisatorischer Veränderungen, sichert die Innovationsfähigkeit des Unternehmens)

- Integrations- und Steuerungsfunktion (Fördert Auseinandersetzung und Identifizierung mit der sowie Integration in die Unternehmenskultur)

- Gesunderhaltungsfunktion (Zielt auf den Schutz der gesundheitlichen Arbeitsfähigkeit der Mitarbeiter $)^{4}$

- Entwicklungsfunktion (Unterstützt die persönliche Entwicklung der Mitarbeiter, fördert das Lernen von Lernen, ermöglicht die Auseinandersetzung mit der eigenen Biografie, primärer Fokus liegt nicht auf der Arbeitsfähigkeit)

bonn.de/institut/dienstleistungen/servicestellen/programmforschung/bibliographie/analyse.a spx) keine zur betrieblichen Weiterbildung.

3 Wir verwenden den Funktionsbegriff nicht für eine soziologische Analyse beispielsweise der Funktionen bWB für gesellschaftliche (Teil)Systeme. Dennoch ist die funktionale Analyse nach Merton (1967) insofern anknüpfungsfähig, als dass deutlich wird, dass Phänomene verschiedene Funktionen ausüben können und dass unterschiedliche soziale Gruppen Phänomene als unterschiedlich (dys)funktional einschätzen können.

4 Dies kann sich auf den Schutz der Gesundheit der Person beziehen, die die Weiterbildung besucht (z.B. „Rückengerechtes Heben“), aber auch (und/oder) auf den Schutz der Gesundheit anderer (wie z.B. bei einem „Fahrsicherheitstraining“" oder „Erste-Hilfe-Kurs“). 


\section{Funktionen mit geringem Bezug zu den Lernergebnissen}

- Image- und Akquisefunktion (Erhöht die Attraktivität des Unternehmens für potentielle Mitarbeiter und Kunden)

- Compliancefunktion (Stellt sicher, dass gesetzliche Anforderungen und Unternehmensrichtlinien erfüllt werden; auch Zertifizierungen)

- Gratifikations-, Motivations-, Mitarbeiterbindungsfunktion (Belohnt und motiviert Mitarbeiter, bindet sie an das Unternehmen, wertet Berufsgruppen gegenüber anderen auf)

- Selektions- und Legitimationsfunktion, soziale Reproduktionsfunktion (Legitimiert Beförderungsentscheidungen im Unternehmen bzw. dient als Filter, reproduziert soziale Machtverhältnisse im Unternehmenskontext)

- Ablenkungsfunktion (Lenkt von anderen Themen wie Arbeitsverdichtung ab (Perspektive Betriebsrat, daher nicht gleich Motivationsfunktion))

- Profilierungsfunktion: Selbstinszenierungs- und Einflussfunktion (Wertet die Tätigkeit der einzelnen WB-Mitarbeiter oder der WB-Abteilung innerhalb des Unternehmens auf)

- Networkingfunktion (Ermöglicht kollegialen Austausch/Networking, stärkt Zusammenhalt)

Eine Programmanalyse der betrieblichen Weiterbildung nicht mit Fokus auf den Themen und Inhalten, sondern auf den Funktionen zu machen, lässt das Spezifische bWB deutlicher werden. Denn die kommunikative Rahmung macht den Unterschied. „Es kann durchaus vorkommen, dass auf der Ebene der Themen und Inhalte selbst öffentliche oder freie Träger der Weiterbildung die gleichen Angebote machen, die man auch in Betrieben findet. Differenzen zwischen den verschiedenen Kontexten der Weiterbildung werden nicht auf der Ebene der Themen und Inhalte markiert. Markiert werden sie auf der Ebene der kommunikativen Rahmung, in die die Themen und Inhalte eingefasst sind." (Harney 1998: 140). Der kommunikative Rahmen der BWB ist die ,ständige Frage nach ihrer innerbetrieblichen Existenzberechtigung“ (ebd.: 140) und die Frage nach Weiterbildungskosten und -erträgen - und ergänzend kann man sagen, die Frage nach ihren zugeschriebenen Funktionen.

\section{Methode und Design}

Das von der DFG geförderte Forschungsprojekt StePrA (Steuerung der Programm- und Angebotsplanung in der betrieblichen Weiterbildung. Eine 
Analyse der Handlungskoordination verschiedener Akteursgruppen $)^{5}$ untersuchte die Programm- und Angebotsplanung in der bWB.

Zentral waren die beiden folgenden Fragestellungen:

(1) welcher Einfluss geht von den unterschiedlichen Akteur/inn/en auf die Programm- und Angebotsgestaltung in der bWB aus?

(2) welche spezifischen Formen der Programmplanung erbringen welche Angebote und Programme?

Diese beinhalten als Fragestellungen zu den Programmen:

a) welche Programmarten lassen sich empirisch differenzieren? (vgl. Ergebnisse Punkt 4.1.)

b) welche Funktionen werden in den Angeboten des zentralen Programmkataloges sichtbar? (vgl. 4.2)

c) welche Funktionen weisen die unterschiedlichen Akteure dem zentralen Programmkatalog zu? (vgl. 4.2)

d) welche Rolle spielen zugeschriebene Funktionen bei der Beschreibung einer Programmplanungskultur? (vgl. 4.2 und 5.)

In einem ersten Schritt wurden somit Akteur/inn/e/n, Programmplanungsprozesse und Wirkungen (Programme und Angebote) beschrieben, um diese in einem zweiten Schritt typisierend zu analysieren. Die Zusammenhänge wurden im Rahmen einer Grounded Theory verdichtet (vgl. von Hippel/Röbel 2016).

Es wurden drei Betriebsfallstudien (Gesundheitsbranche, Einzelhandel, Energie; Auswahl erfolgte kategoriengeleitet nach dem theoretical sampling; alle ausgewählten Unternehmen haben mehr als 2800 Mitarbeiter $^{7}$ ) durchgeführt mit jeweils 9-12 Experten- und problemzentrierten Interviews mit unterschiedlichen Planungsakteuren (Weiterbildungsabteilung, Führungskräfte, Mitarbeiter, Betriebsrat etc.). Jede Betriebsfallstudie beinhaltet in Triangulation zudem eine organisationsbezogene, integrierte Programmanalyse (vgl. Robak 2012; Nolda 2009; Käpplinger 2008) mit den Programmen von 2014. $\mathrm{Zu}$ beachten ist, dass die betrachteten Angebote aus dem zentral geplanten und in Heftform oder als PDF-Datei veröffentlichten Weiterbildungsprogramm des Unternehmens entnommen wurden. Zusätzlich unterjährig oder bereichsspezifisch stattfindende Angebote konnten auf Grund der fehlenden Dokumentation nicht berücksichtigt werden.

5 Das Projekt wurde von der DFG unter dem Kennzeichen HI 1599/1-1 gefördert.

6 Damit wurde eine inhaltliche Repräsentation, aber keine statistische Repräsentativität (vgl. Merkens 1997) angestrebt.

7 Nach Bäumer (1999) ist bei dieser Größe davon auszugehen, dass die Organisation die folgenden Kriterien erfüllt: Funktionsbereich WB ist vorhanden, hauptamtliche WB-Stellen sind vorhanden, WB-Budget ist vorhanden, WB ist formal organisiert. 
Die Erstellung des Kodierleitfadens für die Programmanalyse der drei Programmkataloge erfolgte deduktiv und induktiv. Als Grundlage dienten die Wissensinseln nach Gieseke (2008), die Strukturaspekte der Angebotsentwicklung nach Schlutz (2006) und die Kategorien ,Übertragbarkeit auf differentielle Verwendungszusammenhänge“ sowie „Fokus auf die aktuelle Tätigkeit oder auf die langfristige Beruflichkeit" (Robak et al. 2015). Nachdem in den Interviews im Rahmen der Auswertung nach dem Kodierparadigma der Grounded Theory als zentrales Phänomen „Funktionen als akteursabhängige Zuschreibungen in der Programmplanung" herausgearbeitet wurde, wurde diese induktiv entwickelte Kategorie neu codiert.

$\mathrm{Zu}$ jedem Angebot/Ankündigungstext wurden dabei eine bis mehrere Funktionen (siehe Übersicht unter 2.) codiert (sowie die kommunikative Rahmung durch Vorworte analysiert). Nicht alle Funktionen lassen sich forschungsmethodisch dem Programm bzw. einzelnen Angeboten zuordnen - da die Funktionen nicht den Angeboten per se innewohnen, sondern von den Akteuren zugewiesen werden. Insofern ist mit einer Programmanalyse nur das an Funktionen zu finden, was sich in der Aushandlung der Akteure als dominante zugeschriebene Funktion im Programm niedergeschlagen hat, man kann von ,ausgeschriebenen“ Funktionen sprechen. Einige Funktionen sind auch bewusst nicht in einem Programm offengelegt (z.B. Legitimationsfunktion, Einflussfunktion). Methodisch lassen sich darüber hinaus nicht alle Funktionen codieren (z.B. schwieriger zu codieren sind Legitimationsfunktion, Einflussfunktion, Ablenkungsfunktion, Profilierungsfunktion, diese lassen sich valider in den Interviews identifizieren).

Übergreifendes Ergebnis ist, dass sich die Qualifizierungsfunktion so gut wie allen Angeboten zuweisen lässt, den Unterschied machen weitere Funktionen und die Kombination (Kontingenzanalyse). Mittels Kontingenzanalyse ließ sich feststellen, welche Funktionen typischerweise zusammen in einem Angebot auftreten. Sie war damit der erste Schritt zu einer Clusterung der Angebote nach Funktionen.

\section{Ergebnisse}

\subsection{Differenzierung von Programmarten}

Aus den von den Akteuren unterschiedlichen zugeschriebenen Funktionen und Programmplanungsprozessen ergeben sich differente Programmarten ${ }^{8}$, da

8 Da der Begriff „Programmbereich“ konnotiert ist mit den unterschiedlichen Fachbereichen u.a. an VHS, sprechen wir bei der Ergebnisdarstellung von „Programmarten“, um die unterschiedlichen Programmsegmente, -formen deutlich zu machen. Der Begriff des Angebots 
nicht ein Programm alle zugeschriebenen Funktionen der unterschiedlichen Akteure erfüllen kann. In allen drei Unternehmen gibt es eine Vielzahl an WB-Angeboten jenseits des jeweiligen Programmkatalogs. Diese unterschiedlichen Programmarten in der bWB waren bislang kaum systematisch und empirisch erfasst (s.o.). Die Programmarten (siehe folgende Tabelle) unterscheiden sich hinsichtlich der planenden Akteur/inn/e/n, der Zielgruppen, der Themen, der Finanzierung und des Planungshorizonts.

Tab. 1: Vergleich der Programmarten ${ }^{9}$

\begin{tabular}{|c|c|c|c|c|c|}
\hline & $\begin{array}{l}\text { Programm- } \\
\text { katalog }\end{array}$ & $\begin{array}{l}\text { Bereichs- } \\
\text { interne } \\
\text { Angebote }\end{array}$ & $\begin{array}{l}\text { Externe } \\
\text { Angebote }\end{array}$ & $\begin{array}{l}\text { Maßge- } \\
\text { schneiderte } \\
\text { Angebote }\end{array}$ & \begin{tabular}{|l} 
Weitere \\
Einzel- \\
angebote
\end{tabular} \\
\hline $\begin{array}{l}\text { Beschrei- } \\
\text { bung }\end{array}$ & $\begin{array}{l}\text { die von der } \\
\text { WB-Abteilung } \\
\text { im Voraus } \\
\text { geplanten, } \\
\text { offenen Ange- } \\
\text { bote }\end{array}$ & $\begin{array}{l}\text { von verschie- } \\
\text { denen Unter- } \\
\text { nehmens- } \\
\text { bereichen/ } \\
\text { Gruppen/ } \\
\text { Standorten } \\
\text { im Unter- } \\
\text { nehmen } \\
\text { eigenständig } \\
\text { geplante An- } \\
\text { gebote ohne } \\
\text { Beteiligung } \\
\text { der WB- } \\
\text { Abteilung }\end{array}$ & $\begin{array}{l}\text { Angebote } \\
\text { externer } \\
\text { Anbieter, die } \\
\text { für einzelne } \\
\text { Mitarbeiter } \\
\text { gebucht } \\
\text { werden, } \\
\text { entweder mit } \\
\text { oder ohne } \\
\text { Beteiligung } \\
\text { der WB- } \\
\text { Abteilung }\end{array}$ & $\begin{array}{l}\text { Angebote, } \\
\text { bei denen die } \\
\text { WB- } \\
\text { Abteilung als } \\
\text { Dienstleister } \\
\text { fungiert und } \\
\text { kurzfristig auf } \\
\text { Bedarf aus } \\
\text { unterschied- } \\
\text { lichen Unter- } \\
\text { nehmens- } \\
\text { bereichen } \\
\text { reagiert }\end{array}$ & $\begin{array}{l}\text { Weitere WB- } \\
\text { Angebote } \\
\text { bzw. } \\
\text { Aktivitäten } \\
\text { der WB- } \\
\text { Abteilung }\end{array}$ \\
\hline $\begin{array}{l}\text { Planende } \\
\text { Akteure }\end{array}$ & $\begin{array}{l}\text { WB Abteilung. } \\
\text { Zusätzlich: } \\
\text { Vertreter der } \\
\text { Berufsgruppen } \\
\text { Regionale PE } \\
\text { und Leitungs- } \\
\text { personen }\end{array}$ & $\begin{array}{l}\text { Führungs- } \\
\text { kräfte ver- } \\
\text { schiedener } \\
\text { Ebenen, } \\
\text { Regionale } \\
\text { PE }\end{array}$ & $\begin{array}{l}\text { Tlw. Beteili- } \\
\text { gung WB- } \\
\text { Abteilung, } \\
\text { tlw. direkte } \\
\text { Vorgesetzte } \\
\text { der } \\
\text { Mitarbeiter }\end{array}$ & \begin{tabular}{|l|} 
WB- \\
Abteilung, \\
Leitungs- \\
personen \\
(Abteilungen, \\
regional etc.)
\end{tabular} & $\begin{array}{l}\text { WB- } \\
\text { Abteilung, } \\
\text { tlw. weitere }\end{array}$ \\
\hline $\begin{array}{l}\text { Ziel- } \\
\text { gruppen }\end{array}$ & $\begin{array}{l}\text { Alle } \\
\text { Mitarbeiter }\end{array}$ & \begin{tabular}{|l} 
Jeweilige \\
Mitarbeiter \\
der \\
Führungs- \\
kräfte/der \\
Region bzw. \\
Angehörige \\
einer Berufs- \\
gruppe
\end{tabular} & $\begin{array}{l}\text { Einzelne } \\
\text { Mitarbeiter }\end{array}$ & $\begin{array}{l}\text { Alle } \\
\text { Mitarbeiter } \\
\text { der } \\
\text { jeweiligen } \\
\text { Abteilung/ } \\
\text { des } \\
\text { jeweiligen } \\
\text { Standorts } \\
\text { oder }\end{array}$ & $\begin{array}{l}\text { Verschie- } \\
\text { dene (alle, } \\
\text { oder z.B. nur } \\
\text { Führungs- } \\
\text { kräfte) }\end{array}$ \\
\hline
\end{tabular}

oder der Angebotsform wurde wiederum nicht gewählt, da es sich bei der Differenzierung nicht um eine Differenzierung nach Angebotsformen handelt, sondern um eine Strukturierung nach Planungsaspekten.

9 Für die Zusammenstellung der Tabelle danke ich Tina Röbel. 


\begin{tabular}{|c|c|c|c|c|c|}
\hline & $\begin{array}{l}\text { Programm- } \\
\text { katalog }\end{array}$ & $\begin{array}{l}\text { Bereichs- } \\
\text { interne } \\
\text { Angebote }\end{array}$ & $\begin{array}{l}\text { Externe } \\
\text { Angebote }\end{array}$ & $\begin{array}{l}\text { Maßge- } \\
\text { schneiderte } \\
\text { Angebote }\end{array}$ & \begin{tabular}{|l} 
Weitere \\
Einzel- \\
angebote
\end{tabular} \\
\hline & & & & \begin{tabular}{|l} 
spezielle \\
Gruppen, \\
z.B. \\
Führungs- \\
kräfte
\end{tabular} & \\
\hline $\begin{array}{l}\text { Planungs- } \\
\text { horizont }\end{array}$ & Langfristig & $\begin{array}{l}\text { Kurz- und } \\
\text { mittelfristig }\end{array}$ & $\begin{array}{l}\text { Kurz- und } \\
\text { mittelfristig }\end{array}$ & Kurzfristig & $\begin{array}{l}\text { Kurz-, mittel- } \\
\text { und } \\
\text { langfristig }\end{array}$ \\
\hline Themen & $\begin{array}{l}\text { Große } \\
\text { Spannbreite } \\
\text { (alle Kom- } \\
\text { petenzen) }\end{array}$ & v.a. fachlich & Verschieden & $\begin{array}{l}\text { Verschie- } \\
\text { den, auch } \\
\text { Team- } \\
\text { entwicklung }\end{array}$ & $\begin{array}{l}\text { Anlass- } \\
\text { bezogen } \\
\text { oder Schwer- } \\
\text { punktthemen }\end{array}$ \\
\hline $\begin{array}{l}\text { Finan- } \\
\text { zierung }\end{array}$ & \begin{tabular}{|l} 
Zentrales \\
Budget, \\
interne \\
Verrechnung
\end{tabular} & $\begin{array}{l}\text { Budget der } \\
\text { initiierenden } \\
\text { Führungs- } \\
\text { kraft }\end{array}$ & $\begin{array}{l}\text { Budget der } \\
\text { initiierenden } \\
\text { Führungs- } \\
\text { kraft }\end{array}$ & \begin{tabular}{|l} 
Budget der \\
initiierenden \\
Führungs- \\
kraft
\end{tabular} & verschieden \\
\hline
\end{tabular}

Betrachtet man die Programmarten nach dem Aspekt der Offenheit (für alle Zielgruppen) und dem Aspekt der zumindest möglichen Offenheit für erwachsenenpädagogisches Planungshandeln, lassen sich die Programmarten folgendermaßen zu Typen clustern (vgl. Tab. 3). Damit handelt es sich beim Programmkatalog um die Programmart, die prinzipiell den höchsten Grad an Offenheit für Zielgruppen und erwachsenenpädagogische Planung ermöglicht; die bereichsinternen Angebote wiederum weisen den jeweils geringsten Grad auf. Eine Zunahme von kurzfristigen bedarfsorientierten Angeboten, wie sie in der Literatur beschrieben wird, kann damit auch ein geringeres Gewicht von erwachsenenpädagogischem Planungseinfluss (und auch an Teilnahmechancen für bestimmte Zielgruppen) bedeuten.

Tab. 2: Typen von Programmarten

\begin{tabular}{|l|l|l|}
\hline & $\begin{array}{l}\text { Planende } \\
\text { Akteure }\end{array}$ & $\begin{array}{l}\text { WB-Abteilung + } \\
\text { Leitungspersonen }\end{array}$ \\
\hline alle & $\begin{array}{l}\text { Programmkatalog } \\
\text { Einzelangebote }\end{array}$ & Führungskräfte \\
\hline spezifisch & \multicolumn{2}{|c|}{ Externe Angebote } \\
& Maßgeschneiderte Angebote & Bereichsinterne Angebote \\
\hline
\end{tabular}


In den jeweiligen WB-Angeboten spiegeln sich unterschiedliche funktionale Bezüge, wie nachfolgend am Beispiel des zentralen Programmkatalogs aufgezeigt werden soll.

\subsection{Programmanalyse zu Funktionen}

Es handelt sich im Vorliegenden um eine Analyse der Programmplanung bWB, bei der über zugeschriebene Funktionen die Programmplanung und das Ergebnis Programm näher beschrieben werden können.

Im Folgenden werden exemplarisch die Ergebnisse der Programmanalysen zu Fall A (Gesundheitsbranche), Fall B (Einzelhandel) und Fall C (Energiebranche) einzeln und dann vergleichend dargestellt. Es wurde jedes Einzelangebot mit den Funktionen codiert sowie die kommunikative Rahmung durch Vorworte und übergreifende Gestaltungselemente analysiert.

In Fall A gibt es einen umfangreichen Programmkatalog mit einem Umfang von ca. 400 Seiten, der getrennt jeweils von einer Seite gelesen ein Mitarbeiter- und ein Führungskräfteprogramm enthält. Beide Vorworte verweisen auf die Qualifizierungsfunktion, Gesunderhaltungsfunktion, Entwicklungsfunktion, Image- und Akquisefunktion und Gratifikationsfunktion. Die Gratifikationsfunktion wird an folgenden Punkten sichtbar: Es wird eine fiktive Teilnahmegebühr für nicht im Unternehmen Beschäftigte ausgewiesen, um die Wertigkeit deutlich zu machen; nicht nur für Ärzte, sondern auch für das Pflegepersonal und alle weiteren Mitarbeitergruppen werden eigens Fortbildungspunkte ausgewiesen (genannt „Fall A-Fortbildungspunkte“); das Inhaltsverzeichnis ist auch nach Berufsgruppen sortiert, z.T. gibt es doppelt ausgewiesene Angebote, was zu einem großen Umfang des Programmkatalogs führt - was wiederum günstig für die Image- und Akquisefunktion ist (ebenso wie die fiktiven Teilnahmegebühren). Das Vorwort für die Führungskräfte enthält zusätzlich Verweise auf die Flexibilität- und Bewältigungsfunktion (,Notwendigkeit von Veränderungsprozessen“) und die Integrationsfunktion und Legitimationsfunktion (,Uns ist die Teilnahme an den modular aufgebauten Führungstrainings sehr wichtig. Insbesondere für neue Führungskräfte sollten [...] eingeplant werden."). Diese Funktionen spiegeln sich dann auch in den Angeboten (s.u.).

In Fall B (Einzelhandel) werden die Zielgruppen nicht als Mitarbeiter/innen und Führungskräfte, sondern als Kolleg/innen adressiert. Das einleitende Vorwort betont die Entwicklungsfunktion, die weiteren Einleitungstexte zu den unterschiedlichen Angebotsformen betonen die Qualifizierungsfunktion, die Integrationsfunktion (Betonung der Unternehmenskultur) sowie Entwicklungsfunktion. 
Bei Fall C (Energiebranche) handelt es sich beim Trainingskatalog gleichzeitig um das im Intranet verfügbare Angebot. Die Angebote sind sortiert nach Themengebieten, eine Einleitung/Vorwort ist nicht vorhanden.

In der Programmanalyse wurden nach der Codierung der Einzelangebote die Angebote nach typischen Kombinationen von Funktionen (Kontingenzanalyse) geclustert. Die Qualifizierungsfunktion konnte allen Angeboten zugewiesen werden. Dabei lassen sich empirisch folgende Typen von Angeboten differenzieren:

- Fachliche Einzelthemen: Hier steht die Qualifizierungs- und Versorgungsfunktion im Vordergrund, es wurden meist keine weiteren Funktionen codiert.

- Compliance-Angebote: Hier wurden neben der Qualifizierungsfunktion die Compliancefunktion codiert und teilweise einzelne weitere Funktionen (z.B. Gesunderhaltungsfunktion).

- Angebote zur Gesundheitserhaltung: Hier steht der Erhalt der Arbeitsfähigkeit durch Gesundheitsförderung im Mittelpunkt.

- Angebote zur Bewältigung von neuen Herausforderungen: Hier geht es um die Qualifizierung zur Bewältigung von Entwicklungen (durch neue Methoden, neue Gesetze, veränderte Kunden usw.).

- Lehrgänge mit Abschlüssen/Zertifikaten: Hier findet sich typischerweise eine Kombination von Selektions-/Legitimationsfunktion und Gratifikationsfunktion.

- Angebote zur Integration in die Unternehmenskultur: Dies sind Angebote, die vorrangig auf eine Identifikation mit der Unternehmenskultur zielen.

- Angebote zur persönlichen Entwicklung: Bei diesen Angeboten steht neben der Qualifizierung auch die persönliche Entwicklung im Vordergrund.

- Einführung und Entwicklung von Führungskräften: Diese Angebote weisen typischerweise eine Kombination von Qualifizierungs-, Integrations-, Selektions-, Bewältigungs- und Entwicklungsfunktion auf.

Die folgende Abbildung zeigt die Verteilung der Angebotstypen im Vergleich. Interessant zu sehen ist, dass es in Fall B keine Angebote mit Fokus auf die Bewältigungsfunktion gibt und dass diese auch bei den Führungskräfteangeboten fehlt. Dies ist aus der Unternehmenskultur verständlich, die verstärkt darauf abzielt, Veränderungen durch Lernen im Prozess der Arbeit zu bewältigen (die Angebote sollen vorbereitend das „Lernen lernen“ fördern). Eine weitere mögliche Erklärung ist, dass die Neuerungen und Veränderungen in der Gesundheitsbranche umfassender sind als im Einzelhandel. Ebenfalls nicht vorhanden sind Angebote zum Gesundheitserhalt, dies lässt sich darüber erklären, dass die Arbeit körperlich nicht so anstrengend ist wie in der Gesundheitsbranche. Ein anderer Erklärungsansatz ist, dass über eine am Dialog und an Kreativität orientierte Unternehmenskultur mit einem um- 
fassenderen Gesundheitsbegriff dieser Bereich über Angebote zur dialogischen Unternehmenskultur und kreativen Angeboten abgedeckt wird. In Fall B dominieren nach den fachlichen Einzelthemen Angebote, die eine Identifikation mit der Unternehmenskultur beinhalten (das sind die „Angebote zur Integration in die Unternehmenskultur" für alle Mitarbeitenden sowie die Führungskräfteangebote, die diese Funktion auch immer einschließen), sowie auch Angebote, die die Entwicklungsfunktion beinhalten (auch in allen Führungskräfteangeboten und in den Mitarbeiterangeboten dazu spezifisch). Das Programmangebot von Fall A (Gesundheitsbranche) ist demgegenüber dadurch gekennzeichnet, dass neben den fachlichen Einzelthemen Angebote mit Compliancebezug sowie Angebote zur Gesunderhaltung in großem Umfang vorhanden sind. Angebote zur Bewältigung von Veränderungen und neuen Herausforderungen sind sowohl im Führungskräfte- wie im Mitarbeiterprogramm zu finden. Bei Fall C fällt die hohe Anzahl der Weiterbildungsangebote mit Funktionen in den Bereichen Compliance (meist kombiniert mit Gesundheitserhaltung) und Bewältigung von Herausforderungen auf. Je nach Arbeitsinhalt ist die Gesunderhaltung anders ausgestaltet. Bei Fall C liegen die Gefahren der Arbeit eher im Bereich Arbeitssicherheit (dazu gehören beispielsweise auch Fahrsicherheitstrainings und der Umgang mit Gefahrgut), bei Fall A liegt die Gesundheitsgefährdung bei der Arbeit, z.B. in Stress oder Rückenproblemen. In Fall $\mathrm{C}$ gab es kaum Lehrgänge mit Abschlüssen und Zertifikaten, die gleichzeitig auch eine Selektions-/Legitimationsfunktion und Gratifikationsfunktion beinhalteten, z.B. ein MBA. Es gab zwar viele Lehrgänge mit Abschlüssen, die jedoch hauptsächlich im Bereich Compliance angesiedelt waren (z.B. „Motorkettensägenführer").

Vergleicht man die Ergebnisse mit dem Theorie- und Forschungsstand, lässt sich ein Trend zu weniger Fachkursen nicht feststellen. Die Funktionen, die Schmitz (1980) in seiner industriesoziologischen Studie ausmacht, lassen sich teilweise wiederfinden, insbesondere die Funktion, die technisch-instrumentellen Fähigkeiten der Beschäftigten anzupassen, Wissen an Führungskräfte zu vermitteln, um die hierarchische Struktur aufrechtzuerhalten, sowie Disziplinierung und Motivierung der Beschäftigten für Aufstiege. Die vierte Funktion der Gratifikation und Spaltung betrieblicher Arbeitsmärkte durch höhere Investitionen in die Stammbelegschaft ließe sich eher mit einer Analyse des Budgets in Kombination mit einer Analyse der anvisierten Zielgruppen untersuchen. 
Abb. 1: Verteilung der Angebotstypen nach Funktionen im Fallvergleich Kontingenzanalyse

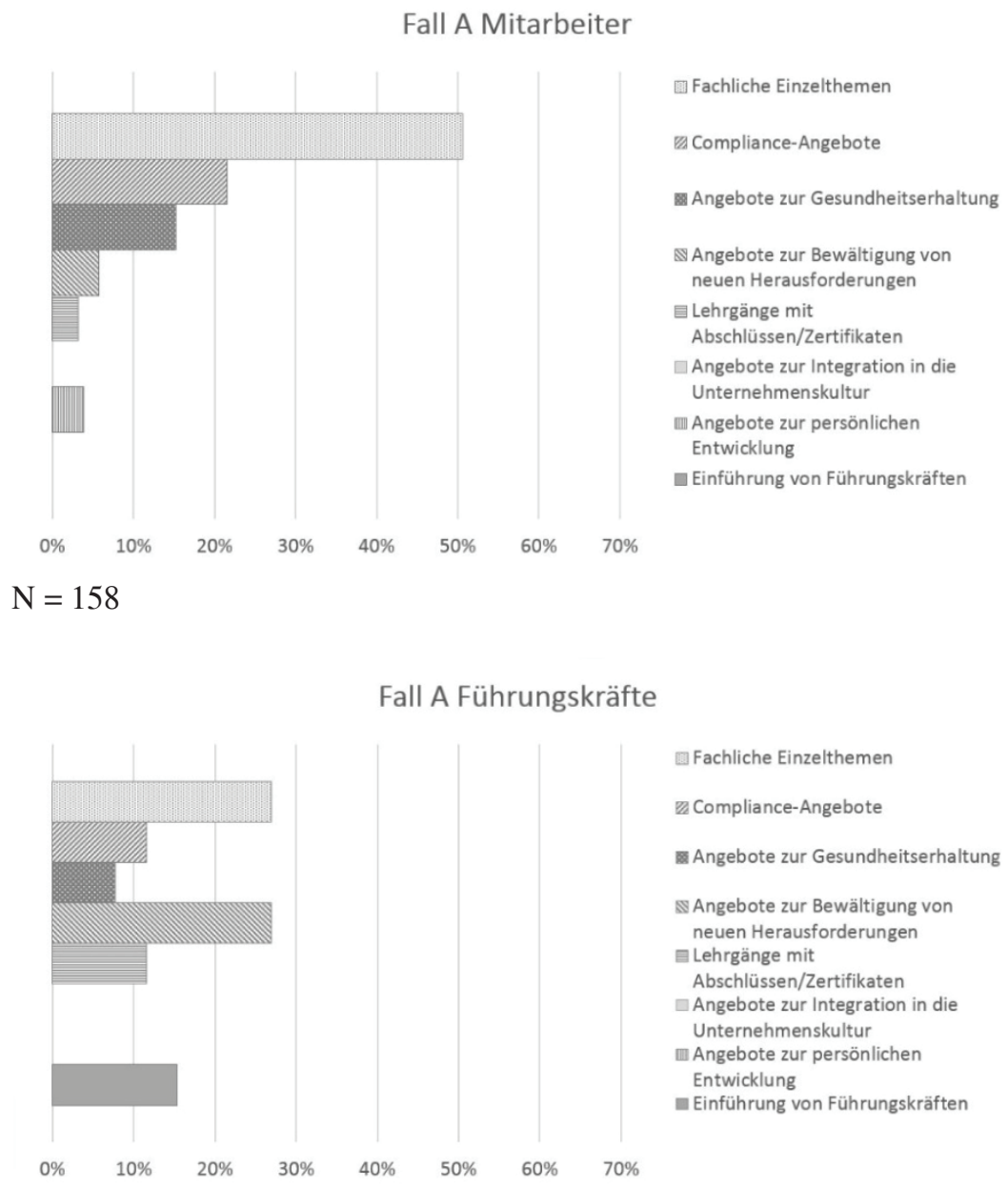

$\mathrm{N}=26$ 


\section{Aiga von Hippel}

Fall B

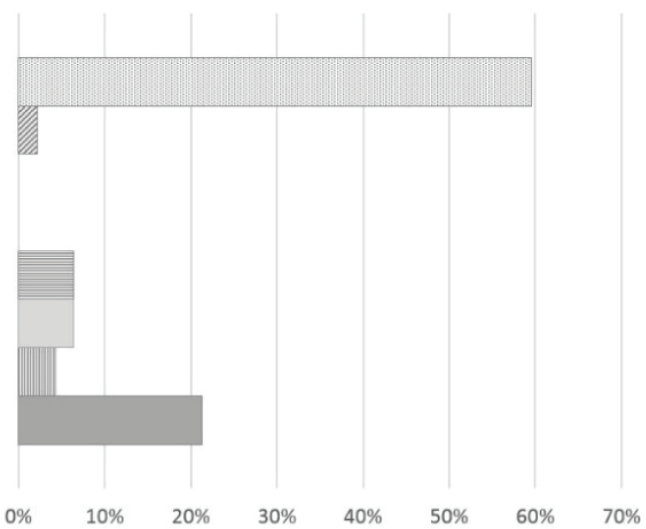

ㅁ. Fachliche Einzelthemen

$\mathbb{Z}$ Compliance-Angebote

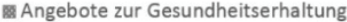

$\mathbb{N}$ Angebote zur Bewältigung von neuen Herausforderungen

目 Lehrgänge mit

Abschlüssen/Zertifikaten

$\square$ Angebote zur Integration in die Unternehmenskultur

$\square$ Angebote zur persönlichen Entwicklung

Einführung von Führungskräften

$\mathrm{N}=47$

Fall C

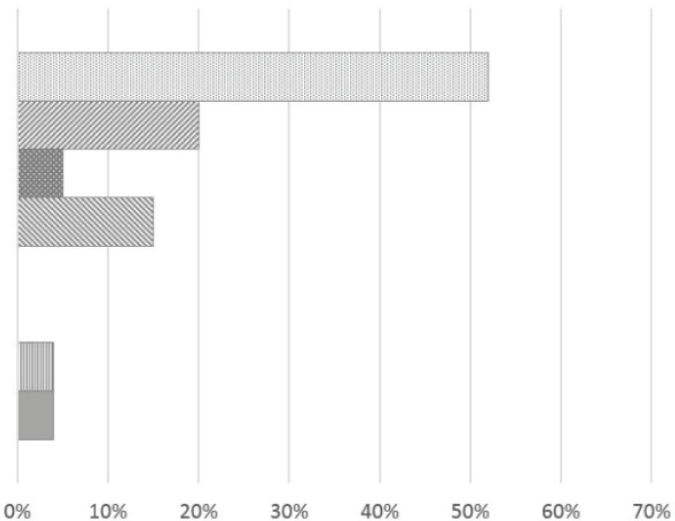

ㅁ. Fachliche Einzelthemen

Compliance-Angebote

Angebote zur Gesunderhaltung

$\mathbb{N}$ Angebote zur Bewältigung von neuen Herausforderungen

目 Lehrgänge mit Abschlüssen/Zertifikaten

$\square$ Angebote zur Integration in die Unternehmenskultur

III Angebote zur persönlichen Entwicklung

E Einführung von Führungskräften

$\mathrm{N}=254^{10}$

10 Für die Unterstützung bei der Programmanalyse zu Fall C danke ich Laura Mecarelli. 
Weiterführend lässt sich die Programmanalyse mit den Interviewergebnissen triangulierend interpretieren. Die folgende Tabelle gibt einen Überblick, welche Funktionen die unterschiedlichen Akteure jeweils dem Programmkatalog - der der obigen Programmanalyse zugrunde lag - zuweisen. Zunächst wird deutlich - und das ist mit dem Begriff der Funktionen als akteursabhängige Zuschreibungen gemeint - dass die unterschiedlichen Akteure dem Programmkatalog unterschiedliche Funktionen zuweisen. Die Leitung der Personalentwicklung in Fall A beispielsweise weist die meisten Funktionen zu. Übereinstimmend weisen alle Akteure die Funktion der Qualifizierung zu. Die in der Programmanalyse ebenfalls umfangreich vorhandenen Angebote zur Erfüllung von Compliance und zur Gesunderhaltung werden nur von einigen Akteuren genannt. Stärker in den Interviews werden die Entwicklungsfunktion und die Image-/Akquisefunktion genannt (die in der Programmanalyse über die übergreifenden Gestaltungselemente sichtbar wird). Viele der hier nicht genannten Funktionen weisen die Akteure den anderen Programmarten (siehe 4.1.) zu (also nicht dem im Voraus zentral geplanten Programmkatalog).

Bei Fall B weisen ebenfalls alle Akteure die Qualifizierungsfunktion zu, zusätzlich liegt der Fokus auf der Entwicklungsfunktion, Integrationsfunktion und Bewältigungsfunktion. Dies entspricht recht gut dem, was die Programmanalyse abbildet; das heißt in diesem Unternehmen - das wurde in den Interviews ebenfalls deutlich - eine Unternehmenskultur mit vielen geteilten Werten in Bezug auf bWB (in Form von zugewiesenen Funktionen). Dabei akzeptieren die Akteure wechselseitig ihre unterschiedliche Begründungslogiken. So argumentiert die Leitung Personalentwicklung erwachsenenpädagogisch und weiß gleichzeitig, dass andere Akteure auf die Wirtschaftlichkeit achten werden. „Dass man einfach gemeinsam auf's große Ganze guckt und jeder aus seiner Professionalität heraus dann sagt: Was ist mein Beitrag und wie können wir's so stricken, dass wir sozusagen in beiden Themen vorankommen." (Regionaler Personalentwickler) (mit beidem ist gemeint: persönliche Entwicklung der Mitarbeiter und betriebswirtschaftliche Sicht).

Bei Fall $\mathrm{C}$ betonen insbesondere die Personalleitungen die Funktionen Gesunderhaltung und Compliance: „Und das is' 'n großes Thema, da wird sehr viel unterrichtet und geschult, weil das 'n hohes Sicherheitsrisiko auch hat." (Personalleitung Tochter 2). Die Funktion der Bewältigung von Herausforderungen wird insbesondere durch die Veränderungen in der Energiewirtschaft begründet. Die Integrationsfunktion wird vorrangig den Führungskräfteangeboten zugewiesen. Insgesamt scheint die Begründungslogik bei Fall C ökonomisch zu sein, mit einem Fokus darauf, kostensparend Arbeitskräfte zu erhalten, dennoch scheinen die Akteure bei der Programmplanung unterschiedliche konflikthafte Interessen zu verfolgen. So sieht sich die WBAbteilung dafür verantwortlich, im Sinne des Gesamtunternehmens durch einen zentralen Einkauf von Trainings Kosten zu sparen. Demgegenüber 


\section{Aiga von Hippel}

nutzen die einzelnen Abteilungen ihre Budgetmacht, um auf dem externen Markt nach kostengünstigeren Alternativen zu suchen.

Tab. 3: Dem Programmkatalog zugewiesene Funktionen nach Akteuren (Basis: Experteninterviews und problemzentrierte Interviews) ${ }^{11}$

Fall $A$

\begin{tabular}{|c|c|c|c|c|c|c|c|c|c|c|c|c|}
\hline $\begin{array}{l}\text { Funktion } \\
\text { Planende Akteure }\end{array}$ & 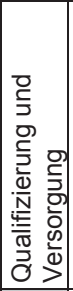 & 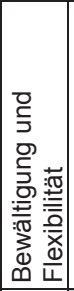 & 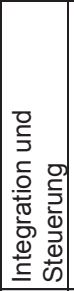 & 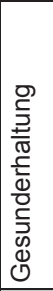 & 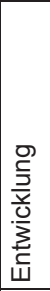 & 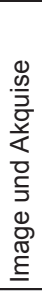 & 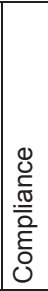 & 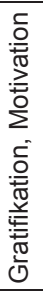 & 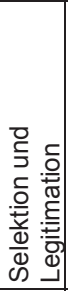 & 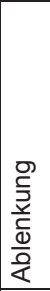 & 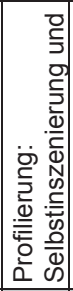 & $\frac{1}{2}$ \\
\hline $\begin{array}{l}\text { Leitung } \\
\text { Personalentwicklung }\end{array}$ & & & & & & & & & & & & \\
\hline $\begin{array}{l}\text { Teamleitung } \\
\text { Konzeption }\end{array}$ & & & & & & & & & & & & \\
\hline $\begin{array}{l}\text { Mitarbeiterin } \\
\text { Personalentwicklung }\end{array}$ & & & & & & & & & & & & \\
\hline $\begin{array}{l}\text { Geschäftsführung } \\
\text { Standort } 1\end{array}$ & & & & & & & & & & & & \\
\hline $\begin{array}{l}\text { Chefarzt Abteilung } \\
\text { XY }\end{array}$ & & & & & & & & & & & & \\
\hline $\begin{array}{l}\text { Pflegedirektion } \\
\text { Standort } 2\end{array}$ & & & & & & & & & & & & \\
\hline Krankenschwester & & & & & & & & & & & & \\
\hline Betriebsrat & & & & & & & & & & & & \\
\hline
\end{tabular}

11 Für die Zusammenstellung der Tabellen danke ich Tina Röbel. 
Fall B

\begin{tabular}{|c|c|c|c|c|c|c|c|c|c|c|c|c|}
\hline $\begin{array}{l}\text { Funktion } \\
\text { Planende Akteure }\end{array}$ & 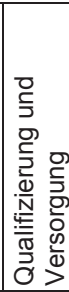 & 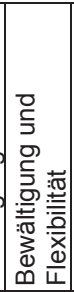 & 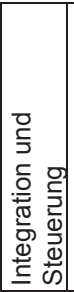 & 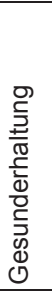 & 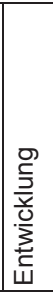 & 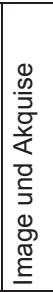 & 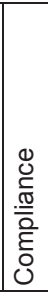 & 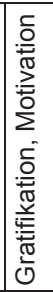 & 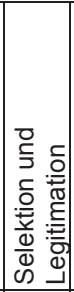 & 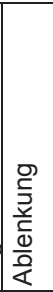 & 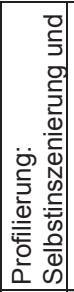 & $\frac{1}{2}$ \\
\hline $\begin{array}{l}\text { Leitung } \\
\text { Personalentwicklung }\end{array}$ & & & & & & & & & & & & \\
\hline $\begin{array}{l}\text { Teamleitung } \\
\text { Organisation }\end{array}$ & & & & & & & & & & & & \\
\hline $\begin{array}{l}\text { Regionale } \\
\text { Personalentwickler (2) }\end{array}$ & & & & & & & & & & & & \\
\hline $\begin{array}{l}\text { Gebietsverantwortliche } \\
\text { (2) }\end{array}$ & & & & & & & & & & & & \\
\hline Filialleitung (2) & & & & & & & & & & & & \\
\hline Mitarbeiter & & & & & & & & & & & & \\
\hline $\begin{array}{l}\text { Betriebsrat }{ }^{*} \text { ) } \\
{ }^{*} \text { keine Angabe }\end{array}$ & & & & & & & & & & & & \\
\hline
\end{tabular}

\section{Fall C}

\begin{tabular}{|c|c|c|c|c|c|c|c|c|c|c|c|c|}
\hline \begin{tabular}{|l} 
Funktion \\
Akteur \\
Akn
\end{tabular} & 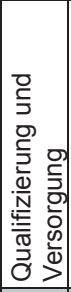 & 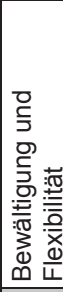 & 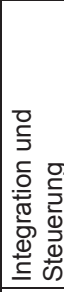 & 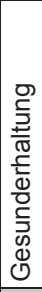 & 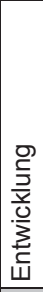 & 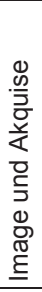 & 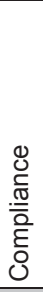 & 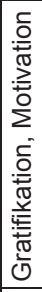 & 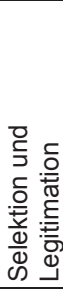 & $\begin{array}{l}\text { D } \\
\frac{1}{2} \\
\frac{2}{0} \\
\frac{0}{0} \\
\frac{0}{2}\end{array}$ & 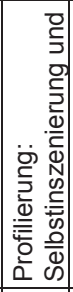 & 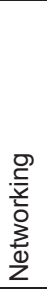 \\
\hline \begin{tabular}{|l|} 
Leitung \\
Personalentwick
\end{tabular} & & & & & & & & & & & & \\
\hline \begin{tabular}{|l|} 
Teamleiter \\
Konzeption
\end{tabular} & & & & & & & & & & & & \\
\hline \begin{tabular}{|l|} 
Teamleiter \\
Organisation \\
\end{tabular} & & & & & & & & & & & & \\
\hline Trainingsmanag & & & & & & & & & & & & \\
\hline $\begin{array}{l}\text { Personalleitung } \\
\text { Tochter } 2\end{array}$ & & & & & & & & & & & & \\
\hline $\begin{array}{l}\text { Personalleitung } \\
\text { Tochter } 3\end{array}$ & & & & & & & & & & & & \\
\hline
\end{tabular}




\begin{tabular}{|c|c|c|c|c|c|c|c|c|c|c|c|c|}
\hline Akteur & 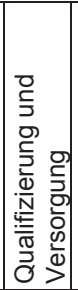 & 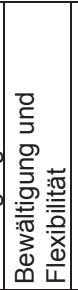 & 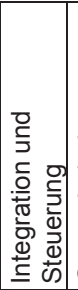 & 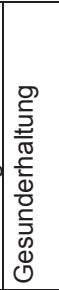 & 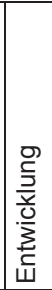 & 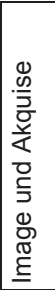 & 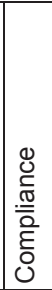 & 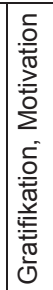 & 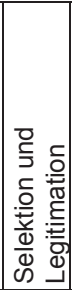 & 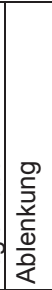 & 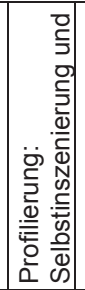 & 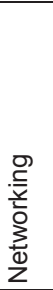 \\
\hline $\begin{array}{l}\text { Personalleitun } \\
\text { Tochter } 1\end{array}$ & & & & & & & & & & & & \\
\hline Betriebsrat & & & & & & & & & & & & \\
\hline $\begin{array}{l}\text { Führungskraft } \\
\text { Tochter } 2\end{array}$ & & & & & & & & & & & & \\
\hline
\end{tabular}

\section{Forschungsertrag und Ausblick zu Programmplanungskulturen in der betrieblichen Weiterbildung}

Mit dem induktiv gewonnenen Begriff der Funktionen als akteursabhängige Zuschreibungen wird ein Beitrag zur weiteren Theoriebildung zur Programmplanung in der betrieblichen Weiterbildung geleistet. Die Analyse von Programmplanungshandeln der Akteure und den Programmen selbst ergab eine empirische Differenzierung der Programmarten, zeigte Angebotstypen nach Funktionen auf und machte die Zuschreibung durch verschiedene Akteure deutlich. Als Anschlussfragestellungen lassen sich folgende Fragen formulieren: Welche Funktionen werden den anderen Programmarten neben dem Programmkatalog zugeschrieben? Welche Funktionen werden in anderen Institutionen beigeordneter Bildung (z.B. Museen) der Weiterbildung zugeschrieben? Schließlich stellt sich die empirisch-theoretische Frage nach der Differenzierung von Programmplanungskulturen in Institutionen der beigeordneten Bildung. Hier scheint es weiterführend, die Kategorie der zugeschriebenen Funktionen in die Analyse aufzunehmen. Mit der Differenzierung von Organisationskulturen $\mathrm{zu}$ arbeiten, erscheint weiterführend. Hierbei handelt es sich um verschiedene Perspektiven zur Charakterisierung von Organisationskulturen, nicht um Typen (vgl. Dollhausen 2008: 40). Empirisches Material wird danach untersucht, ,inwieweit sich dem empirischen Material Anhaltspunkte für kulturelle Integration, Differenzierung oder Fragmentierung entnehmen lassen." (ebd.). Die Integrationsperpektive fragt danach, inwiefern Deutungs- und Interpretationsmuster organisationsweit geteilt werden, eine Planungskultur konsistent ist (organisationsweiter Konsens). Bei Fall B ließe sich beispielsweise sagen, dass die Orientierung an persönlicher Entwicklung und Identifikation mit der Unternehmenskultur 
Konsens ist. Die Differenzierungsperspektive untersucht, ob eine Planungskultur in sich differenziert und inkonsistent ist und ob sich die kulturelle Differenzierung als kulturelles Nebeneinander gestaltet oder als Kollidieren von Kulturen (subkultureller Konsens/Konsens in einer Untergruppe). Bei Fall B ließen sich hier die unterschiedlichen Begründungslogiken für die oben genannten Funktionen anführen (so lässt sich der Fokus auf persönliche Entwicklung erwachsenenpädagogisch begründen, aber auch ökonomisch, da der Mensch/Verkäufer im Einzelhandel mit hohen Beratungskompetenzen eigentlich der unique selling point bei hohem Konkurrenzdruck ist). Die Fragmentierungsperspektive beleuchtet Prozesse eines unabgeschlossenen Deutungs- und Interpretationsgeschehens mit ständiger Neukombination und Variation von Deutungs- und Interpretationsmustern. Hier ließen sich bei Fall B Aushandlungsprozesse über kurz- und langfristige Planung von Angeboten mit unterschiedlichen Interessen der Akteure beleuchten.

\section{Literatur}

Bäumer, Jens (1999): Weiterbildungsmanagement. Eine empirische Analyse deutscher Unternehmen. München: R. Hampp.

Büchter, Karin (2006): Betriebliche Weiterbildung in der Weimarer Republik. http://www.bwpat.de/ausgabe9/buechter_bwpat9.pdf [Zugriff: 28.04.2016].

Bundesministerium für Bildung und Forschung (2014): Weiterbildungsverhalten in Deutschland. AES 2014 Trendbericht. https://www.bmbf.de/pub/Weiterbildungs verhalten_in_Deutschland_2014.pdf [Zugriff: 03.11.2015].

Caffarella, Rosemary S./Ratcliff Daffron, Sandra (2013): Planning Programs for Adult Learners. A Practical Guide. 3. ed. San Fransisco: Jossey-Bass.

Cervero, Ronald M./Wilson, Arthur L. (1994): Planning responsibly for adult education. A guide to negotiating power and interests. San Francisco: Jossey-Bass.

Dewe, Bernd/Feistel, Katharina (2013): Betriebliche Weiterbildung. Materialien in didaktischer und bildungsökonomischer Perspektive. Stuttgart: Franz Steiner.

Diedrich, Andreas (1988): Effizienz betrieblicher Weiterbildung: betriebliche Weiterbildung als einzelwirtschaftliches Entscheidungsproblem. Köln: Wirtschaftsverlag Bachem.

Dobischat, Rolf/Düsseldorff, Karl (2010): Personalentwicklung und Arbeitnehmer. In: Tippelt, Rudolf/Hippel, A. von (Hrsg.): Handbuch Erwachsenenbildung, Weiterbildung. Wiesbaden: VS Verlag für Sozialwissenschaften, S. 917-938.

Dollhausen, Karin (2008): Planungskulturen in der Weiterbildung. Angebotsplanungen zwischen wirtschaftlichen Erfordernissen und pädagogischem Anspruch. Bielefeld: Bertelsmann.

Ennen, Kathrin/Günther, Ullrich (1996): Personalentwicklungsveranstaltungen deutscher Großunternehmen. Eine empirische Analyse der Themen und Trends. In: Zeitschrift für Personalforschung 10, 1, S. 33-47.

Faulstich, Peter (1998): Strategien der betrieblichen Weiterbildung. Kompetenz und Organisation. München: Franz Vahlen. 
Fleige, Marion (2011): Lernkulturen in der öffentlichen Erwachsenenbildung. Theorieentwickelnde und empirische Betrachtungen am Beispiel evangelischer Träger. Münster, New York, München, Berlin: Waxmann.

Gieseke, Wiltrud (2008): Bedarfsorientierte Angebotsplanung in der Erwachsenenbildung. Bielefeld: Bertelsmann.

Gieseke, Wiltrud/Opelt, Karin (2003): Erwachsenenbildung in politischen Umbrüchen. Programmforschung Volkshochschule Dresden 1945-1997. Opladen: Leske + Budrich.

Harney, Klaus (1998): Handlungslogik betrieblicher Weiterbildung. Stuttgart: Hirzel.

Heuer, Ulrike (2010): Betriebliche Weiterbildungsentscheidungen: Aushandlungsprozesse und Bildungscontrolling. Fallstudienbericht. http://www.bibb.de/veroeffent lichungen/de/publication/download/id/6221_[Zugriff: 12.04.2014].

Hippel, Aiga von/Röbel, Tina (2016): Funktionen als akteursabhängige Zuschreibungen in der Programmplanung betrieblicher Weiterbildung. In: Report - Zeitschrift für Weiterbildungsforschung. Springer.

Kade, Jochen/Nittel, Dieter/Seitter, Wolfgang (2007): Einführung in die Erwachsenenbildung/Weiterbildung. 2. Aufl. Stuttgart: Kohlhammer.

Käpplinger, Bernd (2008): Programmanalysen und ihre Bedeutung für pädagogische Forschung. In: Forum Qualitative Sozialforschung 9, 1, Art. 37. http://nbnresolving.de/urn:nbn:de:0114-fqs0801379 [Zugriff: 26.07.2011].

Käpplinger, Bernd (2009): Betriebliche Weiterbildungsentscheidungen: Aushandlungsprozesse und Bildungscontrolling. Abschlussbericht. http://www2.bibb.de/ bibbtools/tools/fodb/data/documents/pdf/eb_22203.pdf_[Zugriff: 06.09.2012].

Käpplinger, Bernd (2016): Theorien und Theoreme der betrieblichen Weiterbildung. In: Tippelt, Rudolf/Hippel, Aiga von (Hrsg.) (2016): Handbuch Erwachsenenbildung/Weiterbildung. 6. überarb. und aktual. Aufl. Springer.

Merkens, Hans (1997): Stichproben bei qualitativen Studien. In: Friebertshäuser, Barbara/Prengel, Annedore (Hrsg.): Handbuch Qualitative Forschungsmethoden in der Erziehungswissenschaft. Weinheim: Juventa, S. 97-106.

Merton, Robert K. (1967): Funktionale Analyse. In: Hartmann, H. (Hrsg.): Moderne amerikanische Soziologie. Stuttgart: Enke (dtv), S. 169-214.

Nolda, Sigrid (2009): Programmanalyse - Methoden und Forschungen. In: Tippelt, Rudolf/Hippel, Aiga von (Hrsg.): Handbuch Erwachsenenbildung, Weiterbildung. Wiesbaden: VS Verlag für Sozialwissenschaften, S. 293-307.

Pullig, Karl-Klaus (1991): Weiterbildungskonzepte deutscher Unternehmen - eine Analyse von Weiterbildungsprogrammen. In: Zeitschrift für Personalforschung 5, 3, S. 219-237.

Robak, Steffi (2012): Programmanalysen: Einführung in die Erstellung von Codesystemen. http://www.die-bonn.de/Institut/Dienstleistungen/Servicestellen/Programm forschung/Methodische_Handreichungen/codiersysteme/ [Zugriff: 10.02.2014].

Robak, Steffi/Rippien, Horst/Heidemann, Lena/Pohlmann, Claudia (2015): Bildungsurlaub - Planung, Programm und Partizipation. Eine Studie zur Perspektivverschränkung. Frankfurt a.M.: Peter Lang.

Rodehuth, Maria (1999): Weiterbildung und Personalstrategien. Eine ökonomisch fundierte Analyse der Bestimmungsfaktoren und Wirkungszusammenhänge. München, Mering: Hampp. 
Schiersmann, Christiane (2007): Berufliche Weiterbildung. Wiesbaden: VS Verlag für Sozialwissenschaften.

Schlutz, Erhard (2006): Bildungsdienstleistungen und Angebotsentwicklung. Münster, New York, München, Berlin: Waxmann.

Schmitz, Enno (1980): Betriebliche Weiterbildung als Personalpolitik. In: Weymann, Ansgar (Hrsg.): Handbuch für die Soziologie der Weiterbildung. Darmstadt, Neuwied: Hermann Luchterhand GmbH \& Co. KG, S. 120-136.

Schrader, Josef (2003a): Berufliche Weiterbildung zwischen Öffentlichkeit und Privatheit. In: Report - Zeitschrift für Weiterbildungsforschung 26, 1, S. 142-152.

Schrader, Josef (2003b): Wissensformen in der Weiterbildung. In: Gieseke, Wiltrud (Hrsg.): Institutionelle Innensichten der Weiterbildung. Bielefeld: wbv, S. 228-253.

Seyda, Susanne/Werner, Dirk (2014): IW-Weiterbildungserhebung 2014. Höheres Engagement und mehr Investitionen in betriebliche Weiterbildung. In: IWTrends - Vierteljahresschrift zur empirischen Wirtschaftsforschung aus dem Institut der deutschen Wirtschaft 41, 4. http://www.iwkoeln.de/studien/iw-trends/ beitrag/susanne-seyda-dirk-werner-iw-continuous-vocational-training-survey2014-201128 [Zugriff: 21.01.2016].

Weber, Wolfgang (1985): Betriebliche Weiterbildung. Stuttgart: Poeschel.

Wittpoth, Jürgen (1997): Recht, Politik und Struktur der Weiterbildung. Baltmannsweiler: Schneider Hohengehren. 



\section{Zur Modellierung transnationaler Lernkulturen anhand der Institutionalisierung von Professionalitäts- und Organisationsstrukturen}

\section{Einleitung}

Globalisierungs- und Transnationalisierungsprozesse betreffen die Erwachsenenbildung und Weiterbildung in mehrfacher Hinsicht und evozieren mehrdimensionale Fragestellungen für die Gestaltung des Systems der Erwachsenenbildung und Weiterbildung, für die Gestaltung der Institutionen und der darin eingebetteten Lernkulturen. Die Ausweitung und Implementation des Lebenslangen Lernens (vgl. Hof 2008) wird sowohl auf europäischer als auch auf globaler Ebene vorangetrieben und bildungspolitisch als ein zentraler Wettbewerbsfaktor platziert. Wie die regelmäßigen Erhebungen von iMOVE (2013) zeigen, gehen immer mehr deutsche Bildungsanbieter über den inländischen Markt hinaus und beteiligen sich an strategischen Allianzen mit ausländischen Bildungsanbietern, anderen deutschen Unternehmen oder auch ausländischen Unternehmen, gründen eigene Niederlassungen oder beteiligen sich an Joint Ventures.

Es lässt sich ein anhaltender Trend feststellen, dass deutsche Bildungsanbieter auch im Ausland gute Wachstumschancen sehen und ihre Programme und Angebote mit einem Schwerpunkt in China und Indien (teilweise auch projektförmig) offerieren (iMOVE 2013). Bewusst gestaltet oder beiläufig können sich sowohl bestehende Lernkulturen in andere Länder und Kontexte hinein öffnen als auch neue bzw. hybride Lernkulturen entstehen. Daher fragt der vorliegende Beitrag aus einer erwachsenpädagogischen Sicht, welche Anforderungen in der Gestaltung von Lernkulturen bei transnationaler Bildungsarbeit relevant werden und bewusst im professionellen Handeln aufzunehmen sind.

Grundlage der Betrachtung bildet das BMBF-geförderte Projekt „EWA“, das sich mit der Analyse und Entwicklung nachhaltiger Transnationalisierungs- und Konstitutionsstrategien der beruflichen Bildung und Weiterbildung beschäftigt und das übergeordnete Ziel verfolgt, ein Weiterbildungsinstitut in Peking, VR China, zu gründen und erfolgreich am Markt zu etablieren. Hierfür werden durch die operativen Partner Konfix (Einrichtung der beigeordneten Bildung) und Weitbild (privater Weiterbildungsanbieter) berufliche (Weiter)Bildungsangebote für internationale und chinesische Unternehmen sowie für öffentliche Einrichtungen offeriert. Das Projekt läuft über einen Zeitraum von drei Jahren (Juni 2013 bis Mai 2016) und ist Teil der 
BMBF-Förderlinie „Berufsbildungsexport durch deutsche Anbieter“. Das Institut für Berufspädagogik und Erwachsenenbildung ist im Rahmen der Begleitforschung für die Analyse der Transnationalisierung, Institutionalisierung und Lehr-Lernebene verantwortlich und arbeitet dabei insbesondere Gelingensbedingungen und Hemmnisse aus.

Am Beispiel der laufenden Begleitforschung zum Aufbau und zur Etablierung einer Weiterbildungseinrichtung in China werden wir im Folgenden darstellen, dass bei solchen internationalen Bestrebungen veränderte bzw. erweiterte Anforderungen im professionellen Handeln $\mathrm{zu}$ integrieren und Wissensstrukturen für die Gestaltung von Lernkulturen zu identifizieren sind. Dazu entwickeln wir im dritten Abschnitt ein Institutionalisierungsverständnis, in dem unter Bezug auf den Neo-Institutionalismus und die Strukturationstheorie davon ausgegangen wird, dass kulturelle Ressourcen und Regeln in transnationalen Lernkulturen über die Akteure eingebunden werden und sich die Weiterbildungsinstitution in dem veränderten organisationalen Feld positionieren muss sowie Angleichungen erfährt. Über die Handlungsfelder des Bildungsmanagements und der Programmplanung werden auf diese Weise Professionalitätsstrukturen und Organisationsstrukturen geformt, die Ausdruck einer modellierten Lernkultur sind. Zunächst bedarf es jedoch einer Annährung, wie Lernkulturen im transnationalen Zusammenhang zu fassen sind.

\section{Lernkulturen im transnationalen Zusammenhang}

Für den deutschsprachigen Raum, aber auch im internationalen Zusammenhang hat die Beschäftigung mit und die Bestimmung von Lernkulturen verschiedene Stadien durchlaufen (Arnold/Schüßler 1998; Schüßler/Thurnes 2005 und in diesem Band), es finden sich etwa themenspezifische und institutionalformspezifische Auslegungen von Lernkulturen (vgl. z.B. Pätzold/ Lang 1999; Friebe 2005; Fleige 2011) und auch diskursanalytische Auseinandersetzungen (vgl. Klingovsky 2009). Über verschiedene Forschungsprojekte, die sich theoretisch und vor allem empirisch mit Lernkulturen beschäftigt haben (u.a. Gieseke 2009; Robak 2009, 2012; Fleige 2011), wurde folgender Lernkulturbegriff entwickelt, der auch für unser Forschungsprojekt herangezogen wird:

„Lernkulturen bauen Brücken zwischen den Individuen mit ihren Bildungsbedarfen, -bedürfnissen, -interessen und Nutzenerwartungen zu den Möglichkeiten an Lernhandlungen in den Organisationen der Erwachsenen- und Weiterbildung. Lernkulturen eröffnen Lernzugänge für Erwachsene durch gestaltete Angebote, Programme, Projekte, Aneignungssituationen. Sie sind damit Bestandteil der Strukturen selbst als auch des Prozesses der Verdichtung dieser Strukturen. Teil des Prozesses sind ei- 
nerseits die Gestaltungspraktiken der professionell Handelnden auf den Ebenen Bildungsmanagement, Programmgestaltung sowie Lehr-Lern-Arrangements und andererseits die Lern-, Bildungs- und Aneignungsprozesse der Individuen. Diese Prozesse und Strukturen rahmen und beeinflussen die Organisationen der Erwachsenen- und Weiterbildung, die wiederum in durch die von Bildungspolitik, regionalem Umfeld und Trägern vorgegebenen Konzepte, Bedingungen und Interessen eingebunden sind. Lernkulturen entstehen somit an der Schnittstelle von Mikro-, Meso- und Makroebene" (siehe Fleige/Robak i.E.).

Letztlich sind daher auch bei der transnationalen Bildungsarbeit alle Ebenen - Makro-, Meso- und Mikroebene - für Gestaltungsprozesse zu bedenken und zu integrieren. Unsere Ausführungen werden sich auf die Makro- und Mesoebene der Gestaltung von Lernkulturen in einem andauernden Institutionalisierungsprozess fokussieren, da ein gesonderter Zugang zur Mikroebene im Beitrag von Gillen und Herzog erarbeitet wird (siehe Beitrag in diesem Band).

Transnationalisierungsprozesse werfen verschiedene Beobachtungsrichtungen auf und müssen für den öffentlichen Erwachsenenbildungsbereich und für den kommerziellen sowie für den betrieblichen Weiterbildungsbereich je spezifisch betrachtet werden (Robak/Enoch i.V.). Wenn - wie im Fall unseres Projektes EWA - eine kommerzielle Weiterbildungsinstitution eine Kooperation mit einer beigeordneten Bildungsorganisation eingeht, und diese in Form einer neu zu gründenden $\mathrm{GmbH}$ ihre Institutionalisierungsbemühungen in anderen Ländern vollziehen, werden zwei Ebenen der Lernkulturen relevant. Zum einen ist zu betrachten, welche Lernkulturen sich aus den Ursprungsorganisationen in die neu zu gründende Weiterbildungsinstitution übertragen. Und zum anderen, welchem Ansatz der länderübergreifenden Aktivitäten sie folgen: ein ethnozentrischer Ansatz würde bedeuten, dass auf allen drei Ebenen die vorhandenen Lernkulturen standardisiert übertragen werden. Für Globalisierungsprozesse von Unternehmen ist bereits untersucht und beschrieben worden, dass der ethnozentrische Ansatz nur begrenzt erfolgreich ist (Hilb 2002; Zinger 2002). Möglich wäre, dass ein regionaler Ansatz für Bildungseinrichtungen eine funktionale Strategie darstellt, der bedeuten würde, dass alle Prozesse regional von vor Ort eingestelltem Personal eigenständig verantwortet werden (ebd.).

Ein transnationaler Ansatz wird in doppelter Hinsicht relevant, einerseits aus organisationaler Sicht eines transnationalen Unternehmens und andererseits aufgrund der zunehmenden grenzüberschreitenden Vernetzung von Lebenswelten. Da in unserem Fall ein Partner eine kommerzielle Einrichtung ist, die gleichzeitig mit einem transnationalen Konzern verbunden ist, kann auf Forschungen über transnationale Unternehmen zurückgegriffen werden. Diese Unternehmen begannen zuerst mit dem Verkauf und Vertrieb von 
Produkten in anderen Ländern und haben aufgrund steigender Nachfrage Standorte in den Zielländern errichtet sowie eine eigene Forschungs- und Entwicklungsabteilung dort angesiedelt. Damit einher geht eine strukturinterne Globalisierung in den Unternehmen, bei der Prozesse so ausgedehnt werden, dass sich auch die internen Strukturen verändern (siehe Köhler 2004). Ein transnationales Unternehmen weist demnach eine höchstmögliche interne Vernetzung auf: die Arbeitsstrukturen der Mitarbeiter vernetzen und verdichten sich derartig, dass die wechselseitigen Abhängigkeiten eine Organisationsform ausbilden.

Daneben geben sozialwissenschaftliche und kulturwissenschaftliche Diagnosen weiterführende Hinweise, dass sich eine umfassende grenzübergreifende Vernetzung der Lebenswelten entwickelt (Mau 2007; Pries 2008), die bildungswissenschaftlich zu deuten und aufzunehmen ist. Mit Hilfe des Begriffes transnational bzw. Transnationalisierung soll hinterfragt werden, wie dies aufeinander bezogen und vernetzt geschehen kann. Es betrifft bildungspolitische Setzungen und das jeweilige Handeln in den Weiterbildungsinstitutionen. Die Forderung kann dabei nicht sein, dass die Bildungsanbieter überall gleich agieren mit denselben Organisations-, Programm- und Angebotsstrukturen. Die Aufmerksamkeit muss vielmehr darauf gerichtet sein, wie diese Strukturen Bezüge zu den anderen Ländern und Kulturen herstellen und welche Balancen zwischen globaler und lokaler Ausrichtung gestaltet werden können. Dafür braucht es Wissen darüber, was man wechselseitig unter Lernen und Bildung versteht, wie Bildungsinteressen jeweils aussehen, wie man Bedarfe, Bedürfnisse und Interessen eruiert sowie welche Spezifika der Länder als Stärken und Ressourcen verstanden und für eigene Gestaltungen berücksichtigt werden können. Das bedeutet für die Gestaltung der Lernkulturen im transnationalen Zusammenhang, dass notwendige professionelle Handlungsstrukturen, Gestaltungsanforderungen sowie die im Prozess vorgenommenen Handlungen und getroffenen Entscheidungen kulturspezifische Justierungen und Modellierungen benötigen, also Standardisierungen des Bekannten und Gegebenen nur im begrenzten Maße transferierbar sind.

Auf europäischer Ebene hat sich bereits in Teilen ein solch diversifiziertes Verständnis für den öffentlichen Erwachsenenbildungsbereich entwickelt: Es werden zunehmend länderübergreifende bildungspolitische Entscheidungen getroffen und auch entsprechende Projektlinien verabschiedet und finanziert (Lattke/Nuissl/Pätzold 2010). Es ist von Angleichungen in Inhalten, Strukturen und Prozessen auszugehen. Jedoch liegt bisher für den asiatischen Raum in keinem Bereich der Weiterbildung fundiertes Wissen über transnationale Vernetzungen vor.

Gehen wir von einem Transnationalisierungskonzept aus, das Vernetzungen des Handelns als Basis eines Institutionalisierungsprozesses in einem anderen Land denkt (Robak/Lorenz/Herzog/Asche 2014), dann schließt sich daran die Frage, auf welchen Ebenen kulturelle Ressourcen wie einzubinden 
sind. Kulturwissenschaftlich folgen wir dabei dem Ansatz der Hybridisierung, der davon ausgeht, dass durch die Einbindung neuer kultureller Ressourcen in bestehende Praktiken veränderte, modifizierte Praktiken entstehen, die auch kreative Neuformungen beinhalten können (vgl. Reckwitz 2006).

\section{Institutionalisierung von Organisations- und Professionalitätsstrukturen}

In den nachfolgenden Ausführungen wird ein Institutionalisierungsbegriff entwickelt, der im Rahmen der Begleitforschung zu einem BMBF-geförderten Projekt entstanden ist. Daran anschließend werden das Bildungsmanagement und die Programmplanung als Handlungsfelder beschrieben, in denen die Akteure die Professionalitätsstrukturen ausbilden und dadurch gestaltend auf die Lernkultur wirken.

\subsection{Institution und Institutionalisierung}

Eine Herausforderung in der Erforschung transnationaler Prozesse im Bildungszusammenhang besteht darin, die Begrifflichkeiten aus vorhandenen nationalen (inter)disziplinären Bezügen heraus zu entwickeln, da internationale Forschungen in diesem Zusammenhang fehlen. Dazu gehört im vorliegenden Projekt die Entwicklung eines Institutionalisierungsbegriffes sowie eine Konzeptualisierung dessen, welche Ebenen im Prozess der Etablierung einer Weiterbildungseinrichtung $\mathrm{zu}$ betrachten sind. Entsprechend unseres Lernkulturbegriffes wurde ein Institutionalisierungsbegriff entwickelt, der insbesondere die Makro- und Mesoebene berücksichtigt. Die professionellen Handlungsstrukturen auf diesen beiden Ebenen betreffen den managementbezogenen Aufbau und die strategische Verankerung sowie die Entwicklung von Angeboten und Programm- sowie Projektstrukturen.

Unsere im Projekt entwickelten leitenden Begrifflichkeiten für Institution und Institutionalisierung orientieren sich an Forschungszielen und den vorhandenen theoretischen und empirischen Rückbezügen. Diese Konzeption erfolgt abduktiv (vgl. Reichertz 2003) in einem rekursiven Abgleich zwischen vorhandenen Wissensstrukturen und Reflexionen im laufenden Forschungsprozess. Der Institutionenbegriff wurde unter Berücksichtigung und Auswertung vorhandener Begriffe (z.B. Faulstich 2010; Hartz/Schardt 2010; Kil/Schlutz 2008; Schäffter 2001; Schrader 2012; Tippelt 2009) folgendermaßen gefasst:

Unter einer (Weiterbildungs)Institution verstehen wir alle gewachsenen und gestalteten organisationalen Strukturen, im Rahmen derer ihre Mitglieder auf der Grundlage einer Professionalitätsstruktur intentionale 
Handlungen zur Erfüllung der (Haupt-)Aufgabe, der kontinuierlichen Offerierung von Bildungsangeboten, Projekten, Bildungs- und Beratungsleistungen vornehmen, um einen Bildungs- und Qualifizierungsauftrag umzusetzen.

Institutionen bieten organisationale Rahmungen und Formen für wissensbasierte Handlungskoordinationen, mithilfe derer Prozesse des Bildungsmanagements, der Bildungsplanung, Angebots- und Projektentwicklung sowie Beratung als Professionalitätsstrukturen aufgebaut und auf Dauer gestellt werden. Institutionen werden strukturiert durch Praktiken. Sie formen nach innen relationale zeitlich stabile Rahmungen, Regeln, Abläufe, Organisationskulturen inklusive Werte und Kommunikationsstrukturen für die Absicherung professionellen Handelns auf den Ebenen Bildungsmanagement, Programmentwicklung sowie Lehr-Lernarrangements.

Institutionen sind keine autonomen Gebilde, sie sind nach außen hin mit der Umwelt verbunden, antizipieren gesellschaftliche Veränderungen bis hin zur Ausrichtung an Regelungen und Gesetzen. Sie greifen besonders in transnationalen Kontexten unterschiedliche kulturelle Ressourcen auf (Organisationskulturen, länderspezifische kulturelle Ressourcen sowie deren gesellschaftliche Kodierungen, differente Lernkulturen) und transponieren diese in die Realisierung des Bildungs- und Qualifizierungsauftrags und die Ausgestaltung der professionellen Handlungsstrukturen und organisationalen Rahmungen.

Als theoretisches Gerüst der Begleitforschung zur Institutionalisierung werden der Neo-Institutionalismus und die Strukturationstheorie herangezogen, um zum einen die Verortung der Weiterbildungseinrichtung im System und potenzielle Angleichungsprozesse fassen zu können und zum anderen einen dezidierten Blick auf die Ebene der Handlungen und Praktiken der professionellen Akteure zu erhalten (Robak et al. 2014).

Laut soziologischem Neo-Institutionalismus sind Organisationen in organisationale Felder eingebettet, welche ,sich aus all den Organisationen zusammen[setzen], die die relevante gesellschaftliche Umwelt und damit den Bezugsrahmen der zu untersuchenden Organisation bilden" (Hasse/Krücken 2005). Organisationen entwickeln formal-rationale Strukturen, um primär gesellschaftliche Legitimität zu erreichen, wobei Rationalitätserwartungen aufgenommen werden und sich in einer Strukturangleichung von Organisation und gesellschaftlicher Umwelt ausdrücken (Hasse/Krücken 2005; Meyer/Rowan 1977). DiMaggio \& Powell bestimmen die Umwelt genauer durch das organisationale Feld, in dem Organisationen in einem Legitimationsverhältnis zueinander stehen und sich wechselseitig angleichen (institutionelle Isomorphien). Die dabei vollzogenen Angleichungen erfolgen mittels 
der drei (eher analytisch zu verstehenden) Mechanismen Zwang, normativer Druck und Imitation (vgl. DiMaggio/Powell 1983; Hasse/Krücken 2005).

Giddens geht in seiner Theorie der Strukturation davon aus, dass menschliches Handeln omnipräsent und für die Entstehung und Veränderung sozialer Systeme und Strukturen verantwortlich ist. Soziale Systeme versteht er ,als reproduzierte soziale Praktiken“ (Giddens 1997: 69), die reproduzierte Beziehungen und Interaktionen beinhalten. Die Struktur als ,raumzeitliches Phänomen“" (ebd.) drückt sich in sozialen Praktiken aus und leitet das Handeln der Individuen. Strukturen dienen damit als Orientierungspunkte des Handelns, die Handlungen sowohl ermöglichen als auch einen einschränkenden Charakter haben können (Giddens 1997). Das Individuum ist dabei in der Lage, Strukturen zu reflektieren und die Strukturen über Handlungen zu reproduzieren oder zu verändern (Vester 2010: 154; Kießling 1988: 290). Damit sind Handlungen und Strukturen immer zeitgleich und bezogen aufeinander zu verstehen, d.h. Strukturen als Regeln und Ressourcen sind zugleich Medium und Ergebnis von Handlungen (die Dualität von Struktur und Handlung) (Giddens 1997).

Aufbauend auf unserem Institutionenbegriff lässt sich daraus folgendes Verständnis von Institutionalisierung ableiten:

Institutionalisierung bezieht sich auf den Prozess der Genese und Gestaltung einer Professionalitäts- und Organisationsstruktur für die Formgebung einer funktionalen Weiterbildungsinstitution. Gegenstand der Institutionalisierung von Weiterbildungsinstitutionen sind Angebote, Programme, Dienstleistungen und Projekte, für die Formen der Auf-DauerStellung entwickelt und veranlasst werden. Institutionalisierung vollzieht sich über Strukturierungen, bei denen die Individuen Regeln und Ressourcen über ihr Handeln aufnehmen, reflektieren und reproduzieren.

Als ein entscheidender Bezugspunkt, der ein leitender Teil der Auswertungsperspektive ist, erweist es sich zu verstehen, dass zwei unterschiedliche Institutionalformen miteinander kooperieren, die über unterschiedliche Strukturen, Zielsetzungen, Finanzierungsmöglichkeiten und Logiken des professionellen Handelns verfügen: Zum einen, wie bereits genannt, eine kommerzielle Einrichtung mit einer Anbindung an einen transnationalen Konzern, zum anderen eine beigeordnete Einrichtung, die Kulturaustausch und Kulturtransfer zum Hauptziel hat und den Bildungsbereich als eine weitere Säule des Geschäftes aufbaut. Diese verschiedenen Institutionalformen führen zugleich zu differierenden Herangehensweisen des Aufbaus einer Professionalitätsstruktur und differierenden Lernkulturen. 


\subsection{Professionalitätsstruktur}

Neben fragilen Organisationsstrukturen, die nach eineinhalb Jahren der Entstehung der Weiterbildungsinstitution sichtbar wurden und sich im Prozess als zentral herausstellten, haben wir als zentrale Beobachtungsperspektive den Aufbau einer Professionalitätsstruktur fokussiert, die die Erstellung von Programmen, Angeboten und Projekten (Programmplanungshandeln) sowie die strategische Etablierung und Verankerung der Leitung (Bildungsmanagement) beinhaltet. Programmplanungshandeln und Bildungsmanagement zeigen sich in der ersten Phase des Projekts als besonders relevante Herausforderungen, die wesentliche Pfeiler für die Modellierung transnationaler Lernkulturen darstellen.

Im Bildungsmanagement werden überwiegend von den Leiter/inne $/ \mathrm{n}$ in Erwachsenen- und Weiterbildungseinrichtungen bzw. beigeordneten Bildungseinrichtungen Handlungswaben konstelliert. Ihr Handeln ist durch einen spezifischen Modus der Gestaltung geprägt, der sich u.a. durch Formen der Entscheidung und Delegation auszeichnet und organisational als Handlungswaben eine strukturelle Einbettung findet, d.h. Ermöglichungsräume für Programme, Projekte und Angebote realisiert. Die Leitung balanciert dabei ordnungspolitische Steuerungsanforderungen von außen und organisationale Anforderungen von innen aus. Die systematischen Aufgabenbereiche des Weiterbildungsmanagements sind organisationsstrukturelles Management, überinstitutionelles Vernetzungsmanagement, Marketing, Öffentlichkeitsarbeit, Schaffung von Rahmenbedingungen für die Programmplanung, finanzielles Management, Selbstmanagement und Qualitätssicherung (vgl. Robak 2004).

Die Institutionenentwicklung und Programmentwicklung beziehen sich aufeinander. Dementsprechend wirken die ,institutionelle Auslegung der trägerspezifischen EB/WB-Vorstellungen, die dadurch vermittelnden Anforderungen oder antizipierten Bildungsinteressen sowie das inhaltliche, konzeptionelle Profil [...] bis in die einzelnen Angebote der jeweiligen Institution, die mit den einzelnen Teilangeboten zu einem Programm gebündelt werden“ (Gieseke 2015: 166). In dem pädagogischen Handlungsfeld der Programmplanung werden Programme und Bildungsangebote von Planenden konzipiert. Unter Einbezug und Abwägung differierender Interessen der beteiligten Akteure werden planungsrelevante Entscheidungen ausgehandelt und getroffen, um für Erwachsene bedarfsorientierte Räume zum Erwerb von Bildung, Kompetenz und Qualifikation zu schaffen (Caffarella/Ratcliff Daffron 2013; Cervero/Wilson 1994; Gieseke 2008; Höffer-Mehlmer 2011). Die Elemente der Programm- und Angebotsplanung stehen dabei in Beziehung zueinander (Sork 1998) und sind miteinander vernetzt, woraus die Komplexität des Planungsprozesses resultiert. 
Sowohl Gieseke $(2000,2008)$ als auch Caffarella \& Ratcliff Daffron (2013) betrachten den Planungsprozess als situativen, nicht linearen, sondern flexiblen Prozess, den der/die Planende unter Anwendung von Wissensressourcen steuern muss. Während bei Gieseke die Wissensinseln als Wissensressourcen und Handlungsschritte verstanden werden, unterscheiden Caffarella \& Ratcliff Daffron zwischen grundlegenden Wissensressourcen und Komponenten, die eine Mischung spezieller Entscheidungen und Aufgaben darstellen, wie beispielsweise die Bedarfsanalyse (ebd.). Besondere Bedeutung kommt in der Erforschung transnationaler Lernkulturen der grundlegenden Wissensressource der kulturellen Differenzen zu, die nach Caffarella \& Ratcliff Daffron auch dazu führen kann, dass das ,Interactive Model of Program Planning" stärker linear ausgelegt wird, spezifische Zuschnitte und Erweiterungen der Komponenten erfolgen oder die Komponenten unterschiedlich ausgedeutet werden (Caffarella/Ratcliff Daffron 2013). Das heißt: Die Planenden müssen beim Aufbau einer Weiterbildungseinrichtung in China in der Programmplanung die kulturellen Ressourcen und Verständnisse aus Deutschland und China als Strukturen aufnehmen und in den Planungsprozess reflexiv integrieren. Eine Möglichkeit kann in der Aufnahme der spezifischen Kulturstandards bestehen, die nach Thomas Merkmale eines universellen Orientierungssystems (Kultur) darstellen. „Unter Kulturstandards werden alle Arten des Wahrnehmens, Denkens, Wertens und Handelns verstanden, die von der Mehrzahl der Mitglieder einer bestimmten Kultur für sich persönlich und andere als normal, selbstverständlich, typisch und verbindlich angesehen werden" (Thomas 2003: 112) (zur weiteren Auseinandersetzung mit Kulturstandards nach Alexander Thomas siehe Robak 2012).

Zwischenergebnisse des Projektes ${ }^{1}$ verdeutlichen, dass auf der Ebene des strategischen Managements die Anforderungen der Vernetzung in China besonderen kulturellen Herausforderungen unterliegen. Es wirken verschiedene Kulturstandards in alle Prozesse hinein. Als besonders relevant erweisen sich die Kulturstandards „Hierarchie einhalten“ und „Beziehungen aufbauen bzw. pflegen“" (chinesisch: guanxi). Das bedeutet, das Vernetzungsmanagement der Weiterbildungseinrichtung muss in besonderer Weise die Formen der hierarchiegestützten Vernetzung einhalten, die mit spezifischen Formen des Beziehungsaufbaus verbunden sind. Eine Konsequenz ist, dass eine Aufgabenteilung zwischen Planung und strategischer Verankerung und damit systematisch gesprochen - zwischen Programmplanung und Bildungsmanagement nicht möglich ist. Die Leitungsebene, und das sind in unserem Fall die Personen, die nicht vor Ort in China sind, müssen Vernetzungen mit auf-

1 Die Forschungsdaten wurden neben Dokumentenanalysen und teilnehmenden Beobachtungen vor allem über leitfadengestützte Expert(inn)eninterviews erhoben und inhaltsanalytisch ausgewertet. Alle Leitfäden wurden abduktiv (vgl. Reichertz 2003) aus dem laufenden Projektstand und nach aktuellem Erkenntnisstand entwickelt. 
bauen und Akquisegespräche selbst führen. Vernetzungen und die besondere Beziehungskomponente im Chinesischen wirken damit bis in die Programmplanung hinein, da die Akquisegespräche einen entscheidenden Zugang zur Planung darstellen. Vernetzungen ermöglichen und limitieren damit zugleich professionelles pädagogisches Handeln (Lorenz/Robak 2016).

\section{Fazit}

Zusammenfassend lässt sich festhalten, dass es insbesondere bei der Gestaltung von Institutionalkontexten Abstimmungen, Aushandlungsprozesse und Angleichungen braucht, in denen die jeweiligen kulturellen Ressourcen identifiziert, eingebracht sowie wechselseitig übersetzt werden können. Die Platzierung kultureller Ressourcen und Besonderheiten führt dazu, dass ein gemeinsamer transnationaler Bildungsraum wachsen und entstehen kann, der transnationale Lernkulturen, d.h. modellierte Lernkulturen, gestaltet. Gemeinsamkeiten erwachsen, weil die kulturellen Besonderheiten Akzeptanz finden, in Gestaltungsentscheidungen eingehen und auch eine Ressource für andere Kulturen darstellen können. Darin realisiert sich die Forderung der Balance von Globalität und Lokalität, wie etwa Robertson (1998) es als Anforderung der Gestaltung einer Weltgesellschaft beschreibt (Robak/Enoch i.E.)

\section{Literatur}

Arnold, R./Schüßler, I. (1998): Wandel der Lernkulturen. Ideen und Bausteine für ein lebendiges Lernen. Darmstadt.

Caffarella, R. S./Ratcliff Daffron, S. (2013): Planning Programs for Adult Learners. A Practical Guide ( $3^{\text {rd }}$ edition). San Francisco: Jossey-Bass.

Cervero, R. M./Wilson, A. L. (1994): The Politics of Responsibility: A Theory of Program Planning Practice for Adult Education. Adult Education Quarterly 45, pp. 249-268. DOI: 10.1177/0741713694045001001.

DiMaggio, P. J./Powell, W. W. (1983): The Iron Cage Revisited. Institutional Isomorphism and Collective Rationality in Organizational Fields. American Sociological Review, 2, pp. 147-160.

Faulstich, P. (2010): Institutionen. In: Arnold, R./Nolda, S./Nuissl, E. (Hrsg.): Wörterbuch Erwachsenenbildung. http://www.wb-erwachsenenbildung.de/ [Zugriff: 25.01.2016].

Fleige, M. (2011): Lernkulturen in der öffentlichen Erwachsenenbildung. Theorieentwickelnde und empirische Betrachtungen am Beispiel evangelischer Träger. Münster: Waxmann.

Fleige, M./Robak, S. (i.E.): Lehr-Lernkulturen in der Erwachsenenbildung/Weiterbildung. Erscheint in: Tippelt, R./Hippel, A. von (Hrsg.): Handbuch Erwachsenenbildung/Weiterbildung. Frankfurt a.M.: Leske und Budrich. 
Friebe, J. (2005): Merkmale unternehmensbezogener Lernkulturen und ihr Einfluss auf die Kompetenzen der Mitarbeiter. Heidelberg.

Giddens, A. (1997): Die Konstitution der Gesellschaft. Grundzüge der Theorie der Strukturierung (3. Aufl.). Frankfurt a.M., New York: Campus.

Gieseke, W. (Hrsg.) (2000): Programmplanung als Bildungsmanagement? Qualitative Studie in Perspektivverschränkung; Begleituntersuchung des Modellversuchs „Erprobung eines Berufseinführungskonzeptes für hauptberufliche pädagogische Mitarbeiter, -innen in der konfessionellen Erwachsenenbildung". Recklinghausen: Bitter.

Gieseke. W. (2008): Bedarfsorientierte Angebotsplanung in der Erwachsenenbildung. Bielefeld: Bertelsmann.

Gieseke, W. (2009): Professionalisierung in der Erwachsenenbildung/Weiterbildung. In: Tippelt, R./Hippel, A. von (Hrsg.): Handbuch Erwachsenenbildung/Weiterbildung. Wiesbaden: VS Verlag, S. 385-403.

Gieseke, W. (2015): Programme und Angebote. In: Dinkelaker, J./Hippel, A. von (Hrsg.): Erwachsenenbildung in Grundbegriffen. Stuttgart: Kohlhammer, S. 165-173.

Hartz, S./Schardt, V. (2010): (Organisations-)theoretische Bezüge in erwachsenenpädagogischen Arbeiten. Eine Bestandsaufnahme. In: Dollhausen, Karin u.a. (Hrsg.): Erwachsenenpädagogische Organisationsforschung. Wiesbaden: VS Verlag, S. 21-44.

Hasse, R./Krücken, G. (2005): Neo-Institutionalismus, 2. überarb. Aufl. Bielefeld: Transcript.

Hilb, M. (2002): Transnationales Management der Human-Ressourcen. Das Modell des Glocalpreneuring. 2. überarb. Aufl. Neuwied u.a.

Hof, C. (2008): Lebenslanges Lernen. Eine Einführung. Stuttgart: Kohlhammer.

Höffer-Mehlmer, M. (2011): Programmplanung und -organisation. In: R. Tippelt/ Hippel, A. von (Hrsg.): Handbuch Erwachsenenbildung/Weiterbildung. Wiesbaden: VS Verlag, S. 989-1002.

iMOVE (2013): TrendBarometer Exportbranche Aus- und Weiterbildung 2013. https://www.imove-germany.de/cps/rde/xbcr/imove_projekt_de/p_iMOVE_ Trendbarometer_2013_sicher.pdf [Zugriff: 28.01.2016].

Kießling, B. (1988): Die ,Theorie der Strukturierung“. Ein Interview mit Anthony Giddens. Zeitschrift für Soziologie 17, 4, S. 286-295.

Kil, M./Schlutz, E. (2008): Veränderungen und Ausdifferenzierungen im Anbieterund Leistungsspektrum der organisierten Weiterbildung. In: Hof, C. u.a. (Hrsg.): Strukturen Lebenslangen Lernens. Hohengehren: Schneider-Verlag, S. 64-75.

Klingovsky, U. (2009): Schöne Neue Lernkultur. Transformationen der Macht in der Weiterbildung. Eine gouvernementalitätstheoretische Analyse. Bielefeld: Transcript.

Köhler, B. (2004): Strukturen und Strategien transnationaler Konzerne: Empirische Soziologie der ,,inneren Globalisierung“. Wiesbaden: Deutscher Universitätsverlag.

Lattke, S./Nuissl, E./Pätzold, H. (2010): Europäische Perspektiven der Erwachsenenbildung. Bielefeld: Bertelsmann.

Lorenz, L./Robak, S. (2016): Interpersonal relationships in China - bridges for transnational adult education. In: Harju, A. /Heikkinen, A. (Eds.): Adult Education and the Planetary Condition (forthcoming Open Access Publication). 
Mau, S. (2007): Transnationale Vergesellschaftung. Die Entgrenzung sozialer Lebenswelten. Frankfurt a.M.: Campus.

Meyer, J. W./Rowan, B. (1977): Institutionalized Organizations: Formal Structure as Myth and Ceremony. American Journal of Sociology 2, pp. 340-363. www.jstor.org/stable/2778293 [Zugriff: 24.06.2014].

Nittel, D. (2000): Von der Mission zur Profession? Bielefeld: Bertelsmann.

Pätzold, G./ Lang. M. (1999): Lernkulturen im Wandel. Didaktische Konzepte für eine wissensbasierte Organisation. Bielefeld.

Pries, L. (2008): Die Transnationalisierung der sozialen Welt. Frankfurt a.M.: Suhrkamp.

Thomas, A. (2003): Psychologie interkulturellen Handelns (2. akt. Aufl.). Göttingen: Hofgrefe.

Tippelt, R. (2009): Institutionenforschung in der Erwachsenenbildung/Weiterbildung. In: Tippelt, R./Hippel, A. v. (Hrsg.): Handbuch Erwachsenenbildung/Weiterbildung. Wiesbaden, S. 453-471.

Reckwitz, A. (2006): Das hybride Subjekt. Eine Theorie der Subjektkulturen von der bürgerlichen Moderne zur Postmoderne. Weilerswist: Velbrück.

Reichertz, J. (2003): Die Abduktion in der qualitativen Sozialforschung. Opladen: Leske und Budrich.

Robak, S. (2004): Management in Weiterbildungsinstitutionen. Eine empirische Studie zum Leitungshandeln in differenten Konstellationen. Hamburg: Dr. Kovac.

Robak, S. (2009): Kulturelle Aspekte von Lernkulturen in transnationalen Unternehmen unter Globalisierungsbedingungen. In: Gieseke, W./Robak, S./Wu, M-L. (Hrsg): Transkulturelle Perspektiven auf Kulturen des Lernens. Bielefeld: Transcript, S. 119-159.

Robak, S. (2012): Kulturelle Formationen des Lernens. Zum Lernen deutscher Expatriates in kulturdifferenten Arbeitskontexten in China - die versäumte Weiterbildung. Habilitationsschrift. Münster/New York/München/Berlin: Waxmann.

Robak, S./Enoch, C. (i.E.): Internationalisierung, Globalisierung und Transnationalisierung der Erwachsenenbildung, Weiterbildung und Personalentwicklung. Studienbrief EB0610 Erwachsenenbildung.

Robak, S./Lorenz, L./Herzog, M./Asche, E. (2014): Perspektiven zum Aufbau einer Weiterbildungseinrichtung in China - Vernetzung zwischen Professionalität und fluider Organisationsstrukturierung. In: Nittel, D./Tippelt, R./Wahl, J.(Hrsg.): Kooperation inner- und außerhalb des Systems des lebenslangen Lernens, S. 1930. URN urn:nbn:de:0111-pedocs-100177 [Zugriff: 23.01.2016].

Robertson, R. (1998): Glokalisierung: Homogenität und Heterogenität in Raum und Zeit. In: Beck, Ulrich (Hrsg.) (1998): Perspektiven der Weltgesellschaft. Frankfurt a.M.: Suhrkamp, S. 192-220.

Schäffter, O. (2001): Organisation. In: Arnold, Rolf u.a. (Hrsg.): Wörterbuch Erwachsenenpädagogik. Bad Heilbrunn, S. 243-246.

Schüßler, I./Thurnes, C. M. (2005): Lernkulturen in der Weiterbildung. Bielefeld: Bertelsmann.

Schrader, J. (2011): Struktur und Wandel der Weiterbildung. Theorie und Praxis der Erwachsenenbildung. Bielefeld: Bertelsmann.

Sork, J. (1998): Program Priorities, Purposes, and Objectives. In: Cookson, P. S. (Eds.): Program Planning for the Training and Continuing Education of Adults: 
North American Perspectives. Malabar, Florida: Krieger Publishing Company, pp. 273-300

Vester, H.-G. (2010): Kompendium der Soziologie III: Neuere soziologische Theorien. Wiesbaden: VS Verlag für Sozialwissenschaften.

Zinger, G. H. (2002): Die Internationalisierung der Belegschaften multinationaler Unternehmen mit Sitz in Deutschland. Berlin. 



\section{Professionalisierung von Lehrhandeln in einer digital geprägten Lernkultur. Bedarf und Ansatzpunkte der Entwicklung einer Online- Lernumgebung}

\section{Einleitung}

In der aktuell geführten Diskussion zur Sicherung der Qualität der Weiterbildung stehen neben den organisationalen Strukturen vor allem die Lehrenden als Zuständige für die Gestaltung von Lehr-Lernprozessen im Fokus der Betrachtung (vgl. z.B. Research voor Beleid 2008; Kraft/Seitter/Kollewe 2009). Bezüglich der Förderung ihrer professionellen Handlungskompetenz stellt sich die Frage der Entwicklung zielgruppengerechter Angebote, die den heterogenen Voraussetzungen und begrenzten zeitlichen und finanziellen Ressourcen gerecht werden sowie eine überinstitutionelle Verständigung über die für Lehrende in der Weiterbildung gegebenen Rahmenbedingungen, relevanten Wissensbestände, Erfahrungen etc. anstreben (vgl. Schrader 2010).

Formen selbstgesteuerten und informellen, kooperativen Lernens können flexible Ansätze für die praxisnahe Weiterbildung und den einrichtungsübergreifenden Austausch von Lehrenden bieten. Am Modell der Communities of Practice orientiert, könnten netzgestützte Lernsettings etabliert werden, die Lehrenden den Rahmen bieten, Wissen zu teilen und sich gemeinsam für arbeitsplatzbezogene Interessen sowie die Weiterentwicklung ihres Lehrhandelns einzusetzen sowie über dieses Zusammenwirken die digitale Lernkultur aktiv mitzugestalten (vgl. Wenger 2001; Gray 2002).

Hier setzt das vom Deutschen Institut für Erwachsenenbildung in Kooperation mit der Universität Tübingen realisierte Vorhaben der Entwicklung und exemplarischen Erprobung eines netzgestützten Weiterbildungsangebots zur Professionalisierung Lehrender unter Konstruktion einer Kultur gemeinschaftlichen Lernens an. Basierend auf den Ergebnissen einer Bedarfsanalyse unter Lehrenden aller Weiterbildungskontexte zu den Lerninteressen und Voraussetzungen des gemeinsamen Austausches werden eine Lern- und Vernetzungsumgebung für Lehrende konzipiert sowie ausgewählte Inhalte in der Nutzung durch Communities of Practice als Lernsetting erprobt. 


\section{Anknüpfungspunkte}

\subsection{Professionstheoretische Grundlagen}

Dem Vorhaben liegt ein Professionalisierungsverständnis zugrunde, das eine individuelle und kollektive Perspektive vereint, bei den Einzelnen und deren Kompetenzentwicklung ebenso wie an einem gemeinsamen Eintreten der Berufsangehörigen für die Professionsinteressen ansetzt und eine Festigung der strukturellen Rahmenbedingungen des Berufsfeldes miteinschließt. Insbesondere die notwendige Wissensgrundlage sowie der konkrete Fallbezug des beruflichen Könnens von Lehrenden in der Erwachsenenbildung/ Weiterbildung, das sich in der Fähigkeit zu einer situationsangemessenen Gestaltung von Lehr-Lernprozessen unter der Einnahme der Perspektiven der Beteiligten zeigt, lassen es angemessen erscheinen, Professionalität handlungsbezogen $\mathrm{zu}$ fassen und über eine individuelle Kompetenzentwicklung Lehrender die Professionalisierung ihrer beruflichen Tätigkeit zu fördern (vgl. z.B. Tietgens 1988). Weiter geht mit der Professionalisierung der Anspruch einer Vereinheitlichung eines Berufsbildes bezüglich seiner Rahmenbedingungen und Handlungsgrundlagen einher (vgl. z.B. Nittel 2000). Dieser Prozess ist mitunter abhängig von der Herausbildung eines kollektiven beruflichen Selbstverständnisses der Berufsangehörigen, das eine Vorstellung über ihr Handlungsfeld, ihre Aufgaben und deren Ausübung (z.B. Berufsethik) einschließt. Dies bedarf neben wissenschaftlichem Wissen als Handlungsgrundlage zum Bestreiten des Feldes auch eines organisierten kollegialen Umfeldes mit der Möglichkeit der professionsspezifischen Sozialisation (vgl. z.B. Kraus 2012).

\subsection{Ausgangslage der Lehrenden}

Über das berufliche Selbstverständnis und die professionellen Kompetenzen von Lehrenden in der Erwachsenenbildung/Weiterbildung liegen kaum empirisch gesicherte Befunde vor (vgl. Schrader 2010). Wir können jedoch davon ausgehen, dass die Kompetenzen dieser Lehrpersonen eher erfahrungs- als theoriebasiert sind. Nur eine zahlenmäßig kleine Gruppe von Lehrenden verfügt über eine grundlegende (erwachsenen-)pädagogische Qualifizierung. Als "Quereinsteiger“ beruht ihr Zugang zur Lehrtätigkeit mehrheitlich vor allem auf fachlicher Expertise oder beruflicher Erfahrung, so dass ihr Unterricht in der Regel ohne expliziten Rekurs auf speziell pädagogisch fundiertes, didaktisch-methodisches Wissen erfolgt (vgl. WSF 2005).

Weiter ist die berufliche und soziale Lage der rund 530.000 lehrenden Personen durch heterogene Beschäftigungsverhältnisse gekennzeichnet (vgl. Martin et al. i.V.). Vor allem die Lage der freiberuflich und nebenberuflich 
Tätigen ist unter finanziellen und versicherungsrechtlichen Gesichtspunkten in hohem Maße als prekär einzustufen. Dabei steht die Teilnahme an Fortbildung trotz der tendenziell vorhandenen hohen Weiterbildungsbereitschaft der Lehrenden (vgl. BMBF 2013) häufig in Konkurrenz zur Ausübung der Kursleitung und damit zur Einkommenssicherung, weswegen Angebote, die hohe Anforderungen an Präsenzzeiten stellen, in der Regel wenig nachgefragt werden (vgl. z.B. Martin/Langemeyer 2014). Die berufliche Situation drängt viele Lehrkräfte zu einer autodidaktischen Qualifizierung und individuellen Professionalisierung (vgl. Nittel 2006), häufig unter Zuhilfenahme von didaktisch-methodischer Ratgeberliteratur oder auch von Informationen aus dem Internet. Die fehlende Einbindung in professionelle Gemeinschaften erschwert den kollegialen, fachlichen und sozialen Austausch zwischen den Lehrenden (vgl. Schrader 2010).

\subsection{Mögliche Ansätze der Förderung professionellen Lehrhandelns}

Angesichts der skizzierten Herausforderungen bedarf es flexibel nutzbarer sowie kontextnah konzipierter Qualifizierungs- und Vernetzungsangebote für Lehrende, die eine Reflexion pädagogischen Handelns selbst zum Thema machen und so eine situationsadäquate Kompetenzentwicklung der Lehrenden über institutionelle Grenzen hinweg ermöglichen. Mit Blick auf den Markt findet man eine große Zahl an eher auf Wissensvermittlung konzentrierte Weiterbildungsangebote mit hohen Anforderungen an Präsenzzeiten vor. Trägerübergreifend und digital konzipierte Angebote von größerer Reichweite bestehen kaum (vgl. Schrader 2010).

Netzgestützte Lernangebote könnten hier einen vielversprechenden Ansatz bieten. Aufgrund ihrer zeitlich und örtlich flexiblen Nutzbarkeit können sie standort- und anbieterübergreifend eingesetzt werden und kommen damit den beruflichen Rahmenbedingungen in der Erwachsenenbildung/Weiterbildung entgegen. Weiter können digitale Lernumgebungen modularisierte Angebote vorhalten, die einen bedarfsgerechten und selbstorganisierten Zugriff ermöglichen sowie Räume für den Austausch von Wissen und Erfahrungen zwischen Lehrenden umfassen. Als kollegiale Netzwerke in Form von Communities of Practice organisiert, könnten Angebote konzipiert werden, die kompetenzorientiertes und erfahrungsbasiertes Lernen gleichermaßen bieten und die Plattform für eine Kommunikation zwischen Lehrenden und die Etablierung einer einrichtungsübergreifenden Lernkultur bilden (vgl. Wenger 2001). Lernkultur wird dabei verstanden als ,die Gesamtheit der Lern- und Entwicklungspotentiale, die über das Zusammenwirken der Mitglieder der Interaktions- und Kommunikationsprozesse auf unterrichtlicher, kollegialer und organisatorischer Ebene arrangiert werden" (Arnold/Schüßler 
1998). So wird in Form der Communities of Practice ein bestimmtes Lernsetting gewählt, das eine Selbstlernarchitektur sowie Austauschinfrastruktur umfasst, deren konkrete Nutzung in der Wahl relevanter Inhalte und Ausgestaltung des gemeinschaftlichen Lernprozesses jedoch den Lehrenden überlässt und diese somit zu Mitgestaltern der digitalen Lernkultur macht (vgl. z.B. Weinberg 1999). Dabei sind für die Communities of Practice drei Merkmale konstituierend: 1) ein geteilter Wissens- und Erfahrungsbereich als inhaltliche Grundlage für die Bildung der Community, 2) gegenseitiges Engagement, das den sozialen Kontext für den Austausch schafft und auf Vertrauen und Respekt basiert sowie 3) die im Laufe der Zeit innerhalb der Community gemeinsam entwickelten Sichtweisen und herausgebildeten Routinen (vgl. Wenger 2001).

Inwiefern über onlinegestützte Communities of Practice eine Professionalisierung des beruflichen Handelns von Lehrenden durch die Weiterentwicklung der individuellen Kompetenzen sowie Herausbildung kollektiver Sichtweisen gelingen kann, stellt das zentrale Untersuchungsinteresse des hier vorgestellten Vorhabens dar.

\section{Ziele, Fragestellungen und Vorgehen}

Vor dem Hintergrund der dargelegten Anknüpfungspunkte zielt das Vorhaben auf die Konzeption und Erprobung eines Angebots für Lehrende in der Erwachsenenbildung/Weiterbildung, das auf einen kollegialen Austausch in netzgestützten Lerngemeinschaften als ein Kernelement setzt und darüber individuelle Kompetenzentwicklung sowie kollegiale Vernetzung gleichermaßen ermöglicht. Als Grundlage für die Konzeption stellt sich dabei einerseits die Frage, welchen Voraussetzungen und Bedarfen das Angebot gerecht werden sollte, um Nachfrage zu erzeugen und Kompetenzentwicklung zu ermöglichen. Andererseits gilt es die Rahmenbedingungen zu eruieren, unter denen es gelingen kann, die Lehrenden für eine aktive Nutzung der digitalen Umgebung und darüber Mitgestaltung der Lernkultur zu gewinnen.

Zur Berücksichtigung der Interessen und der Mitwirkungsbereitschaft der Lehrenden als zentrale Gestaltungselemente erfolgt die Entwicklung des netzgestützten Angebots von Beginn an im Abgleich mit der Praxis. Dabei entspricht das Vorgehen dem Konzept einer anwendungsorientierten Grundlagenforschung, die sich der Wissenschaft und der Praxis als Referenzsysteme gleichermaßen verpflichtet und sowohl praktische Fortbildungsinteressen als auch grundlagenwissenschaftliche Fragestellungen in den Blick nimmt. So werden die Entwicklungsaufgaben ausgehend von dem erhobenen Bedarf der Lehrenden und den strukturellen Gegebenheiten der Weiterbildungslandschaft formuliert, mit offenen Forschungsfragen der Professionalisierung von Lehrhandeln vor dem Hintergrund kompetenztheoretischer 
Überlegungen verbunden und in enger Abstimmung mit der Praxis in Form eines iterativen Entwicklungs- und Erprobungsprozesses bearbeitet (vgl. Schrader/Goeze 2011; s. Abb. 1).

Abb. 1: Schematischer Vorgehensablauf

Die konkrete Umsetzung dieses Ansatzes im Rahmen der Entwicklung einer Lern- und Vernetzungsumgebung für Lehrende in der Erwachsenenbildung gestaltet sich dabei wie folgt:

1) Erhebung von Bedarfen und Rahmenbedingungen: Um das Lern- und Vernetzungsangebot möglichst adressatengerecht und praxisnah auszurichten, wurde im ersten Schritt eine umfassende Befragung der Lehrenden realisiert. Diese wurde über die Träger und Anbieter der Erwachsenenbildung verteilt, so dass eine in Bezug auf die Heterogenität der Weiterbildungslandschaft hinsichtlich der Programmbereiche, institutionellen Kontexte und Beschäftigungsverhältnisse zufällige, aber im Vergleich zu den Daten der WSF-Erhebung von 2005 näherungsweise repräsentative Stichprobe von 1367 Befragten erzielt werden konnte. Die Ergebnisdarstellungen erfolgen in Form von Mittelwertangaben zu den skalierten Fragen (von 1=trifft nicht $\mathrm{zu}$ bis $4=$ trifft $\mathrm{zu}$ ) sowie einer Verdichtung der ergänzend gestellten offenen Fragen.

2) Konzeptionelle Entwicklungen: Aufbauend auf die Erkenntnisse der Befragung sowie auf theoretische Grundlagen zur mediendidaktischen sowie kompetenzorientierten Gestaltung von netzgestützten Weiterbildungsangeboten erfolgte die exemplarische Ausarbeitung einer prototypischen Lern- und Vernetzungsumgebung.

3) Pilotierung der Entwicklungen: Die Konzeption wurde im Kontext von Fokusgruppen diskutiert und mit den Lernpraxen und -gewohnheiten der Lehrenden abgeglichen. Dazu fanden an fünf Standorten halbtägige Veranstaltungen mit jeweils 10-15 Lehrenden aus unterschiedlichen institutionellen Kontexten und Programmbereichen statt $(\mathrm{N}=54)$. Die 
Ergebnisse basieren auf analysierten Gesprächsmitschnitten sowie einer standardisierten Befragung zur Beurteilung des Lernangebots. Ergänzend wurden die prototypischen Entwicklungen durch neun netzgestützte Communities of Practice in Dreiergruppen erprobt. Diese setzten sich aus 27 Lehrenden in fach- sowie kontextübergreifenden Konstellationen zusammen, die sich über einen erfahrungsbasierten sowie theoriegeleiteten Austausch mit Fragen der Gestaltung von Lehr-Lernprozessen beschäftigten. Abschließend wurden sie fragebogengestützt gebeten, die Lerninhalte, das kollegiale Lernsetting sowie den subjektiven Lernertrag einzuschätzen.

4) Umsetzung der Konzeption: Die Befunde der Pilotierung bieten die Basis für den weiteren Feinschliff der Konzeption und deren Umsetzung in Form des Aufbaus des künftig im Netz verfügbaren Lern- und Vernetzungsangebots. Der Aufbau und die Ausdifferenzierung des Angebots wird dabei kontinuierlich weiter durch Befragungen und Erprobungen begleitet werden, um die bisher eher punktuell gewonnenen Eindrücke breiter empirisch zu untermauern und auch die Lernwirkung sowie den Transfer des Gelernten in die Praxis der Lehrenden stärker in den Blick zu nehmen.

Im Folgenden werden zentrale Ergebnisse der einzelnen Vorgehensschritte dargestellt. Abschließend wird ein Zwischenfazit für die weitere Entwicklung des Lern- und Vernetzungsangebots gezogen.

\section{Ergebnisse}

\subsection{Befunde zu Bedarfen und Rahmenbedingungen}

In der Befragung zeigte sich ein hoher Bedarf der Lehrenden nach Angeboten zur didaktischen Konzeption und methodischen Umsetzung von Kursen $(\mathrm{M}=3.40 ; \mathrm{SD}=.910)$. Weiter wäre eine thematische Auseinandersetzung mit Fragen der Gestaltung von Lernprozessen $(\mathrm{M}=3.15 ; \mathrm{SD}=.978)$ sowie der Interaktion und Kommunikation mit Lernenden $(\mathrm{M}=3.32 ; \mathrm{SD}=.912)$ für die Befragten reizvoll. In der Art der Angebote sollte es sich dabei nicht nur um qualitativ hochwertiges theoretisches Wissen handeln, vielmehr sind konkrete Handlungshilfen $(\mathrm{M}=3.21 ; \mathrm{SD}=.859)$ und situative Lösungen $(\mathrm{M}=3,18$; $\mathrm{SD}=.858$ ) wünschenswert. Darüber hinaus besteht bei den Lehrenden ein hoher Bedarf, sich einen Überblick über die rechtlichen, sozialen und finanziellen Rahmenbedingungen der Weiterbildungslandschaft zu verschaffen sowie dazu in kollegialen Austausch zu treten. An Szenarien für eine Kompetenzentwicklung der Lehrenden werden individualisiert nutzbare Lernräume zum Training von Kompetenzen $(\mathrm{M}=3.09$; $\mathrm{SD}=.917)$ gegenüber On- 
line-Arbeitsumgebungen für organisierte Kursgruppen $(\mathrm{M}=2.62 ; \mathrm{SD}=.943)$ bevorzugt. Außerdem werden Instrumente zur Dokumentation und Bilanzierung von Kompetenzen begrüßt $(\mathrm{M}=2.83 ; \mathrm{SD}=.916)$, die eine Überprüfung von Lernständen sowie darauf basierende Lernwege ermöglichen. Der deutliche Wunsch nach Tools zum einrichtungsübergreifenden Austausch mit Lehrenden $(\mathrm{M}=2.96 ; \mathrm{SD}=.841)$ sowie zum Austausch mit Experten $(\mathrm{M}=3.17$; $\mathrm{SD}=.810)$ spricht - ebenso wie die bevorzugte Form des situativen Lernens just-in-time - für die Bereitstellung einer Infrastruktur, die selbstgesteuertes, auf Erfahrungsaustausch basierendes Lernen ermöglicht, wie es für Communities of Practice konstituierend ist.

Blickt man auf die offen genannten Argumente der Befragten für bzw. gegen eine Nutzung der Lern- und Vernetzungsumgebung, zeigt sich folgendes Bild: 462 Befragte geben Zeitmangel bzw. Zeitaufwand als ein ContraArgument an. Weiter schätzen 89 Personen das Verhältnis zwischen Aufwand und Ertrag als kritisch ein bzw. machen ihre Mitarbeit von einer in diesem Punkt positiven Nutzenbilanz abhängig. Damit in Zusammenhang lässt sich die Nennung des Aspekts, das eigene Wissen als eigenes „Kapital“ anderen nicht (ohne Gegenwert) zur Verfügung stellen zu wollen, bringen (22). Bedenken hinsichtlich des Datenschutzes werden von 69 Befragten eingeräumt. Auch zweifeln einige die Expertise der Beteiligten dafür, inhaltlich qualifizierte Beiträge leisten zu können, an (26). Ferner stellt die geringe Vertrautheit mit digitalen Medien für 63 Personen ein Hindernis dar und 49 Lehrende spricht ein rein medial vermittelter und für sie dadurch unpersönlicher Austausch nicht an (s. Abb. 2).

Das grundsätzliche Interesse an einem Austausch von Informationen und Erfahrungen mit anderen Lehrenden stellt das gewichtigste Argument für eine Nutzung der Lern- und Vernetzungsumgebung dar (152). Dabei werden sowohl die Vernetzungsmöglichkeit (67) als auch das Streben nach individueller Professionalisierung (49) als zentrale Motive genannt. Das hohe Austausch- und Weiterbildungsinteresse wird auch anhand der Beteiligungsgründe, über die Lernumgebung Wissen erwerben und weitergeben zu können (40), informiert zu bleiben bzw. Neues zu erfahren (37) sowie sich gegenseitig Unterstützung bieten zu können (19), deutlich. Zeitlich-organisatorische Vorteile eines zentralen Zugriffs auf relevante Informations- und Lernmöglichkeiten im Netz sehen 50 der befragten Personen (s. Abb. 3). 
182 Sabine Schöb/Matthias Rohs/Carmen Biel/Tim Scholze

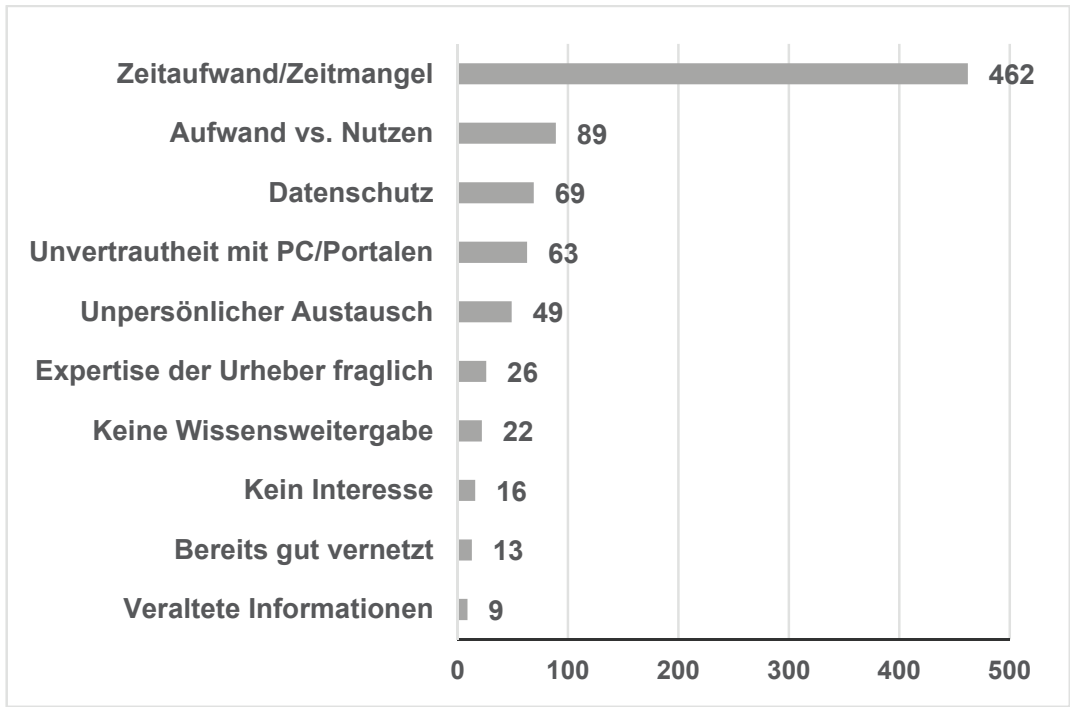

Abb. 2: Argumente gegen die Nutzung

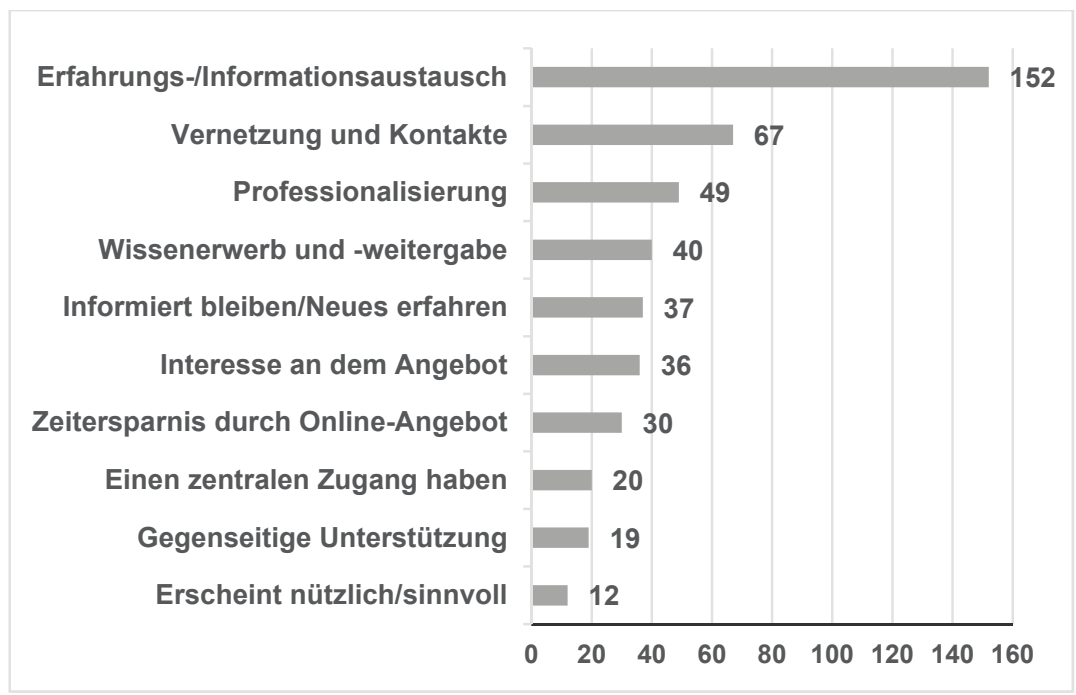

Abb. 3: Argumente für die Mitarbeit 
Dabei korreliert die Bereitschaft zur Mitwirkung nicht nur mit der Medienaffinität sowie dem Alter der Befragten, es zeigen sich auch Unterschiede in Abhängigkeit des Handlungsfelds der Lehrenden. Im innerbetrieblichen sowie privatwirtschaftlichen Kontext Tätige weisen gegenüber den Lehrenden aus dem staatlichen und gemeinschaftlich organisierten Kontext eine etwas höher ausgeprägte Bereitschaft, sich innerhalb einer Community zu engagieren sowie Beiträge einzubringen, auf. In Bezug auf die Bereitstellung eigener Materialien für andere Nutzende ist die Bereitschaft bei Lehrenden in Programmbereichen der Vermittlung von Handlungswissen (Sprache, EDV, berufliche Qualifizierung etc.) am höchsten.

Die Ergebnisse zeigen Erfolgsfaktoren für die Entwicklung eines netzgestützten Angebots für die Zielgruppe auf, die neben einer inhaltlichen Passung in einem technisch einfach und zeitlich flexibel nutzbaren, datenschutzrechtlich geklärten Raum und einem reziproken, in der Qualität gesicherten Austausch bestehen, worüber sich eine Kultur des gemeinsamen Lernens etablieren kann.

\subsection{Konzeptionelle Entwicklung}

Die mediendidaktische Konzeption der Lern- und Vernetzungsumgebung baut auf die Annahmen des problembasierten und situierten Lernens auf (vgl. Lave/Wenger 1991; Boud/Feletti 1997). Dabei werden die Lernenden mit authentischen oder vergleichbaren Problemstellungen aus ihrem Arbeitsoder Berufsalltag konfrontiert, die in einer kooperativen Lernform unter Selbst- sowie Fremdreflexion des Lernprozesses und der Lernergebnisse bearbeitet werden. Der Schwierigkeitsgrad der Aufgaben und das Ausmaß der Instruktionen können dabei durch die Lernenden selbst gesteuert werden, um ein bedarfsgerechtes Lernen zu ermöglichen. Verfügbare Lernstanderfassungen in Form von Assessments wie z.B. Wissenstests, Falldiagnosen und Handlungsproben, die Lernprozesse rahmen und mit Empfehlungssystemen gekoppelt sind, unterstützen darin, die Lernschritte gemäß bestehender Lernvoraussetzung und -motivation zu strukturieren (vgl. Leutner 1992; Kerres 2013). Die Zusammenstellung der verfügbaren Aufgaben und Assessments basiert auf den Topantworten der thematischen Fortbildungsbedarfe der Lehrenden in der Ausgangsbefragung, zu denen Lernfelder ausgearbeitet wurden. In der methodischen Ausgestaltung wird auf Communities of Practice als Lernform gesetzt und es werden Räume sowie Lernmaterialien für einen selbst gestaltbaren Austausch bereitgestellt.

Exemplarisch wird hier das Lernfeld zum Umgang mit heterogenen Gruppen in Bildungsangeboten herausgegriffen, welches zum jetzigen Zeitpunkt neben zwei weiteren Lernfeldern ausgearbeitet vorliegt und im Rahmen von Fokusgruppen sowie Communities of Practice pilotiert wurde. 
Das Lernfeld widmet sich den Herausforderungen, Lernstände zu diagnostizieren, Teilnehmende zum Lernen zu motivieren, die Arbeit mit Gruppen inhaltlich binnendifferenziert und für unterschiedliche Lernstile und -typen methodisch ansprechend zu gestalten sowie die Rolle der Lehrenden in der Begleitung der Lernprozesse zu hinterfragen. Dabei stehen Aufgaben und Assessments zur Planung, Umsetzung und Evaluation der Arbeit mit heterogenen Gruppen zur Verfügung, die der Entwicklung (medien-)didaktischer, methodischer sowie diagnostischer Kompetenz dienen (s. Abb. 4).

Abb. 4: Lernfeld zum Umgang mit heterogenen Gruppen in Bildungsangeboten

\subsection{Befunde zur Einschätzung der Akzeptanz und Lernwirkung}

Aus der Diskussion des exemplarischen Lernfelds in den Fokusgruppen lässt sich festhalten, dass die entworfene mediendidaktische Konzeption durchweg als plausibel und stimmig eingestuft wurde. Die mögliche Erschließung des Lernangebots über einen explorativen und situativen Zugang wurde sehr begrüßt. Dabei stellen die für das Lernfeld ausgewählten Themen für $95 \%$ der Lehrenden zentrale Anforderungen dar, mit denen sie sich gern näher lernend auseinandersetzen würden. Die Lernschritte zur Bewältigung von Planungs-, Umsetzungs- und Evaluationsaufgaben anhand von theoretischen Impulsen sowie Erfahrungsbeispielen sprechen die Lehrenden zu 90\% an, wobei die konkreten Lernaufgaben und Assessments sprachlich in Teilen noch einfacher und in der Berücksichtigung unterschiedlicher Handlungsfelder stärker differenziert werden könnten. Auch haben die Lehrenden zu 30\% 
Bedenken gegenüber der Komplexität der Darstellung und äußern die Unsicherheit, aus dem Angebot schnell und eigenständig eine für sich angemessene und relevante Auswahl treffen zu können.

In den Erprobungssettings der netzgestützten Communities of Practice stieß das Lernangebot bei den Lehrenden auf eine ähnlich positive Resonanz. Dabei wurde sowohl die formale Aufbereitung als auch inhaltliche Kombination der Lerninhalte je Aufgabe und Pfad als ansprechend eingestuft $(\mathrm{M}=3.34$; $\mathrm{SD}=.502$, Skala von $1=$ trifft nicht zu bis $4=$ trifft zu). Die Zeitinvestition stand für die Lehrenden in einem guten Verhältnis zum Nutzen $(\mathrm{M}=3.27 ; \mathrm{SD}=.428)$ und der Austausch mit den Kollegen in den Communities ließ sich überwiegend gut organisieren $(\mathrm{M}=2.98 ; \mathrm{SD}=.688)$, wobei zu einem Drittel synchrone Treffen in Form von Voice-Chat stattfanden und zu zwei Dritteln asynchrone Austauschformen durch schriftliche Kommentare und Forumsbeiträge realisiert wurden. Inhaltlich empfanden die Lehrenden das kollegiale Feedback bereichernd für die eigene Weiterentwicklung $(\mathrm{M}=3.18 ; \mathrm{SD}=.623)$ und haben durch den Austausch neue Einsichten für ihr Handeln und Arbeitsfeld gewinnen können ( $\mathrm{M}=3.50 ; \mathrm{SD}=.422)$. Die Beteiligten schätzen die Praxisgemeinschaften als ein angenehmes $(\mathrm{M}=3.09$; $\mathrm{SD}=.701)$ und ertragreiches $(\mathrm{M}=3.43 ; \mathrm{SD}=.520)$ Lernsetting ein, das sie in dieser Form gern erneut absolvieren würden $(\mathrm{M}=3.48 ; \mathrm{SD}=.612)$.

Für eine auf den Erkenntnissen aufbauende Entwicklung des Angebots erscheinen Communities of Practice als ein Kernelement tragfähig. Weiter sollte überlegt werden, wie das Angebot für einzelne Subgruppen differenziert werden und im Spannungsverhältnis von motivierenden Freiräumen versus unterstützenden Supportstrukturen lernwirksam gestaltet werden kann.

\section{Zwischenfazit und Ausblick}

Zusammenfassend betrachtet lässt sich als Ergebnis eine hohe Akzeptanz des geplanten Lern- und Vernetzungsangebots in Inhalt wie Struktur festhalten. Diese ist jedoch mit konkreten Erwartungen der Lehrenden an eine praxisnahe inhaltliche Ausrichtung und strukturell intuitive Bedienbarkeit für einen maximalen Ertrag bei minimalem Aufwand gekoppelt, um die Lernmotivation zu erhalten und die Räume für eine Vernetzung vertrauenswürdig und attraktiv zu machen. Wie die Befunde aus den Communities of Practice zeigen, ist der in diesem Lernsetting bereitgestellte thematisch vordefinierte Materialkanon sowie dessen Erschließbarkeit über ein erfahrungsbasierten Lernens im kollegialen Austausch unter Herausbildung eigener Lösungsstrategien (vgl. Wenger 2001) von hoher Attraktivität und hohem Ertrag für die Lehrenden. Das Lernsetting entspricht dem Bedarf nach Erfahrungsaustausch und Selbstorganisation gleichermaßen und lässt sich ideal für die Initiierung eines situierten, problembasierten Lernens nutzen (vgl. Lave/Wenger 1991; 
Boud/Feletti 1997). Die Entwicklung von Lerngemeinschaften kann eine Lernkultur etablieren helfen, die von den Lehrenden wesentlich mitgestaltet wird und einen Wissens- und Erfahrungsaustausch begünstigt (vgl. z.B. Weinberg 1999), der neben mikrodidaktischen Fragen auch für das Berufsfeld orientierendes Wissen für eine Professionalisierung der Lehrenden einschließt (vgl. z.B. Kraus 2012). Die in den Praxisgemeinschaften im Kleinen erlernte Form der reziproken und vertrauensvollen Kooperation sowie eine zunehmende Sicherheit im Umgang mit einer chat- bzw. forumsgestützten Kommunikation wiederum könnten die Basis legen für eine höhere Bereitschaft der Lehrenden zur Beteiligung in größeren Online-Communities.

Über die Ermöglichung eines Lernens just-in-time und on-demand kann methodisch ein praxisnaher Erwerb von Wissen und Fähigkeiten angestoßen werden, dessen Transfer in eigenes Handeln schon in den Lernpfaden selbst thematisiert wird. So kann das geplante Lern- und Vernetzungsangebot mit seinen auf die Professionalisierung von Lehrenden angelegten Inhalten einen Beitrag zur Förderung der Qualität der Weiterbildungspraxis leisten. Weiter schafft es Strukturen für eine weiterführende Forschung zum Lernverhalten lehrender Erwachsener im Umgang mit digitalen Medien.

\section{Literatur}

Arnold, Rolf/Schüßler, Ingeborg (1998): Wandel der Lernkulturen. Ideen und Bausteine für ein lebendiges Lernen. Darmstadt: Wissenschaftliche Buchgesellschaft.

Boud, David/Feletti, Graham I. (Eds.) (1997): The Challenge of Problem-Based Learning. 2nd edition. London: Cambridge University Press.

Bundesministerium für Bildung und Forschung (2013): Weiterbildungsverhalten in Deutschland. AES 2012 Trendbericht. http://www.tns-infratest.com/sofo/ _pdf/AES2012_ trendbericht_weiterbildungsverhalten.pdf. [Zugriff: 15.01.2016].

Gray, Bette (2002): Informal Learning in an Online Community of Practice. In: Journal of Distance Education 19, 1, S. 20-35.

Kerres, Michael (2013): Mediendidaktik. Konzeption und Entwicklung mediengestützter Lernangebote. 4. Auflage. München: Oldenbourg.

Kraft, Susanne/Seitter, Wolfgang/Kollewe, Lea (2009): Professionalitätsentwicklung des Weiterbildungspersonals. Bielefeld: Bertelsmann.

Kraus, Karin (2012): Professionalisierung und Beschäftigung in der Weiterbildung. Ein Beitrag unter besonderer Berücksichtigung Deutschlands und der Schweiz. In: Sgier, I./Lattke, S. (Hrsg.): Professionalisierungsstrategien der Erwachsenenbildung in Europa. Entwicklungen und Ergebnisse aus Forschungsprojekten. Bielefeld: Bertelsmann, S. 35-46.

Lave, Jean/Wenger, Etienne (1991): Situated learning: Legitimate peripheral participation. New York: Cambridge University Press.

Leutner, Detlev (1992): Adaptive Lehrsysteme: instruktionspsychologische Grundlagen und experimentelle Analysen. Weinheim: Psychologie Verlags Union.

Martin, Andreas/Jütten, Steffanie/Schömann, Klaus/Koscheck, Stefan/Ohly, Hanna/ Schmitz, Nadja/Alfänger, Julia/Cywinski, Robert/Dobischat, Rolf/Elias, Arne/ 
Rosendahl, Anna (i.V.): Personal in der Weiterbildung. Schlussbericht zum Projekt wb-personalmonitor.

Martin, Andreas/Langemeyer, Ines (2014): Demographie, sozioökonomischer Status und Stand der Professionalisierung - das Personal der Weiterbildung im Vergleich. In: Deutsches Institut für Erwachsenenbildung (Hrsg.): Trends der Weiterbildung: DIE-Trendanalyse 2014. Bielefeld: Bertelsmann, S. 43-88.

Nittel, Dieter (2000): Von der Mission zur Profession? Stand und Perspektiven der Verberuflichung in der Erwachsenenbildung. Bielefeld: Bertelsmann.

Nittel, Dieter (2006): Das Phänomen ,individuelle Professionalisierung‘. In: Nittel, D./Maier, C. (Hrsg.): Persönliche Erinnerung und kulturelles Gedächtnis. Einblicke in das lebensgeschichtliche Archiv der hessischen Erwachsenenbildung. Opladen: Barbara Budrich, S. 370-379.

Research voor Beleid (2008): ALPINE - Adult Learning Professions in Europe. A Study of the Current Situation, Trends and Issues. Final report. Zoetermeer.

Schrader, Josef (2010): Fortbildung von Lehrenden der Erwachsenenbildung: Notwendig? Sinnvoll? Möglich? In: Schrader, J./Hohmann, R./Hartz, S. (Hrsg.): Mediengestützte Fallarbeit - Konzepte, Erfahrungen und Befunde zur Kompetenzentwicklung von Erwachsenenbildnern. Bielefeld: Bertelsmann, S. 25-68.

Schrader, Josef/Goeze, Annika (2011): Wie Forschung nützlich werden kann. In: Report - Zeitschrift für Weiterbildungsforschung 34, 2, S. 67-76.

Tietgens, Hans (1988): Professionalität für die Erwachsenenbildung. In: Gieseke, W. (Hrsg.): Professionalität und Professionalisierung. Bad Heilbrunn: Klinkhardt, S. 28-75.

Weinberg, J. (1999): Lernkultur - Begriff, Geschichte, Perspektiven. In: Arbeitsgemeinschaft für Qualifikations-Entwicklungs-Management (Hrsg.): Kompetenzentwicklung, 99. Aspekte einer neuen Lernkultur. Argumente, Erfahrungen, Konsequenzen. Münster: Waxmann. S. 81-143.

Wenger, Etienne (2001): Communities of Practice: Learning, Meaning, and Identity. 2. Auflage. Cambridge: Cambridge University Press.

WSF Wirtschafts- und Sozialforschung (2005): Erhebung zur beruflichen und sozialen Lage von Lehrenden in Weiterbildungseinrichtungen: Schlussbericht. Kerpen: BMBF. 



\section{Panel III:}

Lernkulturen in Hochschulen 



\section{Lernkulturwandel. Eine Diskursanalyse der Stellungnahmen von ausgewählten zentralen hochschulpolitischen Akteuren zum Deutschen Qualifikationsrahmen (DQR)}

\section{Einleitung}

Der vielzitierte Lernkulturwandel von der Input- zur Outcome- und Kompetenzorientierung ist, so Arnold, ein Paradigmenwechsel. Aus Sicht des Konstruktivismus müssen die Lernsettings so verändert werden, dass sie die „Ausschnitthaftigkeit, Erfahrungsabhängigkeit sowie Selbstgeschlossenheit menschlicher Wahrnehmung" berücksichtigen. Dem wird ein studierendenorientierteres Lernen gerechter, das die Kompetenzentwicklung der Studierenden in den Mittelpunkt rückt und Selbstlernkompetenzen stärkt (Arnold 2012: 89, 15, 48).

Die Kompetenzorientierung wurde mit der Einführung des Europäischen Qualifikationsrahmens (EQR), des Hochschulqualifikationsrahmens (HQR) und des DQR von zentralen Akteuren als hochschulpolitische Zielformulierung verabschiedet, allerdings ohne dass sie sich in der Wissenschaft bereits als einziges Paradigma durchgesetzt hätte. ${ }^{1}$ Der subjektive Interpretationsspielraum ist zudem aufgrund der im Grundgesetz verankerten Freiheit von Lehre und Wissenschaft sehr weit und lässt auch widerständische Lesarten $\mathrm{zu}$.

Die unterschiedlichen Diskurse zum Lernkulturwandel lassen sich idealtypisch am Beispiel des Bildungsverständnisses ausgewählter zentraler hochschulpolitischer Akteure anhand der Entwicklung des DQR herausarbeiten. Anzunehmen ist, dass das Ringen um Positionen als Kräftefeld nach Bourdieu dargestellt werden kann, in dem im Sinne Foucaults soziale Praktiken durchgesetzt werden sollen.

Die Untersuchung erfolgte anhand einer empirischen Auswertung von Stellungnahmen der Hochschulrektorenkonferenz (HRK), der Kultusministerkonferenz (KMK), von Arbeitgeber-, Arbeitnehmer- und Studierendenverbänden. Mit Hilfe einer Kontrastierung der Stellungnahmen werden Unterschiede und Gemeinsamkeiten der politischen Akteure deutlich. Die Aufbereitung der grundsätzlichen Positionen soll zur Reflexion darüber beitragen, warum und wozu Studierende lernen und nach welchen Grundsätzen Studi- 
engänge konzipiert werden. Dazu wird eingangs der verwendete Diskurs- und Feldbegriff ausgeführt, der den DQR kurz erläutert, Sample und Methode werden dargelegt, die Stellungnahmen der hochschulpolitischen Akteure herausgearbeitet und abschließend miteinander kontrastiert und in einen sozialen Raum eingeordnet, um die Komplexität der Perspektiven auf den DQR darzustellen. Abschließend wird aufgezeigt, welche Diskurse sich durchgesetzt haben.

\section{Diskurs- und Feldbegriff am Beispiel von Bologna}

Zur Analyse von Stellungnahmen im wissenschaftlichen Feld ist es notwendig, „den Raum der Werke oder Diskurse im Sinne unterschiedlicher Stellungnahmen - und den Raum der Positionen derjenigen, die jene schaffen" zusammenzuführen (Bourdieu 1992: 17).

Bourdieu versteht „ein Feld als ein Netz oder eine Konfiguration von objektiven Relationen zwischen Positionen“ (Bourdieu/Wacquant 2013: 127). In diesem Sinne ist das Feld ein Kräftefeld, in dem die Akteure um die Kräfteverhältnisse ringen, wie Vester u.a. (2001: 17, 150-155) in Anlehnung an Bourdieu, Ernst Cassirer und Kurt Lewin ausführen. Insofern ist die Forderung nach einem Wandel oder Paradigmenwechsel immer auch ein Ringen um die Neuordnung eines Feldes und seiner Kräfte.

Diese Kräfte sind gerade in demokratischen Systemen nur selten offen autoritär. Mit Foucaults „Konzept der Gouvernementalität“ (Klingovsky 2009: 108) lassen sich auch ,didaktisch-methodische Praktike[n]“ (ebd.: 200) als Machttechniken verstehen, indem ,die traditionelle Unterscheidung in mikro- und makropolitische Analyseebenen“ umgangen wird. „In den Fokus der Analyse rückt die Beziehung von Herrschaftsformen und Subjekt und damit das Verhältnis der Regierung anderer (,gouvernement des autres ${ }^{\natural}$ ) zu Formen von Selbstregierung (,gouvernement des soi')“"(ebd.: 108).

Der Lernkulturwandel erfolgt in einer durch den Bologna-Prozess neustrukturierten Hochschullandschaft, die bereits neue Ansprïche an das Subjekt formuliert hat. Daheim unterscheidet den humanistischen Subjektdiskurs mit einem idealistischen Bildungsbegriff zur Überwindung sozialer und ökonomischer Unsicherheiten, einen unternehmerischen Subjektdiskurs (Stichwort Humankapital und unternehmerische Haltung) und einen Diskurs über „das sozio-technisch vernetzte(s) Subjekt" in einer Mediengesellschaft (Daheim 2012: 196). Klingovsky unterscheidet die Diskurse zum Lernkulturwandel anhand ihrer Fragestellungen, nämlich ,wie ein mehr an Selbststeuerung (Rolf Arnold), Selbstsorge (Hermann Forneck), gesellschaftlicher 
Teilhabe (Joachim Ludwig) oder Selbstverfügung (Erhard Meueler) ${ }^{2}$ in LehrLern-Prozessen zu erreichen ist. Alle fordern und fördern die Beteiligung der lernenden Subjekte“ (Klingovsky 2009: 2000). ${ }^{3}$

Die Subjektdiskurse haben gemeinsam, dass sie Anforderungen an die Subjekte formulieren. Daheim fürchtet, dass diese die Subjekte auch überfordern können, zumal das Versprechen ,für ein stärker strukturiertes, standardisiertes Studium mit größerer Leistungsorientierung und geklärter volkswirtschaftlicher Anbindung" noch nicht eingelöst ist. Noch sind, so Daheim, keine „wegweisenden Strategien“ entwickelt worden. Vielmehr wird noch versucht, ,den institutionellen Wandel zu verarbeiten, momentan allerdings zu Lasten der Studierenden“ (Daheim 2012: 199f.).

\section{Der DQR}

Die Erarbeitung eines DQR erfolgte, um auf nationaler Ebene einen Referenzrahmen für den zuvor im April 2008 verabschiedeten EQR zu formulieren (HRK 2010). Die Diskussion um den DQR, für die ein Arbeitskreis gegründet wurde, war ein Ringen von unterschiedlichen politischen Akteuren um die richtigen Definitionen und Niveaueinordnungen von Bildungsabschlüssen, um „Gleichwertigkeiten und Unterschiede von Qualifikationen transparenter zu machen und auf diese Weise Durchlässigkeit zu unterstützen“ sowie Mobilität zu fördern (AK DQR 2011: 3).

Um diese Ziele zu erreichen, ist im DQR die Vergleichbarkeit von Qualifikationen des Bildungssystems in acht Niveaustufen kompetenzorientiert ausformuliert. Der DQR ist aber nicht nur ein Referenzrahmen zur Vergleichbarkeit von Qualifikationen. Die Besonderheit ist, dass der DQR durch eine politische Entscheidung implementiert und damit der Lernkulturwandel hin zu einer Kompetenz- und Outcomeorientierung jenseits der klassischen Input- und Lehr(er)orientierung beschlossen wurde (siehe zum Beispiel Arnold 2012). Der DQR verwendet einen Handlungskompetenzbegriff mit den Teilbegriffen der „Fachkompetenz“, der in „Wissen“ und „Fertigkeiten“ unterschieden wird, sowie der „Personalen Kompetenz" mit der Unterscheidung in „Sozialkompetenz und Selbständigkeit“ (AK DQR 2011: 4).

2 Die Vornamen habe ich zur besseren Einordnung der genannten Wissenschaftler im Zitat ergänzt.

$3 \mathrm{Zu}$ den unterschiedlichen Diskursen zum Lebenslangen Lernen mit vergleichbaren Implikationen siehe z.B. Dietsche/Meyer 2004. 


\section{Sample und Methode}

Ausgewählt wurden zentrale Akteure des hochschulpolitischen Feldes, die auf die Hochschulpolitik entscheidenden Einfluss nehmen und auch im Arbeitskreis DQR vertreten waren ${ }^{4}$ :

- Die Vertretung der Hochschulen, vor allem der Hochschulleitungen: die HRK;

- die Vertretung der Wissenschaftsminister: die KMK;

- die gewerkschaftliche Arbeitnehmervertretung: der Deutsche Gewerkschaftsbund (DGB);

- die Arbeitgebervertretung: die Arbeitgeberverbände

- und die Vertretung der Studierenden: der freie zusammenschluss von studentInnenschaften (fzs).

Für die vorliegende Analyse wurden zentrale Aussagen der Verbände herausgearbeitet und kontrastiert (zur Fallkontrastierung siehe Kluge 1999: 121130), um die Unterschiede der Stellungnahmen zu veranschaulichen (zu den Stellungnahmen siehe auch Sloane 2008; Klenk 2013). Die unterschiedlichen Stellungnahmen stehen, wenn auch auf der Ebene der politischen Repräsentation, für unterschiedliche Wahrnehmungen (im Sinne des Konstruktivismus) und damit auch für unterschiedliche Erwartungen an das Studium. Die Kontrastierung zeigt auf, wie die Akteure im Sinne Bourdieus relational zueinander stehen und in einen sozialen Raum einzuordnen sind (siehe Bourdieu/Wacquant 2013: 127; siehe auch Bremer 2001: 56).

\section{Ergebnisse}

\subsection{HRK}

Die HRK kritisierte mit ihrer Stellungnahme vom 23.02.2010 zusammenfassend, dass wissenschaftliche Kompetenzen gegenüber berufsbildenden Kompetenzen benachteiligt würden. Insgesamt würde ,,der Druck zur Deregulierung des Zugangs zum Hochschulbereich [...] so groß, dass realistische Anforderungen an die Vorqualifikation aus dem Blick geraten und Studierende

4 Mitglieder der staatlichen Seite waren darüber hinaus Vertreter des Bundesministeriums für Bildung und Forschung (BMBF), des Bundesministeriums für Wirtschaft (BMWi), der Wirtschaftsministerkonferenz (WMK) und weitere Experten (siehe Klenk 2013: 139). BMBF und KMK waren bereits in ,die Entwicklung und Verabschiedung des DQR“ eingebunden (ebd.: 146). Die KMK war wohl der stärkste Vertreter der staatlichen Akteure, vertrat allerdings aufgrund der heterogenen Positionen vor allem ,,übergeordnete Zielsetzungen“" (ebd.: 147). BMBF, BMWi und WMK vertraten leicht voneinander abweichende Positionen, hielten sich aber noch stärker als die KMK zurück (ebd.: 144-149). 
wie Hochschulen überfordert würden. Notwendige Nachqualifikationen wären nicht leistbar und das Qualitätsniveau von Lehre und Studium müsste sinken“ (HRK 2010). Die HRK konnte sich zwar mit ihrer Kritik in weiten Teilen nicht durchsetzen, kann aber auch weiterhin mit dem bereits 2005 verabschiedeten, forschungsorientierteren Qualifikationsrahmen für deutsche Hochschulabschlüsse (HQR) ${ }^{5}$ arbeiten (siehe KMK 2005), weil dieser für die Akkreditierungsverfahren maßgeblich ist (Akkreditierungsrat 2013: 11), während der DQR nur freiwillig als Referenzrahmen hinzugezogen werden kann (Akkreditierungsrat 2014).

\subsection{KMK}

Die KMK befürwortete zwar in ihrer Stellungnahme die „Gleichwertigkeit von allgemeiner und beruflicher Bildung". Sie sprach sich aber auch dafür aus, ,sowohl die Allgemeine Hochschulreife und die Fachgebundene Hochschulreife als auch entsprechende Berufsabschlüsse auf Stufe 5 der europäischen Skala von Bildungsabschlüssen zu verorten" und somit das Abitur im Vergleich mit vielen anderen Berufsabschlüssen höher einzuordnen.

Der damalige KMK-Präsident, der niedersächsische Kultusminister Althusmann,

„,bezeichnete es als vordringliche bildungspolitische Herausforderung der nächsten Jahre, den Anteil von Schülerinnen und Schülern mit geringeren Kompetenzen in zentralen Lernbereichen deutlich zu senken, die Zahl der Schul- und Ausbildungsabbrüche weiter zu vermindern sowie die Bildungsbeteiligung von Kindern mit Migrationshintergrund weiter zu erhöhen“ (KMK 2011).

Der HQR wurde von HRK, KMK und BMBF erarbeitet ,und von der“ KMK ,am 21.04.2005 beschlossen“ (KMK 2005). Er unterscheidet Wissen, Verstehen und Können und enthält nicht nur mehr wissenschaftliche als personale Kompetenzen, sondern auch die „systemische Kompetenz" als Teilkompetenz von Können, um auch bei schwierigen Informationslagen ,wissenschaftlich fundierte Entscheidungen zu fällen und dabei gesellschaftliche, wissenschaftliche und ethische Erkenntnisse [zu] berücksichtigen“" (KMK 2005: 29). 


\section{3 $\mathrm{DGB}^{6}$}

„Der DGB erwartet, dass ein DQR die Durchlässigkeit in und zwischen den Bildungsbereichen, insbesondere die Zugänge zum tertiären Bereich verbessert. Insgesamt geht es um mehr Chancengleichheit und die Herstellung der Gleichwertigkeit von allgemeiner und beruflicher Bildung im Bildungssystem“ (DGB 2008). Der DGB kritisiert, den „Kompetenzbegriff der EU“ mit seiner einseitigen Ausrichtung ,vorrangig auf die Beschäftigungsfähigkeit (Employability)“. Der DGB befürchtet, dass die „Outcome-Orientierung“ zu einem „Lernen ohne gesellschaftliche Planung und Verantwortung und ohne gesellschaftspolitisch-emanzipatorische Zielsetzungen“ führen könnte (DGB 2008). „Die Kompetenzentwicklung soll die berufliche Handlungskompetenz herausbilden sowie zu einer reflexiven Handlungsfähigkeit beitragen, die den Vollzug von Arbeitshandlungen unter weitgehender Mitbestimmung und Partizipation in der Arbeit und in der Gesellschaft ermöglicht“" (DGB 2008). ${ }^{7}$

\subsection{Arbeitgeberverbände}

Die Bundesvereinigung der Arbeitgeberverbände (BDA) u.a. ${ }^{8}$ halten die Ausrichtung des DQR ,am Bedarf der Unternehmen“ für notwendig und fordern, dass der DQR ,einen Mehrwert für alle am Wirtschaftsprozess Beteiligten schafft". BDA u.a. teilen die Ziele des DQR, wie die Durchlässigkeit zwischen beruflichem und hochschulischem Bildungssystem, und den Handlungskompetenzbegriff mit seiner Unterscheidung in Fach- und Personalkompetenz. Auch die Outcomeorientierung wird begrüßt, um ,die Gleichwertigkeiten zwischen Qualifikationen sichtbar zu machen, die in verschiedenen Lernkontexten und an verschiedenen Lernorten erworben werden“. BDA u.a. legen Wert darauf, dass die zentralen Akteure in die Er-

6 Teilgenommen haben Vertreter von DGB, IG Metall, der Gewerkschaft Erziehung und Wissenschaft (GEW) und dem Deutschen Beamtenbund (DBB). Klenk (2013: 153) weist jedoch zu Recht darauf hin, dass der DBB der Arbeitnehmerseite „faktisch“ nicht zuzurechnen ist. Der DGB war in seiner Kritik an der „Modularisierung von Qualifikationen“ und der Kompetenzorientierung im Vergleich mit den Einzelgewerkschaften „moderater“ (ebd.: 154f.).

7 Zu den Kritikpunkten der Gewerkschaften siehe auch zusammenfassend Klenk 2013: 154. Klenk hebt auch hervor, dass die Sozialpartner ,für erhebliche Teile des Entwicklungsprozesses als einheitliche[r], komplexe[r] Akteur zu begreifen" sind (ebd.: 149). Allerdings sind die Unterschiede hierbei auch zu betonen, wie die unterschiedlichen Schwerpunkte verdeutlichen (siehe auch nächster Abschnitt zu den Arbeitgeberverbänden).

8 BDA, Bundesverband der Deutschen Industrie (BDI), Bundesverband des Deutschen Großund Außenhandels e.V. (BGA), Deutscher Bauernverband (DBV), Deutscher Industrie- und Handelskammertag (DIHK), Hauptverband des Deutschen Einzelhandels (HDS) und Zentralverband des Deutschen Handwerks (ZDH). 
stellung und Verabschiedung des DQR eingebunden werden (BDA u.a. 2008).

Allerdings lassen sich auch sehr deutliche Unterschiede zwischen BDA sowie Handwerk und Kammern herausarbeiten. Der BDA fordert

„,die Modularisierung der Ausbildung, die Verbetrieblichung der Prüfungsorganisation sowie die Einführung einer zweijährigen Erstausbildung als Regelfall mit individuellen Fortbildungsmöglichkeiten, entweder in einem längeren Ausbildungsberuf, in der Weiterbildung oder , on the job“".

Handwerk und Kammern (DIHK) hingegen befürchten durch diese Individualisierung

„eine Entwertung der öffentlichrechtlichen Abschlussprüfung in den Kammern“ (Busemeyer: 5).

Die „Verbände BITKOM, Gesamtmetall, VDMA und ZVEI ${ }^{9 ، “}$ traten zudem für eine Ausdifferenzierung der zweijährigen Berufsausbildungen nach Schwierigkeitsgrad ein und forderten ihre unterschiedliche Niveaueinordnung, was die Gewerkschaften aber ablehnten (ebd.: 6).

\section{5 freier zusammenschluss von studentInnenschaften (fzs)}

Der fzs befürwortet in einer Stellungnahme aus dem Jahre 2008 die im Rahmen des Bologna-Prozesses angesteuerte ,Studierendenzentrierung und Kompetenzorientierung“, fordert aber eine Nachsteuerung, um ,individuelle Lernstrategien“ über das Angebot „verschiedene[r] Lern- und Prüfungswege“, eines ,individuelle[n] Studienverlauf[s], flexible[r] Auslandssemester und Teilzeitstudium" sowie eine geringere Prüfungslast auch wirklich zu ermöglichen (fzs 2008).

Der fzs nimmt in seiner Stellungnahme zum KMK-Beschluss kritisch Stellung und fordert

- die „Gleichwertigkeit von beruflicher und allgemeiner Bildung“;

- die durchlässige Gestaltung des Bildungssystems auch für Nichtakademikerinnen und Nichtakademiker als Überwindung eines „ungerechte[n] Zustand[s], der weder aus sozialer Perspektive zu akzeptieren ist, noch dem Anspruch gerecht wird, Deutschland als Bildungsrepublik zu etablieren“;

9 Bundesverband Informationswirtschaft, Telekommunikation und neue Medien (bitkom), Verband Deutscher Maschinen- und Anlagenbau (VDMA), Zentralverband Elektrotechnikund Elektronikindustrie e.V. 
- die sozial gerechte und offene Gestaltung von Bildung auf den einzelnen Niveaustufen und ihre sinnvolle Verzahnung;

- die Zurücknahme des Beschlusses der KMK, die Allgemeine Hochschulreife auf Niveau 5 statt auf Niveau 4 und damit gegenüber vergleichbaren dreijährigen beruflichen Qualifikationen höher einzuordnen (fzs 2011).

\section{Vergleich der Stellungnahmen der Akteure}

Bereits die Auswahl der Zitate in Kapitel 5 erfolgte kontrastierend. Sie zeigt, dass sich die politischen Akteure in einem Kräftefeld an unterschiedlichen Paradigmen orientieren:

- Kulturelle Distinktion: Schwerpunktsetzung auf der Beibehaltung eines hohen Niveaus der Forschungs- und Methodenkompetenz (HRK) sowie der Einordnung des Abiturs als höchstes „Niveau“ der Hochschulzugangsberechtigung (KMK);

- Facharbeiterliche Teilhabe: Betonung von Durchlässigkeit der Bildungssysteme, Beruflichkeit, gesellschaftlicher Verantwortung und Mitbestimmung (DGB 2008).

- Studentische Teilhabe: Betonung der Ermöglichung ,individuelle[r] Lernstrategien“, einer geringeren Prüfungslast, der „Gleichwertigkeit von beruflicher und allgemeiner Bildung" sowie der sozial gerechten Öffnung des Bildungssystems;

- Feld der Wirtschaft und der Arbeitgeberlogik: Forderung nach einer Durchlässigkeit zwischen den Bildungssystemen, einer Ausrichtung ,am Bedarf der Unternehmen“ und der Schaffung eines „Mehrwert[s] für alle am Wirtschaftsprozess Beteiligten“" (BDA u.a. 2008).

Die Unterschiede bestehen in unterschiedlichen Feldlogiken, die jeweils gewahrt werden sollen. Die Gemeinsamkeiten bestehen prinzipiell in der Verbesserung der Durchlässigkeit und der Mobilität sowie einer Kompetenzund Outcomeorientierung, deren Gewichtungen aber sehr unterschiedlich gewertet werden, wie die Grafik noch einmal veranschaulicht: 


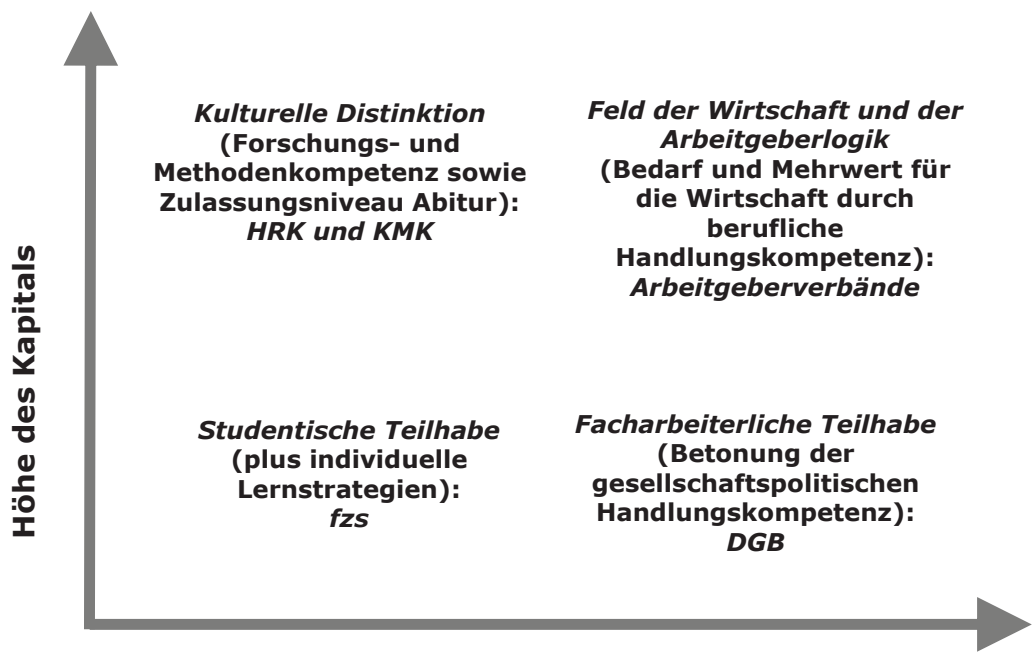

\section{Mehr kulturelles Kapital Mehr ökonomisches Kapital}

Siehe auch die sozialen Raummodelle von Bourdieu 1992; Tippelt u.a. 2008;

Vester u.a. 2001

Abb.1: Einordnung der Stellungnahmen in einen sozialen Raum (eigene Darstellung)

An der Hochschule Kaiserslautern wurde im Rahmen des Projektes „Offene Kompetenzregion Westpfalz" gemeinsam mit der Stabsstelle für Qualität in Studium und Lehre eine Kompetenzdefinition erarbeitet. Dabei wurde darauf geachtet, dass neben dem DQR vor allem auch der verpflichtende HQR Referenzrahmen ist. Die Kompetenzdefinition enthält auch die Dimension der gesellschaftlichen Verantwortung, wie sie die Gewerkschaften gefordert haben: „ethisches Bewusstsein (zivilgesellschaftliche Relevanz beurteilen und moralisch handeln zu können)“ (Fendler/Reinhardt u.a. 2014: 8). ${ }^{10} \mathrm{Zu}$ dem sind die fachlichen und personalen Kompetenzen hochschulbezogen

10 „Die Fachkompetenz wird hierbei als theoretisches sowie methodisches Wissen bzw. Überzeugungen, kognitive und praktische Fertigkeiten zusammengefasst. Wohingegen die Personale Kompetenz überfachliches Wissen, Überzeugungen, Fertigkeiten und Fähigkeiten fokussiert [...]. Die Fachkompetenz und die Personale Kompetenz schließen die Methodenkompetenz jeweils mit ein. Wodurch diese auch als Querschnittskompetenz zu verstehen ist“" (Fendler/Reinhardt u.a.: 9). 
weiter ausgedeutet und konkretisiert worden, um den besonderen Anforderungen wissenschaftlicher Kompetenzen gerecht zu werden.

\section{Schlussfolgerungen}

Die Untersuchung hat gezeigt, dass die Diskurse in einem sozialen Raum als Kräftefeld eingeordnet werden können und sich so die Unterschiede an den Koordinaten ökonomisches und kulturelles Kapital (Bourdieu) veranschaulichen lassen. Die Diskurse stehen also für unterschiedliche Koordinaten und damit auch für unterschiedliche Lernpraktiken (Foucault): stärker kulturell distinktiv (HRK und KMK), stärker an der Wirtschaft orientiert (BDA u.a.), stärker teilhabeorientiert und gesellschaftskritisch (DGB) sowie an individualistischen/partizipatorischen Lernbedarfen der Studierenden orientiert (fzs).

Es konnte herausgearbeitet werden, dass die unterschiedlichen politischen Akteure jeweils unterschiedliche, teils auch gegensätzliche Schwerpunkte haben, so dass die These von den unterschiedlichen Lesarten des DQR und der entsprechenden Diskurse zutrifft. Die verabschiedete Fassung genügt am ehesten der Position der KMK, mit Ausnahme der Einordnung des Abiturs, sowie der Position der Arbeitgeberverbände. Allerdings hatte sich bspw. der BDI vergeblich für eine noch stärker am Bedarf einzelner Unternehmen ausgerichtete Outcomeorientierung und für eine Aufweichung einer eher egalitären Beruflichkeit eingesetzt.

Der Funktionalität der Ausbildung für den Arbeitsmarkt wurde höhere Priorität eingeräumt und die dafür notwendige personale Kompetenz gestärkt, wie sie der Arbeitsmarkt aus Sicht der Arbeitgeber erfordert. Auch die vor allem fachlichen Forschungs- und Methodenkompetenzen zählen zu den zentralen Kompetenzen des DQR.

Reflektiert werden sollte, warum die Forderung nach einer gesellschaftspolitischen Kompetenz bspw. in Form von Teilhabe, wie sie vor allem DGB, aber auch der fzs forderte, im DQR keinen Niederschlag gefunden hat. Zu vermuten ist, dass nach Funktionalität entschieden wurde, d.h. weiterhin Forschungskompetenzen zu vermitteln und für den Arbeitsmarkt auszubilden. Die Lesarten von DGB und fzs haben aber weiterhin ihre Berechtigung und sollten bei der Konzeption von Studiengängen berücksichtigt werden. So mahnt auch Faulstich (2015: 14) dazu, widerständische und kritische Diskurse für eine „Wissenschaft in öffentlicher Verantwortung“ zu stärken.

Der Lernkulturwandel ist zwar nicht das Lösungsmodell zur Überwindung der Unterschiede. Jedoch regt er zur Reflexion darüber an, welche Kompetenzen in den Studiengängen entwickelt werden sollen, und stellt die Position in Frage, nur fachliche Kompetenzen zu fördern. Die Förderung von Selbstlernkompetenzen (Arnold) unterstützt zudem, dass Studierende mit ihren individuellen Wahrnehmungen im Sinne des Konstruktivismus selbst 
aktiver werden und ihre personalen Kompetenzen, auch ihre gesellschaftspolitischen, gezielter (weiter-)entwickeln können. Auch deshalb sollten die Teilhabeforderungen von DGB und fzs stärker berücksichtigt werden, um einen ganzheitlichen Lernkulturwandel zur Stärkung der Selbstlernkompetenzen zu befördern.

$\mathrm{Zu}$ vermuten ist, dass unterschiedliche Hochschulprofile, Studienfachkulturen und Studienformate unterschiedliche Schwerpunkte bei der Studiengangsentwicklung und damit auch blinde Flecken befördern können. Hier besteht weiterer Forschungsbedarf, auch um zu verstehen, wie die Entwicklung von Selbstlernkompetenzen angemessen unterstützt werden kann und wie einseitig formulierte Lernziele und im anderen Extrem überbordende Zielsetzungen für die Studiengangsentwicklung entstehen und im günstigen Fall vermieden werden können.

\section{Literatur}

Akkreditierungsrat (2013): Regeln für die Akkreditierung von Studiengängen und für die Systemakkreditierung. Beschluss des Akkreditierungsrates vom 08.12.2009. Zuletzt geändert am 20.02.2013. http://www.akkreditierungsrat.de/fileadmin/ Seiteninhalte/AR/Beschluesse/AR_Regeln_Studiengaenge_aktuell.pdf [Zugriff: 28.01.2016].

Akkreditierungsrat (2014): Newsletter Ausgabe 3/2014. http://www.akkreditierungsrat. de/fileadmin/Seiteninhalte/AR/Veroeffentlichungen/Newsletter/AR_Newsletter_20 14_03.pdf [Zugriff: 28.01.2016].

Arbeitskreis Deutscher Qualifikationsrahmen (DQR) 2011: Deutscher Qualifikationsrahmen für lebenslanges Lernen. http://www.dqr.de/media/content/Der Deutsche_Qualifikationsrahmen_fue_lebenslanges_Lernen.pdf [Zugriff: 14.01.2016].

Arnold, Rolf (2012): Ermöglichen. Texte zur Kompetenzreifung. Baltmannsweiler: Schneider Verlag Hohengehren.

Arnold, Rolf/Wolf, Konrad/Wanken, Simone (2015): Offene und kompetenzorientierte Hochschule. Band I zur Fachtagung „Selbstgesteuert, kompetenzorientiert und offen?!“ Baltmannsweiler: Schneider Verlag Hohengehren.

BDA u.a. (2008): Deutscher Qualifikationsrahmen (DQR). http://www.kwbberufsbildung.de/fileadmin/pdf/2010_DQR-Position.pdf [Zugriff: 28.01.2016].

Bourdieu, Pierre (1983): Ökonomisches Kapital, kulturelles Kapital, soziales Kapital. In: Kreckel, Reinhard (Hrsg.): Soziale Ungleichheiten. Göttingen: Schwartz Verlag, S. 183-198.

Bourdieu, Pierre (1992) [1984]: Homo academicus. Frankfurt a.M.: suhrkamp taschenbuch wissenschaft.

Bourdieu, Pierre/Wacquant, Loic J.D. (2013) [1987]: Die Ziele der reflexiven Soziologie Chicago-Seminar. Winter 1987. In: dies.: 2013 [1996]: Reflexive Anthropologie. Frankfurt a.M.: suhrkamp taschenbuch wissenschaft, S. 95-249.

Bremer, Helmut (2001): Zur Theorie und Methodologie der typenbildenden Mentalitätsanalyse. Reflexion und Diskussion zweier empirischer Studien. Dissertation 
Universität Hannover. http://edok01.tib.uni-hannover.de/edoks/e01dh01/ 329916602.pdf [Zugriff: 29.01.2016].

Busemeyer, Marius R. (2009): Die Europäisierung der deutschen Berufsbildungspolitik. Sachzwang oder Interessenpolitik? Friedrich-Ebert-Stiftung. Berlin. http://library.fes.de/pdf-files/id/ipa/06512.pdf [Zugriff: 29.01.2016].

Daheim, Susanne (2012): Das lernende Selbst in der Hochschulreform: „Ich“ ist eine Schnittstelle. Subjektdiskurse des Bologna-Prozesses. Bielefeld: Transcript Verlag.

DGB (2008): Deutscher Qualifikationsrahmen. http://www.netzwerk-weiterbildung. info/upload/m48490796666fe_verweis1.pdf [Zugriff: 29.01.2016].

Dietsche, Barbara/Meyer, Heinz H. (2004): Literaturauswertung Lebenslanges Lernen und Literaturnachweis zur Literaturauswertung Lebenslanges Lernen. Anhang 3 und Anhang 4 zur Strategie für Lebenslanges Lernen in der Bundesrepublik Deutschland. Bonn: DIE 2004. http://www.pedocs.de/volltexte/2010/1516/pdf/ 1516dietsche04_02_D_A.pdf [Zugriff: 01.02.2016].

Faulstich, Peter (2015): Aufklärung oder/und Management. Öffentliche und/oder unternehmerische Wissenschaft. In: DGWF Hochschule und Weiterbildung 2/2015, S. 10-15.

Fendler, Jan/Reinhardt, Max/Donner, Noemi/Fleuren, Daniela/Küßner, Viola/Lakatos, Michael/Requadt, Thorsten/Schohl, Susanne/Weber, Anja/Wiemer, Silke (2014): Kompetenzdefinition der Fachhochschule (heute Hochschule) Kaiserslautern. Stand: 16.05.2014. Kaiserslautern: HS Kaiserslautern.

fzs (2011): Offener Brief an die Mitglieder der Kultusministerkonferenz 08.12.2011. http://www.fzs.de/themen/gesetzliche_grundlagen/hochschulzugang/250814.html [Zugriff: 28.01.2016].

Gerhard, Andreas (2012): Kritik der Kompetenz. Zürich: Diaphanes.

GEW/fzs (2009): Lernende und Lehrende fordern Kurswechsel. Pressemittteilung am 18.06.2009. http://www.gew.de/studium/presse/detailseite/neuigkeiten/gew-undfzs-lernende-und-lehrende-fordern-kurswechsel/ [Zugriff: 28.01.2016].

HRK (2010): Zur Weiterentwicklung des Deutschen Qualifikationsrahmens (DQR). Empfehlung des HRK-Senats vom 23.02.2010. Bonn. http://www.hrk.de/ uploads/tx_szconvention/Empfehlung_DQR.pdf [Zugriff: 28.01.2016].

Klenk, Johannes (2013): Nationale Qualifikationsrahmen in dualen Berufsbildungssystemen. Akteure, Interessen und politischer Prozess in Dänemark, Österreich und Deutschland. Berlin: W. Bertelsmann Verlag.

Klingovsky, Ulla (2009): Schöne Neue Lernkultur. Bielefeld: Transcript Verlag.

Kluge, Susann (1999): Empirisch begründete Typenbildung. Zur Konstruktion von Typen und Typologien in der qualitativen Sozialforschung. Opladen: Leske + Budrich.

KMK (2005): Qualifikationsrahmen für Deutsche Hochschulabschlüsse. http://www. kmk.org/fileadmin/Dateien/veroeffentlichungen_beschluesse/2005/2005_04_21Qualifikationsrahmen-HS-Abschluesse.pdf [Zugriff: 29.01.2016].

KMK (2011): Pressemitteilung: Ergebnisse der 335. Plenarsitzung der Kultusministerkonferenz am 20./21. Oktober 2011 in Berlin. http://www.kmk.org/presse-undaktuelles/meldung/ergebnisse-der-335-plenarsitzung-der-kultusministerkonferenzam-2021-oktober-2011-in-berlin.html [Zugriff: 28.01.2016]. 
Sloane, Peter F.E. (2008): Zu den Grundlagen eines Deutschen Qualifikationsrahmens (DQR). Konzeptionen, Kategorien, Konstruktionsprinzipien. Bielefeld: W. Bertelsmann Verlag.

Tippelt, Rudolf/Reich, Jutta/Hippel, Aiga von/Barz, Heiner/Baum, Dajana (2008): Weiterbildung und soziale Milieus in Deutschland. Band 3: Milieumarketing implementieren. Bielefeld. http://www.die-bonn.de/doks/2008-marketing-01.pdf [Zugriff: 29.01.2016].

Vester, Michael/Oertzen, Peter von/Geiling, Heiko/Hermann, Thomas/Müller, Dagmar (2001): Soziale Milieus im gesellschaftlichen Strukturwandel. Zwischen Integration und Ausgrenzung. Frankfurt a.M.: suhrkamp taschenbuch wissenschaft. 

Rüdiger Rhein

\section{Die Universität als Lernort}

\section{Hintergrund}

Universitäten sind Orte zur Befassung mit wissenschaftlichem Wissen (Weber 2002: 7). Sie sind dies nicht nur als Forschungseinrichtungen, sondern auch als Trägerorganisationen des wissenschaftlichen Studiums und somit als Bildungseinrichtungen (vgl. einschlägig Huber 1984). Dieser Nexus erfordert eine Konzeptualisierung, wie wissenschaftliche Praxis und lernende Beschäftigung mit Wissenschaft aufeinander bezogen werden (können). Insofern werfen akademische Lehr- und Lernprozesse grundlegende Fragen auf: Was genau bedeutet es, Wissenschaft zu einem Gegenstand hochschulischen Lehrens und Lernens zu machen? Was ist das Charakteristische $a k a-$ demischer Bildung?

\section{Diskursive Verortungen}

In erwachsenenbildungswissenschaftlicher Hinsicht interessiert die Universität als Lernort zunächst formal, weil die Adressat_innen hochschulischer Bildung Erwachsene sind. In dieser Perspektive findet insbesondere die wissenschaftliche Weiterbildung Beachtung (Jütte/Weber 2005; Hanft/Knust 2007; Graeßner/Bade-Becker/Gorys 2009), ferner die „öffentliche Wissenschaft“, also die „Frage nach der Vermittlung wissenschaftlichen Wissens an diejenigen, die nicht unmittelbar an der Wissenschaftsproduktion beteiligt sind" (Faulstich 2008: IV). ${ }^{1}$

Hochschulen lassen sich ferner als einschlägiger Bestandteil des Bildungssystems auffassen (Teichler 2009) - insbesondere im Kontext des Lebenslangen Lernens (Wolter 2010; Kerres u.a. 2012) - indem sie spezifische Lern- und Bildungsräume eröffnen, in denen entsprechende Bildungsbedürfnisse und Bildungsbedarfe befriedigt werden (sollen). Erwachsenenbildungswissenschaftlich inspirierte Hochschulforschung fragt hier nach angemessenen Lernformen und nach der Funktion von Hochschulbildung (vgl. einschlägig Arnold 2015 - wenngleich aus konstruktivistischer Perspektive und unter Betonung der Kompetenzorientierung argumentierend).

Vgl. ferner zu popularisierenden Vermittlungsformen wissenschaftlichen Wissens Conein/ Schrader/Stadler 2004; zu wissenschaftsverständiger Vermittlung von Naturwissenschaft Bierbaum/Euler/Wolf 2007. 
Gleichwohl ist das Feld bildungswissenschaftlicher Hochschulforschung ein grundsätzlich transdisziplinäres; hochschulisches Lehren und Lernen lässt sich unter bildungswissenschaftlichen, bildungstheoretischen und bildungsphilosophischen Perspektiven betrachten, unter Perspektiven der Wissenschaftstheorie und der Wissenschaftssoziologie, unter Perspektiven der soziologischen, bildungsökonomischen und historischen Hochschulforschung und unter kulturwissenschaftlichen Perspektiven. ${ }^{2}$

\section{Bildungstheoretische Analyse hochschulischer Lernprozesse}

Dieser Beitrag zielt auf eine theoretische Analyse hochschulischen Lernens. Theoriearbeit wird hier als eine Betrachtungs- und Ausdrucksform verstanden, durch die signifikante Aspekte des fokussierten Sachverhaltes identifiziert, analysiert und interpretiert werden, um den Sachverhalt besser zu verstehen. Die Idee der Möglichkeit eines solchen tieferen Verständnisses basiert auf der Annahme, dass der Gegenstand theoretischer Untersuchung einen einschlägigen Eigen-Sinn besitzt, der sich im Zuge von Theoriearbeit explizit ausweisen lässt.

Angestrebt wird hier eine Explikation hochschulischen Lernens, indem durch die systematische Rekonstruktion von Wissenschaft als Lerngegenstand kategorial relevante Aspekte identifiziert werden. Für eine solche Rekonstruktion erweist sich eine kulturtheoretische Perspektive als fruchtbar: Wissenschaftliche Disziplinen können als Wissenskulturen nachgezeichnet werden; sie verweisen auf Grundideen und fachliche Konzepte, mit denen Wirklichkeit erschlossen und verfügbar wird. Lernen im Studium bedeutet (unter anderem) die reflektierte, auf sinnbezogenen Verstehens- und Aneignungsprozessen basierende Einübung in die spezifische Praxis des wissenschaftlichen Handelns, die es in ihren Intentionen und in ihrem Bedeutungsgehalt zu erschließen gilt.

2 Bildungswissenschaftliche Hochschulforschung ist disziplinär, thematisch und methodologisch ein weites Feld. Thematische Schwerpunkte sind u.a. psychologische und pädagogische Lernforschung, Sozialisations- und Biografieforschung, Kompetenzforschung, Lehrund Unterrichtsforschung sowie pädagogische Professionstheorie. Vgl. speziell zu Aspekten der Hochschulentwicklung unter pädagogischer Perspektive Euler 2013, Gerholz/ Sloane 2013, Brahm/Jenert/Euler 2016. Auch unter kulturwissenschaftlicher Perspektive ist Hochschulforschung ein weites Feld, zumal der Forschungshorizont vom zugrunde gelegten Kulturbegriff (vgl. dazu einleitend Moebius 2009) abhängt. Thematisiert wird hier u.a. die Frage, was genau es (für die beteiligten Akteur_innen) bedeutet, wenn Wissenschaft institutionell zu einem Gegenstand des Lehrens und des Lernens gemacht wird; vgl. dazu exemplarisch Darowska/Lüttenberg/Machold 2010, Mecheril u.a. 2013. 
Akademische Bildung nimmt dann Gestalt an in einer je spezifischen, dabei stets kontingenten und polymorphen Koppelung zwischen Wissenschaft als besonderer Praxis der Wissensproduktion einerseits und Studium als performativer Bezugnahme auf Wissenschaft und als Erschließung spezifisch bedeutsamer Sinn- und Handlungsressourcen andererseits.

\subsection{Ausgangspunkt Lernen}

Studieren ist die lernende Bezugnahme auf Wissenschaft zu spezifischen Zwecken. Lernen wird hier aufgefasst als spezifische kognitiv-emotionalsoziale Bezugnahme auf etwas: Jedes Lernen basiert auf einem externen Interaktionsprozess zwischen dem Lernenden und seiner sozialen, kulturellen und physisch-materialen Umgebung sowie einem davon zu unterscheidenden internen psychischen Prozess der Aneignung und Verarbeitung, in dem neue Impulse mit den Ergebnissen früheren Lernens verbunden sind (vgl. Illeris 2006: 29).

Dabei sind die Lerngegenstände in der Regel nicht per se Lerngegenstand, sondern Elemente einer vorgängigen Praxis, wobei unter Praxis menschliche Lebenstätigkeit im Allgemeinen, als tätiger Umgang bzw. als tätige Auseinandersetzung des Menschen mit der ihn umgebenden Wirklichkeit und mit sich selbst verstanden werden soll (vgl. Demmerling 1995).

Die Elemente vorgängiger Praxis werden jedoch zu Lerngegenständen in der Lernsituation, also in demjenigen Tätigkeitsvollzug, in dem sie lernend bearbeitet werden. Dabei sind in die jeweiligen Lerngegenstände und damit in die Lerninhalte immer schon unterschiedliche Aneignungsnotwendigkeiten eingelagert, die aus derjenigen Praxis herrühren, der die Lerngegenstände entstammen. Ferner ist lernende Aneignung immer eingebettet in biographische und sozial-kulturelle Aneignungssituationen, in denen sich Lernende aktuell befinden und die ihnen ihr Lernhandeln auf je spezifisch graduierte Weise als bedeutsam bzw. nicht bedeutsam erscheinen lassen. Untersuchungen des Lernens müssen also die Eigen-Strukturen der Lerngegenstände veranschlagen, und sie müssen danach fragen, auf welche Weise sich Aneignungshandeln als subjekbezogen bedeutsam artikuliert. ${ }^{3}$

3 Diese Überlegungen schließen an subjektwissenschaftliche Lerntheorien (Ludwig 1999) und an die kritisch-pragmatistische Lerntheorie sensu Faulstich $(2003 ; 2013)$ an. Veranschlagt werden ferner Ideen verstehensorientiert-kritischer Ansätze aus der schulischen Unterrichtsforschung (Gruschka 2009; Euler 2013, 2015). Zwar beziehen sich diese Ansätze auf schulisches Lernen (bei Euler explizit auf naturwissenschaftlichen Unterricht unter Rückbindung an Martin Wagenschein), den folgenden Überlegungen liegt aber die Annahme zugrunde, dass die These der Notwendigkeit eines verstehenden und genetischen Zugangs zu wissenschaftlichem Wissen nicht nur für schulisches Lernen und nicht nur für Naturwissenschaften, sondern auch im Studium und für sämtliche Disziplinen Gültigkeit 
Das Lernen an Hochschulen ist geprägt durch (1) die Eigenstrukturen von Wissenschaft, (2) die spezifische Strukturlogik explizit arrangierter LehrLern-Situationen und die Eigen-Arten informellen Lernens, (3) fach-, studiengang- und standortbezogene Lehrkulturen sowie (4) vielfältige idiosynkratische Lernhabitus und den jeweiligen besonderen (auch biographisch bedingten) Eigen-Sinn studentischer Lernprozesse sowie die individuellen Motivstrukturen der Studierenden.

Eine theoretische Rekonstruktion von studierendem Lernen veranschlagt zunächst den Eigen-Sinn von Wissenschaft, und sie versucht auszuweisen, auf welche Weise Sinn und Zweck des Studierens mit Sinn und Zweck von Wissenschaft korrespondieren (können). Dies bedeutet

- das Zurückdenken auf den Eigen-Sinn von Wissenschaft im Allgemeinen bzw. der einzelnen Disziplinen im Besonderen mit ihren Anschluss- und Verwendungsoptionen;

- das Zurückdenken auf Ziel und Zweck der lernenden Auseinandersetzung mit Wissenschaft als Gegenstand des Studiums - sowohl aus der Perspektive der wissenschaftlichen Fächer in Bezug auf die Funktion eines Studiums als auch aus der Perspektive der Studierenden in Bezug auf die Erschließung von Sinn- und Handlungsressourcen;

- die Spezifikation der durch die Studierenden zu erbringenden akademisch-intellektuellen Leistungen - im Nach-Vollzug der Eigen-Logik von Wissenschaft und im Vollzug der Erschließung von Sinn- und Handlungsressourcen;

- die Konzeptualisierung geeigneter Lernräume, in denen Studierende Lern- und Bildungsprozesse vollziehen können.

\subsection{Die Eigenstrukturen von Wissenschaft ${ }^{4}$}

Wissenschaft lässt sich als spezifische Praxis des Vernunftgebrauchs zu Erkenntniszwecken explizieren - also als Institution der methodisch ausgewiesenen und methodologisch begründeten Erzeugung von (gleichwohl potentiell vorläufigem) Wissen. Dabei reklamiert wissenschaftliches Wissen in Abgrenzung zu anderen Wissensformen die Erfüllung spezifischer Kriterien. ${ }^{5}$

beanspruchen kann. Vgl. zu Verstehensprozessen von Wissenschaft auch Pace/Middendorf 2004.

4 In diesem Abschnitt finden Überlegungen des Autors Eingang, die auch an anderer Stelle dargelegt worden sind; vgl. dazu Rhein 2015.

5 Hoyningen-Huene (2009: 22) identifiziert Systematizität ,als das, was Wissenschaft ausmacht“: „Wissenschaftliches Wissen unterscheidet sich von anderen Wissensarten, besonders dem Alltagswissen, primär durch seinen höheren Grad an Systematizität", wobei wissenschaftliches Wissen in neun Dimensionen systematischer ist als andere Wissensarten, nämlich hinsichtlich (1) Beschreibungen, (2) Erklärungen, (3) Vorhersagen, (4) der Vertei- 
Wissenschaft zeigt sich in der Pluralität der verschiedenen Disziplinen, die jeweils einschlägige Gegenstände und Fragestellungen als zur jeweiligen Fachwissenschaft zugehörig und jeweils spezifische Verfahren der Erkenntnisgewinnung als ,wissenschaftlich“ auszeichnen.

Allerdings ist damit der Gegenstand eines akademischen Studiums noch nicht abschließend erfasst: Neben Wissenschaft als epistemischem Projekt finden sich unterschiedliche akademische Praxen als spezifisch wissensbasiertes und begründet-reflektiertes Handeln im Hinblick auf

- den sachkundigen oder verantwortlichen Umgang mit Realgegenständen,

- den sachkundigen Entwurf technischer oder kultureller Artefakte, ihre sachverständige Produktion oder den adäquaten Umgang mit diesen,

- sozial-kommunikative Prozesse in grundlegenden lebensweltlichen Praxen.

Akademische Praxen verwenden zum Zwecke solchen verantwortlichen und sachkundigen Verrichtens, gekonnten Entwerfens, Konstruierens und Gestaltens oder professionellen Kommunizierens, Handelns und Urteilens nicht nur theoretisches, sondern auch praktisches, technisches und reflektorisches Wissen, das aus überindividuell verfügbaren, aktual bewährten Erfahrungen oder aus diskursiv-reflexiven Einsichten gewonnen wurde.

Die einzelnen Fächer sind dann typische integrale Verkoppelungen von wissenschaftlichen Disziplinen und akademischen Praxen: Hier werden analytisch-empirische, theoretisch-explanative, konzeptuell-programmatische, ethische, praxeologisch-operative sowie reflexive Umgangsmodalitäten mit den einschlägigen Gegenständen und Problemhorizonten auf eine je typische Weise konfiguriert.

Das Ziel von Wissenschaft ist Erkenntnis - Wissenschaft ist ein epistemisches Projekt. Davon zu unterscheiden sind die Zwecksetzungen wissenschaftlicher Praxis; dabei muss wiederum differenziert werden zwischen den Erkenntnisinteressen, also der Frage, wozu Wissenschaft betrieben bzw. wozu wissenschaftliches Wissen gewonnen wird, und den Praxen der Verwendung bzw. der Transformation wissenschaftlichen Wissens und den inner- und außerwissenschaftlichen Anschlussoptionen wissenschaftlich orientierter Tätigkeit.

digung von Wissensansprüchen, (5) kritischen Diskurses, (6) epistemischer Vernetztheit, (7) des Ideals der Vollständigkeit, (8) der Vermehrung von Wissen und (9) der Strukturierung und Darstellung von Wissen - ohne dass alle neun Dimensionen für alle Wissenschaften zwingend einschlägig sind (vgl. ausführlich Hoyningen-Huene 2013). Unbenommen bleibt, dass von ,der“" Wissenschaft im Kollektivsingular nur in allgemeiner Hinsicht gesprochen werden kann. 
Es lassen sich idealtypisch mindestens drei Erkenntnisinteressen wissenschaftlicher Tätigkeit rekonstruieren: ${ }^{6}$

- Befriedigung theoretischer Interessen - als Erzeugung theoretischen Wissens in Form von systematischen Beschreibungen, Erklärungen oder Verstehenshorizonten und Ausweis ihrer Geltungsbedingungen;

- Befriedigung poietischer (d.h. auch: technologischer ${ }^{7}$ ) Interessen - als Erzeugung von Verfügungswissen über Ressourcen und Prozesse im Hinblick auf die geschickte Produktion von Artefakten oder auf zielführendes, effizientes Verrichten;

- Befriedigung praktischer Interessen - als Erzeugung von Antwortvorschlägen auf die Frage nach den Formen und Ermöglichungsbedingungen lebenspraktischer Grundlagen und gelingender Lebensvollzüge und den Theoriefiguren zu ihrer Rechtfertigung.

Als Praxen der Verwendung bzw. der Transformation wissenschaftlichen Wissens und als inner- und außerwissenschaftliche Anschlussoptionen wissenschaftlicher Fächer lassen sich typologisch mindestens unterscheiden:

- Wissenschaft selbst - als Bearbeitung selbstreferentiell generierter Forschungsfragen mit dem Ziel der Erweiterung des Wissens;

- wissenschaftliche Expertise - als Bearbeitung von singulären und kontextuell verorteten Fragen, deren Beantwortung zwar wissenschaftliche Methoden erfordert, aber keinen allgemeinen wissenschaftlichen Erkenntnisgewinn erzielt, sondern lediglich einen konkreten Sachverhalt aufklärt;

- Transdisziplinarität - als Vermittlung zwischen Wissenschaft und außerwissenschaftlichen Anliegen; im Unterschied zur Expertise verbleibt das wissenschaftliche Wissen nicht exklusiv bei den wissenschaftlichen Experten, sondern wird geteilt, um durch das Teilen des Wissens zu neuen Einsichten oder Einschätzungen zu kommen;

6 Diese Skizzierung der Ziele wissenschaftlicher Fächer ist ein heuristisch gemeinter Vorschlag, der die habermassche Trias von technischem, praktischem und emanzipatorischem Erkenntnisinteresse (vgl. Habermas 1968) sowie die aristotelische Trias von theoria, poiesis und praxis veranschlagt.

7 Gemeint ist hier ein ,allgemeiner[..] Begriff von Technologie, den man im Anschluss an Luhmann so formulieren kann, dass von Technologie dort die Rede ist, wo ein Verfahren oder eine Handlungsweise wiederholt und von verschiedenen Personen benutzt werden kann, ohne dass die Nutzer einer Technologie alle Sinnimplikationen, die der Technologie zugrunde liegen, nachzuvollziehen gezwungen sind. (Vgl. Luhmann, N.: Macht. Stuttgart 1975, S. 70-74). Dieser Verzicht auf Nachvollzug und Prüfung aller zugrunde liegenden Sinnimplikationen macht gerade die handlungspraktische Ökonomie von Technologien aus und wird zur Bedingung ihrer sozialen Universalisierbarkeit." (Stichweh 2006: 45). 
- akademische Professionalität - als die sachkundige und sachverständige Bearbeitung komplexer Fragen und Problemstellungen in akademisierten Tätigkeitsfeldern und als Prozess der Generierung von Lösungen, dem die Entwicklung und Prüfung von Wissen nicht nur vorausgeht, sondern inhäriert, ohne dass hier der Anspruch erhoben würde, Wissenschaft zu betreiben.

\subsection{Wissenschaft als Lerngegenstand - Anforderungshorizonte im Studium}

Wissenschaftlichen Fächern liegen spezifische Grundideen der Gegenstandskonstitution und der Gegenstandsbehandlung zugrunde, die sich in fachtypischen Denkweisen, Konzepten und Methoden der Generierung von Wissen niederschlagen. Für jede Wissenschaftsdisziplin stellt sich folglich die Frage, auf welche Art und Weise die relevanten Realitätsausschnitte in Theorien, Modellen oder anderen Arten von Rekonstruktionen fasslich und für das Denken operabel gemacht werden, um letztlich die betreffenden Sachverhalte der Wirklichkeit zu verstehen, zu erklären, vorherzusagen, praktisch zu nutzen und zu ändern (Heckhausen 1987: 132).

Wissenschaft als Lerngegenstand fordert von den Studierenden, mit diesen Eigenstrukturen auf eine angemessene Weise umzugehen. Somit stellen sich bestimmte Anforderungen, die als Gelingensbedingungen eines wissenschaftlichen Studiums gelten können: Studieren bedeutet, den Eigen-Sinn von Wissenschaft als epistemischer Praxis zu verstehen, und zwar in theoretischer, in methodischer und in methodologischer Hinsicht. Gegenstandsangemessenes studierendes Lernen erfordert die Aneignung fachwissenschaftlicher Perspektivierungen und Erkenntnisse, die Aneignung legitimierter Verfahren der Genese von Erkenntnissen und die Aneignung der Begründungsmuster für die Auszeichnung von Gegenständen und Verfahren als wissenschaftlich.

Studieren als Erarbeitung des Verstehens des Eigen-Sinns von Wissenschaft bedeutet, zu begreifen, wie Wissenschaft zu ihren Erkenntnissen kommt und wie sie den Anspruch auf Geltung dieser Erkenntnisse begründet. Studieren bedeutet, den spezifischen Charakter wissenschaftlichen Wissens zu begreifen, das heißt

- die Funktion von Theorie und theoretischer Abstraktion zu verstehen;

- das Verhältnis von Theorie und Poiesis/Praxis zu begreifen, also die Zwecke wissenschaftlichen Tuns und die Verwendungsoptionen von Wissenschaft zu erfassen;

- zu wissen, dass - zumindest empirisches - wissenschaftliches Wissen häufig abduktiv gewonnen wird; 
- zu wissen, dass wissenschaftliches Wissen in der Regel paradigmatisch situiert ist;

- zu wissen, dass wissenschaftliches Wissen üblicherweise spezifischen Diskursarenen mit jeweils eigenen Begrifflichkeiten und Gegenstandsprämierungen entstammt;

- zu durchschauen, welche potentiellen Verkürzungen und Vereinseitigungen das disziplinäre Wissen enthält.

Studieren erfordert dabei, mit Zumutungen (und mit Zu-Mutungen) umzugehen, die sich aus der Eigen-Logik von Wissenschaft ergeben:

Eine Herausforderung im Studium ist der Umgang mit (Noch-)NichtVerstehen und (Noch-)Nicht-Wissen. Wissenschaftliche Aussagen stehen ,in einem voraussetzungsreichen, detaillierte Vorkenntnisse erforderlichen Wissenszusammenhang“ (Mittelstrass 1998: 183); dabei sind Prozesse des Verstehens wissenschaftlicher Erkenntnisse bzw. wissenschaftlicher Theoreme und Hypothesen nicht beliebig verkürzbar: „In gewissem, wohlbestimmtem Sinne ist die Wissenschaft, damit auch ihre Sprache, unvermeidlicherweise unverständlich. Wissenschaft befasst sich mit Dingen, die dem nicht-wissenschaftlichen Verstand nicht in gleicher oder in anderer Weise zugänglich sind.“ (Mittelstrass 1998: 183).

Hinzu kommt, dass die Universität - zumindest von ihrer Idee her - ,ein Grenzort [ist], wo die Normalität des ordentlichen Lernens und Wissens überschritten wird durch ein Übermaß des Außerordentlichen und Anomalen“ (Waldenfels 2009: 24). „Die Universität ist ein Ort, an dem Begriffe und Einsichten beständig neu zu denken sind und neu gedacht werden können " (Mecheril/Klingler 2010: 84); die Herausforderung besteht dann darin, sich auf die Universität als Ort, ,,an dem nichts außer Frage steht“ (Derrida 2001: 14), als „transgressive Lebensform“ (Mecheril/Klingler 2010) einzulassen.

\subsection{Das Studium als performative Bezugnahme auf Wissenschaft und als Erschließung von Sinn- und Handlungsressourcen}

Die Studierenden erfahren die Eigen-Logik von Wissenschaft aus der Perspektive ihres je konkreten Studienganges. Die Studiengänge speisen sich freilich aus verschiedenen fachlichen Perspektiven, werden von mehreren Lehrpersonen getragen und stellen sich auf diese Weise hinsichtlich Disziplinaritäten, Paradigmen und den durch die Lehrenden verkörperten Werthaltungen und Maximen als mehr oder weniger heterogen dar. Die Studierenden sind somit aufgefordert, unterschiedliche disziplinäre und paradigmatische Eigenlogiken zu verstehen, deren Heterogenität zu verarbeiten sowie den Gehalt, den Nutzen und die Grenzen inter-, multi- oder transdisziplinärer Verschränkungen und paradigmatischer Vielfältigkeiten nachzuvollziehen. 
Die Eigenstrukturen der Wissenschaft eröffnen Möglichkeiten akademischer Bildung, sofern sich die Studierenden im Laufe ihres Studiums - mehr oder weniger idiosynkratisch - die durch die jeweiligen Fachwissenschaften artikulierten bzw. eröffneten Wissens- und Könnensordnungen aneignen, indem sie den Eigen-Sinn von Wissenschaft zu verstehen suchen, idealerweise eigene Fragestellungen und Erkenntnisinteressen entwickeln und in innerwissenschaftlichen und fachbezogen-außerwissenschaftlichen Praxen Erfahrungen sammeln, diese Erfahrungen systematisch reflektieren und sich Teilhabeoptionen an entsprechenden Praxisgemeinschaften erarbeiten.

\section{Lehr- und Lernkulturen an Hochschulen}

Universitäre Lehrkulturen sind erstens durch die Spezifikation der Anforderungshorizonte charakterisiert, in der sich artikuliert, was Studierende verstehen soll $(\mathrm{t})$ en, welche verschiedenen disziplinären und paradigmatischen Perspektiven sie auf welche Weise integrieren soll(t)en und was sie können soll(t)en, wenn ihr Studium gelingen soll. Universitäre Lehrkulturen sind zweitens durch das veranschlagte Konzept der Studierbarkeit charakterisiert, wobei Studierbarkeit mehr ist als lediglich die zeitliche Bewältigung eines bestimmten Stoffumfangs. Studierbarkeit bedeutet, dass die Gelingensbedingungen des Studiums - Verstehen, Erwerb von profundem Wissen, Aufbau von Können - durch eine geeignete curriculare Struktur und durch das Arrangement adäquater Lernräume wahrscheinlich gemacht werden.

Es bedarf also einer geeigneten Studiengangarchitektur, um Lernräume zu eröffnen, in denen die Bearbeitung komplexer Frage- und Problemstellungen substantiell eingeübt werden kann. Solche Räume müssen nicht immer formal ausgewiesen sein, sie können auch innerhalb von Lehrveranstaltungen geschaffen werden; strukturlogisch handelt es sich dabei mindestens um

- Räume zur Gestaltung von Eingangsphasen, ${ }^{8}$

- Räume zum Aufbau einer fachlichen Wissensbasis und Räume zur Einübung in fachliche Könnensordnungen,

- Räume zur exemplarischen Fokussierung der Bearbeitung komplexer Frage- und Problemstellungen, ${ }^{9}$

8 Die Eingangsphase kann propädeutischen Charakter haben, sie kann aber auch der ErschlieBung der spezifischen Bedeutsamkeit des Gegenstandes, der Entwicklung spezifischer Fragestellungen oder dem Problemaufriss dienen.

9 Der Kern des Studiums - die Disponierung für die Bearbeitung komplexer Frage- und Problemstellungen - erfordert sowohl den Aufbau einer adäquaten Wissensbasis als auch eine an gekonntem Handeln orientierte exemplarische Bearbeitung fachlicher bzw. fachbezogener Fragestellungen. In Anknüpfung an die (durch den Studiengang angestrebten) Praxen der Verwendung bzw. der Transformation wissenschaftlichen Wissens liegen hier verschiedene 
- Räume für spezifische Vertiefungen,

- Räume zur kontinuierlichen Begleitung des Studierens, ${ }^{10}$

- Räume zur Gestaltung von Ausgangsphasen. ${ }^{11}$

Was sich konkret hinter diesen Räumen (in metaphorischem Sinne) verbirgt, hängt von den Entscheidungen über Ziele und Zwecke des jeweils konkreten Studienganges $a b$.

Hochschulische Lehr- und Lernkulturen, so ließe sich abschließend resümieren, sind dadurch charakterisiert, wie das Verhältnis zwischen Zugängen zum Verstehen, der Eröffnung von Teilhabeoptionen, den Zwecksetzungen wissenschaftlicher Praxis und der Gestaltung von Lernräumen und Studiengangarchitekturen konfiguriert ist. In erwachsenenbildungswissenschaftlicher Perspektive ist dabei interessant, wie das Verhältnis zwischen theoretischem, wissenschaftlichem Wissen zu beruflichem Wissen, Erfahrungswissen und lebensweltlicher Praxis konzeptualisiert wird und welche spezifische (auch: bildende) Bedeutung abstraktes, wissenschaftliches Wissen dabei gewinnen kann.

\section{Literatur}

Arnold, R. (2015): Bildung nach Bologna. Die Anregungen der europäischen Hochschulreform. Wiesbaden: Springer VS.

Bierbaum, H./Euler, P./Wolf, B. (Hrsg.) (2007): Naturwissenschaft in der Allgemeinen Weiterbildung. Probleme und Prinzipien der Vermittlung von Wissenschaftsverständigkeit in der Erwachsenenbildung. Bielefeld: WBV.

Brahm, T./Jenert, T./Euler, D. (Hrsg.) (2016): Pädagogische Hochschulentwicklung. Von der Programmatik zur Implementierung. Wiesbaden: Springer VS.

Formate handlungsorientierenden Lernens nahe: forschendes Lernen im Hinblick auf Wissenschaft, fallbezogenes Lernen im Hinblick auf wissenschaftliche Expertise, problembasiertes Lernen im Hinblick auf Transdisziplinarität und projektorientiertes Lernen im Hinblick auf akademische Professionalität.

10 Eine kontinuierliche Begleitung von Lernprozessen kann Folgendes leisten: (1) auf Verstehen zielende dialogisch prozessierte Vermittlung und Zueignung, als Erläuterung und Erörterung des Eigen-Sinns fokussierter Thematiken, Gegenstände oder Praxen; (2) eine gemeinsame Erörterung allgemeiner oder subjektiver Bedeutsamkeiten von Thematiken, Gegenständen oder Praxen; (3) eine kontextadäquate methodische Anleitung, Begleitung oder Supervision und Reflexion von Lern- und Aneignungsprozessen (Klärung von Lernzielen und -anlässen, Lernwegen und Lernschwierigkeiten); (4) Reflexion und Integration von Lernerfahrungen im Studium.

11 Ausgangsphasen dienen zur Reflexion auf (1) den Eigen-Sinn von Wissenschaft im Allgemeinen und der einzelnen Disziplinen im Besonderen (Sachlogik), (2) Sinn- und Handlungsressourcen, die sich in der lernenden Auseinandersetzung mit Wissenschaft prinzipiell erschließen lassen (Anschluss- und Verwendungsoptionen) sowie (3) die Verschränkung dieser beiden Perspektiven. 
Conein, S./Schrader, J./Stadler, M. (Hrsg.) (2004): Erwachsenenbildung und die Popularisierung von Wissenschaft. Bielefeld: WBV.

Darowska, L./Lüttenberg, T./Machold, C. (Hrsg.) (2010): Hochschule als transkultureller Raum? Kultur, Bildung und Differenz in der Universität. Bielefeld: Transcript, S. 83-116.

Demmerling, C. (1995): Praxis. In: Mittelstraß, J. (Hrsg.): Enzyklopädie Philosophie und Wissenschaftstheorie, Bd. 3. Stuttgart: Metzler, S. 336-337.

Derrida, J. (2001): Die unbedingte Universität. Frankfurt a.M.: Suhrkamp.

Euler, D. (2013): Von der Hochschuldidaktik zur Hochschulentwicklung. In: Zeitschrift für Berufs- und Wirtschaftspädagogik 109, 3, S. 360-373.

Euler, P. (2013): Verstehen als pädagogische Kategorie. In: Vierteljahrsschrift für wissenschaftliche Pädagogik 89, 4, S. 484-502.

Euler, P. (2015): Verstehen als Zentrum der Unterrichtsforschung. In: Czejkowska, A./Hohensinner, J./Wieser, C. (Hrsg.): Forschende Vermittlung. Gegenstände, Methoden und Ziele fachdidaktischer Unterrichtsforschung. Wien: Löcker, S. 21-39.

Faulstich, P. (2003): Weiterbildung. Begründungen lebensentfaltender Bildung. München, Wien: Oldenbourg.

Faulstich, P. (2008): Vermittler wissenschaftlichen Wissens. Bielefeld: Transcript.

Faulstich, P. (2013): Menschliches Lernen. Eine kritisch-pragmatistische Lerntheorie. Bielefeld: Transcript.

Gerholz, K.-H./Sloane, P. F. E. (Hrsg.) (2013): Studiengänge entwickeln - Module gestalten. Eine Standortbestimmung nach Bologna. Paderborn: EUSL.

Graeßner, G./Bade-Becker, U./Gorys, B.: Weiterbildung an Hochschulen. In: Tippelt, R./Hippel, A. von (Hrsg.): Handbuch Erwachsenenbildung/Weiterbildung. Wiesbaden: VS Verlag, S. 543-555.

Gruschka, A. (2009): Erkenntnis in und durch Unterricht. Empirische Studien zur Bedeutung der Erkenntnis- und Wissenschaftstheorie für die Didaktik. Wetzlar: Büchse der Pandora.

Habermas, J. (1968): Erkenntnis und Interesse. In: Habermas, J.: Technik und Wissenschaft als „Ideologie“. Frankfurt a.M.: Suhrkamp, S. 146-168.

Hanft, A./Knust, M. (Hrsg.) (2007): Internationale Vergleichsstudie zur Struktur und Organisation der Weiterbildung an Hochschulen. Münster: Waxmann.

Heckhausen, H. (1987): „Interdisziplinäre Forschung“ zwischen Intra-, Multi- und Chimären-Disziplinarität. In: Kocka, J. (Hrsg.): Interdisziplinarität. Frankfurt a.M.: Suhrkamp, S. 129-145.

Hoyningen-Huene, P. (2009): Systematizität als das, was Wissenschaft ausmacht. In: Information Philosophie 37, 1, S. 22-27.

Hoyningen-Huene, P. (2013): Systematicity. The Nature of Science. Oxford: University Press.

Huber, L. (Hrsg.) (1984): Ausbildung und Sozialisation in der Hochschule. Enzyklopädie Erziehungswissenschaft. Bd. 11. Stuttgart: Klett-Cotta.

Illeris, K. (2006): Das „Lerndreieck“. Rahmenkonzept für ein übergreifendes Verständnis vom menschlichen Lernen. In: Nuissl, E. (Hrsg.): Vom Lernen zum Lehren. Lern- und Lehrforschung für die Weiterbildung. Bielefeld: WBV, S. 29-41.

Jütte, W./Weber, K. (Hrsg.) (2005): Kontexte wissenschaftlicher Weiterbildung. Münster: Waxmann. 
Kerres, M./Hanft, A./Wilkesmann, U./Wolff-Bendik, K. (Hrsg.) (2012): Studium 2020: Positionen und Perspektiven zum Lebenslangen Lernen an Hochschulen. Münster: Waxmann.

Krüger, W. (1984): Wissenschaftliche Weiterbildung. Bad Heilbrunn/Obb.: Klinkhardt.

Ludwig, J. (1999): Subjektperspektiven in neueren Lernbegriffen. In: Zeitschrift für Pädagogik 45, 5, S. 667-681.

Mecheril, P./Klingler, B. (2010): Universität als transgressive Lebensform. In: Darowska, L./Lüttenberg, T./Machold, C. (Hrsg.): Hochschule als transkultureller Raum? Kultur, Bildung und Differenz in der Universität. Bielefeld: Transcript, S. 83-116.

Mecheril, P. u.a. (2013): Differenz unter Bedingungen von Differenz. Zu Spannungsverhältnissen universitärer Lehre. Wiesbaden: Springer VS.

Mittelstraß, J. (1998): Wissenschaft verstehen. In: Mittelstraß, J.: Die Häuser des Wissens. Wissenschaftstheoretische Studien. Frankfurt a.M.: Suhrkamp, S. 181-189.

Moebius, S. (2009): Kultur. Bielefeld: Transcript.

Pace, D./Middendorf, J. (Eds.) (2004): Decoding the Disciplines: Helping Students Learn Disciplinary Ways of Thinking. San Francisco: Wiley.

Rhein, R. (2015): Hochschulisches Lernen - eine analytische Perspektive. In: Report - Zeitschrift für Weiterbildungsforschung 38, 3, S. 347-363.

Stichweh, R. (2006): Die Universität in der Wissensgesellschaft. Wissensbegriffe und Umweltbeziehungen der modernen Universität. In: Soziale Systeme 12, 1, S. 33-53.

Teichler, U. (2009): Hochschulbildung. In: Tippelt, R. (Hrsg.): Handbuch der Bildungsforschung. Wiesbaden: VS Verlag, S. 421-444.

Waldenfels, B. (2009): Ortsverschiebungen, Zeitverschiebungen. Modi leibhaftiger Erfahrung. Frankfurt a.M.: Suhrkamp.

Weber, W. E. J. (2002): Geschichte der europäischen Universität. Stuttgart: Kohlhammer.

Wittpoth, J. (1987): Wissenschaftliche Rationalität und berufspraktische Erfahrung. Bad Heilbrunn/Obb.: Klinkhardt.

Wolter, A. (2010): Die Hochschule als Institution des lebenslangen Lernens. In: Wolter, A./Wiesner, G./Koepernik, C. (Hrsg.): Der lernende Mensch in der Wissensgesellschaft: Perspektiven lebenslangen Lernens. Weinheim: Juventa, S. 53-80. 


\section{"Kinder der anderen Kultur". Zur Bedeutung von Kulturalisierungs- und Rassismuskritik für die universitäre Lehramtsausbildung}

Der Kulturbegriff findet trotz seiner Unklarheit und Unschärfe auf vielfältige Weise Verwendung. Kunst, Musik und Literatur als Teil eines kulturellen Guts, Esskulturen, Arbeitskulturen, aber auch eine ethnisierende, an Herkunft und Nationalität gebundene Bezugnahme auf Kultur sind Bestandteile unterschiedlicher Diskurse (vgl. Höhne 2001: 197). Im Feld der Bildung wird zum einen der Frage nach unterschiedlichen Lernkulturen nachgegangen, zum anderen aber auch der Bedeutung von ,natio-ethno-kulturellen Zugehörigkeiten" (Mecheril 2004: 20) für das Bildungssystem und für Bildungsbiographien im Speziellen.

Kulturelle Zugehörigkeit und damit einhergehend kulturelle Differenzen erfahren mitunter seit den UNESCO-Deklarationen (1951) und der damit verbundenen Ablehnung des Rassebegriffs in ihrer Bedeutung eine starke Aufwertung. Wo kulturelle Differenzen bis hin zu einer Unaufhebbarkeit und Unvereinbarkeit thematisiert werden, wird eine kulturrassistische Funktion erfüllt, ,weil statt eines rassischen ein kultureller Determinismus unterstellt wird, aufgrund dessen ein Individuum in seiner Art und Sozialität naturwüchsig festgelegt wird“" (Höhne 2001: 200).

Dieser Beitrag widmet sich der Thematik von Kultur als einer sozial konstruierten Differenzierungskategorie, die der Thematisierung von Kultur als homogen abgeschlossenem System und natürlichem Identitätsmerkmal kritisch gegenübersteht. Die Bedeutung von Kultur als gesellschaftlicher Unterscheidungsdimension wird speziell vor dem Hintergrund des Wissenserwerbs und der Kompetenzentwicklung angehender Lehrer_innen in den Fokus gerückt. Die Notwendigkeit eines reflektierten und sensiblen Umgangs mit der Thematik Kultur ist angesichts einer bestehenden migrationsgesellschaftlichen Realität - auch in der Schule - Ausgangspunkt des im Folgenden vorgestellten Hochschulprojekts in der universitären Lehramtsausbildung. Anknüpfend an die rassismustheoretische Kritik einer Kulturalisierung auch in der Pädagogik (vgl. Messerschmidt 2008: $11 \mathrm{ff}$.) führt das Institut für Allgemeine Pädagogik und Berufspädagogik der Technischen Universität Darmstadt seit 2014 ein solches Projekt unter dem Titel „Heterogenität als Qualitätsherausforderung in der Lehramtsausbildung"1 durch. Ziel ist dabei die Umsetzung von Lehrveranstaltungen, in denen Studierende des gymnasialen und berufli-

Im Folgenden wird die Abkürzung „Heterogenität“ verwendet. 
chen Lehramts eine machtreflexive Perspektive auf Bildung und Migration entwickeln können. Das Projekt wird nachfolgend in seinen gesellschaftlichen Anlässen, seinen theoretischen Zugängen und den damit verbundenen Lehrinhalten vorgestellt und anhand von exemplarischen Einblicken in die seminarbegleitenden Evaluationen präzisiert.

\section{Problemhorizont: Kultur als Bezugsgröße für migrationsgesellschaftliche Unterscheidungsroutinen}

Das Hochschulprojekt „Heterogenität“ richtet sich an junge Erwachsene, welche die eigene Schulgeschichte bereits durchlaufen haben und sich an der Schwelle zur pädagogischen Profession befinden. Es soll die Entwicklung einer kulturalisierungs- und rassismuskritischen Perspektive auf Bildung in globalen Dominanzverhältnissen fördern. Um diese Perspektive systematisch aufzubauen, werden jedes Semester drei Seminare mit jeweils unterschiedlichen Ausrichtungen angeboten: ein Theorieseminar zur Vermittlung von Grundlagen sozialwissenschaftlicher Rassismuskritik und Migrationspädagogik, ein Theorie und Praxis reflektierendes Seminar mit explizitem Bezug zu rassistischen Diskriminierungen in Schule und Unterricht sowie ein biographisch orientiertes Seminar zur Problematik von Ungleichheit und Subjektivierung. In jedem Seminar werden zu Beginn und zum Ende des Semesters qualitative Evaluationen durchgeführt, um Einblicke in die Erwartungshaltungen und den Entwicklungsprozess der teilnehmenden Lehramtsstudierenden zu erhalten. Die Evaluationen sind dabei nicht als systematisch ausgearbeitetes Forschungsdesign zu verstehen, sondern als eine Art Abgleichsinstrument, mit dessen Hilfe z.B. mögliche ,Verstehensklippen " und Lernwiderstände in Bezug auf die besprochenen Seminarinhalte ermittelt und bei darauffolgenden Seminarkonzeptionen entsprechend berücksichtigt werden können.

Bereits die Eingangsevaluationen zeigen, dass insbesondere Kultur bei den Studierenden als wichtiges Thema für die eigene Professionsentwicklung und spätere Lehrtätigkeit aufgefasst wird. Zu beobachten ist hier vorwiegend der Wunsch und die Erwartung der Teilnehmer_innen nach einem Verständnis und Kenntnissen um kulturelle Differenzen und Besonderheiten verschiedener Kulturen. Paradigmatische Einzelaussagen der Eingangsevaluation lassen diese Stimmen deutlich zu Wort kommen:

„Wie geht man als Lehrerin mit Kindern anderer Kulturen um, die durch die Erziehung von zu Hause beigebracht bekommen, dass Frauen nicht ernst zu nehmen sind?! [...] Gründe für Verhalten herausarbeiten“

„Ich erwarte viele hilfreiche Tipps für die Praxis und vor allem Wissen um Problemen entgegenzuwirken.“ 
„Verstehen von Werten in anderen Kulturen - auch für mehr Toleranz untereinander" 2

Dieses Ergebnis, Wissen um die Andersheit der Anderen erwerben zu wollen, deckt sich mit der Beobachtung einer - nicht nur - pädagogischen Praxis. So lassen sich auch in zahlreichen formellen und informellen Bildungskontexten Fortbildungen und Angebote zu interkultureller Kompetenz auffinden, die Kenntnisse und Informationen über andere Kulturen versprechen. Der Wunsch, die ,andere ' und als ,fremd ' markierte Kultur zu verstehen und kennenzulernen, um damit mögliche ,Kulturkonflikte` zu vermeiden, wird dabei zumal seit dem Beschluss über „Interkulturelle Bildung und Erziehung“ der Kultusministerkonferenz (1996) nicht selten insbesondere für das Klassenzimmer formuliert. Wie das erste Beispiel der Evaluation zeigt, spielt bei einem solch kompensatorischen Verstehensanspruch offenbar nicht nur ein kulturtechnisches Wissen über allgemeine gesellschaftliche Lebenspraktiken und Wertvorstellungen eine Rolle, sondern auch ein spezielles Wissen über Geschlechtermodelle, die mit bestimmten Kulturen stereotypisch assoziiert werden.

„Aber diese Wissensbestände haben eine Crux: Durch die Vermittlung
,technischen“ Wissens werden die Anderen als Andere erzeugt: Die mit
dem technischen Wissen [A]usgestatteten [...] werden dem Anderen be-
gegnen. Sie werden sehen, hören, wahrnehmen und interpretieren, was
sie durch die Fortbildung über den ,Fremden' erfahren haben; sie werden
ihren Blick, ihre Wahrnehmung an das Gelernte angleichen und Aspekte,
die diesen Wissensbeständen widersprechen, werden sie ausblenden. Sie
werden sich in der Fortbildung ein Bild vom ,Fremden“ machen - und
siehe da, es funktioniert: Überall begegnet ihnen eben dieser ,Fremde“.
Verstehen konstruiert den Anderen." (Broden 2009: 122, Hervorh. i.O.)

Im Hinblick auf Kulturen und kulturelle Differenzen verweisen Termini wie Kennenlernen, Vermitteln und Verstehen somit auf ein dominanzgesellschaftliches Verständnis von Migration, bei dem die Unterscheidung von ,Dazugehörigen “ und ,Nicht-Dazugehörigen' bzw. ,Anderen“ innerhalb der deutschen Mehrheitsgesellschaft ein tragendes Motiv ist.

Eben der Wunsch und diese Erwartung seitens der Seminarteilnehmer_innen als sich wiederholendes Ergebnis der Eingangsevaluationen im Projekt dienen als Anknüpfungspunkt für die Thematisierung von gesellschaftlichen Zugehörigkeitsordnungen. Zur zentralen Aufgabe wird die Beschäftigung mit der Frage, wie der und die ,Andere' hergestellt werden und welchen Beitrag Pädagogik als wissenschaftliche Theorie und gesellschaftli-

2 Sprecher_innen anonymisiert; Wintersemester 2015/16, Seminar „Rassismuskritische Lehrer_innen? Migrationsgesellschaftliche Realität der Schule“. 
che Praxis hierzu leistet. Denn diese Unterscheidungspraxis und Zuordnung ist bedeutend und gesellschaftsstrukturierend. Vor diesem Hintergrund formuliert Paul Mecheril bei der Beschreibung einer kritischen Pädagogik in der Migrationsgesellschaft durchaus präzise:

„Die migrationspädagogische Perspektive interessiert sich für pädagogische Bedingungen und Konsequenzen einer sozialen Ordnung hierarchisierter Differenzen; sie ist mit Ordnungen des Unterschieds und des Unterscheidens befasst, die immer auch Phänomene der Hierarchisierung und der machtvollen Unterscheidung und Hervorbringung der Differenz zwischen ,Anderen“ und ,Nicht-Anderen“ darstellen." (Mecheril 2004: 19)

Im Kontext von Lernen und Bildung erweist sich diese Unterscheidung als eine machtvolle vor dem Hintergrund von Bildungserfolg und Bildungsbenachteiligung. Spätestens seit den PISA-Ergebnissen Anfang der 2000er Jahre - und ebenso aktuellen - ist die Benachteiligung von Migrant_innen im und durch das Bildungssystem offiziell bestätigt. Deutlich gekennzeichnet wurde das deutsche Bildungssystem als ein äußerst selektives, welches die soziale Herkunft der Schüler_innen reproduziert. Insbesondere Institutionen pädagogischen Handelns wie die Schule weisen eine ,nationale Norm“ (Messerschmidt: in Druck), sowohl hinsichtlich der Bildungsinhalte als auch alltäglicher Abläufe und Routinen, auf. So schreibt auch Paul Mecheril, dass sich die Bundesrepublik und ihre Bildungsinstitutionen mit der migrationsgesellschaftlich bedingten Vielfalt schwertun: „Vor diesem Hintergrund wird nicht nur das Abstraktum Migration, sondern auch die Migrantin, der Ausländer und die ethnisch Andere zur schwierigen Angelegenheit" (Mecheril 2004: 10, Hervorh. i.O.). Schule als wichtiger Teil des migrationsgesellschaftlichen Bildungssystems geht insofern weiterhin stark von einer „Gleichartigkeits-Normalität aus und versteht Differenz und Heterogenität als Störung“" (ebd.: 15).

Eine kulturalisierungs- und rassismuskritische Perspektive und die Bemühung, einen derartigen Umgang mit gesellschaftlicher Heterogenität zu fördern, richtet sich indes weiterhin vorwiegend an Jugendliche und wird in der Erwachsenenbildung vernachlässigt (vgl. Messerschmidt: in Druck). Jugendliche und Schüler_innen zu einem sensiblen und reflektierten Umgang mit kulturellen Zuschreibungen zu befähigen, ist als Anfrage an Lehrer_innen durchaus legitim und von Relevanz. Dennoch stellt sich die Frage, ob pädagogisch Professionelle als Teil der institutionellen Machtstrukturen der Schule nicht selbst höherer Aufmerksamkeit bedürfen. Bedeuten würden dies, als Lehrperson die eigene Involviertheit in kulturalisierende und rassifizierende Praktiken sowie die bislang weitgehend dethematisierte Norm nationalstaatlicher Selbst- und Weltverständnisse explizit zum Thema zu machen. Hierbei gilt es zum einen zu berücksichtigen, wie normalisierte schuli- 
sche Prozesse rassistisches Wissen hervorbringen, indem sie an kulturgeschichtlich vereinseitigte Vorstellungen über natio-ethno-kulturelle Identität festhalten; zum anderen gilt es, rassistische Denk- und Handlungsweisen auf eine Weise sichtbar werden zu lassen, die über die Vorstellung von Rassismus als individuellem Vorurteil bzw. als biologistischer Ideologie hinausgeht und die stattdessen die Funktion kulturell begründeter Differenzsetzungen im gesellschaftlichen Alltag in den Blick nimmt. Das Heterogenitätsprojekt schafft eben diesen Raum der Auseinandersetzung, indem es zwei differenztheoretische Ansätze als Zugänge wählt.

\section{Theoretische Zugänge: (post-)koloniale Kulturalisierungen aus der Perspektive von Dekonstruktion und Intersektionalität}

Verstehen Bildungskonzepte Kulturgeschichte nicht schlicht als idealistische Fortschrittslinie menschlicher Errungenschaften, sondern als herrschaftsförmige „Praxis des Vergleichens“ (Höhne 2001: 199), dann rücken unweigerlich auch Konstruktionen von kultureller Höherwertigkeit und Hierarchisierung in den Blick. Für ein solches Verständnis ist die Auseinandersetzung mit einer postkolonialen gesellschaftlichen Realität unabdingbar: Denn trotz des aufklärerischen Gleichheitsgedankens legitimierte gerade die Idee einer Überlegenheit Weißer Europäer_innen die koloniale Expansion, Unterdrückung und Ausbeutung der ,nicht-kultivierten minderwertigen und fremden Anderen“ (vgl. Dietrich/Strohschein 2011: 117). Edward Said hat diese ,gewaltvolle hegemoniale Praxis“ (Castro Varela/Mecheril 2010: 42) bereits in den 1970er Jahren unter dem dialektischen Begriff des Othering gefasst, nach dem die naturalisierende und homogenisierende Festschreibung nicht-westlicher Kulturen grundsätzlich die Funktion der Beanspruchung einer westlichbürgerlichen Identität erfüllt. Aufklärung und Kolonialismus als historisch und systematisch miteinander verwobene Entwicklungslinien europäischer Kulturgeschichte zu begreifen, ist demnach ein wichtiges Grundanliegen der im Heterogenitätsprojekt verankerten Lehrveranstaltungen. Hierbei geht es über die Auseinandersetzung mit westlicher Kolonialgeschichte hinaus vor allem auch darum, wie diese hierarchisierenden Deutungsmuster in gegenwärtigen gesellschaftlichen Selbst- und Weltverständnissen noch immer fortwirken. Insbesondere in aktuellen gesellschaftspolitischen Debatten um Migration und Flucht ist derzeit eine Rhetorik der Abwehr zu beobachten, die strukturell an binäre Praktiken im Sinne des Othering anknüpft und anhand von „Grenzziehungen [...] entlang der kulturell bestimmten Herkunft" (Messerschmidt 2008: 6) einer nationalstaatlichen Überfremdungslogik Vorschub leistet. 
Einen angemessenen Zugang zu diesem historisch-systematischen $\mathrm{Zu}-$ sammenhang bietet der differenztheoretische Ansatz der Dekonstruktion, der parallel zur Etablierung in den Gender Studies seit den 1980er Jahren auch verstärkt Eingang in die Postcolonial und Cultural Studies sowie seit den 1990er Jahren auch in rassismuskritische und migrationspädagogische Perspektivenwicklungen gefunden hat (vgl. Mecheril/Plößer 2009: 2). Ausgehend von einem solchen Standpunkt gilt es, bildungsbürgerliche Geschichte entgegen ihrem aufklärerischen Egalitätspostulat nicht als ungebrochen positive zu verstehen, sondern mit ihren inneren Widersprüchen und Ambivalenzen in den Blick zu nehmen. ${ }^{3}$ Entsprechend der identitäts- und subjektkritischen Grundhaltung dekonstruktivistischer Positionen rückt hierbei die Frage in den Vordergrund, an wen sich Aufklärung als politisches und soziales Projekt in seinen realgesellschaftlichen Umsetzungen konkret richtet und wer von diesen Umsetzungen jeweils kategorisch ausgeschlossen wird. Universalistische Vorstellungen eines ,mündigen Subjekts ${ }^{4}$ werden auf diese Weise als partikulare und monokulturelle Adressierungen entkleidet, die nicht zuletzt in sprachlich vermittelten Begriffen und Argumentationsweisen tiefe Spuren hinterlassen haben (vgl. Arndt/Ofuatey-Alazard 2011). Gleichzeitig wird es durch diesen historisch-dekonstruierenden Blick möglich, OtheringPraktiken auch in zeitgenössischen gesellschaftlichen Diskursen wahrzunehmen und kritisch auf ihre machtvollen Funktionen zu befragen. Ein wichtiger Anknüpfungspunkt besteht dabei in der Beobachtung, dass gegenwärtig ,die Praxis des Othering in der Bezugnahme auf beispielsweise, die muslimischen Anderen“ bedeutsam [ist]" (Castro Varela/Mecheril 2010: 42). Die Konstruktion von scheinbar ,homogenen' und ,natürlichen' Gruppen wird im Rahmen des Projektes daher aktuell verstärkt unter dem Aspekt religiöskultureller Zuordnungen thematisiert und Antimuslimischer Rassismus als durchschlagende Unterscheidungsform der migrationsgesellschaftlichen Gegenwart ins Zentrum der Auseinandersetzung gerückt.

In enger Verknüpfung mit dekonstruktivistischen Annäherungen an Rassismus findet das Projekt einen zweiten wichtigen Zugang im Ansatz der Intersektionalität, der als theoretische Position ursprünglich aus sozialen Bürgerrechtsbewegungen ab Mitte der 1950er Jahre hervorgegangen ist (vgl. Krüger-Potratz 2011: 183). Dieser Ansatz ergänzt den vorgestellten Zugang der Lehrveranstaltungen insofern, als er die explizit rassismuskritische Per-

3 Mit dieser gesellschafts- und kulturgeschichtlichen Problematisierung überschneiden sich dekonstruktivistische Überlegungen jedoch auch mit Perspektiven, die im deutschsprachigen Raum bereits seit Ende der 1940er Jahre durch die Kritische Theorie sowie seit den 1970er Jahren durch die von dieser geprägten Kritischen Bildungstheorie entwickelt worden sind.

$4 \mathrm{Zu}$ betonen sei an dieser Stelle, dass Subjekt- und Menschsein nicht nur Weißsein, sondern auch Mannsein bedeutet. 
spektive postkolonialer Theorien aufgreift, diese aber gleichzeitig in Richtung einer insgesamt diskriminierungs- und machtkritischen Perspektive erweitert. Anknüpfend an die juristischen Einzelfalluntersuchungen Kimberlé Crenshaws ergibt sich diese Perspektiverweiterung aus der Annahme, dass Rassismus als gesellschaftliche Unterscheidungs- und Diskriminierungspraxis grundsätzlich auch in seiner Überlagerung mit unterschiedlichen Verhältnissen von Macht und Unterdrückung zu betrachten ist, die soziale Ungleichheiten im Spannungsverhältnis von Benachteiligung und Privilegierung (vgl. Krüger-Potratz 2011: 192) hervorbringen. Die Frage, wer gesellschaftlich wie partizipieren kann, ist demnach immer abhängig vom Zusammenwirken bestimmter Unterscheidungskriterien, die diese Partizipationsmöglichkeiten funktional bedingen und gesellschaftlich rechtfertigen. Differenzierungskategorien wie Kultur, Hautfarbe, Herkunft, Geschlecht, Sexualität, Klasse und Religion sind in diesem Sinne nicht als isolierte ,natürliche Wesensmerkmale" und damit als vermeintlich ,objektive Anlässe " für Diskriminierungen $\mathrm{zu}$ verstehen, sondern als gemeinsame Legitimationsgrundlage für materielle und symbolische Zugeständnisse. So wurde durch intersektionale Forschungspositionen bereits mehrfach herausgestellt, dass etwa kulturalistische Ethnisierungsprozesse häufig parallel zu Geschlechterkonstruktionen verlaufen (vgl. Kasatschenko 2015: 2), wodurch strukturell bedingte Ungleichheiten unbemerkt in scheinbar ,natürliche" Positionszuweisungen übersetzt werden. Der Intersektionalitätsansatz ermöglicht es indes nicht nur, die strukturellen Verflechtungen einzelner Differenzierungsdimensionen in den Blick zu nehmen, sondern beleuchtet beispielsweise auch, wie klassen- und geschlechtsbezogene Benachteiligungen durch kulturalisierende Argumentationslinien als „Ungleichheitsfaktoren dethematisiert“ (Krüger-Potratz 2011: 192) werden.

In Bezug auf schulische Bildungs- und Selektionsprozesse ist dieser $\mathrm{Zu}-$ sammenhang von differenzbegründeten Zuordnungs- und Diskriminierungspraktiken von zentraler Bedeutung. Dies gilt vor allem dann, wenn es darum geht, als Lehrperson ein problematisches Verhalten von Schüler_innen zu erklären und damit unterschiedliche Leistungsbewertungen zu rechtfertigen. Gerade im Schulunterricht greifen kultur- und geschlechtsstereotype Rollenbilder wie etwa ,emanzipiert, westlich, modern'vs. ,unterdrückt, rückständig, traditionell` (vgl. Kasatschenko 2015: 48-66) verstärkt als Erklärungsoptionen ineinander, was insbesondere für muslimisch identifizierte Schülerinnen folgenreich in Bezug auf „eine Prognose schulischer Leistungsfähigkeit" (Weber 2008: 52) sein kann. Mit der Verschränkung von Dekonstruktion und Intersektionalität als theoretischen Zugängen soll es angehenden Lehrkräften ermöglicht werden, machtvolle Differenzierungsmechanismen bereits vor dem ersten Referendariat wahrzunehmen und einen differenzreflexiven Umgang mit migrationsgesellschaftlicher Heterogenität jenseits von naturalisierenden Fremdzuschreibungen $\mathrm{zu}$ entwickeln. Hinsichtlich der 
Professionalisierung von Lehramtsstudierenden erfordert dies neben dem Erwerb von Wissens- und Kompetenzbeständen zunächst, auf subjektiven Theorien beruhende Haltungen und Handlungsweisen zu erkennen und als solche zu analysieren. Letzteres bedeutet unter anderem auch, den ,eigenen Subjektivierungsprozess bewusst zu reflektieren, negative Gefühle ins Bewusstsein und damit in ihre Bearbeitbarkeit zu holen“ (Zitzelsberger 2014: 67). Eine sich dekonstruktivistisch und intersektional verstehende pädagogische Zugangsweise kann dabei zu einer Entdramatisierung von Konflikten beitragen, indem diskriminierende Implikationen und Effekte im eigenen Denken und Handeln nicht als individuelle Fehleinstellungen, sondern als Ausdruck geschichtlich gewachsener „Ordnungen des Unterschieds und des Unterscheidens“" (Mecheril 2004: 19) angesprochen werden.

\section{Ausblick: pädagogische Professionsentwicklung zwischen Differenzreflexivität und konstitutiver Unbestimmtheit}

Ergänzend zur Eingangsevaluation werden die im Projekt durchgeführten Lehrveranstaltungen regelmäßig durch abschließende Evaluationen im Hinblick auf einen möglichen Reflexionsprozess und eine Perspektiverweiterung seitens der Studierenden ausgewertet. Ein Blick auf Einzelaussagen dieser Abschlussevaluationen ${ }^{5}$ zeigt, dass die Auseinandersetzung mit kulturalisierungs- und rassismuskritischen Perspektiven durchaus $\mathrm{zu}$ weitreichenden Denkbewegungen führen kann, die in der Folge nicht zuletzt auch die Einschätzung der eigenen pädagogischen Lehrprofessionalität in ausschlaggebenden Aspekten relativieren können. Besonders überraschend empfanden dabei viele Studierende ,die Verankerung des Rassismus in der Gesellschaft ". Rassismus und rassistische Diskriminierungen werden auch aktuell in der Öffentlichkeit skandalisiert und oftmals in den (Rechts-)Extremismus verlagert. Mithilfe dieses Täterschematismus und des „Gebrauch[s] des Extremismusbegriffs“ erscheint „die Mitte der Gesellschaft unproblematisch und demokratisch integriert" (Messerschmidt 2010: 45). Vor diesem Hintergrund lässt sich ein kritisch-reflexiver Erkenntnisgewinn der Seminarteilnehmer_innen in der Evaluationsaussage „Rassismus ist kein Extremum " lesen. Auch begegneten den Studierenden Irritationen im Hinblick auf die eigene Verstrickung in rassistische Argumentationen und Begrifflichkei-

5 Sprecher_innen anonymisiert; Sommersemester 2015, Seminare „Heterogenität im schulischen Unterricht“ und „Diskriminierung als Normalfall? Bürgerliche Bildung zwischen Egalität und Ungleichheit". 
ten, die sich unter anderem in der Beobachtung ausdrücken, ,dass wir plötzlich in rassistische Äußerungen verwickelt waren“".

Eben solche kritischen Relativierungen sind es, die sich nun auch in Einschätzungen über die spätere Lehrpraxis und das eigene Professionalitätsverständnis wiederfinden. Teilweise verabschiedet haben sich die Studierenden von den zu Beginn der Lehrveranstaltungen häufig geäußerten Wünschen und Erwartungen nach einem instrumentellen ,Methodenkoffer' für die schulische Praxis: Dass es nicht um einen „Praxisleitfaden“ gehen kann und es „keine Rezepte für den Schulalltag" gibt, wurde von vielen Teilnehmer_innen erkannt. Äußerungen dieser Art zeigen, dass die kompensatorische Frage nach einem ,richtigen Umgang ' mit Heterogenität im Sinne einer gesellschaftlichen Sonderform durch kulturalisierungs- und rassismuskritische Betrachtungsweisen bewusst an Relevanz verliert. In diesem Sinne verweist die Auseinandersetzung mit einer solchen Perspektive auf die Einsicht, dass Rassismus kein individuelles und pädagogisch ,korrigierbares ' Problem von Stereotypen, sondern ein gesellschaftliches Verhältnis und Teil einer gesellschaftlichen Struktur darstellt. Nicht zuletzt findet sich in diesem $\mathrm{Zu}-$ sammenhang eine ebenso aussagekräftige wie grundsätzliche Erkenntnis in der Feststellung, dass „Heterogenität mehr als unterschiedliches Leistungsniveau bedeutet". Eine solche Aussage kann nicht nur als Ausdruck einer speziell rassismuskritischen, sondern als allgemein diskriminierungskritische Haltung im pädagogischen Umgang mit Differenzen verstanden werden: Sie unterstreicht ein differenzreflexives Verständnis in pädagogischer Bildungsarbeit dahingehend, sich von einer homogen gedachten Gruppe ,der Schüler_innen zu distanzieren, dieser Heterogenität aber gleichzeitig auch ohne das Muster einer Problematisierung, der Anderen' gerecht zu werden.

Die Aufrechterhaltung einer binären Ordnung vom ,Eigenen' und ,Fremden' verschließt die Möglichkeit zur Anerkennung der Existenz hybrider Identitäten und „Mehrfachzugehörigkeiten“ (Mecheril 2010: 185) und damit zur angemessenen Berücksichtigung einer bestehenden migrationsgesellschaftlichen Realität. Der bewusste Verzicht auf vollständige bildungspraktische Planbarkeit und Kontrolle im Sinne einer ,hermeneutischen Unzugänglichkeit des Anderen“ (Mecheril nach Broden 2009: 130) ist daher keineswegs als Indiz für die mangelnde Professionalität von Pädagog_innen zu bewerten. Vielmehr erweist sich gerade diese konstitutive Unsicherheit als professionelle Reflexion von Kultur als gestalterischer Praxis: ein dynamisches Verständnis von kultureller Identität, bei der „das Fremde im Anderen und das Fremde im Eigenen bedacht wird“" (ebd.). 


\section{Literatur}

Arndt, S./Ofuatey-Alazard, N. (2011) (Hrsg.): Wie Rassismus aus Wörtern spricht. (K)Erben des Kolonialismus im Wissensarchiv deutsche Sprache. Ein kritisches Nachschlagewerk. Münster: Unrast Verlag.

Broden, Anne (2009): „Verstehen der Anderen? Rassismuskritische Anmerkungen zu einem zentralen Topos interkultureller Bildung“. In: Scharathow, W./Leiprecht, R. (Hrsg.): Rassismuskritik, Band 2: Rassismuskritische Bildungsarbeit. Schwalbach: Wochenschau Verlag, S. 119-134.

Castro Varela, M./Mecheril, P. (2010): „Grenze und Bewegung. Migrationswissenschaftliche Klärungen“. In: Andresen, S. u.a. (Hrsg.): Migrationspädagogik. Weinheim/Basel: Beltz Verlag, S. 23-53.

Dietrich, A./Strohschein, J. (2011): „Kolonialismus”. In: Arndt, S./Ofuatey-Alazard, N. (Hrsg.): Wie Rassismus aus Wörtern spricht. (K)Erben des Kolonialismus im Wissensarchiv deutsche Sprache. Ein kritisches Nachschlagewerk. Münster: Unrast Verlag, S. 114-120.

Höhne, Thomas (2001): „Kultur als Differenzierungskategorie“. In: Lutz, H./ Wenning, N. (Hrsg.): Unterschiedlich verschieden. Differenz in der Erziehungswissenschaft. Opladen: Leske + Budrich, S. 197-213.

Mecheril, Paul (2004): „Migrationspädagogik. Einführung in eine Perspektive“. In: Mecheril, Paul: Einführung in die Migrationspädagogik. Weinheim/Basel: Beltz Verlag, S. 15-26.

Mecheril, Paul (2010): „Anerkennung und Befragung von Zugehörigkeitsverhältnissen. Umriss einer migrationspädagogischen Orientierung“. In: Andresen, S. u.a. (Hrsg.): Migrationspädagogik. Weinheim/Basel: Beltz Verlag, S. 179-191.

Mecheril, P./Plößer, M. (2009): „Differenz und Pädagogik“. In: Casale, R. u.a. (Hrsg.): Handwörterbuch Pädagogik der Gegenwart. Weinheim/Basel: Beltz Verlag, S. 1-14.

Messerschmidt, Astrid (2008): „Pädagogische Beanspruchungen von Kultur in der Migrationsgesellschaft. Bildungsprozesse zwischen Kulturalisierung und Kulturkritik“. In: Zeitschrift für Pädagogik 54, 1, S. 5-17.

Messerschmidt, Astrid (2009): „Distanzierungsmuster. Vier Praktiken im Umgang mit Rassismus“. In: Borden, A./Mecheril, P. (Hrsg.): Rassismus bildet. Bildungswissenschaftliche Beiträge zu Normalisierung und Subjektivierung in der Migrationsgesellschaft. Bielefeld: Transcript Verlag, S. 41-58.

Messerschmidt, Astrid (in Druck): „Kontextualität und Reflexivität rassismuskritischer Bildung“.

Kasatschenko, Tatjana (2015): „Das Geschlecht (in) der Migration. Er, Sie, Wir und die Anderen: Die soziale Konstruktion von Ethnie und Geschlecht als Macht- und Strukturkategorien“. Darmstadt: Technische Universität/Institut für Allgemeine Pädagogik und Berufspädagogik.

Krüger-Potratz, Marianne (2011): „Intersektionalität“. In: Faulstich-Wieland, H. (Hrsg.): Umgang mit Heterogenität. Baltmannsweiler: Schneider-Verl. Hohengehren, S. 183-200.

Weber, Martina (2008): „Intersektionalität sozialer Unterscheidungen im Schulalltag“. In: Seemann, M. (Hrsg.): Ethnische Diversitäten, Gender und Schule. 
Geschlechterverhältnisse in Theorie und schulischer Praxis. Oldenburg: BISVerlag, S. 41-59.

Zitzelsberger, Olga (2014): ,,Was sagst Du als Marokkanerin dazu?‘ Zum Umgang mit Heterogenität im Schulalltag“. In: Eisenbraun, V./Uhl, S. (Hrsg.): Geschlecht und Vielfalt in Schule und Lehrerbildung. Münster/New York: Waxmann, S. 59-74. 



\section{Innovationen in den Lehr-Lernkulturen an Hochschulen}

In der Erwachsenenbildung wird der Begriff Lernkultur häufig mit Begriffen $n e u$, Wandel oder Innovation in Verbindung gebracht (vgl. Schüßler/Thurnes, 2005). Dies gilt auch im Feld der Hochschule, in dem seit einigen Jahren die Qualität der Hochschullehre, auch angesichts verschiedener großer Förderprogramme, zunehmend in den Fokus rückt.

Unterstellt man, dass Lehr-Lernkulturen sich in verschiedenen organisationalen Kontexten und Fachdisziplinen unterscheiden (vgl. Huber 1991), so ist gute oder innovative Lehre nicht absolut, sondern nur relational zu bestimmen - in Abhängigkeit vom jeweiligen organisationalen und fachspezifischen Kontext.

Ausgangspunkt für unsere wissenschaftliche Begleitforschung zu den „Fellowships für Innovationen in der Hochschullehre“"1 war daher die Perspektive einer multiperspektivischen und multimethodischen Lehr-Lernforschung. Mithilfe eines Mixed Methods Designs wurden Anlässe, Ziele und Wirkungen innovativer Lehre aus der Perspektive der beteiligten Akteure, Studierende und Lehrende, in den Blick genommen. Im Folgenden werden ausgewählte Ergebnisse aus dem Projekt vorgestellt. Im Fokus steht dabei die Kontextabhängigkeit innovativer Lehre, die exemplarisch anhand der Innovationsverständnisse von Lehrenden sowie des Innovationsempfindens von Studierenden verschiedener Fachdisziplinen aufgezeigt wird.

\section{Eine relationale Perspektive auf Lehr-Lernkulturen}

Wie bereits einleitend angedeutet, geht der vorliegende Text von einem dynamischen Verständnis von Lehr-Lernkultur aus. Ihm liegt die systemtheoretische Annahme zu Grunde, dass Lehr-Lernkultur sich nicht als ein substanzhaft zeitbeständiger Zusammenhang spezifischer Sinngebungen behandeln lässt, sondern vielmehr als ein entsprechend formbares Medium ver-

1 Die von der Baden-Württemberg Stiftung finanzierte wissenschaftliche Begleitforschung (2012-2015) zu den Fellowships für Innovationen in der Hochschullehre nahm Anlässe, Gestaltungsformen und Wirkungen innovativer Lehre in den Blick. Das von mehreren Stiftungspartnern gemeinsam geförderte Programm ist darauf angelegt, durch eine individuelle, personengebundene Förderung eine Anschubfinanzierung für die Entwicklung und Erprobung innovativer Lehr- und Prüfungsformate und die Neugestaltung von Modulen und Studienabschnitten zu gewährleisten. http://stifterverband.info/wissenschaft_und_ hochschule/lehre/fellowships/index.html 
standen werden muss. Ihr kommt die Funktion zu, selektiver kontingenter Deutungskontext für zeitgebundene Sinngebungen zu sein (vgl. Krause 2005: 186).

Das Selektiv-Kontingente drückt sich darin aus, dass die Strukturen von Lehr-Lernkulturen von gesellschaftlichen und institutionellen Rahmenbedingungen sowie von beteiligten Akteuren immer wieder neu ausgewählt bzw. erzeugt werden. Es handelt sich somit um ein soziales System, das als spezielles Teilprogramm des allgemeinen Kulturprogramms einer Gesellschaft angesehen werden kann. Dieses Teilprogramm umfasst alle Bezugnahmen von Aktanten in Lehr-Lernsystemen auf Momente, die im Wirklichkeitsmodell einer Organisation für Lehr-Lernprozesse jeder Art relevant sind (vgl. Schmidt 2005: 106).

Die Strukturen von Lehr-Lernkulturen stellen keine Konstanten dar, sondern sind sowohl in ihren jeweiligen Merkmalen als auch in ihren Relationierungen dynamisch, also durch Veränderung gekennzeichnet. Die Bewertung von Veränderungsimpulsen, die von außen auf das Lehr-Lernkultursystem einwirken, erfolgt jeweils auf Basis des aktuellen strukturellen Zustands. Wir gehen deshalb davon aus, dass die Qualität der Veränderungsimpulse nur relativ zum sozialen System Lehr-Lernkultur beantwortet werden kann, nicht von einer außenstehenden ,,(be)wertenden“ Instanz. In unserem empirischen Zugriff wurden daher zunächst die Innovationsverständnisse, -formen und geplanten Wirkungen anhand von Dokumentenanalysen der Projektanträge und Interviews mit Lehrenden (Lehrendenperspektive) herausgearbeitet.

$\mathrm{Ob}$ ein Veränderungsimpuls eine Lehr-Lerninnovationen darstellt, hängt folglich nicht von den „Innovationserfindern“ ab, sondern vielmehr von der relationalen Einbettung des Impulses in ein bestehendes Lehr-Lernkultursystem. Innovation stellt in diesem Sinne keine objektivierbare Qualität dar, sondern vielmehr ein sich stetig veränderndes Ergebnis eines zirkulären Wechselspiels zwischen Innovationspostulat und Innovationsakzeptanz/ignoranz. Die Innovativität und die Wirkungen von Lehrinnovationen galt es auf methodischer Ebene daher anhand von Studierendendaten zu ermitteln. Das dafür eingesetzte Instrument wurde aus den Ergebnissen der Dokumentenanalyse entwickelt, um die Lehrenden- und Studierendenperspektiven in einem Multi-Method Design miteinander zu verzahnen.

In der Analyse ging es zum einen darum, Lehrinnovationen von Lehrenden in Hochschulen zu identifizieren und zu klassifizieren sowie Einblicke in ihre Innovationsverständnisse zu gewinnen, und zum anderen, die Bewertung der Innovationen aus Perspektive der Studierenden zu erfassen. Das Innovationserleben der Studierenden wird zusätzlich mittels lehr-lernkulturellen Kontextfaktoren analysiert, um so Rückbezüge auf die Entstehungskontexte von Lehr-Lerninnovationen zu ermöglichen. 


\section{Innovationsverständnisse der Lehrenden}

Im Rahmen einer qualitativen Interviewstudie mit Lehrenden wurden dazu anhand von dreizehn leitfadengestützten Interviews u.a. die Innovationsverständnisse der Lehrenden exploriert und mithilfe der qualitativ orientierten Inhaltsanalyse nach Mayring $(2000,2008,2010)$ ausgewertet. Wenngleich die von ihnen angestrebten Innovationen sowie deren Begründungen, Auslöser und Zielsetzungen ganz unterschiedlicher Natur waren, ließen sich auch Schnittmengen finden, die einen gemeinsamen Bedeutungshof innovativer Lehre im untersuchten Sample umreißen. Die Lehrenden betonen dabei vor allem drei Aspekte: die Kontextgebundenheit, die Bedarfsausrichtung und die Qualitätsorientierung innovativer Lehre.

\section{Kontextgebundenheit der Innovation}

In der Beschreibung ihrer Lehrinnovationen kamen die Lehrenden immer wieder auf ihre organisationalen Kontexte und die bestehenden Lehr-Lernroutinen bzw. Lehr-Lernkulturen im hochschulbezogenen Alltag zu sprechen. In einigen - vorwiegend naturwissenschaftlich-technischen - Fachkulturen gilt z.T. eine interaktive, seminaristische Lehrform gegenüber der üblicherweise als Vorlesung abgehaltenen Lehre bereits als innovativ. Private Hochschulen zeigen sich in den untersuchten Fällen offen für neuartige Lehrformate und verfügen zudem meist über die entsprechenden Ressourcen, diese $\mathrm{zu}$ verwirklichen. Öffentliche Universitäten erweisen sich dagegen insbesondere in sehr traditionsbewussten Fächern mitunter als regelrecht ,innovationsfeindlich“ (KH8, Absatz 242) und unterstützen die Durchführung innovativer Lehre und die nachhaltige Veränderung der Lehrgewohnheiten in den untersuchten Fällen häufig nicht. Es kommt vor, dass sich dann die Einführung von Innovationen ,auch sehr, sehr schwierig im Kollegen-Kreis“ (KH8, Absatz 14) gestaltet. Lehrende in sogenannten Massenstudiengängen sehen sich meist einer sehr heterogenen Studierendenschaft gegenüber, gleichzeitig mangelt es an individualisierten Betreuungsmöglichkeiten. Hier kommen bspw. Tutorenprogramme zum Einsatz, die zwar seitens der Befragten als nicht unbedingt innovativ empfunden werden, in diesem Format aber bisher keinen Einsatz im Studiengang gefunden haben und so eine „neue“ Möglichkeit darstellen, die Studierendenbetreuung zu verbessern. Insgesamt betonten die Lehrenden meist explizit, dass es ihnen nicht darum gehe, etwas grundsätzlich Neues zu erfinden: 
[Die Methode] gibt es schon aber es ist in der Umsetzung für diesen Standort hier, mh, auf jeden Fall innovativ und neu. und hat so einen Mehrwert (BU7, Absatz 91).

also [...] wir haben jetzt nicht geglaubt, dass das noch nirgendwo jemals (LACHT) existiert hat, sondern einfach der Neuheitscharakter war so für unser Feld hier gegeben, das war das Innovative daran (WL7, Absatz 112).

Ja, also ich würde schon, also an meiner Fakultät ist das schon eine Innovation. Also ich habe hier schon studiert und promoviert, ich kenne den Laden schon relativ lange, ich habe noch nie gehört, dass es so etwas gegeben hätte. Dann muss man den Begriff Innovationen relativieren, weil, was vielleicht in der sozialwissenschaftlichen Fakultät gang und gäbe ist, ist für eine [technische] Fakultät etwas vollkommen radikal Neues. Und umgekehrt (DM5, Absatz 30).

Es wird deutlich, dass die Lehrenden selbst ein relationales Verständnis von Innovation anlegen. Nach Schüßler und Thurnes (2005: $89 \mathrm{ff}$.) ,,[stehen] hier nicht grundlegend neue wissenschaftliche Errungenschaften im Vordergrund [...], sondern die geeignete Kombination bereits vorhandener Strategien, Konzepte, Vorgehensweisen und Methoden. Es geht also entsprechend dem Wortsinn nicht um die Erfindung von Neuem - Invention -, sondern um die neue wirksame Nutzbarmachung von bereits Erfundenem insbesondere durch Kombination, Adaption und Weiterentwicklung - Innovation". Was innovativ ist, lässt sich also nur an der bestehenden Lehr-Lernkultur messen. Anhand der Interviews lässt sich aufzeigen, dass insbesondere der Fachdisziplin, dem Hochschultypus und der Studienganggröße besondere Bedeutung für die Innovationsverständnisse der Lehrenden zukommt. Diese Kontextspezifika nehmen auch Einfluss auf die wahrgenommenen Bedarfe, auf die die Lehrenden mit ihren Innovationen reagieren.

\section{Innovation als Antwort auf erlebte Bedarfe}

Gemeinsam ist allen Lehrinnovationen, dass sie als Antwort auf erlebte Bedarfe verstanden werden. Diese äußeren Anforderungen können dabei auf unterschiedlichen Ebenen liegen. Es finden sich u.a. Handlungsproblematiken, die sich aus organisationalen Kontextbedingungen ergeben, beispielsweise die Vorgabe bestimmter Ziele durch die Hochschule oder RessourcenEngpässe.

Also es war eigentlich wirklich so eine Bedarfssituation oder so eine Situation, wo man einfach dachte, so, ich würde es gerne machen, aber es ist einfach die Ausstattung nicht da (LA6, Absatz 11). 
Ebenso werden aber Bedarfe auch auf curriculare Strukturen und Merkmale konkreter Lehr-Lerninteraktionen mit den Studierenden zurückgeführt.

Ich leide ein bisschen daran, dass das, was die Leute mitbringen an Leidenschaften und an Begeisterungen und an Interesse, nach meiner Wahrnehmung im Studium relativ systematisch vernichtet wird (BE5, Absatz 28).

Innovationen lassen sich dann als Ergebnis von Problemlöseprozessen deuten, in denen Maßnahmen entwickelt oder adaptiert werden, um den erlebten Bedarfen zu begegnen. Innovation in der Lehre kann also als Hilfsmittel verstanden werden, um (vorgegebene und/oder neue) didaktische, organisationale aber auch persönliche Ziele zu erreichen.

Allgemein ist meine Erwartung da dran, dass ich ein Ziel, was prinzipiell besteht, durch eine Lehrinnovation besser einmal, oder vielleicht auch bezüglich der Ziele erweiternd, erreiche (EF7, Absatz 42).

Innovation in der Lehre besitzt also keine eigene Qualität (vgl. WL7, Absatz 112), sondern wird als Mittel zum Zweck verstanden:

Also, Innovation der Innovation willen, das, wie gesagt, würde für mich eher nicht in Frage kommen, es sei denn man macht es am Rande des gesamten Studienbetriebs und probiert so etwas aus. (EF7, Absatz 44).

Also es muss nicht per se neu sein (BE5, Absatz 28).

Es lässt sich festhalten, dass Innovation als Antwort auf erlebte Bedarfe verstanden wird, die aus einem Missverhältnis zwischen einem angestrebten Zielzustand und dem diagnostizierten Ist-Zustand resultieren.

\section{Innovation als Verbesserung/Mehrwert}

Die Zielsetzung wird von den Lehrenden je nach Kontext und Problemdiagnose unterschiedlich gerahmt, zielt aber im Grundsatz vor allem auf die Qualität der Lehre:

[...] die Qualität der Lehre. Ich glaube das sollte das Ziel der Lehre sein. Aber das ist nicht unbedingt innovativ. Ich glaube das war schon immer das Ziel der Lehre, bloß geht es manchmal verloren, wenn man keine Ressourcen hat und nicht genug Lehrende hat und viel zu viel Studenten hat und keine Räume hat und keine Mittel hat [...]. Also es hat viel mit Ressourcen zu tun (WP6, Absatz 41).

Und Innovation finde ich erst mal nicht wichtig, ich finde wichtig, also mir liegen die Menschen am Herzen. Und wenn wir das hinkriegen, eine Lehre $\mathrm{zu}$ machen, die, die Leute mitnimmt und begeistert und wo wir nachher begeisterte Berufsträger haben, die mit eigenem Ethos und mit 
eigenen Ideen mit ihren Leidenschaften an ihrem Ort, die Sachen vorantreiben, das ist das Ziel und das würde ich sagen, treibt mich zu Lehrinnovation, nicht weil es innovativ ist, sondern weil das das Ziel ist (BE5, Absatz 28).

Folgt man diesen Aussagen, dann ist es nicht das primäre Ziel, innovative Lehre anzubieten: „Es ist jetzt eben zufälligerweise Innovation“ (HK7, Absatz 19). Vielmehr steht die Qualität der Lehre im Fokus. Dieser Abgleich zwischen den Zielsetzungen und Qualitätsansprüchen der Lehre und dem aktuellen Ist-Zustand stellt sich dann nicht als punktuelles Innovationsereignis dar, sondern vielmehr als kontinuierlicher Reflexions- und ggf. Innovationsprozess. Innovation in der Lehre stellt sich dann als ein Prozess der fortwährenden Analyse, Reflexion und Anpassung an aktuelle Anforderungen und Erkenntnisse dar mit dem Ziel, die Qualität in der Lehre zu erhalten und/oder zu verbessern: „Natürlich ist man davon überzeugt, dass das einfach eine Bereicherung ist" (BU7, Absatz 41). Diesen Mehrwert für das LehrLerngeschehen herauszustellen und auch zu belegen, ist den Lehrenden wichtig, damit Innovation nicht zur luftleeren Blase wird, die zwar ,irgendwelche Presse“, aber keinen „,substantiellen Wert“ (vgl. DM5, Absatz 30) bringt.

Zusammenfassend heißt das: Innovative Lehre ist kein Selbstzweck. Das „Neue“ besitzt keine eigene Qualität, sondern gewinnt erst einen Mehrwert, wenn es eine Antwort auf erlebte Bedarfe darstellt, die aus einem Missverhältnis zwischen einem angestrebten Zielzustand und dem Ist-Zustand resultieren. Der gewünschte Zielzustand orientiert sich dabei an den Vorstellungen der Lehrenden von Qualität in der Lehre. Diese Zielvorstellungen, Bedarfe und Lösungsansätze sind ebenfalls nicht absolut zu bestimmen, sondern nur relational vor dem Hintergrund der jeweiligen Lehr-Lernkulturen, Fachdisziplinen, Hochschultypen etc. verstehbar.

\section{Innovationsempfinden der Studierenden nach verschiedenen lernkulturellen Merkmalen}

Wenn innovative Lehre sich nicht absolut, sondern nur relational bestimmen lässt, so kann die Innovation auch nur von Vertreter/innen desselben lehrlernkulturellen Kontextes beurteilt werden.

Im Rahmen einer Online-Erhebung wurden die Studierenden befragt, die an den innovativen Lehrformaten der geförderten Fellows im Rahmen des Fellowship-Programms im Untersuchungszeitraum von 2013 bis 2014 teilgenommen haben. Die Studierendenbefragung war als Vollerhebung konzipiert und konnte in 30 Veranstaltungen unterschiedlicher Fachdisziplinen eingesetzt werden. Sie basierte auf einem selbst entwickelten Fragebogen, der aufbauend auf einer qualitativen Inhaltsanalyse der Projektanträge der geförderten Lehrenden konzipiert wurde und verschiedene innovierte Veranstal- 
tungsmerkmale und angestrebte Lerneffekte erfasste. Die Stichprobe belief sich auf N=850 Studierende. Die Analyse stützte sich neben bivariaten Verfahren schwerpunktmäßig auf Hauptkomponentenanalysen (vgl. Backhaus et al. 2016) und Clusteranalysen (vgl. Schendera 2010).

Geht man angesichts eines relationalen Innovationsverständnisses davon aus, dass ein und dasselbe Veranstaltungsmerkmal in dem einen Kontext als innovativ wahrgenommen werden kann, während es in einem anderen Kontext zum Veranstaltungsalltag gehört, müsste sich eine positive Korrelation zwischen dem Innovationserleben der Studierenden und ihrer Veranstaltungszugehörigkeit zeigen, da die Studierenden derselben Veranstaltung denselben organisationalen Kontext teilen. Im Fragebogen wurden die Studierenden gebeten, anhand des Items „Ich fand die Veranstaltung insgesamt innovativ" auf einer vierstufigen Skala abschließend die Gesamtinnovativität der Veranstaltung zu beurteilen. Setzt man dieses Item ins Verhältnis zur Veranstaltungszugehörigkeit der Studierenden, so ergibt sich ein Korrelationskoeffizient von 0,422 (Eta), d.h. eine mittlere Zusammenhangsstärke. Die Veranstaltungszugehörigkeit und damit das Teilen desselben lehr-lernkulturellen Kontextes steht also durchaus im Zusammenhang mit dem Innovationsempfinden der Studierenden.

Welche Merkmale des Kontextes prägen dabei dieses gemeinsame Erleben? Die Lehrenden rekurrieren bezüglich möglicher Unterscheidungsmerkmale in den Interviews u.a. auf die Fachkultur (vgl. Kapitel 2). Es wurde daher geprüft, ob - und wenn ja wie - sich Studierende verschiedener Fachdisziplinen im Hinblick auf ihr Innovationsempfinden unterscheiden.

Im Rahmen der Befragung wurde die Einschätzung der Studierenden zur Ausprägung verschiedener Veranstaltungsmerkmale erfasst, die von den Lehrenden in ihren Projektanträgen als Charakteristika ihrer geplanten Innovationen beschrieben wurden. Anhand von Hauptkomponentenanalysen ließ sich die Vielfalt unterschiedlicher Veranstaltungsmerkmale zu wenigen Merkmalsdimensionen verdichten ${ }^{3}$.

Die ermittelte Lösung mit sechs Hauptkomponenten ist in der Lage, 58\% der Gesamtvarianz zu erklären. Tabelle 1 veranschaulicht, welche Items zu Hauptkomponenten zusammengefasst werden und wie die Hauptkomponenten benannt wurden. Die Werte geben an, wie stark ein Item auf der jeweiligen Hauptkomponente lädt. Je höher der Wert ist, desto stärker repräsentiert ein Item die gebildete Hauptkomponente. Es werden nur Ladungen angezeigt, die über 0,3 liegen. Items, die auf keinem Faktor über 0,5 laden, wurden in die Darstellung ebenfalls nicht aufgenommen.

3 Zuvor wurden die Voraussetzungen für die Durchführbarkeit einer Hauptkomponentenanalyse anhand des Kaiser-Meyer-Olkin-Tests und des Barlett-Tests auf Sphärizität geprüft. Der KMO-Wert beträgt 0,933; der Bartlett-Test ergibt einen nicht-signifikanten Wert. Somit sind die Voraussetzungen für die Durchführung einer Hauptkomponentenanalyse erfüllt. 
Tab. 1: Merkmalsdimensionen innovativer Lehrveranstaltungen (Rotierte Hauptkomponentenmatrix für die Items aus Teil A des Fragebogens)

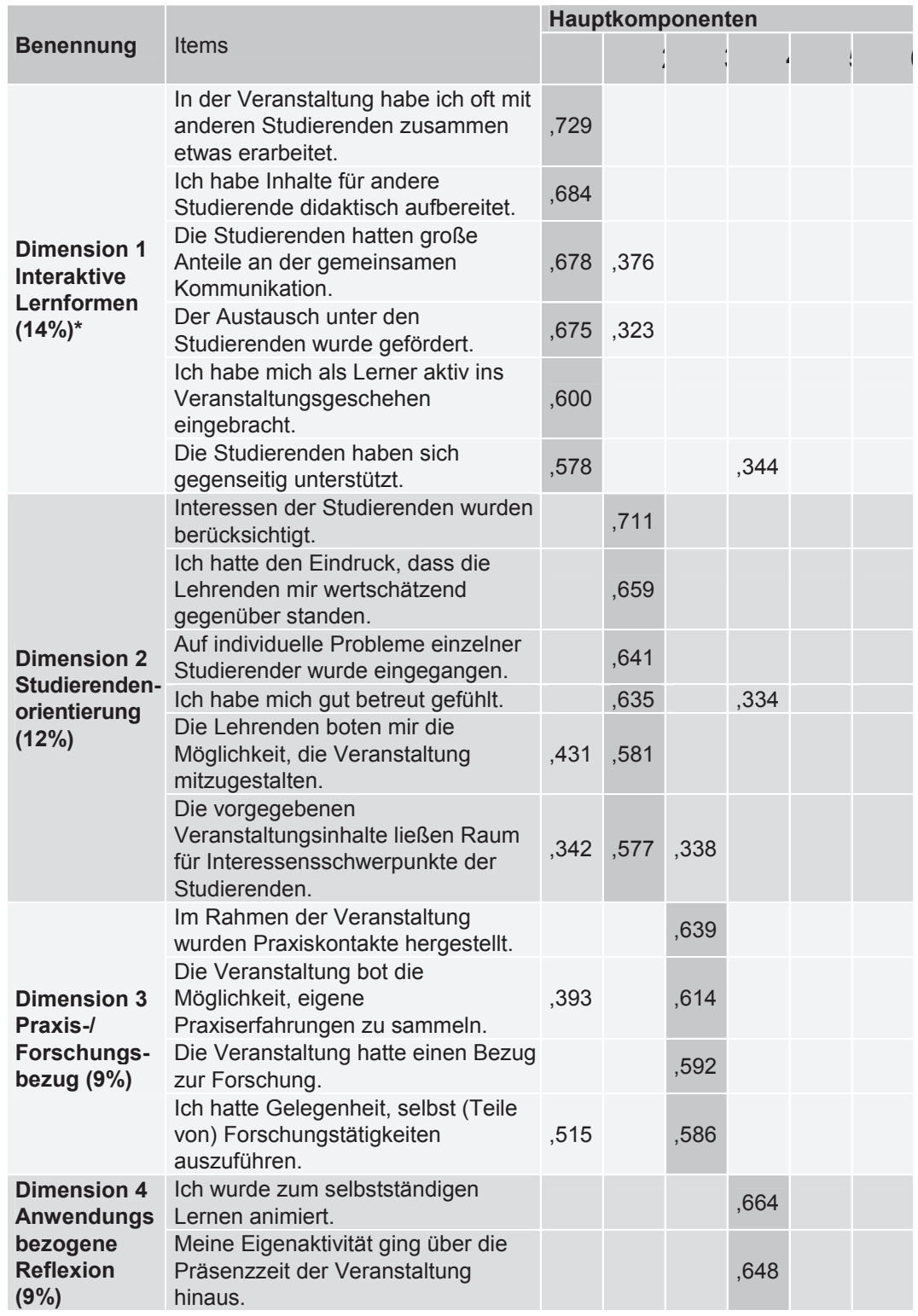




\begin{tabular}{|c|c|c|c|c|c|c|}
\hline & $\begin{array}{l}\text { Die Praxisrelevanz der } \\
\text { Veranstaltungsinhalte wurde } \\
\text { deutlich. }\end{array}$ & ,342 & & 639, & & \\
\hline & $\begin{array}{l}\text { Zu den Inhalten wurden praktische } \\
\text { Anwendungsbezüge hergestellt. }\end{array}$ & & 313, & ,556, & & \\
\hline $\begin{array}{l}\text { Dimension } 5 \\
\text { Medien- }\end{array}$ & $\begin{array}{l}\text { Medien/Technik kamen häufig zum } \\
\text { Einsatz. }\end{array}$ & & & & 809, & \\
\hline einsatz $(7 \%)$ & $\begin{array}{l}\text { Es kamen unterschiedliche } \\
\text { Medien/Technik zum Einsatz. }\end{array}$ & & & & 695, & \\
\hline $\begin{array}{l}\text { Dimension } 6 \\
\text { Neue }\end{array}$ & $\begin{array}{l}\text { In der Prüfung wurde ein besonderer } \\
\text { Bewertungsmodus eingesetzt (z.B. } \\
\text { Selbsteinschätzung der eigenen } \\
\text { Leistung, Berücksichtigung von } \\
\text { Aktivität während der Veranstaltung } \\
\text { etc.). }\end{array}$ & & & & & 696, \\
\hline $\begin{array}{l}\text { Prüfungs- } \\
\text { formen }(6 \%)\end{array}$ & $\begin{array}{l}\text { In der Veranstaltung wurde ein } \\
\text { besonderes Prüfungsformat } \\
\text { eingesetzt (z.B. Gruppenprüfung, } \\
\text { Dokumentation eines } \\
\text { durchgeführten Tutoriums, Fallarbeit } \\
\text { etc.). }\end{array}$ & & & & & 676, \\
\hline
\end{tabular}

* Durch die Hauptkomponente aufgeklärte Varianz der Korrelationsmatrix

Im Fächervergleich wurde deutlich, dass sich die Ausprägungen dieser Merkmalsdimensionen innerhalb verschiedener Fachdisziplinen deutlich unterschieden. Bei Studierenden der Rechtswissenschaften $(\mathrm{N}=82)$ sind fast alle Merkmalsdimensionen unterdurchschnittlich ausgeprägt. Auch wenn das Ausmaß der meisten Abweichungen nicht als überzufällig angesehen werden kann, ist das Ergebnis vor dem Hintergrund auffällig, dass die Fachdisziplin auch in den Interviews mit Lehrenden als „maximal innovationsfeindlich“ und ,strukturkonservativ" beschrieben wurde. Die Veranstaltungen von Studierenden der Wirtschaftswissenschaften $(\mathrm{N}=84)$ zeichnen sich beispielsweise insbesondere durch einen unterdurchschnittlichen Praxis-/Forschungsbezug aus. Die Studierenden der Ingenieurwissenschaften $(\mathrm{N}=109)$ empfinden in den besuchten Veranstaltungen tendenziell fast alle Merkmalsdimensionen als überdurchschnittlich ausgeprägt; insbesondere den Praxis-/Forschungsbezug.

Für die einzelnen Fachdisziplinen ergeben sich also unterscheidbare Merkmalsprofile. Da es sich bei allen Veranstaltungen um explizit innovierte Veranstaltungen handelte, die möglicherweise gerade nicht oder nur bedingt ein typisches Veranstaltungsprofil der eigenen Fachdisziplin repräsentieren, stellt sich die Frage, welche der Merkmalsdimensionen von den Studierenden der verschiedenen Fachdisziplinen als besonders innovativ erlebt werden. Daher wurden für jede Fächergruppe die Korrelationen der einzelnen Merk- 
malsdimensionen mit der Beurteilung der Gesamtinnovativität der Veranstaltung durch die Studierenden ermittelt ${ }^{4}$ (s. Abb.1).

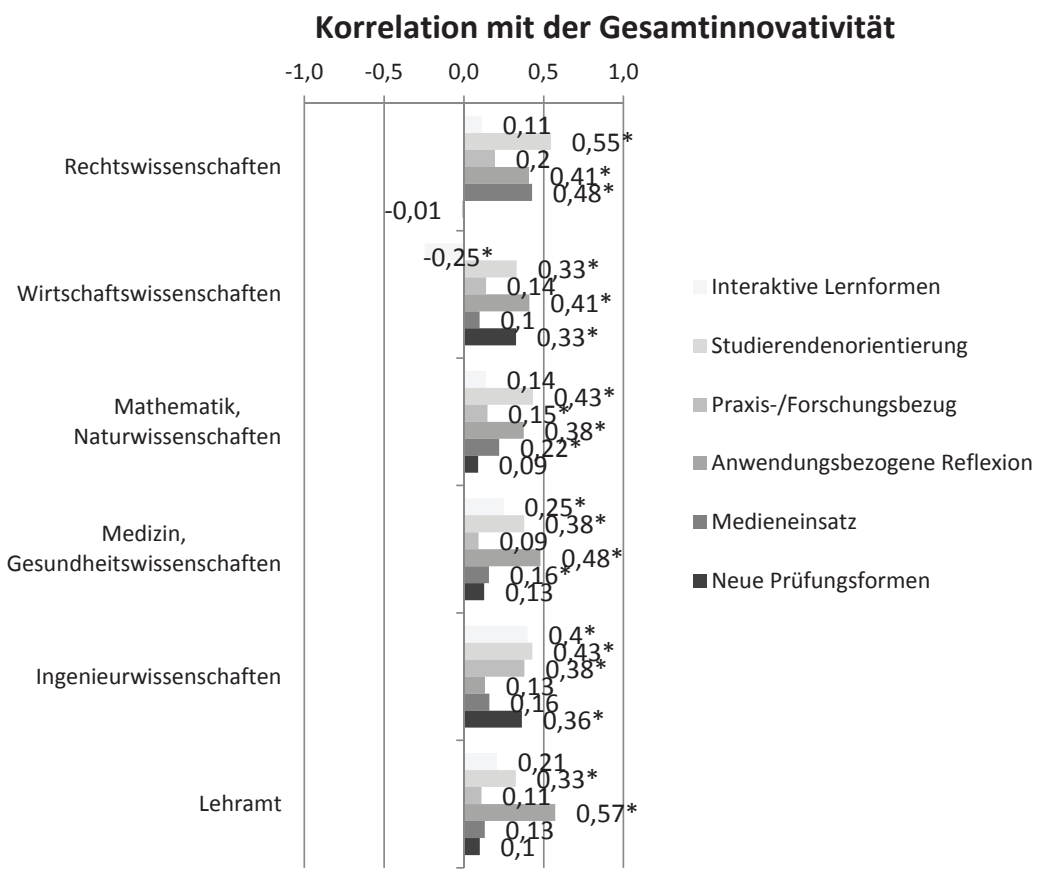

Abb. 1: Korrelationskoeffizienten zwischen Merkmalsdimensionen und Gesamtinnovativität für die Fächergruppen (*=signifikant auf 5\%-Niveau)

Für die Fächergruppen gilt insgesamt: Alle Merkmalsdimensionen korrelieren positiv mit der wahrgenommenen Gesamtinnovativität der Veranstaltungen. Eine Ausnahme bildet die Merkmalsdimension „Interaktive Lernformen" bei den Studierenden der Wirtschaftswissenschaften. Hier gilt: Je höher die Dimension „Interaktive Lernformen“ ausgeprägt ist, desto niedriger schätzen die Studierenden die Gesamtinnovativität der Veranstaltung ein. Dies könnte darauf hindeuten, dass ein hohes Maß an interaktiven Lernformen in den Lehrveranstaltungen wirtschaftswissenschaftlicher Studiengänge

4 Aufgrund der geringen Fallzahlen ist die Berechnung für die Sprach- und Kulturwissenschaften $(\mathrm{N}=3)$ und die Sozialwissenschaften $(\mathrm{N}=2)$ hier nicht möglich. 
bereits zum Veranstaltungsalltag gehört, allerdings war die Ausprägung dieser Dimension im Fächervergleich bei den untersuchten Veranstaltungen wirtschaftswissenschaftlicher Studierender eher unterdurchschnittlich ausgeprägt. Hier wären ergänzende, qualitative Analysen mit Studierenden weiterführend, um diesen Zusammenhang genauer zu beleuchten.

Betrachtet man die positiven Korrelationen im Fächervergleich genauer und legt besonderes Augenmerk auf die signifikanten Zusammenhänge, die mindestens eine mittlere Zusammenhangsstärke von $>.3$ aufweisen, zeigt sich ein überraschendes Ergebnis:

In allen untersuchten Fachdisziplinen weisen die Merkmalsdimensionen „Studierendenorientierung" und "Anwendungsbezogene Reflexion“ die stärksten Zusammenhänge mit der wahrgenommenen Veranstaltungsinnovativität auf.

Zwar ergeben sich für einzelne Fächer weitere, ähnlich starke Zusammenhänge - so stellt sich für Studierende der Rechtswissenschaften auch der Medieneinsatz als besonders innovativ dar, für Studierende der Wirtschaftswissenschaften neue Prüfungsformen und für Studierende der Ingenieurwissenschaften auch eine Betonung von interaktiven Lernformen sowie veränderte Prüfungsformen - trotzdem herrscht unter den Studierenden eine bemerkenswerte Einhelligkeit in der Innovationseinschätzung der verschiedenen Veranstaltungsmerkmale.

\section{Fazit und Ausblick}

In einem relationalen Verständnis von innovativer Hochschullehre lässt sich diese nicht absolut bestimmen, sondern nur im Verhältnis zur bestehenden Lehr-Lernkultur im organisationalen Kontext beschreiben. Hochschullehrende selbst sind sich im Hinblick auf die von ihnen implementierten Innovationen dieser Relationierung durchaus bewusst. Ihr Anspruch ist es nicht, etwas Neues zu erfinden, sondern als Antwort auf erlebte Bedarfe die Qualität der Lehre in ihrem Fach zu erhalten oder zu verbessern. Dazu übertragen sie Konzepte oder Ideen aus anderen Kontexten und passen sie in die bestehende Lehr-Lernkultur ein. Inwiefern es ihnen dabei gelingt, einen spürbaren Unterschied zur bestehenden Lehr-Lernkultur herzustellen, lässt sich nur anhand der Einschätzungen anderer Akteure bestimmen, die an derselben Lehr-Lernkultur teilhaben. Dies wurde in der vorliegenden Untersuchung anhand von Studierendenbefragungen berücksichtigt.

Ein von den Lehrenden geäußertes Wesensmerkmal verschiedener LehrLernkulturen ist die Fachkultur. Die Studierendendaten bestätigen, dass sich in Veranstaltungen verschiedener Fachdisziplinen z.T. sehr unterschiedliche Merkmalsprofile der besuchten Veranstaltungen ergeben. Gleichzeitig ist ein auffälliges Ergebnis, dass über alle Fachkulturen hinweg dieselben Veran- 
staltungsmerkmale die höchsten Korrelationen mit der empfundenen Gesamtinnovativität der Veranstaltung aufweisen: „Studierendenorientierung“ und „Anwendungsbezogene Reflexion“. Die Ergebnisse relativieren die Bedeutung der Fachkultur für das Innovationserleben der Studierenden und sprechen dafür, dass der Studienalltag der befragten Studierenden unabhängig von ihrer Fachdisziplin derzeit überwiegend nicht durch eine Orientierung an den Interessen und Bedürfnissen der Lernenden und entsprechenden Betreuungsformaten gekennzeichnet ist.

Die vorgelegte Analyse zeigt, dass Fachkultur als Differenzmerkmal zur Beschreibung von Lehr-Lernkultur scheinbar weniger Bedeutung zukommt, als allgemein angenommen wird. Diese Ergebnisse könnten auf eine konvergierende hochschulische Lehr-Lernkultur hindeuten, aber auch als Aufforderung zur Suche nach weiteren Differenzkriterien auf den unterschiedlichen Systemebenen verstanden werden. Erste empirische Hinweise liegen bereits vor und zeigen, dass beispielsweise die Unterschiede auf der Ebene der Interaktionssysteme (Veranstaltungsebene) das im Innovationserleben der Studierenden stärker abbilden als Unterschiede auf Hochschulebene (vgl. Jütte et al. 2015; Jütte/Walber/Lobe 2016). Insgesamt zeigt sich ein hohes Potenzial für Anschlussforschung im Feld der hochschulbezogenen Lehr-Lernforschung.

\section{Literatur}

Bacher, Johann (2002): Clusterananlyse. München: Oldenbourg.

Backhaus, Klaus et al. (2016): Multivariate Analysemethoden: Eine anwendungsorientierte Einführung. 14. Auflage. Berlin, Heidelberg: Springer.

Huber Ludwig (1991): Fachkulturen: über die Mühen der Verständigung zwischen den Disziplinen. Neue Sammlung 31, 1, S. 3-24.

Jütte, Wolfgang/Walber, Markus/Lobe, Claudia (2016): Das Neue in der Hochschullehre. Lehrinnovationen aus der Perspektive der hochschulbezogenen Lehr-LernForschung. Wiesbaden: Springer VS (im Erscheinen).

Jütte, Wolfgang/Walber, Markus/Lobe, Claudia/Emas, Nathalie (2015): Innovativer Lehre auf der Spur. Ausgewählte Ergebnisse der wissenschaftlichen Begleitforschung. Arbeitspapier der Baden-Württemberg Stiftung - Bildung Nr. 10. Stuttgart. http://www.bwstiftung.de/uploads/tx_news/Arbeitspapier_Nr._10_ Fellowshipprogramm_final.pdf [Zugriff: 02.03.2016].

Krause, Detlef (2005): Luhmann-Lexikon. Stuttgart: Lucius \& Lucius.

Mayring, Philipp (2000): Qualitative Inhaltsanalyse. Forum Qualitative Sozialforschung 1, 2, Art. 20. http://nbn-resolving.de/urn:nbn:de:0114-fqs0002204. [Zugriff: 27.07.2015].

Mayring, Philipp (2008): Qualitative Inhaltsanalyse. Grundlagen und Techniken. 10. neu ausgestattete Auflage. Weinheim: Beltz.

Mayring, Philipp (2010): Qualitative Inhaltsanalyse. In: Mey, G/Mruck, K. (Hrsg.): Handbuch Qualitative Forschung in der Psychologie. Wiesbaden: VS, S. 601-613.

Schendera, Christian FG (2010): Clusteranalyse mit SPSS. Mit Faktorenanalyse. München: Oldenbourg. 
Schmidt, Siegfried Jochen (2005): Lernen, Wissen, Kompetenz, Kultur. Heidelberg: Carl-Auer.

Schüßler, Ingeborg/Thurnes, Christian (2005): Lernkulturen in der Weiterbildung. Bielefeld: W. Bertelsmann. 



\section{Panel IV: \\ Politik, multi-nationale Projekte}





\section{Kultur als Praxis in europäischen Vergleichsstudien. Kritische Reflexion eines multilateralen Projekts ${ }^{1}$}

\section{Einleitung}

Die Teilnahme an Erwachsenenbildungsmaßnahmen unterscheidet sich in Europa nicht nur von Mitgliedsstaat zu Mitgliedsstaat, sondern auch von Region zu Region teils maßgeblich. So reichte die Teilnahmerate der 25- bis 64-Jährigen 2015 von $36 \%$ in der Region Zürich bis zu 0,8\% in der rumänischen Region Sud-Est (EUROSTAT 2016). Schon länger beschäftigen sich verschiedene europäische Institutionen mit der Teilnahme, aber auch mit den Maßnahmen selbst und den Prozessen, die zu diesen Maßnahmen führen: Die Generaldirektion Bildung und Kultur der Europäischen Kommission beschreibt 2012, dass ,große regionale Unterschiede bezüglich Bildungsmöglichkeiten und der Bildungsleistung zwischen aber auch in den EU-Mitgliedstaten und Regionen fortbestehen" (Ballas 2012: 1, unsere Übersetzung). Der Ausschuss der Regionen stellt bereits 2008 fest, ,,dass in vielen Mitgliedstaaten die regionale und lokale Ebene nicht nur für die Erwachsenenbildung zuständig sind, sondern auf ihnen auch die Bildungs- und Ausbildungsentscheidungen getroffen und in die Praxis umgesetzt werden" (Ausschuss der Regionen 2008). Bereits 2006 arbeitet die Europäische Kommission heraus: „Die Barrieren, die einer Teilnahme entgegenstehen, können [...] politisch bedingt sein" (Europäische Kommission 2006). Diese Berichte heben die Wichtigkeit regionaler Prozesse in der Erwachsenenbildungspolitik hervor und betonen gleichzeitig, dass zwischen den Regionen durchaus nennenswerte Unterschiede in der Erwachsenenbildung bestehen. Um herauszuarbeiten, wie regionale Unterschiede politisch ausgeglichen werden können, ist es wichtig, vorab die Entscheidungsprozesse zu analysieren, die in der Erwachsenenbildungspolitik ablaufen. Genau dies tat das EU-geförderte Projekt Comparative Analysis of Regional Policies for Adult Learning (REGIONAL) in den Jahren 2014 und 2015, indem es die Prozesse der Formulierung, Implementierung und Finanzierung von Erwachsenenbildungspolitik in $21 \mathrm{Re}-$ gionen in sechs Ländern Europas untersuchte und verglich.

1 Dieses Projekt wurde mit Unterstützung der Europäischen Kommission finanziert. Die Verantwortung für den Inhalt dieser Veröffentlichung trägt allein der Verfasser; die Kommission haftet nicht für die weitere Verwendung der darin enthaltenen Angaben. 
Dieser Beitrag wird nur kurz auf die Ergebnisse des Projekts eingehen, die an anderer Stelle einzusehen sind. ${ }^{2}$ Eher soll hier eine kulturkritische Metaperspektive eingenommen werden, die sich mit kulturellen Aspekten in REGIONAL auseinandersetzt, insbesondere am Beispiel von Sprache. Diese wird dabei nicht nur als Problem des kulturübergreifenden Vergleichs verstanden (vgl. u.a. Reischmann 2009: 146; Mitter 2009: 97), sondern auch als dessen Gegenstand, denn in Sprache manifestieren sich auch im zusammenwachsenden Europa wesentliche kulturelle Unterschiede.

Sprachen spielen sowohl auf der Ebene des Vergleichs der politischen Prozesse als auch in der konkreten Projektarbeit, die von sieben Partnereinrichtungen aus sechs Ländern durchgeführt wurde, eine Rolle. Um beide Ebenen zu verbinden und angemessen zu analysieren, werden wir das Konzept Kultur als Praxis einführen, mit dem man sowohl den Vergleich und die Unterschiede in den Ergebnissen als auch den Einfluss der Akteure (inkl. der Geldgeber) auf die Ergebnisse erfassen kann. Es dient dann zur Feststellung und Analyse kultureller Unterschiede bzw. zur Erfassung von Unterschieden als kulturell bedingt und liefert somit als Analyseinstrument die Möglichkeit zu bestimmen, wer auf welche Weise zu kulturellen Eigenarten beiträgt und wie Ansätze aus anderen Kulturräumen verglichen und übertragen werden können. Gleichzeitig dient dieser weite Kulturbegriff der Analyse der Projektarbeit selbst, denn Kultur als Praxis erlaubt es, den Einfluss Einzelner und vor allem des Geldgebers auf europäische Bildungskultur zu analysieren. Wir werden so anhand von REGIONAL zeigen, wie wichtig kulturelle Unterschiede in europäischen Vergleichen trotz der vermeintlich abnehmenden Bedeutung von Nationalkulturen und der vermeintlich zunehmenden kulturellen Vermischung und Vereinheitlichung (vgl. Welsch 2010: passim) sind sowie wie nötig und hilfreich ein auf Praktiken bezogener Kulturbegriff ist, um diese Unterschiede zu erfassen. Auf dieser Metaebene werden wir zudem zeigen, dass die EU als Geldgeber und Rahmen der Untersuchung eine Katalysatorfunktion im europäischen Vergleich ausübt. Die ohnehin bestehende Tendenz kulturübergreifender Vergleiche zur Ambivalenz wird von der europäischen Dichotomie der gleichzeitigen Einheit und Diversität (vgl. Mitter 2009: 87) gesteigert.

Im Folgenden wird das Projekt REGIONAL kurz vorgestellt. Danach setzen wir uns mit Kulturtheorien auseinander, um das hier zugrunde gelegte Konzept von Kultur als Praxis zu diskutieren, und geben einen kurzen Einblick in die Funktionsweise kulturübergreifender Vergleiche im Bildungsbereich und darüber hinaus. Anschließend wird der Kulturbegriff auf organisa-

2 Alle Projektergebnisse und -veröffentlichungen sind auf der Projektwebsite www.regionalproject.eu verfügbar. Methodik, Länderprofile und Vergleich sind zudem in der Publikation Policy Making in Adult Education: A Comparative Approach across $21 \mathrm{Eu}$ ropean Regions veröffentlicht. 
torischer und auf inhaltlicher Ebene auf das Projekt REGIONAL angewendet, um dieses anhand des Themenbereichs Sprache und Begriffe kritisch zu reflektieren. Dieser Beitrag schließt mit einem Fazit zum Stellenwert der EUFörderung aus kulturkritischer Perspektive.

\section{Projekt REGIONAL}

Wie eingangs angesprochen setzte sich das Projekt REGIONAL zum Ziel, die Formulierung, Implementierung und Finanzierung von Erwachsenenbildungspolicies in 21 Regionen in sechs Ländern vergleichend zu analysieren. Auf diese Weise sollten stichprobenartige Einblicke in erwachsenenbildungspolitische Prozesse in ganz Europa genommen werden, um daraus Good-Practice-Beispiele abzuleiten, die die an solchen Prozessen Beteiligten unterstützen können. Das übergeordnete Ziel dabei ist die qualitative Verbesserung von Erwachsenenbildungsangeboten durch eine Verbesserung der institutionellen Rahmenbedingungen. Das Projektteam des im LebenslangenLernen-Programm der EU geförderten, 22-monatigen Projekts bestand aus insgesamt sieben Partnereinrichtungen aus Deutschland, Irland, Italien, Serbien, der Slowakei und Ungarn, die politische Prozesse in insgesamt 21 Regionen dieser sechs Länder untersuchten. Die Partnereinrichtungen waren so gewählt, dass sie bezüglich ihrer Herkunft die unterschiedlichen Entwicklungsstufen der Europäischen Union abbilden (EU15, EU28 und Beitrittskandidaten) sowie dass sowohl öffentliche, private und gemeinnützige Organisationen als auch die Wissenschaft vertreten waren.

Die untersuchten Regionen wurden danach ausgewählt, dass sie nach unterschiedlichen Kriterien (wie Bevölkerungsdichte, BIP, Arbeitslosigkeit, Beteiligung an Erwachsenbildungsangeboten, gesetzliche und administrative Grundlagen für die Erwachsenenbildung) die gesamte Bandbreite der jeweils im Vordergrund stehenden Staaten repräsentieren. In den ausgewählten Regionen wurden dann auf zweierlei Weise Daten erhoben: Erstens durch fragebogengestützte, offene Interviews mit an erwachsenenbildungspolitischen Prozessen Beteiligten (z.B. in Deutschland mit Mitarbeiter/-innen der zuständigen Fachministerien, Landtagsabgeordneten, Vertreter/-innen von Verbänden) und zweitens durch die Analyse relevanter politische Dokumente aus dem Zeitraum zwischen 2011 und 2015. Daraus erstellten die Partnereinrichtungen Länderprofile, die im Folgenden vergleichend analysiert wurden. Schließlich leitete das Projektteam daraus Good-Practice-Beispiele und konkrete Hinweise für an politischen Prozessen Beteiligte ab, die in einem Toolkit zusammengefasst wurden. 


\section{Kulturbegriff und kulturübergreifender Vergleich}

\subsection{Kultur als Praxis}

Bevor nun die Rolle kultureller Unterschiede in REGIONAL diskutiert werden kann, muss zunächst die theoretische Grundlage erörtert werden, auf der dies geschieht. Wir möchten dafür von einer Kulturdefinition des britischen Kulturwissenschaftlers Stuart Hall ausgehen:

Culture, it is argued, is not so much a set of things [...] as a process, a set of practices. Primarily, culture is concerned with the production and the exchange of meanings $[\ldots]$ between the members of a society or group (Hall 1997: 2, Hervorhebung im Original). ${ }^{3}$

Hall betont in seiner Definition das praktische Element von Kultur, das Bedeutung schafft. In der Semiotik bezeichnet Bedeutung das Verhältnis zwischen Bezeichnendem und Bezeichnetem; erweitert gesprochen verhalten sich Akteure durch Aktionen zu ihrer Umwelt, produzieren also durch Handlung Bedeutung und tauschen diese aus. Am besten verdeutlicht man diese Auffassung von Kultur mithilfe des Beispiels Sprache: Bezeichnet man einen Gegenstand oder Sachverhalt sprachlich, so produziert man kulturell spezifische Bedeutung, die zwischen Mitgliedern einer mehr oder weniger eng zusammenhängenden Gruppe, nämlich derer, die die Sprache beherrschen, ausgetauscht werden kann.

Hall gibt der Kulturanalyse mit seinen Ausführungen eine wichtige akteurszentrierte Dimension, die über die Analyse statischer Kulturprodukte hinausgeht. Kulturanalyse darf sich demnach nicht (nur) mit kulturellen Artefakten oder Wertevorstellungen auseinandersetzen, sondern muss sich mit den Wegen beschäftigen, wie diese geprägt werden. Sie dient somit dazu, zu verstehen, warum Akteure handeln, wie sie handeln. Dadurch wird sie auch für Bildungsprozesse relevant. Dies beschreibt der Erziehungswissenschaftler Volker Schubert, der den produktiven Wert des Verständnisses von Kultur als Praxis für vergleichende Bildungswissenschaften anhand internationaler Bildungsstudien verdeutlicht:

Das gute Abschneiden japanischer Schülerinnen und Schüler hat durchaus auch ,kulturelle" Gründe. Aber dabei handelt es sich nicht um ein von der sozialen Praxis abgelöstes Bündel von Wertorientierungen, das

3 Unsere Übersetzung: „Kultur, so wird argumentiert, ist nicht so sehr eine Sammlung von Dingen, sondern vielmehr ein Prozess, eine Sammlung von Praktiken. In erster Linie beschäftigt sich Kultur mit der Produktion und dem Austausch von Bedeutung zwischen Mitgliedern einer Gesellschaft oder Gruppe.“ 
eben ,japanisch" und deshalb nicht übertragbar ist; die ,kulturellen“ Gründe verweisen vielmehr auch auf spezifische pädagogische Arrangements, die als Produkte von Interaktionsprozessen, von pädagogischem Handeln oder auch Nichthandeln, zu einem guten Teil mehr oder (meist) weniger bewusst inszeniert werden (Schubert 2005: 8).

Begreift man Kultur also als Praxis, dann entfernt man sich von dem Konzept starrer, unveränderlicher Kulturen, denn Praxis impliziert, dass ein ständiger Prozess stattfindet, der von Akteuren beeinflusst und somit verändert werden kann. Auf Grundlage dieses Verständnisses von Kultur lässt sich Bildung ebenfalls als kulturelle Praktik analysieren. Konkret heißt das im Hallschen Sinn z.B., dass Lernende sich mit Gegenständen beschäftigen und so Bedeutung schaffen. Zum Austausch solcher Bedeutungen trägt das Bildungssystem bei, also z.B. auch die politischen Rahmenbedingungen, wie sie in REGIONAL im Vordergrund stehen. Auch Policyformulierung selbst kann als Produktion von Bedeutung und somit als kulturelle Praktik interpretiert werden. Diese Austauschprozesse und weitere bildungsbezogene Praktiken werden sowohl auf regionaler und nationaler Ebene als auch auf europäischer Ebene (z.B. durch das Projekt REGIONAL als Teil gemeinsamer Bildungspraxis) ausgeführt, die Systeme koexistieren und greifen ineinander. Durch seine Akteurszentrierung bildet dieses Kulturverständnis auch den Rahmen, um die Beteiligung von Geldgebern in internationalen Vergleichsstudien zu erfassen, die sich durch (finanzielle) Förderung an kulturellen Praktiken beteiligen. Insgesamt hilft Halls akteurszentrierter Kulturbegriff dabei, die Ansprüche zu erfüllen, die Jost Reischmann an den Vergleich in der Erwachsenenbildungsforschung stellt - dieser soll ,die Hintergründe und Bedeutung der Ähnlichkeiten und Unterschiede verstehbar machen“ (Reischmann 2009: 146).

\subsection{Kulturübergreifende Vergleiche}

Kern der Arbeit in REGIONAL ist der Vergleich der erwachsenenbildungspolitischen Prozesse in 21 europäischen Regionen. Dabei hatte das Projektteam mit den gleichen Schwierigkeiten zu tun, die viele Vergleiche in der Bildung, aber auch in anderen Feldern hervorrufen, nämlich, dass sie gegenteilige Effekte haben können. António Nóvoa und Tali Yariv-Mashall stellen z.B. fest, dass vergleichende Bildungsstudien das Feld sowohl verarmen als auch bereichern und vertiefen können (vgl. Nóvoa/Yariv-Mashall 2014: 1617). Wolfgang Mitter ordnet Vergleiche in einem etwas weniger wertenden Sinne in Europas oben bereits angesprochene Dichotomie der Einheit und Diversität ein (Mitter 2009: 87).

Nóvoa und Yariv-Mashall nehmen in ihren Ausführungen Bezug auf literatur- und kulturwissenschaftliche Forschung, in der vergleichende Arbeit 
einen großen Stellenwert hat. Entsprechend intensiv setzt sich diese Disziplin mit Stärken und Schwächen des Vergleichs auseinander. Rajagopalan Radhakrishnan arbeitet heraus, dass Vergleiche Hierarchien abbauen und Wissen in Frage stellen können (Radhakrishnan 2009: passim), Peter Zima betont, dass sie Untersuchungsergebnisse allgemeingültiger machen (Zima 2011: 16). Jedoch kann diese Allgemeingültigkeit wiederum zum Problem werden, denn allgemeingültige Ergebnisse können Sachverhalte stark vereinfachen, Raum für Wiedersprüche geht verloren und Hierarchien können aufgebaut werden. Letzteres ist insbesondere bei kulturübergreifenden Vergleichen relevant, wie Robert Stam und Ella Shohat herausarbeiten. Sie verweisen darauf, dass dabei schnell eine Kultur an der anderen gemessen wird: „one culture as normative and the other as aberrant" (Stam and Shohat 2009: 473). Vergleiche können bestimmte Aspekte verdecken oder betonen, weshalb Vorüberlegungen offengelegt werden müssen: ,who is doing the comparing, in relation to which social groups, along what axes, and to what ends" (Stam/Shohat 2009: 485). Insbesondere bzgl. politischer Maßnahmen, die in REGIONAL verglichen werden, ist eine Kontextualisierung der Vergleichsgegenstände notwendig, um unkritische und unpassende Übertragung von Konzepten zu vermeiden und eben nicht Prozesse, die in einem kulturellen Raum vermeintlich gut funktionieren, vorbehaltslos auf andere kulturelle Räume zu übertragen.

Es wird deutlich, dass Vergleiche diffizile Angelegenheiten sind, die die Qualität von Untersuchungsergebnissen sowohl erhöhen als auch schmälern können. Betrachtet man die positiven und negativen Facetten, die Radhakrishnan, Zima, Stam und Shohat erörtern, so fällt auf, dass diese fast deckungsgleich sind. Vergleiche können Hierarchien ab- und aufbauen, sie können allgemeingültig machen und verallgemeinern. Hier ist das Reflexionsvermögen des Vergleichenden entscheidend, um den Anstoß in die gewünschte Richtung zu geben. Außerdem ist es wichtig, die Kriterien und Methoden des Vergleichs offenzulegen, damit im Nachhinein klar wird, welches die gewünschte Richtung eigentlich war. Kultur als Praxis lässt sich dafür nutzbar machen, denn damit kann man kulturelle Prozesse (also auch Bildungsprozesse) kontextualisieren und transparent vergleichen sowie der Tatsache gerecht werden, dass Vergleiche in beide Richtungen tendieren können (auch gleichzeitig, bzgl. unterschiedlicher Aspekte) und dass sie sich ebenso wie Europa selbst zwischen den Polen Einheit und Diversität bewegen. 


\section{Kulturelle Unterschiede in Ergebnissen und Projektarbeit}

Im zusammenwachsenden Europa sind nationalstaatliche Abgrenzungen und damit verbundene kulturelle Unterschiede an vielen Stellen immer weniger präsent. Dennoch bestehen kulturelle Unterschiede nach wie vor, insbesondere in Sprache und Begrifflichkeiten, die im Sinne von Hall und Hörning geteilte kulturelle Praktiken sind. Die Durchführung des Projekts REGIONAL zeigte dies sowohl in den Projektergebnissen als auch in der Projektzusammenarbeit.

Der Vergleich von Erwachsenenbildungspolicies in REGIONAL zeigt deutlich, wie sprachliche Unterschiede die Ergebnisse beeinflussen und prägen. Sprache vermittelt kulturspezifische Konzepte. Das heißt, dass sie nicht nur Bedeutungen prägt, sondern dass sie auch festlegt, was überhaupt denkbar und ausdrückbar ist. Ein oberflächliches aber einschlägiges Beispiel dafür sind die Begriffe Bildung und Erziehung. Diese auf Deutsch klar abzugrenzenden Begriffe haben auf Englisch mit education eine gemeinsame Entsprechung. Hier werden bereits die Schwierigkeiten kulturübergreifender Vergleiche deutlich, denn die deutschen Begriffe implizieren Deutungsnuancen, die dem englischen Begriff fehlen.

Von zentraler Bedeutung in der Vergleichsarbeit in REGIONAL waren die Begriffe Erwachsenenbildung und berufliche Bildung. Insbesondere in den süd- und osteuropäischen Ländern der REGIONAL-Studie wurden diese Konzepte von Interviewpartnern deckungsgleich verwendet, in Deutschland und Irland jedoch nicht (REGIONAL 2015: Comparative Analysis). Hier läuft man dann Gefahr, die sprichwörtlichen Äpfel mit Birnen zu vergleichen (die natürlich außerhalb ihrer sprichwörtlichen Bedeutung sehr wohl vergleichbar sind), denn sprechen unterschiedliche europäische Akteure von Erwachsenenbildung, dann werden - je nach kultureller Prägung - unter Umständen völlig unterschiedliche Aspekte betont.

Ein Verständnis von Kultur als Praxis hilft an dieser Stelle, praktisch damit umzugehen. Denn anstatt Kulturen trotz ihrer meist klar voneinander abgrenzbaren Sprachen als statisch und unveränderbar wahrzunehmen, kann man dann davon ausgehen, dass europäische (Bildungs-)Kulturen sich aufeinander zubewegen können. Ein Projekt wie REGIONAL kann dazu beitragen, europäische Bildungskultur zu prägen und diese kulturellen Gegensätze zu überbrücken. Konkret können in solchen Fällen übergreifende Definitionen Abhilfe verschaffen, wie etwa der mehrsprachige CEDEFOP-Glossar, der auf europäischer Ebene durchaus für seine Begriffsdefinitionen im Bildungsbereich anerkannt ist. Allerdings muss man dann in seiner Analyse die Prozesse und vor allem die beteiligten Akteure mit einbeziehen. CEDEFOP legt als Europäisches Zentrum für die Förderung der Berufsbildung einen Fokus auf einen bestimmten Bildungssektor. Dieser spiegelt sich auch im 
Glossar wider, in dem sich z.B. kein Eintrag zur kulturellen Bildung findet. Es zeigt sich, dass standardisierte Begriffe und kulturübergreifende Vergleiche mit denselben zentralen Schwierigkeiten kämpfen, nämlich damit, interessengeleitet zu sein.

Diese Schwierigkeit setzt sich auch in der Projektarbeit selbst fort. Die beteiligten Partner in REGIONAL sprechen unterschiedliche Sprachen und benutzen dementsprechend unterschiedlich konnotierte Begriffe. Diese Konnotationen bleiben in der gemeinsamen Arbeitssprache Englisch durchaus erhalten, sodass verschiedene beteiligte Akteure gemeinsame Begriffe unter Umständen mit unterschiedlicher Bedeutung aufladen. Es reicht also nicht, sich auf eine gemeinsame Arbeitssprache zu einigen, vielmehr ist die Aushandlung von Begriffen nötig. Dadurch schaffen die Beteiligten eine europäische Bildungskultur bzw. nähern sich dieser an. Dennoch bleibt das Problem der unterschiedlichen Arbeitssprachen bestehen, denn alle Partner haben Daten in ihren Landessprachen erhoben, diese ins Englische übersetzt, die Ergebnisse verglichen und diesen Vergleich wieder in die Landessprachen zurückübersetzt. Die Gefahr, Konzepte zu übersetzen, ohne den entsprechenden kulturellen Konnotationen gerecht zu werden, ist enorm und es bedarf höchster kritischer Distanz und kulturellen Reflexionsvermögens, um überhaupt nutzbare Ergebnisse zu generieren. An dieser Stelle wird deutlich, wie schmal der Grat zwischen differenzierten, allgemeingültigen Ergebnissen und Übersimplifizierung im europäischen Vergleich ist und wie stark der kulturübergreifende Vergleich die europäische Dichotomie zwischen Gleichheit und Diversität befeuert.

Wie in der Datenerhebung, der Übersetzung und dem Vergleich können auch im Bereich der Zusammenarbeit der Partnerinstitutionen europäische Organisationen eine Brücke schlagen, indem sie allgemeine Begrifflichkeiten zur Verfügung stellen. Verdeutlicht man sich jedoch die Rolle solcher Institutionen als Akteure in kulturellen Praktiken, dann wird offensichtlich, dass sie eben keine neutralen Kategorien bereitstellen, sondern ebenso kulturell verortet sind und dass sie interessengeleitete Kategorien auf- und bereitstellen. Zusätzlich sind auch vermeintlich neutrale Kategorien immer noch sprachlich verortet und somit mit kulturell spezifischer Bedeutung aufgeladen, sodass sie u.U. eher Hierarchien auf- als abbauen.

\section{Fazit: Stellenwert der EU-Förderung}

Sprache zeigt, wie sehr kulturelle Unterschiede gleichzeitig die Untersuchungsergebnisse in REGIONAL und die Zusammenarbeit der Projektpartner prägen. Sprache ist ein besonders geeigneter Indikator dafür, denn kulturelle Unterschiede manifestieren sich oft in sprachlichen Konzepten. Aus kulturwissenschaftlicher Sicht kommt also sprachlichen Repräsentationen von 
Erwachsenenbildungsstrukturen und Sprache als kultureller Praktik in Verbindung mit Bildung als kultureller Praktik entscheidende Bedeutung zu.

Versteht man Kultur als Praxis wird deutlich, wie stark der Einfluss europäischer Institutionen in vergleichender Kulturanalyse ist (vgl. Mitter 2009: 96). Oben wurde dies insbesondere auf das Bereitstellen von Begriffen bezogen. Die EU und ihre Institution beeinflussen Projekte wie REGIONAL aber auch als Geldgeber und greifen so auf einer weiteren Ebene als Akteure in Produktion und Austausch von Bedeutung, also in gewisser Weise in den Prozess der Genese europäischer Bildungskultur ein. Dies lässt sich ebenfalls auf den Ebenen der Projektpartnerschaft und der Projektergebnisse betrachten. Bezüglich der Partnerschaft lässt sich feststellen, dass die EU durch ihre Förderung den Austausch überhaupt ermöglicht. Dies bezieht sich gleichermaßen auf finanzielle wie auf ideelle Förderung, denn einerseits ist die finanzielle Förderung grundlegend dafür, dass das Projekt durchgeführt werden kann, andererseits spornt viele Partnereinrichtungen aber auch allein das Prestige der Teilnahme an einem EU-Projekt an. Die EU beeinflusst den Austausch, den sie durch ihre Förderung initiiert, jedoch auch massiv durch politische Interessen - sie entscheidet, welche Projekte gefördert werden und sie sitzt im übertragenen Sinn mit am Tisch und lenkt die Diskussion, sowohl als allgemeiner Referenzrahmen (z.B. bzgl. Begriffe) als auch durch das (bevorstehende) Gutachten und die Finanzierung.

Inhaltlich schafft die EU Raum für Auseinandersetzung mit verschiedenen Kulturen und einen Rahmen der Akzeptanz von Vielfalt - das multinationale Konsortium ermöglicht differenzierte Projektergebnisse, die aus vielen verschiedenen Perspektiven generiert und geprüft werden. Außerdem ermöglicht die im Konsortium angelegte Mehrsprachigkeit die Erhebung von Daten in den einzelnen Landessprachen und somit Ergebnisse, die nationale Projekte nicht leisten können. Auch hier hat die EU-Förderung jedoch wieder gegenteilige Effekte, denn die politischen Ziele der EU priorisieren die Ergebnisse und hierarchisieren sie damit - zumindest implizit. Die Neuorganisation der Europäischen Kommission 2014 zeigt deutlich auf, welche Prioritäten die EU dabei setzt: Im Zuge der Umstrukturierung einiger Bereiche wurde die Erwachsenenbildung von der Generaldirektion Bildung und Kultur in die Generaldirektion Beschäftigung verschoben. Dies setzt den gleichen Fokus auf berufliche Bildung, den z.B. CEDEFOP auf begrifflicher Ebene ebenfalls setzt.

EU-Förderung ist offensichtlich eine vielschichtige Angelegenheit, die Chancen und Risiken birgt. Mit Bezug auf Radhakrishnan, Zima, Stam und Shohat haben wir oben festgestellt, dass kulturübergreifende Vergleiche Hierarchien hinterfragen und aufbauen können und dass sie Ergebnisse universeller machen, sie aber auch stark vereinfach können. EU-Förderung kann, wie oben gezeigt, alle Facetten des kulturübergreifenden Vergleichs steigern - sie wirkt wie ein Katalysator, der hilfreiche und kritische Aspekte kultur- 
übergreifender Vergleiche gleichermaßen verstärkt. EU-geförderte Projekte wie REGIONAL bieten große Chancen für überaus weitreichende und tiefgehende Einblicke, müssen aber extrem reflektiert durchgeführt werden, um nicht ins Gegenteil umzuschlagen. Ein Verständnis von Kultur als Praxis hilft dabei, denn es fokussiert die Analyse auf die beteiligten Akteure und ihre Interessen.

\section{Literatur}

Agentur für Erwachsenen- und Weiterbildung (2016): Policy Making in Adult Education: A Comparative Approach across 21 Regions. Bielefeld: WBV.

Ausschuss der Regionen (2008): Stellungnahme des Ausschusses der Regionen „Aktionsplan Erwachsenenbildung - Zum Lernen ist es nie zu spät“" (2008/C 257/11). http://eur-lex.europa.eu/legal-content/DE/TXT/?uri=uriserv:OJ.C_.2008.257.01. 0070.01.DEU [Zugriff: 26.01.2016].

Ballas, Dimitris et al. (2012): Mind the Gap - Education Inequalities Across EU Regions. NESSE/Europäische Kommission. http://www.nesse.fr/nesse/activities/ reports/mind-the-gap-1 [Zugriff: 26.01.2016].

CEDEFOP (2014): Terminology of European Education and Training Policy: A Selection of $130 \mathrm{Key}$ Terms. Luxembourg: Publications Office of the European Union.

Europäische Kommission (2006): Erwachsenenbildung: Man lernt nie aus. http://eurlex.europa.eu/legal-content/DE/TXT/HTML/?uri=CELEX:52006DC0614\&from =EN [Zugriff: 26.01.2016].

EUROSTAT (2016): Teilnahmequote an Bildung und Weiterbildung (letzte 4 Wochen) nach NUTS-2-Regionen. http://appsso.eurostat.ec.europa.eu/nui/show.do [Zugriff 29.04.2016].

Hall, Stuart (1997): Introduction. In: Hall, Stuart (Hrsg.): Representation: Cultural Representations and Signifying Practices. London: Sage, S. 1-11.

Mitter, Wolfgang (2009): Comparative Education in Europe. In: Cowen, R./Kazamias, A. M. (Hrsg.): International handbook of Comparative Education. Dordrecht, Heidelberg et al: Springer.

Nóvoa, António/Yariv-Mashal, Tali (2014): Comparative Research in Education: A Mode of Governance or a Historical Journey? In: Fenwick, Tara,/Margez, Eric/Ozga, Jenny (Hrsg.): Governing Knowledge - Comparison, Knowledgebased Technologies and Expertise in the Regulation of Education. World Yearbook of Education 2014. London und New York: Routledge.

Radhakrishnan, R. (2009): Why Compare? In: New Literary History 40.3, S. 453-471.

REGIONAL (2015): Länderprofil: Deutschland. http://www.regionalproject.eu/ results.php [Zugriff 26.01.2016].

REGIONAL (2015): Comparative Analysis of Regional Policies for AL. http://www.regionalproject.eu/results.php [Zugriff 26.01.2016].

REGIONAL (2015): AL Policy Making Toolkit. http://www.regionalproject.eu/ results.php [Zugriff 26.01.2016].

Reischmann, Jost (2009: Comparative Adult Education: Der Internationale Blick auf die Erwachsenenbildung. In: Hessische Blätter 2.2009, S. 140-151. 
Schubert, Volker (2005): Pädagogik als vergleichende Kulturwissenschaft - Erziehung und Bildung in Japan. Wiesbaden: Verlag für Sozialwissenschaften.

Stam, Robert/Shohat, Ella (2009): Transnationalizing Comparison: The Uses and Abuses of Cross-Cultural Analogy. In: New Literary History 40.3, S. 473-499.

Welsch, Wolfgang (2010): Was ist eigentlich Transkulturalität? In: Darowska, Lucyna/ Lüttenberg, Thomas/Machold, Claudia (Hrsg.): Hochschule als Transkultureller Raum? Bielefeld: Transcript, S. 39-65.

Zima, Peter V. (2011): Komparatistische Perspektiven - Zur Theorie der Vergleichenden Literaturwissenschaft. Tübingen: Francke. 



\section{Transnationale Lernkulturen beruflicher Aus- und Weiterbildung in Deutschland und China am Beispiel handlungsorientierten Lernens}

\section{Lehr-Lernkonzepte im Rahmen des Berufsbildungsexports}

Seit den 1980er Jahren besteht ein Interesse an deutscher Aus- und Weiterbildung in China (Zhao 2013: 10). Berufsausbildungen, berufliche Qualifizierungen und Angebote der Management-Qualifizierung stehen dabei im Fokus und Bildungsinhalte und -formate werden von Deutschland nach China exportiert (iMOVE 2010; BMBF 2015). Im Mittelpunkt steht das Bestreben deutscher Weiterbildungsanbieter, auf dem chinesischen Weiterbildungsmarkt Fuß zu fassen. Bisher ist jedoch kaum untersucht worden, nach welchen didaktischen Konzepten und Modellen deutsche Anbieter in China vorgehen und was derartige transnationale Lernkulturen überhaupt ausmacht.

Der vorliegende Beitrag untersucht diesen Kontext anhand der Unterscheidung von Inter- und Transnationalisierung sowie des Modells handlungsorientierten Lernens und stellt Zwischenergebnisse des Forschungsprojekts „EWA“ vor. Dazu werden Forschungskontext sowie Begriffsverständnisse vorgestellt. Nachdem dargelegt wird, warum handlungsorientiertes Lernen in China evident erscheint, werden die empirischen Grundlagen reflektiert. Ausgehend von der Hypothese, dass handlungsorientiertes Lernen in China prinzipiell anwendbar ist, wird eingeschränkt, dass dies angesichts der rasanten Entwicklungen in China zunächst nur modifiziert funktioniert und besonders stark von dem Bedarf der Lerngruppe abhängig ist. Dabei spielt der Übergang von internationaler Lernkultur hin zu transnationaler Lernkultur eine wichtige Rolle. Die Zwischenergebnisse verdeutlichen lernkulturelle Ambivalenzen anhand von fünf Grundsätzen handlungsorientierten Lernens.

\section{Strukturen und Begriffe im Projekt "EWA in China"}

Worin liegen eigentlich die Herausforderungen im Institutionalisierungsprozess, wenn deutsche Bildungsangebote auf dem chinesischen Markt platziert werden? Ausgehend von dieser Frage und angetrieben von dem Vorhaben, sich im chinesischen Markt der Aus- und Weiterbildung erfolgreich zu etablieren, wurde im Jahr 2014 von zwei deutschen Weiterbildungs- bzw. Kultureinrichtungen eine Weiterbildungsinstitution gegründet. Den Rahmen 
dieser Unternehmung bildet das Forschungs- und Entwicklungsprojekt EWA (Etablierung einer Weiterbildungsakademie in China, vgl. Robak et al. 2014). Gefördert durch das Bundesministerium für Bildung und Forschung, kooperieren beide Einrichtungen mit dem Institut für Berufspädagogik und Erwachsenenbildung der Leibniz Universität Hannover, das die wissenschaftliche Begleitforschung durchführt.

In Bezug auf Transnationalisierung wird im Projekt EWA, ebenso wie im vorliegenden Beitrag, dem Ansatz von Welsch (2005) gefolgt (vgl. Robak 2012: 117-119), der einen alten und neuen Kulturbegriff voneinander abgrenzt. Nach altem Verständnis existieren Kulturen vorwiegend aufgrund eines nationalen Kerns: „Kulturen, die im Prinzip als autonom [...] aufgefaßt sind, können einander letztlich nicht verstehen, sondern müssen sich der Logik dieser Auffassung gemäß voneinander absetzen..." (Welsch 2005: 320), während dies nach einem neueren Verständnis in Auflösung begriffen ist (Interkulturalität, Transkulturalität). Transkulturalität wird dabei durch gegenseitige Durchdringung und Durchmischung gekennzeichnet (Welsch 2005: 322) und lässt den interkulturellen Zugang erschließen und weiterentwickeln.

Nicht nur in allgemein kultureller Hinsicht, auch bezogen auf die Gestaltung von Lehr-Lernprozessen, kommt dabei den Fragen der Rahmung von Lernkultur eine besondere Bedeutung zu (Schüßler/Thurnes 2005: 15). Lernkulturen $^{1}$ - so das Begriffsverständnis des vorliegenden Beitrags (vgl. Dehnbostel 2010) - sind dynamisch, da sie sozial konstruiert sind. Dabei sind die früheren Lehr- und Lernerfahrungen der Kursbeteiligten wie auch die pädagogische Atmosphäre von Bedeutung. Diesbezüglich gehen Schüßler und Thurnes davon aus, dass Lernkulturen den Beteiligten Orientierung geben können, wenn es gelingt, dass Normen, Werte und Deutungsmuster von allen Beteiligten geteilt werden (ebd.) und implizite Einflüsse ebenso wie explizite Aspekte Berücksichtigung finden (vgl. Abb. 1).

1 Lernkulturen geben Grundlagen, Hintergründe, gesellschaftliche, soziale und institutionelle Dimensionen des Lernens an. Der Begriff Lernkultur ist als übergreifender Gattungsbegriff anzusehen. In der realen Arbeits- und Lebenswelt ist von jeweils spezifischen Lernkulturen auszugehen, die die Modalitäten des Lernens und die damit verbundenen individuellen und sozialen Dispositionen erfassen. 


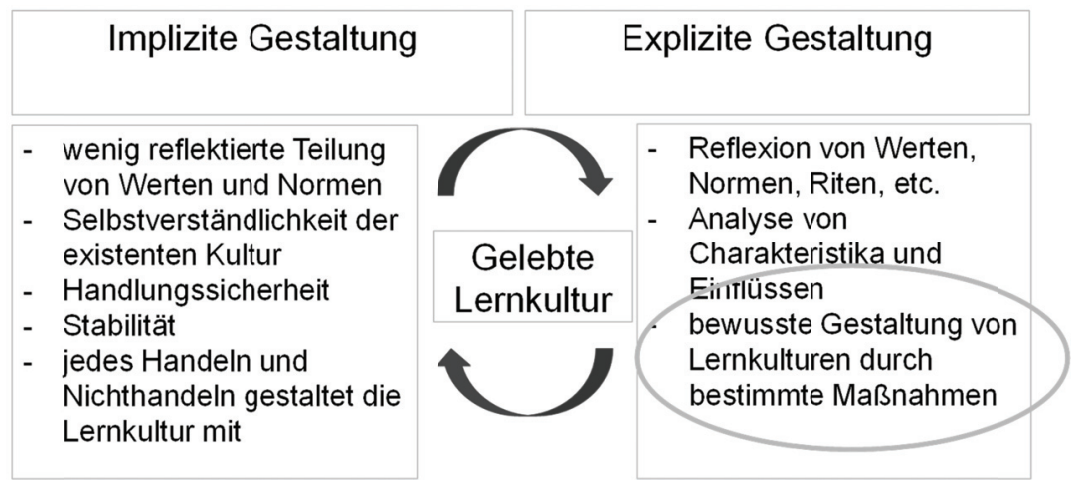

Abb. 1: Explizite und Implizite Gestaltung von Lernkulturen (vgl. Schüßler/Thurnes 2005: 322)

Bezogen auf die Entwicklung von Transkulturalität gehen wir hypothetisch davon aus, dass Maßnahmen, wie sie in der bewussten Gestaltung von Lernkulturen nach Schüßler/Thurnes konzipiert sind, sich zunächst in der ,autonomen" Form von Interkulturalität entwickeln (Phase interkultureller Absetzung). Erst danach und in Auflösung dieser Phase können Maßnahmen transkultureller Lernkultur entstehen (vgl. Abb. 2).

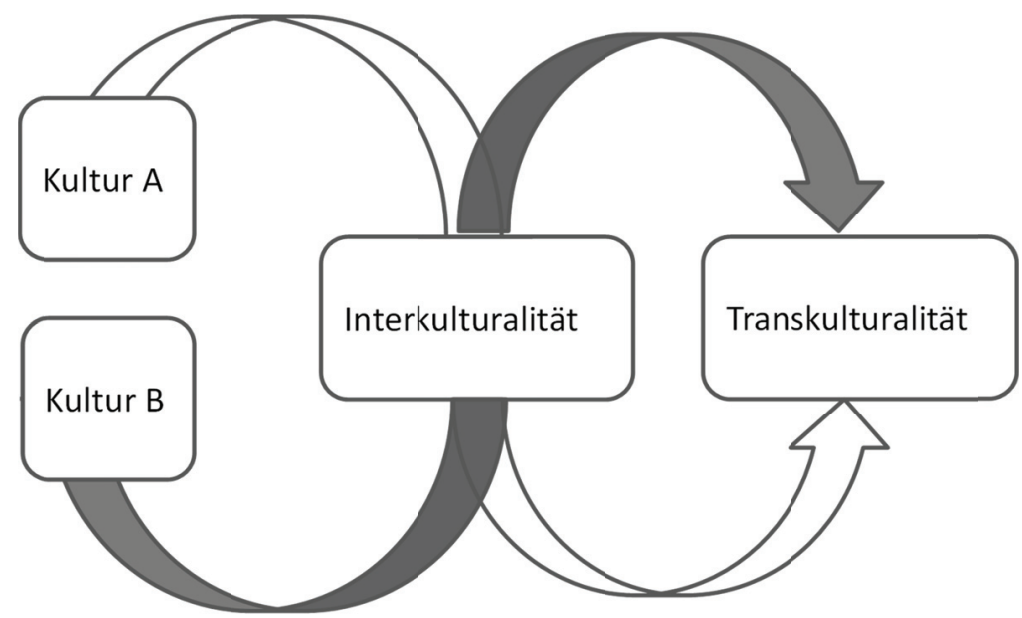

Abb. 2: Transkulturalität in Vermittlung durch Interkulturalität (eigene Darstellung) 


\section{Handlungsorientierung als Konzept expliziter transnationaler Lernkulturgestaltung und empirischer Hintergrund}

Wie lassen sich nun Lehr- und Lernkonzepte finden, die adäquat, erfolgreich und richtungsweisend für den Einsatz im Bildungsexport nach China sind? Dem bereits genannten Verständnis von Transkulturalität folgend, stellt sich zunächst die Frage, auf welcher Lernkultur aufgebaut wird, bevor ein Konzept begründet ausgewählt werden kann. Auch wenn hier nicht ausführlich auf die Lehr- und Lernkulturen Deutschlands bzw. Chinas in der beruflichen Aus- und Weiterbildung eingegangen werden kann (vgl. etwa Watkins/Biggs 1996; Seufert/Metzger 2013), so lassen sich skizzenartig folgende Charakteristika festhalten:

- Für die deutsche Seite kann von einem umfangreichen Verständnis und vielschichtigen Zugängen ausgegangen werden, die häufig reformpädagogisch geprägt sind, individualisiertes und kompetenzorientiertes Lernen anstreben und auf die Förderung der beruflichen Handlungsfähigkeit der Lernenden in der betrieblichen Praxis abzielen (lernen \& lehren 2010, Gillen 2013).

- Chinesisches Lernen dagegen ist traditionell an Frontalsituationen und einer Lehrerzentrierung orientiert. Gegenwärtig kann noch immer aufgrund der konfuzianischen Tradition, nach der das Akademische einen ungleich höheren gesellschaftlichen Stellenwert einnimmt als das praktische Tun, von einer starken Theorie-Ausrichtung und einer Distanz zu berufspraktischen Tätigkeiten ausgegangen werden (Herzog/Bai 2015: 14). Diese Einstellungen könnten sich zukünftig durch politische Reformen und arbeitsmarktbezogene Entwicklungen ändern, die weitere Öffnungseffekte erwarten lassen (Facharbeitermangel, Aufhebung der Ein-KindPolitik, Durchführung der großen Bildungsreform in China vgl. z.B. Schmidt-Dörr 2015; BMBF 2015; iMOVE 2010).

Vor diesem Hintergrund stellt der Ansatz handlungsorientierten Lernens für die berufliche Aus- und Weiterbildung (z.B. bHO 2013, Meyer/Oelke 2013) einen Ausgangspunkt für die Entwicklung transnationaler Lehr-Lernkonzepte dar, um die Bedarfe an berufspraktischem Handlungswissen Chinas zu decken. Er nimmt berufspraktische Handlungsfelder systematisch zur Grundlage der Ausbildung und orientiert sich an einer komplexen Problemstellung aus dem beruflichen Alltag. Diese bildet die Grundlage des Lehr-Lernprozesses, an dessen Ende in der Regel ein konkretes Handlungsprodukt entsteht, das sich unter realen betrieblichen Anwendungsbedingungen bewähren sollte (vgl. Abb. 3). 


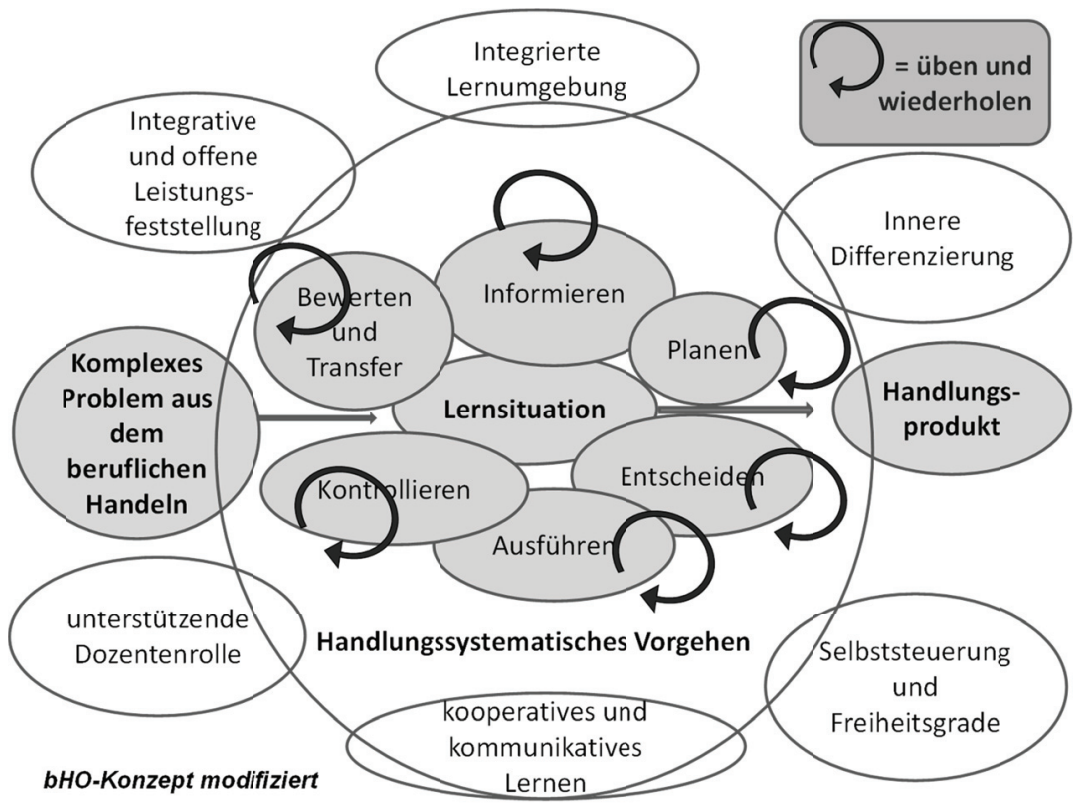

Abb. 3: Konzept beruflicher Handlungsorientierung (modifiziert nach bHO 2013)

Doch wie erfolgreich kann ein solches Konzept unter den Bedingungen transnationaler Lernkulturen sein? Welche Anpassungen sind nötig, um nicht nur ein Konzept zu exportieren, sondern es im Raum der Transkulturalität zu verändern?

Um diese Fragen zu beantworten, werden in Kapitel vier didaktische Grundsätze handlungsorientierter Lernsituationen empirischen Reflexionen für eine Anwendung in China gegenübergestellt. Der dazu verwendete Methoden-Mix (vgl. Herzog/Gillen 2016) vereint Dokumentenanalyse, teilnehmende Beobachtung, Videoaufzeichnungen der durchgeführten Seminare sowie leitfadengestützte Experteninterviews sowohl mit den Lehrenden als auch mit den Lernenden in qualitativer Auswertung. Diese empirische Grundlage muss allerdings kritisch reflektiert werden. So hatten die durchgeführten Seminare eher einen Test-Charakter, z.B. in Bezug auf Dozenten (unterschiedliche Sprach- und Fachkompetenzen), Seminarthemen oder Unterrichtssprache (Deutsch/Englisch mit Übersetzung ins Chinesische). Überwiegend ähnlich waren die Seminare in Bezug auf Geschlecht (eher ausgeglichene Verteilung), Gruppengröße (8-16 Teilnehmer), Bildungshintergrund (akademisch) und berufliche Position (mittleres Management). Alle Kurse 
wurden gedolmetscht und unterlagen einem Qualitätsmanagement (Feedback-Bögen an Teilnehmer).

Da der chinesische Bildungsmarkt unbekannt und das Vorwissen eher gering war, wurde seitens der Projektpartner zunächst mit Frontalunterricht gearbeitet. Im Laufe der verschiedenen Seminardurchführungen wurden jedoch zunehmend Elemente des handlungsorientierten Unterrichts behutsam getestet. Eine komplett diesem Konzept entsprechende Seminargestaltung gab es im untersuchten Zeitraum nicht. Auch aus anderen Gründen fungieren die hier gewonnenen Zwischenergebnisse als momentane Anhaltspunkte, da China gegenwärtig folgende Dynamiken prägen (vgl. Heilmann 2016):

- starke gesellschaftliche und kulturelle Veränderungen in kurzer Zeit

- steigende Diversifizierung der Lebenssituationen in der Bevölkerung

- wachsende Internationalisierung in allen Lebensbereichen (Orientierung an internationalen Standards z.B. durch Akademiker mit Auslandserfahrung)

- Arbeitsmärkte mit hoher Fluktuation

- Bildungssystem im Umbruch (Ausbau beruflicher Bildung)

Insgesamt kann festgehalten werden, dass Aussagen zu „Chinese Learners“ zunehmend weniger eindeutig zu treffen sind. Die Ergebnisse der Untersuchung sind unterschiedlichen methodischen Einschränkungen unterworfen und dienen vor allem der Bildung von Annahmen, um die Entwicklung transnationaler Lernkulturen in der chinesischen Aus- und Weiterbildung besser verstehen zu können.

\section{Didaktische Grundsätze handlungsorientierter Lernsituationen und empirische Reflexionen in Bezug auf die Anwendung in China}

Inwieweit handlungsorientiertes Lernen als Teil „,deutscher Lernkultur“ sich mit der „chinesischen Lernkultur“" vergleichen lässt, soll an den didaktischen Grundsätzen handlungsorientierter Lernsituationen (KMK 1998) verdeutlicht werden.

Von der Ausbildungssituation in Deutschland ausgehend spielen schulische Lernfelder und Lernsituationen sowie betriebliche Handlungsfelder über Handlungssituationen des Berufs ineinander. Im Konzept der Handlungsorientierung wird davon ausgegangen, dass (1) die didaktischen Bezugspunkte des Lernens durch Situationen gegeben sind, die für die Berufsausübung bedeutsam sind (KMK 1998: 6). Danach wird von einem umfänglichen System deutscher Beruflichkeit ausgegangen, in dem viele Parameter gesellschaftlich und rechtlich geregelt sind (z.B. Berufsbild, berufliche Tätigkeiten etc.). 
Beruf und Beruflichkeit sind in China dagegen nicht so stark ausgeprägt wie in Deutschland (Herzog/Bai 2015). Arbeitsplätze sind z.T. eher funktionsbezogen als berufsorientiert. In China gibt es z.B. das Studium des Brückenbaus, nicht aber eine Ausbildung oder einen Studiengang zum Brückenbauer (Zheng/Rützel 2014). Die Kenntnis von Ziel- und Lernergruppe gehört daher zu den wichtigsten Voraussetzungen adäquaten Dozenten-Handelns in handlungsorientierten Lernsituationen, um beruflich bedeutsame Situationen überhaupt einschätzen zu können.

Ein weiterer Aspekt der Handlungsorientierung in der beruflichen Bildung besteht darin, dass sich (2) Lernen in vollständigen Handlungen vollzieht, möglichst selbst ausgeführt oder zumindest gedanklich nachvollzogen werden soll (KMK 1998: 6). Die Orientierung am Prinzip der vollständigen Handlung bezieht sich auf die methodische Vollständigkeit von Arbeitsaufträgen, in denen möglichst der gesamte Arbeitsprozess (Planung, Durchführung und Kontrolle) integriert ist (vgl. Sloane/Twardy/Buschfeld 1998: 107). Lehrende sollen vollständige Handlungen thematisieren, die für die Lernenden interessant und berufsrelevant sind. Organisiertes Lernen in China vollzieht sich typischerweise in großem Fleiß und hoher Arbeitsmoral, eher passivem Lernverhalten und ausgeprägter Bildungsaffinität (vgl. z.B. Yeung 2015: 126-127). Selbst ausgeführte Handlungen sind dabei weniger üblich, das Lernen findet klassischerweise im theoretischen Kontext statt. Praktische Handlungen sind weniger anerkannt (vgl. z.B. Pilz/Li 2014: 20f.; Lui/Leung 2013: 36; Wang 2008; Aulig 2008). Das Berufsausbildungssystem Chinas ist gegenüber dem Deutschlands nicht so stark entwickelt, so dass der Umfang praxisnaher Ausbildungsteile von lokal ausgehandelten Partnerschaften zwischen Schulen und Arbeitgebern abhängt (Li/Pilz 2011: 14). Um vollständige Handlungen im Zielland identifizieren zu können, sind Kenntnisse der realen Arbeitsweisen nötig, da sich die Vorstellungen einer vollständigen beruflichen Handlung z.T. deutlich unterscheiden können.

Zusätzlich folgt das Konzept handlungsorientierten Lernens einem (3) konstruktivistischen Leitbild, in dem die Lernenden den eigenen Lern- und Arbeitsprozess selbst planen (KMK 1998: 6), durchführen und auswerten. Lernen wird dabei im Sinne einer konstruktivistischen Lernorientierung als konstruktiver, selbstgesteuerter und sozialer Prozess verstanden, bei dem es eine lernförderliche Verknüpfung von Führung und Selbständigkeit gibt. Während Lernprozesse von den Lernenden in der Regel selbst gestaltet werden, nehmen Lehrende eher die Rolle eines Moderators selbstgesteuerter Lernprozesse ein.

Die Verknüpfung von Führung und Selbständigkeit ist im chinesischen Ausbildungssystem eher unüblich. Die Lernenden verstehen es dagegen, nach Vorgaben zu arbeiten. Ganzheitlichkeit und Selbststeuerung nach deutschem Verständnis sind offenbar (noch) nicht Teil gegenwärtiger Lernkulturen, wie ein deutscher Experte aus der Autobranche ausführt: 
„Und es war ganz interessant, ich habe erst letzte Woche ein Gespräch gehabt mit einem Teilnehmer, der zu mir gesagt hat: Ja das Training war schon sehr gut. Aber er glaubt, dass wir die Leute eigentlich überfordern, indem wir einfach zu viel, ja Eigenverantwortung, Selbstständigkeit voraussetzen. Also da muss man eher noch bisschen runterfahren und einfache Trainings machen, längere Praxiseinheiten, sonst trifft es einfach nicht den Markt.“ (Int 50_36).

Für die Vermittlung handlungsorientierten Lernens sind demnach Kenntnisse beruflicher Lernkulturen und entsprechender Bedarfe bezogen auf den angezielten Bildungsmarkt ebenso unabdingbar wie das Wissen um die Fähigkeit der Zielgruppe, selbständig zu handeln.

Nach dem Verständnis handlungsorientierten Lernens greifen Handlungen (4) die Erfahrungen der Lernenden auf und reflektieren sie in Bezug auf ihre Auswirkungen (KMK 1998: 6). Erfahrungen, Reflexionsbereitschaft sowie Reflexionsvermögen der Lernenden sind somit zentrale Elemente handlungsorientierten Lernens. Dabei ist die Abfolge von Handlung - Erfahrung - Reflexion und deren kontinuierliche Fortführung unter Berücksichtigung vorheriger Erfahrungs- und Erkenntnisprozesse als Prozess des Aufbaus von Erfahrungswissen anzusehen. In chinesischen Lehr- und Lernprozessen sind Handlungen als Gegenstand von institutionalisierten Lernprozessen bisher eher ungewöhnlich (vgl. allerdings Wagner 2001, der auf den gelungenen Einsatz solcher Konzepte in Einzelfällen schon seit den 1980er Jahren hinweist). Reflexionen eigener Handlungen und deren Auswirkungen werden weniger offen ausgetauscht. Dieses Verhalten, so lässt sich vermuten, ist auch nicht so stark ausgeprägt, weil dies dem Ansehen der Lehrenden schaden könnte, was einem Gesichtsverlust dieser Respektspersonen gleichkäme (Henze 2008). So sind Erfahrungen der Lernenden aus deutscher Perspektive kaum über direkte Ansprachen zu erfahren, wie ein Dozent berichtet:

„...eine Teilnehmerin [...] hatte mich in der Pause dann beiseite genommen und sagte, ich soll ja nicht böse sein und auf keinen Fall so direkt die Leute ansprechen, das mögen sie nicht. [...] Die Teilnehmer haben Angst, bei Fragestellungen das Gesicht zu verlieren, weil die Frage vielleicht ungeschickt ist..."(Int 48_18)

Es lässt sich somit festhalten, dass offener Erfahrungsaustausch und Reflexion kulturbedingt Hürden bei der Umsetzung handlungsorientierten Lernens darstellen können.

Eine Herausforderung für die Lehrenden besteht nach dem Ansatz handlungsorientierten Lernens nicht zuletzt darin, (5) Wissens- und Kompetenzvermittlung in Einklang zu bringen und dabei auch soziale Prozesse sowie unterschiedliche Perspektiven der Berufs- und Lebensplanung zu vereinbaren (KMK 1998: 6). Dabei geht es im Sinne sozialer Kompetenz darum, mikro- 
politische Prozesse im beruflichen Alltag zu erkennen und adäquat zu gestalten. Sozialkompetenz wird demnach verstanden als „Bereitschaft und Fähigkeit, soziale Beziehungen und Interessen zu erfassen und zu verstehen sowie sich mit Anderen verantwortungsbewusst auseinanderzusetzen und zu verständigen. Personalkompetenz bezeichnet schließlich die Bereitschaft und Fähigkeit, die eigene Entwicklung zu reflektieren und in Bindung an individuelle und gesellschaftliche Wertvorstellungen weiter zu entfalten" (KMK 1998: 4). In China werden Handlungen vornehmlich als gemeinschaftliche Prozesse betrachtet. Subjektorientierte Handlungen (Interessenerklärung, Konfliktbewältigung sowie Berufs- und Lebensplanung) stehen dabei weniger im Vordergrund (vgl. z.B. Chiao 2003). Allerdings wandelt sich allgemein auch hier die Einstellung, insbesondere in der jüngeren Generation. So treten die moralischen und gesellschaftlichen Werte mittlerweile hinter dem persönlichen Fortkommen zurück (Herzog/Bai 2015: 11).

Die Berücksichtigung sozialer Prozesse ist allerdings gerade auch auf Seiten deutscher Dozenten von Belang. Eine solche Subjektorientierung erfordert mehr als die Kenntnis nationalstaatlicher Stereotype. Erst, wenn Dozenten wie Lernende verstehen, worin die Unterschiede in den Kulturen liegen, so lässt sich hier vermuten, können hybride, neue, individuelle und transkulturell geprägte Lernkulturen entwickelt werden.

\section{Ausblick: Transkulturelle Lernkulturen als Ergebnis handlungsorientierten Lernens}

Die deutsche „Berufsgesellschaft“, charakterisiert durch umfangreiche Regulierungen, steht einer chinesischen Beruflichkeit gegenüber, die sich noch in der Entwicklung befindet: Berufliche Bildung ist noch eher theoretisch als praktisch gestaltet. Kooperationen zwischen Berufsschulen und Unternehmen sind nicht die Regel; Selbständigkeit und offene Diskussionen stehen in der Gestaltung von Lernprozessen nicht im Mittelpunkt.

Methoden des erfahrungs- und handlungsorientierten Lernens ermöglichen sowohl die Entwicklung fachlicher als auch die Förderung sozialer und personaler Kompetenzen und stellen die Lernenden mit ihren Erfahrungen und ihrer Reflexionsfähigkeit in den Mittelpunkt. Im Bildungsexport erfordert das Konzept die Fähigkeiten der Dozierenden, Inhalte adäquat auf berufspraktische Kontexte des Ziellands zu übertragen. Genau in dieser Übertragung liegen die Herausforderungen im Rahmen transkultureller Lehr- und Lernprozesse. Somit sind folgende Erkenntnisse entsprechend der Grundsätze handlungsorientierten Lernens festzuhalten:

1) Wesentliche Kenntnisse der Lernenden sowie ihrer Berufswelt stellen einen wichtigen Zugang dar, um beruflich bedeutende Situationen einschätzen zu können. 
2) Der Einblick in Arbeitsweisen der beruflichen Realität ermöglicht es, das Konzept der vollständigen Handlungen im Zielland adäquat zu realisieren.

3) Das Wissen um die Lernkultur, Bedarfe des Bildungsmarktes und Voraussetzungen in Bezug auf selbständiges Handeln ist bedeutsam, um dem konstruktivistischen Leitbild handlungsorientierten Unterrichts angemessen entsprechen zu können.

4) Offener Erfahrungsaustausch und die Bereitschaft zur Reflexion sind kulturell bedingt nicht selbstverständlich.

5) Größtmögliche Kenntnis um die „Innenperspektive“ in Bezug auf das Zielland ist nötig, um die sozialen Aspekte in der Berücksichtigung von Berufs- und Lebensplanung im Sinne handlungsorientierter Wissens- und Kompetenzvermittlung in Einklang zu bringen.

Im letzten Punkt wird besonders deutlich, dass spätestens an dieser Stelle eine direkte Anwendung des Konzepts handlungsorientierten Unterrichts im Zielland nicht funktionieren kann und transnationale Lernkulturen erforderlich sind. Diese bauen offenbar zunächst auf Interkulturalität und damit auf Abgrenzung: Erst wenn Trennendes markiert und reflektiert wird, lässt sich die kulturelle Andersartigkeit begreifen und verstehen. Dieses interkulturelle Verständnis erscheint als Voraussetzung für transnationale Lernkulturen. Dem pragmatischen Motto Deng Xiaopings ,,von Stein zu Stein tastend den Fluss zu überqueren" folgend, können Ergebnisse, wie sie hier vorgestellt wurden, dazu beizutragen, transnationale Lernkulturen besser in ihrer Bedeutung zu verstehen. Trotz der bisherigen Erkenntnisse, die das Projekt EWA mit handlungsorientiertem Lernen auf deutscher wie auf chinesischer Seite erbracht hat, stehen solche transnationalen Lernkulturen erst am Anfang. Wo sich bewusst gestaltete Lernformen in diesem Sinne dauerhaft entwickeln, können transnationale Lernkulturen mittels interkultureller Erkenntnisse neue Wege herkömmlichen Lernens freilegen.

\section{Literatur}

Aulig, Thomas G. (2008): Wirtschaft und Bildung in der VR China. Die Qualifikationsanforderungen des Kfz-Handwerks in der VR China; aufgezeigt an Fallstudien in Lanzhou und Weifang. Univ., Diss. München, 2006. Saarbrücken: VDM Verlag Dr. Müller.

bHO (2013): Handlungsorientierung in der beruflichen Bildung. Ein Konzept zur Umsetzung in der curricularen Arbeit und im Unterricht. http://nibis.de/nibis3/ uploads/2nlq-a2/files/bHO-Gesamtkonzept.pdf [Zugriff: 05.01.2016].

BMBF (2015): China-Strategie des BMBF 2015-2020 I. Strategischer Rahmen für die Zusammenarbeit mit China in Forschung, Wissenschaft und Bildung. https://www.bmbf.de/pub/china_strategie_bmbf.pdf [Zugriff: 05.01.2016]. 
Chiao, Wei (2003): Quellen interkultureller Missverständnisse. In: Osterwalder, A. (Hrsg.): Interkulturelle Kompetenz in der beruflichen Bildung. Ergebnisse eines Expertengesprächs. Bielefeld: Bertelsmann W.-Verl., S. 28-30.

Dehnbostel, Peter (2010): Betriebliche Bildungsarbeit. Kompetenzbasierte Aus- und Weiterbildung im Betrieb. Studientexte Basiscurriculum Berufs- und Wirtschaftspädagogik, Bd. 9. Baltmannsweiler: Schneider-Verl. Hohengehren.

Gillen, Julia (2013): Kompetenzorientierung als didaktische Leitkategorie in der beruflichen Bildung - Ansatzpunkte für eine Systematik zur Verknüpfung curricularer und methodischer Aspekte. In: BWP@ Ausgabe 24/ 2013. www.bwpat.de/ ausgabe24/gillen_bwpat24.pdf [Zugriff: 05.01.2016]

Henze, Jürgen (2008): Die Rolle von Vertrauen in sozialen Beziehungen - das Beispiel chinesischsprachiger Kulturräume. In: Jammal, E. (Hrsg.): Vertrauen im interkulturellen Kontext. Wiesbaden: VS Verlag für Sozialwissenschaften, S. 193-211.

Heilmann, Sebastian (Hrsg.) (2016): Das politische System der Volksrepublik China. Wiesbaden: Springer Fachmedien Wiesbaden.

Herzog, Marius/Bai, Bin (2015): Chinesische und Deutsche Beruflichkeit im Vergleich. In: BWP@ Ausgabe 29/ 2015. http://www.bwpat.de/ausgabe/29/herzogbai [Zugriff: 05.01.2016].

Herzog, Marius/Gillen, Julia (im Erscheinen): Methodische Herausforderungen prozessorientierter Begleitforschung beim Aufbau beruflicher Aus- und Weiterbildung in China. In: Zeitschrift Bildung und Erziehung. Themenheft 1/2016 „Auswärtige Kulturpolitik“.

iMOVE (2010): Marktstudie China für den Export beruflicher Aus- und Weiterbildung. https://www.google.de/search?q=Marktstudie+Chinaf\%C3\%BCr+den+Export+ beruflicher+Aus-+und+Weiterbildung $\&$ ie $=$ utf-8\&oe $=$ utf-8\&gws_rd=cr\&ei $=$ B2TMVfe5LMH0auS_huAF [Zugriff: 13.08.2015].

KMK (1998): Rahmenlehrpläne für die Berufsausbildung in der Bauwirtschaft. (Beschluss der Kultusministerkonferenz vom 05.02.1998). http://www.kmk.org/ fileadmin/pdf/Bildung/BeruflicheBildung/rlp/WaermeKaelteSchallisolierer.pdf [Zugriff: 05.01.2016].

lernen \& lehren (Elektrotechnik-Informatik/Metalltechnik) (2010): Schwerpunktthema: Handlungsorientiertes Lernen - ein Streitthema, 25. Jahrgang, 98.

Li, Jun/Pilz, Matthias (2011): Vorberufliche Bildung in Deutschland und China. Ein curricularer Vergleich. In: Tertium comparationis 17, 2, S. 137-158.

Lui, Ka Wai/Leung, Frederick Koon Shing (2013): Curriculum traditions in Berlin and Hong Kong: a comparative case study of the implemented mathematics curriculum. In: ZDM the international journal on mathematics education 45, 1 , S. 35-46.

Meyer, Hilbert/ Oelke, Uta (2013): Didaktik und Methodik für Lehrende in Pflegeund Gesundheitsberufen. Berlin: Cornelsen Verlag.

Pilz, Matthias/Li, Junmin (2014): Das duale Ausbildungssystem im Gepäck? Eine Untersuchung deutscher Tochterunternehmen in China und den USA. In: Berufsbildung in Wissenschaft und Praxis 43, 6, S. 18-21.

Robak, Steffi (2012): Kulturelle Formationen des Lernens. Zum Lernen deutscher Expatriates in kulturdifferenten Arbeitskontexten in China; die versäumte Wei- 
terbildung. Humboldt-Univ., Habil.-Schr.-Berlin, 2011. Internationale Hochschulschriften, Band 578. Münster: Waxmann.

Robak, Steffi et al. (2014): Perspektiven zum Aufbau einer Weiterbildungseinrichtung in China - Vernetzung zwischen Professionalität und fluider Organisationsstrukturierung. In: Nittel, D./Tippelt, R./Wahl, J. (Hrsg.): Kooperation inner- und außerhalb des Systems des lebenslangen Lernens, S. 19-30.

Schmidt-Dörr, Thomas (2015): Neue Strukturen für das Hochschulsystem in China. Akademikerarbeitslosigkeit versus Fachkräftemangel. In: Forschung \& Lehre 10, S. 838-841.

Schüßler, Ingeborg/Thurnes, Christian M. (2005): Lernkulturen in der Weiterbildung. Studientexte für Erwachsenenbildung. Bielefeld: Bertelsmann.

Seufert, Sabine/Metzger, Christoph (2013): Kompetenzentwicklung in unterschiedlichen Lernkulturen. Festschrift für Dieter Euler zum 60. Geburtstag. 1. Aufl. 2013. Paderborn: Eusl.

Sloane, Peter/Twardy, Martin/Buschfeld, Detlef (1998): Einführung in die Wirtschaftspädagogik. Paderborn: Eusl.

Wagner, Hans-Günter (2002): Einige praktische Erfahrungen mit handlungsorientierten Lehr- und Lernformen in der chinesischen Berufsbildung. In: Berufsbildung 55,69, S. 41-43.

Wang, Jianchu (2008): Lernfeldorientierte berufliche Curricula und deren Entwicklungspotenziale für die Modernisierung der Berufsbildung in der V.R. China. Darmstädter Beiträge zur Berufspädagogik, Band 29. Paderborn: Eusl.

Watkins, David/Biggs, John B. (1996): The Chinese learner. Cultural, psychological, and contextual influences. Hong Kong, Camberwell, Melbourne, Vic.: CERC; ACER.

Welsch, Wolfgang (2005): Auf dem Weg zu transkulturellen Gesellschaften. In: Allolio-Näcke, L. (Hrsg.): Differenzen anders denken. Bausteine zu einer Kulturtheorie der Transdifferenz. Frankfurt a.M.: Campus-Verl., S. 314-341.

Yeung, Yau Yuen (2015): Characteristics of Chinese Learners as Revealed from Their Affective Domain and Choices of Science Learning in China. In: Khine, Myint Swe (Hrsg.): Science Education in East Asia. Pedagogical Innovations and Research-informed Practices. 1st ed. 2015. Cham, s.l.: Springer International Publishing, S. 123-146. http://dx.doi.org/10.1007/978-3-319-16390-1 [Zugriff: 05.01.2016]

Zhao, Zhiqun (2013): Berufsforschung in China. In: Pahl, J.-P./Herkner, V. (Hrsg.): Handbuch Berufsforschung. Bielefeld: W. Bertelsmann Verl, S. 923-932.

Zheng, Jianping/Rützel, Josef (2014): Aktuelle Herausforderungen in der Berufsschullehrerbildung in China. In: Berufsbildung 68, 148, S. 47-50. 


\section{Regionalität von Erwachsenenbildung. Eine qualitative Untersuchung aus Organisationssicht in den Euroregionen Großregion SaarLorLux und Neiße-Nysa-Nisa}

Dieser Beitrag fragt, wie Organisationen der Erwachsenen- und Weiterbildung (nachfolgend EB) auf den Fakt reagieren, dass sie in einer Grenz- und Euroregion liegen. Ausgehend von divergierenden Anforderungen an EB in Grenzregionen in Bezug auf deren regionale Ausrichtung (1.) untersucht er die Auswirkungen von Grenzlage auf EB-Organisationen. Er stellt dazu die Untersuchung sowie die Untersuchungsgebiete kurz vor (2.), Kern des Beitrags ist die Vorstellung zweier Textauszüge (3.) sowie einer Auswertungsheuristik (4.), anhand derer vier Typen sozialer Praktik entwickelt werden (5.). Im Fazit (6.) wird der Begriff organisationale Regionalität eingeführt und der Bezug zu einer räumlich reflektierten Lernkulturforschung hergestellt.

\section{Fragestellung und Begründung}

Die Frage nach dem Verhalten von EB-Organisationen in Grenzregionen zeigt sich aus theoretischen wie auch aus empirischen Gründen relevant. Theoretisch ist der Begriff Region aus Organisationssicht zu beleuchten. Dieser in der Erwachsenenbildungswissenschaft sehr unterschiedlich verwendete Begriff begleitet aktuell eine stark politische Steuerungslogik (Ahrens 2010; Bernhard 2014). Die „Wiederentdeckung der Bedeutung der Region“ (Martin/Schömann/Kuper/Schrader 2015: 26) legt eine weitere Ausarbeitung des Konzepts Region aus Organisationssicht nahe, da diese die Regionalisierung von Politik letztlich schultern (Höhne 2010). Eine Theoretisierung von Region aus Organisationssicht fehlt bisher (Bernhard 2014; Gieseke 2014). Dieses Desiderat nimmt der Beitrag auf. Empirisch zeigen sich in Zeiten der Europäisierung einerseits und den nationalen und föderalen Bildungspolitiken andererseits divergierende bildungspolitische Anforderungen an EB-Organisationen in Grenzregionen (ähnlich Höhne 2012). Hinzu treten lebensweltliche Anforderungen der lernenden Subjekte, die sich regional u.a. als atypische Migration oder Grenzpendlertum äußern. $\mathrm{Zu}$ diesen divergierenden Anforderungen müssen sich Organisationen in ihrer regionalen Verortung verhalten, indem sie die Grenze in ihren Aktivitäten überschreiten oder nicht überschreiten. Grenzregionen eigenen sich daher gut, um regionale Aspekte von EB zu beleuchten. 
Der vorliegende Beitrag ist abzugrenzen von einer Reihe von Untersuchungen, die die „Übersetzung von Organisationen“ (Engel 2014: 421) und die Grenze selbst in den Mittelpunkt stellen. Er fokussiert nicht das Phänomen Grenze, sondern die Ausgestaltung von Region durch EB-Organisation. Grenze wird als eines der vielen zu beleuchtenden, regionalen Gegebenheiten genutzt, da sie Phänomene wie Pendlertum sichtbar macht.

\section{Empirisches Design und Sampling}

Um das Verhalten von Organisation zur grenzregionalen Lage zu beleuchten, wurden Leitungspersonen von EB-Organisationen in Einzelfallstudien befragt. Die Datenerhebung erfolgte in explorativen Experteninterviews (Bogner/Menz 2005) und wurde in Anlehnung an Grounded Theory mit dem Ziel der Typenbildung (Kelle/Kluge 2010) ausgewertet. Stellenweise wurden auch Programme der untersuchten Organisationen einbezogen (siehe auch Abschnitt 3).

Die untersuchten Organisationen (Tab. 1) liegen in den Euroregionen Großregion SaarLorLux sowie Neiße-Nysa-Nisa. Beide Regionen beschreiben politische Konstrukte, die sich über Teile von drei oder mehr Ländern erstrecken. In der gut funktionierenden, euroregionalen Zusammenarbeit der Politik werden so Anforderungen an EB herangetragen. Ansonsten unterscheiden sich die beiden Gebiete stark: Die Großregion SaarLorLux ist mit 450 km Nord-Süd-Ausdehnung sehr groß und umfasst städtische Ballungsgebiete sowie dünn besiedelte ländliche Räume. Darüber hinaus zeigen sich in der euroregionalen Zusammenarbeit der Großregion zwei Sprachen, Deutsch und Französisch, relevant; es besteht ein enorm großes Grenzpendlertum nach Luxemburg. Die Euroregion Neiße-Nysa-Nisa ist mit einer Nord-SüdAusdehnung von ca. $80 \mathrm{~km}$ tendenziell klein und zeichnet sich durch eine weitgehend ländliche Siedlungsstruktur aus. Das Grenzpendlertum ist gering. Offiziell werden vier Sprachen gesprochen, von denen besonders Deutsch, Tschechisch und Polnisch relevant sind; Sorbisch wird als anerkannte Minderheitensprache gepflegt.

Es wurden zehn Interviews ausgewertet, die sich über die beiden Regionen und die verschiedenen Segmente der EB verteilen (Tab. 1). Nur wenige der untersuchten Organisationen verfolgen eine explizit grenzüberschreitende Programmatik, so dass die Grenze aus dem Fokus und Region in den Fokus der Untersuchung rückt. Ebenso sind wenige Organisationen Teil des Programms „Lernende Regionen“ gewesen, so dass auch die Setzung von Region als Netzwerk aufgelöst wird (ähnlich Alke 2014). Die Ergebnisse der Auswertung werden nachstehend dargestellt. 
Tab. 1: Sample der Untersuchung nach den Kriterien Region und Art der Organisation

\begin{tabular}{llll}
\hline & $\begin{array}{l}\text { Allgemeine, politi- } \\
\text { sche, kulturelle } \\
\text { Erwachsenenbildung } \\
\text { (VHS Segment, } \\
\text { direkter Kontakt mit } \\
\text { Teilnehmern) }\end{array}$ & $\begin{array}{l}\text { Berufliche } \\
\text { Weiterbildung } \\
\text { (SGB III, SGB II, SGB } \\
\text { IX) }\end{array}$ & $\begin{array}{l}\text { Netzwerk- } \\
\text { koordinierungs- } \\
\text { stellen }\end{array}$ \\
\hline $\begin{array}{l}\text { Großregion } \\
\text { SaarLorLux }\end{array}$ & 4 & 0 & 1 \\
\hline $\begin{array}{l}\text { Euroregion } \\
\text { Neiße-Nysa- } \\
\text { Nisa }\end{array}$ & 2 & 2 & 1 \\
\hline
\end{tabular}

\section{Ergebnisse}

Exemplarisch werden nun zwei Interviewauszüge diskutiert. Daran werden sowohl die Interpretationspraxis der Studie gezeigt als auch erste Ergebnisse der Studie dargestellt.

„Wir planen hier unser Programm, natürlich schon für den Landkreis, aber wenn wir sagen, ,Okay, das gehört jetzt in den Bereich, wo wir glauben, dass es für die luxemburger Bevölkerung speziell von Interesse ist $^{6}$, gehen wir auch in die luxemburger Medien zum Beispiel explizit rein und bewerben diese Kurse" (Interview 1, Absatz 8).

Dieser Interviewausschnitt zeigt ein Beispiel aus der allgemeinen, politischen und kulturellen EB im deutschen Grenzraum zu Luxemburg und Frankreich.

Die interviewte Person beschreibt mit „wir“ die gesamte Organisation und somit eine beobachtbare, wiederholte und institutionalisierte soziale Praktik (Reckwitz 2004). Deren Regelhaftigkeit wird durch den Allgemeinheitsgrad der Aussage deutlich und daran, dass die interviewte Person die Regelhaftigkeit auch an Beispielen erläutert. So spricht sie an anderer Stelle vom Gesundheitsbereich, für den in Luxemburg keine Angebote existieren; daher kommt die luxemburgische Bevölkerung bei solchen Angeboten zur deutschen Einrichtung. Die Organisation nutzt dies: Um Teilnahmezahlen zu generieren, schaltet sie Anzeigen in luxemburgischen Medien. Die Handlung kann als routinisiert beschrieben werden.

Dabei unterscheidet die interviewte Person zwei Teilschritte pädagogischen Handelns: 1. die Programmplanung, 2. die Kommunikation einzelner Kurse. In diesen Einzelhandlungen schlagen sich verschiedene Ausprägungen von Region nieder. In der Programmplanung zeigt sich der Landkreis als 
ausschlaggebend. Für dieses administrative Territorium wird das Programm „,natürlich“ (s.o.) geplant. Die damit geäußerte Selbstverständlichkeit ist Ausdruck des Auftrags: Den Landkreis gilt es zu versorgen und das Programm auf diesen anzupassen. Die Organisation produziert in diesem Fall die im Auftrag festgeschriebene Region - ihr Zuständigkeitsgebiet.

Die Kommunikation der Angebote hingegen erfolgt regelmäßig - nämlich immer dann, wenn es in der Einschätzung der interviewten Person für die luxemburgische Bevölkerung interessant ist - in einem anderen Erdausschnitt, der weitaus größer ist als das Zuständigkeitsgebiet. Dies ist als ein Ausschöpfen von Märkten mit den angebotenen Produkten zu interpretieren. Die Organisation weitet somit ihre Region über das Zuständigkeitsgebiet hinaus und schafft einen größeren Einzugsbereich, auf den sie sich immer wieder bezieht, ohne die Zielgruppe ihres Angebots zu verändern. Die Einrichtung reproduziert so das Zuständigkeitsgebiet, allerdings produziert sie auch ein Einzugsgebiet auf Basis von Marktmechanismen.

So werden im Beispiel zwei Ausprägungen von Region in den sozialen Praktiken der Leitung deutlich: zum einen ein gesetzliches Territorium - das Zuständigkeitsgebiet -, zum anderen ein physischer Raumbegriff, der sich als das Einzugsgebiet beschreiben lässt und eine große Nähe zu Marktmechanismen und metrischer Entfernung zeigt.

Interessant ist hierbei, dass im gesamten Interview der Luxemburgbezug immer wieder relevant wird, ein Frankreichbezug der Organisation - obwohl das Land nahezu gleich nah ist - allerdings kaum in Erscheinung tritt. Luxemburg gehört also zur Region, genauer zum Markt - Frankreich nicht.

Anders verhält sich das Netzwerk in der Euroregion Neiße-Nysa-Nisa zur grenzregionalen Lage:

„Entstanden ist es erstmal auf der deutschen Seite mit vorrangig deutschen Akteuren, [...] aber mit einem klaren inhaltlichen Bezug immer wieder zu dieser grenzüberschreitenden Kooperation, zu den Themen Nachbarsprache, interkulturelle Kompetenz. Das sind so die Kernthemen, an denen wir dann auch so arbeiten und die dann eben auch die Brücke zu den Nachbarn bahnen“ (Interview 9, Abschnitt 38).

Die interviewte Person spricht ebenfalls von „wir“ und von „immer wieder“. Sie verweist damit ebenfalls auf eine routinisierte, soziale Praktik. Sie spricht von einem Territorium, auf dem sie selbst angesiedelt ist („deutsche Seite“), äußert aber im Gegensatz zu Beispiel 1 eine klare Strategie, über die Grenze des Territoriums hinweg den Weg zu den Nachbarn zu bahnen. Dies tut sie über inhaltliche Bezüge mit Themen, die die grenzüberschreitende Region einen und als Gesamtes interessieren können. Das heißt: Sie weitet ihre Zielgruppe über das Territorium hinweg und bietet eine Deutung von Region an, die quer zu den gesetzlichen Zuständigkeiten und somit den institutionalisierten Deutungen von Region liegt. Hier wird ein dritter Regionsbegriff 
deutlich, der Region nicht als Einzugsgebiet oder als Zuständigkeitsgebiet definiert, sondern als eine Bildungsvorstellung, die ein positives Zusammenleben mit Menschen auf der anderen Seite der Grenze anstrebt und sich über Inhalte äußert. Dies zeigt sich auch durch die intensive Verwendung des Begriffs „Nachbar“, eine Beschreibung, die einen Bezug zum eigenen Handeln herstellt und die im gesamten Interview, aus dem Beispiel 1 stammt, nicht fällt. Das Netzwerk beschreibt kein Einzugsgebiet, auch die Beschreibung eines gesetzlich vorgeschriebenen Territoriums erhält die Form einer $\mathrm{zu}$ überwindenden und eher lästigen Bedingung.

In der kontrastierenden Beobachtung und Beschreibung der genannten Beispiele wird also bereits deutlich, dass EB-Organisationen sehr unterschiedlich in Grenzregionen agieren und in der Konsequenz auch unterschiedliche Regionsausprägungen in unterschiedlicher Art und Weise (re)produzieren.

Dabei dehnen sich die gezeigten Verhaltensweisen über weite Teile der Organisationen aus. Zwar werden zunächst Einzelpersonen befragt und Aspekte der individuellen Professionalität bestimmen das Bild, aber die Regelmäßigkeit und die Institutionalisierung der benannten Praktiken lässt sich anhand von Programmen und erfolgter Öffentlichkeitsarbeit belegen; sie bestehen über Jahre hinweg. Die Konzentration auf verbale Daten begründet sich mit der handlungszentrierten Geografie, die hier den Status einer Heuristik (Kelle/Kluge 2010: 36) erhält, entlang derer Kategorien entwickelt und inhaltlich gefüllt wurden. Andere Studien entwickeln organisationsethnografische Ansätze, die stärker auf der Interpretation von performativen Mustern fußen (Engel/Göhlich 2013). Mit dieser sehr starken Betonung auf Artefakten verliert sich allerdings die Deutung seitens der Subjekte, die im vorliegenden Ansatz im Vordergrund steht. Mit der handlungszentrierten Geografie lassen sich die aufgezeigten Handlungsweisen und deren Routinisierung als subjekthaft theoretisch beleuchten und systematisieren (Werlen 1997).

\section{Handlungszentrierte Geografie}

Laut Werlen kranken die meisten theoretischen Ausarbeitungen zu Raum und Region ist nichts anderes als ein Raumausschnitt mittlerer Größe (Sinz 2005; Freytag/Jahnke/Kramer 2014) - daran, dass sie den Raum selbst in den Mittelpunkt stellen und Handlung darin verorten. Aus dieser Kritik heraus dreht Werlen das Verhältnis von Handlung und Raum um: Er stellt Handlung in den Mittelpunkt seiner Ausarbeitung und beschreibt deren Konsequenzen auf Raum. Er definiert (2013: 6-8) Raum als nur eine von drei Dimensionen von Handlung: 1. mentaler Bereich (Wissen, Deutung), 2. sozial-kulturelle Begebenheiten (Normen), 3. physisch-materieller Kontext (Raum). Raum wird so zum Attribut von Handlung: zur Räumlichkeit. Ebenso wird Region 
zur Regionalität von Handlung, die sich in jeder Handlung als Deutung von Raum aktualisiert. Raum besteht daher nicht an sich, denn physische Gegebenheiten tragen keine gegebene Bedeutung für Handlung, sondern werden physischen Gegebenheiten auferlegt und materialisieren sich in sozialer Praktik. Handelnde Subjekte institutionalisieren oder deinstitutionalisieren diese Bedeutungen und machen so Geographie (Werlen 2002: 13). Entsprechend gilt es individuelle Handlung in den Blick zu nehmen, was auch den Fokus dieser Studie begründet. Der Routinisierung der impliziten Bedeutung von Raum in Handlung verleiht der Begriff ,alltägliche Regionalisierung“ (Werlen 2000: 611) Ausdruck.

Dies lässt sich an den bereits dargestellten Interviewauszügen illustrieren:

Die Organisation aus Beispiel 1 (re)produziert den Landkreis in ihrer Programmplanung, indem sie sich weitgehend auf dessen Gebiet bezieht. Sie institutionalisiert dieses Gebiet somit als ,ihre Region', dessen Bedeutungsgehalt bezieht sie aus dem institutionellen Auftrag, die Bevölkerung des Landkreises zu versorgen. Daher bezieht sie sich inhaltlich auf den Landkreis. Anlassbezogen produziert die Einrichtung durch ihre Werbemaßnahmen einen Einzugsbereich. Durch das regelmäßige Inserieren in luxemburgischen Zeitungen nimmt sie Einfluss auf das Einzugsgebiet. Den Bedeutungsgehalt für diese Handlung bezieht die handelnde Person hierbei aus einer Marktlogik in kalkulatorischen Überlegungen. Die Einrichtung aus Beispiel 2 setzt eine eigene Region und sucht Inhalte, die diese einen, um so eine Region zu produzieren. Dabei treten Marktüberlegungen und Zuständigkeitsgebiet in den Hintergrund. Die Einrichtung produziert einen eigenen Bedeutungsgehalt durch die Inhalte, die sie setzt, und somit eine eigene Region. Die beiden Einrichtungen regionalisieren sehr unterschiedlich, die einzelnen Handlungen lassen sich orientiert an den gesetzlichen Zuständigkeiten, marktorientiert und an Deutung orientiert bezeichnen.

Entsprechend Werlens Forschungsentwurfs gilt es nun diese drei Arten des Regionalisierens in Relation zueinander zu setzen und so soziale Praktiken als Ganzes im Zusammenspiel von Markt, inhaltlicher Deutung und territorialer Zuständigkeit zu rekonstruieren.

\section{Vier Typen sozialer Praktiken}

Durch das systematische In-Beziehung-Setzen zweier der drei beschriebenen Handlungsdimensionen entstehen die dargestellten Typen sozialer Praktik (Tab. 2). Es handelt sich um eine exemplarische Darstellung der beiden Handlungsdimensionen Markt und Zuständigkeit. Dadurch, dass es sich im Modell der handlungszentrierten Geografie um drei Dimensionen handelt und 
im vierten Typ die beiden erstgenannten Dimensionen in den Hintergrund treten, tritt in Typ 4 (Bearbeitung) die dritte Dimension in den Vordergrund.

Tab. 2: Typen organisationaler Praktiken in Bezug auf Region

\begin{tabular}{|c|c|c|c|}
\hline & & \multicolumn{2}{|l|}{ Zuständigkeitsgebiet steht im } \\
\hline & & Vordergrund & Hintergrund \\
\hline \multirow[b]{4}{*}{ 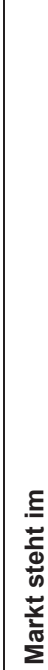 } & & $\begin{array}{l}\text { Abdeckung eines } \\
\text { territorialisierten Marktes }\end{array}$ & $\begin{array}{l}\text { Weitung des } \\
\text { Zuständigkeitsgebiets durch } \\
\text { Verträge }\end{array}$ \\
\hline & 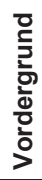 & & \\
\hline & \multirow[b]{2}{*}{ 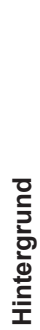 } & $\begin{array}{l}\text { Sicherung von Ressourcen zur } \\
\text { Versorgung des } \\
\text { Zuständigkeitsgebiets }\end{array}$ & $\begin{array}{l}\text { Bearbeitung: Inhaltliche } \\
\text { Dimensionen der Region stehen } \\
\text { im Vordergrund }\end{array}$ \\
\hline & & & \\
\hline
\end{tabular}

\subsection{Abdeckung eines territorialisierten Marktes}

Diese Praktiken treten in Organisationen auf, die stark im Bereich Sozialgesetzbuch (SGB) II und SGB III aktiv sind. Sie unterliegen einem starken gesetzlichen Regelwerk, das wenig Freiraum lässt sowie Teilnahme und Kunde entkoppelt. Die Planenden orientieren sich in ihrer regionalen Ausrichtung an den Territorien der Kunden (z.B. Agenturbezirken) und versuchen, diese über Außenstellen abzudecken. Ebenso erfolgt die Kommunikation von Angeboten über die Auftraggeber. Innerhalb der Territorien fallen Begriffe, die eine physische Nähe zum Vertragspartner (z.B. Jobcenter) beschreiben (Interview 7, Absatz 14), was einen metrischen Raumbezug und somit einen Markt in den Vordergrund stellt. Ein bewusstes Setzen von regionalen Inhalten findet nicht eigenmächtig statt, sondern erfolgt durch die vorgegebenen Inhalte des Auftraggebers (z.B. Agentur für Arbeit). 
Auf territoriale Beschränkung sozialer Praktiken in EB-Organisationen wirkt sich begünstigend aus, dass die Anbahnung von Verträgen mit Kunden gute Netzwerke zu entsprechenden Schlüsselpersonen voraussetzt. Ein solches Netzwerk über verschiedene Agenturbezirke zu unterhalten, erfordert große Ressourcen. Somit ist es unter den gegebenen Bedingungen effektiver, sich auf ein Territorium eines Auftraggebers zu beziehen.

\subsection{Weitung des Zuständigkeitsgebietes durch Verträge}

Soziale Praktiken des Typs Weitung des Zuständigkeitsgebiets durch Verträge finden sich im Sample bei zwei französischen EB-Organisationen im Sample. Grund für ein Verlassen des Zuständigkeitsgebiets mit Angeboten und im gegebenen Fall sogar mit Außenstellen sind Überlegungen zur größeren, wirtschaftlichen Unabhängigkeit gegenüber der Auftrag gebenden Kommune. Die Weitung des Zuständigkeitsgebietes geht einher mit Marktüberlegungen, somit spielt vor allem der Einzugsbereich an Kunden eine wichtige Rolle. Im Rahmen dessen werden neue Angebote konzipiert und auf neue Zielgruppen hin angepasst. Diese werden über das Zuständigkeitsgebiet hinaus kommuniziert. Dabei entfernen sich die Organisationen vom Auftrag, das ursprüngliche Zuständigkeitsgebiet zu versorgen. Die Weitung des $\mathrm{Zu}$ ständigkeitsgebiets setzt eine Trägerstruktur und Gesetzeslage voraus, die erlaubt, auch an Märkten außerhalb des Territoriums aktiv zu werden. Zudem ist die erfolgreiche Etablierung im neuen Markt eine Bedingung, die wiederum durch wenig Konkurrenz begünstigt wird.

\subsection{Sicherung von Ressourcen zur Versorgung des Zuständigkeitsgebiets}

Soziale Praktiken dieses Typs konzentrieren sich stark auf ihr angestammtes Territorium, das im Auftrag festgeschrieben ist. Die Handelnden sehen hier einen Versorgungsauftrag für das Zuständigkeitsgebiet, dem sie unbedingt nachkommen. Um dies zu tun, verlassen sie sehr gezielt ihr eigenes Territorium, wenn Märkte und Auftrag dies erlauben oder sogar verlangen. Das Verlassen des Zuständigkeitsgebiets geschieht unter der Prämisse, den eigenen Auftrag im ursprünglichen Territorium besser nachkommen zu können. Es erfolgt somit keine Weitung des Auftrags. Hier ist insbesondere die Einrichtung aus Beispiel $1 \mathrm{zu}$ nennen, die die Nähe zum (zahlungskräftigen) Luxemburg nutzt, um sich zu finanzieren (Mitnahmeeffekt) und sich sonst sehr stark am eigenen Territorium orientiert. Ebenfalls beschreibt eine Kammer Schulungen mit französischen Jugendlichen, um den Fachkräftemangel auf der deutschen Seite der Grenze zu bekämpfen. Beide Einrichtungen ver- 
lassen somit das Territorium, dies wird allerdings legitimiert über den Mehrwert für die eigene Referenzgruppe vor Ort.

\subsection{Bearbeitung: Inhaltliche Dimensionen der Region stehen im Vordergrund}

Wenn sowohl Territorium als auch Markt in den Hintergrund treten, tritt vor allem die symbolische Signifikation (Werlen 2013) als die an Inhalten orientierte Deutung in den Vordergrund. Bei sozialen Praktiken dieses Typs werden bewusst spezifische Raumdeutungen durch die angebotenen Inhalte vermittelt. Idealtypisch können hier zum Beispiel eine Weiterbildung „Gästeführer SaarLorLux" stehen oder ein angebotenes Sprachenzertifikat in Neiße, das alle Nachbarsprachen umfasst. Beide Angebote produzieren eine Region durch ein spezifisches Arrangieren von Inhalten, die spezifisch nur für die jeweilige Euroregion Sinn ergeben. Die inhaltliche Dimension von Region wird insbesondere im Falle der Netzwerkagentur deutlich. Sie versucht, die eigene Region nicht mehr als Ende der Welt, sondern als Anfang der Welt darzustellen. Hier stehen symbolisch-kommunikative Prozesse im Vordergrund, die im Fall von regional orientierten, sozialen Praktiken versuchen, entsprechende Deutungsmuster zu etablieren. Nationale Territorien werden durch grenzüberschreitende Kooperation überwunden, um entsprechende Inhalte anbieten zu können.

\section{Organisationale Regionalität als Lernkultur}

Es lässt sich festhalten: Organisation lässt sich regional reflektieren und als organisationale Regionalität beschreiben. In Grenzregionen sind Organisationen sehr unterschiedlich in Bezug auf die Grenze aufgestellt: Während einige Organisationen vereinzelte Aktivitäten über die Grenze im Sinne eines Bildungsbegriffs beschreiben und begründen, bezieht sich die Mehrzahl der Einrichtungen eher formal auf Marktmechanismen und ihr eigenes Territorium. Die Arten, wie sie sich auf die Region beziehen, können mit 4 Typen (5.) beschrieben werden. Die organisationale Regionalität oder die Regionalität von Organisationen äußert sich in den Daten sowohl formal als auch in Inhalten: Formal lassen sich besonders die Außenstellen und Marketingaktivitäten von Organisationen als Artefakte von regionalem Handeln beschreiben. Ebenfalls äußert sich organisationale Regionalität in Netzwerken. Diese dienen als Vehikel, um Marketingaktivitäten zu realisieren oder organisationale Infrastrukturen zu schaffen. In der Sicht der Interviewten stellen Kooperationen allerdings kein eigenes Kriterium für die Regionsdefinition dar. Es zeigt sich ebenfalls bei den Handelnden ein recht großes Bewusstsein für die 
Räumlichkeit ihres Handelns in Bezug auf Märkte und Territorien; sehr viel weniger besteht ein Bewusstsein für inhaltliche Aspekte von Regionalität.

In Bezug auf Lernkultur lässt sich festhalten, dass organisationale Regionalität einen Beitrag dazu leisten kann, Lernkultur zu beschreiben. Durch die Definition von Region als soziale Praktik (Werlen 2013) lässt sich ein Anschluss finden an Schäffters (2010) Lernkulturbegriff: Dieser beinhaltet, soziale Praktiken als Heuristiken zu verstehen, um Lernkulturen in Organisationen zu beschreiben und zugrundeliegende Bildungsbegriffe zu rekonstruieren. Dies ist im vorliegenden Beitrag basierend auf Interviews mit Einzelpersonen geschehen und kann auch auf eine Interpretation organisationaler Strukturen, die sich als Artefakte äußern können, ausgedehnt werden (Engel 2014). Ebenso wäre in weiteren Arbeiten der Einsatz qualitativer, sozialräumlicher Methoden (z.B. Deinet 2009) zu berücksichtigen. Durch die Rückbindung an Werlen erhält Region eine inhaltliche Bestimmung. Dies beugt einer Verkürzung von Regionalisierung auf eine reine Effektivierungsstrategie (Ahrens 2011) vor.

\section{Literatur}

Ahrens, Daniela (2011): Region. In: Reutlinger, C./Fritsche, C./Lingg, E. (Hrsg.): Raumwissenschaftliche Basics. Eine Einführung für die Soziale Arbeit. Wiesbaden: VS, S. 221-229.

Alke, Matthias (2014): Kooperation als Medium der Selbststeuerung und Reproduktion von Organisationen der Weiterbildung. In: Report - Zeitschrift für Weiterbildungsforschung 4, S. 69-82.

Bernhard, Christian (2014): Region $\neq$ Region. Vom normativen Regionsbegriff zur interpretativen Regionalität. In: Report - Zeitschrift für Weiterbildungsforschung 4, S. 83-96.

Bogner, Alexander/Menz, Wolfgang (2005): Das theoriegenerierende Experteninterview. Erkenntnisinteresse, Wissensformen, Interaktion. In: Bogner, A./Littig, B./Menz, W. (Hrsg.): Das Experteninterview. Theorie, Methode, Anwendung. Wiesbaden: VS, S. 33-70.

Deinet, Ulrich (2009): Methodenbuch Sozialraum. Wiesbaden: VS.

Engel, Nicolas (2014): Die Übersetzung der Organisation. Pädagogische Ethnographie organisationalen Lernens. Organisation und Pädagogik, Band 14. Wiesbaden: VS.

Engel, Nicolas/Göhlich, Michael (2013): Kulturelle Differenz und Übersetzung in Organisationen. Eine ethnographische Studie in Einrichtungen grenzüberschreitender Jugendarbeit und Weiterbildung. In: Zeitschrift für Pädagogik 59, 5, S. 691-705.

Freytag, Tim/Jahnke, Holger/Kramer, Caroline (2014): Bildung und Region. Eine Expertise aus bildungsgeografischer Perspektive. https://www.neps-data.de/ Portals/0/Working\%20Papers/WP_XLVII.pdf. [Zugriff: 05.01.2016].

Gieseke, Wiltrud (2012): Bildungstheoretische Begründungen von Themenkulturen und Partizipationsportalen der Erwachsenenbildung. In: ebForum 4, S. 43-47. 
Höhne, Thomas (2010): Bildungsregionen. Zur bildungspolitischen Konstruktion neuer Bildungsräume. In: Tertium Comparationis. Journal für International und Interkulturell Vergleichende Erziehungswissenschaft 16, 1, S. 179-199.

Höhne, Thomas (2013): Cross Border Identities. Zur (Inter)Kulturalität grenzüberschreitend agierender Organisationen. In: Göhlich, M./Weber, S. M./Öztürk, H./Engel, N. (Hrsg.): Organisation und kulturelle Differenz. Diversity, Interkulturelle Öffnung, Internationalisierung. Organisation und Pädagogik, Band 12. Wiesbaden: VS, S. 209-217.

Kelle, Udo/Kluge, Susann (2010): Vom Einzelfall zum Typus. Fallvergleich und Fallkontrastierung in der qualitativen Sozialforschung. Wiesbaden: VS.

Martin, Andreas/Schömann, Klaus/Schrader, Josef/Kuper, Harm (Hrsg.) (2015): Deutscher Weiterbildungsatlas. Bielefeld: wbv.

Reckwitz, Andreas (2004): Die Entwicklung des Vokabulars der Handlungstheorien. Von den zweck- und normorientierten Modellen zu den Kultur- und Praxistheorien. In: Gabriel, M. (Hrsg.): Paradigmen der akteurszentrierten Soziologie. Wiesbaden: VS, S. 303-328.

Schäffter, Ortfried (2010): Institutionalformen für das lebenslange Lernen. Eckpunkte eines erwachsenenpädagogischen Forschungsprogramms. In: Dollhausen, K./Feld, T. C./Seitter, W. (Hrsg.): Erwachsenenpädagogische Organisationsforschung. Wiesbaden: VS, S. 293-316.

Sinz, Manfred (2005): Region. In: Akademie für Raumforschung und Landesplanung (Hrsg.): Handwörterbuch der Raumordnung. Hannover: Verlag der ARL, S. 471-486.

Werlen, Benno (1997): Gesellschaft, Handlung und Raum. Grundlagen handlungstheoretischer Sozialgeographie. Stuttgart: Steiner.

Werlen, Benno (2000): Allägliche Regionalisierungen unter räumlich-zeitlich entankerten Lebensbedingungen. In: Informationen zur Raumentwicklung 9/10, S. 611-622.

Werlen, Benno (2002): Handlungsorientierte Sozialgeographie. Eine neue geographische Ordnung der Dinge. In: Geographie heute 22, 200, S. 12-15.

Werlen, Benno (2013): Gesellschaft und Raum: Gesellschaftliche Raumverhältnisse. Grundlagen und Perspektiven einer sozialwissenschaftlichen Geographie. In: Erwägen - Wissen - Ethik 1, S. 3-16. 



\section{Differente Sichtweisen auf Grundbildung Erwachsener in der öffentlichen Bildungsverwaltung}

\section{Einleitung}

Seit einigen Jahren zeigt sich eine intensivierte Auseinandersetzung der Erwachsenen- und Weiterbildung mit dem sich zunehmend etablierenden Gegenstandsbereich einer Grundbildung Erwachsener. Ausgehend von der Alphabetisierungsarbeit seit Ende der 1970er Jahre und einer bereits in den 1990er Jahren proklamierten Einbettung der Alphabetisierung in eine Elementarbildung Erwachsener, wird nun in Deutschland erneut eine Ausweitung der Alphabetisierung hin zu dem offensichtlich umfassender verstandenen Konzept der Grundbildung Erwachsener gefordert (vgl. Abraham/Linde 2010: 890). Dabei ist allerdings bislang nicht geklärt, was eine Grundbildung Erwachsener umfasst und wie sich diese von dem Konzept der Alphabetisierung unterscheidet: Welche Inhalte, Ziele und Kompetenzen umfasst Grundbildung Erwachsener? Welche Zielgruppen werden angesprochen? Wie lässt sich Grundbildung in Form von Praxisangeboten realisieren? Es geht dabei um die Aushandlung einer als grundlegend notwendig angesehenen Bildung und um die begründete Auswahl ihrer Inhalte, Kompetenzen sowie Formate. Vor allem der öffentlichen Bildungsverwaltung kommt hier derzeit eine wichtige Schlüsselrolle zu, denn mit der Überführung der nationalen Strategie für Alphabetisierung und Grundbildung in eine nationale Dekade kann angenommen werden, dass über die verstärkte öffentliche Förderung von Grundbildungsangeboten gerade die Kultusministerien der Länder einen nicht unbedeutenden Einfluss auf die Gestaltung der als ,angemessen“ bzw. legitim betrachteten Grundbildungskonzepte in der Praxis ausüben werden.

Gegenstand dieses Beitrags ist die Frage, inwieweit sich in der öffentlichen Bildungsverwaltung aufgrund länderspezifischer Förderbedingungen differente Sichtweisen auf Grundbildung formieren und somit zur Entstehung spezifischer Lernkulturen im Feld der Alphabetisierung und Grundbildung beitragen. Damit wird in Anlehnung an Ingeborg Schüßler und Christian Thurnes ein umfassender Lernkulturbegriff herangezogen, wonach Lernkulturen gesellschaftlich und institutionell geprägte Orientierungen des Lehr-/ Lernhandelns darstellen und dabei sowohl die innere Struktur als auch Wechselwirkungen mit äußeren Faktoren in den Blick nehmen (vgl. Schüßler/Thurnes 2005: $13 \mathrm{f}$.). Vor allem der bildungspolitische Kontext von Lernkulturen, welcher als Makroebene über bildungspolitische Leitlinien, 
Gesetzgebungen und Deutungsmuster den Rahmen für die Entwicklung und Gestaltung von Lernkulturen absteckt (vgl. Schüßler/Thurnes 2005: 45 ff.; Fleige 2011: 10f.), wird hier näher betrachtet. Dazu wird zunächst ein Einblick in den erwachsenenbildnerischen Diskurs zum Grundbildungsbegriff sowie zur Durchsetzung legitimer Sichtweisen im Feld der öffentlichen Bildungsverwaltung gegeben, um im Anschluss daran das methodische Vorgehen sowie empirische Ergebnisse vorzustellen, die im Rahmen einer Studie zum Grundbildungsverständnis im Kontext von Interessen und Macht im Feld der öffentlichen Bildungsverwaltung erhoben wurden.

\section{Der Grundbildungsbegriff im Kontext von Interessen und Machtverhältnissen}

Der Grundbildungsbegriff wird laut Ellen Abraham und Andrea Linde insbesondere seit den 1990er Jahren im Kontext der Alphabetisierung Erwachsener verwendet und markiert eine Bewegung hin zu Bildungskonzepten, die über reine Lese- und Schreibfähigkeiten hinausgehen (vgl. Abraham/Linde 2010: 890; Linde 2007: 90). Allerdings existiert bislang kein übergreifender Konsens darüber, was eine Grundbildung Erwachsener mindestens umfasst. So wird Grundbildung auf der einen Seite offen definiert als „Minimalvoraussetzungen an Wissensbeständen, Kenntnissen, Fertigkeiten, personalen und sozialen Kompetenzen, die für Orientierung und aktives Handeln in der Gesellschaft notwendig sind“ (Döbert 1999), allerdings vom inhaltlichen Umfang her aufgrund gesellschaftlicher und individueller Anforderungen und Bedürfnisse nicht fix definierbar seien. Auf der anderen Seite existiert eine Fülle an Grundbildungskonzepten, die divers, wenn nicht sogar kontrovers zueinander stehen. Im Rahmen der nationalen Strategie beispielsweise wird Grundbildung verstanden als

„Kompetenzen in den Grunddimensionen kultureller und gesellschaftlicher Teilhabe, wie: Rechenfähigkeit (Numeracy), Grundfähigkeiten im IT-Bereich (Computer Literacy), Gesundheitsbildung (Health Literacy), Finanzielle Grundbildung (Financial Literacy), Soziale Grundkompetenzen (Social Literacy)“ (BMBF/KMK 2012: 1).

Im Kontrast zu diesem im Wesentlichen auf die Anwendung von Schriftsprache bezogenen Grundbildungsbegriff stehen Modelle, die beispielsweise auch politische Kompetenzen, nachholende Schulabschlüsse und berufliche Qualifikationen als Teil einer Grundbildung berücksichtigen.

Theoretisch anschlussfähig an diese vorzufindende Pluralität von Grundbildung ist Monika Trösters Ansatz, Grundbildung als einen relationalen Begriff $\mathrm{zu}$ verstehen, der im Spannungsfeld von Ansprüchen und Interessen verschiedener Akteure stehe (vgl. Tröster 2000: 17f.). Allerdings suggeriert die 
so proklamierte Offenheit und Vielfalt meines Erachtens eine scheinbare Gleichwertigkeit und Neutralität der Grundbildungsdefinitionen, indem die hinter diesen normativen Setzungen befindlichen Kontroversen und Interessenkonflikte ausgeblendet werden (vgl. Euringer 2016: $25 \mathrm{ff}$.; Grotlüschen et al. 2014: $54 \mathrm{ff}$.). Auch die New Literacy Studies weisen auf die Verwobenheit von Literalität mit sozialen Praktiken und Interessen sowie die daraus folgende Reproduktion gesellschaftlicher Machtverhältnisse hin (vgl. Street 2003; Barton/Hamilton 2000). Demnach sei Literalität zwar situationsbezogen und vielfältig, zugleich aber immer auch hierarchisch. Insbesondere mächtige soziale Institutionen, wie z.B. staatliche Akteure, haben Einfluss auf die Durchsetzung gesellschaftlich ,wertvoller“ Literalitätskonzepte. Entsprechend identifizieren auch Anke Grotlüschen u.a. (2009) in Anlehnung an Pierre Bourdieu eine „legitime Literalität“, welche die Interessen der Oberschicht an Distinktion erfülle.

\section{Durchsetzung legitimer Sichtweisen im Feld der öffentlichen Bildungsverwaltung}

Für die bildungspolitische Durchsetzung legitimer Grundbildungskonzepte spielen aufgrund der Kulturhoheit der Länder in Deutschland die für allgemeine Weiterbildung zuständigen Kultusministerien der Länder eine bedeutsame Rolle. Die Frage, im Kontext welcher Interessen und Machtverhältnisse bestimmte Sichtweisen auf Grundbildung durch diese hervorgebracht und legitimiert werden, macht eine Untersuchung der dort bedeutsamen Entscheidungs- und Handlungsmechanismen notwendig: Mit Bezug auf welche bildungspolitischen Leitlinien, Gesetzgebungen, Normen und Werte sowie Deutungsmuster werden Handlungsspielräume genutzt bzw. legitime Entscheidungen im Hinblick auf (Grund-)Bildung getroffen? Aus dem Steuerungs- und Regulationsdiskurs der Erwachsenen- und Weiterbildung erscheinen folgende Aspekte besonders relevant:

Gesetzgebung und Recht: In vierzehn der insgesamt sechzehn Bundesländern ist die Zuständigkeit der Länder für allgemeine Weiterbildung - und damit auch Grundbildung - über Erwachsenen- und Weiterbildungsgesetze festgehalten. Diese regeln das Verhältnis des Staates zu den Einrichtungen und Trägern sowie Fragen zur Programmatik, Organisation, Finanzierung und Qualität von Weiterbildung (vgl. Faulstich/Haberzeth 2007: 57). Insbesondere rechtliche Normierung und finanzielle Förderung können als zentrale Steuerungsinstrumente zur Durchsetzung von Inhalten und Zielen angesehen werden (vgl. Grotlüschen et al. 2010: 359). Allerdings unterscheiden sich die Bundesländer im Hinblick auf das Verständnis des politischen Bildungsauftrags und des politischen Gestaltungswillens (vgl. Frischkopf 2005: 34). 
Zudem zeige sich zunehmend eine Kompensation landespolitischer Mittel durch europäische Mittel oder Mittel des Bundes (Kuhlenkamp 2005: 27).

Soziale Institutionen: Die öffentliche Bildungsverwaltung ist zudem mit Blick auf neo-institutionalistische Ansätze als ein System institutioneller Regeln aufzufassen (vgl. Meyer/Rowan 1977), d.h. soziale und kognitive Strukturen und Praktiken spielen ebenfalls eine wichtige Rolle bei der Steuerung und Gestaltung von Weiterbildung. Diese konstituieren einen gemeinsam geteilten, vorstrukturierten ,legitimatorische[n] Kontext organisationalen (Steuerungs-)handelns“ (Koch 2011: 85), welcher die Möglichkeiten und Grenzen des legitimierungsbedürftigen Handelns steuernder und gesteuerter Organisationen formiert. Auf die Bedeutung weiterbildungspolitischer Orientierungen internationaler Akteure wie OECD, EU, UNESCO und Weltbank für das Handeln auf nationalen Politikebenen - insbesondere über finanzielle Mittel wie z.B. den Europäischen Sozialfonds (ESF) - und damit einhergehende Spannungsfelder weist zudem Michael Schemmann (2007: 233) hin.

Akteurskonstellationen und Netzwerke: Mit Blick auf Governance- und Netzwerkansätze ist die öffentliche Bildungsverwaltung außerdem als ein vernetzter Akteur zu verstehen, der sein Handeln mit einer Vielzahl von Akteuren abstimmt und auf diese Weise Gestaltungsspielräume und Begrenzungen erfährt (vgl. Kussau/Brüsemeister 2007; Faulstich 2010; Ioannidou 2011). Gerade die Zerstreuung der bildungspolitischen Akteure auf unterschiedliche Ressorts sowie das Zusammenwirken von Ministerien, Gesetzen, Programmen, Koordinierungsrunden und Einrichtungen zeige laut Wolfgang Seitter, dass Weiterbildung als ein „bedeutsames Querschnittsthema“ (Seitter 2011: 58) zu denken sei.

Dass es dabei im Feld der öffentlichen Verwaltung auch um die machtbasierte Durchsetzung von (partikularen) Interessen geht, zeigt Pierre Bourdieu in seinem Werk „Über den Staat“ (2014) eindrucksvoll auf: Allgemeine Interessen würden hier vorgeschoben, um Partikularinteressen unbemerkt durchzusetzen (Bourdieu 2014: 71).

\section{Methodisches Vorgehen: Leitfadengestützte Interviews}

Zur Analyse des Grundbildungsverständnisses in der öffentlichen Bildungspolitik wurde ein empirisch-qualitativer Zugang gewählt, der eine komplexe Annäherung an die Verwobenheit von Grundbildung, Interessen und Macht im Feld der öffentlichen Bildungsverwaltung ermöglicht. Dazu wurden von Dezember 2013 bis Juni 2014 zwölf telefonische Leitfadeninterviews mit Akteuren aus der öffentlichen Bildungsverwaltung der Länder geführt und durch sechs weitere Interviews mit Schlüsselakteuren der Nationalen Strategie kontrastiert. In einem möglichst offenen Gespräch wurden die Interview- 
ten nach ihrem Verständnis von Grundbildung sowie legitimierenden Begründungen befragt, um die dahinter liegenden Abgrenzungsinteressen zu erforschen. Die Auswahl der Befragten aus der öffentlichen Bildungsverwaltung erfolgte nach formalen Zuständigkeiten für Grundbildungspolitik, wird hier allerdings zur Sicherung von Anonymität und kritischer Diskussion nicht näher spezifiziert.

Die Auswertung der Interviews erfolgte in Anlehnung an die drei Kodierschritte (offenes, axiales, selektives Kodieren) der Grounded Theory (Strauss/Corbin 1996), wobei dem axialen Kodieren ein begründungslogisch gewendetes Kodierparadigma zugrunde gelegt wurde. Ausgehend vom Standpunkt des interviewten Subjekts lassen sich die deterministisch konnotierten Begriffe Kontext, ursächliche Bedingungen, intervenierende Bedingungen sowie Konsequenzen nur jeweils in ihrer Bedeutung für das Subjekt begründen und müssen folglich auch aus dieser Perspektive nachvollzogen werden (vgl. Euringer 2016: 128). Die von den Interviewten geäußerten Grundbildungsverständnisse und Begründungen wurden folglich mithilfe des Kodierparadigmas in einen begründungslogischen Zusammenhang gebracht, indem untersucht wurde, welche Interessen und Ziele zur Begründung der genannten Grundbildungsdimensionen herangezogen wurden und ob hier systematische Gemeinsamkeiten und Unterschiede erkennbar sind.

\section{Empirische Ergebnisse: Was ist Grundbildung?}

Die Frage, was eine „angemessene“ Grundbildung Erwachsener umfasst, wird aus Perspektive der Interviewten in der öffentlichen Bildungsverwaltung mit Blick auf vier verschiedene Dimensionen beantwortet, die mit Verweis auf bestimmte Ziele und Interessen jeweils unterschiedlich konkretisiert und legitimiert werden (siehe Abb. 1). Demnach umfasst Grundbildung aus Perspektive der Befragten bestimmte Inhalte und Kompetenzen (Dimension I), die im Hinblick auf als mindestens notwendig betrachtete Kompetenzstufen bzw. Mindestniveaus (Dimension II) differenziert werden. Zudem wird auf zielgruppenspezifische Kontexte (Dimension III) sowie spezifische Angebotsformate (Dimension IV) hingewiesen. Begründet werden diese Dimensionen und Aspekte von allen Befragten zunächst mit Verweis auf allgemeine Ziele, die insbesondere an Partizipation, Teilhabe und lebenslangem Lernen orientiert sind. Eher partikulare, bundeslandspezifische Interessen werden als Begründung herangezogen, wenn es um die Rechtfertigung solcher Aspekte geht, die von den Befragten gerade nicht als Teil von Grundbildung angesehen werden. Hier ist der Verweis auf die Eingrenzung offizieller Zuständigkeit und Verantwortlichkeit, die Bereitstellung zusätzlicher Fördermittel sowie die Erhöhung der Teilnehmendenzahlen dominant - stellt damit aller- 
dings den tatsächlichen Beitrag einer solchermaßen verstandenen Grundbildung für umfassende Teilhabe und Chancengleichheit aller infrage.

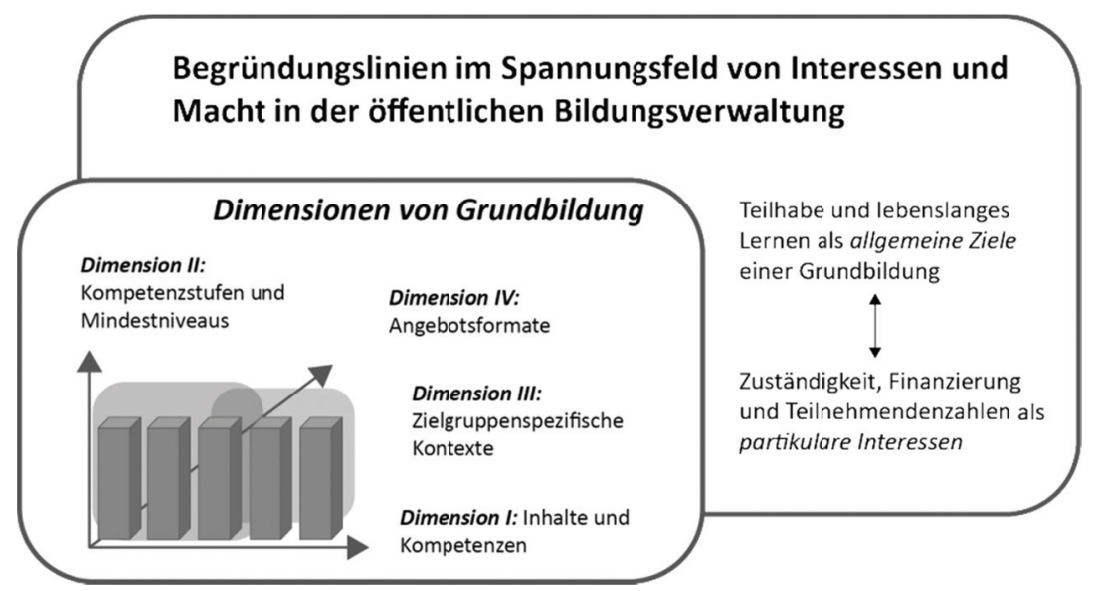

Abb. 1: Grundbildung aus Sicht der öffentlichen Bildungsverwaltung: Dimensionen und Begründungslinien (eigene Darstellung)

Besonders deutlich zeigt sich die hier angesprochene Ambivalenz bei Inhalten und Kompetenzen (Dimension I) sowie Angebotsformaten (Dimension IV) von Grundbildung, welche daher im Folgenden einer näheren Betrachtung unterzogen werden.

\subsection{Inhalte und Kompetenzen (Dimension I)}

Die erste Dimension umfasst Inhalte und Kompetenzen, die aus Perspektive der Befragten unter einer Grundbildung Erwachsener zu fassen sind. Dominant ist dabei die Ansicht, dass Lesen und Schreiben zwar den Kern einer Grundbildung Erwachsener ausmache, Grundbildung zugleich aber breiter zu denken sei, d.h. über Lesen und Schreiben hinausgehen müsse:

„Ich würde auch immer sagen, im Mittelpunkt der Diskussion steht sicherlich erst einmal Lesen und Schreiben als der Kern von Grundbildung. [...] Zu dem müssen dann noch weitere hinzukommen. Also es ist ja nicht nur das Lesen und Schreiben, sondern muss breiter angelegt sein, um die Zielsetzung des Gesetzes - nämlich sich beruflich weiterentwickeln zu können, sich persönlich weiterentwickeln zu können, aber auch gesellschaftlich sich einbringen zu können - auch erfüllen zu können“ (Interview 04, Z. 12). 
Neben dem Lesen und Schreiben werden weitere Inhalte und Kompetenzen als angemessen betrachtet, nämlich Rechnen und finanzielle Grundbildung, Umgang mit neuen Medien, Gesundheitsgrundbildung, politische Grundbildung, Selbst- und Sozialkompetenzen, Lernen lernen, Englisch sowie Deutsch als Fremd- bzw. Zweitsprache. Dabei zeigt sich allerdings in einigen der Fälle - trotz der proklamierten Ausweitung von Grundbildung - eine deutliche Fokussierung auf Alphabetisierung bzw. die Anwendung von Schriftsprache im Alltag. Begründet wird dies mit Verweis auf knappe öffentliche Gelder sowie die Notwendigkeit, angesichts öffentlichkeitswirksamer Literalitätsstudien (PIAAC, leo. - Level-One Studie) bekannte Begrifflichkeiten wie z.B. „funktionaler Analphabetismus“ aufzugreifen, denn die Politiker und Geldgeber seien schließlich darauf sensibilisiert und bereit, hierfür finanzielle Unterstützung aufzubringen. Das Interesse an einer Ausweitung der Finanzierung, auch durch den arbeitsmarktorientierten Europäischen Sozialfonds (ESF), sowie an öffentlich sichtbarer Wirksamkeit ist hier besonders deutlich, hat allerdings auch eine individualistische, anpassungsorientierte Verkürzung der Grundbildungskonzepte zur Folge, indem Inhalte und Kompetenzen ohne Rücksicht auf bestehende Fachdiskurse an wirtschaftliche, arbeitsmarktbezogene Anforderungen ausgerichtet werden. So wird das Lernen lernen behavioristisch auf Lerntechniken reduziert und politische Grundbildung umfasst neben dem Wissen zum politischen System und Wahlen nur selten bürgerschaftliche Ansätze zur Durchsetzung kollektiver Interessen mit dem Ziel umfassender, die Handlungsfähigkeit erweiternder Teilhabe - wie sie aber an anderer Stelle durchaus gefordert werden.

\subsection{Angebotsformate (Dimension IV)}

Diese Dimension umfasst spezifische Angebotsformate, die im Hinblick auf die Umsetzung einer Grundbildung Erwachsener als angemessen bzw. förderwürdig angesehen werden. Unterschieden werden hier in Anlehnung an den Trendbericht 2014 zum Weiterbildungsverhalten in Deutschland (BMBF 2015) drei Formate, nämlich non-formale, informelle und formale Angebotsformate. In den Interviews wird deutlich, dass Grundbildung aus Sicht der öffentlichen Bildungsverwaltung überwiegend mit klassischen, non-formalen Kursen - insbesondere der Volkshochschulen - in Verbindung gebracht wird, da diese im Rahmen der Weiterbildungsgesetze primär förderfähig sind. Um möglichst viele Teilnehmende erreichen zu können, werden zudem informelle Formate wie Beratungs- und Vernetzungsmaßnahmen auch außerhalb der primären Erwachsenen- und Weiterbildungseinrichtungen forciert. Formale Formate wie z.B. nachholende Schulabschlüsse, berufliche Nachqualifizierung und BAMF-Sprachkurse, die immer wieder von gesellschaftlichen Akteuren als Teil einer Grundbildung gefordert werden, werden je nach bun- 
deslandspezifischen Förderbedingungen unter einer Grundbildung subsumiert bzw. ausgeklammert. So wird zwar der nachträgliche Erwerb eines Hauptbzw. Realschulabschlusses mit Blick auf die Ermöglichung von Teilhabe häufig als grundlegend betrachtet. Das trifft vor allem dann zu, wenn nachholende Schulabschlüsse von der Verantwortlichkeit und Zuständigkeit her der allgemeinen Weiterbildung zugeordnet sind. Liegt die Zuständigkeit allerdings woanders, z.B. im Bereich der Schulgesetze, so werden nachholende Schulabschlüsse formal aus dem Grundbildungsverständnis ausgeklammert, um Kompetenzübertretungen und damit möglicherweise verbundene Schuldzuweisungen zu vermeiden:

„Die Schulabschlüsse sind von der Verantwortlichkeit hier im Kultusministerium angesiedelt, gehören aber nicht in den Bereich der Weiterbildung, sondern fallen unter das Schulgesetz. Und das ist eine ganz andere Ebene. [...] Inhaltlich hat das schon Berührungspunkte, [...] aber von der Organisation und Finanzierung ist es völlig getrennt“" (Interview 07, Zeile $50 \mathrm{ff}$.).

Ähnliches zeigt sich auch im Hinblick auf berufliche Nachqualifizierung und BAMF-Integrationskurse, deren Zuständigkeiten primär nicht auf Landesebene liegen, sondern bei dem Bundesministerium für Arbeit und Soziales (BMAS) und den Jobcentern bzw. dem Bundesamt für Migration und Flüchtlinge (BAMF).

\section{Fazit und Ausblick}

Es zeigt sich, dass sowohl innerhalb der öffentlichen Bildungsverwaltung als auch im Vergleich zu weiteren Akteuren differente Sichtweisen auf Grundbildung Erwachsener vorhanden sind, die auf unterschiedlichen Interessen und Ansprüchen basieren. Auf der Makroebene von Lernkulturen spielen dabei vor allem die gesetzlich fixierten Zuständigkeiten und finanziellen Ressourcen für die aufgezeigten Differenzen zwischen den Ländern eine bedeutsame Rolle und führen zu teils enggeführten und funktionalistischen Grundbildungskonzepten. Gerade mit Blick auf die immer wieder betonte Bedeutung von Grundbildung für Partizipation, Teilhabe und lebenslanges Lernen bleibt abschließend allerdings kritisch anzumerken, dass die teilweise Ausblendung nachholender Schulabschlüsse und beruflicher Nachqualifizierung dem Ziel der Teilhabe tendenziell entgegensteht, wenn nämlich die Lernenden ohne den Erwerb weiterqualifizierender Zertifikate und Abschlüsse im non-formalen System der Alphabetisierung/Grundbildung verbleiben. Die Gestaltung der Übergänge von der Alphabetisierung zur Grundbildung zur abschlussorientierten Weiterbildung scheint eine wichtige, noch zu klärende Aufgabe darzustellen. Zudem bleibt zu fragen, inwieweit die 
Interessen der Adressat/inn/en von der öffentlichen Bildungsverwaltung angemessen berücksichtigt werden oder vielmehr hinter administrativen Interessen verloren gehen.

\section{Literatur}

Abraham, Ellen/Linde, Andrea (2010): Alphabetisierung/Grundbildung als Aufgabengebiet der Erwachsenenbildung. In: Tippelt, R./Hippel, A. v. (Hrsg.): Handbuch Erwachsenenbildung/Weiterbildung. Wiesbaden: VS Verlag für Sozialwissenschaften, S. 889-903.

Barton, David/Hamilton, Mary (2000): Literacy practices. In: Barton, D./Hamilton, M./lvanic, R. (Hrsg.): Situated literacies. Reading and writing in context. London: Routledge, S. 7-15.

Bourdieu, P. (2014): Über den Staat. Vorlesungen am Collège de France 1989-1992. Berlin: Suhrkamp.

Bundesministerium für Bildung und Forschung (BMBF) (2015): Weiterbildungsverhalten in Deutschland 2014. AES 2014 Trendbericht. http://www.bmbf.de/ pubRD/BMBF_Trendbericht_AES2014_2015-03-16.pdf [Zugriff: 28.09.2015].

BMBF/KMK (2012): Vereinbarung über eine gemeinsame nationale Strategie für Alphabetisierung und Grundbildung Erwachsener in Deutschland 2012-2016. http://www.bmbf.de/pubRD/NEU_strategiepapier_nationale_alphabetisierung $\% 2$ 81\%29.pdf [Zugriff: 27.01.2014].

Döbert, Marion (1999): Grundbildung. In: Lexikon. Wissenswertes zur Erwachsenenbildung. http://socioweb.leuphana.de/lexikon/lex_geb/begriffe/grundbil.htm [Zugriff: 10.06.2014].

Euringer, Caroline (2016): Was ist „Grundbildung Erwachsener“? Das Grundbildungsverständnis der öffentlichen Bildungsverwaltung im Kontext von Interessen und Machtverhältnissen. Dissertation. Hamburg: Universität Hamburg/ Fakultät für Erziehungswissenschaft.

Faulstich, Peter (2010): „Was können wir gemeinsam machen?“ Kooperationen: Euphorie und Risiken in der Rückblende. In: DIE Zeitschrift für Erwachsenenbildung 1, S. 43-45.

Faulstich, Peter/Haberzeth, Erik (2007): Recht und Politik. Bielefeld: Bertelsmann.

Fleige, Marion (2011): Lernkulturen in der öffentlichen Erwachsenenbildung. Theorieentwickelnde und empirische Betrachtungen am Beispiel evangelischer Träger. Dissertation, Berlin, 2009. Münster: Waxmann.

Frischkopf, Arthur (2005): Gesetz auf dem Prüfstand. Evaluation und Wirksamkeit von Weiterbildungsgesetzen am Beispiel NRW. In: DIE Zeitschrift für Erwachsenenbildung III, S. 33-35.

Grotlüschen, Anke/Bonna, Franziska/Euringer, Caroline/Heinemann, Alisha M. B. (2014): Konsequenzen der Konstruktion von Literalität hinsichtlich der Vergleichbarkeit der Alpha-Levels mit den Niveaustufen des Europäischen Referenzrahmens Sprachen. In: Pätzold, H./Felden, H. v./Schmidt-Lauff, S. (Hrsg.): Programme, Themen und Inhalte in der Erwachsenenbildung. Dokumentation der Jahrestagung der Sektion Erwachsenenbildung der DGfE vom 19. bis 21. September 2013. Baltmannsweiler: Schneider-Verlag Hohengehren, S. 51-65. 
Grotlüschen, Anke/Haberzeth, Erik/Krug, Peter (2010): Rechtliche Grundlagen der Weiterbildung. In: Tippelt, R./Hippel, A. v. (Hrsg.): Handbuch Erwachsenenbildung/Weiterbildung. Wiesbaden: VS Verlag für Sozialwissenschaften, S. 347-366.

Grotlüschen, Anke/Heinemann, Alisha M. B./Nienkemper, Barbara (2009): Die unterschätzte Macht legitimer Literalität. In: Report - Zeitschrift für Weiterbildungsforschung 32, 4, S. 55-67. http://www.die-bonn.de/doks/report/2009-sprache01.pdf [Zugriff: 22.12.2015].

Grotlüschen, Anke/Riekmann, Wibke (Hrsg.) (2012): Funktionaler Analphabetismus in Deutschland. Ergebnisse der ersten leo. - Level-One Studie. Münster: Waxmann.

Grotlüschen, Anke/Riekmann, Wibke/Buddeberg, Klaus (2012): Hauptergebnisse der leo. - Level-One Studie. In: Grotlüschen, A./Riekmann, W. (Hrsg.): Funktionaler Analphabetismus in Deutschland. Ergebnisse der ersten leo. - Level-One Studie. Münster: Waxmann, S. 13-53.

Ioannidou, Alexandra (2011): Governance im transnationalen Bildungsraum: Von der nationalstaatlichen Steuerung zur transnationalen Governance. In: Schmid, J./Amos, K./Schrader, J./Thiel, A. (Hrsg.): Welten der Bildung. Vergleichende Analysen von Bildungspolitik und Bildungssystemen. Baden-Baden: Nomos, S. 267-286.

Koch, Sascha (2011): Steuerung aus neoinstitutionalistischer Perspektive. Die Legitimation des Zweiten Bildungsweges zwischen Weiterbildung und Schule. In: Hof, C./Ludwig, J./Schäffer, B. (Hrsg.): Steuerung, Regulation, Gestaltung. Governance-Prozesse in der Erwachsenenbildung zwischen Struktur und Handlung. Dokumentation der Jahrestagung der Sektion Erwachsenenbildung der DGfE vom 23. bis 25. September 2010. Baltmannsweiler: Schneider-Verlag Hohengehren, S. 81-92.

Kuhlenkamp, Detlef (2005): Ambivalente (De-)Regulierung. Weiterbildungsrecht der Länder und SGB III. In: DIE Zeitschrift 3, S. 27-29. http://www.diezeitschrift.de/ 32005/kuhlenkamp0501.pdf [Zugriff: 18.12.2013].

Kussau, Jürgen/Brüsemeister, Thomas (2007): Educational Governance. Zur Analyse der Handlungskoordination im Mehrebenensystem der Schule. In: Altrichter, H./Brüsemeister, T./Wissinger, J. (Hrsg.): Educational governance. Handlungskoordination und Steuerung im Bildungssystem. Wiesbaden: VS Verl., S. 16-54.

Linde, Andrea (2007): Alphabetisierung, Grundbildung oder Literalität? In: Grotlüschen, A./Linde, A. (Hrsg.): Literalität, Grundbildung oder Lesekompetenz? Beiträge zu einer Theorie-Praxis-Diskussion. Münster: Waxmann, S. 90-99.

Meyer, John W./Rowan, Brian (1977): Institutionalized Organisations: Formal Structure as Myth and Ceremony. In: American Sociological Review 83, 2, S. 340-363.

OECD (2013): OECD Skills Outlook 2013: First Results from the Survey of Adult Skills. OECD Publishing. http://skills.oecd.org/documents/OECD_Skills_ Outlook_2013.pdf [Zugriff: 11.10.2013].

Schemmann, Michael (2007): Internationale Weiterbildungspolitik und Globalisierung. Orientierungen und Aktivitäten von OECD, EU, UNESCO und Weltbank. Bielefeld: Bertelsmann.

Schüßler, Ingeborg/Thurnes, Christian M. (2005): Lernkulturen in der Weiterbildung. Bielefeld: Bertelsmann. 
Seitter, Wolfgang (2011): Ministerielle Steuerungsformen. Adressierungen, Gestaltungsimpulse und Steuerungsmodi hessischer Ministerien für die Weiterbildung in Hessen. In: Hof, C./Ludwig, J./Schäffer, B. (Hrsg.): Steuerung, Regulation, Gestaltung. Governance-Prozesse in der Erwachsenenbildung zwischen Struktur und Handlung. Dokumentation der Jahrestagung der Sektion Erwachsenenbildung der DGfE vom 23. bis 25. September 2010. Baltmannsweiler: SchneiderVerlag Hohengehren, S. 53-65.

Strauss, Anselm/Corbin, Juliet (1996): Grounded Theory. Grundlagen qualitativer Sozialforschung. Weinheim: Beltz, PsychologieVerlagsUnion.

Street, Brian (2003): What's ,new“ in the New Literacy Studies? Critical approaches to literacy in theory and practice. In: Current Issues in Comparative Education 5, 2, S. 77-91.

Tröster, Monika (2000): Grundbildung - Begriffe, Fakten, Orientierungen. In: Tröster, M. (Hrsg.): Spannungsfeld Grundbildung. Bielefeld: Bertelsmann, S. 12-27. 

Marion Fleige

\section{Zum Zusammenhang von Lernkulturen, Programmen und Organisationen in der Erwachsenenbildung/Weiterbildung. Begriffsbestimmungen und Befunde}

\section{Thema und Zielstellung des Beitrags}

Bislang wurden differente Verständnisse und Verwendungsweisen von „Lernkulturen“ in der Erwachsenenbildung/Weiterbildung (EB/WB) entwickelt (vgl. Schüßler in diesem Band; Schüßler/Thurnes 2005). Die Jahrestagung 2015 der Sektion Erwachsenenbildung der DGfE hob auf diese Vielfalt ab. Sie fragte aber auch nach ,Abhängigkeit- und Beziehungsstrukturen (von Lernkulturen, M. F.) in Regionen, von Trägern, Institutionen, Organisationen und Lehr-Lernarrangements“ und nach dem ,Zusammenspiel verschiedener Akteursgruppen, für Bildungsmanagement, Programmentwicklung, Gestaltung von Lehr-/Lernarrangements" (Ausschreibungstext). Der vorliegende Beitrag nimmt diese Fragen auf. Er markiert die Schnittstelle von Mikrodidaktik und Makrodidaktik, die im Diskurs bislang noch nicht sehr umfassend beleuchtet wurde. Auch die Institutionalformen der Organisationen - Einrichtungstypen/Lernorte wie Akademien beruflicher oder kultureller Bildung, Bildungsstätten, Volkshochschulen - sowie das Zusammenspiel von Lernkulturentwicklung mit Steuerung durch Bildungspolitik und Träger und mit den (regionalen) Bedarfs-/Bedürfnisstrukturen sowie mit Erwartungshaltungen, welche die Adressat/innen kanalisiert über Nachfrage an die Organisationen der EB/WB herantragen, scheinen für Lernkulturen in der Praxis eine Rolle zu spielen und werden hier aufgegriffen.

Dazu werden im Folgenden in den Abschnitten 2 und 3 Befunde aus Lernkulturanalysen und Begriffsbestimmungen aus unterschiedlichen Arbeiten zusammengeführt und kulturtheoretisch erweitert (Abschnitt 2.2), um einen mehrperspektivischen Lernkulturbegriff zu entfalten. In Abschnitt 4 wird schließlich die systematische Erarbeitung einer Träger- und Bereichssystematik von Lernkulturen vorgeschlagen.

\section{Entfaltung eines mehrperspektivischen Lernkulturbegriffs}

Wir gehen davon aus, dass sich in Lernkulturen die Bildungsrealitäten und die professionellen Handlungsrealitäten in Organisationen der EB/WB im 
Spiegel von pädagogischen Annahmen, von Normenstrukturen und Interessenlagen der Träger, von Erwartungsstrukturen aufseiten der Adressat/innen, Teilnehmer/innen, regionalen Umfelder und von bildungspolitischen Vorgaben - bzw. das Wechselspiel der genannten Faktoren - zeigen. Auf der pädagogischen Handlungsebene drückt sich dieses aus in einer spezifischen Aufnahme von Bedarfs- und Bedürfnisstrukturen über Programmentwicklung, über die Entwicklung von zum Programm passenden Institutionalformen, wie auch über eine entsprechende Ausgestaltung der Lehr-/Lernarrangements und der Lehr-/Lern-Situationen. Im Folgenden gehen wir besonders auf die Programmstrukturen und Institutionalformen als Aspekte von Lernkulturen ein, die im Diskurs bislang noch nicht sehr stark behandelt sind. ${ }^{1}$

\subsection{Programme und Institutionalformen als Aspekte von Lernkulturen}

(Regionale) Weiterbildungsmärkte weisen differente Strukturen von Trägern, Bildungsorganisationen und Programmen auf. Die Organisationen decken entweder mit verschiedenen Schwerpunktsetzungen die Breite der Themenbereiche ab (kommunal im Rahmen der Weiterbildungsgesetze der Länder geförderte Einrichtungen), oder sie fokussieren sich auf einen der Bereiche (vor allem freie Träger und privat-kommerzielle Anbieter) (vgl. z.B. Schrader 2011). Die EB/WB ist ein offenes Feld: ohne festes Curriculum oder Lehrplan, ohne Teilnahmepflicht, immer am Brennpunkt von Angebot und Nachfrage operierend (vgl. z.B. Gieseke 2008; Käpplinger 2007; von Hippel 2013).

Programme bündeln in diesem offenen Feld ein adressat/innenorientiertes Angebot gemäß einer ermittelbaren Nachfrage. Diese resultiert aus: (Kompetenz-)Bedarfen/Bedürfnissen/Interessen/(Nutzen-)Erwartungen, Lebensbedingungen in Lebens- und Arbeitswelten, regionalen Umfeldern und ausdifferenzierten Lebensläufen, zivilgesellschaftlichen Rahmungen und gesellschaftlichen Diskursen, Normen, Werten, Kulturen und Bildungsbegriffen der Träger, bildungspolitischen Rahmungen, Stützungen und Anforderungen des Feldes, inhaltlichen Ideen der Planenden und möglicher Dozent/innen, pädagogischem Handlungswissen, sich kontinuierlich erweiterndem fachspezifischem (wissenschaftlichem) Wissen, fachlichen Überzeugungen und Handlungsethiken sowie fachlichen Diskursen, beispielsweise in der kulturellen Bildung um Kunst und Kultur und ihren unterschiedlichen Domänen (vgl. Fleige/Gieseke/Robak 2015: 28, hier leicht modifiziert).

1 S. dazu auch, und z.T. in analogen Formulierungen, Handbuchbeitrag von Fleige/Robak (2016 i.E.). 
Lernkulturen bündeln die Formen der Planung und Realisierung des Angebots bzw. Programms und klammern dazu auch das darauf bezogene konzeptionelle Handeln und die in der Organisation dazu geführten Diskurse ein. Trotz aller Einflussnahmen von außen werden Entscheidungen über den Inhalt und damit auch über den Bildungsbegriff, welcher sich in Programmen und in der Ausgestaltung des Angebots in den Lernkulturen zeigt, nicht von irgendeiner Distanz durchgesteuert. Zwar werden die Vorgaben in das planerische Handeln integriert, doch die Entscheidungen über das Angebot und das Programm treffen die Planenden als hauptamtliches pädagogisches Personal. Lernkulturen bilden das Scharnier der konzeptionellen Planung und der Realisierung von Angeboten bzw. Programmen, während das Programm(heft) für sich genommen auch eine Kommunikation zu den Adressat/innen herstellt (vgl. Gieseke 2000: 12).

Zeitdiagnostisch ist dabei zu berücksichtigen, dass Programme und Strukturen der EB/WB - und damit auch die Lernkulturen - einem flexiblen Veränderungsmodus unterliegen. Programmanalysen in den 2000er Jahren ergaben eine Ausdehnung der Anbieter „beigeordneter Bildung“, deren Hauptaufgabe nicht Bildung ist (vgl. Gieseke u.a. 2005; Gieseke/Opelt 2005a, b). Dazu gehören etwa Kulturinstitutionen wie Museen und Theater, aber auch Betriebe und beispielsweise auch Kirchengemeinden. Inzwischen gibt es noch weitergehende Hypothesen zur Entwicklung der Programmstrukturen und der Institutionalformen der EB/WB. So ergab eine Sichtung der Bestände im Weiterbildungsprogramm-Archiv Berlin/Brandenburg an der Humboldt-Universität zu Berlin im Jahr 2009 Hinweise auf neue Ausdifferenzierungen: Neben einer Feststellung der Kontinuität etablierter Weiterbildungseinrichtungen und ihrer Programmstrukturen fiel eine weitere Zunahme der Angebote „,beigeordneter Bildung“ auf, ebenso wie ein Anwachsen der Angebote von Kammern, aber auch von privat-kommerziellen und vernetztintermediär operierenden Anbietern der beruflichen Bildung. Die Landschaft dieser Anbieter wirkt fluide und ,rhizomartig“, wie Gieseke im Anschluss an Arbeiten von Deleuze formulierte (vgl. Enoch/Gieseke 2011). Erkennbar wird ein neuer Grad der Marktförmigkeit der EB/WB. Anzunehmen ist eine Mischung aus und ein neuer Grad der Steuerung durch die Förderinstanzen, die Träger wie auch die Nachfrage (vgl. Gieseke 2011: $314 \mathrm{f}$.).

Die Anschlussthese dazu ist, dass durch die Gestaltung von Lernkulturen in den Organisationen der EB/WB diese Formen der Steuerung in Wechselwirkung mit der relativen Planungs- und Handlungsfreiheit der Pädagog/innen nachvollzogen werden, dass neue Schwerpunktsetzungen in den Programmen und die Ausbildung spezifischer, zum Programm passender Institutionalformen und Profile unterstützt werden, und dass die Bedürfnisse, Interessen und Erwartungen der Teilnehmenden in der Nachfrage- wie in der konkreten Lehr-Lernsituation gebündelt werden. Zugleich ist die Gestaltung von Lernkulturen als ein Scharnier der Realisierung von Programmen ein 
Instrument, um Spannungen zwischen dem Programm bzw. den darin aufgehobenen Bildungsvorstellungen und den Interessen von Trägern und Bildungspolitik auszugleichen (vgl. Gieseke 2008: 81, 85; Gieseke/Opelt 2005b).

\subsection{Definition von "Lernkulturen" und theoretische Erweiterungen}

Vor diesem Hintergrund wird im Folgenden ein Lernkulturbegriff vorgeschlagen, der in einem abduktiven Verfahren entwickelt wurde. Der Kontext waren Fallanalysen in Organisationen der öffentlich-kommunal geförderten EB/WB, hier im Bereich Evangelischer Träger (Evangelische Erwachsenenbildung, EEB). In die fünf Fallstudien flossen dabei Programmanalysen für die Jahrgänge 1997 und 2007, halb-strukturierte Interviews sowie im begrenzten Rahmen Dokumentenanalysen und teilnehmende Beobachtung ein (vgl. Fleige 2011).

Lernkulturen wurden in dieser Studie bestimmt als „Kulturen des Lehrens und Lernens, Planens, Disponierens und Partizipierens in Institutionen der Erwachsenenbildung im Modus institutionalformenspezifischer Praktiken, Deutungsmuster, Werte/Normen, Interaktionen/Rituale und Beziehungsstrukturen“" (ebd.: 15). Zur Charakterisierung der Handlungs- und Deutungsanteile von Pädagog/innen, Leitenden, Verantwortlichen bei Trägern und Verbänden sowie von Teilnehmenden wurde ferner formuliert, dass Lernkulturen ,professionell pädagogisch gestaltet und individuell rekonstruiert, ausgehandelt und gesteuert" werden und ,einen gesellschaftlich-kulturellen Kontext" haben (ebd.). Sie realisieren differente und gleichwohl überindividuell geteilte Konzepte der Bildung und des Lernens und differente Muster der Aneignung und des Lernens in Organisationen der EB/WB. Hinzu kommt eine Umsetzung von Bildungsbedarfen und -bedürfnissen der Adressat/innen, die diese aus Lebenslagen und sozialen Lagen sowie Deutungen heraus, etwa milieugebunden, einbringen. Ein Zusatz beschreibt den Einfluss von Steuerungsgrößen innerhalb von Lernkulturen speziell in der öffentlichen EB/WB: „Bei partikular-gemeinnützigen Institutionen der Erwachsenenbildung äußern sie sich in balancierten Lernkulturformen von Trägerkulturen, Zivilkulturen, Partizipationskulturen und Verbandskulturen.“ Dabei beziehen sich „Partizipationskulturen“ auf die Bedürfnisse und Interessen der Teilnehmenden. Beigefügt ist die theoretische Bestimmung, dass sich die vier genannten Aspekte in der öffentlich-kommunal geförderten EB/WB gemäß deren Prinzipien in einer Balance befinden müssen.

Der Lernkulturbegriff schließt eine systematische Auslegung des Kulturbegriffs ein. Dieses war bereits der Fall in frühen Arbeiten von beispielsweise Arnold/Schüßler (1998), die mit „Kultur“ die impliziten Muster der Lehr- 
und Lerngestaltung meinen. Im Sinne eines auf die verschiedenen didaktischen Handlungsebenen bezogenen Lernkulturbegriffs wurde „Kultur“ als in Organisationen der EB/WB und den von ihnen vorgehaltenen und realisierten Angeboten überindividuell geteilte Muster, Praktiken und Deutungen verstanden (siehe dazu auch: Gieseke/Robak/Wu 2009; Fleige 2011). Wir folgen hier grundlegend dem kultursoziologischen Ansatz von Reckwitz und seiner Theorie der Praktiken (vgl. Reckwitz 2006), als welche sich das Planen, Partizipieren, Lehren und Lernen interpretieren lassen. Praktiken sind von sozialen Gruppen ausgeübte Handlungen, die mit Deutungen und damit etwa auch mit Normen in Verbindung stehen. Anschließen lassen sich hier etwa auch Vorstellungen über Einflüsse von Lebenswelten sozialer Gruppen (etwa Milieus) in der gelebten Lernkultur. Diese werden in der heutigen Gesellschaft zunehmend transkulturell und hybrid ausgelebt und ausgestaltet (vgl. Welsch 2005; Robak 2013; Fleige/Gieseke/Robak 2015).

Dabei ist das Individuum Trägerin oder Träger von Kultur. Diese beeinflusst individuelle Überzeugungen und Auslegungen (Deutungen, Normen). Organisationen sind Transmissionsriemen von Kultur. Sie setzen kulturelle Vorstellungen in Angebote um und gestalten Lehr-Lernsituationen vor - welche dann durch die Lernkulturen erfahr- und erlebbar sowie erlernbar werden. Die Organisationen und ihre Institutionalformen selbst sind Ausdruck kultureller Prägungen von Bildung in einer Zeit. Insofern wird auch verständlich, warum erwachsenenpädagogische Organisationsanalysen, die die Wechselwirkungen von Organisation, Programm und Lehr-Lernarrangements - oder eben von Organisation, Programm und Lernkulturen - behandeln, neo-institutionalistische Ansätze nutzen: Diese fokussieren ebenfalls das Verhältnis von Struktur und Kultur (vgl. z.B. Gieseke/Opelt 2003, siehe auch Koch/ Schemmann 2009).

Hier schließt insgesamt auch das Lernkulturverständnis an, das im Sammelband von Gieseke/Robak/Wu (2009) herausgearbeitet wurde. Insbesondere die Beiträge von Gieseke (2009) und Robak (2009; vgl. auch Robak 2012) umreißen ein Verständnis von „Kultur“, nach dem sich diese in den Deutungen, Lebensweisen und Alltagshandlungen zeigt, zu denen auch die Praktiken des Lehrens und Lernens gehören. Diese sind durch Institutionalformen und Organisationskulturen/Unternehmenskulturen, aber auch durch Lebensweisen geprägt. Lernkulturen kristallisieren sich dabei über eine Vielfalt von Handlungsakten entlang von Lernangeboten heraus. Insbesondere in transkulturellen Zusammenhängen, etwa in den Lernkulturen in transnationalen Unternehmen, spielen dabei auch die kulturellen Prägungen von Individuen bzw. deren transkulturellen Ligaturen eine Rolle für die gelebte Lernkultur. 


\subsection{Beispielhafte Befunde}

In der erwähnten fallstudienbasierten Lernkulturanalyse in der öffentlich geförderten EEB (Fleige 2011), die hier beispielhaft herangezogen werden soll, ${ }^{2}$ wurde vor allem gefragt nach: den kulturellen Prägungen der Deutungen und Praktiken des Planens, Partizipierens, Lehrens und Lernens in protestantischen Milieus, aus denen (auch unter dem Gedanken der Öffentlichkeit) die Personen häufig kommen; den Profilen von Lernkulturen der Falleinrichtungen $^{3}$; sowie besonders nach dem Spanungsverhältnis von Trägerkulturen und Programm sowie Trägerkulturen und Partizipationskulturen.

In den Befunden zeigte sich, dass sich das „Evangelische“ in den LehrLernsituationen gerade in einer großen Offenheit für unterschiedliche Lebensformen und Bildungsbedürfnisse bei einem sowohl diskursiven als auch biographischen Anspruch in den mikrodidaktischen Zugängen realisiert eine Mischung, die den Grundlegungen in den protestantischen Normen und Menschenbildern entspricht. Allerdings zeigten sich aber auch leichte Einflüsse von religiös-konfessionellen Ritualen, wie etwa in einem Kurs das Aufstellen einer Kerze und das Sprechen eines Gebets zum Ende. Eine teilnehmende Beobachtung verdeutlichte, dass die wenigen Nicht-Kirchenmitglieder unter den Teilnehmenden zu diesen Ritualen keinen Zugang fanden und somit faktisch nicht partizipierten, obwohl sie seitens der Kursleitung dazu eingeladen waren. Weniger trifft solches zu bei dekorativ-gestalterischen, atmosphärisch wirksamen Elementen wie etwa Tüchern im Seminarraum, die säkularer wirken.

Hinsichtlich der Programmstrukturen zeigten die Falleinrichtungen Strukturmuster, die auch aus anderen Programmanalysen in der EEB (vgl. zusammenfassend Gieseke 2008) bekannt sind: Schwerpunkte wurden insbesondere im Bereich der kulturellen und politischen Bildung und im Bereich von Ethik, Philosophie und Religion/Theologie gesetzt. Diese thematischen Ligaturen sind für die EEB zwischen öffentlicher und kirchlicher Trägerschaft insgesamt typisch (vgl. Fleige/Gieseke/Robak 2015: $30 \mathrm{f}$.). Allerdings wiesen die Falleinrichtungen untereinander auch erhebliche Differenzierungen auf, die insbesondere mit der Größe bzw. Kleinheit gerade der Einrichtungen im ländlichen Raum zusammenhingen, aber offenkundig auch mit der Nachfrage und der Bedarfs- und Bedürfnisstruktur, die vor Ort bestand (,Partizipationskulturen“). Auch jüngere Programmanalysen bei diesem Trä-

2 Ebenso wäre als Beispiel für Lernkulturanalysen an der Schnittstelle von Programm, Organisation und Teilnahmeverhalten die Untersuchung von Robak (2012) zum Lernen von Expatriats in transnationalen Unternehmen zu nennen (siehe Fleige/Robak 2016 i.E.).

3 Ein kirchliches Werk, der Dachverband mit den Mitgliedseinrichtungen Evangelische Akademie und regionale Arbeitsstellen. 
ger weisen in diese Richtung (vgl. Robak/Fleige/Sterzig/Seifert/Teichmann/ Krueger 2015; siehe auch Robak/Petter 2014).

Entsprechend wurden auch in der Lernkulturstudie von 2011 für die Falleinrichtungen differente Lernkulturprofile wie „Frauenbildungskulturen in vernetzen Regional- und Wissensbezügen“ oder auch „Förderkulturen in ästhetischer und aktivierender Perspektive" erschlossen. Im letzteren Fall war der Träger der Einrichtung die Diakonie, was den Schwerpunkt auf dem fördernden Ansatz erklärt. Hinzu kam eine besondere Schwerpunktsetzung auch im Bereich der Fremdsprachen und der Gesundheitsbildung. Die Erklärung dieser für die EEB eher ungewöhnlichen Akzentuierung lag in den fachlichen Hintergründen der Planenden/Kursleitenden und einer entsprechenden Nachfrage vor Ort.

Deutlich zutage trat in der Gesamtuntersuchung das Spannungsverhältnis zwischen den Trägerkulturen und dem Programm. In den meisten Falleinrichtungen, insbesondere in einem kirchlichen Werk, wurde im Zehnjahresvergleich eine Zunahme von Angeboten zu Religion und Theologie wie auch von Lehrgängen zur Qualifizierung ehrenamtlichen kirchlichen Personals festgestellt und auf den tiefgreifenden Organisationswandel und die neuen Profilbildungsanstrengungen der Trägerorganisation Kirche in den späten 1990er und den 2000er Jahren zurückgeführt. Diese Profilierungsanstrengungen sind zum einen aus den Programmen und den Diskursen aus dieser Zeit rekonstruierbar, und sie sind zum anderen auch in kirchlichen Strategiepapieren fixiert. Eine solche Entwicklung konfligiert mit dem Gründungsauftrag der EEB als öffentlicher Erwachsenenbildung aus den 1960er Jahren (,Verbandskulturen"), denn sie führt zu einem anteiligen Rückgang von Angeboten in den anderen Themenbereichen und damit zu einer Verengung der Profile. Das Resultat ist eine Ausdehnung und damit Disbalance der „Trägerkulturen“. Dieses kam einer Durchsteuerung von innerorganisationalen Interessen des Trägers gleich, welche für einen Anbieter im Bereich der öffentlichen EB/WB problematisch ist. Dabei ist diese Entwicklung für den Untersuchungszeitraum auch auf den Rückgang der öffentlichen Finanzierung zurückzuführen.

\section{Anhaltspunkte aus dem Lernkulturdiskurs}

Auch der Lernkulturdiskurs, wie er in den 1990er und 2000er Jahren in der Disziplin der EB/WB im deutschsprachigen Raum geführt wurde, bezog unterschiedliche Handlungsebenen, Inhaltsbereiche und Organisationen mit ein. Allerdings beschrieben die meisten Beiträge nur eine der Handlungsebenen, meistens die Mikroebene. Auch die Bestimmungen des „Kultur“-Begriffs waren unterschiedlich stark ausgearbeitet. Zudem transportierten viele Beiträge Soll-Bestimmungen zur Implementierung und Ausgestaltung einer 
bestimmten „modernen“ Lernkultur. Sie waren damit vielfach eingebunden in modernisierungstheoretische Annahmen und bildungspolitische Diskurse. In lerntheoretischer Hinsicht wurde in der Zielperspektive eines selbstgesteuerten Lernens vor allem mit konstruktivistischen Annahmen operiert. Unter anderem Schüßler/Thurnes (2005) und Klingovsky (2009) haben diesen Diskurs in seinen grundlagentheoretischen Annahmen und Differenzierungen umfassend aufgearbeitet, wobei gerade letztere selbst kritische Zugänge, etwa aus der machttheoretischen Perspektive, einbringt.

Im Zuge der pädagogisch-theoretischen und praktischen Anstrengung für eine Erhöhung der Partizipation von Individuen an der Lernkulturgestaltung entstanden dabei auch empirische Beiträge - Analysen und Begleitforschung - deren Zusammenschau erste Anhaltspunkte für ein differenziertes Bild von Lehr-Lernarrangements und ihrer Gestaltung in unterschiedlichen Organisationen/Institutionalformen (z.B. VHS und Betriebe) sowie Träger- und Inhaltsbereichen ergab.

So wurde für die Mikroebene unter anderem deutlich, dass sich in den Betrieben die non-formalen Lernformen, auch in der Verbindung von Arbeiten und Lernen, ausdifferenzieren (vgl. Dehnbostel 2005). Auch die informellen Lernformen wurden in diesem Zusammenhang in vielfältigen Arbeiten beschrieben. In Unternehmen mit Standorten in verschiedenen Ländern differieren nach der Untersuchung von Egetenmeyer (2008) die Lernstile. Für die Volkshochschulen ist nach der Analyse von Gieseke/Käpplinger (2001) von einer veränderten Mikrodidaktik vor allem im Zusammenhang mit einer Zunahme der medialen selbstgesteuerten Lernformen auszugehen (vgl. auch Dust 2006). Diese Lernformen verändern sich vor allem auch im Bereich von Organisationen, die einen Schwerpunkt in der beruflichen Bildung haben (vgl. stellvertretend Arnold/Schüßler 1998; Klingovsky 2009). Insgesamt wurde über eine Entgrenzung des Lernens auch in nicht-organisationale Kontexte diskutiert (vgl. zusammenfassend Meisel/Heuer/Botzat 2001; siehe für eine neuere Studie auch Fahrenwald 2011).

Für die Mesoebene wurde vor allem das Lernen von Organisationen im Rahmen von Organisationentwicklungsprozessen als „Lernkulturentwicklung“ konturiert (vgl. Dollhausen 2006), bei welcher der Leitung eine bündelnde Funktion zukommt. Aber auch der Diskurs zu „Lernenden Regionen“ nahm Anklang an „Lernkulturen“. Für die Makroebene entstand ein Diskursstrang, bei dem vor allem der Wandel von Systemen und gesetzlichen Rahmenbedingungen von Weiterbildungslandschaften als "Lernkulturwandel“ beschrieben wurde (vgl. Niemeyer 2005; Solarczyk-Szwec 2007). 


\section{Fazit und Ausblick}

Die im Beitrag vorgenommene Zusammenführung von Studien und Diskursen sollte den Zusammenhang von Lernkulturen mit Programmen, Institutionalformen, bildungspolitischen Einflüssen sowie Interessenlagen der Teilnehmenden bzw. Adressat/innen, der Träger und der handelnden Pädagog/innen in Organisationen der EB/WB verdeutlichen. Lernkulturen erscheinen dabei als die Klammer für die Planung und Umsetzung von Angeboten/Programmen unter Einbezug der Praktiken und Deutungen auch der Kursleitenden und der Teilnehmenden. Auch Passungs- und Spannungsverhältnisse können dabei analysiert und für die einzelnen Träger- und Inhaltsbereiche der EB/WB nachvollzogen werden. Weitergehend könnten dabei international-vergleichende Studien hilfreich sein, um die Spannungsfelder zu konturieren. Auch eine Untersuchung zur Transkulturalität in Lernkulturen bei Trägern der EB/WB steht, gerade aufgrund der aktuellen gesellschaftlich-kulturellen Entwicklungen, an (vgl. Fleige/Robak 2016 i.E.). Die Frage ist hier, wie sich die beschriebenen Spannungsfelder in den Lernkulturen verändern, wenn neue Teilnehmendengruppen hinzukommen, und ob hierdurch neue Entwicklungen freigesetzt werden. Insgesamt ist danach $\mathrm{zu}$ fragen, wie die Einrichtungen durch die Gestaltung und Entwicklung von Lernkulturen auf regionale Lern- und Bildungsbedarfe einerseits und individuelle Bildungsbedürfnisse andererseits in Zukunft konzeptionell und inhaltlich reagieren und dabei Differenzierungen ausprägen. Eine noch systematischere Analyse und Differenzierung für unterschiedliche Träger, Organisationen und Institutionalformen und daran anschließende Detailstudien scheinen erforderlich, um zu diesen Fragen differenziert Auskunft zu geben. Eine über Forschung generierte analytische Kompetenz wie auch ein instrumentelles Handlungswissen scheinen hier angesichts der Gestaltungsherausforderungen von hoher Bedeutung zu sein.

\section{Literatur}

Arnold, Rolf/Schüßler, Ingeborg (1998): Wandel der Lernkulturen. Ideen und Bausteine für ein lebendiges Lernen. Darmstadt: Wiss. Buchgesellschaft.

Dehnbostel, Peter (2005): Lernen - Arbeiten - Kompetenzentwicklung. Zur wachsenden Bedeutung des Lernens und der reflexiven Handlungsfähigkeit im Prozess der Arbeit. In: Wiesner, G./Wolter, A. (Hrsg.): Die lernende Gesellschaft. Lernkulturen und Kompetenzentwicklung in der Wissensgesellschaft. Weinheim u. München: Juventa Verlag, S. 111-126.

Dollhausen, Karin (2006): Neue Lernformen - neue Lernkulturen - organisationales Lernen in Bildungseinrichtungen. http://www.die-bonn.de/esprit/dokumente/doc2006/dollhausen06_01.pdf [Zugriff: 06.01.2016].

Dust, Martin (2006): ,Neue Lernkulturen' und Volkshochschule - Doch was ist letztlich neu? In: Gamm, H.-J. u.a. (Hrsg.): Jahrbuch für Pädagogik 2006: Infantilisie- 
rung des Lernens? Neue Lernkulturen - ein Streitfall. Frankfurt a.M.: Lang, S. 257-267.

Egetenmeyer, Regina (2008): Informal Learning in betrieblichen Lernkulturen. Eine interkulturelle Vergleichsstudie. Baltmannsweiler: Schneider Verlag Hohengehren.

Enoch, Clinton/Gieseke, Wiltrud (2011): Wissensstrukturen und Programmforschung. Programmforschung als empirischer Zugang zur Bildungsarbeit der Weiterbildungsinstitutionen. http://www.ifbe.uni-hannover.de/fileadmin/ifbe/enoch/ enoch_gieseke14_Juli2011.pdf [Zugriff: 09.10.2015].

Fahrenwald, Claudia (2011): Erzählen im Kontext neuer Lernkulturen. Eine bildungstheoretische Analyse im Spannungsfeld von Bildung, Lernen und Subjekt. Wiesbaden: VS Verlag.

Fleige, Marion (2011): Lernkulturen in der öffentlichen Erwachsenenbildung. Theorieentwickelnde und empirische Betrachtungen am Beispiel evangelischer Träger. Münster u.a.: Waxmann.

Fleige, Marion/Gieseke, Wiltrud/Robak, Steffi (2015): Kulturelle Erwachsenenbildung. Bielefeld: W. Bertelsmann.

Fleige, Marion/Robak, Steffi (2016 i.E.): Lehr-Lernkulturen in der Erwachsenenbildung. Erscheint in: Tippelt, R./Hippel, A. von (2016): Handbuch Erwachsenenbildung/Weiterbildung. 6. Aufl. Wiesbaden: Springer VS.

Gieseke, Wiltrud (2000): Perspektivverschränkung als Ansatz für die Analyse von Programmplanungshandeln. In: Dies. (Hrsg.): Programmplanung als Bildungsmanagement? Qualitative Studie in Perspektivverschränkung. Recklinghausen: Bitter, S. 30-58.

Gieseke, Wiltrud/Gorecki, Claudia (2000): Programmplanung als Angleichungshandeln - Arbeitsplatzanalyse. In: Gieseke, W. (Hrsg.): Programmplanung als Bildungsmanagement? Qualitative Studie in Perspektivverschränkung. Recklinghausen: Bitter, S. 59-114.

Gieseke, Wiltrud/Käpplinger, Bernd (2001): Lehren braucht Support. ExpertInnenInterviews zu neuen Lehr- und Lernkulturen in der Weiterbildung. In: Heuer, U. (Hrsg.) (2001): Neue Lehr- und Lernkulturen in der Weiterbildung. Bielefeld: W. Bertelsmann, S. 233-270.

Gieseke, Wiltrud/Opelt, Karin (2003): Erwachsenenbildung in politischen Umbrüchen. Das Programm der Volkshochschule Dresden 1945-1997 (unter Mitarbeit von Ulrike Heuer). Opladen: Leske + Budrich.

Gieseke, Wiltrud/Opelt, Karin/Stock, Helga/Börjesson, Inga (2005): Kulturelle Erwachsenenbildung in Deutschland. Exemplarische Analyse Berlin/Brandenburg (Europäisierung durch Kulturelle Bildung. Bildung - Praxis - Event. Bd. 1. Hrsg. von W. Gieseke und J. Kargul). Münster u.a.: Waxmann.

Gieseke, Wiltrud/Opelt, Karin (2005a): Programmanalyse zur kulturellen Bildung in Berlin/Brandenburg. In: Gieseke, W./Opelt, K./Stock, H./Börjesson, I. (Hrsg.): Kulturelle Erwachsenenbildung in Deutschland - Exemplarische Analyse Berlin/Brandenburg. (Europäisierung durch kulturelle Bildung. Bildung - Praxis - Event. Bd. 1). Münster u.a.: Waxmann, S. 43-108.

Gieseke, Wiltrud/ Opelt, Karin (2005b): Zusammenfassung: Bildungstheoretische Ansätze für kulturelle Bildung - Aspekt Programm. In: Gieseke, W./Opelt, K./Stock, H./Börjesson, I. (Hrsg.): Kulturelle Erwachsenenbildung in Deutsch- 
land. Exemplarische Analyse Berlin/Brandenburg. Münster u.a.: Waxmann, S. 317-332.

Gieseke, Wiltrud (2008): Bedarfsorientierte Angebotsplanung in der Erwachsenenbildung. Bielefeld: W. Bertelsmann.

Gieseke, Wiltrud (2009): Organisationstheoretische Überlegungen zur Lernkultur. Der übersehene institutionelle/organisatorische Faktor im Lernkulturdiskurs. In: Dies./Robak, S./Wu, M.-L. (Hrsg.): Transkulturelle Perspektiven auf Kulturen des Lernens. Bielefeld: Transcript, S. 49-86.

Gieseke, Wiltrud/Robak, Steffi/Wu, Ming-Lieh (Hrsg.) (2009): Transkulturelle Perspektiven auf Kulturen des Lernens. Bielefeld: Transcript.

Gieseke, Wiltrud (2011): Programme und Programmforschung als spezifisches Steuerungswissen für Weiterbildungsorganisationen? In: HBV 4, S. 314-322.

Hippel, Aiga von (2013): Programmplanung als professionelles Handeln - „Angleichungshandeln“ und „Aneignungsmodi“ im aktuellen Diskurs der Programmund Professionsforschung. In: Käpplinger, B./Robak, S./Schmidt-Lauff, S. (Hrsg.): Engagement für die Erwachsenenbildung - Ethische Bezugnahmen und demokratische Verantwortung. Festschrift für Wiltrud Gieseke. Wiesbaden: Springer VS, S. 131-143.

Käpplinger, Bernd (2007): Abschlüsse und Zertifikate in der Weiterbildung. Bielefeld: W. Bertelsmann.

Klingovsky, Ulla (2009): Schöne Neue Lernkultur? Transformationen der Macht in der Weiterbildung. Bielefeld: Transcript.

Koch, Sascha/Schemmann, Michael (Hrsg.) (2009): Neo-Institutionalismus in der Erziehungswissenschaft. Grundlegende Texte und empirische Studien. Wiesbaden: VS Verlag.

Niemeyer, Beatrix (Hrsg.) (2005): Neue Lernkulturen in Europa? Prozesse, Positionen, Perspektiven. Wiesbaden: VS Verlag.

Reckwitz, Andreas (2006): Das hybride Subjekt. Eine Theorie der Subjektkulturen von der bürgerlichen Moderne zur Postmoderne. Weilerswist: Velbrück Wissenschaft.

Robak, Steffi (2009): Kulturelle Aspekte von Lernkulturen in transnationalen Unternehmen unter Globalisierungsbedingungen. In: Gieseke, W./Robak, S./Wu, M.-L. (Hrsg.) (2009): Transkulturelle Perspektiven auf Kulturen des Lernens. Bielefeld: Transcript, S. 119-150.

Robak, Steffi (2012): Kulturelle Formationen des Lernens. Zum Lernen deutscher Expatriates in kulturdifferenten Arbeitskontexten in China - die versäumte Weiterbildung. Münster/New York: Waxmann.

Robak, Steffi (2013): Interkultur - Transkultur - Hybridkultur. Spannungsfelder und (weiter)bildungsrelevante Implikationen. In: HBV 1, S. 14-28.

Robak, Steffi/Petter, Isabell (2014): Programmanalyse zur interkulturellen Bildung in Niedersachsen. http://www.die-bonn.de/doks/2014-interkulturelle-bildung-01.pdf [Zugriff: 27.07.2015].

Robak, Steffi/Fleige, Marion/Sterzik, Linda/Seifert, Jennifer/Teichmann, Anne-Kristin/ Krueger, Anneke (2015): Die Konstitution Kultureller Bildungsräume. Fünf überregionale Institutionen der non-formalen Kulturellen (Erwachsenen-)Bildung: Bildungsangebotsentwicklungen, innovative Impulse, Planungsspielräume. Recherche im Auftrag des Rates für Kulturelle Bildung. http://www.ratkulturelle-bildung.de/index.php?id=59 [Zugriff: 06.01.2016]. 


\section{Marion Fleige}

Schrader, Josef (2011): Struktur und Wandel der Weiterbildung. Bielefeld: W. Bertelsmann.

Schüßler, Ingeborg/Thurnes, Christian M. (2005): Lernkulturen in der Weiterbildung. Studientexte für Erwachsenenbildung. Bielefeld: W. Bertelsmann.

Solarczyk-Szwec, Hanna (2007): Lernkulturen in der polnischen Erwachsenenbildung: Alte, neue und moderne. In: Heuer, U./Siebers, R. (Hrsg.): Weiterbildung am Beginn des 21. Jahrhunderts. Festschrift für Wiltrud Gieseke. Münster u.a.: Waxmann, S. 284-292.

Welsch, Wolfgang (2005): Auf dem Weg zu transkulturellen Gesellschaften. In: Allolio-Näcke, L./Kalscheurer, B./Manzeschke, A. (Hrsg.): Differenzen anders denken. Bausteine zu einer Kulturtheorie der Transdifferenz. Frankfurt a.M.: Campus Verlag, S. 314-341. 


\section{Autorinnen und Autoren}

Asche, Eike, M.A., Leibniz Universität Hannover, Institut für Berufspädagogik und Erwachsenenbildung. Arbeitsschwerpunkte: Bedarfsforschung und Bedarfsanalyse, Qualifizierung für die digitale Arbeitswelt, Digitales Lernen, Diversität.

Becker, Sara, M.A., FernUniversität in Hagen, Interdisziplinäres Fernstudium Umweltwissenschaften (infernum), Arbeitsschwerpunkte: Blended-Learning, E-Learning, Virtuelle Mobilität, (Virtuelle) Studierendenberatung, Studiengangskoordination.

Bernhard, Christian, Dipl. Päd., Nationale Agentur Bildung für Europa beim Bundesinstitut für Berufsbildung. Arbeitsschwerpunkte: Erwachsenenbildung und Sozialraum, erwachsenenpädagogische Organisationstheorie, Subjektorientierung, qualitative Forschung, Erwachsenenbildung in europäischen Zusammenhängen.

Biel, Carmen, Dipl.-Päd., wissenschaftliche Mitarbeiterin, Deutsches Institut für Erwachsenenbildung, Leibniz-Zentrum für Lebenslanges Lernen e.V. Arbeits- und Forschungsschwerpunkte: Lehren und Lernen mit digitalen Medien, formative Beurteilung, Learning Analytics.

Buddeberg, Klaus, M.A., Universität Hamburg, Fakultät für Erziehungswissenschaft, Arbeitsbereich Lebenslanges Lernen. Arbeitsschwerpunkte: Literalitäts- und Grundbildungsforschung, Large Scale Assessment, Inklusion in der Erwachsenenbildung, Leichte Sprache.

Damm, Christoph, M. A., wissenschaftlicher Mitarbeiter, Otto-von-GuerickeUniversität Magdeburg. Arbeitsschwerpunkte: Anerkennungs- und Anrechnungspraxen in der wissenschaftlichen Weiterbildung, Kooperationen und Netzwerke in der Erwachsenenbildung, Dokumentarische Evaluationsforschung, Qualitative und quantitative Erwachsenenbildungsforschung.

Dörner, Olaf, Juniorprofessor für Erziehungswissenschaft, Schwerpunkt Erwachsenen-/Weiterbildung, Otto-von-Guericke-Universität Magdeburg. Arbeitsschwerpunkte: Wissenschaftliche Weiterbildung, Bildung Erwachsener, (Nicht-)Beteiligung an Eb/Wb, Umgang mit Wissen, Methoden und Methodologien qualitativer Erwachsenenbildungsforschung.

Dust, Martin, Dr., Agentur für Erwachsenen- und Weiterbildung. Arbeitsschwerpunkte: Erwachsenenbildung, Organisationspädagogik, Historische Bildungsforschung. 
Euringer, Caroline, Dr. des., Universität Hamburg. Arbeitsschwerpunkte: Alphabetisierung und Grundbildung, Weiterbildungspolitik, Kompetenzdiskurs, Subjektwissenschaft, Grounded Theory.

Fleige, Marion, Dr., Deutsches Institut für Erwachsenenbildung. Arbeitsschwerpunkte: Programm-, Beteiligungs- und Lernkulturforschung in verschiedenen Institutionen und Inhaltsbereichen der EB/WB.

Franz, Julia, Prof. Dr., Eberhard Karls Universität Tübingen. Arbeitsschwerpunkte: Lehrkulturen der Erwachsenenbildung, Intergenerationelles Lernen, Didaktik, Organisationale Aspekte der Erwachsenenbildung.

Freieck, Lisa, M.A., wissenschaftliche Mitarbeiterin am Institut für Allgemeine Pädagogik und Berufspädagogik, TU Darmstadt. Arbeitsschwerpunkte: Rassismuskritische und migrationsgesellschaftliche Pädagogik, Diskriminierungskritische Menschenrechtsbildung und Social Justice, Kulturelle Bildung im Kontext postkolonialer und postnationalsozialistischer Erinnerungsarbeit, Kritische Bildungs- und Subjekttheorie.

Gillen, Julia, Prof. Dr., Professur für Berufspädagogik: Organisations- und Qualitätsentwicklung in der beruflichen Bildung, Institut für Berufspädagogik und Erwachsenenbildung (IfBE) Leibniz Universität Hannover. Arbeitsschwerpunkte: Kompetenzentwicklung und Kompetenzerfassung in schulischen und betrieblichen Kontexten, Kompetenzorientierte Didaktik in der beruflichen Bildung, Qualitäts- und Organisationsentwicklung in der beruflichen Bildung.

Hanak, Helmar, M.A., Servicestelle Offene Hochschule Niedersachsehen gGmbH. Arbeitsschwerpunkte: Anerkennung und Anrechnung, Hochschulzugang, Beratung.

Herzog, Marius, Dr., Universität Hamburg, Fakultät für Erziehungswissenschaft. Arbeitsschwerpunkte: Lehrerbildung, Bildungsforschung, Organisationssoziologie, Hochschulforschung.

Kasatschenko, Tatjana, M.A., wissenschaftliche Mitarbeiterin am Institut für Allgemeine Pädagogik und Berufspädagogik, TU Darmstadt. Arbeitsschwerpunkte: Rassismuskritische Bildung und Migration, Postkoloniale Theorie, Gender Studies.

Lobe, Claudia, Dr., Universität Bielefeld, Erwachsenen- und Weiterbildung. Arbeitsschwerpunkte: Wissenschaftliche Weiterbildung, Teilnehmerforschung, Transitionen, Hochschule.

Lorenz, Lisa Marie, M.A., Leibniz Universität Hannover, Institut für Berufspädagogik und Erwachsenenbildung. Arbeitsschwerpunkte: Programmpla- 
nung, Berufliche Weiterbildung, Kooperation und Vernetzung, Internationalisierung der Erwachsenenbildung, Digitalisierung.

Mania, Ewelina, Dipl.-Päd., wissenschaftliche Mitarbeiterin am Deutschen Institut für Erwachsenenbildung - Leibniz-Zentrum für Lebenslanges Lernen (DIE) in Bonn im Programm „Programme und Beteiligung“. Arbeitsschwerpunkte: (Finanzielle) Grundbildung und Literalität, Weiterbildungsbeteiligung, Sozialraum in der Erwachsenenbildung.

Marquardt, Henning, Dr., Agentur für Erwachsenen- und Weiterbildung. Arbeitsschwerpunkte: Erwachsenenbildung, europäische Projekte, Migration, Integration.

Otto, Daniel, Dr., FernUniversität in Hagen. Arbeitsschwerpunkte: Internationale Beziehungen, Klimapolitik, Nachhaltige Entwicklung, E-Learning, virtuelle Mobilität.

Reinhardt, Max, Dr. phil., freiberuflicher Coach und Berater, zuvor wiss. Mitarbeiter Hochschule Kaiserslautern, Arbeitsschwerpunkte: Kompetenzforschung, Biografieforschung, Habitusforschung, soziale Milieus und gesellschaftspolitischer Lager.

Rhein, Rüdiger, Dr. phil., Institut für Erziehungswissenschaft/Leibniz Universität Hannover. Arbeitsschwerpunkte: Hochschulisches Lehren und Lernen, Theorie der Hochschuldidaktik, Bildungstheorie.

Riekmann, Wibke, Dr., Hochschule Fresenius, Fachbereich Gesundheit und Soziales. Arbeitsschwerpunkte: Jugend- und Jugendverbandsarbeit, Literalitätsforschung, Politische Bildung, Freiwilliges Engagement und Ehrenamt.

Robak, Steffi, Prof. Dr., Leibniz Universität Hannover, Institut für Berufspädagogik und Erwachsenenbildung. Arbeitsschwerpunkte: Transnationale Personalentwicklung, Kulturelle Bildung, Diversität und Inter-/Transkulturelle Bildung, Programmforschung, Bildungsmanagement.

Rohs, Mathias, Jun.-Prof. Dr., Technische Universität Kaiserslautern. Arbeits- und Forschungsschwerpunkte: Informelles Lernen, Lernen mit digitalen Medien, Professionalisierung, betriebliche Weiterbildung.

Rothe, Daniela, Univ.-Prof. Dr., Alpen-Adria-Universität Klagenfurt. Arbeitsschwerpunkte: Lernen in der Lebensspanne, Methoden der qualitativen Sozialforschung (Diskursanalyse, Biographieforschung, Ethnographie), rekonstruktive Praxisforschung.

Schlüter, Anne, Prof. für Erwachsenenbildung/Bildungsberatung an der Universität Duisburg-Essen. Arbeitsschwerpunkte: Weiterbildung und Biographie, Bildungsberatung, Bildungsmobilität, Mentoring. 
Schöb, Sabine, Dipl.-Päd., wissenschaftliche Mitarbeiterin, Abteilung Erwachsenenbildung/Weiterbildung der Universität Tübingen. Arbeits- und Forschungsschwerpunkte: Videofallarbeit, Professionalisierung, Empirische Lehr-Lernforschung (mit neuen Medien), Curriculumentwicklung, Wissensmanagement.

Scholze, Tim, Dr., Wissenschaftlicher Mitarbeiter, Deutsches Institut für Erwachsenenbildung, Leibniz-Zentrum für Lebenslanges Lernen e.V. Arbeits- und Forschungsschwerpunkte: Lehren und Lernen mit digitalen Medien, Informelles Lernen, Kompetenzvalidierung.

Schüßler, Ingeborg, Prof. Dr., PH Ludwigsburg, Institut für Erziehungswissenschaft, Abteilung Erwachsenenbildung und Berufliche Bildung. Arbeitsschwerpunkte: Professions- und Organisationsforschung, Lernkultur- und Qualitätsentwicklung, Didaktik und Methodik in der Erwachsenenbildung, Lehr-Lernforschung, Nachhaltigkeit in der Weiterbildung.

Stimm, Maria, M.A., Humboldt-Universität zu Berlin. Arbeitsschwerpunkte: Lehr-Lernkulturforschung, AdressatInnen-, Teilnehmenden- und Zielgruppenforschung, Beratungsforschung.

Sturm, Nico, M.A., Philipps-Universität Marburg, Fachbereich Erziehungswissenschaften. Arbeitsschwerpunkte: Anerkennung und Anrechnung, Durchlässigkeit, Beratung, Netzwerkarbeit.

Tröster, Monika, wissenschaftliche Mitarbeiterin am Deutschen Institut für Erwachsenenbildung - Leibniz-Zentrum für Lebenslanges Lernen (DIE) in Bonn im Programm „Programme und Beteiligung". Arbeitsschwerpunkte: Alphabetisierung, (Finanzielle) Grundbildung und Literalität.

von Hippel, Aiga, Prof. Dr., Institut für Erziehungswissenschaften, Humboldt- Universität zu Berlin, Arbeitsschwerpunkte: Programmforschung, Adressatenforschung, Professionsforschung, Absolventenverbleib, internationale Bildungszusammenarbeit 